www.anatomy.org.tr

doi:10.2399/ana.15.075s

\title{
Abstracts for the XXIV International Symposium on Morphological Sciences 2nd-6th September, 2015, Istanbul, Turkey
}

\section{Invited Lectures}

$$
(\mathrm{I}-0-\mathrm{I}-53)
$$

\section{I-1}

\section{New perspectives in neurosciences}

\section{Yaşargil G}

Department of Neurosurgery, Yeditepe University, Faculty of Medicine, Istanbul, Turkey

Intensive research activities within the past 200 years have revealed that the central nervous system (CNS) is a heterogeneous, heteromorphic, multidimensional and multifunctional compound organ system. Scientists working in Comparative Anatomy and Embryology determined that the transformative adaptive evolution of the CNS occurred over an extensive time period of 500 million years and continues to unfold and progress. The phylogenic, ontogenic, histogenic, angiogenic, endocrinogenic and immunogenic defined compartments of the CNS present a unique macro- and microarchitecture along the spinal cord, brain stem, diencephalon and telencephalon with archipallial, paleopallial and neopallial intertwining architecture. The continuing upswing in scientific technology is providing medicine and surgery with tools of immense value; the availability of achromatic microscope, free from spherical aberration, invented by J.J. LISTER, 1830, in the United Kingdom, later the formulation of the equation "Angular Aperture" by Ernst ABBE, in 1880, Zeiss Company, Jena, Germany, followed by the Electron Microscope in 1933, Scanning Tunneling Microscope in 1981 and Positron Microscope in 1987. Scientists in biology and neurobiology have thus been compelled and stimulated to initiate profound studies on morphology and function of all organs and their cells. Scientists in molecular biology and genetics were ultimately able to reveal a fascinating inter- and intracellular microarchitecture such as receptors, microtubules, mitochon- dria and synapses. Scientists in physics and chemistry finally succeeded to bypass Abbes optical limitation of 0.2 micrometers and the nanoscope was born. This latter achievement opens a new era in biology and pathology, allowing the study in-vivo of ultrastructures. The visualization of living ultramicro-structures is a great accomplishment, a culmination of centuries of scientific endeavors. In contrast, the phylogenic aspects of vascular, neoplastic, degenerative, toxic and viral diseases of the CNS had hitherto found only marginal attention. The clinical, neuroradiological and neuropathological observations on a large number of patients with neurovascular and neoplastic lesions, degenerative diseases and viral infections alerted us to specific predilection sites of these diseases, which affect certain compartments of the CNS, in relation to their phylogenic, ontogenic, histogenetic and angiogenic order. The adjacent compartments remain unaffected. The conduct of these lesions teaches us new aspects in neuropathology. These observations will be presented and discussed.

\section{I-2}

Ultrastructural neuropathology in transgenic models of Alzheimer's disease

Erisir A

MD, PhD, Professor of Psychology, University of Virginia

Transgenic animal models that replicate the symptoms, the lesions or the possible causes of human disease are prolific tools, aiding attempts to understand the cause, and to alleviate the destruction of Alzheimer's Disease. Numerous mouse transgenic lines have now succeeded in partially reproducing its lesions: the diffuse extracellular deposits of $\mathrm{A} \beta$ peptide, the neuritic plaques, 
and the intracellular accumulation of tau protein (or neurofibrillary tangles). Because these models also develop the clinical signs of the human disorder, they allow for testing of the effectiveness of any therapeutic candidates in alleviating the behavioral deficits in correlation with subdued progression of neuropathologic signs. As neuropathologic changes observed in transgenic models provide a confirmation of the validity of genetic and molecular precursors of impending behavioral decline, electron microscopy provides a powerful alternative approach for routine methods of staging the brain pathology. In this talk, I will summarize the advantages and limitations of several current transgenic rodent models. I will also outline a transgenic staging protocol modeled after the AA guidelines for the neuropathologic assessment of Alzheimer's disease in humans. Because the use of electron microscopy allows direct discrimination of neuritic plaques from amyloid precursor protein and $\mathrm{A} \beta$ aggregates, and reveals early subcellular pathology that may elude light microscopic approaches, it provides a sensitive quantitative assay for correlation of ultrastructural pathology and the behavioral deficits in transgenic models of the Alzheimer's Disease.

\section{I-3}

\section{Property issues of deceased}

Brenner E

Division of Clinical and Functional Anatomy, Medical University of Innsbruck, Austria

Mors auxilium vitae - death helps the life. Therefore, anatomy requires deceased. Cutting into deceased is commonly seen as violation. These short sentences describe the cornerstones of the area of conflict, when anatomists use the bodies of deceased. In order to dissect legally and ethically firm, we have to clarify, who owns that cadaver. Having defined this issue, we can proceed to the question, when and why anatomists are allowed to perform a dissection and avoid serious allegations of violation. The question of ownership has two possible, but contradictory answers. The first answer is based on the assumption that the cadaver is a mere thing, an item. With this, a cadaver may be inherited or even traded like any other good. It is obvious that this resolution to our problem is false. Almost all, if not really all societies deny that a cadaver - or parts of it - are a tradable good and consequently interdict trading of cadavers or parts of it. The second answer is based on the assumption that the cadaver is - still - part of the immaterial personal rights of the once living person. This means that the person itself - and nobody else - has the right to decide on the cadaver's future, of course within the framework of legal and ethical rules. This person might decide that his or her cadaver has to be buried at a certain cemetery or has to be handed over to the anatomists for their specific purposes. On the other hand, this person must not decide that his or her cadaver has to be used for commercial purposes, with or without reimbursement. Such a decision to hand over the cadaver to the anatomists requires an appropriate informed consent, which also allows for - severely - harming the cadaver. Of course, also the obligation to entomb is transferred.

\section{I-4}

From the clinical practice to the Anatomy lab: clinical question and anatomical reply

Duparc F

Rouen University Hospital, Laboratory of Anatomy, Rouen, France

Objectives: Through clinical and typical examples, the role of the clinical anatomist is emphasized. The conditions of a "bed question" are exposed, the different possibilities of a "lab reply" are listed, and the pedagogical aspects are shown.

Methods: Five types of questions related to clinical practice are developed. A question by a student about physical examination; One by a resident in orthopedic and trauma surgery has examined a patient and finds clinical aspects that do not deal with what he has learned in Anatomy; An interrogation by an orthopedic surgeon asks about a patient who complains about pain in the lateral part of the forearm without any local sign; a technical difficulty in regional anesthesia; one by a physiotherapist about the loss of sensory of the dorsum of the foot in an ice-hockey player.

Results: Numerous tools have been used for carrying an anatomical reply, at the bed-side, in the operative room, in the clinical department, by e-mail, or in the laboratory of Anatomy: simple drawing, books, slides, videos, websites, virtual dissection, dissection.

Conclusions: The clinical anatomist has a great place, as a reference person, who knows or who is able to search and inform about the reply. Facing a clinical question, the role of the anatomist is to provide an anatomical explanation, but not only. The pedagogic relevance is strong. The anatomical knowledge is reinforced by the increasing of the process of memorization that is more developed when the basic knowledge is associated with a live experience. Teaching the ability to go from the bed side to the lab is a way for self-learning of excellence.

\section{1-5}

Professor Moscovici: Brazilian anatomist of the world

Prates NEVB*, Prates JC**

*Department of Anatomy, Institute of Biomedical Sciences, University of Sao Paulo, Brazil; **Department of Morphology and Genetics, Paulista Medical School- Federal University of Sao Paulo, Brazil

Professor Moscovici was born in São Paulo, Brazil, on February 28, 1925, son of European immigrants. He married the professor of Psychology, Fela Moscovici and following the example of his father he was graduated Surgeon Dentist, from the University of Brazil Dentistry, current Federal University of Rio de Janeiro. Defendedhis Ph.D. thesis under the title "Contribution to the study of the ascending mandibular branch." Became Professor at the same University, defending the thesis "Contribution to the study of the nervusfacialis in man" and later Full Professor of Anatomy with the thesis "Blood angioarchitecture of the papillary muscles of the left heart ventricle in Man". In 1965 he received the title of Professor Honoris Causa from the Federal Fluminense University in the city of Rio de Janeiro. In 1975 during the X Congress of the International Federative Association of Anatomists (IFAA), held in Tokyo, Japan, under the chairmanship 
of Professor Nakayama, he became Member of the Permanent Subcommittee of Finance of International Anatomical Nomenclature Committee (IANC). From this date, Brazil's Anatomy Teacher opened up to the world presiding in the following year XI Congress of the Brazilian Society of Anatomy and II Luso-Brazilian Anatomy Congress in Niteroi, Rio de Janeiro, Brazil. He begins to preside over the great congresses of Morphology. In 1982 he became President of the V International Symposium of Morphological Sciences (ISMS) in Rio de Janeiro. During the XII Congress of the IFAA, held in London in 1985 , he was elect President of IFAA. In 1989 chairs the XIII Congress of IFAA in the city of Rio de Janeiro, Brazil. Finally I can say that Professor Moscovici ascended from his condition of an anatomist of Brazil to an Anatomist of the world and all of us are proud of having spent with him great moments of our life.

\section{$1-6$}

Anatomy of the head and neck to new trends in its surgery

Le Floch-Prigent $\mathrm{P}$

Versailles-Saint Quentin University, Paris, France

Since more than a hundred years, the surgery of the head and neck made tremendous progress, particularly after the two world wars (Thorek, 1949; d'Allaines, 1956). The anatomical studies on the arteries of the skin (Salmon in Marseille) were a first, important landmark in the surgical knowledge of the repairs. However the two main factors of improvement were the development of the flaps and specially the free one since the end of the seventies. The perfection of the osteosynthesis and the use of new biomaterials were also important factors. We will focus on the description of the anterior flap of the temporal muscle in the correction of the facial nerve injuries. Then we will study the description of the arteries of the head and neck as reported in the book of Christian Vacher. Last we will review the use of the great pectoralis muscle flap, the scapular one and the latissimus dorsi muscular flap. The later can be also a cutaneous and an osseous one isolated or combined; its basis is the subscapular artery (2 to $3 \mathrm{~mm}$ in diameter). Without numerous and extensive dissections in the departments of gross-anatomy, these flaps will never have been discovered, nor surgically used. The anatomical details of the arterial vascularisation of the skin and nearby tissues, their variations could be only studied by extensive dissections or injection and corrosions in the anatomy laboratories. By that way the gross anatomy of the head and neck, which was supposed to be well known since several centuries, has been surgically «re-discovered» and analyzed for the surgical needs and new possibilities offered by the development of the flaps: local, translocation or free ones.

\section{I-7}

\section{Hypoxic-ischemic brain injury in neonatal mice is associated with autophagy}

Uchiyama Y

Department of Cell and Molecular Neuropathology, Juntendo University School of Medicine, Tokyo, Japan
By genetic studies using knockout mice of Atg7, capase-3, and caspase-activated DNase (CAD), we have shown that since hypoxic-ischemic $(\mathrm{H} / \mathrm{I})$ injury-mediated pyramidal neuron death is blocked in the hippocampus of neonatal mice by Atg7 deficiency, it is deeply associated with autophagy (Koike et al., 2008). However, since autophagy is essential in the maintenance of cellular metabolism, it still remains unknown whether autophagyrelated cell death is present. To further understand autophagyrelated neuron death, we produced double knockout mice of LC3A and LC3B. Although LC3, a mouse homologue of yeast Atg8, has mainly been used for a marker protein of autophagy, little is known about roles of subtypes of LC3, LC3A and LC3B, or whether these proteins compensate their roles for each other during autophagic processes. For this, we produced LC3A and LC3B (LC3A/B)-double deficient (KO) mice, which were born normally and were grown-up to be capable of reproduction. Using these KO mice, both mRNA and protein levels of LC3 homologous proteins, a GABARAP family of proteins, were examined in brain and other tissues, and found that they did not significantly alter between wild-type and LC3A/B-double deficient mice, except for that the expression level of GABARAP was significantly higher in liver tissue than in brain tissue. These results indicate that basic autophagy may work normally in neurons of LC3A/B-deficient brains. Then, LC3A/B-double deficient mice were applied to H/I model studies. As previously reported, hippocampal pyramidal neurons in wild-type mice (wt) were vulnerable to H/I injury, while the protein amount of LC3-II was significantly more elevated in the ipsilateral hippocampus than in the contralateral hippocampus from 9 to 24 hours after H/I injury. In contrast, hippocampal pyramidal neurons deficient in $\mathrm{LC} 3 \mathrm{~A} / \mathrm{B}$ were resistant to the H/I injury, while the protein amount of LC3 II in the double KO mouse hippocampus was not increased after H/I injury, compared to that in the wt hippocampus. These results suggest that LC3A/B, but not GABARAP family of proteins, are involved in $\mathrm{H} / \mathrm{I}$ injury-mediated pyramidal neuron death that occurs in the hippocampal pyramidal layers of neonatal mouse brains. As shown in our previous study, excess autophagic stress may induce neuron death. Our present data using LC3A/B-deficient mice strongly support the presence of $\mathrm{H} / \mathrm{I}$ injury-mediated autophagic neuron death in neonatal brains.

\section{I-8}

\section{Imaging-based signatures of macrophage metabolism linked to atheromatous detection}

Boscá $\mathrm{L}^{*}$, Mojena $\mathrm{M}^{*}$, Rosales EC*,**, Ramos GS*, Chinchilla $\mathrm{P}^{*}$, Velasco FM*, Regadera $\mathrm{J}^{* * *}$

*Instituto de Investigaciones Biomédicas Alberto Sols, CSIC-UAM, 28029 Madrid, Spain; **Departamento de Bioquímica y Medicina Molecular. Facultad de Medicina, Universidad Autónoma de Nuevo León. Francisco I Madero y Eduardo Aguirre Pequeño S/N, Colonia Mitras Centro, CP 64460 Monterrey, Nuevo León, México; ***Facultad de Medicina, Universidad Autónoma de Madrid (UAM), 28029 Madrid, Spain

Macrophages are present in a large variety of locations, playing distinct functions that are determined by its origin and by the nature of the activators present in the environment, ranging from host defense to atherogenesis. This variety of phenotypes have 
been classified as pro-inflammatory (M1 polarization) or antiinflammatory/pro-resolution/ deactivation (M2), coexisting in the course of inflammation. We have profit these specific macrophage signatures to take advantage in the analysis of the presence of these cells in the atheromatous lesions, increasing the sensitivity of detection using image-based approaches. PET/CT analysis of ${ }^{18}$ fluorine-2-deoxyglucose uptake (FDG) has been used as a tool to trace the presence and activity of macrophages in the atheromas. By using metabolic reprogramming of macrophages we provided the rationale to increase sensitivity and selectivity in the detection of the macrophages in atheromatous lesions that avidly incorporate FDG. Our data provide evidencebased approaches to target these cells, modulating their viability, their avidity to incorporate FDG as well as to contribute to atheroma stabilization preventing their instability and capacity to cause thrombotic events. In this context, we have investigated the role of macrophages in the development of atherogenesis, its diagnosis and its contribution to plaque stability and culprit and non-culprit acute coronary events. These studies allowed us to develop new strategies to provide specific metabolic-based signatures associated to the evaluation of plaque integrity.

\section{I-9}

\section{Ultrastructural morphology of white matter axons as a function of age and ischemia}

Katharine S, Chinthasagar B, Grahame K, Sylvain B, Selva B

Department of Neurosciences, Lerner Research Institute, Cleveland clinic, Cleveland, $\mathrm{OH} 44120$, USA

Aging has global effects on axons, rendering white matter (WM) more susceptible to neurodegenerative diseases and stroke. We hypothesized that age-related alterations in axon-myelin architecture, mitochondrial function, and mitochondrial - sarcoplasmic reticulum (SER) interactions lead to changes in $\mathrm{Ca}+2$ homeostasis and ATP production, which predisposes axons to more profound effects of aging and severe injury during stroke. We utilized a pure WM tract, mouse optic nerve (MON), obtained from 2- or 12-month-old wild-type and Thy-1 mito CFP (+) transgenic mice, where mitochondria are intrinsically fluorescent, to quantify axon structure and function using immunoblots, ATP assays, electrophysiology, and three-dimensional electron microscopy. Evoked compound action potentials (CAPs) were larger in aging axons but recovered less after ischemia compared to young axons. Structurally, aging axons became thicker with lower G-ratios and had increased internodal distances and nodal length changes such as smaller clusters of voltage-gated $\mathrm{Na}+$ channels and thinner and longer CASPR overlapping with voltage-gated $\mathrm{K}+$ channels. Aging axonal mitochondria were larger and thicker with a lower incidence of SER association. A mismatch of mitochondrial shaping proteins with aging resulted in aggregation and fusion of mitochondria, leading to lower ATP production capacity, which suggests mitochondrial dysfunction. Our results suggest that aging alters axonal morphology as well as axonal mitochondrial structure and function, leading to reduced ATP production that may disrupt $\mathrm{Ca}+2$ homeostasis, which likely underlies the increased vulnerability of aging axons to ischemia and neurodegenerative diseases.
I-10

The effects of methylene blue on autophagy and apoptosis in MRI-defined normal tissue, ischemic penumbra and ischemic core

Jiang $Z^{* *}$, Watts $\mathrm{L}^{* * *}$, Huang $\mathrm{S}^{* * *}$, Shen $\mathrm{Q}^{* * *}$, Rodriguez $\mathrm{P}^{* * *}$, Chen $\mathrm{C}^{*}$, Zhou $\mathrm{C}^{*}$, Duong $\mathrm{T}^{* * *}$

*Peking University Health Science Center, Beijing, China; **Peking University Health Science Center, Beijing, China; University Of Texas Health Science Center At San Antonio, San Antonio, TX, USA; $* * *$ University Of Texas Health Science Center At San Antonio, San Antonio, TX, USA

Objectives: Methylene blue (MB) USP, which has energyenhancing and antioxidant properties, is currently used to treat methemoglobinemia and cyanide poisoning in humans. We recently showed that $\mathrm{MB}$ administration reduces infarct volume and behavioral deficits in rat models of ischemic stroke and traumatic brain injury. This study reportsthe underlying molecular mechanisms of MB neuroprotection following transient ischemic stroke in rats.

Methods: Rats were subjected to transient ischemic stroke. Multimodal MRI during the acute phaseand at $24 \mathrm{hrs}$ was used to define three regions of interest (ROIs): i) the perfusion-diffusion mismatch salvaged by reperfusion, ii) the perfusion-diffusion mismatch not salvaged by reperfusion, and iii) the ischemic core. These ROIswere extracted for western blot analyses of autophagic and apoptotic markers.

Results: The major findings were: 1) MB treatment reduced infarct volume and behavioral deficits, 2) MB improved CBF to perfusion-diffusion mismatch tissue after reperfusion and minimized harmful hyperperfusion24hrs after stroke, 3) MB inhibited apoptosis and enhanced autophagyin the perfusion-diffusion mismatch tissue, 4) MB inhibited apoptotic signaling cascades (p53-Bax-Bcl2-Caspase3), and 5) MB enhanced autophagic signaling cascades (p53-AMPK-TSC2-mTOR).

Conclusions: $\mathrm{MB}$ induced neuroprotection, at least in part,by enhancing autophagy and reducing apoptosis in perfusion-diffusion mismatch tissue following ischemic stroke.

\section{I-11}

\section{Transcriptional control of serotonergic neuron development}

\section{Song NN, Ding YQ}

Department of Anatomy and Neurobiology, Tongji University Faculty of Medicine, Shanghai 200092, China

Serotonin is an important neurotransmitter involved in many physiological functions, and dysfunction of this system has been reported to be involved in the some psychological diseases such as depression, anxiety, fear memory and autism. We identified a Lim-homeodomain transcription factor Lmx1b is required for the differentiation of serotonergic neurons during embryonic development as well as for normal expression of serotonin in adulthood as evidenced by data from knockout (KO) mice. We generated conditional Lmx1b KO (cKO) mice 
lacking serotonergic neurons, and inducible Lmx1b cKO mice with lowered level of serotonin but normal number of serotonergic neuron in the brain. These mice showed no obvious alterations in forced swimming and tail suspension test, and it is also observed in mice lacking tryptophan hydroxylase 2 (Tph2, the key enzyme for serotonin synthesis), suggesting that depression-like behaviors are not changed and therefore supporting the idea that central serotonin deficiency is not required for the development of depression at least in mouse. However this mice displayed enhanced contextual fear memory with impaired extinction of fear memory, which can be rescued by intraventricular administration of serotonin. It has been shown enhancing serotonin activity by using selective serotonin reuptake inhibitors promotes adult hippocampal neurogenesis, but we found that lowering serotonin in adult brain showed a similar effects on this process. We therefore demonstrate complex roles of central serotonin in regulating brain functions.

\section{I-12}

The easily ignored role of autophagy in early events of embryo development

H. Lu W*, Wang $\mathrm{G}^{*}$, Cheng $\mathrm{X}^{*}$, Cao L** ${ }^{* *}$ Yang $\mathrm{X}^{*}$

*Division of Histology \& Embryology, Key Laboratory for Regenerative Medicine of the Ministry of Education, Medical College, Jinan University, Guangzhou 510632, China; *Key Laboratory of Medical Cell Biology, China Medical University, Shengyang 110001, China

Autophagy is important for cell renewing for its contribution to the degradation of bulk cytoplasm, long-lived proteins, and entire organelles and its role in embryonic development is largely unknown. In our study, we investigated the function of autophagy in gastrulation of the chick embryo using both in vivo and in vitro approaches, especially in the EMT process, and we found that autophagy gene Atg7 was expressed on the apical side of the ectoderm and endoderm. Over-expression of Atg7 could enhance the expression of Atg8 and the E-cadherin, the latter of which is a crucial marker of the EMT process. We also found that the disturbance of autophagy could retard the development of chick embryos in $\mathrm{HH} 4$ with shorter primitive steak than that in the control group, which is a newly formed structure during EMT process. So we assumed that autophagy could affect EMT process by adhesion molecule expression. Moreover, more molecules, such as slug, chordin, shh et., which were all involved in EMT process, were detected to address the mechanism of this phenomena. We established that the inhibition of autophagy could cause developmental delay by affecting EMT process in gastrulation of chick embryos.

\section{$1-13$}

\section{Morphological studies on the central antinociceptive effects of endomorphin}

\section{Li YQ}

Department of Anatomy and KK Leung Brain Research Centre, The Fourth Military Medical University, No.169, West Changle Road, Xi'an 710032, Shaanxi Province, P.R. China
Pain is a common and severe disease that affects millions of people worldwide. $\mathrm{Mu}$-opioid receptor (MOR), a G-protein coupled receptor expressed in the nervous system, could be activated by endomorphin (EM), which has high affinity and selectivity for MOR and is considered to be its endogenous ligand. Activation MOR by binding with EM initiates signaling cascades which result in potent analgesia and possess fewer side effects. In order to provide evidence for the antinociceptive effects of EM, the morphological methods were used in the present study on 54 adult male SD rats. The main results are as follows: (1) EM might affect both somatic sensory information transmission and visceral reflex by modulating other neurotransmitters releasing from somatic and vagal afferent terminals through binding to presynaptically localized MOR in both spinal dorsal horn and solitary tract nucleus; (2) In diabetic rats, the reduced endogenous $\mathrm{EM}$ and MOR in the spinal dorsal horn might induce ongoing diabetic mechanical allodynia; (3) Ovariectomy (OVX) in the female rats induces long lasting thermal hyperalgesia, spinal EM2 decrease as well as exacerbated inflammatory pain responses. EM can dose dependently attenuate the OVX induced thermal hyperalgesia as well as the second phase pain responses in the s.c. formalin induced pain among these OVX rats, mainly via spinal MOR signal pathway; (4) Down-regulated spinal EM is an important contributor to the neuropathological process of bone cancer pain and enhancing activation of EM/MOR signaling might provide a therapeutic alternative to optimizing the treatment of cancer-induced bone pain; (5) In the periaqueductal gray, EM binds to MOR expressing GABAeregic inhibitory interneurons and then results in disinhibition of 5-HTergic projection neurons to exert analgesic effects at the spinal cord level. These results have provided morphological evidence for the mechanisms and functional roles of EM in antinociception in the CNS.

\section{I-14}

\section{Rapid steroid effects in the brain}

Jirikowski GF* ${ }^{*}$, Caldwell J**

*Inst. Anatomie II, FSU Jena, Germany; **Department of Pharmacology, LECOM, Erie, USA

Steroid hormones are known to affect cerebral development and function. Interestingly many of the steroid responsive brain regions are in part devoid of nuclear steroid receptors. We could show that steroid binding globulins (SHBG, ABP, CBG and DBP) are expressed in the brain of rat, mouse and humans. With immunocytochemistry, in situ hybridization and with RT-PCR we found that SHBG, CBG and DBP are in part co-localized with hypothalamic neuropeptides. In rat brain, steroid binding globulins seem so be compartmentalized in secretory vesicles suggesting that they are subject to axonal transport and storage in a neurohormone like way. SHBG has been shown to facilitate female sexual receptivity when infused into the brain indicating that it may have co-functions with oxytocin. Fluorescence labelled SHBG is rapidly internalized into neurons and glial cells in specific brain regions. With physiological and biochemical we obtained evidence for the existence of SHBG receptors in rat 
hypothalamus. Similar membrane associated and G-protein coupled receptors may exist for other steroid binding globulins. They may be part of the mechanisms that control known rapid, nongenomic responses of the brain to neurosteroids. These may include neuroendocrine, neuroimmune and behavioral effects of systemic and central steroids hormones.

\section{I-15}

\section{Glucocorticoid actions and CBG expression in human heart}

\section{Schäfer HH}

Roche Diagnostics, Basel, Swiss

Glucocorticoids are known to be involved in both myocardial regeneration and destruction. Cardomyocytes are mostly devoid of nuclear glucocorticoid receptors (GR) and it is generally assumed that effects of adrenals steroids in heart are mediated through the mineralocorticoid receptor (MR). Here we used immunocytochemistry to study localization of corticosteroid binding globulin (CBG) in semithin sections of human cardiac tissue samples. With staining of consecutive sections we examined colocalization with GR and MR immunoreactivities. While GR staining was almost undetectable we found a portion of myocytes with MR immunostained nuclei. Almost all cadiomyocytes exhibited CBG immunostaining in cytoplasm and on the cell membrane. Most pronounced CBG immunoreactivities were found in Purkinje fibres and in smooth muscle cells of arterial walls. With RTPCR we found in homogenates of cardiac tissue detectable levels of CBG encoding mRNA. Our findings indicate that CBG is expressed in human heart. Known cardiac effects of adrenal steroids may in part be mediated through the binging globulin and it's putative membrane receptor in addition to nuclear steroid receptors and direct genomic action. This may be of some importance in the ethiology of glucocorticoid dependent cardiac ailments including stress induced chronic heart failure.

\section{I-16}

\section{Impact of gonadal steroids on neuronal development} in vitro

Gebhart V

Institute of Anatomy II, Friedrich Schiller University, Jena, Germany

Rat pheochromocytoma cells PC 12 are known to develop features of dopaminergic neurons upon treatment with Nerve growth factor NGF. They express in part estrogen receptors (ER) alpha, ERbeta, and G-protein coupled receptor 30 (GPR30). Estrogens promote development of these cells and excert neuroprotective effects. Here we treated NGF differentiated PC 12 cells with physiological concentrations of 17 betaestradiol (E2) and with the anti-estrogen Tamoxifen. With immunocytochemistry we observed cytoplasmic staining for SHBG and nuclear staining for ER- $\$$, in a portion of these cells. With RT-PCR we found SHBG encoding transcripts as well as ER-B specific m-RNA indicating intrinsic expression of both the steroid binding globulin and the steroid receptor. With immunoassays of supernatants of E2 treated cultures, we found measurable amounts of SHBG, suggesting stimulus dependent release. Double immunostaning with ER- $\beta$ revealed that only a small group of PC 12 cells contained both antigens. Numbers of ER-13 positive cells were significantly higher after E2 treatment. These effects could not be inhibited by pretreatment of cultures with Tamoxifen. Our findings suggest that SHBG expression in PC 12 cells is in part estrogen dependent, perhaps also mediated by factors independent from classical nuclear estrogen receptors.

\section{I-17}

\section{Gonadal steroid hormones as olfactory ligands}

Ploss V

Inst. Anatomie II, FSU Jena, Germany

Derivates of gonadal steroids known as volatile and nonvolatile pheromones have multiple effects on the rodent brain via the olfactory system. Their primary port of entry seems to be the vomeronasal organ (VNO). The underlying cellular and molecular mechanisms are unclear so far. Recently we localized sex hormone binding globulin (SHBG) in both the main and the accessory olfactory system of rat and mouse with immunocytochemistry and RT-PCR. The accessory olfactory system consisting of VNO and accessory olfactory bulb showed particularly high expression of SHBG. Here we studied in greater detail SHBG expression in the rat VNO. In semithin sections we found SHBG immunostaining confined to the apical parts of sensory cells and in their axons. Many of the sustentacular cells contained abundant SHBG immunofluorescence. A portion of the basal cells and some of the goblet cells in the nonsensory epithelium also showed intense SHBG staining. SHBG staining was most abundant in exocrine cells of the vomeronasal glands. Our findings indicate that SHBG expressed in the VNO may be liberated into nasal secretions to bind pheromones. SHBG in sensory cells may be involved in signaling actions of gonadal steroids.

\section{I-18}

\section{Myocardial bridges in adults: facts and fantasy - The Belgrade study}

Parapid GT ${ }^{* * * * * * * *}$ Jankovic $\mathrm{R}^{* *}$, Kanjuh $\mathrm{V}^{* * *}$,

Nedeljkovic $\mathrm{I}^{* * * *}$, Prates $\mathrm{N}^{* * * * *}$, Virmani $\mathrm{R}^{* * * * * *}$, Danchin $\mathrm{N}^{* * * * * *}$, Simic $\mathrm{D}^{* * * *}$, Ostojic $\mathrm{M}^{* * *}$

*Institute of Anatomy "Niko Miljanic", Faculty of Medicine, University of Belgrade, Belgrade, Serbia; **Institute of Pathology, Faculty of Medicine, University of Belgrade, Belgrade, Serbia; ***Serbian Academy Of Sciences And Arts, Belgrade, Serbia; $* * * \star$ Division of Cardiology, CCS, Faculty of Medicine, University of Belgrade, Belgrade, Serbia; $* * * * *$ Institute of Biomedical Sciences, University of Sao Paulo, Sao Paulo, Brazil; ******Cardiovascular Pathology Inc., Gaithersburg MD, USA; $* * * * * *$ Département De Cardiologie, Hôpital Européen Georges Pompidou, Assistance Publique Des Hôpitaux De Paris; Faculté De Médecine, Université Paris-Descartes, Paris, France; $* \star \star \star \star \star \star \star \star$ Division of Cardiology, Clinical Center of Serbia, Belgrade, Serbia 
Myocardial bridge (MB) is a congenital anomaly. Reported incidence on autopsies extensively varies (4.7\%-86\%) likely related to geographical regions. Our retrospective study performed at the Institute of Pathology, Faculty of MedicineUniversity of Belgrade (IPFMUB) revealed the prevalence of $0.8 \%$ which we doubted to be the true one in Serbian population. This prospective study was carried out on 96 hearts from cadavers of individuals (18-86 yrs; mean age: $66.45 \pm 13.89$ yrs) of both sexes $(51 \mathrm{~m})$ deceased from natural causes, obtained at IPFMUB. Tunneled coronary arteries (TA) were serially sectioned with the adjacent myocardium. Tissue blocks were processed, serially sectioned $(5 \mu \mathrm{m})$ and stained (HE, Masson trichrome, PTAH, Weigert van Gieson, Verhoeff van Gieson). Images were recorded (Olympus DP70) and measured (ImageJ). The total of $14 \mathrm{MBs}$ were found in $13(13.54 \%)$ hearts. In 1 heart we noted $2 \mathrm{MBs}$ (left anterior descending artery, LAD, and the left marginal, LMA, were overbridged). They were equally common in male and in female $(13.72 \%$ vs. $13.33 \%, \mathrm{p}>0.05$ ) (mean age: $66.69 \pm 18.01 \mathrm{yrs}$ ). None of the MBs has been diagnosed during life. Diabetes mellitus was present in 2/13 cases; hypertension - in all cases with MB. The most common causes of death were cardiac ones. MBs were located over: $\operatorname{LAD}(50.0 \%), \operatorname{LCx}(28.6 \%), \mathrm{LMA}(14.3 \%)$ and right coronary artery (RCA) $(7.1 \%)$. In $92.3 \%$ of the cases, the dominant artery was the RCA. The only heart with balanced type had $2 \mathrm{MBs}$. In $30.8 \%$ of hearts with MBs we found additional CA variations/anomalies. The median MB length was 20 $\mathrm{mm}(10-80 \mathrm{~mm})$. The differences between average MB thicknesses (2.5-3.5 mm) over different arteries was insignificant ( $>0.05)$. All TAs were surrounded by adipose and loose connective tissue with nerves.

The described prevalence of MBing in Serbian population (13.54\%) compared to previous report $(0.8 \%)$ stresses the importance of broader education of younger pathologists MBs are the entities that should be born to mind. (Supported by MESRSerbia Grants:III41022;175030).

\section{|-19}

\section{Effects and mechanism of activating microglia} on da neuron injury

\section{$\underline{\mathrm{Su}}^{*}$, Shan $\mathrm{M}^{* *}$, Lin $\mathrm{S}^{*}, \mathrm{Li} \mathrm{S}$}

*Development and Regeneration Key Lab of Sichuan Province, Department of Anatomy and Histology and Embryology, Chengdu Medical College, Chengdu 610500, China; **Department of Pathology, Development And Regeneration Key Lab Of Sichuan Province, Chengdu Medical College, Chengdu, China

Microglial mediated inflammation plays striking roles in Central nervous system degenerative diseases, like Parkinson's disease. The dynamic changes of two phenotypes of microglia, M1 and M2, are critically associated with the neuro-degeneration and repair. In our study, it was found that TRIF one adaptor molecule downstream TLR3 and TLR4 innate immunity receptor that was able to inhibit microglial M1/M2 transformation by the stimulation of IL-4 and LPS. After LPS and IL-4 treatment fol- lowed by siRNATRIF interference, the mRNA expression of TNF- $\alpha$, IL-6, CD206 and Arg1 decreased significantly, which suggested siRNATRIF inhibition altered the microglia M1/M2 dynamic transformation. In a MPP+ treatment in vitro model, conditional medium was added to the MN9D cells with or without MPP+ treatment. As a result, it was indicated that the interference of TRIF in BV2 inhibits the transformation of BV2 to M2 phenotype which play some protective role in $\mathrm{PD}$ degenerative process. While the conditional medium treated MN9D cells were detected by flow cytometry to explore the apoptotic ratio of MN9D cells by different BV2 treatment medium, i.e., $\mathrm{MPP}+, \mathrm{MPP} / \mathrm{siNC}-\mathrm{CM}, \mathrm{MPP}+/$ siTRIF-CM and control group. The siRNATRIF treated group had increased apoptotic ratio of MN9D cells compared with control and siNC-CM groups, which indicates the inhibition of TRIF deteriorate the MN9D cell survival ratio. In conclusion, it was found that TRIF is a key switch that may contribute to microglial M1 and M2 phenotype transformation, the interruption of TRIF expression may decrease survival ratio of MN9D cell, classical DAT and TH protein secretion and microglial migration. This work was supported by NSFC (NO: 31371215; 81241019).

\section{I-20}

\section{The value of applied clinical embryology in the 21st century assisted reproductive technology}

\section{Ashiru O, Ogbechi RO}

Medical Art Center, 21 Mobolaji Bank Anthony Way, Ikeja, Lagos

Objectives: Assisted Reproductive Technology (ART) with the breakthrough research by embryologists has witnessed remarkable scientific and clinical advances since the birth of the world's first in vitro fertilization baby, Louise Brown (1978) in the United Kingdom. The scientists further introduced techniques such as intracytoplasmic sperm injection (ICSI) that now allows better manipulation of the fertilization process in order to bypass some pathological obstacles such as blocked vas deferens and low sperm count in the males. Other procedures such as assisted hatching, Embryo biopsy, pre-implantation genetic diagnosis, time lapse imaging and other techniques, have now been employed in ART with very good results.

Results: A speedy advancement in the development of different assisted reproductive techniques makes infertility problem more treatable than it ever had been.

Conclusions: With all these scientific advances, challenges are still being faced. There are challenges which scientists have to deal with. There are challenges to the health services. There are challenges for which the health profession, as a group, has to take responsibility. And last but not least, society has to cope with new challenges brought to the forefront by this technological revolution and its social implications. One challenge that cuts across all others is how to make ART more widely available and affordable for all who need it, particularly in developing countries. 
I-21

Cell death signaling by caspase activation during neural development and inflammation

Miura M

Department of Genetics, Graduate School of Pharmaceutical Sciences, The University of Tokyo and CREST, JST, Tokyo, Japan

Cell death is widely observed during development, tissue homeostasis and disease condition. However, it is still difficult to detect dying cells and study its physiological roles in vivo. Live cell imaging using fluorescence resonance energy transfer (FRET) technology permits us to monitor cell-signaling activities simultaneously with cell behavior in real time. We succeeded in monitoring caspase-3 activation by SCAT3 in living mouse embryo. We found that caspase activated cells showed typical and atypical apoptotic behavior in a region-specific manner during neural tube closure. Caspase activation regulates the kinetics of neural tube closure. Furthermore, caspase-mediated elimination of brain organizing center was shown to be an efficient mechanism for the switching of morphogen during brain development. In addition to the SCAT sensor for apoptotic caspase, we have generated FRET sensor for inflammatory caspase, caspase-1, and named this probe as SCAT1. Analysis of spatial and temporal activation of caspase- 1 revealed that IL- $1 \mathrm{~b}$ is secreted from dying macrophages, suggesting the close relationship of cell death and cytokine secretion. Combination of live imaging and genetics revealed the active roles of cell death signaling by caspase during brain development and inflammation.

\section{$1-22$}

\section{The clinical anatomy of Langer's lines}

Carmichael S* , Waldorf $\mathrm{JC}^{* *}$

*Mayo Clinic, Rochester, MN, USA; Mayo Clinic, Jacksonville, FL, USA

In the early 1860s Karl Langer published 4 scholarly articles in German on the anatomic characteristics of the human skin (Carmichael, 2014). His most notable contributions were maps of the skin tension lines that are still known as Langer's lines. Langer's articles did not appear in English until 1978 although Langer's lines were reproduced in surgery textbooks, etc. for many years before that in several languages, including English. Many times Langer's lines were interpreted to be guidelines for elective surgical incisions, although we now know that skin relaxation lines, not skin tension lines, are more reliable for cosmetically successful results. Adding to the confusion is the fact that in parts of the body, such as the neck, thorax, and upper abdomen Langer's lines are mostly parallel with minimal surgical scars, specifically they are transverse in these regions. However in the lower abdomen Langer's lines become more vertical whereas minimal surgical scars are transverse. Also on the forehead Langer's lines are vertical but more successful results are from transverse incisions. However on the glabella both Langer's lines and successful incisions are vertical. We will show the results of several surgical incisions that illustrate that Langer's lines are not reliable guidelines to obtaining suc- cessful cosmetic results from an elective incision. Skin relaxation lines, which can be determined by the "pinch test" (Waldorf et al., 2002) are the most reliable guidelines to a result that will be most pleasing to the patient who often judges the surgeon's work on the basis of the resulting scar.

\section{$1-23$}

General angiomorphological imaging using SEM vascular corrosion casts

Pais D, Regadera J

NOVA Medical School, Faculdade de Ciências Médicas-UNL, Lisbon, Portugal

One of the difficulties that the less experienced researcher meets, when starting the study of the microvasculature of a given tissue or viscera, is the correct interpretation of the images he obtains. This is particularly significant in interpreting SEM images of microvascular corrosion casts of parenchymatous organs, much more than in flat tissues or viscera or in those where the microvascular architecture is arranged in successive layers, as may be found in the study of the microvasculature of the wall of hollow viscera. One of the viscera that presents a greater challenge to the interpretation of those images is undoubtedly the testis, especially in species such as human, where the fibrous septa are incomplete or nonexistent. In this presentation the author will briefly present some of the aspects essential to the interpretation of SEM microvascular corrosion casts mainly of human testis and epididymis. These aspects will be addressed: 1) characterizing features helping the identification of blood arterioles, venules and capillaries as well as lymphatic microvessels; 2) Specific findings resulting from the imprinting of smooth muscle fibers, sphincteric constrictions, 'intra-arterial cushions', arterial vasa vasorum and filling of the interstitial spaces with resin; 3) technical artifacts that often lead to misleading interpretations, such as incomplete filling of vessels, sudden vascular endings and 'plastic strips'; 4) Testiculo-epididymal Microvascular Classification, where caliber of the SEM microvascular corrosion casts are compared to findings obtained in microangiography. clearing technique and histology.

\section{$1-24$}

Effects of enviromental pollutants on cultured mouse oocytes and granulosa cells

Palmerini $\mathrm{MG}^{*}$, Zhurabekova $\mathrm{G}^{* *}$, Balmagambetova $\mathrm{A}^{* *}$, Miglietta $\mathrm{S}^{* * *}$, Belli $\mathrm{M}^{*}$, Bianchi $\mathrm{S}^{*}$, Cecconi $\mathrm{S}^{*}$, Nottola $\mathrm{S}^{* * *}$, Macchiarelli $\mathrm{G}^{*}$

${ }^{*}$ Dept. of Life, Health and Environmental Sciences, University of L'Aquila, L'Aquila, Italy; **Dept. of Normal and Topographical Anatomy, West Kazakhstan Marat Ospanov State Medical University, Aktobe, Kazakhstan; ***Dept. of Anatomy, Histology, Forensic Medicine and Orthopaedics, La Sapienza University, Rome, Italy

The drying of the Aral Sea for irrigation, followed by an excessive pesticide exposure, is one of the greatest man-made environmental disaster. High levels of reproductive pathologies 
have been observed in this region for more than 20 years. The responsible include Hexachlorocyclohexanes, as the herbicide Lindane, and Dithiocarbamates, as the fungicide Mancozeb. We demonstrated the toxic effect of Mancozeb on mouse oocytes characterized by alterations in spindle morphology, a reduction in the formation of male/female pronuclei and a reduced ability to complete meiotic maturation and fertilization. Lindane altered the formation of the first meiotic spindle and polar body extrusion on mouse oocytes in vitro. This was probably due to gap junction inhibition between oocyte and surrounding granulosa cells (GCs). To understand the ultrastructural morphological alterations on GCs after pesticide exposure, increasing dose of Lindane and Mancozeb were tested on GCs collected from prepubertal CD1 mice ovaries and matured in vitro and studied by transmission and scanning electron microscopies (TEM and SEM). Results indicated dosedependent detrimental effects on GCs subjected to Lindane or Mancozed exposure, as per the presence of abundant presence of cell debris, the lost of cell contacts, the decrease of microvilli and cytoplasmic processes. In addition, peripheral aggregation of chromatin under the nuclear membrane, extensive plasma membrane blebbing, abundant GC remnants were also present, but more evident after Lindane exposure. In conclusion, our data showed a different dose-dependent toxicity for Lindane and Mancozeb on GCs, associated to morphological signs of cell death. Since alterations of GCs may be associated to impaired oocyte competence and sterility, these results may be of interest in the studies addressed to the preservation of fertility, both in animals and humans.

\section{I-25}

\section{Ovarian reserve markers of women living in the Aral sea area}

Zhurabekova G* ${ }^{*}$, Balmagambetova A*, Abdelazim I**, Macchiareli $G^{* * *}$

*Department of Normal and Topographical anatomy, West Kazakhstan Marat Ospanov State Medical University, Aktobe, Kazakhstan; **Department of Obstetrics \& Gynecology, Ain Shams University, Cairo, Egypt and Ahmadi Hospital, Kuwait Oil Company (KOC), Kuwait; ***Department of Life, Health and Environmental Sciences and Center of Electron Microscopies, University of L'Aquila, L'Aquila, Italy

Introduction: Ecological disaster and death of the Aral Sea now create a tense situation in the region lead to high levels of various chemicals, pesticides, and heavy metals in soil, water and air. Ecological disaster resulted in high incidence of congenital abnormalities, nutritional problems during pregnancy and infertility.

Objectives: The study comparative prospective study designed to identify the ovarian reserve of women living in the Aral Sea area (Shalkar) included in group I (study group) and compared to women living in Aktobe in group II (control group). Women included in this study were evaluated using trans-vaginal ultrasound (TVS) to detect Antral Follicle Count (AFC) as well as Basal Ovarian Volume (BOV). Followed by hormonal evalua- tion including; Follicle stimulating hormone (FSH) and Antimullerian hormone (AMH).

Results: There was no significant difference between two studied group as regarding biochemical markers of ovarian reserve including; FSH and $\mathrm{AMH}$, while, the number of $\mathrm{AFC}$ was significantly reduced in the group of women in Aral Sea region (Shalkar) compared to Aktobe group. In addition, the BOV reduced in women living in Aral Sea region (Shalkar) compared with Aktobe women of same age group. The ovarian reserve as indicated by $\mathrm{AFC}$ and $\mathrm{BOV}$ reduced in women living in Aral Sea region (Shalkar) compared with Aktobe women of same age group.

Conclusions: the results of this study suggests possible environmental effect on ovarian reserve of women living in Aral Sea area. This study, also, concluded that, AFC and BOV is a simple, rapid, non-invasive method for assessment of ovarian reserve.

\section{I-26}

\section{Recent insights in the ultrastructure of human oocyte}

Macchiarelli $G^{*}$, Familiari G**, Nottola SA**

*Department of Life, Health and Environmental Sciences, University of L'Aquila, L'Aquila, Italy; **Department of Anatomy, Histology, Forensic Medicine and Orthopaedics, Sapienza University, Rome, Italy

The positive outcome of assisted reproductive technologies (ART) depends on the oocyte quality, in terms of maintenance of full morphofunctional integrity during its complex and long maturation. In fact, the completion of maturation, in the preovulatory period, both in vivo and in vitro, as well as the preservation of cytoplasm, permit the oocyte to become competent for fertilization. The oocyte ultrastructure reflects the developmental competence of this cell that, therefore, has been object of several studies, especially after ART. Since, after completion of its maturation, the human oocyte acquires very peculiar fine structural features, likely influenced by the application of ART, we studied the above changes in human oocytes, from young and aged women, after informed consent, subjected to the most recent ART protocols including, in vitro maturation and or cryopreservation. In this paper, we reviewed by electron microscopy and correlated techniques, the changes of the nucleus, cytoplasm and zona pellucida, occurring in the human oocyte during the final stages of maturation, after ART. We could observe that the human oocyte undergoes a reorganization of its genome, ooplasm and extracellular matrix characterized by neogenesis, modification and redistribution of organelles, membranes and glycoproteins of the ZP. Our analysis showed that the completion of the maturation in the preovulatory period and the absence of cytoplasmic degenerative alterations, make the female gamete competent for fertilization. However, several subcellular aberrations, detectable only by electron microscopy analysis, occur in the oocyte as the consequence of the application of ART protocols (IVM and cryopreservation, especially) and could be responsible for ART failures, or even affecting early embryo development. Electron microscopy observations, associated with other 
morphological studies, and analyzed together with clinical, epidemiological, biological, molecular/ biochemical data, are useful in the assessment of the cell structural integrity, in order to define the proper "oocyte health" state.

\section{I-27}

\section{Comparison of calcium and barium microcapsules as scaffolds in the development of artificial dermal papillae}

$\underline{\text { Lin } \mathrm{C}^{*}}$, Li $\mathrm{Y}^{* *}$, Huang $\mathrm{K}^{* * *}$

*Shantou University Medical College, Shantou, Guangdong, China; **1st Affiliated Hospital of Shantou University Medical College, Shantou, Guangdong, China; $* * * 2 n d$ Affiliated Hospital of Shantou University Medical College, Shantou, Guangdong, China

Although dermal papilla transplantation is one of the most promising treatments for baldness, lack of dermal papilla with hair-follicle inductive properties still remains a major obstacle. Previously, we developed artificial dermal papilla by enclosing dermal papilla cells within microcapsules. In this study, we used barium and calcium microcapsules as candidates for scaffolding in the development of artificial dermal papilla. We compared both microcapsules in terms of biocompatibility, structural stability, permeability, cell viability, and hair-follicle inductive properties in the short term. Both barium and calcium microcapsules maintained xenogenic dermal papilla cells in an immunoisolate environment and induced the formation of hair-follicle structures. Biocompatibility, permeability and cell viability were better with calcium microcapsules compared with barium microcapsule. Especially, biocompatibility was better for calcium microcapsules compared with barium microcapsules. Calcium microcapsules were assessed for long-term inductive properties with xenotransplantion into SpragueDawley rat ears. Before 18 weeks, calcium microcapsules gathered together, with no substantial immune response. After 32 weeks, some microcapsules were near inflammatory cells and were wrapped with fiber. A few large hair follicles were found. The control samples showed no marked changes at the implantation site. Barium microcapsules are advantageous for structural stability. The cells encapsulated in hydrogel barium microcapsules exhibited higher short-term viability compared with the cells encapsulated in calcium microcapsules. Barium microcapsules showed higher mechanical stability compared with calcium microcapsules. Barium microcapsules may be of use in short-term transplantation study. Calcium microcapsules may provide an effective scaffold for the development of artificial DP

\section{I-28}

\section{Brain, behaviour and evolution}

Paxinos $\mathrm{G}$

Neuroscience Research Australia, Sydney, Australia

Pre-Socratic philosphers rejected supernatural explanations for the existence of the physical world and the nature of the soul.
These philosophers rejected gods and magic. Later Hippocartes said that men aught to know that from the brain, and only from it, derive our pleasures, happiness, laughter as well as pain and sorrow. After the long battle to find the seat of the soul, psychology lost its soul in the 1930s. According to Hebb (1958), the mind is the integration of the activity of the neurons of the brain. That is, there is no ghost in the machine. If the relation between brain and behavior is 1 to 1 , then there is no need to hypothesize the presence of the soul to understand behavior and modify it. We have constructed two types of brain atlases: histological and MRI. Standard atlases using identical nomenclature enable scientists to navigate seamlessly between the brain of humans and experimental animals to test hypotheses inspired by human considerations and relate data from experimental animals to humans. In the histological atlases, we make use of genes that are responsible for the segmentation of the brain in development (hox genes). Using evidence from transgenic mice and birds we are proposing a new plan for the organization and function of certain brain regions of mammals. The brainstem, for instance, can no longer be considered as a container of haphazardly arranged nuclei (as potatoes in a sac), but instead as regions which co-vary (start and end) with their neighbours. The human brain features many more homologies with the brain of monkey (eg, virtually all areas of the cortex are homologous), of the rat and of the bird than previously thought. Areas which are shown to be homologous are likely to have similar function as for example are 9/46 of the prefrontal cortex which is homologous in human and monkey and is involved in executive processing in working memory in both species. Using MR images in mice and non-human primates we are providing $3 \mathrm{D}$ volumes of canonical brains against which transgenic varieties with clinical significance can be compared. Finally, the human brain contains homologous parts to brains of reptiles and conflicts between human societies and the extinctions caused by humans may be in part attributable to this heredity.

\section{$1-29$ \\ New insights into the role of Olig1 and Olig2 in oligodendroglial differentiation \\ Xiao L, Niu J, Mei F \\ Department of Histology and Embryology, Third Military Medical University (TMMU), Chongqing, China}

Objectives: Oligodendroglial cells undergo rapid transcriptional and dynamic morphological transformation in order to effectively myelinate neuronal axons. We aimed to investigate the regulation role of Olig1 and Olig2, two basic helix-loophelix transcription factors essential for oligodendroglial genesis during later stages of oligodendroglial development.

Methods: Using immunocytochemisty, we compared the oligodendroglial differentiation between wide type mice and transgenic mice in which Olig2 is deleted in OPCs and OLs; we also examined the underlying mechanism by using molecular cell biological methods. 
Results: We found that Olig2 deletion in OPCs dramatically represses OPCs differentiation into OLs and myelination. However, deletion of the Olig2 gene in newly generated OLs significantly enhances the maturation process and accelerates the kinetics of myelination/remyelination. Underlying the differing stage-specific roles for Olig2 is the compensatory expression and function of Olig1. We observed that translocation of Olig1 into the cytosol highly correlates with differentiation of oligodendrocytes and serine 138 in the helix-loophelix domain of Olig1 is phosphorylated and that this form resides in the cytosol. Mutating serine 138 studies indicated that phosphorylated cytosolic Olig1 in membrane expansion and maturation of oligodendrocytes.

\section{I-30}

\section{Body donations today and tomorrow - a challenge for best practice}

\section{Riederer BM}

Platform for Morphology \& Department of Fundamental Neurosciences, Faculty of Biology and Medicine, University of Lausanne, Centre des Neurosciences Psychiatriques, University Hospital Canton de Vaud, Lausanne, Switzerland

There is much agreement that the use of human bodies for teaching and research is still of importance. Yet, not all Universities use dissection to teach human gross anatomy. From a historical point-of-view the concept of body donation has evolved over centuries and there is still a considerable discrepancy between countries regarding the way of acquisition and use of human bodies for education and research. Many countries have well-established donation programs and use more or less or not at all body dissection to teach human gross anatomy. There are those countries that have no donation programs, and use unclaimed bodies only or perhaps a few donated bodies. In several countries a use of cadavers for dissection is unthinkable for cultural or religious reasons. It is of interest to concentrate on successful donation programs and to define what makes these programs successful. On this basis we will look in the future what best practice may look like and how to replace the use of unclaimed bodies for anatomy teaching. From an ethical point of view, countries that need unclaimed bodies of dubious provenance may take advantage of these reports and adopt strategies how to develop successful donation programs. In many countries the act of body donation was guided by laws and ethical frameworks, and has evolved together with a need for medical knowledge and to improve teaching human anatomy. Undoubtedly, also in the future there will be a need for human bodies to assure optimal pre- and post-graduate training, but also to progress biomedical research. Good body donation practice should be applied wherever possible and we should come away from the use of unclaimed bodies of dubious provenance and adopt strategies how to favor settingup successful donation programs.

\section{I-31}

Ultrastructural modifications in the organular pattern of human metaphase II oocytes subjected to freeze-thawing

Nottola $\mathrm{SA}^{*}$, Borini $\mathrm{A}^{* *}$, Levi-Setti $\mathrm{PE}^{* * *}$, Palmerini $\mathrm{MG}^{* * * *}$, Macchiarelli $\mathrm{G}^{* * * *}$

*Department of Anatomy, Histology, Forensic Medicine and Orthopaedics, Sapienza University, Rome, Italy; **Tecnobios Procreazione, Centre for Reproductive Health, Bologna, Italy; $* * *$ Department of Gynecology and Reproductive Medicine, IRCCS Istituto Clinico Humanitas, Rozzano, Milan, Italy; $* * * *$ Department of Life, Health and Environmental Sciences, University of L'Aquila, L'Aquila, Italy

Human metaphase II (MII) oocyte is very susceptible to cryodamage. In this study we evaluated the modifications occurring in the organular pattern of human MII oocytes subjected to freezethawing and examined by light and transmission electron microscopy (TEM) at different time intervals during thawing (beginning, group A; middle, group B; end, group C). Fresh MII oocytes were used as controls. Morphometric and TEM analysis revealed that vacuoles were only occasionally detected in fresh controls. Conversely, vacuoles were numerous in the cryopreserved oocytes of group A and appeared to reach an even larger number in group B oocytes. Mitochondria-smooth endoplasmic reticulum (M-SER) aggregates, large and abundant in fresh controls, significantly decreased in number following freezing, particularly in the oocytes belonging to groups A and B. Mitochondria-vesicle (MV) complexes were instead small and scarce in fresh controls but augmented after freezing, being especially abundant in the oocytes belonging to group B. Vacuoles and MV complexes both diminished in the oocytes belonging to group C, whereas M-SER aggregates increased in number. Cortical granules (CGs) were scarce in all cryopreserved oocytes in respect to those found in fresh controls and gradually diminished as thawing progressed. In conclusion, this study proves that vacuoles, generally regarded as markers of oocyte cryodamage during slow cooling, may form during freezing, but become numerous during thawing, particularly in the middle phase. Significant variations in the number of M-SER aggregates and MV complexes occurred during freeze-thawing, suggesting a dynamic process of transition between these two forms of organelle associations. This study also evidences that a premature CG exocytosis progressively occurs during the whole freezethawing procedure. Thus, all ooplasmic membranes appear significantly concerned by freeze-thawing but, except for CGs, their alterations seem to undergo a partial or, more rarely, an almost complete recovery at the end of thawing.

\section{I-32}

\section{Neuropeptide transmitters in health and disease: a translational perspective}

\author{
Hökfelt T, Adori C, Barde S, David Xu ZQ \\ Department of Neuroscience, Karolinska Institutet, S-17177, Stockholm, \\ Sweden
}

Neuropeptides are in terms of numbers the most abundant type of transmitter in the brain $(>100)$, with a correspondingly large 
number of receptors ( $>200)$, virtually always belonging to the 7 transmembrane, G protein-coupled receptor family. Neuropeptides coexist with classic transmitters: for example, in the rat, galanin with noradrenaline (NA) in the locus coeruleus (LC) and with 5-hydroxytryptamnine (5-HT) in the dorsal raphe nucleus (DRN). Galanin exerts its actions via three receptors, GalR1-3. Functionally, neuropeptides seem to be of particular significance, when the nervous system is challenged, such as by injury or stress; and loss of a single receptor may have dramatic consequences. Animal experiments suggest that peptides and their receptors may be associated with various diseases. For example, both GalR1 and GalR2 have been proposed to influence depression-like behavior. However, in order to establish the relevance of these findings for human disease, and for drug development, such results have to be validated by studies on human tissues. To this end we have explored the situation in the human brain, demonstrating distinct species differences: for example, many 5-HT neurons in the DRN synthesize galanin in rat, but none in human; and in human the NA neurons in LC express GalR3 vs. GalR1 and -2 in rats (Le Maitre et al., PNAS 110, E536, 2013). These findings indicate that GalR3 may be a target for development of antidepressants. Interestingly, our recent genetic association study has reported that variants in genes for galanin and its three receptors confer increased risk of depression and anxiety in those who experienced childhood adversity or recent negative life-events (Juhasz et al., PNAS 111, E1666, 2014). Further studies from our laboratory indicate involvement of neuropeptides, including the galanin system, also in Alzheimer's disease. A major advancement in the neuropeptide field is the approval by FDA in the USA of a new type of sleeping pill, suvorexant (Belsomra, from Merck), acting at receptors of the neuropeptide orexin/hypocretin (Yang, Drugs 74, 1817, 2014). Moreover, monoclonal anti-CGRP antibodies have been developed for successful treatment of pain (Dodick et al., Lancet Neurol. 13, 1100, 2014). Taken together, evidence for distinct roles of neuropeptides in nervous system function is accumulating, including progress in development of drugs acting on peptidergic mechanisms for treatment of various diseases.

\section{I-33}

Electrochemical processing of charged molecules: pathway to engineering of complex tissue structures

Akkuş O

Department of Mechanical and Aerospace Engineering, Biomedical Engineering and Orthopaedics, Case Western Reserve University, Cleveland, $\mathrm{OH}$, USA

Electrochemical compaction of collagen molecular solutions as dense tissue forms is a method that was invented in the Akkus lab. The process will be presented as an enabling modality which holds significant promise for reconstruction of a wide spectrum of topographically complex systems such as tendons, joint surfaces, craniofacial defects, ears, nose or urogenital forms. Collagen solutions are phase-transformed to mechanically robust shell structures with curviplanar topographies using electrochemically induced $\mathrm{pH}$ gradients. The process enables rapid layer-by-layer deposition of collagen-rich mixtures over the entire field simulta- neously to obtain compositionally diverse multilayered structures. Electrocompaction of collagen molecules by $\mathrm{pH}$ gradients increases the in-plane and out-of-plane mechanical properties by one to three orders of magnitude. Cells proliferate, adhere, spread and establish focal adhesions on electrocompacted collagen (ELCOM) at significantly greater rates than on regular collagen hydrogel. The capacity of the method to fabricate nonlinear curved topographies with compositional heterogeneity is demonstrated by sequential deposition of a collagen-hydroxyapatite layer and collagen-GAG layer to obtain a hemispherical shell emulating the bone to soft tissue transition observed in joints. In another demonstration, the complex curved topography of the nasal structure is replicated by the electrochemical compaction method. Cumulatively, reported improvements in the cell-matrix interactions would serve to expedite the population of electrocompactedcollagen scaffolds by cells and improve the associated tissue repair timeline.

\section{I-34 \\ Surgical anatomy for retroperitoneal lymph node dissection}

Biasutto SN

Chair and Institute of Normal Anatomy, Faculty of Medical Sciences, National University of Cordoba, Cordoba, Argentina

Retroperitoneal lymph node dissection (RLND) is a surgical procedure indicated in certain pelvic tumors (mainly in nonseminoma testicular and some gynecological carcinomas). It extends laterally between both ureters, upward to the renal vessels and downward to the imaginary line passing at the level of the common iliac artery divisions. The main difficulties for the performance of this procedure are the wide extension and the precise anatomical knowledge necessary to avoid injuries and to completely remove lymph node groups. The success is evaluated by the number of lymph nodes removed and the lack of complications. A xifo-pubic incision lets the access to the abdominal cavity and the retroperitoneum approach is done by externally surrounding the ascending and descending colon, including the release of both colonic flexures. Then the posterior parietal peritoneum (with the colon) is moved to the medial line following an avascular cutting plane in such way that it will remain completely free, if RLND is bilateral (with a particular consideration on the inferior mesenteric artery). Lymph-adipose tissue will be removed "in block" with special care on ureters, left spermatic vein, pancreas and lumbar vessels. In this instance, knowing the position of the lymph node groups acquires a particular importance, mainly because of its variations. (We'll describe the different groups, positions and number variations). Lateral Inferior Vena Cava, inter cava-aortic and latero-aortic lymph nodes are very variable in number and position, and its proximity with the lumbar vessels increase the risk of mortality associated to this procedure. Most of these nodes are located immediately below the renal pedicle and this position makes them very difficult to be completely resected and avoid lymphorraghia. This procedure is indicated in some specific circumstances, but the anatomical landmarks may be considered at the time of lymphomas, sarcomas and other retroperitoneal surgical interventions. 
I-35

Mechanical signals and cellular morphology

Missirlis Y

Department of Mechanical Engineering and Aeronautics, University of Patras, Patras, Greece

Mechanical signals are omnipresent in the living body. They are of different modalities and interrogate a variety of cell receptors at various times. They travel from outside the cells, through the membrane, cytoplasm, nuclear membrane all the way to the chromatin in the nucleus to prepare it for its actions. This statement implies that the mechanical signaling is faster than the chemical one, and that such appropriate mechanotransduction expresses relevant genes for cell differentiation, growth and morphogenesis.

\section{I-36}

\section{Midline crossing of sensory pathways}

Erzurumlu RS

Department of Anatomy and Neurobiology, University of Maryland School of Medicine, Baltimore, MD 21201, USA

In the mammalian brain, the left and the right hemispheres command the opposite sides of the body. Sensory inputs also partially or completely cross in the brain to reach the opposite neocortex. Crossed sensory-motor pathways may have evolutionary significance in escape behaviors of the organism away from danger or predators. During development midline crossing of sensory and motor axonal pathways is governed by a number of molecular signals emitted from the floor or roof plates. These signals attract and/or repel growing axons in temporally and spatially regulated manners. Genetic defects that impair expression of midline signals that traffic axons can lead to aberrant sensory or motor pathways. In this presentation, I will focus on the somatosensory system, particularly the facial/trigeminal system, and present consequences of midline crossing defects along this pathway.

\section{I-37}

\section{Multimodality imaging of the lower extremity arterial disease}

\section{Costa Nuno}

Faculty of Medical Sciences, New University of Lisbon

Peripheral artery disease (PAD) is underdiagnosed, undertreated and poorly understood by the medical community. Clinical symptoms such as claudication, ischemic rest pain and ulcerations lead to poor quality of life, repeated and longtime hospitalizations and sometimes limb loss or even death. Patients with $\mathrm{PAD}$ are initially evaluated with an ankle brachial index (ABI) which is an excellent screening test. Imaging of the lower limb is important to provide additional anatomic information, confirming the location and degree of stenosis prior to vascular revascularization. The characterization of peripheral arteri- al disease can be made by noninvasive methods: computed tomography angiography (CTA), magnetic resonance angiography (MRA) or duplex-ultrasound (DUS) according to patient characteristics. The high accuracy of this methods, the possibility of post processing imaging replaced the catheter-based angiography as the gold standard. This presentation will review the main imaging modalities in the diagnosis of lower extremity $\mathrm{PAD}$, advantages, disadvantages and recent advances in noninvasive imaging techniques.

\section{I-38}

\section{Clinical IVM and possible role in fertility preservation for cancerous patients}

Khalili MA

Researc and Clinical Center for Infertility, Shahid Sadoughi University of Medical Sciences, Yazd, Iran

Currently, in vitro maturation (IVM) of GV oocytes is the only choice besides cryopreservation of ovarian tissue for whom ovarian stimulation is not possible due to life circumstances (cancer). These oocytes represent pool of germ cells that can be cryopreserved and be used after patients' recovery from cancer. Therefore, it becomes necessary to optimize both cryopreservation technique as well as IVM condition for GV oocytes in clinical setting. Our recent study showed that only $30.2 \%$ of oocytes aspirated from cancerous patients reached maturity after IVM. Furthermore, no oocytes were found in $34.6 \%$ of women, and $29.5 \%$ of the oocytes were degenerated at the time of aspiration. With aid of Polscope, the meiotic spindle (MS) could be visualized in IVM oocytes. From these matured oocytes, $61.5 \%$ oocytes presented a high/positively scoring $\mathrm{ZP}$ birefringents. Also, GV appeard more suitable for vitrification than $\mathrm{MII}$ oocytes, as indicated by good ultrastructural preservation of important structures that are present only in GVs, like the nucleus and migrating cortical granules (CG). Also, with PI/Hoechst immunostaining technique, the oocyte viability rate was shown to reduce in vitrified GV (56.0\%), when compared with fresh samples $(86.8 \%)$ from infertile cases. In addition, upon supplementation of IVM culture media with GDF-9, as an oocytesecreted growth factor, resulted in $66.1 \%$ of maturation rate and $55.3 \%$ of good embryo development. However, both stress and apoptosis related genes, as assessed by real time PCR, increased post vitrification of human immature oocytes. The differential expression of these genes profile may be usful to evaluate the outcome of these germ cells after cryopreservation. Furhter investigation should be aimed at optimizing the IVM methodology for patients with malignant diseases.

\section{I-39}

The evolutionary logics of networks in motion from microcircuits to selection of behaviour

Grillner S

Department of Neuroscience, Karolinska Institute, Stockholm

The basic features of the vertebrate nervous system are conserved throughout vertebrate phylogeny to a much higher 
degree than previously thought. In this lecture, we show that not only the organization of the different motor programs underlying eye, orienting, locomotor, and respiratory movements are similarly organized, but also that the basic structure of the forebrain engaged in the control of movement is conserved. In the lamprey," which diverged already 560 million years ago from the vertebrate line of evolution leading up to primates, the basic components of the basal ganglia are similar to those of mammals in considerable detail. Moreover, the properties of the synaptic input are similar as well as transmitters/peptides in the direct and indirect pathway throughout the basal ganglia. The membrane properties of the striatal projection neurons with D1 and D2 receptors, respectively, are also similar, as are those of the pallidal output neurons. Our evidence suggests that the basal ganglia can be subdivided into functional modules controlling different motor programs, like locomotion and eye movements. What has happened during evolution is that the number of modules has increased in parallel with a progressively more complex behavioral repertoire. For value-based decisions, the circuitry through the lateral habenulae to the dopaminergic modulator neurons is also conserved, as well as the relay inhibitory interneurons involved. The habenular input is from a pallidal glutamatergic nucleus in lamprey as well as mammals, and this nucleus in turn receives input from the striosomal compartment within striatum and also from pallium (cortex in mammals). Most recently we have shown that also the efferent projections from pallium (corresponding to the mammalian cortex) are organized in a similar way. This applies to the monosynaptic glutamatergic connections to striatum, thatlamus, tectum, reticulospinal neurons and the rostral spinal cord. All in all, the basic design of the forebrain, brainstem and spinal cord is similar throughout vertebrate phylogeny - and had already evolved at the time the lamprey diverged from the vertebrate line of evolution leading up to mammals and man.

\section{I-40}

New trends in biomedical imaging of erectile dysfunction

Pereira JA

Erectile dysfunction (ED) affects more than 300 million men worldwide and nearly $50 \%$ of men between ages 45 and 75 years1. ED is a multifactorial disease but penile arterial insufficiency $(\mathrm{PAI})$ is present in more than $55 \%$ of the cases 1 . $\mathrm{PAI}$ is believed to result from atherosclerotic disease of the erectile related arteries (ERAs)1. Endovascular revascularization (EVR) of male pelvic arteries, namely the common iliac and internal pudendal arteries has been suggested as a potential therapeutic alternative in PDE5i refractory ED due to PAI. Patient selection is paramount before EVR, excluding ED patients without PAI and including only those with EVR accessible PAI lesions. Until now, patient selection for EVR has been based on Digital Subtraction Angiography (DSA), considered the gold-standard for characterization of the male pelvic arteries. CT Angiography (CTA) is non-invasive and has shown promising preliminary results in the diagnosis and characterization of PAI-ED. In this presentation we will review the angiographic anatomy of the male pelvic arteries and the different patterns of penile vascularisation. We will also review CTA protocols and assess the technique's role in the evaluation of patients with ED.

\section{I-41}

\section{Peripheral nerve repair and regeneration}

\section{Geuna S}

Neuroscience Institute of the Cavalieri Ottolenghi Foundation \& Department of Clinical and Biological Sciences, University of Turin Faculty of Medicine, Orbassano, Italy

Although peripheral nerves have an intrinsic potential for spontaneously regenerating after trauma or disease, the clinical outcome in patients who suffered for a severe nerve lesion is often unsatisfactory. Recently much interest has been dedicated to the perspective of improving peripheral nerve repair and regeneration by means of tissue engineering and, similarly to many other fields of regenerative medicine, great expectations have risen within the general public. However, in spite of the scientific advancements, applications to the patients is still very limited and it appears that to optimize the strategy for the tissue engineering of the peripheral nerves in the clinical view, more basic science research is needed and neuroscientists have to strive for a new level of innovation which will bring together (in a multi-translational approach) different regenerative approaches. In this presentation, the basic concepts of repair and regeneration of peripheral nerves are introduced. In addition, a brief overview of the today's key strategies to peripheral nerve tissue engineering is carried out in order to throw a light on the most promising future perspectives in combining the different approaches for improving posttraumatic recovery in patients. Finally, examples of successful translational research in peripheral nerve repair are provided together with a critical discussion on some of the pitfalls which may arise in this intriguing scientific field.

\section{$1-42$}

\section{Role of integrin-interacting proteins in the control of} EMT in cancer and fibrosis

\section{Zhang $\mathrm{H}$}

Department of Anatomy, Histology and Embryology, School of Basic Medical Sciences, Peking University Health Science Center \# 38 Xue Yuan Avenue, Beijing 100191, China

Integrins are transmembrane proteins that link extracellular matrices with cytoskeleton and mediate a variety of biological functions including cell adhesion and migration as well as tumor cell invasion and metastasis. In this report, we identified 
a novel role of an integrin-interacting protein Kind-2 in the control tumor metastasis in human breast carcinoma via activation of canonical Wnt/TCF pathway. An array based systematic analyses indicated an overall initiation of tumor invasion and metastasis program including upregulation of key Wnt/TCF signaling targets TCF4, LEF1 and HOXB9 as well as epithelial-mesenchymal transition (EMT) regulatory transcriptional factors Snail, Slug, Twist and Zeb1. The Kind-2/beta-catenin signaling greatly accelerates tumor cell proliferation, migration, and invasion in vitro, and promotes tumor growth and lung-metastasis in mice, demonstrating a role of Kind-2 in cancer progression. On the other hand, we also indentified that Kind-2 is involved in the regulation of TGF- $\beta$ signaling and promotes renal interstitial fibrosis by inducing EMT. Depletion of Kind-2 in kidney reduces renal interstitial fibrosis in UUO mice including reduced collagen deposition and decreased $\alpha$-SMA and fibronectin expressions. Overall, our results indicate an important role of Kind-2 in the regulation of tumor metastasis and kidney fibrosis via an EMT program.

\section{I-43}

\section{Histopathological changes in animal models of ALS}

Ulupinar E

Department of Anatomy and Interdisciplinary Neuroscience, Faculty of Medicine, Eskisehir, Eskişehir Osmangazi University, Eskişehir, Turkey

Amyotrophic lateral sclerosis (ALS) is a debilitating neurodegenerative disorder for which there is no effective cure. While $5 \%$ to $10 \%$ of ALS patients have a family history (FALS), remaining patients have no clear family history and are considered as sporadic ALS (SALS). FALS and SALS are clinically indistinguishable displaying muscle weakness, atrophy, and spasticity as a consequence of the loss of both upper and lower motor neurons. Since studying a sporadic disease is very difficult, majority of the research focus on the genetic causes of FALS and the disease models begin with a mutated gene known to cause disease in humans. In some the rodent models, spontaneous or induced mutations of these genes might experimentally induce ALS. In other models, expression of mutated proteins is at low levels; therefore, the gene is often "overexpressed" to make more protein than usual, in order to speed up the disease process. Although no model is a perfect representation of the human disease, each of them offers distinct advantages and insights into disease pathology. In addition, these genetic rodent models play a crucial role in testing and selecting potential therapeutics that can be used to treat ALS and/or other motor neuron disorders. In this talk, I give a systematic overview of the most important genetic rodent models that show motor neuron degeneration by giving a special emphasize to histo-pathological changes. I also present our current research data from a new mouse model displaying progressive upper motor neuron degeneration. By discussing the value and limitations of these different models, I conclude that it remains a challenge to find more and better rodent models based on mutations in new genes causing ALS. Hopefully, in the near future, effective use of these animal models will provide a unique opportunity to tackle this incurable and fatal human disease both clinically and etiopathologically.

\section{I-44 \\ Novel regulators of autophagy and relevance to human diseases}

Gözüaçık D

Department of Molecular Biology, Genetics and Bioengineering Program, Sabancı University, Tuzla, Istanbul, Turkey

Autophagy is a cellular stress mechanism characterized by sequestration of bulk cytoplasm, proteins and organelles in double or multimembrane vesicles, and their delivery to and subsequent degradation by the cell's own lysosomal/vacuolar system. This biological phenomenon occurs at low basal levels in all cell types (from yeast to mammals) under non-deprived conditions, performing homeostatic functions like protein degradation and organelle turnover. Autophagy is rapidly upregulated under conditions leading to cellular stress like nutrient or growth factor deprivation, to provide an alternative source of intracellular building blocks and substrates for energy generation. Autophagy dysregulations play a critical role in the pathogenesis and progress of several human health problems, including neurodegenerative disorders (i.e. Parkinson's and Huntington's diseases), degenerative syndromes (i.e. IBMPFD), lysosomal storage disorders (i.e. Gaucher's disease) and cancer. In this speech, results from our recently published and unpublished studies will be presented. *This work was supported by The Scientific and Technological Research Council of Turkey (TUBITAK) 1001 Grant number: 112T272 and Sabancı University.

\section{I-45}

\section{Apoptosis: where it starts? Where it stops?}

\section{Vatansever S}

Department of Histology and Embryology, Faculty of Medicine, Celal Bayar University, Manisa, Turkey; Research Center of Experimental Health Science, Near East University, North Cyprus

Apoptosis or programmed cell death is very important mechanism in the embryonic development and tissue homoeostasis. Apoptosis is responsible for balancing cell proliferation and maintaining constant cell numbers in tissues undergoing turnover. In addition, during development, apoptosis plays a key role by eliminating unwanted cells from a variety of tissues. While apoptosis observe during normal embryonic development in most of the fetal tissue from blastocyt stage of embryos to birth, it is also triggered from several pathways to obtain integrity of the normal tissues. The fetal human ovary contains millions of germ cells, with a peak number observed at fifth month of pregnancy, however, up until birth, the number of germ cells decreases dramatically. In the ovary, the mechanisms underlying decisions of life or death involve interaction between pro-apoptotic and anti-apoptotic molecules. Apoptosis is found in ovarian 
follicles throughout fetal and adult life. During fetal life, apoptosis localized to the oocytes, whereas in adult life, it is detected in granulosa cells of secondary and antral follicles. Many of members of the Bcl-2 family have been isolated in the ovary. Bax, Bcl2 and Bcl-XL expression is highly restricted to the ovary. In addition, during organogenesis, apoptosis also allows proper development. Apoptosis observe destroying abnormal cells during cellular abnormalities clearing the cells from an injured kidney, brain, lung, bowel, cardiac muscle etc. or after ischemia of any tissues. Apoptosis is induced after chemotherapy and radiotherapy for eliminating the cancer cells. Cancer cells escape apoptotic cell death by avoiding the activation of upstream apoptotic signals and/or by upregulating of inhibitory factors in the apoptotic signal pathway. Many cellular factors involved in apoptotic signaling were discovered and their roles in the regulation of the apoptotic pathway have been elucidated. Apoptosis is initiated when the cells receive negative signaling, such as growth factor withdrawal, DNA damage by oxidants, ultraviolet light etc. Activation of apoptotic signaling is achieved by either an extrinsic or an intrinsic pathway. However, in different cellular condition, different apoptotic signaling molecules secrete and try to repair of the tissue. It is important that which signaling pathway is play a role in tissue, and also, different abnormal cells such as cancer cells can be evaluate with apoptotic pathway.

Keywords: Apoptosis, early embryonic development, organogenesis, tissue remodeling, cancer cells.

\section{I-46}

Initial mechanism of intimal fibrosis in saphenous vein with variocose disease. Immunohistochemical and morphometric quantifications and molecular oxidative stress evaluation

Juan PVM

Department of Anatomy, Histology and Neuroscience, Faculty of Medicine, Universidad Autónoma de Madrid, Spain

Chronic Venous Disease (CVD) is a common clinical event with a significant impact on both afflicted individuals and the health care system. A varicose vein affects more than 25 million adults in the United States. Histological features of varicose veins are intimal hypertrophy, subendothelial fibrosis, luminal dilation and a progressive vascular thickening. The aim of this study was the quantification of the immunohistochemical expression of alpha smooth muscle actin (SMA), collagen type I, collagen type III and the distribution of elastic fibers in the intimal layer. In addition we studied oxidative stress mechanisms that can be implied in the evolution of the varicose vein disease. 20 distal and proximal segments of saphenous veins from patients with CVD in CEAP grade 2 were studied. Expression at intimal level of SMA, collagen type I, collagen type III and elastic fibers were quantified by image analysis at histological level, comparing segments. These molecules and NOX-4, COX-2, mPGES y MAC3 were also studied by RT-PCR. Additionally, H2O2 production and NADPH oxidase activity was compared between both proximal and distal saphenous vein segments. Our histological study demonstrated differences between the proximal segment, the most injured segment, that showed a statistical higher expression of total SMA, collagen type I, collagen type III and elastic fibers than in distal segment. As soon as the intimal layer gets thickening, the proportion of SMA and elastic fibers decreased, but the proportion of collagen type I and collagen type III get increased compared to full intimal area. mRNA expression of SMA, collagen type I, collagen type III, elastin, NOX-4 and COX-2 is higher at proximal segment. The increased production $\mathrm{H} 2 \mathrm{O} 2$ and NADPH oxidase activity at proximal level with the upregulation of NOX-4 y COX-2 shown that oxidative stress mechanism could be implied in the fibrosis that occurs at first steps in varicose disease.

\section{I-47 \\ Cholinergic brainstem modulation of striatal circuits: multiple levels of synaptic integration}

Segovia JM

Center for Molecular and Behavioral Neuroscience, Rutgers University

In the brainstem, neurons of the pedunculopontine nucleus $(\mathrm{PPN})$ and the laterodorsal tegmental nucleus (LDT) have been involved in a variety of functions, ranging from the regulation of the waking state to the modulation of reward systems and locomotion. Their projections innervate several regions of the basal ganglia and thalamus following a topographical gradient. Using novel tracing technologies we have mapped the circuits that originate in the cholinergic neurons of PPN and LDT. We recently identified a direct cholinergic projection that innervates wide areas of the striatal complex, where PPN preferentially contact postsynaptic structures in the dorsolateral striatum and LDT mainly innervates the dorsomedial striatum and nucleus Accumbens. Furthermore, we found that the axon collaterals of the striatal-projecting cholinergic neurons innervate the dopaminergic midbrain and the thalamus, both of which in turn project to the striatum. The convergence of the direct and indirect innervation from the cholinergic brainstem in the striatal complex suggests a high level of integration of multimodal information that may shape how striatal circuits operate. I will be discussing our recent optogenetic studies that illustrate this novel neuromodulatory influence and its implications for the striatal function.

\section{I-48}

Teaching the anatomical dogma new tricks: challenging common knowledge - a few examples of common knowledge in anatomy that turned out to be inaccurate

Thomas RG

Texas Tech University Health Sciences Center Paul L. Foster School of Medicine, EI Paso, TX, USA

Science progresses through a process of building upon the accumulated knowledge of previous researchers. Dogma is defined as a principle or set of principles laid down by an 
authority as incontrovertibly true. In every branch of science, including anatomy, dogma exists, and it is our duty to be on constant vigil to challenge known truths that do not agree with what we observe. Four examples of anatomical dogma that turned out to be inaccurate are presented, two from the realm of embryology and two from gross anatomy. Current embryology texts explain the development of the blood supply of the gut as remodeling of a set of vitelline arteries arising from the dorsal aorta. Despite this common knowledge, however, hindgut arterial supply, the inferior mesenteric artery, arises by a different mechanism. Similarly, most gross anatomists know and teach a story of innervation of the lacrimal gland that involves a communicating branch of the zygomaticotemporal nerve carrying parasympathetic fibers to the lacrimal nerve, and then this nerve completing the course to reach the gland. Again, despite this pattern being shown in the majority of texts, it only occurs a third of the time. Anular pancreas is a very rare congenital anomaly, reported in from 1 in 1,000 to 1 in 10,000 individuals, and yet this very rare congenital anomaly is found in nearly $80 \%$ of embryology texts. On the other hand, abnormal development of the pancreatic ducts called pancreas divisum is found in approximately $10 \%$ of the population, and yet is mentioned in less than $20 \%$ of the embryology texts surveyed. The thoracic splanchnic nerves are commonly depicted as penetrating the diaphragm in three locations distributed craniocaudally. However, the thoracic splanchnic nerves actually usually penetrate the crura of the diaphragm together, passing through a single hiatus, to reach their preaortic ganglia targets.

\section{$1-49$}

3D cell culture platforms for optogenetic treatment of epilepsy

Sokullu E

Department of Bioengineering, Faculty of Engineering, Izmir Katip Çelebi University, İmir, Turkey

Optogenetics provides a possible alternative treatment for epilepsy by allowing for the reversible excitation and inhibition of neurons with millisecond time resolution using light-activated ion channels and pumps expressed in target cell populations. Cell activation can be achieved using channelrhodopsin2 (ChR2), a light-gated cation channel isolated from the algae Chlamydomonas reinhardtii that has been successfully expressed in mammalian neurons. After illumination with blue light, ChR2 opens to allow the passive movement of $\mathrm{Na}, \mathrm{H}, \mathrm{Ca}$ and $\mathrm{K}$ ions, causing depolarization of the cell membrane. The light-activated chloride pump, halorhodopsin ( $\mathrm{NpHR}$ ), that is naturally expressed by the halobacterium Natronomonas pharaonis, can cause membrane hyperpolarization and inhibition of action potential firing in neurons after exposure to yellow light. Given that seizure disorders result from excessive neuronal activity, common optogenetic strategies currently being investigated for the treat- ment of epilepsy are to inhibit excitatory neurons using $\mathrm{NpHR}$ or to excite inhibitory neurons using ChR2 that is selectively expressed in these cells.
Previously, NpHR expression in the hippocampal formation was shown to provide sufficient inhibition to curtailexcessive hyper-excitability induced by an electrical stimulus burst in organotypic slice cultures. Similarly, optical activation of NpHR in neurons at the site of an epileptic focus transduced using lentiviral gene delivery can attenuate electrographic seizures in a rodent model of focal neocortical epilepsy using open-loop optical stimulation paradigms. Closed-loop control using seizure detection algorithms to apply optical stimulation only at seizure onset has also been shown to be effective to suppress seizures either by temporarily inhibiting pyramidal neurons or through the activation of a sub-population of GABAergic inter-neurons [13-21]. These studies indicate that seizures can be aborted and hyper- excitability suppressed by optical stimulation that could induce either neural activation or inhibition. In this study we prepared Neuron encapsulated 3D - Gels. This platform counteract many difficulties with animal studies by using photopolymerized cell encapsulated structures to trap neurons in close proximity to electrodes, without inhibiting their growth. The use of 3D Gel encapsulation techniques minimizes the fabrication and scaling complexities, improving the device yield. In this study, 3D Gels will be fabricated on glass substrates, with a variety of electrical insulation materials (GELMA). Constant number of neurons are placed into the 3D-Gels with a pressure-driven micropipette. 3D gel environement composed by hydrogel and provide the neurites to extend out of the gels and form synaptic connections with their neighboring neurons. Each 3D gel structure will has its own electrode, which is platinized to increase its capacitance. Long-term cell survival rate is expected and the neurons inside the 3D-Gels will be stimulated, with both current and voltage pulses. Action potentials, both spontaneous and resulting from a current stimulus, will be recorded from neurons comprising the neural networks.

Keywords: Epilepsy, Optogenetic, IPS, 3D Cell Culture, Microelectrode Array

\section{I-50}

\section{Biotechnology in morphological sciences mapping the nervous system with CLARITY}

Şengül G

Department of Anatomy, Faculty of Medicine, Ege University, Izmir, Turkey

CLARITY is a newly-developed technique developed by Deisseroth's group in Stanford (K. Chung et al. Nature Methods 10:508-513, 2013), and is considered one of the most important advances for doing neuroanatomy. The method is used to transform an intact tissue into an optically transparent and permeable hydrogel-hybridized form that can undergo immunostaining, in situ hybridization, and high resolution 3-D imaging without damage to the sample. It is designed to provide visualization of long-range cellular projections for threedimensional tissue mapping of a variety of tissue types. Thus far, CLARITY has shown to be a sustainable method for intact tissue imaging of long-range projections, local circuit wiring, 
cellular relationships, subcellular structures, protein complexes, nucleic acids, and neurotransmitters. Research using CLARITY for the brain, and our first results revealing new information for the spinal cord anatomy will be presented.

\section{I-51}

\section{Body donation in Turkey}

\section{Şehirli ÜS}

Department of Anatomy, Faculty of Medicine, Marmara University, Istanbul, Turkey

The lack of cadaver in Turkey has been started to be since 1990s due to increase number of medical schools and students, and also the progress in funeral services by municipalities. In previous years, unclaimed bodies were the only source of cadaver in anatomy education. This problem made me to search the ethical and historical aspect of cadaver donation in other countries. The history of the transition from unclaimed bodies to donated bodies in the anatomy education in Europe has shown that the main solution to lack of cadaver is to increase the body donation. An oral presentation about how to solve cadaver problem in Turkey at National Anatomy Congress 2001 was made by myself. This was the first talk about the approach to cadaver donation in Turkish Anatomy Society meetings. In the following years, Turkish anatomists started to discuss how to increase body donation. Many interviews have took place at the media. The body donation information has put on the websites of many medical schools. The documents to be filled to make was prepared. The positive comments about body donation of theologists made great contribution to explain the religious aspect. The funeral ceremonies of the donated bodies were also released to press. A body donation campaign was carried out by Turkish Society of Anatomy and Clinical Anatomy. As a result of all these activities the awareness of the people has started to increase. Unfortunately the number of donated bodies is still not enough to meet the need of anatomy education in Turkey but the number of donation has been increasing year by year. It was not expected to solve all the problems all of a sudden because it is a long term solution.

\section{I-52}

Translational research, from cadaver to the operating room, what we have achieved so far

Loukas M, Tubbs S, Bhusnurmath S, Bhusnurmath B

Departments of Anatomy and Pathology, St. George's University, Grenada, West Indies

Translational research continues to be an under investigated area of science. One discipline that has had scant attention by those who practice translational research is gross and clinical anatomy. As a clinicians and anatomists the authors have experienced success with reverse and direct translational research model in various clinical settings, as well as across disciplines. This paradigm identifies surgical, pathological and clinical problems, which are further explored in the anatomy and pathology laboratory in order to investigate ways of answering these issues. The goal of such research is to improve patient care and improve clinical and surgical outcomes, which eventually will improve health care. The authors will review their 20-year experience with identifying common clinical, pathological and surgical issues and addressing their feasible solution. In addition, the authors will correlate translational research in inter and transdisciplinary fashion as well as it the applications in medical education.

\section{I-53}

\section{Using biomaterials and 3D culture systems to regulate in vitro cell morphology}

\section{Ürkmez AŞ}

Department of Bioengineering, Faculty of Engineering, Ege University, izmir, Turkey

Traditional two-dimensional (2D) cell culture system was a convenient way to study cell behavior in vitro, but it fails to mimic the in vivo cellular microenvironment in terms of cell-cell communications, cell-extracellular matrix (ECM) interactions, expression of cell surface receptors, cell proliferation characteristics, cell polarity, growth factor synthesis and cell differentiation characteristics, which all play key roles in cell morphology, behavior and functionality. Thus, to develop in vitro culture models and to investigate mammalian cell behavior for basic science, diagnostic and therapeutic purposes, use of different biomaterials and three dimensional (3D) cell culture systems are gaining importance. The extracellular matrix in mammalian tissues is a complex mixture containing glycoproteins, collagens and proteoglycans. This ECM forms structural framework that stabilizes tissues and provides mechanical support for cell attachment; and particularly plays important role in cell functionality and differentiation. 3D substrates used to mimic ECM must exhibit the right chemistry and microstructure on regulating mammalian cell morphology as well as the right mechanical and electrical properties. There are several techniques to produce 3D scaffolds, which need to be selected according to the properties of targeted tissue. Characterisation of cell behavior on these substrates is not trivial, since overwhelming majority of characterization techniques were designed to be used on petri dishes, slides or multi-well plates. High throughput assays are also designed for $2 \mathrm{D}$ culture systems. There is an urgent need to modify existing techniques and investigate new technologies to monitor cell behavior in $3 \mathrm{D}$. 


\section{Oral Presentations \\ (0-01 - 0-99)}

\section{0-01}

\section{A study of the patterns of digito-palmar dermatoglyphics} in Esan ethnic group of Edo State, Nigeria

\section{Ujaddughe $\mathrm{MO}$}

Ambrose Alli University, P.M.B. 14, Ekpoma, Edo State, Nigeria

This study was carried out to find out the possibility of a unique pattern of palm and fingerprints (Dermatoglyphics) of 192 adults of Esan origin who were at the time of the study residing in Esanland, in the central senatorial district of Edo State, Nigeria. The 192 subjects were selected via a multi-stage sampling technique from the fifty two (52) wards (making up five, five (5) Local Governments) that make up the study area. Their fingerprints were obtained using the Indian ink method, the prints obtained were analyzed for fingerprint types and the palms observed for the angles connecting the triradii at the roots of the fingers (a-index finger, b-middle finger, c-ring finger $\mathrm{d}$-small finger and t-the most proximal triradii in the palm) taken as atd, tad and tda angles. Using the student's t-test, chi square test and ANOVA (Analysis of variance) as statistical tools, the results obtained were analyzed. The results showed that loop pattern had the highest frequency of $61.7 \%$ followed by whorl with $24.9 \%$, the arch with $12.8 \%$ and then the double whorl $0.6 \%$. The mean atd angles were 43.49 for males and 44.02 for females, tad angles were 75.11 for males and $74.71 \%$ for females then tda were $61.22 \%$ for males and $61.35 \%$ females. This study therefore shows that there is no clear-cut Digito-palmer prints pattern for Esan people, it is rather in conformity with universal findings except for few uniqueness. However digito-palmer prints studies remain relevant in Anthropologic/Genetic studies.

Keywords: Dermatoglyphics, esan people, atd, tad and tda angles, palmer and finger prints

\section{0-02}

\section{Chlorella vulgaris alleviates lead-induced testicular toxicity better than Zingiber officinale: an ultrastructural study}

\section{Hesham NM}

Department of Anatomy, Faculty of Medicine, King Abdulaziz University, Jeddah, Saudi Arabia

Natural products are studied to combat reproductive alterations of Lead. The current work aim to disclose the efficacy of Chlorella vulgaris and Zingiber officinale to alleviate lead acetate induced toxicity. Sixty adult male Wistar rats were distributed into four groups. Group 1 was considered control, group 2 received $200 \mathrm{mg} / \mathrm{l} \mathrm{PbAc}$ water, group 3 received $10 \%$
C. vulgaris and $200 \mathrm{mg} / ; \mathrm{PbAc}$ water, and group 4 received 100 $\mathrm{mg} / \mathrm{kg}$ of $\mathrm{Z}$. officinale and $200 \mathrm{mg} / \mathrm{l} \mathrm{PbAc}$ water for 90 days. Testis samples were subjected to ultrastructural examination. It is observed that $\mathrm{PbAc}$ caused degenerative alterations in the spermatogenic series in many tubules, with a loss of germ cells and vacuoles inside the cytoplasm and between the germ cells. Mitochondria exhibited ballooning, with lost cristae and widening of the interstitial tissue, destructed nuclear envelopes of primary spermatocytes, and distortion of the axonemes of the mid-pieces of the sperms. With the treatment with C. vulgaris or Z. officinale, there were noticeable improvements in those modifications. It has concluded that both $\mathrm{C}$. vulgaris and Z. officinale represent convincing medicinal components that may be used to ameliorate testicular toxicity in those exposed to lead in daily life with superior potentials revealed by C. vulgaris due to its chelating action.

\section{0-03}

Effect of using ethylene glycol and DMSO as anti-freezing agents on the oocyte development to blastocyst in mice

Mahmoudi R

Cellular and Molecular Research Center, Yasuj University of Medical Sciences, Yasuj, Iran

Objectives: Cryopreservation of oocytes, which is an interesting procedure to conserve female gametes, is an essential part of reproductive biotechnology. The objective of the present study was to investigate the effects of exposure to combination of cryoprotectants and vitrification on immature mouse oocytes in presence or absence of cumulus cells.

Methods: Immature oocytes (germinal vesicles) with and without cumulus cells isolated from ovaries of normal mice (4-6W of age) were either vitrified in conventional straw using $15 \%$ ethylene glycol (EG),15\% dimethyl sulfoxide (DMSO) and $0.5 \mathrm{M}$ sucrose as vitrification solution or exposed to vitrification solution without subjected to liquid nitrogen. After warming, oocytes were investigated for nuclear maturation following in vitro maturation and for developmental capacity to blastocyst following in vitro fertilization. Fresh immature oocytes were used as the control group.

Results: Fertilization and development to blastocyst rates in vitrified oocytes with cumulus cells were significantly lower than control group $(\mathrm{p}<0.05)$, maturation rates in exposure groups were significantly lower than vitrified and control groups $(\mathrm{p}<0.05)$. Presence of cumulus cells in all experiments and control groups significantly increased fertilization rate than denuded oocytes $(\mathrm{p}<0.05)$. Also in vitrified groups survival 
rate of oocytes that vitrified with cumulus cells was significantly higher than denuded oocytes $(\mathrm{p}<0.05)$.

Conclusion: Germinal vesicle stage oocytes in presence or absence of cumulus cells can be vitrified successfully. Exposure to cryoprotectants can be decrease the developmental competence of GV oocytes. Presence of cumulus cells have positive effects on maturation and fertilization rate in IVF procedure.

Keywords: Germinal vesicle oocyte, cumulus cells, vitrification, cryoprotectants combination

\section{0-04}

\section{The morphology and haemodynamics of vessels in kidney with a single and aberrant renal arteries in norm and in hypertension}

Dgebuadze $M$

Department of Human Normal Anatomy, Tbilisi State Medical University, Tbilisi, Georgia

Till now debatable is the question about the role of multiple renal arteries in the development of hypertension and biological reliability of such kidney as a graft. Many authors highlight the importance of studying the function of both kidneys separately. Nowadays diagnostic tests for renal artery stenosis, performed for inpatients with hypertension, are based upon concept of the left-right differences in renal blood flow; however, it is still unclear, whether asymmetry of renal haemodynamics is an inborn phenomenon or a result of hypertension. The aim of the present work was to detect the peculiarities of morphology and haemodynamics of vessels in kidney with aberrant renal artery, as well as to study right and left renal blood flow in normo- and hypertensive patients. Using injection, histological, histochemical and morphometric methods superior segments of 12 autopsic kidneys with a single and aberrant renal arteries of healthy people at the age of I period of maturity (from 21 to 35 years of age) were investigated. Using Doppler renal investigation in color duplex scan mode and multislice computed tomographyc angiography, retrospective analysis of angiograms and dopplerograms of right and left renal vasculature (32 kidneys) of normoand hypertensive patients at the same age period were also performed. Our study has revealed evidences of hypovascularity in the segments of autopsic kidneys with aberrant renal artery: statistically significantly were decreased number of glomeruli, as well as mean volume of normal glomeruli; were marked atrophic, hypertrophied, partly or completely hyalinized glomeruli; in the wall of sclerotic and hyalinized vessels, as well as in the area of deposits of excessive amounts of connective tissue, was revealed increasing amount of glycoproteins; in renal medulla are observed avascular zones. In hypertensive patients internal diameter of renal artery is statistically significantly decreased in comparision with the normotensive patients $(6.48 \pm 0.04$ and $6.68 \pm 0.03 \mathrm{~mm}$ accordingly, $\mathrm{p}<0.002)$. Comparisson of multislice computed tomographic angiography data did not show any statistical significant differences between length of right and left renal arteries, as well as between diameters of these arteries. By comparision of results from Doppler investigation of right and left renal arteries asymmetry of renal hemodynamic parameters was not found neither in normotensive patients ( Vps - peak sys- tolic velocity: on right $-89 \pm 1.5$, on left $-91.8 \pm 1.7 \mathrm{~cm} / \mathrm{s}, \mathrm{p}>0.1$; Ved - end diastolic velocity: on right $-39 \pm 4.6$, on left $-41.6 \pm 5$ $\mathrm{cm} / \mathrm{s}, \mathrm{p}>0.1$ ), nor in hypertensive patients (RI-resistive index: on right $-0.6 \pm 0.009$, on left $-0.5 \pm 0.009, \mathrm{p}>0.1$; AT - acceleration time: on right $-0.08 \pm 0.05$, on left $-0.09 \pm 0.03$ second, $\mathrm{p}>0.1$ ). According to morphometric study of glomeruli of autopsic right and left kidneys no significant differences were found.

\section{0-05}

\section{Protective effects of thrombomodulin on microvascular permeability after subarachnoid hemorrhage in mouse model}

Yan J*, Xu T*, Zhang $\mathrm{WG}^{*}$, Yang $\mathrm{L}^{*}$, Zhang $\mathrm{Y}^{*}, \mathrm{Lu} \mathrm{JF}^{*}$, Han H.B**, Zhou CM**, Yan JH*

*Department of Anatomy and Histology, Faculty of Basic Medical Sciences, Peking University, Beijing, China; **Beijing Key Lab of Magnetic Resonance Imaging Technology, Beijing China

The enhanced vascular permeability is a major early brain injury following subarachnoid hemorrhage (SAH).However, its mechanism is not clear yet. In this work, we explored its potential mechanism and investigated the roles of thrombomodulin (TM) in maintaining microvascular integrity after SAH. SAH models were established in adult male ICR mice (28-32 g, n=185) by endovascular perforation. TM was immediately administered by femoral vein injection following SAH. The brain water content, Evans blue content and neurological functions were evaluated. Brain edema was also detected by Magnetic Resonance Imaging (MRI) (T2 map). The siRNA technique, enzyme linked immunosorbent assay (ELISA), immunofluorescence staining and western blotting were performed to explore the potential mechanism of TM treatment. The number of microthrombus in hippocampus microvessels was also recorded. TM significantly decreased brain water content and Evans blue content, alleviated brain edema and neurological deficits after SAH. The plasma concentration of activated protein $\mathrm{C}$ was increased after TM treatment. In addition, the levels of phospho-p38MAPK, phospho-p53, cleaved caspase-3, phospho-NF- $\mathrm{B}$ (p65) were markedly decreased. Additionally, the loss of VE-cadherin and Occludin (markers of vascular integrity) and the number of microthrombus in hippocampus were also reduced. Our results indicated that TM has protective effects on preserving microvascular integrity following SAH partly through preserving endothelial junction proteins and quenching apoptosis/inflammation in endothelial cells via blocking $\mathrm{p} 38 \mathrm{MAPK}-\mathrm{p} 53 / \mathrm{NF}-\mathrm{\kappa B}$ (p65) pathway.

Keywords: Microvascular permeability, mouse; subarachnoid hemorrhage, thrombomodulin

\section{0-06}

\section{Hypothalamic circuits in rapid stress response}

Sivukhina E, Jirikowski GF

Inst. Anatomie II, FSU Jena, Germany

The hypothalamo-neurohypophyseal system has a main role in maintenance of homeostasis and regulation of numerous adap- 
tive reactions e.g., response to stress in most mammalian species. The nonapeptides vasopressin and oxytocin are major hormones of this system. Magnocellular vasopressin is known as one of the main physiological regulators of water-electrolyte balance. In addition its role in a control of the hypothalamo-pituitary-adrenal axis has widely been described. The labor inducing and milk ejecting posterior lobe hormone oxytocin has complex actions in systemic and central stress response. The neuroendocrine basis of stress response includes activation of the hypothalamo-pituitary-adrenal axis. Corticotropin-releasing hormone, a central neurohormone of this system, is synthesized by parvocellular neurons of the paraventricular hypothalamic nucleus. The significant role of vasopressin, produced by the same neurons, in a complex mechanism upon stress has been postulated. Glucocorticoids - the end products of the hypothalamo-pituitary-adrenal axis - have both central and peripheral effects. Their availability to target tissues is mainly dependent on levels of corticosteroid-binding globulin. Intrinsic expression of this protein in different brain regions in neurons and glial cells has been recently demonstrated. Regulation of the hypothalamopituitary-adrenal axis and hypothalamo-neurohypophyseal system is highly complex. There may be a significant role of both systems in various chronic health issues in humans including psychiatric, autoimmune and cardiovascular pathologies.

\section{0-07}

\section{Neuroplastin 65 mediates cognitive functions via excitatory/inhibitory synapse imbalance and ERK signal pathway}

Amuti $\mathrm{S}^{* *}, \mathrm{Wu} \mathrm{S}^{* *}$, Liu L** Li H** Yuan $\mathrm{Q}^{*}$

*Department of Neurology, Tongji University Faculty of Medicine, Shanghai Tongji Hospital, China; **Department of Anatomy and Neurobiology, Tongji University Faculty of Medicine, Shanghai, China

Neuroplastin 65 (NP65) is a brain-specific glycoprotein component of synaptic membrane. It is predominantly located in the forebrain such as the cerebral cortex, amygdala and striatum and hippocampus. Previous studies show that NP65 is implicated in synaptic plasticity, so it was hypothesized to play a role in cognitive functions. To test this hypothesis, we generated NP65 knock-out (KO) mice and found that the null mice exhibited enhanced hippocampus-dependent learning and memory as manifested by Morris water maze test and stepthrough passive avoidance test, but showed anxiety-like behaviors as manifested by open field test and light/dark exploration test. In addition, molecular and cellular studies revealed several alterations including: (1) the enhanced ratio of excitatory to inhibitory synapses; (2) increased expression of NMDA receptors NR2A; (3) enhanced activation of ERK signaling; (4) increased astrocytes in KO mice; (5) lowered number of the mushroom- and bifurcate-shaped dendritic spines in NP65 KO mice. In addition, the maintenance of long-term potentiation (LTP) was impaired while the basal synaptic transmission was normal in KO hippocampal slices. Together, our findings suggest that NP65 may mediate cognitive functions.
0-08

Sexism and anatomy, as discerned in images of anatomy by medical students at Cardiff University and University of Paris Descartes

Morgan $\mathrm{S}^{*, * *}$, Plaisant $\mathrm{O}^{* * *}$, Lignier B ${ }^{* * * *}$, Moxham B*****

${ }^{*}$ Cardiff University, Cardiff, Wales, UK; **Cardiff Faculty of English, Communication and Philosophy, Cardiff University, Cardiff, Wales, UK; $* * *$ University of Paris Descartes, Sorbonne Paris Cité, Paris, France, $* * * *$ Laboratoire Evaclipsy, Université Paris Ouest Nanterre La Défense, Paris, France; $* * * * *$ Cardiff Faculty of Biosciences, Cardiff University, Cardiff, Wales, UK

Contemporary textbooks of anatomy and surface anatomy were evaluated to ascertain whether they are gender neutral. The evidence of this, and previous studies (Morgan et. al., 2014, J. Anat. 224, 352-365), suggests that, both in terms of imagery and text, many textbooks lack neutrality. To further investigate such matters, we provided second year medical students studying at Cardiff University $(n=293)$ and at the Sorbonne Cité Paris Université $(n=142)$ with a questionnaire inviting them to address the possibility that social/gender factors hinder the dispassionate representation of anatomy. Ethical approval was obtained from both Cardiff and Paris universities. The hypothesis tested is that medical students perceive a gender bias that is reflected in the anatomical images to which they are exposed. Our findings suggest that, while students recognise the importance of gender issues and do not wish to associate with sexism, most are unaware of the possible negative aspects of sexism within the culture of anatomy as discerned in anatomical imagery. In this respect, the findings do not support our hypothesis. We consequently recommend that teachers of anatomy and authors of anatomy textbooks should be aware of the possibility of adverse effects on professional matters relating to equality and diversity issues when using imagery. This study is being complemented by analyses of the reactions of professional anatomists and clinicians to anatomical imagery.

\section{0-09}

\section{Confocal microscopy analysis of granulovacuolar degeneration in transgenic mice}

\section{Köhler C, Dinekov M}

Institute II for Anatomy, University of Cologne, Cologne, Germany

Granulovacuolar degeneration (GVD) is a neurodegenerative change that appears as granule-containing vacuoles in the cytoplasm of neurons. Severe GVD in the hippocampus is a histopathological hallmark of Alzheimer's disease. Granulovacuolar inclusions are present in neurons together with hyperphosphorylated tau and can be labeled with markers of the activated unfolded protein response (UPR), a stress response that becomes activated by the accumulation of unfolded proteins in the endoplasmic reticulum. The relationship between GVD, tau pathology and the UPR is not well understood and only few models for GVD exist. We performed light microscopy immunostaining, immunofluorescence double labeling and confocal microscopy to study the presence of 
GVD and the UPR in transgenic pR5 mice that express human tau and in mice with an A,-plaque pathology. pR5 mice and non-transgenic littermates were analyzed at 5 weeks $(\mathrm{pR} 5: \mathrm{n}=3$, non-tg: $\mathrm{n}=3$ ), 3-4 months (pR5: $\mathrm{n}=3$, non-tg: $\mathrm{n}=3$ ), 8.5 months (pR5: $\mathrm{n}=6$, non-tg: $\mathrm{n}=3$ ), 18.5 months (pR5: $\mathrm{n}=8$, non-tg: $\mathrm{n}=6$ ), 24 months (pR5: $\mathrm{n}=5$, non-tg: $\mathrm{n}=5$ ), and 28 months (pR5: $\mathrm{n}=4$, non-tg: $n=2$ ) of age. Three APPSLxPS1mut mice with an A,plaque pathology were analyzed at 20 months of age. Neurons that developed an advanced stage of tau hyperphosphorylation and early tau fibrillary pathology in pR5 mice displayed inclusions with the morphology of GVD, but we rarely saw GVD in neurons with mature neurofibrillary tangles or early tau hyperphosphorylation. We confirmed the GVD nature of the granulovacuolar inclusions by immunolabeling with established GVD markers. In addition, the granulovacuolar inclusions in pR5 mice were labeled with markers of the activated UPR. We did not find neurons with GVD in mice with an A,-plaque pathology and only exceptionally in very old non-transgenic mice. These findings show that human tau transgenic mice develop GVD and suggest that GVD and the activated UPR accompany neurofibrillary tangle formation in pR5 mice.

\section{0-10}

The role of antibody receptor TSH (TRAb) and goiter size in Graves' disease patients in Padang, West Sumatra, Indonesia

Dwitya E

Department of Internal Medicine, Faculty of Medicine, Andalas University, Padang, Indonesia

Objectives: Graves' disease is an organ-specific autoimmune disease with several clinical manifestations such as hyperthyroidism, diffuse goitre, ophtalmopathy and dermopathy. Expression of thyroid stimulating hormone (TSH) receptor on the surface of thyroid cell plays pivotal role in regulation of thyroid hormone and thyroid gland growth. Thyroid overactivity in Graves' disease were caused by increasing of antibody receptor TSH (TRAb) from stimulation of Th2, thus increased thyroid hormone production, proliferation of thyroid gland, which later became goiter. Recent studies shown that goitre size may related with severity and prognosis of Graves' patients. Aim of this study was to investigated the correlation between antibody receptor TSH (TRAb) and goitre size in Graves' disease patients.

Methods: In this cross sectional study, we studied 30 patients with Graves' disease without any history of anti-thyroid medication. Serum were collected from patients to measure TSH receptor antibody using assay method. Goitre size were measured based on WHO classification (Grade I, II and III) and ultrasonography. Data analysis were using SPSS program version 20 (SPSS.Inc)

Results: Antibody receptor TSH (TRAb) was found increased in Graves' patients with mean serum TRAb $5.6 \pm 3.7 \mathrm{pg} / \mathrm{ml}$. Size of goitre by using WHO Classification were mostly found in grade II and III (40\% dan 36.7\%) while thyroid volume found less than 10 gram. From our analysis, there is no significant differences occured between level of TRAb with goitre size $(p>0.05)$.
Conclusion: There was no significant differences between antibody receptor TSH (TRAb) value and size of goitre in Graves' disease patients, nevertheless, further studies are required to compare the role of TRAb during and after treatment with thyroid enlargement.

Keywords: Graves' disease, TRAb, goitre size

\section{0-11}

Sca-1+Lin-CD117-Mesenchymal stem/stromal cells induce the generation of novel IRF8-controlled regulatory dendritic cells through notch-RBP-J signaling

$\underline{\text { Zhao RC}}^{*}$, Liu $\mathrm{X}^{* *}$, Ren $\mathrm{S}^{* *}$, Ge $\mathrm{C}^{* *}$, Cheng $\mathrm{K}^{* *}$, Zenke $\mathrm{M}^{* * *}$, Fan $\mathrm{E}^{* * * *}$, Keating $\mathrm{A}^{* * * * *}$

*Institute of Basic Medical Sciences Chinese Academy of Medical Sciences, Faculty of Basic Medicine Peking Union Medical College, Peking Union Medical College Hospital, Center of Excellence in Tissue Engineering Chinese Academy of Medical Sciences, Beijing, People's Republic of China; **/nstitute of Basic Medical Sciences Chinese Academy of Medical Sciences, Faculty of Basic Medicine Peking Union Medical College, Center of Excellence in Tissue Engineering Chinese Academy of Medical Sciences, Beijing, People's Republic of China; $* * *$ Department of Cell Biology, Institute For Biomedical Engineering, Rhenish-Westphalian Technical University, Aachen University Medical School, Aachen, Germany; $* * * *$ Beijing Huayuyunsheng Health Technology Co., Ltd. Beijing, People's Republic of China; $* * * *$ Princess Margaret Hospital, Institute of Biomaterials and Biomedical Engineering and Department of Medicine University of Toronto, Toronto, Canada

Mesenchymal stem/stromal cells (MSCs) can influence the destiny of hematopoietic stem/progenitor cells (HSCs) and exert broadly immunomodulatory effects on immune cells. However, how MSCs regulate the differentiation of regulatory dendritic cells (regDCs) from HSCs remains incompletely understood. In this study, we show that mouse bone marrow-derived Sca1(+)Lin(-)CD117(-) MSCs can drive HSCs to differentiate into a novel IFN regulatory factor (IRF)8-controlled regDC population (Sca(+) BM- MSC-driven DC [sBM-DCs]) when cocultured without exogenous cytokines. The Notch pathway plays a critical role in the generation of the sBM-DCs by controlling IRF8 expression in an RBP-J-dependent way. We observed a high level of $\mathrm{H} 3 \mathrm{~K} 27 \mathrm{me} 3$ methylation and a low level of $\mathrm{H} 3 \mathrm{~K} 4 \mathrm{me} 3$ methylation at the Irf8 promoter during sBM-DC induction. Importantly, infusion of sBM- DCs could alleviate colitis in mice with inflammatory bowel disease by inhibiting lymphocyte proliferation and increasing the numbers of CD4(+)CD25(+) regulatory T cells. Thus, these data infer a possible mechanism for the development of regDCs and further support the role of MSCs in treating immune disorders.

\section{0-12}

\section{The neuroprotective effect of a triazine derivative in an Alzheimer's rat model}

Hassanzadeh $\mathrm{G}^{*}$, Alipour $\mathrm{F}^{* *}$, Oryan $\mathrm{S}^{* *}$, Sharifzadeh $\mathrm{M}^{* * *}$, Karimzadeh $\mathrm{F}^{* * * *}$, Kafami $\mathrm{L}^{* * * * *}$, Irannejad $\mathrm{H}^{* * * * * *}$, Amini $M^{* * * * * *}$ 
*Department of Anatomy, Faculty of Medicine, Tehran University of Medical Sciences, Tehran, Iran; **Department of Animal Physiology, Faculty of Biological Sciences, Tehran University of Medical Sciences, Tehran, Iran; ***Department of Pharmacology and Toxicology, Faculty of Pharmacy, Tehran University of Medical Sciences, Tehran Iran; $* * * *$ Department of Neuroscience, Faculty of Advanced Medical Technology, Tehran University of Medical Sciences, Tehran, Iran; $\star * * * *$ Department of Pathobiology, Faculty of Medicine, Alborz University of Medical Sciences, Alborz, Iran; ******Department of Medicinal Chemistry, Faculty of Pharmacy, Mazandaran University of Medical Sciences, Mazandaran, Iran; $* * * * * * *$ Department of Medicinal Chemistry, Drug Design \& Development Research Center, Tehran University of Medical Sciences, Tehran, Iran

Introduction: Alzheimer's disease (AD) is the most prevalent neurodegenerative disorder. It is characterized by formation of amyloid plaques and neurofibrillary tangles in the brain, degeneration of the cholinergic neurons and neural cell death.

Objectives: This study was aimed to investigate the effect of a triazine derivative, $\mathrm{C} 16 \mathrm{H} 12 \mathrm{Cl} 2 \mathrm{~N} 3 \mathrm{~S}$, on learning in an Alzheimer's rat model.

Methods: Animals were divided into seven groups; each group contained seven animals. Control group: animals received no surgery and treatment; saline group: animals received normal saline after recovery; sham group: animals received 10\% DMSO after recovery; STZ group (Alzheimer's model) : animals received streptozotocin (STZ) in four and six days after recovery; T5, T10 and T15 groups: animals were treated with triazine derivative, $\mathrm{C} 16 \mathrm{H} 12 \mathrm{Cl} 2 \mathrm{~N} 3 \mathrm{~S}$, at doses of 5, 10 and $15 \mu \mathrm{M}$, respectively. All drugs were injected intracerebroventricular. The spatial learning and histological assessment were performed in all groups.

Results: Animals in STZ group had more deficits in spatial learning than the control group in Morris water maze. C16H12Cl2N3S improved spatial learning significantly compared to STZ group. The CA1 pyramidal layer thicknesses in STZ group were reduced significantly compared to control group. $\mathrm{C} 16 \mathrm{H} 12 \mathrm{Cl} 2 \mathrm{~N} 3 \mathrm{~S}$ increased the CA1 pyramidal layer thickness in T15 group compared to STZ group.

Conclusion: Current findings suggest $\mathrm{C} 16 \mathrm{H} 12 \mathrm{Cl} 2 \mathrm{~N} 3 \mathrm{~S}$ may have a protective effect on learning deficit and hippocampal structure in $\mathrm{AD}$.

Keywords: Triazine, learning, Alzheimer's disease

\section{0-13}

\section{The effect of atrovastatin on apoptosis of hippocampal CA1 pyramidal cells following transient global ischemia in male rat}

Faghani $\mathrm{M}^{*}$, Ejlali $\mathrm{F}^{*}$, Movaseghi $\mathrm{S}^{* *}$, Molladost $\mathrm{H}^{*}$

*Anatomical Sciences Department, Faculty of Medicine, Guilan University of Medical Sciences, Rasht, Iran; **Department of Anatomical Sciences, Faculty of Medicine, Islamic Azad University of Medical Sciences, Tehran, Iran

Introduction: CA1 hippocampus is one of the most sensitive parts of the brain to ischemia. Atorvastatin has antioxidants property and can prevent cell damage consequent cell death by omitting free radicals and component resulted from cell damage.
Objectives: Evaluating the protective effect of atorvastatin on hippocampal CA1 cells following transient global ischemia in male rat

Methods: This experimental study was conducted on a total of 24 male adult NMRI rats weighting 250-300 $\mathrm{g}$ with average age of 8-10 weeks. They were randomly divided into four groups: control, sham, vehicle and treatment. For production ischemia reperfusion model, common carotid artery in both side were blocked for 20 minute. First dose atorvastatin (10 $\mathrm{mg} / \mathrm{kg}$ ) in treatment group was administrated after 6 hours flowing ischemia reperfusion, then 24,48 and 72 hours intraperitoneal. After four days, all rats were killed, and the brain were dissected and processed for nissl histological staining. The numbers of CA1 healthy pyramidal cells were considered. The results were analyzed by analysis of variance (ANOVA) and Tukey's tests.

Results: Our data showed that there was no significant difference in number viable pyramidal cell and apoptotic cells in CA1hippocampus in sham and Atrovastatin treated groups but the number of viable pyramidal cell and apoptotic cells between ischemic group and sham group was significantly diffrent $(\mathrm{p}<0.05)$.

Conclusion: These finding reveals using atorvastatin 10 $\mathrm{mg} / \mathrm{kg}$ after transient ischemia reperfusion, decrease severity of damage and cell death in CA1hippocampus cells.

\section{0-14}

Quantification of cortical neurons: a neuroanatomical myth in neuroanatomy

Aguirre JFG ${ }^{*}$,** , Parra JED***, De Mejía FR**

* Universidad Autónoma De Manizales, Departamento De Ciencias Basicas Biológicas, Caldas, Colombia; **Universidad Autónoma De Manizales, Caldas, Colombia; $* * *$ Universidad De Caldas, Departamento De Ciencias Basicas, Universidad Autónoma De Manizales, Colombia

Objectives: The objective was to analyze whether there was a definite number of cerebral cortical neurons for humans.

Methods: Data was examined for the total number of brain neurons in the literature related to the field of neuroscience. This suggested that the human cerebral cortex has a large number of neurons whose value changes with the life cycle due to several factors that lead to have an indefinite number.

Results: an analysis based on the literature showed the main factors influencing the total number of cortical neurons such as neurogenesis, apoptosis during life cycle, gender and pathological conditions. The inconsistent assessment of the number of neurons in the human cortex has been discussed by several authors, emphasizing that the values that have been displayed in the literature are presumably uncertain and are based on the views of some authors or the conceptions of others without carrying out replicable scientific studies. The above described has exhibited variable and inconsistent values in different texts about this issue. It has been concluded that the total number of cortical neurons varies due to diverse factors that play a role in determining the number of neurons in the human cerebral cortex. 


\section{0-15}

Relationship between consentration of alkaline phosphatase with bone destruction in periodontal disease patients

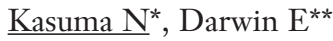

*Department of Oral Biology, Faculty of Dentistry University of Andalas, West Sumatera, Indonesia; **Department of Histology, Faculty of Medicine University of Andalas, West Sumatera, Indonesia

Periodontitis is a chronic inflammatory process which affect connective tissues surrounding the tooth (gums, periodontal ligaments,and alveolar bone) leading to attachment loss. Periodontitis may progress to bone destruction and tooth loss if it is left untreated. Clinical characteristics of periodontal disease include bleeding and friable gums, gingival recession, deepening pockets surrounding the tooth (indicating loss of anchoring attachments), and eventual tooth loosening. Alkaline phosphatase is a hydrolase enzyme, which is synthesized and secreted by polymorphonuclear neutrophils during inflamation and by osteoblast during bone formation and also by periodontal ligament fibroblast during periodontal regeneration. Creating a local bone environment of alkalinity to help bone mineralization. Acute infection causes bone destruction mechanism. Chronic periodontal inflammation increase levels of acid and alkaline phosphataseand by all products from bacteria and the destruction of tissues that support the teeth. When the inflammation spread along the transeptal fribres, it will shows a resorption of the alveolar bone crest. Due to the severity of the periodontal inflammation and bone turnover rate will increase ALP concentration. In severe periodontitis, the increasing bone turnover intensifies bone destruction by osteoclast. The purpose of this study is to examine the relationships between consentration of alkaline phosphatase with bone destruction in periodontal patients disease. This research involved 60 people with 20 healthy samples, 20 mild gingivitis samples, and 20 mild periodontitis samples. To see a normal distribution, Kolmogorov Smirnof Test is used ( $p>0.05)$. Post-hoc Bonferroni test is taken to test the differences each variables. Conclussion of this research is there are significant differences in the levels of Alkaline Phosphatase on the terms.

\section{0-16}

Effect of tryptophan on testosterone, estradiol and luteinizing hormone levels and on Leydig cells in male rats

Darwin $\mathrm{E}^{*}$, Putri A** Aryaneta $\mathrm{Y}^{* *}$

*Department of Histology, Faculty of Medicine, Andalas University, Padang, West Sumatra, Indonesia; **Biomedic Programe, Faculty of Medicine, Andalas University, Padang, West Sumatra, Indonesia

Introduction: Tryptophan is an essential amino acid found in many plant and animal proteins, that can be synthesized into serotonin and be converted to melatonin. Since tryptophan is the precursor of serotonin, its dietary amount has important effects on stress, mood, memory, and male sexual behavior. Melatonin is a hormone that regulates diurnal rhythms and influences the immune, gastro intestinal and reproductive systems.

Objectives: To determine the effect of tryptophan on testosterone, estradiol, luteinizing hormone levels and the number of Leydig cells in rats.
Methods: Male Rattus norvegicus were divided into four groups of seven rats, one served as control and three as treatment $(\mathrm{P})$ groups. The treatment groups were given 14 days intraperitoneal injection of 40, 50 and $60 \mathrm{mg} / \mathrm{kg} \mathrm{BW}$ tryptophan for groups P1, P2, and P3. Blood was collected at day 15 to determine the level of testosterone, estradiol, and luteinizing hormone, and testis were excised and processed histopathologically to determine the number of Leydig cells

Results: Testosterone level of P1, P2 and P3 were no difference from control $(12.95 \pm 1.55 \mathrm{nmol} / 1,11.03 \pm 0.54 \mathrm{nmol} / 1,13.57 \pm 1.79$ $\mathrm{nmol} / \mathrm{l}$ and $13.78 \pm 2.33 \mathrm{nmol} / \mathrm{l})$, respectively). Estradiol level was significantly higher in P2 than control $(10.17 \pm 0.85 \mathrm{pg} / \mathrm{dl}$ and $8.65 \pm 0.74 \mathrm{pg} / \mathrm{dl}$ respectively). Meanwhile, there was no difference between P1 and P3 from control $(9.87 \pm 1.01 \mathrm{pg} / \mathrm{dl}$, $8.08 \pm 0.53 \mathrm{pg} / \mathrm{dl}$ and and $8.65 \pm 0.74 \mathrm{pg} / \mathrm{dl}$ respectively). The level of luteinizing hormone was significantly lower in P1, P2 and P3 than control $(3.78 \pm 0.29 \mathrm{nmol} / \mathrm{l}, 3.32 \pm 0.35 \mathrm{nmol} / \mathrm{l}, 2.96 \pm 0.28$ $\mathrm{nmol} / \mathrm{l}$ and $5.60 \pm 0.30 \mathrm{nmol} / \mathrm{l}$ respectively). The number of Leydig cells was significantly lower in P3, but no difference between P1 and P2 from control $(17.66 \pm 0.81 \%, 21.00 \pm 1.09 \%$, $19.66 \pm 1.03 \%$, and $22.50 \pm 1.22 \%$ respectively).

Conclusion: Higher dose of tryptophan in the diet led to an increase of serotonin and melatonin, which led to an effect on the level of estradiol and luteinizing hormone. However, there were no effect on testosterone and on the number of Leydig cells.

\section{0-17 \\ Uric acid induces glomerulosclerosis, tubular injury and renal fibrosis through transforming growth factor, 1 elevation and fibroblast expansion}

\section{$\underline{\text { Romi MM}}^{*}$, Arfian N*, Tranggono U**, Sari DCR*}

*Department of Anatomy, Faculty of Medicine, Universitas Gadjah Mada, Yogyakarta, Indonesia; ** Department of Surgery, Faculty of Medicine, Universitas Gadjah Mada, Yogyakarta, Indonesia

Uric acid (UA) is an independent factor of cardiovascular diseases and induces renal damage. Transforming Growth Factor $\beta 1$ (TGF $\beta 1$ ) is well known as a profibrotic factor in kidney and associated with fibroblast expansion. Here, we elucidate TGF $\beta 1$ modulation of hyperuricemia induced renal fibrosis in mice. Hyperuricemia is induced in Swiss Background mice (3-4 month, 30-35 gram, $\mathrm{n}=21$ ) using intraperitoneal injection of $125 \mathrm{mg} / \mathrm{kg}$ of uric acid daily. $\mathrm{NaCl}$ injection was used in control mice. Mice were sacrificed in 7 (UA7) and 14 days (UA14) injection. Uric acid and creatinine serum is measured from retro-orbital blood serum before renal harvesting. Paraffin section is made, deparaffinized, then stained for Periodic Acid Schiff (PAS) and Sirius Red for glomerulosclerosis, tubular injury and fibrosis quantification. We extracted RNA and made cDNA, then run Reverse Transcriptase PCR (RT-PCR) for nephrine, podocine, MCP-1 and ICAM-1. PDGFR, immunostaining was done for quantification of fibroblast number. TGF, 1 was measured using ELISA. $\mathrm{p}<0.05$ was used as significant difference during data analysis. Injection of UA induced significant elevation of uric acid and creatinine level after 7 and 14 days followed by significant increase of glomerulosclerosis and tubular injury score in uric acid group compared to control $(\mathrm{p}<0.05)$. Both UA7 and UA14 groups also 
had higher fibrosis with significant increase of fibroblast cell number. Prolonged UA injection induced severe injury as shown by higher fibrosis and glomerulosclerosis in UA14 compare to UA7 group $(\mathrm{p}<0.05)$. RT-PCR measurement revealed reduction of nephrine and podocine ( $\mathrm{p}<0.05 \mathrm{vs}$ control) and up-regulation of MCP- 1 and ICAM-1 expression ( $\mathrm{p}<0.05$ vs control). UA7 and UA14 also had higher TGF,1 level compare to control $(\mathrm{p}<0.05)$. In conclusion, UA induced glomerulosclerosis, tubular injury and renal fibrosis with reduction of podocytes function and elevation of inflammatory mediators. TGF, 1 and fibroblast expansion might modulate hyperuricemia induced renal fibrosis.

\section{0-18}

The role of student surgical interest groups and surgical olympiads in anatomical and surgical undergraduate training in Russia

Dydykin $\mathrm{S}^{*}$, Kapitonova $\mathrm{M}^{* *}$

*Sechenov First Moscow State Medical University, Moscow, Russia; **MARA University Of Technology, Jalan Hospital, Selangor, Malaysia

Traditional department-based surgical interest groups for students in Russian medical schools have proven to be useful tools for student-based selection of specialty training. They also form a nucleus for initiating research activities among undergraduate students. In Russia, the Departments of Topographical Anatomy and Operative Surgery play an important role in initiating student-led research and providing learners with advanced, practical surgical skills. In tandem with department-led activities, student surgical interest groups prepare learners through surgical competitions, known as "Surgical Olympiads," which have been conducted in many Russian centers on a regular basis since 1988. Surgical Olympiads stimulate student interest in the development of surgical skills before graduation and encourage them to choose surgery as their postgraduate specialty. In student scientific interest groups, students have a chance to select their future specialty and begin practical skills training at the age of 19-20 years, rather than 24-25 years of age as in other countries. This arrangement allows students to master practical surgical skills at an earlier, more pliant age, which may result in higher quality surgical training in Russia Many of the participants in these surgical Olympiads have become highly qualified specialists in general surgery, orthopedic surgery, neurosurgery, urology, gynecology, and emergency medicine. The present article emphasizes the role of student interest groups and surgical Olympiads in clinical anatomical and surgical undergraduate training in Russia.

\section{0-19}

Maternal transplantation of epidermal neural crest stem cells provides prenatal therapy in entorhinal cortex of methylazoxymethanol exposed mice

\section{Omidi A, Kashani IR, Akbari M}

Tehran University of Medical Sciences, Tehran, Iran

Objectives: Malformations of cortical developmOents (MCDs) are one of the most usual causes of neurological dis- orders. The goal of the current study was to quantify the morphological changes in the entorhinal cortex in the mice that exposed in utero to methylazoxymethanol (MAM) and then received EPI-NCSCs prenatally.

Methods: The young mice were divided into four groups, each including 5 animals and following intervention was performed during in utero life: group I: control animals which received $\mathrm{NaCl}$; group II: sham animals which received vehicle; group III: MAM-exposed animals which received intraperitoneal (ip) injection of MAM (25 mg/kg maternal body weight); group IV: MAM-exposed animals which received EPI-NCSCs. 30 days after birth, the brain tissues were removed. Then cresyl violet staining and also immunohistochemistry for NeuN, a mature neuronal marker was performed. The thickness of entorhinal cortex, number and mean cell volume of this area of cortex were estimated using stereological methods.

Results: Remarkable reduction in the thickness of the entorhinal cortex and mean cell volume of mature neurons was observed following ip injection of MAM as compared to control mice. But the number of neurons in the entorhinal cortex was not statistically significant difference among groups. In prenatal stem cell- treated animals, there was a significant increase in the width of entorhinal cortex. Moreover, the mean cell volume of NeuN-immunostained cells was significantly higher in the entorhinal cortex of stem cell transplanted mice in comparison with those of MAM- exposed mice.

Conclusion: Prenatal administration of EPI-NCSCs prevents the reduction in the entorhinal thickness following in utero exposure to MAM. As a result, EPI-NCSCs may provide clinically applicable source of stem cell in transplantation treatment of MCDs.

Keywords: Malformation of cortical development, epidermal neural crest stem cells, entorhinal cortex

\section{0-20}

\section{Anatomy learning comics for the interested laypeople and students}

Chung MS, Chung BS

Department of Anatomy, Ajou University Faculty of Medicine, Suwon, Republic of Korea

The laypeople who are interested in their health as well as the students who are going to take the anatomy course are recommended to know anatomy at basic level. For them, the comics might be an optimum educational media, thanks to its familiarity and simplicity. However, existing comics, drawn by cartoonists ignorant of anatomy, are not proper for the target readers. The objective of this study was to promote the laypeople and students to understand anatomy without other's assistance. Based on the teaching experience for three decades, anatomy comics were elaborated in a simple style. Title of comics was named after two main characters, "Anna \& Tommy". The comics, which were arranged by the systems, delivered anatomical knowledge in concise and interesting manner. Because the comics were logical, memorable, and comprehensible, its educational effect was expected to be enhanced. Actually the evalua- 
tion by the medical students indicated the usefulness of the comics as a learning material. On the authors' homepage (anatomy.co.kr), the comics could be watched online or downloaded to be printed with no charge. Moreover, other entertaining anatomy comic strips (Dr. Anatophil), made by the same team, complemented the learning comics.

\section{0-21}

\section{Surgical anatomy of the left triangular ligament and 'fibrous appendix' of the liver}

Mirilas P, Demesticha T, Skandalakis P

Department of Anatomy and Surgical Anatomy, University of Athens Medical School, Athens, Greece

Objectives: To reexamine the left triangular ligament (LTL) and distinguish from the so-called 'fibrous appendix of the liver' or 'appendix fibrosa hepatis' (atrophied hepatic tissue at the left liver edge containing remnants of hepatic tissue).

Methods: Dissection, measurements and transillumination of LTL in 24 human cadavers (16 males and 8 females, 79+8.6, y.o). Histologic examination followed, by dividing LTL in proximal, middle, and distal parts.

Results: LTL had an average length of $11.2 \mathrm{~cm}$ (range 7-17.5 $\mathrm{cm}$ ), with no significant difference between genders. LTL was mostly triangular in shape, attached to the superior surface of the left liver edge, but also extending in its posterior (more often) or anterior surface. In transillumination, LTL adjacent to the liver contained liver-like tissue and vessels, then just vessels, while distally seemed empty. Histologic examination revealed that capillaries, arteries, veins and nerves were common findings in proximal, middle and distal LTL (24/25 specimens). The proximal part contained bile ducts in 20 specimens and liver tissue in 4 ; in contrast, these were absent in the middle and distal parts. No histo-morphologic distinction between proximal LTL and 'fibrous appendix' of the liver was possible.

Conclusions: We agree with others who did not report any 'fibrous appendix' of the liver; in truth, this is the proximal LTL. The latter commonly contains liver tissue and bile ducts, and the middle and distal LTL vessels. Therefore, LTL is of surgical significance in mobilization of left liver lobe. To avoid leakage, transection between ligations is advised, especially in proximal LTL.

\section{0-22}

\section{Radiological investigation of brain volume changes in} migraine disease

Palancı Ö**, Kalaycığglu A* , Eyüpoğlu I*, Çakmak VA****, Acer $\mathrm{N}^{* * * * *}$

*Department of Anatomy, Faculty of Medicine, Karadeniz Technical University, Trabzon, Turkey; **Department of Anatomy, Bayburt Vocational Faculty of Health Services, Bayburt University, Bayburt, Turkey; ***Department of Radiological, Faculty of Medicine, Karadeniz Technical University, Trabzon, Turkey; ****Department of Neurology, Faculty of Medicine, Karadeniz Technical University, Trabzon, Turkey; $* * * * *$ Department of Anatomy, Faculty of Medicine, Erciyes University, Kayseri, Turkey
Introduction: Migraine is a neurological disease which is characterized by headache attacks accompanied by neurological and gastrointestinal symptoms. In most of the cases neurological examination of migraine patient appears normal and Magnetic Resonance Imaging (MRI) shows no evidence that explain migraine headaches. In literature, there exist some studies that tried to observe volume changes of brain structures by using MRI. In these studies brain white matter and gray matter volume measurements take the first places.

Objectives: In those studies, there were no study that examine volumes of whole brain structures. In our study, we intended to compare volume measurements of migraine patients with control people by using brain parcellation with MR T1 images.

Methods: 6 migraine patients ( 2 males, 4 females $)$ and 6 controls (4 males, 2 females) with no neurological history are included in this study. Brain parcellation maps are prepared by using MriStudio software and then volumes of brain regions are obtained. Statistical analysis is done by using SPSS 15.0 version.

Results: We observed statistically significant volume decrease at right hypothalamus and right occipital sulcus in migraine patients compared to control group $(\mathrm{p}<0.05)$. In addition to these two brain region volumes, there appeared volume decrease also in other brain regons $(\mathrm{p}>0.05)$.

Conclusion: Using MriStudio we found evidence of structural some brain area changes in patients with migraine. As a matter of fack, a we should been in mind that, the number of subjects in the current study is relatively small and these findings need to be confirmed in future studies.

\section{0-23}

\section{Anthropometric characteristics of craniums in residents of Qazvin, Iran and Gazi Khan, Pakistan: a comparative study}

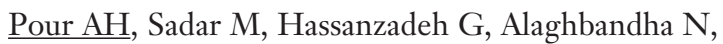
Dehbashipour A, Abbasi M, Zeidi OH

Department of Anatomy, Tehran University of Medical Sciences (TUMS), Tehran, Iran

Objectives: The purpose of present study is to compare anthropometric characteristics of the adult cranium between Iranian and Pakistani residents in two specific areas where people have special characteristics in the features of their heads and faces.

Methods: This cross-sectional investigation was performed on 300 adult inhabitants of Qazvin, Iran and 356 residents of Dera Ghazi Khan, Pakistan. Participants were selected randomly and did not have any physical deformities or any previous history of trauma. Measurements were performed in an anatomical position on the cephalic length, cephalic breadth, and auricular height.

Results: Cephalic length, cephalic breadth and auricular height in females from Qazvin were significantly higher than those of DG Khan $(\mathrm{p}<0.05)$. According to the findings, $82 \%$ of males and $58 \%$ of females from Qazvin were megacephalic 
while $25 \%$ of males and only $1 \%$ of females of DG Khan were megacephalic. The prominent cranium form in males from DG Khan was mesocephaly (41\%), whereas it was microcephaly (85\%) in females from DG Khan. The mean cephalic index was as follows: Qazvin males $(88.19 \pm 5.78)$ and females $(86.54 \pm 3.23)$; DG Khan males $(84.11 \pm 3.7)$ and females $(85.27 \pm 6.09)$. For DG Khan residents, the cranial capacity was $1348.4 \pm 122 \mathrm{~cm}^{3}$ for males and $1189.3 \pm 180.5 \mathrm{~cm}^{3}$ for females. At the same time, the brain weight was $1395.5 \pm 126.2 \mathrm{~g}$ in males and $1230.9 \pm 186.8 \mathrm{~g}$ in females.

Conclusion: This study shows the existence of differences in cranium dimensions between residents of Qazvin, Iran and DG Khan, Pakistan as well as in different regions of each country.

Keywords: Skull, anthropometry, Iran, Pakistan

\section{0-24}

\section{Anthropometric features of body index in natives of Qazvin, Iran}

Saberi K${ }^{*}$, Sabbaghziarani $F^{*}$, Hassanzadeh $\mathrm{G}^{*}$, Alaghbandha $\mathrm{N}^{* *}$, Dehbashipour $\mathrm{A}^{* *}$

*Department of Anatomy, Faculty of Medicine, Tehran University of Medical Science, Tehran, Iran; **Faculty of Medicine, Qazvin University of Medical Science, Qazvin, Iran

Introduction: The purpose of the present study was to evaluate anthropometric characteristics of weight, sitting \& standing height, cormic index and Body Mass Index(BMI) in the adult residents of Qazvin, Iran.

Methods: In this cross-sectional study, 300 adult inhabitants aged 18-55 years (180 males and 120 females) of Qazvin, Iran were evaluated. The participants were selected randomly and without any physical deformities or any previous history of trauma. Measurements were performed in an anatomical position.

Results: Their mean \pm SD weight was $72.64 \pm 11.03$ and $66.53 \pm$ $9.48 \mathrm{~kg}$, mean \pm SD standing height was $171.41 \pm 5.33$ and $158.24 \pm$ $5.2 \mathrm{~cm}$, mean \pm SD sitting height was $90.22 \pm 4.04$ and $86.24 \pm 2.45$ $\mathrm{cm}$, mean \pm SD cormic index was $52.51 \pm 2.07$ and $54.52 \pm 1.57 \mathrm{~cm}$ and mean \pm SD BMI was $24.67 \pm 3.2$ and $26.57 \pm 3.64$ in males and females, respectively.

Conclusion: The result of the present study showed that the mean dimensions of weight, sitting height and BMI parameters were higher than the most of other accomplished studies.

Keywords: Cormic Index, BMI, Anthropometry, Iran

\section{0-25}

\section{Determination of stature from forearm length in medical students}

Navid S, Mokhtari T, Hassanzadeh G

Tehran University of Medical Sciences, Tehran, Iran

Introduction: Stature is important for skeletal remains identification in unknown individuals. Body segments can be used for prediction of stature. In the present study, anthropometric relationships between forearm length and stature were evaluated between medical students.

Methods: This cross-sectional study was investigated on 100 medical students (50 males and 50 females and aged 19-25) of Tehran University of Medical Sciences. Participants were chosen randomly and they did not have any deformities or any previous history of disease. Measurements were performed in an anatomical position.

Results: The results showed that there was a significant relationship between the length of forearm and stature $(\mathrm{P}=0.0001)$. According to the results, the length of the forearm in the population was $26.63 \pm 2.89 \mathrm{~cm}$ and the length of males forearm $28.42 \pm 1.73 \mathrm{~cm}$ was longer than the length of females forearm $24.84 \pm 20.72 \mathrm{~cm}$. The correlation between the stature and forearm height was significant in the population $(\mathrm{r}=0.638$, $\mathrm{p}=0.0001)$, and in addition in males $(\mathrm{r}=0.427, \mathrm{p}=0.002)$. However, that was not significant in the females $(r=0.14$, $\mathrm{p}=0.325$ ).

Conclusion: The results showed that there was a reliable relationship between the length of forearm and stature between medical students and this relation was reliable in the males, too.

Keywords: Anthropometry, forearm, body segment, stature

\section{$0-26$}

\section{Implications of fibre orientation of the Achilles tendon on surgical rupture and repair}

\section{Blyth P, Sanders R}

Department of Anatomy, University of Otago, Dunedin, New Zealand

Objectives: The Achilles tendon is the most commonly ruptured tendon. Common descriptions portray simply a spiral arrangement of its fibres. Spiraling has implications for blood supply, diagnosis and surgical treatment. To further characterise this spiraling, a morphological study was undertaken.

Methods: 15 tendons from 12 embalmed cadavers, donated under the Human Tissue Act 2008, were excised. Tendons were cleaned and 11 predetermined $(5$ gastrocnemius and 6 soleus) fibres per specimen were identified and pins placed at $1 \mathrm{~cm}$ intervals using a standardized photographic setup. The protocol allowed for fibres that either sunk deep into the tendon or passed from anterior to posterior. Photographs were analysed, and pin location measured using ImageJ software. Fibre locations were normalised for tendon width, and the degree of spiraling calculated. Repeat measurements were performed.

Results: The mean gastrocnemius and posterior soleus fibre lengths were $190 \mathrm{~mm} \pm 40 \mathrm{~mm}$, and anterior soleus $83 \pm 39 \mathrm{~mm}$. The $>5000$ measurements revealed significant variation in spiraling between specimens. The most lateral fibres of anterior soleus had the largest range of spiraling of $226^{\circ}$ with a mean (range) of $68\left(0-226^{\circ}\right)$, The most lateral aspect of gastrocnemius had the smallest range of $90^{\circ}$, with a mean of $55^{\circ}$ (range 6-98). There was little consistency of fibre spiraling within par- 
ticular tendons, with specimen 10 having the closest range of spiraling $21^{\circ}$ to $88^{\circ}$.

Conclusion: This study shows the large amount of variation between and within tendons. No two tendons were similar enough to suggest a classification system that could be used to stratify risk of rupture. It was not possible to predict which quadrant of a tendon belonged to which muscle portion. Therefore imaging of partial tears must be able to track the fibre to the musculo- tendinous junction. Similarly it is not possible to give assurances of which portion of the tendon belongs to which muscle within the surgical field at repair.

Keywords: Achilles, orthopaedics, surgery

\section{0-27}

Time-course changes in PCNA and DCX expression in the juvenile and adult Japanese quail brains

Nkomozepi $\mathrm{P}^{*}$, Mazengenya $\mathrm{P}^{* *}$, Manger $\mathrm{P}^{* *}$, Ihunwoa**

*University of Johannesburg, Johannesburg, South Africa;

**University of the Witwatersrand, Johannesburg, South Africa

Neurogenesis is a multi-step process that involves progenitor cell proliferation, differentiation, migration and cell death. Most avian studies focused on the song system in songbirds. The objective of this study was to qualitatively and quantitatively assess changes in neurogenesis with post-hatching age in the Japanese quail brain. Cell proliferation and differentiation were investigated in the quail brains using immunohistochemistry for proliferating cell nuclear antigen (PCNA) and doublecortin (DCX) respectively. Quails were euthanized by an overdose of euthanase and perfusion fixed with $4 \%$ paraformaldehyde at post hatching ages 3-12 weeks. Brains were carefully removed, fixed overnight in $4 \%$ paraformaldehyde, cyroprotected in 30\% sucrose before being sectioned at $50 \mu \mathrm{m}$ on a cryostat at -22 ?C.Free-floating sections ( 1 in 5 series) were immunohistochemically processed for PNCA (1:500) and DCX (1:300). Another series of sections were Nissl stained for anatomical orientation. We report here preliminary qualitative results obtained from brains of juvenile (3-5 weeks) quails. PCNA-ir cells were observed mainly in the ventricular zones of the olfactory bulbs, telencephalic ventricular zones, cerebellum, periventricular zones of third and fourth ventricles and the optic tectum. In the olfactory bulb and telencephalic ventricular zones, PCNA-ir cells were mostly concentrated at the ventral and dorsal regions. DCX-ir cells were mostly concentrated in the olfactory bulb, parenchyma of the telencephalon, diencephalon, optic tectum and cerebellum. In the telencephalon, DCX-ir cells were scattered throughout the nidopallial, hyperpallial, mesopallial and entopallial areas but most frequently near the external surface. DCX-ir cells near the ventricular zones were mostly small and bipolar cells whereas those observed in the pallial areas were mostly multipolar. DCX-ir cells were also observed in the hippocampus and medial striatal areas. In conclusion, cell proliferation appears to be restricted to the ventricular zones whereas neuronal recruitment is more widespread in the telencephalon.

\section{0-28}

Broad distribution of LYVE-1-expressing endothelial cells and reticular cells with special reference to the reticulo-endothelial system (RES)

Zheng $M$, Kishimoto A, Iwanaga $T$

Hokkaido University Graduate Faculty of Medicine Laboratory of Histology and Cytology, Sapporo, Hokkaido, Japan

Objectives: LYVE-1, a receptor molecule for hyaluronic acid, is selectively expressed in the lymphatic endothelium, hepatic sinusoidal endothelial cells and some macrophage lineages. However, our preliminary examination showed more broad distribution of LYVE-1 in endothelial cells of several organs. The present study aimed to identify such LYVE-1-expressing endothelial cells at light and electron microscopic levels.

Methods: Mice were used and perfusion-fixed for immunohistochemistry using LYVE-1 antibody and F4/80 antibody. We carried out in situ hybridization analysis using 33P- or fluorescein-labeled oligonucleotide probes for LYVE-1 and hyaluronidase mRNAs. Although quantitative analysis was not done, images on X-ray films for mRNA expression gave useful information concerning the comparative intensity of expression.

Results: Immunohistochemical study revealed more broad distribution of LYVE-1 in the endothelium of the lung, adrenal gland, spleen, and heart of mice. In addition, reticular cells in the medulla of the lymph node intensely expressed LYVE-1. These cells are largely classified as reticulo-endothelial system (RES) for eliminating foreign particles. The LYVE-1-immunoreactive cells were topographically associated with a dense distribution of macrophages in each tissue: Kupffer cells, alveolar macrophages in the lung, macrophages within both sinusoidal lumen and parenchyma of the adrenal gland, macrophages in the splenic red pulp, and macrophages in auricular wall. Ultrastructually, the immunogold particles for LYVE-1 were localized on the plasma membrane of all cell types. Abundant expression of hyaluronidase mRNA was detectable in some organs possessing LYVE-1-expressing endothelial and reticular cells: the liver, lung, adrenal cortex, and medulla of the lymph node. However, macrophages but not RES cells were a predominant cell type for the expression of hyaluronidase, as shown in the lymph node.

Conclusion: LYVE-1 is a novel marker substance of RES. Function of the LYVE-1-expressing cells may be uptake of hyaluronate circulating in blood and lymph and subsequent degradation in relay with adjacent macrophages.

\section{0-29}

Volume calculation of midbrain structures in Parkinson's patients with Diffeomap software

Ocak H$^{*}$, Palancı $\mathrm{O}^{* *}$, Kalaycıŏglu A***, Acer $\mathrm{N}^{* * * *}$, Yeğinoglu $\mathrm{G}^{* * *}$

*Hacettepe University, Medical Faculty, Department of Anatomy, Ankara, Turkey; **Department of Anatomy, Bayburt Vocational Faculty of Health Services, Bayburt University, Bayburt, Turkey; $* * \star$ Department of Anatomy, Faculty of Medicine, Karadeniz Technical University, Trabzon, Turkey; **** Department of Anatomy; Faculty of Medicine, Erciyes University, Kayseri, Turkey 
Parkinson is a disease related with mainly dopamine decrease in some brain structures that result with some symptoms such as rigidity, bradikinesia, tremor and etc. Magnetic Resonance Imaging (MRI) is an imaging method which has been gaining more and more attention with development of new softwares and analyzing tools. In our study, we intended to compare volumes of midbrain structures in 5 Parkinson patients (1 female, 4 males) with 5 control people ( 1 female, 4 males) who have no neurological history. Processing of MRI data was performed by using Diffeomap Software. Brain parcellation maps were prepared with MriStudio Software. Then we obtained volumes of brain regions. Statistical analysis was done by using SPSS 15.0 version. We observed statistically significant volume decrease at red nucleus and cerebellar peduncles in Parkinson patients compared to control group $(\mathrm{p}<0.05)$. In addition to these two brain regions, there existed volume decrease also in other brain regions but this decrease was not statistically significant ( $>0.05)$. Evaluation of Parkinson and other neurological diseases will be easier as these types of studies are performed. For having more reliable results, more studies are required with larger data sets.

\section{0-30}

\section{Pre- and para-proliferative features of ductular reaction following biliary obstruction}

Makaridze D**, Kordzaia $M^{*}$, Kakabadze $M^{*}$, Kordzaia D*

${ }^{*}$ Al. Natishvili Institute of Morphology, Iv. Javakhishvili Tbilisi State University, Tbilisi, Georgia; ${ }^{*} /$ V. Javakhishvili Tbilisi State University, Tbilisi, Georgia

Recent studies confirm that Ductular reaction" (DR) in rodent livers, following common bile duct ligation (CBDL), except the "typical bile duct proliferation", contains "atypical" ones: "progenitor/stem cells proliferation" and "hepatocytes transdifferentiation into biliary epithelial cells". We studied interrelationship between typical-atypical components of DR considering CBDL terms. 54 male Wistar rats (150-200 g) subjected to CBDL were studied on 6th, 12 th hours and 2 nd -7 th days after intervention. Control and 8 target groups were created according to experimental terms; 4 rats' livers from each group were studied histologically, immunohistochemically (CK7, CK19, OV6, NCAM, AE1AE3 and Ki67) and by immunoblotting analyses. The microcorrosion casts of vascular and biliary systems of next 2 animals per each group were studied by Scanning Electron Microscopy. We revealed that early DR (1st-2nd days after CBDL) could not be result of proliferation: CBDL derived biliary pressure leads to widening subtle bile ductules manifested by appearance of new ductular profiles in different areas of liver lobules ("Pre-proliferative" stage). At 3rd-7th days the "Proliferative" stage of DR is manifested: cholangiocytes, hepatocytes, also stem/progenitor cells (but with lesser extent) proliferate. Proliferated cholangiocytes and hepatocytes participate in creating neoductules; proliferating progenitors differentiate to biliary cells lineage. Along with pre- and proliferative stages of DR, develops biliary transdifferentiation of hepatocytes, manifested on 12 th h and remaining at all studied terms from CBDL. In the first 24 hours processes coinside with significant increase of cytokeratins (37\%) with molecular weight 56 and $40 \mathrm{Kda}$ corresponding to cholangiocite-specific cytokeratins CK19 and CK7 ("Para-proliferative" stage). Thus, DR following CBDL is characterized by "Pre-proliferative", "Proliferative" and "Para-proliferative" features interfering in time. Ductular profiles revealed in different areas of liver lobules may be resulted from widening of existed ductules as well as developing of proliferation and/or transdifferentation processes; Intralobular biliary network could be considered as plastic and suitable to remodeling.

\section{0-31 \\ Histopathologic features of adverse reactions to facial dermal fillers}

Galuza $^{*}$, Markovs J*, Jursevic E**, Krumina D*, Knipse G*

*Department of Anatomy and Histology, Faculty of Medicine, University of Latvia, Riga, Latvia; ${ }^{* *}$ Clinic of Aesthetic Medicine, Riga, Latvia

Introduction: Over the past years, there has been an increase in number of biodegradable cosmetic dermal filler injections. Early or delayed side effects can occur with any dermal filler, but the role of immunocompetent cells in development of unfavorable results is not specified.

Objectives: To evaluate the facial tissue histopathological reactions after injections of biodegradable cosmetic fillers Restylane (hyaluronic acid), Radiesse (calcium hydroxylapatite $\mathrm{CaHA}$ ) and autologous fat (AF), during development of late side effects.

Methods: Tissues obtained during corrective surgeries 4-8 months after biodegradable filler injections for lip countouring purposes were histologically examined. Research included 12 patients who complained about long-term bruising, swelling, lip contour irregularity and nodules in lip mucosa. Tissue sections were stained with hematoxylin - eosin, Giemsa and immunohistochemically investigated for CD68, CD31 and CD4.

Results and Conclusions: Granulomatous inflammation plays important role in the case of late complications after injections of biodegradable fillers. In all 12 cases CD68 positive foreign body gigant cells (FBGC) and structural changes that indicates chronic inflammation - CD31 positive high endothelial venules and lymphoplasmacytic infiltrate - were found. After injections of Radiesse, histology slides demonstrated CaHA microspheres surrounded by epithelioid histiocytes, FBGC, lymphocytic infiltratrate and fibrous tissue. After injections of Restylane, FBGC with vacuoles and increased amount of mast cells were found, which indicates allergic nature of the inflammation. Increased amount of $\mathrm{CD} 4$ positive dendritic cells were observed in mucosa. That suggests activation of immunocompetent cells, which are not directly involved in formation of inflammation focus. Following $\mathrm{AF}$ injections lipogranulomas with numerous CD 68 and CD 31 positive lipophages and occasional FBGC around free lipids were observed. We believe that by placing too much AF is hindered grafts blood supply and additional free lipids will be released from necrotic adipocytes. If any filler's quantity exceeds macrophages ability to eliminate it, despite the biocompatibility, foreign body reaction will occur. 
S104 XXIV International Symposium on Morphological Sciences, 2nd-6th September, 2015, Istanbul, Turkey

\section{0-32}

Gross variations of groove for superior petrosal sinus in humans

Aguirre $\mathrm{JFG}^{* * *}$, Parra JED ${ }^{* * *}$

*Universidad Autónoma De Manizales, Departamento De Ciencias Basicas Biológicas; **Universidad Autónoma De Manizales, Caldas, Colombia; $* * *$ Departamento De Ciencias Básicas Biológicas. Universidad Autónoma De Manizales Departamento De Ciencias Básicas. Universidad De Caldas, Colombia; Departamento De Ciencias Básicas. Universidad De Manizales, Caldas, Colombia

Introduction: Classical anatomical descriptions considered the groove for the superior petrosal sinus, as a constant area, present on the upper edge of the petrous temporal bone. However anatomical scans allow us to infer that this anatomical region can present a wide variety in terms of their frequency, length and anatomical way.

Objectives: To describe the morphology of the groove for the superior petrosal sinus of human, identifying anatomic variations that may arise.

Methods: Gross anatomy of 48 adult human skulls was assessed, after which the groove is marked by dough molds, the length was measured and the respective photographic records were taken of the structure.

Results: Three varieties of presentation of the groove for the superior petrosal sinus were found Type I: lateral variety, with an overall frequency of $45.7 \%$, being this the most common. Type II: medial variety, with a frequency of $33.2 \%$. Type III: selection, if the groove takes a journey along the entire upper edge of the petrous temporal frequency of $14.6 \%$, there was finally no groove in $6.25 \%$ of cases. The groove for the superior petrosal sinus is a structure with a significant anatomical variation, which allows us to suggest indirectly, the variations that can present the superior petrosal sinus. The knowledge of these anatomical variations have relevance in the fields of neuroanatomy and neurosurgery.

\section{0-33}

Satellite glial cells promote regeneration of the nerve injury in vivo

Yi XN, Zhang QP, Zhao JH, Zhang X.F

Department of Anatomy, Hainan Medical College, Haikou, China

Objectives: To study the influence of satellite glial cells on nerve regeneration and the role of Slit1 protein in this process, in vivo.

Methods: Satellite glial cells were proliferated via drug stimulation. Sciatic nerve crush model, together with fluorescence gold retrograde tracer, immunofluorescence and thermal radiation experiment were used to observe the influence of satellite glial cells on nerve regeneration; the role of Slit1 protein in the process of nerve regeneration was detected via immunofluorescence and Western blotting.

Results: Satellite glial cells can be stimulated to proliferate by drugs in vivo; comparing with control group, the number of nerve fibers and the function of nerve regeneration were significantly increased at nerve injury $14 \mathrm{~d}, \mathrm{p}<0.05$; after nerve injury, Slit1 protein was increased, peaked at 14d; comparing with no injection of Slit1 protein, Injection of Slit1 protein can significantly promote the restoration of nerve fiber function, $\mathrm{p}<0.05$.

Conclusion: Satellite glial cells can promote nerve regeneration and Slit - Robo signaling pathways play a role in this process.

\section{0-34}

\section{Comparative effect of apple, date and balsamic vine-} gar's on liver histopathology in rats under high fat diet

Mohammadghasemi $F^{*}$, Abbasi $M^{* *}$, Aghajaninasab $M^{* * *}$, Atrkarroshan $Z^{* * * *}$, Rudkhaneei $\mathrm{K}^{* * * * *}$

*Cellular\&Molecular Research Center, Faculty of Medicine, Guilan University of Medical Sciences, Rasht, Iran; **Student Research Center, Guilan University of Medical Sciences, Rasht, Iran; $* * *$ Biochemistry Department, Faculty of Medicine, Guilan University of Medical Sciences, Rasht, Iran; ****Biostatistic Department, Faculty of Medicine, Guilan University of Medical Sciences, Rasht, Iran; $* * * * *$ Student Research Center, Guilan University of Medical Sciences, Rasht, Iran

Objectives: Obesity is considered as a risk factor for various chronic diseases. One of the ways for reducing of obesity is change in diet regime. We were aimed to Compare effect of apple, date and balsamic vinegar on obesity and liver histopathology in rats under high fat diet.

Methods: Male Wistar rats $(\mathrm{n}=40)$ were divided into 5 groups: control, high fat diet (HFD), HFD \& apple vinegar (AV), HFD $\&$ date vinegar (DV), HFD \& balsamic vinegar (BV). Control group received $16.6 \% \mathrm{Kcal}$ per day, and groups $2-5$ received high fat diet (HFD) $51.6 \%$ Kcal per day containing canola oil. After 4 months groups 3-5 in addition to HFD received apple, date or balsamic vinegar $(0.51 \mathrm{mg} / \mathrm{kg})$ for 6 weeks orally through their water. In the end of experiment rats were dissected and evaluations were made by photometry and histochemistry for study of serum liver enzymes of alanine aminotransferase (ALT), aspartate aminotransferase (AST) and alkaline phosphatase (ALP), serum glucose level and study of liver histopathology respectively. The data were analyzed with ANOVA and Tuckey post hoc test. A value of $\mathrm{p}<0.05$ was considered significant.

Results: All types of vinegars significantly reduced animals weight compared to HFD ( $\mathrm{p}<0.02)$. No significant differences were recorded in experimental groups in ALT level. Just apple vinegar reduced AST, ALP $(\mathrm{p}<0.05)$ and glucose level $(\mathrm{p}<0.01)$ significantly compared to HFD. However treatment with all kinds of vinegars in groups 3-5 significantly reduced fibrosis around central vein, steatosis and inflammatory cell numbers in liver tissue compared to HFD ( $\mathrm{p}<0.05)$.

Conclusion: This study showed that daily receiving of apple, date and balsamic vinegar with the same concentration induces weight loss and ameliorates histopathology of liver in obese rats. However Apple vinegar is more effective in comparison to date and balsamic vinegar. 


\section{0-35}

Morphometric evaluation of long bones of the Caspian horse by radiography

$\underline{\text { Nazhvani SD }}^{*, * *}$, Taravat $\mathrm{S}^{* *}$, Khafi SA**, Jalali $\mathrm{Y}^{* *}$, Hassanpour $\mathrm{A}^{* *}$

*Department of Surgery, Faculty of Veterinary Medicine, Shiraz University, Shiraz, Iran; ** Department of Surgery, Faculty of Veterinary Medicine, Shiraz University, Shiraz, Iran

Objectives: The Caspian horse is a small indigenous breed of north of Iran. A morphometric study was contacted to determine the size of different long bones by radiographic evaluation.

Methods: 10 Caspian horse of both sex (5 under 10 and 5 more than 10 years old) were used in this study. Radiographs were obtained from all the long bones in Anterior posterior and lateral view in standing position. The various dimensions of the bone were measured manually by ruler and cullies. The data was analyzed by Spss.

Results: The measurements of length, width of the tibia bone showed no significant difference between young and adult horses and also between anterior posterior and lateral radiographic views. Similar results were obtained from the other bones such as metatarsal bone, Radial bone, and Metacarpal bone.

Conclusion: In conclusion there was no differences between radiologic morphometric evaluation of the young and old Caspian horses. This horse is not a pony but a small breed of horse specific to the north of Iran, The Caspian area south of Caspian sea, therefore the clinical relevance of this morphometric study can be used for identification of this horse and it's authenticity.

Keywords: Caspian horse, morphometric study, long bone, radiography.

\section{0-36}

Innervation pattern of the masseter muscle by Sihler technique: a descriptive cadaver study

Hernandez JM, Hernandez R*, Lemus J*, Aguilar $W^{* *}$, Cordova $\mathrm{R}^{*}$, Sandoval $\mathrm{M}^{*}$, Rosa $\mathrm{A}^{*}$, Cisternas $\mathrm{M}^{*}$, Hernandez $\mathrm{A}^{*}$

*Human Anatomy Program, University of Chile, Santiago, Chile, **Human Anatomy and Developmental Biology Program, Faculty of Medicine, University of Chile Santiago, Santiago, Chile

Objectives: While it's true that messeter's muscle anatomy has been widely described in the classical anatomy texts, different variables underlying the muscle's innervation have not been precisely demonstrated yet The objective of this study is to characterize the innervation pattern of the masseter muscle given by the maseter nerve using the Sihler technique. This anatomical technique, different from the conventional, allows to evaluate the intramuscular nerve distribution in the three dimensions of space. This is possible since muscle tissue's transparency is achieved after violet staining of the intramuscular nerve branch- es, which allows an easy identification of neural structures without structural disruption caused by anatomical dissection. Innervation pattern of the masseter muscle is essential for understanding several clinical procedures, such as, facial paralysis surgery and botulinum toxin treatment.

Methods: Anatomical dissection of eigth masseter muscles in the right side of the parotid-masseteric region in preserved human cadaver was performed. The muscles were submitted to Sihler staining method and then observed under stereomicroscope and transillumination. A standardized photographic protocol was performed, arbitrarily dividing the muscle in 9 topographical zones for analysis, the pattern of innervation was characterized by computer software.

Results: The masseter muscle is innervated by a single main trunk corresponding to masseteric nerve, whose passage has a well-defined direction and sense, that is, from posterior to anterior and from superior to inferior between the surface and deep portion of the muscle. Additionally collateral branches that communicate within it, branching toward the terminal portion were observed.

Conclusion: There is a common pattern of innervation to the muscles studied, being small variations between them. The surgical approach suggested in the topographic zone $\mathrm{N}^{\mathrm{o}} 4$, since the total samples the main trunk passed through this region. In addition it is proposed as an ideal for the application of botulinum toxin the topographical zone $\mathrm{N}^{\circ} 5$ and 8 , because these areas are densely innervated.

\section{0-37}

\section{Quantitative morphology of the subcortical structures in ADHD using an atlas-based analysis: a diffusion tensor imaging study}

Acer $N^{*}$, Dolu N** Zararsı G***, Doğan M.S ${ }^{* * * *}$, Gümüş $\mathrm{K}^{* * * * *}$, Özmen $\mathrm{S}^{\star * * *}$, Kara $\mathrm{AY}^{* * * * * *}$, Per $\mathrm{H}^{* * * * * * *}$

${ }^{*}$ Department of Anatomy, Faculty of Medicine, Erciyes University, Kayseri, Turkey; **Department of Physiology, Faculty of Medicine, Erciyes University, Kayseri, Turkey; $* * \star$ Department of Biostatistics, Faculty of Medicine, Erciyes University, Kayseri, Turkey; ****Department of Radiology, Faculty of Medicine, Erciyes University, Kayseri, Turkey; $* * * * *$ Department of Biophysics, Faculty of Medicine, Erciyes University, Kayseri, Turkey; $* * * * *$ Department of Child Psychiatry, Faculty of Medicine, Erciyes University, Kayseri, Turkey; $* * * * * * \star$ Department of Pediatrics, Faculty of Medicine, Erciyes University, Kayseri, Turkey

Objectives: To examine brain diffusion characteristics in pediatric patients with attention deficit hyperactivity disorder (ADHD) using diffusion tensor imaging (DTI) and an atlasbased anatomical analysis of the whole brain and to investigate whether these images have unique characteristics that can support functional diagnoses.

Methods: The institutional ethic board approved this study. Seventeen children with ADHD and ten control subjects (all age-matched) underwent MRI scans. The written informed consent was obtained from the parents of each patient. Morphometric analysis was performed using MriStudio software. The diffusion images were normalized using a linear 
transformation, followed by large deformation diffeomorphic metric mapping. For 189 parcellated brain regions, the volume, fractional anisotropy (FA), mean diffusivity (MD), axial diffusivity (AD), and radial diffusivity (RD) were measured.

Results: Children with ADHD were found to have increase in the body of lateral ventricle volumes compared to the control. Increased MD was found in the deep gray matter, amygdala, thalamus, substantia nigra, and also the cerebellum left and right side. Increased RD was found in the deep gray matter, caudate, thalamus, substantia nigra and hippocampus left and right side compared to the control. Significant elevated FA were found in bilateral splenium of corpus callosum in ADHD patients.

Conclusion: Children with ADHD display abnormal diffusion characteristics and anatomical features compared to healthy controls. DTI can provide sensitive information on integrity of white matter (WM) and intra-WM structures in ADHD.

Keywords: ADHD, Diffusion tensor imaging, Atlas based analysis, MriStudio

This work is funded by the Scientific and Technological Research Council of Turkey (TUBITAK) 1002 Project (114S149).

\section{0-38}

Effect of forced swimming in different temperatures on ovary

Mohammadghasemi $F^{*}$, Ebrahimi $\mathrm{H}^{* *}$

*Cellular\&Molecular Research Center, Faculty Of Medicine, Guilan University of Medical Sciences, Rasth, Iran; **Student Research Center, Guilan University of Medical Sciences, Rasht, Iran

Objectives: Physical activity as a stressor inhibits female reproductive functions. We investigated whether water temperature and chronic swimming is effective on mouse ovary.

Methods: Adult female mouse $(\mathrm{N}=42)$ were randomly divided into 7 groups: 1 -controls 2 -cold water $\left(10^{\circ} \mathrm{C}\right)$ with swimming 3cold water $\left(10^{\circ} \mathrm{C}\right)$ without swimming 4 - luke warm water $\left(23^{\circ} \mathrm{C}\right)$ with swimming 5 - luke warm water $\left(23^{\circ} \mathrm{C}\right)$ without swimming 6warm water $\left(36^{\circ} \mathrm{C}\right)$ with swimming 7 - warm water $\left(36^{\circ} \mathrm{C}\right)$ without swimming. All animals in swimming groups were submitted to forced swimming in a pool for 5 minutes daily for 5 days/week during two weeks. However the groups of 3.5 and 7 were just standing on a raised platform inside the water in pool without swimming for the same time and duration. Ovarian tissue were evaluated for study of apoptosis using terminal deoxynucleotidyl transferase (TdT) dUTP nick-end labeling (TUNEL) assay. Serum FSH and estradiol level were assayed using an enzymelinked immunosorbent assay (ELISA). The data were analyzed with ANOVA and Tuckey post hoc test. A value of $\mathrm{p}<0.05$ was considered significant.

Results: In all experimental groups Serum FSH and estradiol level were reduced significantly compared with the controls $(\mathrm{p}<0.05)$ except in cold water $\left(10^{\circ} \mathrm{C}\right)$ and luke warm water $(23$ ${ }^{\circ} \mathrm{C}$ ) groups without swimming (groups $3 \& 5$ ). Apoptotic index in granulose cells in controls were a few $(3.9 \pm 0.02)$. Warm water with and without swimming increased significantly the index of apoptotic cells compared with the controls.
Conclusion: This study indicates chronic swimming in any temperature disturbs secretion of FSH and estradiol hormones in adult mice. Additionally warm water itself with or without swimming is associated with increased apoptotic index in granulose cells. These alterations may be associated with subfertility or infertility in female mice. Chronic immersion in cold and luke warm water itself has no adverse effects on abovementioned parameters in mouse ovary.

\section{0-39}

Carotid arteriography and jugular veinography in equine head

Nazhvani SD*,**, Hassanpour A**, Khafi AA**

*Department of Surgery, Faculty of Veterinary Medicine, Shiraz University, Shiraz, Iran; * Department of Surgery, Faculty of Veterinary Medicine, Shiraz University, Shiraz, Iran

Objectives: Angiography in normal state would reveal normal pattern of the vessels in an organ and the angiography in pathologic conditions would show the diseased situation of the vessels to detect abnormalities. The purpose of this study was to show the normal angiogram of the head and brain in the horse to be used for the future angiographic study of the brain or skull or head of the equine species.

Methods: Five horses were euthanized due to incurable disease conditions, their heads were collected fresh. The carotid artery and jugular vein were dissected and catheterized. Contrast media was injected and radiographs were obtained in lateral and dorsoventral positions.

Results: The arteriogram were remarkable and showed arterial supply of the different parts of the head, to name the auricular, palpebra, occular, lingual, linguofacial, facial, laryngeal, glandular, occipital and brain branches. There were vascular anastomoses such as major palatine arterial anastomose were clear on both sides.

Conclusion: Normal vascular angiogram is helpful to compare the diseased situation in pathologic conditions and to diagnose the abnormalities. There are fewer occasions for the use of cerebral angiography in the horse, but it is indicated as an aid to the differential diagnosis of some abnormalities of the central nervous system.

\section{0-40}

Effects of exogenous estrogen treatment on hippocampal neurogenesis of diabetic ovariectomized adult rats

Jameie $B^{*}$, Mehdinejadiani $S^{* *}$, Soleimani $M^{* * *}$, Kerdari $M^{*}$, Jalilipoor $\mathrm{M}^{* * * *}$, Hayat $\mathrm{P}^{* * * * *}$

*Department of Medical Basic Sciences, Faculty of Allied Medicine, Iran University of Medical Sciences, Tehran, Iran; ${ }^{* * D e p a r t m e n t}$ of Anatomy, Faculty of Medicine, Tehran University of Medical Sciences, Tehran, Iran; ${ }^{* * *}$ Department of Medical Basic Sciences, University of Social Welfare and Rehabilitation, Tehran, Iran; $* * * *$ Medical School, Tehran University of Medical Sciences; $* * * * *$ Cellular\&Molecular Research Center, IUMS, Tehran, Iran 
Objectives: Diabetes Mellitus (DM) is the most important metabolic diseases. The incidence of DM is prone to increase. Vasculopathy, retinopathy, central and peripheral neuropathy are the most important reported complications of disease. Cognitive dysfunction with sex dependent dominancy was also reported in DM. Regarding cognitive dysfunction in DM and with attention to the role of hippocampus in cognitive processing and its ability in neurogenesis it might be affected by DM. Neuroprotective effects of female sex steroids are reported elsewhere. In order to answer the question of whether female sex steroid are able to suppress the effects of DM on neurogenesis of dentate gyrus (DG) in diabetic ovariectomized rat hippocampus the present study designed.

Methods: Sprague-Dawley adult female rats were used in this study. The animals randomly was divided in 8 groups including; control, diabetic (Diab), ovariectomy (OVX), Diab+OVX, estrogen treated $(\mathrm{E} 2 ; \mathrm{Diab}+\mathrm{OVX}+\mathrm{E} 2)$, surgical and vehicle sham. Intrapritoneal injection of STZ, subcutaneous injection of E2 and routine bilateral surgery were used respectively to induce diabetes, estrogen treatment and OVX. Nissl staining, Brdu immunohistochemistry (IHC) and western blotting were used in this study. Statistical analysis was done and the results presented in mean $\pm S D, p<0.05$ considered significant.

Results: Brdu IHC showed that the neurogenesis significantly decreased in OVX, Diab and OVX-Diab groups $(\mathrm{Pv}<0.05)$ in comparison with control and sham groups. Western blotting showed significant increase of $\mathrm{Bax}$ and decrease of $\mathrm{Bcl} 2$ proteins of trial groups comparing to control. Estrogen treatment significantly improved neurogenesis in animals of Diab+OVX+E2 group. The neurogenesis impairment was more "sever" in OVX + Diab animals than OVX and Diab ones merely.

Conclusion: Based on our data, cognitive dysfunction caused by $\mathrm{DM}$ is related to hippocampal neurogenesis reduction and might improve under the influence of ovarian steroidal hormone therapy.

Keywords: Diabetes mellitus, ovarian steroidal hormones, cognitive function, neurogenesis

\section{0-41}

\section{Effects of total light deprivation on hippocampal neurogenesis and memory of adult rats: a sexual dimorphism study}

Jameie $B^{*}$, Moosavi $M^{* *}$, Soleimani $M^{* * *}$, Jalilipoor $M^{* * * *}$, Kerdari $\mathrm{M}^{\star * * * *}$, Mehraeein $\mathrm{F}^{* * * * * *}$, Nasirneghad $\mathrm{F}^{* * * * * *}$

*Department of Medical Basic Sciences, Faculty of Allied Medicine, Iran University of Medical Sciences, Tehran, Iran; ${ }^{* *}$ Department of Anatomy, Faculty of Medicine, Tehran University of Medical Sciences, Tehran, Iran; ***Department of Medical Basic Sciences, University of Social Welfare and Rehabilitation, Tehran, Iran; $* * * *$ Faculty of Medicine, Tehran University of Medical Sciences; ***** Department of Physiology, Faculty of Medicine, Iran University of Medical Sciences, Tehran, Iran C Sciences, Faculty of Allied Med, Iran University of Medical Sciences, Tehran, Iran; ******Department of Anatomy, Faculty of Medicine, Iran University of Medical Sciences, Tehran, Iran; $\star * \star * * * \star$ Department of Physiology, Faculty of Med, Iran University of Medical Sciences, Tehran, Iran
Objectives: Hippocampus is well-known for its role in memory processing and learning. It is also identified as a unique area in mammalian nervous system for its ability of neurogenesis. Many factors influence the neurogenesis process in hippocampus, subsequent deficiency in memory and learning may happen. Light is one of the factor that insert powerful effects on hippocampus structure and function. There might be sexual dimorphism in neurogenesis following certain intervention. Due to importance of neurogenesis, effect of light-dark cycle and also sex dependent differences of memory and learning deficiency in human cases, we decided to study the possible sex dependent neurogenesis under changes of dark-light cycle in hippocampus of adult rats that received two months of total light deprivation (2mTLD).

Methods: Forty male and female adult Wistar rats that received two months of TLD were used in this study. The animals randomly divided in four groups. To study possible sexual differences in neurogenesis male and female rats separated. TLD started one week prenatally and continued for seven weeks later. Nissl staining, BRDU IHC and Morris Water Maze (MWM) were used respectively for studying cell density, neurogenesis in dentate gyrus (DG) and evaluation of spatial memory. The results subjected to statistical analysis and presented in Mean \pm SD. The $(\mathrm{p}<0.05)$ considered significant.

Results: BRDU IHC showed significant decrease in neurogenesis following $2 \mathrm{~m}$ TLD in both sexes with more severity in male compared to female rats but not in animals of control groups. Results of Nissl staining and MWM also confirmed the Brdu findings.

Conclusion: Based on our findings we believe that sex dependent reduction in neurogenesis following TLD might be acceptable reason for sex dependent differences of cognitive disorders under similar situation.

Keywords: Neurogenesis, Sexual dimorphism, Light deprivation, steroid neuroprotection

\section{0-42}

Neuroethics: neuroscience for ethics or ethics for neuroscience

Jameie $\mathrm{B}^{*}$, Jameie $\mathrm{M}^{* *}$

*Department of Medical Basic Sciences, Faculty of Allied Medicine, Iran University of Medical Sciences, Tehran, Iran; *Department of Anatomy, Faculty of Medicine, Shahid Beheshty University of Medical Sciences, Tehran, Iran

In the late 1960s, a new field of philosophical and moral enquiry began to grow. Bioethics, as it soon came to be called, quickly expanded and developed its own association, journals, professional, conferences, degree programs and experts. Neuroethics, a recently modern field of bioethics is founded on the decades of challenges of ethical items related to mind and behavior. Generally speaking, neuroethics has two aspects; one is concerned with ethical, legal and social policy implications of neuroscience and another one is for neuroscience research itself. Rapidly growth of neuroscience in the last century put more demand on neuroscience to answer and explain the nature of many critical individual and social human behaviors especially for the ones 
which categorized as psychopathic behaviors that may need forensic and legal intervention. Increasing the rate of crimes in different societies has encountered neuroscience with the problem of responsibility and interpreting the nature of crimes, an intersection field between judgment and science. During the last recent years, Neuroscience, as a new field of study and research has been added to educational curriculum of some of the Iranian Anatomy Department and its graduates are gradually joining to academic and research centers. Despite this rapid growth, it seems that the place and importance of neuroethics is neglected and it needs more attention on this very important and implicational aspect of neuroscience. As conclusion, neuroethics should consider as the responsibility of neuroscience for human rights and society.

Keywords: Bioethics, neuroethics, social and individual behaviors, criminal behaviors

\section{0-43}

Neuroprotective effect of coenzyme Q10 in chronic constriction injury-induced neuropathic pain the rat

Jameie B* ${ }^{*}$, Janzadeh A**, Nasirneghad $F^{* *}$

*Department of Medical Basic Sciences, Faculty of Allied Medicine, Iran University of Medical Sciences, Tehran, Iran; ** Department of Physiology, Faculty of Medicine, Iran University of Medical Sciences, Tehran, Iran

Introduction: Oxidative stress contributes to neuropathic pain. Coenzyme Q10 (CoQ10) is used for the treatment of neurodegenerative diseases. However, there is no report regarding the therapeutic effect of CoQ10 on neuropathic pain.

Objectives: The present study investigated the effects of CoQ10 on pain alleviation in animals with chronic constriction injury (CCI) of the sciatic nerve.

Methods: Wistar male rats were randomly allocated to four groups. Nerve injury was induced by four loose ligatures around the sciatic nerve. CoQ10 (200 mg/kg) or vehicle were daily injected intraperitoneally for 10 days. Behavioral tests were performed before nerve injury and on fifth and tenth days after injury. Radiant heat, Randall Selitto test, and Von Frey filaments were used to assess the pain threshold. At the end of the experiment, the animals were perfused transcardially. Lumbar spinal cord was removed and prepared for Nissl staining. Analysis of variance (ANOVA) and Mann-Whitney were used for statistical analysis. P-value below 0.05 was considered statistically significant.

Results: Reduction in pain threshold and the number of neurons in dorsal horn of the spinal cord after nerve injury $(p \leq 0.001)$ were resulted. Injection of CoQ10 and vehicles increased the pain threshold $(\mathrm{p} \leq 0.001$ and $\mathrm{p} \leq 0.01)$ and prevented cell loss in neuropathic rats compared with injured animals with no treatment $(\mathrm{p}<0.05)$. Significant differences observed between animals that had received coQ10 compared with vehicle treated ones $(\mathrm{p}<0.05)$.

Conclusions: CoQ10 abolished neuropathic pain in CCI rats. Prevention of cell loss might be attributed to the analgesic effect of CoQ10. Hence, it might be used as a therapeutic intervention for neuropathic pain.

Keywords: Chronic constriction injury, sciatic nerve, pain; coenzyme Q10

\section{0-44}

The significance of dentition in maintaining the morphology of the human adult mandible

Hutchinson $E^{*}$, Farella $\mathrm{N}^{* *}$, Kramer B*

*Faculty of Anatomical Sciences, Faculty of Health Sciences, University of the Witwatersrand, Johannesburg, South Africa; **Discipline of Orthodontics, Department of Oral Sciences, Sir John Walsh Research Institute, Faculty of Dentistry, University of Otago, Dunedin, New Zealand

Edentulism negatively affects both the masticatory performance and the morphology of the mandible, influencing dietary intake and the general biomechanical loading on the edentulous bone. However, little is known about the focal areas of change in the human mandible during the transition from the dentate state to full edentulism. Thus this study investigated changes in the morphology of skeletonized human mandibles across the various states of the dentition, which included fully dentate, partially edentulous and edentulous mandibles. In total, 498 mandibles between the 20th and 80th years of life were sourced from the Raymond A. Dart Collection of Human Skeletons. A series of twelve linear measurements of the mandible were derived from digitized landmarks located on its external surface. Data analysis included conventional statistics namely a two-way ANOVA, MANOVA and MANCOVA as well as geometric morphometrics and comparisons across the various states of the dentition. The measures of the mandible significantly conformed to those of an edentulous individual, namely a short alveolar height $(-0.4 \mathrm{~mm}, \mathrm{p}<0.00)$ and mandibular body length $(-4 \mathrm{~mm}, \mathrm{p}<0.005)$ as well as an enlarged gonial angle $(+40, \mathrm{p}<0.001)$ when compared to dentate individuals. While the significantly obtuse mental angle $(+4.70, \mathrm{p}<0.000)$ and broader bicondylar width $(+3.8 \mathrm{~mm}, \mathrm{p}<0.000)$ conformed to the edentulous group, the taller ramus height $(+1.6 \mathrm{~mm}$, $\mathrm{p}<0.000)$ did not. The onset and progression of edentulism thus significantly alters the morphology of the mandible and hence will impact on its biomechanical functioning.

\section{0-45}

\section{Combined therapeutic effects of low power laser (980nm) \& CoQ10 on neuropathic pain in adult male rats}

Jameie $^{*}$, Masoumipoor $M^{*}$, Kerdari $M^{*}$, Soleimani $M^{* *}$

*Department of Medical Basic Sciences, Faculty of Allied Med, Iran University of Medical Sciences, Tehran, Iran; ${ }^{*}$ Department of Medical Basic Sciences, Faculty of Allied Med, University of Social Welfare and Rehabilitation, Tehran, Iran

Objectives: Neuropathic pain (NP) is one of the most suffering medical conditions that often fail to respond to certain pain therapy. Although its exact etiology is still unknown the role of reactive oxygen species (ROS) and oxidative stress were explored by many researchers. Neuropathies either central or peripheral lead to painful condition as well as social and economic isolation, thus various therapies were used to treat or reduce the pain. Laser therapy and antioxidant drugs have sep- 
arately considered as treatment for NP, but the combination of them have not been used yet. In order to study the combination effects of Low Level Laser Therapy (LLLT) and Coenzyme Q10 (CoQ10) the present study was designed.

Methods: Sixty adult male rats (230-320g) were used in this experimental study that divided into six groups $(\mathrm{n}=10)$. Chronic constriction injury (CCI) was used to induce neuropathic pain. The CoQ10 or vehicle, a low level laser of 980nm was used for two consecutive weeks. Thermal and mechanical paw withdrawal thresholds were assessed before and after surgery on 7th and 14th days.

Results: As we expected CCI decreased the pain threshold, whereas CoQ10 administration for two weeks increased mechanical and thermal threshold. The same results obtained for laser therapy using the CCI animals. Combination of laser 980nm with CoQ10 also showed significant differences in CCI animals.

Conclusion: Based on our findings the combination of CoQ10 with LLLT showed better effects than each one alone. In this regard we believe that there might be cellular and molecular synergism in simultaneous use of CoQ10 and LLLT on pain relief.

Keywords: Low level laser therapy, neuropathic pain, chronic constriction injury, coenzyme Q10

\section{0-46}

Effects of coenzyme Q10 on the ratio of $\mathrm{TH} 1 / \mathrm{TH} 2$ in experimental autoimmune encephalomyelitis model of multiple sclerosis in C57BL/6

$\underline{\text { Soleimani } M^{*}}$, Jamei $\mathrm{SB}^{* *}$, Barati $M^{* * *}$, Mehdizadeh $M^{* * * *}$, Kerdari $M^{* * * * *}$

*Department of Basic Sciences, University of Social Welfare and Rehabilitation Sciences, Tehran, Iran; **Department of Anatomy, Faculty of Medicine, Iran University of Medical Sciences, Tehran, Iran; ***Department of Pharmaceutical Biotechnology, Shahid Beheshti University of Medical Sciences, Tehran, Iran; $* * * *$ Cellular and Molecular Research Center, Iran University of Medical Sciences, Iran $* * * * *$ Department of Medical Basic Sciences, Faculty of Allied Medicine, Iran University of Medical Sciences, Tehran, Iran

Objectives: Multiple sclerosis (MS) is known as a progressive central nervous system inflammatory disease. Certain factors, such as interleukins, inflammatory cells, and oxidative stress are supposed to involve in MS etiology. Because of the important role of oxidative stress, antioxidant therapy for MS has received more attention. Although coenzyme Q10 (CoQ10) acts as an antioxidant, there is a lack of enough research on its effects on MS. Therefore, the present research was designed.

Methods: C57BL/6 female adult mice $(n=30)$ were used in this study. The animals were randomly divided into trial and control groups. To induce MS, routine procedure for experimental autoimmune encephalomyelitis (EAE) was used, and scoring was performed based on clinical signs. By detecting score one, CoQ10 administration was started $(10 \mathrm{mg} / \mathrm{kg} /$ three weeks). By using ELISA and real-time PCR, the brain levels of TNF- $\alpha$, IL-10, IL-4, and IL-12 were studied. Statistical tests were used to analyze the data and the Pvalue less than 0.05 was considered to be significant.
Results: Clinical symptoms in EAE animals were significantly decreased $(\mathrm{p}<0.05)$ as compared to control ones. In addition, the level of the TNF- $\alpha$ was significantly decreased following CoQ10 administration versus IL-10. The ratio of TH1/TH2 interleukins in treated animals was significantly less than that in non-treated animals $(\mathrm{p}<0.01)$.

Conclusion: Regarding the effect of CoQ10 on IL-4, IL-10, IL12, and TNF-. in EAE, our finding is similar to different studies. For example, Premkumar et al (2007) reported that CoQ10 suppressed the production of inflammatory mediators such as IL-6 that is similar to what we found for TNF- $\alpha$. Following CoQ10 administration, IL-4 (TH2) remained unchanged but the expression of IL-10 (TH2) was increased significantly. Our result regarding IL-10 was similar to what reported by Zhou et al (2007). Our findings showed that CoQ10 is capable of suppressing

\section{0-47}

\section{Vitamin D3 influence the Th1/Th2 ratio in C57BL/6 in model of experimental autoimmune encephalomyelitis}

Soleimani $\mathrm{M}^{*}$, Jameie $\mathrm{SB}^{* *}$, Keradi $\mathrm{M}^{* * *}$

*Department of Basic Sciences, University of Social Welfare And Rehabilitation Sciences, Tehran, Iran; **Department of Anatomy, Faculty of Medicine, IUMS, Tehran, Iran; ***Department of Medical Basic Sciences, Faculty of Allied Medicine, Iran University of Medical Sciences, Tehran, Iran

Objectives: Multiple Sclerosis (MS) is known as a progressive inflammatory disease in CNS. Cytokines belong to Th1 or Th2 family and inflammatory cells, play significant role in pathophysiology of MS. Thus any treatment supposed to influence the relation between Th1 to Th2 cytokines expression. Although it is so long time that vitamin D3 prescribed as a therapeutic supplement of MS, it is not clear that how much it may affect the Th1/Th2 ratio. In order to answer this question the present research designed.

Methods: Thirty C57BL/6 adult female mice were used. The animals randomly divided into trial and control groups. Experimental Autoimmune Encephalomyelitis (EAE) modeling for MS and clinical scoring as cited by others were used. Based on scoring and step of the disease vitamin D3 application $(5 \mathrm{mg} / \mathrm{kg}$ ) started and continued for three weeks.

Results: Levels of TNF- $\alpha$, IL-10, IL-4 and IL-12 in the brain were determined by using ELISA and RT-PCR. Significant decrease of clinical symptoms in trial group which received vitamin D3 was seen comparing to control animals $(\mathrm{p}<0.05)$. The level of the TNF- $\alpha$ but not IL-10 significantly decreased following vitamin D3 administration. By comparing the level of Th1 and Th2 Interleukins and counting the ratio of them we found that in treated animals the ratio was significantly less than non-treated $(\mathrm{p}=0.01)$.

Conclusion: Based on our findings, vitamin D3 could suppress the inflammatory process involve in MS, Whether it has clinical value needs more research in human clinical trials.

Keywords: EAE, vitamin D3, Th1/Th2 ratio 


\section{0-48}

Method of housing and transfer and experimental autoimmune encephalomyelitis: an experimental study on C57BL/6

Jameie SB${ }^{* * *}$, Soleimani $M^{* * *}$, Kerdari $M^{*}$

*Department of Medical Basic Sciences, Faculty of Allied Medicine, Iran University of Medical Sciences, Tehran, Iran; **Department of Anatomy, Faculty of Medicine, IUMS, Tehran, Iran; ***Department of Basic Sciences, University of Social Welfare and Rehabilitation Sciences, Tehran, Iran

Objectives: Multiple Sclerosis (MS) is an autoimmune inflammatory demyelinating disease of human central nervous system. Although experimental autoimmune encephalomyelitis (EAE) is the most commonly used method to induce MS, there are unexpected results in the modeling outcomes, which led to inappropriate clinical score scaling. Recent studies focused on the possible factors that may affect the final outcome of EAE modeling. Some of these factors were observed and discussed in our experiment on C57BL/6 model. The present research was carried out to find the possible effects of environmental factors, including transfer, handling, housing, and dark-light cycle on EAE modeling scoring.

Methods: Twenty female mice (C57BL/6) were used that divided into two groups $(n=10)$ by random. The routine method of MS induction in mammals was used in both groups. Following induction, animals of group one were placed in a separated room with the least local translocation and handling, whereas animals of the second group were placed in the same room as the other animals with normal local allocation as others. The animals were observed and scored using routine clinical scoring for EAE.

Results: Our data showed that the EAE induction in group one was significantly more successful than group two (with the mean score $>3$ ).

Conclusion: Although the EAE is still a scientific method to induce MS in rodents, it requires more attention to environmental factors that might influence the result. The mechanisms of these factors are unknown, but it seems that the role of housing environment should be take into consideration.

Keywords: Housing, experimental autoimmune encephalomyelitis, animal

\section{0-49}

Every anatomist can and should contribute to better anatomical nomenclature

$\underline{\text { Kachlik D }}^{*}$, Musil V**, Baca V*

*Department of Anatomy, Third Faculty of Medicine, Charles University in Prague, Praha, Czech Republic; **Department of Health Care Studies, College of Polytechnics Jihlava, Jihlava, Czech Republic **Centre of Scientific Information, Third Faculty of Medicine, Charles University in Prague, Czech Republic; **Institute of Information Studies and Librarianship, Faculty of Arts, Charles University in Prague, Czech Republic

Terminologia Anatomica was a huge step forward in the Latin anatomical nomenclature. From 1998 further effort was done by FCAT, FICAT but mainly by FIPAT (Federative International
Programme on Anatomical Terminology). 17 years after publication of the newest revision of the Latin anatomical nomenclature (TA) revealed several weak points of the nomenclature. They cover terminological (grammatical) discrepancies (in adjectives, in concordant and non-concordant attributes and in synonyms), anatomical discrepancies, multiplication of terms, abundant synonyms for one structure, identical terms for different structures. But the weakest point are missing terms, concerning systemic and topographic anatomy. This fact opens space for every anatomist to contribute to amelioration of TA. Any expert on certain anatomical structure can recommend the precise and easy terms of until now not officially denominated structures, providing an article or results of a study with short discussion to any member of FIPAT. This contribution should evoke such activity among all anatomist throughout the world. Supported by Charles University Grant 236070/IPUK/2015.

\section{0-50}

\section{Johannes Jessenius and his book on public dissection performed in 1600}

$\underline{\text { Kachlik D }}^{*}$, Musil V**, Baca $V^{*}$, Stingl J***

*Department of Anatomy, Third Faculty of Medicine, Charles University in Prague, Praha, Czech Republic; ${ }^{* *}$ Department of Health Care Studies, College of Polytechnics Jihlava, Jihlava, Czech Republic **Centre of Scientific Information, Third Faculty of Medicine, Charles University in Prague, Czech Republic; 4Institute of Information Studies and Librarianship, Faculty of Arts, Charles University in Prague, Czech Republic $* * *$ Department of Anatomy, Third Faculty of Medicine, Charles University in Prague, Praha, Czech Republic

Johannes Jessenius (Ján Jesenski) was an interesting and complicated personality of mannerism Central Europe. He was a polymath, physician, anatomist, teacher, philosopher and politician. He was born in Breslau (now Wroclaw in Poland) in 1566, his family stemming from Upper Hungary (now Slovakia), he studied in Germany (Leipzig, Wittenberg) and Italy (Padua). He was a dean and rector of the medical faculty in Wittenberg but then he met Tycho Brahe and craved for the emperor's court. In 1600, he performed a public dissection in Prague and published a detailed book describing it (Anatomiae, Pragae, anno M.D.C. abs se solenniter administratae historia (History of the Dissection Done By Him Solemnly in Prague A.D. 1600)" to gain the emperor's attention. Although he did not achieve his personal goals, he moved to Prague and worked as a mere physician, later as a court physician in Vienna. Finally he was twice elected rector of Prague university. Unfortunately, he joined the Czech protestant side in politics and after the battle at Bílá hora (8.11. 1620), the protestant side was defeated, Jessenius was arrested and beheaded (21.6. 1621).

\section{0-51}

Anatomy of the radial grooves for the first extensor compartment of the wrist on 3D reconstructed CT images

Gürses İA $^{*}$, Türkay $\mathrm{R}^{* *}$, İnci $\mathrm{E}^{* *}$, Örs $\mathrm{S}^{* *}$, Önal $\mathrm{Y}^{* * *}$, Özel $\mathrm{S}^{* * * *}$,Vural $\mathrm{M}^{* * * * *}$

*Department of Anatomy, istanbul Faculty of Medicine, istanbul University, Istanbul, Turkey; **Department of Radiology, Istanbul 
Bakırköy Dr. Sadi Konuk Training and Research Hospital, İstanbul, Turkey; ***Department of Radiology, Istanbul Faculty of Medicine, istanbul University, istanbul, Turkey; $* * * *$ Department of Biostatistics and Medical Informatics, Istanbul Faculty of Medicine, Istanbul University, Istanbul, Turkey; $* * * * *$ Istanbul Physical Medicine and Rehabilitation Training Hospital, İstanbul, Turkey

Objectives: De Quervain tenosynovitis affects the first extensor compartment of the wrist. Although the compartment is a fibro-osseous canal, presence of a septum and/or tendon variations are usually attributed as a cause for tenosynovitis. Recently, the osseous part of the compartment is gaining importance. We aimed to investigate the anatomy of tendon grooves at the distal radius with $\mathrm{CT}$.

Methods: We investigated wrists of 244 patients (72 women and 172 men) that undergone CT evaluation. We investigated the presence of a bony ridge at the floor of the compartment and measured the width of the groove on $3 \mathrm{D}$ reconstructed images.

Results: We observed a bony ridge (Type I) on 58 (23.8\%) and a single groove (Type II) on $186(76.2 \%)$ wrists. The average width was $12.52 \pm 1.3 \mathrm{~mm}$ for men and $11.5 \pm 1.4 \mathrm{~mm}$ for women, respectively. The width of the groove was significantly smaller in women. Type I groove was present on 24 (33.3\%) wrists among women and $34(19.8 \%)$ wrists among men. The presence of a Type I groove was statistically associated with women.

Conclusion: We found that a bony ridge was more frequent in women. This difference may have a role on the high frequency of de Quervain disease among women. The first extensor compartment is a fibro-osseous canal and knowledge on both aspects of this anatomic structure has importance in the management of de Quervain disease.

\section{0-52}

Right-left and sex dependant differences of hippocampus and amygdala sizes and their relation to the clinical symptoms in schizophrenics: a comparative brain segmentation study

Elfaki A* ${ }^{*}$ Ali TO**, Osman A***, Güdek MA*, Gölpınar M*, Şahin B*

*Department of Anatomy, Faculty of Medicine, Ondokuz Mayıs University, Samsun, Turkey; **Department of Anatomy, Faculty of Medicine, National Ribat University, Burri 55 Khartoum, Sudan; $* * \star$ Department of Psychiatry, Faculty of Medicine, International University of Africa, Khartoum, Sudan

Introduction: The hippocampus and amygdala are believed to be central to the cognitive deficits associated with the Schizophrenia.

Objectives: In the present study, the volumes and volume fractions of the hippocampus and amygdala have been examined depending on the right and left sides and sex in the schizophrenics and control. We also evaluated the relation between the quantitative data and their relation to the clinical symptoms.

Methods: 88 control and 57 schizophrenics were participated in the study. Structural magnetic resonance imaging was performed and the DICOM images were evaluated using auto- matic brain segmentation software (BrainSuite). The volume and volume fraction of the region of interest were evaluated.

Results: The mean volumes of right hippocampus and total hippocampi $\left(2.07\right.$ and $\left.3.83 \mathrm{~cm}^{3}\right)$ were smaller in schizophrenics than that of the controls $\left(2.30\right.$ and $\left.4.25 \mathrm{~cm}^{3}\right),(\mathrm{p} \leq 0.05)$. The mean volume of right amygdala was also smaller in schizophrenics when compared to the controls $\left(0.57\right.$ and $\left.0.65 \mathrm{~cm}^{3}\right)$, $(\mathrm{p} \leq 0.05)$. There were no differences for the other volumes. Female patients tend to have significant negative correlation between; the volume of hippocampus and negative symptoms and the volume of amygdala and positive symptoms $(\mathrm{p}<0.05)$.

Conclusion: There is a significant association between chronic schizophrenic female and reduced volume in the right hippocampus and amygdala. Our findings indicate that the size of hippocampus and amygdala shows sex and side differences in the schizophrenics.

\section{0-53}

\section{Mirtazapine affects the ion channels and neuropeptides levels in diabetic neuropathy}

Bektur NE, Şahin E, Dönmez DB, Baycu C

Department of Histology and Embryology, Faculty of Medicine, Eskişehir Osmangazi University, Eskişehir, Turkey

Type 1 diabetes mellitus is a form of diabetes that results from autoimmune destruction of insulin- producing $\beta$-cells. Diabetic neuropathies are nerve damaging disorders associated with diabetes mellitus. Despite significant advances in glucose monitoring and insulin therapy, people with both type 1 and type 2 diabetes still spend significant portions of the day with hyperglycemia, placing them at risk for the development of diabetic complications including peripheral diabetic neuropathy. A new generation of safer antidepressants with a better tolerability and fewer side effects are increasingly used for treating chronic pain. Mirtazapine is a nonadrenergic and specific serotonergic antidepressant drug. However the mechanism of the analgesic properties of antidepressants are poorly understood (Shaul et al. 2002). We aim that how mirtazapine affect the ion channels and neuropeptides levels in diabetic neuropathy. So $55 \mathrm{mg} / \mathrm{kg}$ streptozotocin (STZ) in 0,1 M citrate buffer injected the rats (Xiaofeng et al. 2012) and 4 weeks later, $20 \mathrm{mg} / \mathrm{kg}$ mirtazapine administrated by oral gavage for 2 weeks. We quantified mechanical and thermal nociception using thermal plantar, cold plate and RandallSelitto Paw Pressure Test. We isolated lumbal dorsal root ganglions and medulla spinalis and expressions in the spinal cord and DRG were quantified with western blot and immunohistochemistry analysis. Our results show that the pain thresholds are decreased in diabetic neuropathy, exhibited both mechanical and thermal hypersensitivity (Zhang et al., 2013). Levels of ASIC1, TRPV1, STT, NPY and SP levels, which are increased by diabetic neuropathy (Khomula et al., 2013), are restored by mirtazapine in DRG and medulla spinalis. Mirtazapine administration to the diabetic neuropathy may be worth considering as a new candidate for the treatment of diabetic neuropathy. 


\section{0-54}

Effects of streptozotocin induced diabetes mellitus on hippocampal volume in rats

$\underline{\text { Onur } S^{*}}$, Akdoğan I*, Yonguç GN**, Özdemir MB*

*Department of Anatomy, Faculty of Medicine, Pamukkale University, Denizli, Turkey; **Department of Anatomy, Faculty of Medicine, Dokuz Eylül University, Denizli, Turkey

Diabetes Mellitus is a chronic disease that affects many organs and it causes neuronal damage in central nervous system. Also hippocampus is one of these structures. The aim of this study is to evaluate hippocampal volume alterions in streptozotocin induced diabetic rats. In this experiment, we used 18 adult Wistar rats, 4 months old, were divided in to three groups: Control (C), Sham (S) and Diabetes (D). In group D, diabetes was induced by intraperitoneally injection of $60 \mathrm{mg} / \mathrm{kg}$ streptozotocin dissolved in saline. In group D, at the 3 rd and 10th days after streptozotocin injection, blood glucose levels of rats were above $300 \mathrm{mg} / \mathrm{dl}$. Six rats were selected randomly from each group and decapitated. Hippocampal volume in rats was estimated by Cavalieri Method that a stereological method. Left hippocampal volumes estimated in C group $2.26 \mathrm{~mm}$ ?, S group $2.24 \mathrm{~mm}^{3}$, D group $1.73 \mathrm{~mm}^{3}$ meanly. Right hippocampal volumes estimated in $\mathrm{C}$ group 2.26 $\mathrm{mm}^{3}$, S group $2.17 \mathrm{~mm}^{3}$, D group $1.73 \mathrm{~mm}^{3}$ meanly. The results showed that in D group the hippocampal volume where less than $\mathrm{C}$ and $\mathrm{S}$ group both left and right hippocampal volumes $(\mathrm{p}<0.05)$. According to the present results, it has been shown to reduce hippocampal volume in streptozotocin induced diabetic rats.

Keywords: Hippocampal volume, streptozotocin, diabetes mellitus, stereology, Cavalieri method

\section{0-55}

\section{Association between the number of pregnancies and bone mineral density in postmenopausal women: is less better?}

\section{Gürlek YS ${ }^{* *}$, Gürlek B*** Kalaycioğlu A ${ }^{*}$}

*Department of Anatomy, Karadeniz Technical University Faculty of Medicine, Trabzon, Turkey; **Department of Anatomy, Karadeniz Technical University Institute of Health Sciences, Trabzon, Turkey; ***ŞAR Hospital Gynecology and Obstetrics Clinic, Rize, Turkey

Objectives: The aim of the study was to analyze the bone mineral density of postmenopausal women and elucidate the association between the number of pregnancies and postpartum breast-feeding with bone mineral density.

Methods: The study was included 121 postmenopausal individuals aged between 47-80. Participants with metabolic diseases (Turner syndrome, anorexia nervosa, hiponadizm, Cushing's syndrome, inflammatory bowel disease ), on treatment for osteoporosis (Vitamin D, hormone replacement therapy, etidronate, alendronate, risedronate on, bisphosphonates such as ibandronate, selective estrogen receptor modulators, strontium ranelate, calcitonin and fluorides) and who has a history of previous hysterectomy were excluded. Bone mineral density was measured with Dual-Energy X-Ray Absorptiometry from L1L4 vertebras. OneWay ANOVA test was used to determine the association between the number of pregnancy and the bone mineral density T-Scores. PostHocTests and Tukey HSD were used to find the group that causes the difference. Participants were divided into three groups (0-2 children, 3-5 children and 6+ children) based on number of completed pregnancies.

Results: There was 28 patients in $6+$ group, 67 patients 3-5 group and 26 patients in $0-2$ group respectively. Our results demonstrated that $6+$ pregnancies group has significantly lower bone mineral density T-Score than both 3-5 pregnancy group $(\mathrm{p}=0.014)$ and $0-2$ pregnancy group $(\mathrm{p}=0.001)$. There was no difference between the groups who has $3-5$ and $0-2$ pregnancies $(\mathrm{p}>0.05)$

Conclusion: Participants who has more than 6 children have significantly lower T-Scores than the other groups. This can be secondary to various factors such as hormonal changes during the pregnancy and the loss of minerals during breast-feeding period. Our results showed that number of pregnancies is another important factor that has a critical impact on bone strength and should be considered for screening postmenopausal osteoporosis.

Keywords: Postmenopausal osteoporosis, bone mineral density, pregnancy

\section{0-56}

\section{The effect of growth hormone on the 6-OHDA model of Parkinson's disease}

Kirazli Ö$^{*}$, Arman A**, Gülhan R***, Özkanm*, Şehirli ÜS*

*Department of Anatomy, Faculty of Medicine, Marmara University, Istanbul, Turkey; **Department of Medical Genetics, Faculty of Medicine, Marmara University, Istanbul, Turkey; $* * *$ Department of Pharmacology and Clinic Pharmacology, Faculty of Medicine, Marmara University, Istanbul, Turkey

Parkinson's disease (PD) is a progressive neurodegenerative disorder characterized by motor and cognitive dysfunction, mood disturbances mainly due to the loss of dopamine neurons in nigrostriatal pathway. Growth hormone (GH) shows functions through GHR. Both GH and GHR exist in different regions of the brain. This shows that GH-GHR signaling may play an important role for function of the brain. In recent studies, in animals and humans, a positive effect of GH has been shown on brain reparation following brain injury. In this study we examined the effect of GH treatment in 6-hydroxydopamine (6OHDA) model of PD. Adult male Sprague-Dawley rats $(n=8)$ were used in the experiments. Following right medial forebrain bundle (MFB) injection of 6-OHDA, animals were divided in saline control $(n=4)$ and $\mathrm{GH}$ treatment $(n=4)$ groups and they received GH $(0.15 \mathrm{mg} / \mathrm{kg} /$ day, s.c.) or saline for 3 weeks. Motor behavior of the animals was tested by apomorphine $(0.25 \mathrm{mg} / \mathrm{kg}$, i.p.) rotation test at the end of 3 weeks. Subsequently, the rats were deeply anesthetized and transcardiacly perfused to remove brains for immunohistochemistry staining. Thyrosine hydroxylase $(\mathrm{TH})$ positive neurons were semi-quantitavely evaluated in all animals. Following 3 weeks of GH or saline treatment, rotational behavior of $\mathrm{GH}$ treatment group was dramatically decreased compared to control saline group (1.4 turn/min versus $9.9 \mathrm{turn} / \mathrm{min}$ ). In accordance with the rotational data, unilateral 
MFB lesions of 6-OHDA resulted in significant loss of TH immunoreactivity in the striatum of the control group. In contrast, the GH treatment group did not show a homologous loss of TH immunoreactivity in the striatum. Early GH treatment showed a neuroprotective effect in 6-OHDA model of PD, prevented loss of dopaminergic innervation in the striatum and corrected motor impairment of the animals. Further studies are needed to evaluate its long term effects.

\section{0-57}

Differentiation of bone marrow mesenchymal stem cells to germ cells by sertoli cells conditioned medium

Rastegar T*${ }^{*}$, Hajian $M^{*}$, Baazm $M^{* *}$

*Department of Anatomical Sciences, Faculty of Medicine, Tehran University of Medical Science, Tehran, Iran; **Department of Anatomical Sciences, Faculty of Medicine, Arak University of Medical Science, Tehran, Iran

Objectives: Sertoli cells are responsible for differentiation of germ stem cells to spermatozoa by secreting some factors in vivo. Mesenchymal stem cells could differentiate into germ cells under appropriate conditions. This study investigated differentiation of bone marrow derived mesenchymal stem cells (BMSCs) to germ line cells using Sertoli cells conditioned medium.

Methods: Mesenchymal stem cells were isolated from the bone marrow of adult male mice and cultured for 7 days until $80 \%$ confluence. On the other hand, Sertoli cells were enriched using lectin coated plates, cultured for 48 hours and collected medium. Mesenchymal stem cells cultured using Sertoli cells conditioned medium.

Results: We have found that conditioned medium prepared from adult sertoli cells supports the differentiation of BMSCs into putative germ cells. BMSCs cultured with Sertoli cells condition medium, expression of meiotic and post-meiotic genes was observed (Mvh, ID4, Piwil2) by RT-PCR and protein expression of germ-cell-specific marker Scp3 identified by immunocytochemistry. Gel Electrophoresis of PCR showed expression Mvh, ID4, Piwil2 transcripts in BMSCs differentiated to germ like cells while expression of these molecular marker didn't observed in control cells ( $\mathrm{p} \leq 0.05)$.

Conclusion: Sertoli cells conditioned medium may allow induction of BMSCs to germ cells for treatment of male infertility.

Keywords: Bone marrow mesenchymal stem cells, sertoli cell conditioned medium, differentiation, germ cell

\section{0-58}

Protective effect of pentoxifylline on male Wistar rat testicular germ cell apoptosis induced by 3.4-methylenedioxymethamphetamine

Sharifi $Z^{*}$, Movassaghi $S^{* *}$, Nouri $M^{* * *}$

*Department of Anatomy, Faculty of Medicine, Tehran Medical Sciences Branch, Islamic Azad University, Tehran, Iran; **Department of Anatomy, Faculty of Medicine, Tehran Medical Sciences Branch, Islamic Azad University, Tehran, Iran; ***Department of Medical Sciences, Shahrood Branch, Islamic Azad University, Shahrood, Iran

Objectives: 3.4 methylenedioxymethamphetamine (MDMA) is one of the methamphetamine derivatives that is prevalent in young individuals. A growing number of young people are consumers of drugs such as MDMA which can affect their reproductive capability. Apoptosis is a main mechanism for male infertility. Pentoxifylline (PTX) increases cAMP intracellularly and reduces tumor necrosis factor- $\alpha$. This study aimed to investigate the effect of PTX administration on MDMA that induces apoptosis in testis of male Wistar rats.

Methods: Thirty male Wistar rats weighing 250-300 g were randomly divided into five groups: control group, the group received MDMA ( $7.5 \mathrm{mg} / \mathrm{kg}$, three times at every two hours), first experimental group received PTX $(100 \mathrm{mg} / \mathrm{kg}$ Just in the time of the third injection of MDMA), second experimental group received PTX $(100 \mathrm{mg} / \mathrm{kg}$ a week before MDMA administration) and group received saline. Two weeks after interventions, testes were removed and prepared for $\mathrm{H} \& \mathrm{E}$ staining, TUNEL and western blot techniques.

Results: In first and second experimental groups, the mean testicular biopsy score was increased significantly compared with the MDMA group. The number of TUNEL-positive cells/ tubule was increased significantly in MDMA and saline groups. A significant difference was revealed in the mean number of TUNEL-positive cells between the rats treated with PTX before MDMA administration and MDMA group. Expression of active caspase-3 was significantly increased in the MDMA group.

Conclusion: Pentoxifylline can significantly reduce the severity of lesions in the testis following administration of MDMA.

\section{0-59}

The neuroprotective effect of propofol on CA1 area of male Wistar rat hippocampus following transient global ischemia/reperfusion

Movassaghi $^{*}$, Sharifi $N^{* *}$, Farhangi $D^{* * *}$

*Department of Anatomy, Faculty of Medicine, Tehran Medical Sciences Branch, Islamic Azad University, Tehran, Iran; **Department of Anatomy, Faculty of Medicine, Tehran Medical Sciences Branch, Islamic Azad University, Tehran, Iran; ***Young Researchers and Elite Club, Tehran Medical Sciences Branch, Islamic Azad University, Tehran, Iran

Objectives: Cerebral ischemia is a major problem in the world and its subsequent reperfusion makes cell death or apoptosis. CA1 pyramidal cells of hippocampus are highly vulnerable to ischemic results. Lives of patients are threatened by cerebral ischemia and its neurological injury. Recent studies reported that propofol has neuroprotective effect on neuron but it needs more studies. The goal of this study is to evaluate the neuroprotective effects of propofol on CA1 pyramidal cells of hippocampus

Methods: The male rats ( $\mathrm{n}=18)$ were randomly divided into the 3 groups: Control group, IR- induced group, Propofol-treated group. Propofol $(40 \mathrm{mg} / \mathrm{kg}$ ) was administrated intraperitoneally for $1 \mathrm{~h}$ before the induction of ischemia. Global Cerebral ischemia was induced by clamping the bilateral common carotid 
arteries for $20 \mathrm{~min}$. Brains were removed and prepared for $\mathrm{H} \& \mathrm{E}$ staining, TUNEL and western blot techniques.

Results: Data showed that propofol limited apoptosis and caspase-3 activities in rats' hippocampi. There were no significant differences between the number of apoptotic bodies in the CA1 region of the hippocampus in the control and propofol -treated groups. The results revealed that ischemia significantly increased caspase- 3 levels in the hippocampus $(\mathrm{p}<0.05)$; however, the level of caspase- 3 in propofol -treated rats was less than the ischemic group.

Conclusion: Ischemia makes apoptotic cells and morphologic impairments, while administration of propofol can significantly decrease cell death and protect neurons against ischemic injury in CA1 region of Hippocampus

\section{0-60}

Morphometry of the anterolateral brainstem as a guide for surgical approaches to intrinsic brainstem lesions

$\underline{\text { Koçer IB }}$, Oner M, Ertürk M, Şengül G

Department of Anatomy, Faculty of Medicine, Ege University, Izmir, Turkey

Objectives: Surgery of the brainstem is challenging due to the complexity of the area with cranial nerve nuclei, the reticular system, extrapyramidal nuclei and ascending and descending fibers located here. Safe entry zones are required to reach the intrinsic lesions of the brainstem. The study gives detailed measurements for anatomical landmark zones of the ventrolateral surface of the human brainstem related to previously defined safe entry zones such as the peritrigeminal area, the pontomedullary sulcus between the exit of the rootlets of facial and glossopharyngeal nerves or the anterior aspect of crus cerebri lateral to the oculomotor nerve.

Methods: 53 whole brainstems and 34 midsagittal brainstems from the cadaver collection of Ege University, Faculty of Medicine, Department of Anatomy were measured using a stainless caliper with an accuracy of $0.01 \mathrm{~mm}$. Thirteen parameters were measured in the anterolateral brainstem area.

Results: Pontomesencephalic sulcus - bulbopontine sulcus distance was measured as $26,94 \mathrm{~mm}$, sulcus basillaris - lateral side of pons (CN V) distance as $17.23 \mathrm{~mm}$, transverse length of the pyramis $5.42 \mathrm{~mm}$, vertical length of the pyramis as $21.36 \mathrm{~mm}$, lateral mesencephalic sulcus as $12.73 \mathrm{~mm}$, distance of the lateral mesencephalic sulcus to the oculomotor nerve as $13.85 \mathrm{~mm}$, distance of $\mathrm{CN} \mathrm{V}$ to the upper tip of pyramis $17.58 \mathrm{~mm}$, olivary body (transverse length) at midpoint $5.21 \mathrm{~mm}$, the olivary body vertical length $14.77 \mathrm{~mm}$, the thickness of superior colliculus $4.36 \mathrm{~mm}$, the thickness of inferior colliculus $5.06 \mathrm{~mm}$, the length of tectum $14.50 \mathrm{~mm}$, and the length of the interpeduncular fossa as $11.26 \mathrm{~mm}$.

Conclusion: Profound anatomical knowledge and careful analysis of preoperative imaging are mandatory before surgery of the brainstem

\section{0-61}

Identifying the best position to reach the longest drill depth for anchor insertion in arthroscopic repair of slap lesion: a 3D reconstruction study

$\underline{\text { Ísmailoğlu } \mathrm{AV}^{*}}$, Kocaoğlu B**, Bayramoğlu A*, Yıldız YZ ${ }^{* * *}$, Karahan $M^{* *}$

*Department of Anatomy, Faculty of Medicine, Acıbadem University, istanbul, Turkey; **Department of Orthopedics and Traumatology, Faculty of Medicine, Acıbadem University, Istanbul, Turkey; ***Department of Anatomy, Cerrahpaşa Medical Faculty, Istanbul University, Istanbul, Turkey

Introduction: During drilling of glenoid rim and anchor insertion to repair SLAP (superior labrum anterior to posterior) lesion, perforation of glenoid wall might occur. Perforation may lead to injury of the suprascapular nerve as reported previously.

Objectives: The purpose of this study was to identify axis of the longest drill depth that passing through 12.00 o'clock in relation with acromion and coracoid process to avoid the risk of the suprascapular nerve injury.

Methods: Sectra IDS7 multi-touch screen visualization table, a commercially available $3 \mathrm{D}$ reconstructor of CT\&MR images, was used to reconstruct CT images in 3D. 87 shoulder girdles from 85 individuals were included. Coraco-acromial line (CA), extended between the posterior tip of coracoid process and the antero-lateral corner of acromion, was drawn to represent coracoacromial arch. Just under and parallel to CA line, a transvers section was obtained at 12.00 o'clock. The longest drill depth without penetrating glenoid wall and its axis according to CA line were determined in that transverse plan. CA line is divided into three parts as one third posterior $(1 / 3-\mathrm{P})$, one third middle $(1 / 3-$ $\mathrm{M})$ and one third anterior (1/3-A) to determine practically axis of the longest drill depth.

Results: Totally five different the longest drill depth axis position was found. 7 of 87 axes were next to the tip of acromion (1), 4 of 87 axes were in the middle of 1/3-P (2), 35 of 87 axes were at intersection of 1/3-P and 1/3-M (3), 27 of 87 axes were in the middle of $1 / 3-M(4), 12$ of 87 axes were at intersection of 1/3-M and 1/3-A (5). Average of the longest drill depth was measured as $35.8 \mathrm{~mm}$.

Conclusion: In conclusion, the best position to reach the longest drill depth for anchor insertion is the intersection point of the one third posterior and two thirds anterior parts of CA line.

\section{0-62}

Topographical anatomy of cricothyroid membrane and its relation with invasive airway access

Develi S, Yalçın B , Yazar F

Department of Anatomy, Gülhane Military Medical Academy, Ankara, Turkey

Objectives: Cricothyroidotomy is a surgical procedure performed through the skin and cricothyroid membrane to maintain airway during life-threatening emergency conditions. As a result of this procedure, complications such as bleeding, laryn- 
geal stenosis might be seen. In this study we aimed to identify the anatomic structures located on the membrane and describe if there is a safe area on the membrane for this procedure.

Methods: 10\% formaldehyde fixed 30 larynx specimens in the department of Anatomy were dissected for this study.

Results: Cricothyroid artery, cricothyroid vein, a common vein formed by cricothyroid veins, pyramidal lobe of thyroid gland and lymph nodes were observed on the membrane. Cricothyroid membrane was divided into four quadrants and the most common locations of these anatomic structures on the membrane were examined. Cricothyroid artery and cricothyroid vein were located on the upper half of the membrane, common vein was found in the midline and coursing to the thyroid gland, lobus pyramidalis and lymph nodes were located on the left side of the midline.

Conclusion: As a result; anatomical structures mostly located on the upper half and lower left quadrant of cricothyroid membrane. Thus, lower right quadrant of the membrane seemed to be safer for invasive procedures such as needle cricothyroidotomy or other cannulation techniques. If needle punction or cannulation is performed through the lower right quadrant of cricothyroid membrane, fewer anatomic structures will be damaged and complications due to degradation of the vascular supply of this region might be seen lesser.

\section{0-63}

\section{Coordinated multiple use of fresh frozen cadavers}

$\underline{\text { Akca } \mathrm{P}^{*}}$, Aktekin $\mathrm{M}^{* *}$

*Department of Anatomy, Faculty of Medicine, Acıbadem University, istanbul, Turkey **Department of Orthopedics and Traumatology, Faculty of Medicine, Acıbadem University, Istanbul, Turkey

Introduction: Using fresh frozen human cadavers in surgical training has several advantages such as real tissue quality and handling. Fresh frozen cadavers are used in courses of various surgical departments in our university. Also, finding embalmed cadavers for undergraduate anatomy education is a hard process in our country.

Objectives: Here, we aimed to present the process of coordinated multiple use of fresh frozen cadavers in both the graduate and undergraduate anatomy education.

Methods: Two fresh frozen lower extremities were used in three separate surgical hip arthroscopy courses. Thus, the legs had the thawing-freezing process for three times. After the third surgical course, we embalmed them with formalin and kept them in formalin tanks.

Conclusion: In countries such as ours, providing cadaver for anatomy education has always been a great problem. On the other hand, fresh frozen cadavers can be obtained for graduate education by several clinical associations. After their usage for this sort of education, cadavers can be embalmed and kept for long term anatomy education, which can be termed as 'coordinated multiple cadaver use'. We presented here that after three times usage of fresh frozen cadavers, it is still possible to use them as embalmed cadavers. We also discussed the criterion for effective protection of the cadavers and for protection from infections.

\section{0-64}

Prevalence of Chiari type I malformation: a retrospective study from cervical MRI

Öktem H*${ }^{*}$ Dilli A**, Soysal H*, Kürkcüoğlu A*, Yazıcı $\mathrm{C}^{* * *}$, Pelin $\mathrm{C}^{*}$

*Department of Anatomy, Başkent University, Ankara, Turkey; **Department of Radiology, Dışkapı Yıldırım Beyazıt Training and

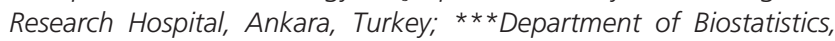
Başkent University, Ankara, Turkey

Objectives: Chiari malformation is characterized by the caudal herniation of cerebellar tonsils from foramen magnum to cervical canal in radiographical images. Type I Chiari malformation is the herniation of cerebellar tonsils more than $3 \mathrm{~mm}$ and does not give any symptoms up to adulthood. In this study our objective was to assess the prevalence of Chiari Type I malformation in male and females.

Methods: In this retrospective study cervical MR images of 2480 male and female patients aged between 18-70 years old were examined. All the MR images were available in the archive of MR imaging center of Dışkapı Yıldırım Beyazıt Education and Research Hospital between the years 20132015. Type I Chiari malformation is defined as an inferior displacement of the cerebellar tonsils more than $3 \mathrm{~mm}$ below the opisthion-basion line. The patients with intracranial lesions, hydrocephalus and craniotomy were excluded.

Results: Chiari Type I malformation prevalence was 3.3\% in females and $0.7 \%$ in males. We observed that Chiari Type I malformation was associated by syringomyelia in $0.5 \%$ of the female patients and in $0.2 \%$ of the males. In the present study syringomyelia associated Chiari Type I malformation was not observed as much as it was reported in the literature $(0.7 \%)$. On the other hand it was observed that the incidence of Chiari tip I malformation was relatively higher in the patients with scoliosis $(15.4 \%)$. The ratio of this alignment was also more in females $(11.4 \%)$ than in males (4\%).

Conclusion: In the present study the prevalence of Chiari Type I malformation has been evaluated on a large sample. This preliminary study will enlighten the authors for the later studies on this subject.

Keywords: Chiari Tip I malformation, cervical MRI, syringomyelia, scoliosis

\section{0-65}

\section{The effect of high fat diet on the adrenal gland morphology}

$\underline{\text { Topal F }}^{*}$, Aydar $\mathrm{Y}^{* *}$, Peker $\mathrm{H}^{* *}$, Yücel F** Ortadeveci A**, Peker $\mathrm{S}^{* * *}$

*Bilecik Şeyh Edebali University, Health Services Vocational School, Bilecik \& Eskişehir Osmangazi University, Institute of Medical Sciences, Eskişehir, Turkey; ${ }^{*}$ Department of Anatomy, Faculty of Medicine, Eskişehir Osmangazi University, Eskişsehir, Turkey; ${ }^{* *}$ Department of Histology and Embriyology, Faculty of Veterinary Medicine, Uludağ University, Bursa, Turkey 
Objectives: The diet containing high amount of fat stimulates development of obesity. The incidence of obesity and obesityassosiated pathologies continues to increase with profound adverse effects on health status in the developed countries. Obesity is shown to poorly affect physiology of the adrenal gland. However, the effect of obesity on the morphometrical parameters of the adrenal gland has not been well studied. We aimed to investigate the effect of high fat diet on the morphometrical parameters of the adrenal gland.

Methods: We fed the mice with either high-fat diet $(60 \% \mathrm{kcal}$ from fat, Altromin, Germany ) or low-fat diet (10\% kcal from fat; Altromin, Germany) for nine weeks. Unbiased stereological methods were used to evaluate the adrenal gland morphology. The mice were anasthesized by intraperitoneally injection and killed with cervical dislocation. Tissue samples were processed by routine and standard paraffin embedding and were serially sectioned in $5 \mu$ thickness. Then, every seventh section was saved and stained with Crossman's triple stain. The sections were evaluated using Cavalieri's method and volume fraction approach. We calculated total volume of adrenal gland, ratio of cortex to medulla volume $(\mathrm{Vv})$, the volume of zona glomerulosa, zona fasciculata, zona reticularis $(\mathrm{Vv})$, total number of cromaffin cells, number of cromaffin cells per $1 \mu^{3}$ volume and mean diameter of cromaffin cells.

Results: The weight of adrenal gland $(\mathrm{p}=0.01)$, body weight and intraperitoneal adipose tissue $(\mathrm{p}=0.001)$ in the obese mice significantly increased when compared with the control group. No changes were observed in the total volume of adrenal gland, ratio of cortex to medulla volume $(\mathrm{Vv})$, the volume of zona glomerulosa, zona fasciculata, zona reticularis $(\mathrm{Vv})$, the number of cromaffin cells and mean diameter of cromaffin cells. However, number of cromaffin cells per $1 \mu \mathrm{m} 3$ volume in the obese mice considerably increased compared with the control group $(\mathrm{p}=0.015)$.

Conclusion: The present results suggest that obesity causes accumulation of adipose tissue in the adrenal gland.

\section{0-66}

The effect of oxytocin and kisspeptin-10 on ovary and uterus of ischemia/reperfusion injured rats

$\underline{\text { Aslan }}^{*}$, Akkaya $\mathrm{H}^{* *}$, Şahin $\mathrm{S}^{* * *}$, Y1lmaz B ${ }^{* * * *}$, Şentürk GE*****

*Faculty of Medicine, Bahçeşehir University, Göztepe, Istanbul, Turkey; **Experimental Research Center, Faculty of Medicine, Yeditepe University, İstanbul, Turkey; *** istanbul Zeynep Kamil Women Health Hospital, Üsküdar, Istanbul, Turkey; ****Department of Physiology, Faculty of Medicine, Yeditepe University, Istanbul, Turkey; $\star * * * *$ Department of Histology and Embryology, Faculty of Medicine, Bahçeşehir University, İstanbul, Turkey

Ischemia/reperfusion (I/R) injuries result in damage to endothelial and parenchymal cells, accompanied by reactions of granulocytes and macrophages. Oxytocin (OXY), a nonapeptide, produced in the paraventricular and supraoptical nuclei of the hypothalamus, stimulates uterine contraction during parturition and myoepithelial cells during suckling. OXY has been used as a pro- tective antioxidant. Kisspeptin (metastin) is a 145 amino acid protein which plays a key role in the central control of reproductive functions and onset of puberty. This study investigated the effects of Kisspeptin and oxytocin on I/R injured ovary and uterus of female rats. Sprague Dawley rats were separated into five groups. Group 1, is sham-operated control group; Group 2, rats were subjected to $45 \mathrm{~min}$ ischemia followed by $45 \mathrm{~min}$ reperfusion. Group 3, OXY administration (i.p.) $30 \mathrm{~min}$ prior to I/R applied rats; Group 4, Kisspeptin administration (i.p.) $30 \mathrm{~min}$ prior to I/R applied rats; Group 5, OXY and Kisspeptin administration (i.p.) $30 \mathrm{~min}$ prior to I/R. After application ovary and uterus were removed and stained with Hematoxylen and Eosin for histopathological observations. Histopathologic scoring was compared using an analysis of variance (ANOVA) statistical test. Histopathological results show that stromal and granulosa cells in ovary, endometrial cells in uterus were damaged in I/R group when compared to control group. The cellular damage of ovary and uterus were reduced in OXY and Kisspeptin administered I/R group when compared to only Kisspeptin injected I/R group and I/R group. There is no significant difference between OXY and $\mathrm{OXY}+$ Kisspeptin injected I/R groups. In consequence, Kisspeptin cannot act as an antioxidant, whereas oxytocin can play an important role in protecting ovary and uterus from ischemia/reperfusion injury.

\section{0-67}

\section{Vitamin D attenuates kidney fibrosis via reducing fibroblast expansion, inflammation and epithelial cell apoptosis}

Arfian $\mathrm{N}^{*}$, Muflikhah $\mathrm{K}^{* *}$, Soeroyo SK${ }^{* *}$, Sar1 DCR*, $\underline{\text { Tranggono }}^{* * *}$, Romi $\mathrm{MM}^{*}$

*Department of Anatomy, Embryology, Anthropology, Faculty of Medicine, Universitas Gadjah Mada, Yogyakarta, Indonesia; **Department of Physiology, Faculty of Medicine, Universitas Gadjah Mada, Yogyakarta, Indonesia; $* * *$ Department of Surgery, Faculty of Medicine, Universitas Gadjah Mada, Yogyakarta, Indonesia

Objectives: Kidney fibrosis is the common final pathway of Chronic Kidney Diseases (CKD) that is characterized by myofibroblast formation, inflammation and epithelial architecture damage. Vitamin D is known as a renoprotective agent although the precise mechanism is not well understood. This study is to elucidate effect of Vitamin D in fibroblast expansion, inflammation and apoptosis in kidney fibrosis.

Methods: We performed Unilateral Ureteral Obstruction (UUO) model in male Swiss background mice (3 months, 3040 grams) to induce kidney fibrosis. The mice $(n=25)$ were divided into five groups: UUO, oral Vitamin D treatment with dose: $0.125 \mathrm{mg} / \mathrm{kg}$ (UUO+VitD-1), $0.25 \mathrm{mg} / \mathrm{kg}$ (UUO+VitD2) and $0.5 \mathrm{mg} / \mathrm{kg}$ (UUO+VitD-3) and Sham operation (SO) with ethanol $0.2 \%$ supplementation. We sacrificed the mice in day14 after operation, harvested the kidney, made paraffin section for histology analysis and kept in RNA Later solution for protein and RNA extraction. Tubular injury and fibrosis were quantified based on PAS and Sirius Red (SR) stainings. Immunostaining was done for examination of myofibroblast 
( $\alpha$ SMA), fibroblast (PDGFR,), TLR4 and apoptosis (TUNEL). Reverse Transcriptase PCR (RTPCR) was done for measuring MCP-1, ICAM-1, TLR4, and Collagen1 expression. TGF $\beta 1$ level was quantified using elisa.

Results: We observed significant lower of fibrosis $(\mathrm{p}<0.001)$, tubular injury score $(\mathrm{p}<0.001)$ and myofibroblast area $(\mathrm{p}<0.001)$ in group treated with Vitamin D compare to UUO group. Those findings were similar to measurement of TGF, 1 level and fibroblast quantification. Meanwhile, we didn't find any significant difference among Vitamin D treated groups. Concerning the dose independent effect, we only compared the UD- 1 group and found that UUO+VitD-1 had significant lower of epithelial cell apoptosis by TUNEL assay. RT PCR analysis showed significant lower expression of collagen 1 as well as inflammation mediator expression (MCP-1, ICAM-1, TLR4) in UD-1 group.

Conclusion: Vitamin D reduces kidney fibrosis through inhibition of fibroblast activation and ameliorate epithelial cell architecture.

\section{0-68}

\section{Morphologic changes in ovarium of mice fed with high fat diet}

$\underline{\text { Peker }}^{*}$, Topal F** Ortadeveci A*, Yücel F* Aydar Y*, Peker $\mathrm{S}^{* * *}$

*Department of Anatomy; Faculty of Medicine, Eskişehir Osmangazi University, Eskişehir, Turkey; *Bilecik Şeyh Edebali University, Health Services Vacotional School, Bilecik \& Eskişsehir Osmangazi University, Institute of Medical Sciences, Eskişehir, Turkey; $* * \star$ Department of Histology and Embriyology, Faculty of Veterinary Medicine, Uludağ University, Bursa, Turkey

Objectives: Increased food consumption rich in fat and carbonhydrate and sedantery lifestyle have seriously increased the rates of obesity and obesity -assosiated diseases in developed countries. Obesity causes many diseases such as metabolic sendrome, cardiovasculer disease, demantia in addition to impaired reproductive system functions in both sexes. Female mice with diet-induced obesity (DIO) exhibit infertility and thus can serve as a model for human polycystic ovary syndrome (PCOS). The aim of present study was to examine how ovarium is affected by diet-induced obesity.

Methods: The effects of high fat diet (HFD) on ovarium morphology in mice fed with HFD were investigated using unbiased stereological methods. The ovarium of mice fed with HFD (Altromine, C1090-60, Germany) for nine weeks, were compared with that of mice fed with standart chow diet (Altromine, C1090-10, Germany). The mice were anasthesized and killed by cervical dislocation. Stereologic parameters were obtained from the ovarium removed from the mice in dioestrous cycle. The samples were processed through routine and standard paraffin embedding and were serially sectioned in $5 \mu$ thickness then, every tenth section was saved, stained with Crossman's triple stain for counting and measuring. In all sampled sections mean follicle diameters (primordial, primary, sec- ondary, tertier), total ovarium volume, cortex to medulla ratio $(\mathrm{Vv})$, ovum to cell ratio in secondary follicule were examined in all sampled sections.

Results: The present results showed that weight of ovarium $(\mathrm{p}=0.009)$ and amount of intraperitoneal adipose tissue $(\mathrm{p}=0.001)$ and the body weight $(\mathrm{p}=0.01)$ markedly increased in obese mice when compared with these control groups. Morever, follicule diameters (primordial, $\mathrm{p}=0.000$; primary, $\mathrm{p}=0.001$; secondary, $\mathrm{p}=0.000$; tertier, $\mathrm{p}=0.000$ ) were significantly increased in obese mice. Cortex to medulla ratio $(\mathrm{Vv})$ $(\mathrm{p}=0.000)$ and ovum to cell ratio in secondary follicule ( $\mathrm{p}=0.002$ ) were also considerably different between experimental and the control grups.

Conclusion: The present findings indicate that obesity adversely affects overall ovarium morfology.

\section{0-69}

Improvement of memory performance after Centella asiatica (Gotu kala) ethanol extract treatment associates with upregulation of brain-derived neurotrophin factor (BDNF), TrkB and ERK1/2 signaling in chronic electrical stress model in rats

Sarı DCR*, Ar-Rochmah $\mathrm{M}^{* *}$, Juananda D*, Tranggono $\mathrm{U}^{* * *}$, Romi MM*, Emoto $\mathrm{N}^{* * * *}$, Arfian $\mathrm{N}^{*}$

*Department of Anatomy, Embryology, Anthropology, Faculty of Medicine, Universitas Gadjah Mada, Yogyakarta, Indonesia; **Department of Neurology, Faculty of Medicine, Universitas Gadjah Mada, Yogyakarta, Indonesia; ***Department of Surgery, Faculty of Medicine, Universitas Gadjah Mada, Yogyakarta, Indonesia; $\star * * \star$ Division of Cardiovascular, Department of Internal Medicine, Graduate Faculty of Medicine, Kobe University, Kobe, Japan; Department of Clinical Pharmacy, Kobe Pharmaceutical University, Kobe, Japan

Impairment of hippocampus function as a memory processing occurs due to stress. Gotu kola (Centella asiatica L.) is known to improve memory, intelligence and neural protection although the precise mechanism is not well understood. Mitogen Activated Protein Kinase (MAPK) is a group of transcription factor that involves in physiology and pathology of nervous system. This study aimed to investigate the effects of ethanol extracts of Gotu kola toward MAPK (p38 protein, ERK1/2, and JNK) expression as downstream signaling of Brain-Derived Neurotrophin Factor (BDNF) and its association with memory retention in the rat hippocampus after administration of electrical stress. We performed chronic electrical stress model in 20 male Sprague Dawley rats (1 month old, 100-120 grams), then rats were divided into four groups: normal control group $(\mathrm{KN})$ which received distilled water, and three treatment groups receiving oral gotu kola ethanol extracts in oral doses of $150 \mathrm{mg} / \mathrm{kgBW}$ (P1), 300mg/kg $\mathrm{BW}(\mathrm{P} 2)$, and $600 \mathrm{mg} / \mathrm{kgBW}(\mathrm{P} 3)$ four weeks. Memory retention was assessed with the Morris water maze. Hippocampus is harvested, then extracted for protein and RNA analysis. MAPK proteins (p38, ERK1/2, JNK) were measured using 
S118 XXIV International Symposium on Morphological Sciences, 2nd-6th September, 2015, Istanbul, Turkey

western blot, meanwhile BDNF and TrkB receptor were analyzed with Real Time PCR (RTPCR). We observed an increased of memory performance after treatment with Gotu kala in P3 group significantly as shown by significant elevation of probe test after treatment compare to before treatment. This finding associated with significant elevation of BDNF and $\operatorname{TrkB}$ mRNA level in P3 group compare to control. Westernblot analysis showed significant upregulation of MAPK pathway especially ERK1/2 in P3 group $(\mathrm{p}<0.05)$ compare to control. Here, we revealed contribution of BDNF signaling trough TrkB and ERK1/2 pathway in amelioration of memory performance after Gotu kala treatment in electrical stress model.

\section{0-70}

\section{Neural mechanisms underlying time perception and reward prospect}

Apaydın $\mathrm{N}^{*}$, Üstün $\mathrm{S}^{* *}$, Kale $\mathrm{E}^{* * *}$, Çiçek $\mathrm{M}^{* *}$, Özgüven $\mathrm{HD}^{* * * *}$

*Department of Anatomy and Ankara University Brain Research Center, Faculty of Medicine, Ankara University, Ankara, Turkey; **Department of Physiology and Ankara University Brain Research Center, Faculty of Medicine, Ankara University, Ankara, Turkey; ***Faculty of Engineering and Ankara University Brain Research Center, Ankara University, Ankara, Turkey; **** Department of Psychiatry and Ankara University Brain Research Center, Faculty of Medicine, Ankara University, Ankara, Turkey

With the present functional magnetic resonance imaging (fMRI) study, we sought to elucidate the neural processes that are shared and distinct between brain regions responsible for time perception and reward prospection. Participants were 20 young adults (age 19-36, mean 25 years), 7 men and 13 women. All were right handed. The methods and procedures used in the study had approval from the Ankara University Institutional Review Board. We employed a temporal attention task in which observers had to extrapolate the velocity of an occluded moving object in reward vs. no-reward sessions. On each trial, the target displayed on the left side of the screen and moved toward right side becoming "invisible" in the center of the screen under an occluding surface and the velocity of the target was slightly increased or decreased in this invisible period. After the period of invisible motion, the target reappeared and participants were asked to make perceptual judgments whether it reappeared slightly too early or too late compared with its predicted velocity. Before the fMRI procedure, the participants were informed that they would be rewarded for each correct answer in reward sessions and they gained 1.5 TL (0.5?) per correctly done trial. Inside the scanner, the participants performed four 7-minute runs, yielding a total of 128 trials in each session. Two of the runs were rewarded and 2 were non-rewarded. Accordingly, fMRI images were acquired using a 3-T scanner with a 32-channel head-coil array. The most prominent activations associated with time perception included the bilateral prefrontal cortex (middle frontal gyrus),inferior parietal lobule and left middle temporal gyrus. And the most prominent activations associated with reward as compared with no-reward sessions included the left caudate nucleus. In addition to the main effects, the results revealed a significant interaction between the 2 factors in the left insula and bilateral posterior cingulate cortex.

\section{0-71}

The need of vertical integration of anatomy education in medical schools

Songur A, Ekinci S, Gülsarı Y, Gönül Y, Turamanlar O

Department of Anatomy, Faculty of Medicine, Afyon Kocatepe University, Afyonkarahisar, Turkey

In many studies, it is stated that medical students' anatomy knowledge decrease with time and "the negligence of vertical integration in anatomy teaching" is counted among the reasons. In this study, we tried to draw attention to the need of vertical integration of anatomy education in medical schools with the questionnaire. The study was conducted with $3 \mathrm{rd}-$ 6th grade students, practicing physicians, research fellows, specialist and lecturers from medical schools of Afyon Kocatepe, Pamukkale and Namık Kemal universities. The participants are asked to answer 16 questions with 5 options, prepared as a Likert test. 344 volunteers attended the study. $90 \%$ of them are medical students, $6 \%$ of them are practicing physicians or research fellows, $\% 1$ of them are specialists and $3 \%$ of them are lecturers. The majority of participants responded positive for the questions "there must be repeat lessons of 1 st and 2nd grade at lessons in 4th and 5th grades" (by 45\%), "practice hours of anatomy must be increased" (by 59\%), "anatomy can be made easier for me to learn with clinic knowledge" (by $85 \%)$, "along with theoretical and practical anatomy courses, students must be given radiologic and cross sectional anatomy lessons" (75\%). Anatomy education given in the first years is usually forgotten in the following years because it relies on memorization. Therefore, a reminder lessons in clinic internships will be useful in terms of vertical integration. We also think it will be useful to update the curriculum, by making minor changes in it regarding the method, content and clinic relation of the anatomy education (appropriate with the vertical and horizontal integration). We think it is necessary to prepare a standard for anatomy, compatible with the one that is planned to be made according to National Core Education Program (UCEP-2014) in undergraduate medicine education.

\section{0-72 \\ Effect of herbal extract of the Viola odorata on morphogenesis of mice}

Mehrannia $K^{*}$, Vardasbi $S^{* *}$, Kazemzadeh $S^{*}$, Shayan $M^{* * *}$

*Department of Anatomy, Faculyt of Medicine, Tehran University, Iran; **Department of Biochemistry, Faculyt of Medicine, Tehran University, Iran; ***Shahid Beheshti University of Medical Sciences Faculty of Medicine, Tehran, Iran 
The Viola odorata is a species of the genus viola, native to America and Australia. The herb has been used in our country from past till now but recently prescribed in migration encouraged us study effect of this herb and survey the probably teratogenic effect on morphogenesis of mice. In this study four group of adult mice Balb/c female received herbal extract daily in four concentration. $2.5,12.5$ and $25 \mathrm{mg}$ in $0.25 \mathrm{ml}$ distilled water by oral gavage. The control group only received distilled water $(0.25 \mathrm{ml})$ in gastrulation period (6-15 pregnancy). After killing maternal mice the fetuses were first studied microscopically regarding gross anomalies and then histologically and clearing method to survey malformation in organ and skeletal system. We didn't see any malformation in low dosages but we saw bleeding in abdominal region and around the heart and respiratory system in upper dosage. Comparing this result with control group indicated that the high dosage of this herb maybe has the ability to produce malformation.

\section{0-73}

\section{Investigation of the effects of aging on the expression of aquaporin 1 and aquaporin 4 protein in heart tissue}

Bıçakçı $\mathrm{H}^{*}$, Sarsılmaz M*, Ocaklı S**, Uysal M**, Sapmaz Hí ${ }^{* * *}$, Acar T**, Demirtaş $\mathrm{I}^{*}$, Açıkgöz R*

*Department of Anatomy, Faculty of Medicine, Sifa University, Izmir, Turkey; **Department of Anatomy, Faculty of Medicine, Gaziosmanpaşa University, Tokat, Turkey; ***Department of Histology and Embryology, Faculty of Medicine, Gaziosmanpaşa University, Tokat, Turkey

Objectives: Fluid loss occurs in all tissues with aging. This fluid loss, may be related with changes in the intensity and functions of aquaporin (AQP) which are the water channel proteins facilitating the passage of water and some small rigid molecules in tissue. Especially the presence of AQP 1 and AQP 4 has been indicated in human heart and the studies have been focused on these two aquaporin. It has been reported that the AQP 4 mostly exist in muscle cells while the cardiac AQP 1 mostly exist in micro-vascular vessels. For this purpose, the present study is aimed to research the effects of the aging on AQP 1 and AQP 4 on the heart tissue.

Methods: In this study, $14 \mathrm{Balb} / \mathrm{C}$ type white mice (50-80 gr) were used. Animals were divided into two equal groups. Group I was arranged as two months young animals group $(n=7)$ and Group II as eighteenmonths old animals group $(n=7)$. The heart tissue was removed and half of it was used for western blotting application while the other half was used for immunohistochemical and histopathological evaluations. Density of AQP1 and AQP4 was determined by western blot method. After routine histological procedures, AQP1 and AQP4 were stained immunohistochemically. Also hematoxylin-eosin $(\mathrm{H} \& \mathrm{E})$ were stained and all sections were examined under the light microscope. Findings: In this study, the expression of AQP1 and AQP4 were shown with western blot and immunohistochemistry in hearts of young and old mice. With either method, increased AQP1 and AQP4 expression was determined in heart tissue with aging.

Conclusion: Increased AQP1 and AQP4 levels in the heart tissue may be correlated with the maintenance of water and electrolytes balance which decreasing with aging.

Keywords: Mice, heart, aging, aquaporin 1and aquaporin 4

\section{0-74}

\section{Evaluation of eyeball and orbit in relation to gender and age: preliminary results}

Özer $\mathrm{CM}^{*}$, Öz İI**, Şerifoğlu I**, Büyükuysal MÇ***, Barut Ç* *Department of Anatomy, Faculty of Medicine, Bülent Ecevit University, Zonguldak, Turkey; ${ }^{*}$ Department of Radiology, Faculty of Medicine, Bülent Ecevit University, Zonguldak, Turkey; ${ }^{* *}$ Department of Biostatistics, Faculty of Medicine, Bülent Ecevit University, Zonguldak, Turkey

The orbital aperture is the entrance to the orbit in which most important visual structures, as the eyeball and the optic nerve are found. It is vital not only for visual system but also for the evaluation and recognition of the face. Eyeball volume is essential for diagnosing the microphthalmos or buphthalmos in several eye disorders. The length of the optic nerve is necessary in the decision of the right scissors for the enucleation. Therefore, the aim of this study is to evaluate eyeball volume, orbital aperture and optic nerve dimensions in a Turkish population sample according to sex and body side for a morphologic description. Paranasal sinus CT scans of 198 individuals ( 83 females, 115 males) aged between 5-74 years were evaluated retrospectively. Dimensions of orbital aperture, axial length and volume of eyeball, diameter and length of intra-orbital part of optic nerve were measured. CT examinations were performed on Activion 16 CT Scanner (Toshiba Medical Systems, 2008 Japan). The CT measurements were calculated by using OsiriX software in personal computer. All parameters were evaluated according to gender and right/left sides. Statistically significant difference between genders was found with respect to axial length of eyeball, optic nerve diameter, dimensions of orbital aperture on both sides and right optic nerve length. Furthermore certain statistically significant side differences were also found. There were statistically significant correlations between age and axial length of eyeball, optic nerve diameter, and transverse length of orbital aperture on both sides for the whole study group. In this study we determined the certain morphometric parameters of orbit. These outcomes may be helpful to develop a database to determine normal orbit values for Turkish population so that quantitative assessment of orbital disease and orbital deformities will be evaluated both for preoperative planning and for assessing postoperative outcomes.

\section{0-75 \\ Morphometry of the petrous bone as a guide for suboccipital retrosigmoid surgical approach}

Illhan $\mathrm{P}^{*}$, Kayhan $\mathrm{B}^{*}$, Erturk $\mathrm{M}^{* *}$, Şengül $\mathrm{G}^{* *}$

*Department of Neuroscience, Faculty of Medicine, Ege University, Izmir, Turkey; ${ }^{* *}$ Department of Anatomy, Faculty of Medicine, Ege University, Izmir, Turkey 
Objectives: The surgical anatomy of the temporal bone, specifically the posterior surface of the petrous bone, is important for suboccipital retrosigmoid and retrosigmoid transmeatal approaches in the removal of acoustic neuromas protruding from the internal acoustic meatus. We aimed to provide morphometry of this region to establish anatomical guidelines for safe removal of lesions here.

Methods: We performed morphometric analysis of 217 (123 left side, 94 right side) temporal bones from the bone collection of Ege University, Faculty of Medicine, Department of Anatomy and measured ten different parameters using a stainless steel caliper with an accuracy of $0.01 \mathrm{~mm}$.

Results: The distance between the uppermost point on posterior wall of the sigmoid sulcus and the lateral wall of internal acoustic meatus was measured $41.07 \pm 2.37 \mathrm{~mm}$, distance between the mid-point of upper margin of internal acoustic meatus and the superior border of petrous bone $4.72 \pm 0.85 \mathrm{~mm}$, distance between the opening of vestibular aqueduct and the lateral edge of internal acoustic meatus $10.29 \pm 1.25 \mathrm{~mm}$, distance between the midpoint of upper margin of jugular foramen (corresponds to highest point of jugular bulb) and the level of lower margin of internal acoustic meatus (ILIAM) $9.81 \pm 1.44 \mathrm{~mm}$, distance between ILIAM to the lateral margin of the trigeminal impression $8.05 \pm 1.58 \mathrm{~mm}$, distance between ILIAM to petrosal sulcus $5.36 \pm 1.11 \mathrm{~mm}$, distance between the subarcuate fossa to ILIAM $4.46 \pm 0.95 \mathrm{~mm}$, and distance between the arcuate eminence and ILIAM $16.25 \pm 1.76 \mathrm{~mm}$. Vertical and horizontal diameters of the internal auditory meatus were measured $3.86 \pm 0.71 \mathrm{~mm}$ and $5.80 \pm 0.94 \mathrm{~mm}$, consequently.

Conclusion: This study provides anatomical guidelines for safe removal of the lesions in the petrous bone posterior surface region during suboccipital retrosigmoidal surgical approaches.

\section{0-76}

3D analysis of facial mask in the sitting, standing and supine body positions

Özsoy U, Şekerci R, Ögüt E

Department of Anatomy, Faculty of Medicine, Akdeniz University, Antalya, Turkey

Objectives: The head and body position is an important issue to acquire reliable data in cephalometric analysis. Medical imaging techniques require various body positions. Gravity causes changes in facial soft tissue and acts in different directions according to the position of the head during imaging. The aim of this study is to evaluate the effect of positional changing in facial soft tissue.

Methods: Facial masks of 70 Caucasian subjects (35 male, 35 female) were scanned in the sitting, standing and supine body positions. The differences in the positions were compared by using Root Mean Square (RMS), Mean Absolute Deviation (MAD) and Mean Signed Distance (MSD).

Results: RMS, MAD and MSD values of the sitting/standing comparison were significantly lower than the sitting/supine and standing/supine. There were no significant differences between the sitting/supine and standing/supine ( $<<0.0001)$.
Conclusion: Our results demonstrated a significant change in the facial soft tissue caused by the body position. More similar measurements were acquired between sitting-standing body positions comparison than sitting-supine or standing-supine.

\section{0-77}

Investigation of the effects of glutamate stress in a model central nervous system cell line on graphene oxide sheets

$\underline{\text { Kayhan B }}^{*}$, Taşdemir Ş** Çoruk P*, Görgün $C^{* * *}$,

Şendemir Ürkmez $A^{* * * * * *}$, Şengül $G^{*, * * * *}$

${ }^{*}$ Department of Neuroscience, Institute of Health Sciences, Ege University, izmir, Turkey; ${ }^{*}$ Department of Bioengineering, Faculty of Engineering, Ege University, Izmir, Turkey; ${ }^{* *}$ Department of Biomedical Technologies, Graduate Faculty of Natural and Applied Sciences, Ege University, Izmir, Turkey; $* * * *$ Department of Anatomy, Faculty of Medicine, Ege University, Izmir, Turkey

Objectives: Graphene, a monolayer of sp2-bonded carbon atoms, is a quasi-two-dimensional (2D) material with unique electrical and chemical properties. In terms of the biomedical applications of graphene and graphene oxide (GO), nervous system would be an ideal breakthrough model, because neural cells are electro-active. Glutamate is one of the important neurotransmitters in the central nervous system (CNS) and it plays a vital role in neural pathways. Extreme glutamate concentrations may cause excitotoxicity, which is defined as the pathological process by which nerve cells are damaged or killed by excessive stimulation. The aim of this work is to investigate whether culture of CNS neurons on GO has any neuroprotective effect toward glutamate stress.

Methods: B35 neuroblastoma cells were grown in DMEM F12 containing $10 \%$ fetal bovine serum. Graphene oxide powder was coated onto glass slides as a thin film. B35 cells were cultured on graphene oxide (GO) sheets. Cultivated cells on glass slides were used as the control group. After 24 hours of cell culture, L-glutamic acid induced excitotoxicity was applied on B35 cells on both surfaces. After 24 hours of post-stress culture, morphologies of cells were examined by scanning electron microscopy (SEM). Cell viability was measured by MTT assay and toxicity was shown by LDH assay.

Results: The effects of glutamate stress on cell viability were visible as early as 1 hour on both surfaces. The cell viability of B35 cells on GO sheets was higher than on glass slides, and cells recovered from the stress within 6 hours on GO surfaces while viability on glass surfaces was lower than $50 \%$ after 24 hours. Cell morphology and toxicity measurements also supported this observation.

Conclusion: We demonstrated that glutamate is toxic to B35 cells above the concentration of $100 \mathrm{mM}$. The culture on GO increases the resistance to glutamate stress on B35 cells, and helps fast recovery. This study aimed to show the potential use of GO, with the increasing knowledge about the role of glutamate, as a therapeautic biomaterial helping the treatment for excitotoxicity induced neurodegenerative diseases.

Keywords: Glutamate stress, B35 cell line, graphene oxide, excitotoxicity 


\section{0-78}

Investigation of the effects of hypoxia on SH-SY5Y cells on graphene oxide sheets

Illhan $\mathrm{P}^{*}$, Görgün $\mathrm{C}^{* *}$, Kayhan $\mathrm{B}^{*}$, Taşdemir Ş***,

Şengül G, ${ }^{* * * * *}$, Şendemir Ürkmez A**,***

*Department of Neuroscience, Institute of Health Sciences, Ege University, Izmir, Turkey; ${ }^{*}$ Department of Biomedical Technologies, Graduate Faculty of Natural and Applied Sciences, Ege University, Izmir, Turkey; *** Department of Bioengineering, Faculty of Engineering, Ege University, Izmir, Turkey; $* * * *$ Department of Anatomy, Faculty of Medicine, Ege University, Izmir, Turkey

Objectives: In the central nervous system (CNS), neurons are highly sensitive to the availability of oxygen. If oxygen availability is decreased, neurons can degenerate, leading to injury and cell death. Hypoxia is involved in several CNS pathologies, including stroke, head trauma, neoplasia, vascular malformations and neurodegenerative diseases. The human neuroblastoma SH-SY5Y cell line is a well-studied cellular model of neuronal differentiation in vitro. Graphene is one of the most attractive nanostructured materials. Due to its unique electrical properties, graphene and graphene oxide are considered in potential treatments for regulating neural cell behavior in CNS degeneration. The aim of this study is using graphene oxide (GO) sheets as a scaffold, seeding SHSY5Y cells on it and differentiating them with and without retinoic acid, and evaluating the effects of graphene oxide on neuronal behavior under hypoxic stress conditions.

Methods: Graphene oxide powder was coated onto glass slides as a thin film. SHSY5Y neuroblastoma cell line was cultured in DMEM- HG with $10 \%$ fetal bovine serum (FBS), and seeded on graphene oxide sheets. Cells seeded on glass slides were used as the control group. $10 \mu \mathrm{M}$ retinoic acid was used for neuronal differentiation. Cells were incubated at hypoxic $(3 \%$ $\left.\mathrm{O}_{2}\right)$ and normoxic $\left(21 \% \mathrm{O}_{2}\right)$ conditions in an hypoxia chamber for five days. Cell viability was shown by MTT test. Cell morphologies were examined by scanning electron microscopy (SEM) and actin staining.

Results: According to MTT results our cells are more resistant to hypoxia on GO sheets. Actin staining and SEM images indicated more distinct cytoskeletal organization and more neurite formation on GO.

Conclusion: The study demonstrates that GO may have potential use in therapy of hypoxia induced neurodegenerative diseases.

\section{0-79}

Evaluation of relationship between prevalence of palmaris longus muscle and handedness

Karacan K, Akcaalan M, Baylan H

Department of Anatomy, Faculty of Medicine, Sakarya University, Sakarya, Turkey

Objectives: Palmaris longus (PL) is a fusiform flexor muscle of forearm with a short belly and a long tendon that crosses over superficially flexor retinaculum. It flexes wrist and tenses palmar aponeurosus. The absence of PL prevalence varies from $0.6 \%$ to $63.91 \%$ in literature. The existence of $\mathrm{PL}$ on right hand is more common than the existence of PL on left hand. Thus, we aimed to determine the prevalence of PL in Turkish population and the relation of PL absence with gender and handedness in this study.

Methods: 1050 students of Sakarya University between ages 18-28 participated in the study (525 males, 525 females). After completing a questionnaire including handedness, the volunteers were examined on their both hands by using some standart tests to find out PL absences. Pearson chi-square and Fischer's exact tests were used for required statistics in SPSS 20 software.

Results: 937 subjects were right-handed (89.2\%), 70 subjects were left-handed $(6.6 \%)$ and 43 subjects were ambidextrous (4.1\%). The presence of bilateral PL was found in 653 subjects, while the absence of bilateral PL was found in 186 subjects. The absence of PL on right and left hand was detected respectively on $108(10.3 \%)$ and $103(9.8 \%)$ subjects. Left-handedness was more common in males $(7.2 \%)$ than in females $(6 \%)$. PL was absent unilaterally and bilaterally in 397 (37.8\%) subjects.

Conclusion: The study showed that our population has similar prevalence of PL absence with literature. Moreover, there was no statistical correlation between absence of PL and gender or handedness.

\section{0-80}

\section{Morphometric analysis of iliocaval region related to lumbar vertebra and aortic bifurcation on multidetector computed tomography}

Keskinöz EN*, Salbacak A**, Akın D***, Kabakçı ADA***, Yılmaz MT*** Çiçekçibaşı $\mathrm{AE}^{* * *}$, Özbek $\mathrm{O}^{* * * *}$

*Department of Anatomy Faculty of Medicine, Acıbadem University, Istanbul, Turkey; **Department of Anatomy, Faculty of Medicine, Ordu University, Ordu, Turkey; ***Department of Anatomy, Faculty of Medicine, Necmettin Erbakan University, Konya, Turkey; $* * * * *$ Department of Radiology, Meram Faculty of Medicine, Necmettin Erbakan University, Konya, Turkey

The aim of this study is measuring the value of morphometric data such as distance and location of the inferior vena cava (IVC) and comparing these with demographic values. The study was performed on the Multidetector Computed Tomography (MDCT) images for various medical resasons of 200 cases (100 male and 100 female) that ranged in age from 50 to 84 years. The morphometric data were obtained from the coronal, sagittal and axial images. As a result of the assesments, the distance of IVC to 1st lumbal vertebra (L1) and 2nd lumbal vertebra (L2) were measured as $(2.65 \mathrm{~cm}$ and $1.81 \mathrm{~cm})$ in males and $(2.11 \mathrm{~cm}$ and $1.42 \mathrm{~cm}$ ) in females with a high level of significance between the sexes; the distance of IVC to iliocaval region, 3 rd lumbal vertebra (L3) and 4rd lumbal vertebra (L4) were measured as $(0.65$ $\mathrm{cm}, 1.05 \mathrm{~cm}$ and $0.69 \mathrm{~cm})$ in males and $(0.49 \mathrm{~cm}, 0.91 \mathrm{~cm}$ and $0.55 \mathrm{~cm})$ in females with significance between the sexes. The 
average distance of IVC to promontorium was measured as 6.2 $\mathrm{cm}$. The confluence level of IVC was detected $46 \%$ in males, 39 $\%$ in females and average $42.5 \%$ in all individuals at level of lower third of L4. It was measured that IVC located on the right side of the abdominal aorta in all cases, iliocaval region was at right side of vertebral column in $73.5 \%$ and located below the aortic bifurcation $(\mathrm{AB})$ in $97 \%$ of the cases. The vertical distance of iliocaval region to $\mathrm{AB}$ was measured as $20.8 \mathrm{~mm}$ in males and $19.3 \mathrm{~mm}$ in females and negative correlation between the genders based on age was detected. We believe that the obtained results will contribute to the radiologist and surgeons during planning of new surgery and magnetic resonans imaging.

\section{$0-81$}

The effects of maternal hypothyroidism on the immunoreactivity of cytochrome p450 aromatase in the postnatal rat testes

$\underline{\text { Yildırım AB}}^{*}$, Özdamar $S^{*}$, Kaymak E*, Şakalar Ç* ${ }^{* *}$, Sezen $S^{* *}$ *Department of Histology Embryology, Faculty of Medicine, Erciyes University, Kayseri, Turkey; ${ }^{*}$ Department of Medical Biology, Faculty of Medicine, Erciyes University, Kayseri, Turkey

Hypothyroidism is that thyroid hormone is produced less and it occurs in approximately $2.5 \%$ of pregnancies. Mother hypothyroidism negatively affects newborns. Today, thyroid hormones are known to affect spermatogenesis and male infertility. Estrogen is very effective in male gonadal function. Aromatase is the enzyme that converts androgens to estrogens. For men the aromatase deficiency can cause infertility. However, there is not enough information about the interaction between thyroid hormones and aromatase. In this study, it is aimed to investigate the effect of maternal hypothyroidism on offspring testicular morphology and cytochrome-P450-aromatase (P450arom) immunreactivity. 18 Wistar albino pregnant rats were divided into three groups, namely A, B and C groups. Hypothyroidism was induced by adding drinking water which contains \%0.01 of propyl thiouracil (PTU). Hypothyroid mothers Group A: given PTU for 21 days during pregnancy (between gestational day 0-21), hypothyroid mothers Group B: given PTU for 21 days prior to pregnancy, control mothers Group C: given only water. Hypothyroid and control group mothers pups at postnatal day (PND) 15 and 60 were sacrificed. To determine immunreactivity intensity of P450arom and mRNA levels by RT-PCR were performed in the testis tissues. Structure of seminiferous tubule were evaluated by hematoxylin-eosin staining. Furthermore, it has been detected that there is an increase in P450arom immunoreactivity and P450arom mRNA levels in testiculars of PND 15 pups of group A and B when compared to the control group while there is a reduction in aromatase immunoreactivity with an increase in P450arom mRNA levels in testiculars of PND 60 pups of group $\mathrm{A}$ and $\mathrm{B}$ when compared to the control group. As a result of the research, it has been observed that while maternal hypothyroidism increases $\mathrm{P} 450$ arom immunoreactivity in offspring testicular cells in immature period, it decreases P450arom mmunoreactivity in mature period. In this case, it is determined that the testicular development and spermatogenesis process are affected negatively.

\section{0-82}

The anatomical relationship between the myofibriles of accessory iliac - psoas muscle and the femoral nerve

Unat F* $^{*}$ Şirintürk S, Cagimni P, Pınar Y, Gövsa F, Chatzioğlu GN

Department of Anatomy, Faculty of Medicine, Ege University, Izmir, Turkey

Objectives: Compression of the femoral nerve in the iliac fossa has been reported as a consequence of several pathologies. Besides, this may be as a result of aberrant muscular band of iliac and psoas muscles. The purpose of this research is to introduce aberrant muscle band of accessory iliac - psoas muscles and the femoral nerve as detailed.

Methods: Fifty semipelvis of human cadavers (14 female, 36 male specimens), (8 female and 26 male specimens as bilaterally) were dissected in the Department of Anatomy at the University of Ege.

Results: Three left and one right specimens have accessory psoas muscle. Four left and seven right specimens were determined as posterior accessory iliac muscle. Also one left specimen has both posterior and middle iliac muscle. In this specimen, accessory muscle was attached to anterior face of quadratus lumborum muscle with aponeurosis band. Additionally, the accessories muscles relationship with the nerve bundles of femoral nerve was determined.

Conclusion: These important variation may lead to an explanation about deviation of bundles and the migration of femoral nerve along the process of muscle coursing. It may be defective attachments of the myofibriles during fetal life. Especially aberrant fibers or accessory muscles in the pelvis may cause to compression onto femoral nerve especially following hip replacement and during pregnancy. The knowledge of the possible variations of the iliopsoas muscle complex and the femoral nerve may also give surgeons confidence during pelvic surgery. Keywords: Iliac muscle, psoas major muscle, femoral nerve

\section{0-83}

Multidetector computed tomography (MDCT) study of inferior vena cava related to lumbar vertebra

Keskinöz EN*, Salbacak A**, Akın D***, Kabakçı ADA***, Yılmaz MT ${ }^{* * *}$, Çiçekcibaşı $\mathrm{AE}^{* * *}$, Özbek O ${ }^{* * * *}$

*Department of Anatomy Faculty of Medicine, Acibadem University, istanbul, Turkey; ${ }^{*}$ Department of Anatomy, Faculty of Medicine, Ordu University, Ordu, Turkey; ${ }^{* * *}$ Department of Anatomy, Faculty of Medicine, Necmettin Erbakan University, Konya, Turkey; $* * * * *$ Department of Radiology, Meram Faculty of Medicine, Necmettin Erbakan University, Konya, Turkey

Inferior vena cava (IVC) is under the risk during the anterior surgical approach. This study was performed on 200 cases (100 male and 100 female) that ranged in age from 50 to 84 years to investigate the morphometric values such as diameter, angle, height, width of the IVC and compare these with demographic values such as gender, height, body weight and age based on 
Multidetector Computed Tomography (MDCT) images. As a result of the assesments, the transvers and anteroposterior diameter of the iliocaval region was mesured, respectively 29.9 $\mathrm{mm}$ and $16.2 \mathrm{~mm}$. The iliocaval angle of the IVC was $60^{\circ}$ in all individuals $\left(56.53^{\circ}\right.$ in males and $64.01^{\circ}$ in females), and the average height of angle of the ilioacaval region of IVC was as $23.3 \mathrm{~mm}$. The the lordosis angle was measured as $45.64^{\circ}$ in males and $52.14^{\circ}$ in females a high level of significance between the sexes. The width of the right common iliac vein, $1 \mathrm{~cm}$ below the iliocaval region, was measured as $14.1 \mathrm{~mm}$ in males and $13.1 \mathrm{~mm}$ in females; the width of the left common iliac vein, $1 \mathrm{~cm}$ below the iliocaval region, was measured as $17.3 \mathrm{~mm}$ in males and $15.8 \mathrm{~mm}$ in females. The width of IVC $1 \mathrm{~cm}$ and $2 \mathrm{~cm}$ above iliocaval region were measured as, respectively 22.7 $\mathrm{mm}$ and $21.0 \mathrm{~mm}$. Hence, the detailed knowledge about the morphological values of IVC may be significant both surgeons and radiologist during magnetic resonans imaging and interventional procedure.

\section{0-84}

\section{Using social media in anatomy education}

$\underline{\text { Yücel K}}^{*}$, Kaspar EÇ**, Özdemir S***, Aydıner IE ${ }^{* * * *}$, Çelik B****, Gergin $\mathrm{S}^{*}$

*Department of Anatomy, Faculty of Medicine, Yeditepe University, istanbul, Turkey; ${ }^{* *}$ Department of Biostatistics and Medical Informatics, Faculty of Medicine, Yeditepe University, İstanbul, Turkey; ${ }^{* * *}$ Department of Medical Education, Faculty of Medicine, Yeditepe University, Istanbul, Turkey $* * * \star$ First Year Medical Student, Faculty of Medicine, Yeditepe University, istanbul, Turkey; $* * * * *$ Second Year Medical Student, Faculty of Medicine, Yeditepe University, Istanbul, Turkey

Objectives: In the era of social media, it is now possible to be at where your students are and provide education anywhere, anytime. Here we present different social media and Web 2.0 tools used in anatomy education as well as feedback of our students.

Methods: The survey was prepared by Google Docs and sent to 336 healthcare students via Whatsapp. 302 students from four classes (82 first year medical students, 60 second year medical students, 58 first year pharmacy students, and 102 first year nursing and second year dietitian students) answered to our survey with a mean age of $20.1 \pm 1.2$ and a response rate of 90\%. We used Likert-type items in our survey.

Results: The tools used in anatomy education were as follows: 1 ) Wordpress Blog, 2) Whatsapp group, 3) E-Lab Blog, 4) On- line video lectures, 5) Twitter class, 6) YouTube videos and 7) online games in the blog, 8)Lab demonstration videos, 9) Vine Lab, 10) Mp3 lectures; lecturing each sentence in the class notes , 11) Answers to questions at Whatsapp moved to a blog as mp3 files, 12) Skype classes. E-Lab was the mostly used tool by the students, followed by Whatsapp group and lab demonstration videos. According to the students the greatest contribution to the learning process was from E-lab and online lectures, as well as having the blog. However, sharp differences among classes existed $(\mathrm{p}<0.05)$. The main reason for not using the online tools was lack of time. Students liked the easy access to the materials and to the instructor, and also online lectures. Too many options and requirement of too much time were the disadvantages reported.
Some complained about the irrelevant messages in the Whatsapp groups.

Conclusion: The social media strategy in education should be customized according to students' needs and requirements.

\section{0-85}

\section{The relationship of white/grey substances areas with sex and age: human autopsy study}

$\underline{\text { Aydın } \mathrm{AG}^{*}}$, Dereli AK ${ }^{* *}$, Acar K**, Adıgüzel E*

* Department of Neuroscience, Institute of Health Sciences, Pamukkale University, Denizli, Turkey; ${ }^{* *}$ Department of Forensic Science, Faculty of Medicine, Pamukkale University, Denizli, Turkey

Corpus callosum is the biggest part of the fiber groups form the interhemispheric connectivity between cortical neurons. Corpus callosum morphology may be affected by neurodegeneration/ demyelination or vice versa. White matter of telencephalon is important for its functions. The corpus callosum morphology may provide information about the telencephalon physiology. So, we investigated the relation between midsagittal surface area of corpus callosum and cerebral cortical thickness, and the effect of sex, age and handedness on this relationship in post mortem autopsy materials. Total 18 brains (13 male, 5 female; ranging in age $14-81$ years) obtained from forensic autopsy material were included for study. The brain hemispheres were divided by midsagittally cutting after weighting. Then the hemispheres were coronally cut at interval of $2 \mathrm{~cm}$. The photos were taken from medial surface of hemispheres for corpus callosum, and same directed surfaces of the brain slices for white and grey matter of hemispheres. SHTEREOM ${ }^{\odot}$ software was used for measurement of the corpus callosum, white and gray substances' areas on the photographs. Mean area of corpus callosum, grey substance, white substance were $11+/-2.1 ; 136+/-23.1 ; 178.6+/-36.3 \mathrm{~cm}^{2}$ consecutively. Average Brain Weights were $1411.1+/-133.1 \mathrm{gr}$. There was no statistically significant differences between two sexes about area of the corpus callosum, white and grey substances of the brain. There were statistically significant differences between sex and brain weight (Male: 1448.5+/- 38.1; female $1314+/-49.2 ; \mathrm{p}=0.03)$. There was a positive relation between area of white substances and brain weight $(r=0.67$, $\mathrm{p}=0.02$ ) and also negative relation between age and area of grey substances $(\mathrm{r}=-0.53, \mathrm{p}=0.02$; Pearson correlation). As a result, average brain weights of females are lower than those of males. And also the white substances is positively contributed the brain weights, and the area of grey substances decreases with age.

Keywords: Corpus callosum, human brain, postmortem, stereology

\section{0-86 \\ Proximal femoral focal deficiency}

Karaca L, Keleș P, Kara A, Kantarcı M

Department of Anatomy, Faculty of Medicine, Atatürk University, Erzurum, Turkey

Objectives: This study aims to charaterize the short femur and describe typical findings of proksimal focal femoral deficiency 
Methods: In this report, an asimetric short femur lenght was found, during a routin second trimester ultrasonogrphic examination of a twentieth week pregnant woman. There wasn't any other sonographic anomalies. After the premature birth on thirtyfifth week, on an anteroposterior direct radiographic examination, the left femur was clearly hypoplasic with regard to the right femur. (A) At the same image, left acetabulum was shallow and displasic. At the systematical and genetic examination of the new born, we couldn't find any anomalies. Two years after the birth, on the serial radiographic evaluations, new bone mineralisation areas observed on the proximal femur region. (B; C) On the following evaluations, the mineralisation was progressing, and, femoral head was ossificated.

Results: In second trimester, some ultranonographic biometric measurements should be obtained to assess fetal anatomy and detect the presence of any fetal anomalies. Femur lenght measurement is one of the biometric assessments. Short femur lenght on second trimester sonography, could represent a normal varient, or could be a marker of congenital malformation, skeletal dysplasia, fetal growth restriction. Also it may indicate inaccurate pregnancy dating. If there isn't these manners, it could be regarded as an isolated short femur lenght. We report a case of izolated short femur lenght at child. Proximal focal femoral dysplasia should be considered in the differential diagnosis when a short femur is discovered.

\section{0-87}

An exoteric narrative of an esoteric disorder: on the anatomical codes of the temporomandibular joint diseases

\section{Balcıoğlu HA}

Department of Anatomy, Faculty of Dentistry, Istanbul University, istanbul, Turkey

Widely acknowledged as being the most complicated joint in human body, temporomandibular joint has been introduced, in an esoteric manner, to promise disorders that are highly unlikely to be overcome. Debates between the camps of rehabilitative approaches concerning how temporomandibular joint disorders should be managed is typically focused around attempts to overcome the symptoms other than to evaluate the pathogenetic mechanisms offered by certain anatomical key points. In and of itself the process of how the practitioner thinks about clinical problem solving may not be important to him or her, however, it may turn out to be a poisoned chalice. Therefore, assessment of temporomandibular joint disorders should not be based simply on an expostulation to the former occlusionist thinking or old-fashioned prosthetic based prescriptions, but on a solid clinical anatomy knowledge. The goal of the presentation is to investigate the rationale behind the claim that evident superiority in anatomical knowledge is incompatible with failed treatment of temporomandibular disorders. Outline of the lecture will be as follows: starting with some general remarks about temporomandibular joint and its disorders, the above-mentioned arguments will be presented. After that, from a certain scientific point of view, neither theo- retically nor practically but rather exoterically, attention will be devoted to the unique anatomical features of the effected joint, that will point out the best practice guide for the treatment.

\section{0-88}

\section{The effects of different calcium concentration on osteocytes like MLOY4 cell}

Dönmez BO${ }^{*}$, Jung $\mathrm{HJ}^{* *}$, Younesi $\mathrm{M}^{* *}$, Akkuş $\mathrm{O}^{* *}$

*Department of Nutrition and Dietetics, Antalya Faculty of Health, Akdeniz University, Antalya, Turkey; **Department of Mechanical and Aerospace Engineering, Case Western Reserve University, Orthopaedic Bioengineering Laboratories, Cleveland, $\mathrm{OH}$, USA

Osteocytes, which are the most abundant cells in bone and they are embedded in bone matrix. Osteocytes is one of the most important cell to regulate both bone modeling and remodeling and they can be send signals for regulate bone metabolism. Osteocytes produce some of the most important factors like receptor activator of nuclear factor Kappa-B ligand (RANKL) that plays a role for osteoclasts to remove bone and some cytokines like inteleukin 1, (IL-1 $\beta$ ) increase RANKL activitiy in osteocytes. Also IL-1 $\beta$ and tumor necrosis factor alpha (TNF $\alpha$ ) are produced by osteocytes as pro-inflammatory cytokines. The aim of the study is to show which calcium concentration is prompt signal system for cytokines. In the present study, different calcium concentration was used on MLOY4 cells. After calcium treatment at 30th minutes and 24th hours, the number of live-dead cells was counted, production of nitrit oxide was measured with nitrit oxide kit and some cytokines levels were measured with ELISA. While amount of calcium increased, number of dead cells were increased. These result with together knowledge of the calcium mechanism on the MLOY4 cells may play important role of the relationship of calcium and cytokines production.

\section{0-89}

The localization of the supratrochlear, supraorbital and zygomaticotemporal nerves: an alternative measurement to use in migraine treatment

\begin{abstract}
Gilan IY'* Tezer MS ${ }^{* *}$, Aktekin $\mathrm{M}^{* * *}$, Özcömert VB****
*Department of Anatomy, Faculty of Medicine, Mersin University, Mersin, Turkey; **Department of Otorhinolaryngology, Faculty of Medicine, Mersin University, Mersin, Turkey; $* * \star$ Department of Anatomy, Faculty of Medicine, Acibadem University, Istanbul, Turkey; $* * * *$ Department of Biostatistics, Faculty of Medicine, Mersin University, Mersin, Turkey
\end{abstract}

Objectives: Treatment of migraine using injections of Botulinum toxin (Botox), and surgical decompression of the trigger points have been shown to reduce or eliminate migraines in patients who are incompletely treated by traditional medical management. This study was performed to suggest a different and more practical detection parameter for the locations of the supraorbital (SON), supratrochlear (STN), and the zygomaticotemporal (ZTN) nerves. 
Methods: 11 embalmed and 5 fresh frozen cadavers were used in this study. Distances between the exit points of SON and STN and several reference points such as midline and the medial and lateral canthi were measured. The distance between the ZTN and the lateral canthus and lateral orbital margin were also measured. Additionally, the angles between a horizontal line passing through both canthi and the exits of the SON and STN were determined. Similarly, the angle between the ZTN and the lateral canthus were measured.

Results: We found statistically significant differences between male and female subjects in the STN-lateral canthus distance on both sides. Also, there are statistically significant differences between fresh frozen and embalmed cadavers in the STN-lateral canthus distances on the right as well as left side, angle of STNmedial canthus on the left and angle of ZTN-lateral canthus on the right. In addition, we calculated the averages of all datas.

Conclusion: In the present study, we determined the exit points of SON and STN from the orbit and defined the angles between their exits and the medial and lateral canthi. Thus, we provide an easier parameter for the detection of the exits of the SON and STN. Similarly, the exit of ZTN through the temporal fascia was determined and the angle between its exit and the lateral orbital margin was provided. These data may be helpful for surgeons particularly in forehead lift operations and in migraine treatment as well as in local surgical interventions.

\section{0-90}

Creating three dimensional anatomy atlases by using augmented reality technique

Kapakin S

Department of Anatomy, Faculty of Medicine, Atatürk University, Erzurum, Turkey

Augmented Reality rapidly spreading in all areas of the life is a state of art technology in which real world is enriched by computer generated contents (auditory and visual files) and made stunning. It is obvious that computer generated contents have a lot of advantages to clasical anatomy atlases. Firstly, this technique provides three-dimensional imaging of any organ. Secondly, it enables students, researchers, and surgeons to perceive spatial position and neighbourly relationship of any organ. Finally, it allows to make simulations of surgical procedures. The aim of this study is to define the notion of the augmented reality and examine potential uses in anatomy atlases. Three fundamental components of the augmented reality technology are the head mounted display, tracking system and mobile computer. The camera connected to computer captures the images in traditional way. When the camera detects the marker in the image, captures the information encoded in it and it sends this information to the computer. The computer decode information and projects $3 \mathrm{D}$ objects on the marker. It appears as if $3 \mathrm{D}$ objects were parts of the real world. The computer can pursue the size and movements of the $3 \mathrm{D}$ objects. The augmented reality makes lectures more attractive. It makes boring lectures funny. It makes hard topics easier to learn. It provides easy access to course material which is difficult to obtain. It improves quality of education. Augmented reality enables a mobile technology user to interact with virtual comput- er generated contents embedded within the physical anatomy atlases. Augmented Reality can be valuable for both teaching anatomy and learning anatomy. Physical learning environment are replaced with online learning environments through Augmented Reality technology.

Keywords: Anatomy, atlas, augmented reality (AR)

\section{0-91}

\section{Virtual reality modeling language (VRML) form of the rat cerebral ventricular system}

\author{
Kapakin S \\ Department of Anatomy, Faculty of Medicine, Atatürk University, \\ Erzurum, Turkey
}

The rat cerebral ventricular system keeps the brain healthy. This study was conducted to reconstruct the rat cerebral ventricular system three-dimensionally and generate VRML form of it. Images obtained from the atlas titled "The Rat Brain in Stereotaxic Coordinates" by Paxinos and Watson (2005) were used to reconstruct the rat cerebral ventricular system. Surfaces were rendered by using Surfdriver 3.5 software. These models were subjected to different processes in $3 \mathrm{D}$-Doctor software for creating the VRML form of them. The components of the rat cerebral ventricular system were similar to that of the human cerebral ventricular system. There were one central ventricle (the third) associated with two lateral ones (first and second) and a caudal one (fourth). But fourth ventricle had lateral extensions paralel to lateral ventricles. The total volume and surface area of the rat cerebral ventricles were calculated to be $52,6645 \mathrm{~mm}^{3}$ and $262,2931 \mathrm{~mm}^{2}$ using 3D-Doctor software. The present study has defined enigmatic and complicated morphology of the rat cerebral ventricular system, which has clinical significance in diagnosis, treatment and surgical intervention. It can be possible to detect morphological changes in the rat cerebral ventricular system. Clinicians can follow up diseases altering the shape and size of the rat cerebral ventricular system.

Keyword: Rat, rat cerebral ventricular system, VRML

\section{0-92}

\section{The ankle bones: three dimensional reconstruction, photorealistic imaging and physical biomodeling}

Kapakin S

Department of Anatomy, Faculty of Medicine, Atatürk University, Erzurum, Turkey

There are 7 bones in ankle complex of the foot. The ankle bones are positioned so as to provide stability and movement for foot. The motion of the ankle bones takes place on all three axes $\mathrm{X}, \mathrm{Y} \& \mathrm{Z}$. It is complicated and it is not fully clarified. To understand the shape, neighbourly relationships and mobility of the ankle bones are extremely important in terms of surgical interventions. The goal of this study is to create tangible hard copies of the ankle bones with rapid prototyping and use them 
in surgical applications. In the study, the Visible Human Male Dataset was used. High resolution color anatomical cryosections were preferred because these images have higher quality than the others. The Surfdriver software package is applied to the anatomical cryosectional images to reconstruct the ankle bones as three-dimensional DXF (data exchange file) models. These models are post-processed in Cinema 4D software for virtual reality modelling language (VRML) and STL (Standard Triangulation Language) formats. Solid replicas are manufactured in a rapid prototyping machine by using the STL format. This technique was reliable enough to manufacture the ankle bones. It enabled us to reveal structural neighborly relations formed amoung the bones in detail during movements made by ankle bones on $\mathrm{X}, \mathrm{Y}$, and $\mathrm{Z}$ axes. The volumes of the calcaneus and talus were $85,190,6815 \mathrm{~mm}^{3}$ and $51779,1205 \mathrm{~mm}^{3}$ the surface areas of the calcaneus and talus were $13195,3927 \mathrm{~mm} 2$ and $8821,7834 \mathrm{~mm}^{2}$ respectively. The surgeons can use these implants as a medical device to replace missing bones. They can practice on these custom made implants prior to surgery. These implants provides surgeons to be ready for undesirable conditions in the surgery.

Keywords: Ankle bones, rapid prototyping, implant

\section{0-93}

The effects of single dose ketamine application on the hippocampal volume of prenatally stressed juvenile rats $\underline{\text { Aydin Ö}}^{*}$, Polat E*, Aydın EG ${ }^{* *}$, Ulupınar E***

*Department of Interdisciplinary Neuroscience, Faculty of Medicine, Eskişehir Osmangazi University, Eskişehir, Turkey; **Department of Pharmacology, Faculty of Medicine, Eskişehir Osmangazi University, Eskişehir, Turkey; ***Department of Anatomy, Faculty of Medicine, Eskişehir Osmangazi University, Eskişehir, Turkey

Stress exposure during gestation can exert a major impact on brain development and thereby contribute to the pathogenesis of depression. Since depressive disorders are prevalent and disabling with high economic burden for society, it has a major impact on public health. Ketamine, in contrast to delayed action of antidepressants, offers a unique antidepressant property with rapid onset of response and high rate of responders. The aim of this study was to investigate both behavioral and morphological effects of single dose ketamine application in prenatally stressed juvenile rats. In experimental groups, pregnant Sprague-Dawley rats were immobilized between gestational day 14 and 21, for 3 hours. At postnatal day 40, offspring from both gestationally stressed $(\mathrm{n}=16)$ and control dams $(\mathrm{n}=16)$ were received a single dose of ketamine $(10 \mathrm{mg} / \mathrm{kg}$, i.p.) or equal volume of saline. Effects of stress exposure were evaluated behaviorally by sucrose preference, forced swim, open field and modified tail suspension tests. Following intracardiac perfusion, brains were dissected and embedded in paraffin. Nissl-stained sections were used to calculate the volume of hippocampal subfields by using Cavalieri volume estimation method. Our results showed that depressive-like behavior, anhedonia and endurance of animals were not affected from the applied stress protocol. In addition, volumes of the dentate gyrus and pyramidal cell layer of CA1-3 were similar in the experimental and control groups. Ketamine application did not significantly alter those parameters. Current results suggest that prenatally stressed animals were resistant to depression at this specific period of their development. Their tendency to develop depression at later ages and individual responsiveness of animals to rapid-acting antidepressant treatments need to be addressed by future studies.

Keywords: Hippocampus, Cavalieri volume estimation, prenatal stress, ketamine

\section{0-94}

Activation of neurons in the medial prefrontal cortex of prenatally stressed rats was not altered by single dose ketamine injection

Polat E* ${ }^{*}$ Aydın OÖ*, Aydın EG**, Ulupınar E***

*Department of Interdisciplinary Neuroscience, Faculty of Medicine, Eskişehir Osmangazi University, Eskişehir, Turkey; **Department of Pharmacology, Faculty of Medicine, Eskişehir Osmangazi University, Eskişehir, Turkey; ${ }^{* * *}$ Department of Anatomy, Faculty of Medicine, Eskişehir Osmangazi University, Eskişehir, Turkey

Objectives: Despite widely clinical usage of N-methyl-Daspartic acid (NMDA) receptor antagonist ketamine as an anesthetic agent, its rapid antidepressant effect only recently has been reported. Its effectiveness has been shown in adult stress models, but relatively little is known in prenatal stress models. Prefrontal cortex (PFC) is one of the two brain regions containing the largest concentrations of glucocorticoid receptors and an important target for investigation of stress-related alterations. In this study, we examined the effects of single dose ketamine injection on the activation of neurons located in the medial prefrontal cortex of prenatally stressed juvenile rats.

Methods: Pregnant Sprague-Dawley rats were immobilized in wire-meshed cylinders at their last week of pregnancy, for 3 hours. Half of the animals from stressed group $(n=8)$ received a single dose of ketamine injection $(10 \mathrm{mg} / \mathrm{kg}$, i.p.) and the others $(n=8)$ received similar amount of serum physiologic injections. Similar treatments protocols were applied to control groups ( $n=16)$ which were not stressed during their pregnancy period. At postnatal day 40, after stimulation by forced swimming for 6 minutes, animals were perfused to investigate the immediate gene expression by c-fos immunohistochemistry.

Results and Conclusion: The density of activated neurons in the medial PFC of prenatally stressed animals was not significantly different than those of control groups. Application of single dose ketamine injection did not cause a significant alteration in the number of c-fos expressing neurons. These results suggest that stressful stimuli might evoke only minimal changes in the immediate gene expression during the stress hypo-responsive period, possibly to protect the rapidly developing brain from the impact of elevated glucocorticoids.

Keywords: Prefrontal cortex, c-fos, prenatal stress, ketamine 


\section{0-95}

Evaluation of the relationship between metatarsophalangeal, metatarsocuneiform and naviculocuneiform joint angles

Tuğtağ Demir B*, Üzel $M^{* *}$, Gümüşalan $Y^{*}$, Çağlar $V^{* * *}$

*Department of Anatomy, Faculty of Medicine, Fatih University, Istanbul, Turkey; **Department of Orthopaedics and Traumatology, Faculty of Medicine, Kahramanmaraş Sütçü Imam University, Kahramanmaraş, Turkey; ***Department of Anatomy, Faculty of Medicine, Namık Kemal University, Tekirdağ, Turkey

First metatarsophalangeal (MTP) and first metatarsocuneiform (MC) joints are the key structures in a variety of disorders, including osteoarthritis associated with hallux limitus and rigidus, hallux varus, severe hallux abductovalgus, recurrent hallux valgus, and rheumatoid foot deformity. But function of the first naviculocuneiform (NC) joint has long been the subject of discussion, debate and controversy. In this study, investigation of the effects of the tendons of peroneus longus and tibialis anterior muscles as well as the association of those interactions with the metatarsophalangeal, metatarsocuneiform and naviculocuneiform joints was aimed. While the foot was located in anatomical position, the angles for metatarsophalangeal, metatarsocuneiform and naviculocuneiform joints in the metatarsal and sagittal planes were photographed after inserting $8-10 \mathrm{~cm}$ long needles. These angles were calculated by using Corell Draw 5.00. In the horizontal plane, it was found that the angle for metatarsophalangeal joint was between $4.5^{\circ}$ $10^{\circ}$ in females and $3,8^{\circ}-9,9^{\circ}$ in males and the angle for metatarsocuneiform joint was between $5.1^{\circ}-12.6^{\circ}$ in females and $2.7^{\circ}$ $12.3^{\circ}$ in males. Similarly, the angle for naviculocuneiform joint was measured between $2.1^{\circ}-9.4^{\circ}$ in females and $3^{\circ}-11.4^{\circ}$ in males. Intermetatarsal angle varied between $6^{\circ}-11.2^{\circ}$ in males and $5.5^{\circ}-11^{\circ}$ in females. Angle of the first MTP joint is directly deformed in hallux valgus and hallux varus cases. However, this abnormality indirectly affects MTC and NC joints as well and leads to the deformation of the medial longitudinal arch and causes foot pains. Normal values of the angles of those joints should be kept in mind since evaluation of patient's foot deformity will be based on these results. Hence, the severity of patient's foot deformity will be assessed according to the results of those analyses in deciding the conservative or surgical treatment.

\section{$0-96$}

\section{The foot architecture and biomechanics from viewpoint of shoe design}

Durgun B

Department of Anatomy, Faculty of Medicine, Çukurova University, Adana, Turkey

This presentation aims to review and analyze the foot architecture and biomechanics with emphasis on the shoe design. The foot has an architecture which provide to perform its dual static and dynamic role. The foot architecture evolved to provide: (1) mechanisms to limit the deviation of the center of mass dur- ing locomotion and thereby minimize the expenditure of energy; (2) a base of support of sufficient dimensions for the stability necessary to maintain the upright position without excessive muscular activity; (3) mechanisms for flexibility to absorb the shock of the body weight and for accommodation to uneven terrain; (4) rigidity of the foot when it acts as a lever in the push-off period of stance. The foot is presented in all its complexity as an integrity with its different bones, joints, ligaments and muscles. This complex integrity is analogous to architecture of vaults and arches. The bones as "key stone", the ligaments as "metal staples" and muscles as "tie beams" or as "suspension bridge", all play an important role in biomechanics. They respond to cyclical demands for flexibility and resilience, the foot having to either absorb or transmit dynamic forces. The slight mobility of foot arches when weight is applied to and removed from the foot makes walking and running more economical in terms of energy. When the muscles are fatigued by excessive exercise by standing for long periods by being overweight or by illness, the muscular support gives way, the ligaments are stretched, and pain is produced. Shoe comfort has by far the highest priority for males and females, and high pressures under the foot are directly related to discomfort. Therefore the perfectly suited shoes should be designed to support the arches of foot, to decrease the plantar surface area, and to minimize the pressure due to body weight.

Keywords: foot, foot anatomy, foot biomechanics, shoe design

\section{0-97 \\ Effect of morphine-induced CPP on the number of neurons containing serotonin 5-HT2A receptors in adult male rats' hippocampus}

Mohamadi $\mathrm{R}^{*}$, Jameie $\mathrm{SB}^{* *}$, Jahanshahi $\mathrm{M}^{* * *}$

*Department of Anatomy, Faculty of Medicine, Shahid Beheshti University of Medical Sciences, Tehran, Iran; **Department of Anatomical Sciences, Faculty of Medicine, Iran University of Medical Sciences, Tehran, Iran; ***Department of Anatomy, Golestan Neuroscience Research Center, Golestan University of Medical Science, Gorgan, Iran

Objectives: The conditioned place preference (CPP) is a standard preclinical behavioral model used to study the rewarding and aversive effects of drugs. Morphological alterations of the hippocampus due to opium were reported in humans and animals. Therefore, this study was done to determine the CPP effects on the number of neurons containing serotonin 5HT2A receptors in the rats' hippocampus by immune-fluorescent technique.

Methods: 56 male Wistar rats weighted average 200-220 g was used. For behavioral tests, rats distributed into eight groups [Control, Control-saline, Sham morphine $(2.5,5,10 \mathrm{mg} / \mathrm{kg}$ ) and CPP groups $(2.5,5,10 \mathrm{mg} / \mathrm{kg})]$. The rats in sham and CPP groups were received morphine at different doses for three days by subcutaneous injection and control-saline group received saline $(1 \mathrm{ml} / \mathrm{kg})$ and then Conditioned Place Preference test in them were investigated. 48 hours after behavioral testing animals were decapitated under chloroform anesthesia and their brains fixed and after tissue processing, 
sections were stained with immune-fluorescent techniques for 5-HT2A receptors. The density of 5-HT2A receptor containing neurons was measured in all hippocampal areas and their significance was compared among groups $(\mathrm{p}<0.05)$.

Results: Our data showed that morphine injection lonely, and CPP phenomenon increased 5-HT2A receptor neurons in all hippocampal areas compared to controls. The differences between CPP groups and sham-morphine groups were significant too. On the other hands, the CPP groups had more 5-HT2A receptors than the sham-morphine groups, and also the shammorphine groups had more 5-HT2A receptors than the controls.

Conclusion: We concluded that the phenomenon of conditioned place preference induced by morphine can cause a significant increase in the number of neurons containing serotonin 5HT2A receptor in a hippocampus compared to controls.

\section{0-98}

Morphological characteristics of stromal and glandular components of adenomyosis and adenomyosis development stages, focused on stroma invasion theory

Kim A

Faculty of General Medicine, Asfendiyarov Kazakh National Medical University, Almaty, Kazakhstan

Introduction: Adenomyosis is a common condition in women of middle age. This myometrial lesion has ectopic endometrial glands and stroma invading myometrium. The aims of this study were to evaluate the features of stromal and glandular components in case of adenomyosis and to define the stage of adenomyosis, depending on the depth of invasion the stromal and glandular components. Indeed, morphological research would allow understanding what components play a role in the beginning of the process of invasion.

Objectives: We analyzed morphological features of stromal and glandular components in case of adenomyosis in 20 patients (35-50 years old), who were diagnosed with adenomyosis at the Research Center for Obstetrics, Gynecology and Perinatology of the Republic of Kazakhstan.

Methods: We analyzed surgical material of patients who underwent hysterectomy during 2013 and 2014. Tissues were stained by hematoxylin and eosin stain (HE).

Results: In the myometrium we observed adenomyosis foci, including two components: the stromal and glandular. Stromal component was characterized by the presence of fibers and spindle-shaped cells. In different parts of myometrium stromal components varied in size (from single fibers to massive fields of stromal component). And in cases of massive stromal component, there were no glands. Glandular component was characterized by presence of various shapes and sizes of gland, pre- dominantly in medium caliber, with proliferating epithelium and surrounded by varying size of stroma.

Conclusion: The pattern of growth of stromal component suggests that in cases of adenomyosis, invasion of myometrium starts with stromal component then glands follow after storm, and this confirms the invasive theory of endometriosis.

\section{0-99}

Topographical distribution of cholinergic brainstem axons in the thalamus and their relationship with the thalamostriatal systems

Hacioğlu Bay H*, Dautan $\mathrm{D}^{* *}$, Bolam $\mathrm{P}^{* *}$, Huerta-Ocampo ${ }^{* *}$, Segovia JM**

*Department of Anatomy, Faculty of Medicine, Marmara University, IIstanbul, Turkey, **Department of Pharmacology, Medical Research Council Anatomical Neuropharmacology Unit, University of Oxford, United Kingdom

Objectives: A Cre-driver rat-line has here been used to identify thalamic nuclei as targets of the cholinergic brainstem. A second aim of this study was to find out which thalamic nuclei host the thalamostriatal neuron bodies and influence the activity of the striatum as a result of the cholinergic impact from the brainstem proposed as an indirect route from cholinergic brainstem to the striatum.

Methods: Three groups of ChAT::Cre + rats received single injections of adeno-associated virus serotype2 (AAV2) into the rostral part of the PPN (PPNr), the caudal part of the PPN $(\mathrm{PPNc})$ or the LDT. Three further groups received double injections of AAV2 virus as above and also a Fluoro-Gold injection in the dorsal striatum for the PPNr\&PPNc injections and in the ventral striatum for the LDT injections. A fluorescent Nissl immuno stain used to show the borders of thalamic nuclei and outlined with Paxinos and Watson, 2007 as a guide. Cholinergic axons were mapped after Anti-GFP immunohistochemistry according to Dautan et al. (2014). No further stain was needed for the FG labelled neurons and all the FG labelled neuron bodies were marked. The density of the cholinergic axons also the number and the size of the varicosities in each thalamic nucleus were calculated from confocal images with the ImageJ programme.

Results: We observed a high correlation between striatal-projecting thalamic regions and dense cholinergic innervation from the cranial to caudal brainstem, with a motor to limbic gradient. These results coincide with the topographical organisation of the direct brainstem cholinergic projections to the striatal systems, where $\mathrm{PPNr}$ targets dorsolateral striatum and LDT targets nucleus accumbens, suggesting the convergence of a direct and an indirect cholinergic brainstem pathway to the corresponding functional regions of the striatum.

The EM level of the study is still in process. 


\title{
Poster Presentations
}

\author{
(P-1 - P-327)
}

\section{P-1}

Neurons from the adult human dentate nucleus

$\underline{\text { Maric D }}^{*}$, Milosevic N**, Grbatinic I***, Smiljenic D*, Krstonosic B*

*Department of Anatomy, Faculty of Medicine, University of Novi Sad, Novi Sad, Serbia; **Department of Biophysics, Faculty of Medicine, University of Belgrade, Belgrade, Serbia; ***Laboratory of Digital Image Processing, Faculty of Medicine, University of Belgrade, Serbia

Objectives: Although many relevant findings regarding to the structure, morphology and cytoarchitectural development of the dentate nucleus have been presented so far, very little qualitative and quantitative information has been collected on neuronal morphology in the adult human dentate nucleus.

Methods: The neurons were labelled by Golgi staining from thirty human cerebella, obtained from medico-legal forensic autopsies of adult human bodies and free of significant brain pathology. Two-dimensional digital image of each dentate neuron was recorded by a digital camera connected to a light microscope.

Results: The soma and dendritic field size of the human dentate neurons were qualitatively analyzed initially, and these cells were classified into two main class: the small and the large multipolar neurons. Taking into account the shape of the cell body, number of the primary dendrites, shape of the dendritic tree and their position within the dentate nucleus, three subclasses of the large multipolar neurons has been recognized. The results pointed out that the small multipolar neurons represent a unique cell class of the dentate nucleus, having in mind that they have significantly different soma size, dendritic field size and dendritic branching pattern than the large multipolar neurons. As for three subclasses of the large neurons, they can be considered as distinct cell classes since they have different soma size and dendritic branching pattern.

Conclusion: The hypothesis concerning the classification of neurons from the human dentate nucleus has so far been qualitatively confirmed in foetuses and premature infants. From this point of view, this study represents the first qualitative analysis and classification of the large multipolar neurons in the dentate nucleus of the adult human, as well as the first quantitative analysis of the cell types in the human dentate nucleus.

Keywords: Cell classification, cluster analysis, dentate nucleus, human, neuron types

\section{P-2}

Histo-morphological changes in bruises in human

Petrova I, Milenkova L, Kostovska N, Mitevska E, Gerasimovska Z

Institute For Histology and Embryology, Faculty of Medicine, Skopje, Macedonia
Introduction: Histological structure of normal skin includes intact blood vessels and absence of inflammatory cells in the dermis and hypodermis. When a bruise occurs there is extravasation of blood and influx of inflammatory cells in the layers of the skin.

Objectives: The objective of this study was to determine the histo-morphological changes in skin when a bruise is formed.

Methods: Total of 20 samples of human skin were divided in 2 groups, the first one includes samples with normal skin and the second one samples with bruised skin. The skin tissue was processed with usual hematoxylin-eosin and special Perl's Prossian blue method of dyeing and assessed with light microscopy for qualitative histo-morphological analysis.

Results: Qualitative histo-morphlogical analysis demonstrated presence of wide fibrous septa in the bruised skin as result of bleeding. Also there was presence of neutrophils and macrophages in the bruised skin. These changes were located exactly in the area of bruising, in the connective and adipose tissue of dermis and hypodermis.

Conclusion: When a bruise is formed, there is not just bleeding in the tissue. There is a large cascade of reactions in the body attempting to resume the tissue damage. In that manner the immune response is activated, delivering neutrophils and macrophages into the bruise, which phagocyte erythrocytes and hemoglobin. Detecting these cells and pigments with specific histological methods of dyeing we can further discuss about the age of bruises.

Keywords: Bruises, histo-morphological changes, human skin

\section{P-3}

Prompt response of the lymphoid tissue in Wistar rats' lungs after single dose dexamethasone application

Mitevska E, Gerasimovska Z, Petrova I, Kostovska N, Milenkova L

Institute for Histology and Embryology, Faculty of Medicine, Skopje, Macedonia

Objectives: The work was made to determine the eventual reaction of lymphoid tissue in the Wistar rats' lungs after application of single high dose of dexamethasone.

Methods: 24 female Wistar rats were divided into two groups, each one comprising 12 animals: control group that was given physiological solution and experimental group that was administered dexamethasone of $3 \mathrm{mg} / \mathrm{kg}$ bw. Substances were applied once, intramuscularly, 24 hours before the animals were sacrificed. Paraffin sections of the lungs were stained according to the methods: hematoxylin-eosin, Van-Gieson and Masson. 
Results: Histological analysis of the mucous associated lymphoid tissue of the lungs conducting airways has shown that there was reduced infiltration with lymphocytes in lamina propria of the mucosa of bronchi and bronchioles, after dexamethasone application. Lymph follicles near the bronchi and bronchioles were smaller and had lower lymphocyte density, but present germinal centers. It was noted that there was a reduction in the accumulations of lymphocytes in the adventitia of conducting airways blood vessels in the lungs. The presence of lymphocytes in the lungs interstitial connective tissue was also reduced.

Conclusion: The results of the work showed that the single administration of high-dose dexamethasone causes prompt response of lymphoid tissue in terms of reduction of lymphocyte cell population in the Wistar rats' lungs.

\section{P-4}

\section{Reactive changes of lymphoid tissue in the intestines wall after application of single high dose of dexamethasone}

Gerasimovska Z, Mitevska E, Petrova I, Milenkova L, Kostovska N

Institute For Histology and Embryology, Faculty of Medicine, Skopje, Macedonia

Objectives: The aim of our work was to determine the eventual alteration of lymphoid tissue in the Wistar rats' intestines wall after application of single high dose of dexamethasone.

Methods: The experiment was performed on 24 female Wistar rats divided into two groups, each one comprising 12 animals: control group that was given physiological solution and experimental group that was administered single high dose of dexamethasone of $3 \mathrm{mg} / \mathrm{kg}$ bw, intramuscularly. Paraffin sections of the intestines were stained according to the methods: hematoxylin-eosin, Van-Gieson and Masson.

Results: Histological analysis of the intestines in the control group of rats has shown that intestines mucous associated lymphoid tissue was consisted of solitary and aggregated lymph follicles (Peyer's plates) that most commonly include 2-3 lymph follicles. Lymph follicles had distinct germinative centers with lymphoblasts as well as clear cortex of mature lymphocytes. After application of a single high dose dexamethasone, lymph follicles in Peyer's plates and their germinative centers were with smaller dimensions in comparison with those in the control group of rats. Some of the lymph follicles have lost the regular nodulation that was replaced with diffusely distributed lymphoid tissue without germinative centers. The density of lymphocytes present in the lamina propria of the intestine mucosa was reduced.

Conclusion: The results showed that single high dose of dexamethasone causes alteration of lymphoid cell potential in the intestines wall in terms of its reduction.
P-5

The O-linked $\mathrm{N}$-acetylglucosamine containing epitope $\mathrm{H}(\mathrm{O}-\mathrm{GlcNAcH})$ is present in the hypertrophic and absent in the resting/reserve chondrocytes in the ossification centers of human embryos

Zibis A, Nikoalou M, Nikolaou G, Arvanitis D

Department of Anatomy, University of Thessaly, Medical School, Larissa, Greece

Objectives: Epitope $\mathrm{H}$ contains an O-linked $\mathrm{N}$ Acetylglucosamine residue in a specific conformation and / or environment recognized by the igM mouse monoclonal antibody $\mathrm{H}$ (mabH). The epitope $\mathrm{H}$ is present in several types of cells and in several polypeptides. The post-translational modification of serine and threonine residues of polypeptides by the addition of the sugar moiety N-Acetylglucosamine (O-GlcNAc) occurs in many nuclear, cytoplasmic and mitochondrial proteins which are involved in cell processes such as transcription, translation, protein compartmentalization, proteasomal degradation, competition with phosphorylation which influence cell division, differentiation, development, apoptosis, resistance to stress and are engaged in major diseases such as cancer, diabetes mellitus, cardiovascular and neurodegenerative diseases. Chondrocytes in the ossification centers pass from the resting/reserve state after multiplication to the hypertrophic state before their final death. The transition of chondrocytes to the hypertrophic state takes place under the induction of several biochemical properties characteristic for the hypertrophic status eg. Production of collagen X etc.

Methods: In the present work, we examined immunohistochemically the expression of $(\mathrm{O}-\mathrm{GlcNAcH})$ in the chondrocytes of the ossification centers of the long bones of the extremeties, of the ribs and the vertebrae in twenty human embryos of different ages using the indirect immunoperoxidase method and the monoclonal antibody $\mathrm{H}$.

Results: $3^{n}$ all cases examined, the hypertrophic chondrocytes in the ossification centers showed strong cytoplasmic staining for the O-GlcNAcH, whereas the resting/reserve chondrocytes remained unstained.

Conclusion: The O-GlcNAcH can be considered a biological marker for the hypertrophic chondrocytes of the ossification centers and further isolation and characterization of the polypeptide/s bearing the epitope $\mathrm{H}(\mathrm{O}-\mathrm{GlcNAcH})$ might shed more light into the investigation of the role of O-GlcNAc in endochondral ossification.

\section{P-6 \\ Integration of donor mesenchymal stem cell-derived neuron-like cells into host neural network in rat spinal cord transected}

Zeng YS

Department of Histology and Embryology, Zhongshan Faculty of Medicine, Sun Yat-sen University, Guangzhou 510080, China

Functional deficits following spinal cord injury primarily attribute to neural cell death and loss of connectivity. Therefore, we 
designed tissue engineered neural networks acting as bridges to rebuilt signal connection between the cut ends of the ruptured spinal cord. Rat bone marrow-derived mesenchymal stem cells (MSCs) with genetically enhanced expression of $\operatorname{TrkC}$ were induced into neuron-like cells after cocultured with Schwann cells overexpressing neurotrophin-3 in a gelatin sponge scaffold for 14 days. The formation of MSC-derived neural networks was confirmed by electron microscope and recording of spontaneous postsynaptic currents by whole-cell patch clamp. Then, this neural network scaffold was grafted acutely into rats with removal of 2-mm spinal cord tissue. Eight weeks later, MSC-derived neuron-like cells of the grafts maintained their synaptic connection or formed new connection with regenerative axons. Although biotinylated dextran amine labeled fibers of corticospinal tract only formed a few connections with MSC-derived neuron-like cells in the grafts, 5-HT labeled fibers formed much more connections. Furthermore, a part of these connections were confirmed to be synaptic connections by double-labeled immunoelectron microscope. Animals with neural networks scaffold transplantation resulted in higher BBB score and were improved cortical motor evoked potential. These findings indicate that MSC-derived neuron-like cells resulting from NT-3/TrkCinduced differentiation can partially integrate into host neural network in rat spinal cord transected.

\section{P-7}

\section{Decreased efferents of mesencephalic raphe nuclei to striatum in animal model of streptozocin-induced diabetes}

Mokhtari T* ${ }^{*}$, Hassanzadeh $\mathrm{G}^{*}$, Ghorbani $\mathrm{R}^{* *}$, Bayat $\mathrm{M}^{* * *}$, Zendedel A*, Mohammadi $\mathrm{Y}^{*}$, Barzroodi $\mathrm{M}^{* * *}$

*Department of Anatomy, Tehran University of Medical Sciences, Tehran, Iran; **Department of Anatomy, Kermanshah University of Medical Sciences, Kermanshah, Iran; ***Department of Anatomy, Arak University of Medical Sciences, Arak, Iran

Introduction: Diabetes mellitus is a metabolic disease which affects the brain as well as other organs. Connection of mesencephalic raphe nuclei to striatum is important, and reduction of these projections can play a negative role in regulatory connection between them...

Objectives: The main objective of this study was to determine the effects of diabetes on projections of mesencephalic raphe nuclei to striatum of rats...

Methods: Forty eight male adult Wistar rats were divided into four groups (1.control, 2. two-month diabetic rats, 3. fourmonth diabetic rats and 4. six-month diabetic rats). HRP was injected into the dorsal and ventral striatum separately and after perfusion with normal saline, fixation, and postfix solutions, the brains were sectioned coronaly $(40 \mu \mathrm{m})$. Frozen sections were reacted with TMB and counterstained with $1 \%$ neutral red.

Results: This study showed reduction of labeled neurons in both mesencephalic raphe nuclei following induction of diabetes. After injection of HRP to ventral striatum this reduction was prominent in DR nucleus in comparison to median raphe projections to ventral striatum affected by diabetes more than that of those to dorsal striatum.

Conclusions: Diabetes mellitus decreases the projections of mesencephalic raphe nuclei to striatum.

Keywords: Diabetes mellitus, mesencephalon, raphe nuclei, corpus striatum, streptozocin

\section{P-8}

\section{Could Melissa officinalis extract restore streptozotocin-induced spatial memory impairment in rats?}

Sabbaghziarani $\mathrm{F}^{*}$, Rasapour $\mathrm{S}^{* *}$, Mortezaee $\mathrm{K}^{*}$, Oryan $\mathrm{S}^{* *}$, Sharifzadeh $M^{* * *}$, Khanavi $M^{* * *}$, Hassanzadeh $G^{*}$

*Department of Anatomy, Faculty of Medicine, Tehran University of Medical Sciences, Tehran, Iran; **Faculty of Biological Sciences, Khwarizmi University, Tehran, Iran; ***Department of Pharmacology, Faculty of Pharmacy, Tehran University of Medical Sciences, Tehran, Iran

Introduction: Alzheimer disease is a progressive and irreversible neuropsychiatric disorder. Melissa officinalis improves anxiety and clinical dementia symptoms caused by $\mathrm{AD}$; therefore, the purpose of the present study was to evaluate the effect of Mellisa extract on spatial memorydeficit induced by STZ in MWM examination of male Wistar rats.

Methods: In the present study, 112 male Wistar rats (220-270 g) were used. Spatial learningdeficit was induced by bilateral ICV injection of STZ (3 $\mathrm{mg} / \mathrm{kg})$ via cannula; then, the Melissaextract in different doses administrated by gavage; and Morris Water Maze for measurement ofspatial learning parameters was performed.

Results: The results of this study demonstrated that gavage of the $200 \mathrm{mg} / \mathrm{kg}$ of Melissaofficinalis in combination with STZ, followed by significant reduction in two parameters: Timeand Distance.

Conclusion: Totally, the data indicate possible therapeutic value of $M$. officinalis extract onspatial learning improvement.

Keywords: STZ, Melissa officinalis, spatial memory

\section{P-9}

Aquaporin 4 (AQP4) is expressed in the stomach, small and large intestine of the pig

Arciszewski $M^{*}$, Arciszewski $\mathrm{MB}^{*}$, Matysek $M^{*}$, Szalak R*, Sienkiewicz $W^{* *}$

*Department of Animal Anatomy and Histology, Faculty of Veterinary Medicine, University of Life Sciences, Lublin, Poland; **Department of Animal Anatomy, Faculty of Veterinary Medicine, University of Warmia and Mazury in Olsztyn, Olsztyn, Poland

The water channel aquaporin-4 (AQP4) is a protein widely expressed on plasma membrane of a variety of epithelial cells. In this study we investigated the expression of AQP4 in the gastrointestinal tract of the pig using immunohistochemical staining. We found no presence of AQP4 in the different 
regions of the pig stomach. In the porcine small intestine moderate immunoreactivity to AQP4 was detected in enterocytes (along the villi and in the bottom of the crypts), duodenal Brunner's glands and in enteric ganglia in cells lying in close vicinity to myenteric as well as submucous neurons. In superficial epithelial cells of the colonic mucosa as well as of cecal and colonic glands a very strong immunoreactivity to AQP4 was found. Both in the large intestine's myenteric and submucous ganglia AQP4-positive cells surrounding enteric neurons were observed. We concluded that AQP4 expression in the porcine gastrointestinal tract showed some species-dependent differences in relation to other species. Based on the presented distribution pattern of AQP4, it is likely that in the pig the aquaporin plays a role in mucous (but not acid) secretion and intestinal absorptive processes.

\section{P-10}

\section{Promastigotes of different species of Leishmania exhibit distinguishable morphology by scanning electron microscopy}

Fernandes MF** Pires GA* ${ }^{*}$, Rodrigues A** , Mateus DS**, Bolas AV** ${ }^{* *}$ Pedrosa ARS ${ }^{* *}$, Passero LF** ${ }^{* *}$, Gomes GS ${ }^{* *}$

*CIISA-FCT-Faculty of Veterinary Medicine, University of Lisbon, Portugal; **Unidade De Ensino E Investigação De Parasitologia Médica, Global Health and Tropical Medicine, Instituto De Higiene E Medicina Tropical, Universidade Nova De Lisboa, Portugal; ***Laboratório De Patologia De Moléstias Infeciosas, Faculdade De Medicina Da Universidade De São Paulo, Brazil

Introduction: Leishmaniasis is a mammal parasitic disease caused by protozoan of genus Leishmania transmitted by sand flies. During the life cycle Leishmania presents two morphological stages, the amastigote in mammalian host and the promastigote in sand fly. Clinically may present visceral, cutaneous or mucocutaneous manifestations depending on the infective species. Under optical microscopy Leishmania species present identical morphological features, being indistinguishable. Therefore, Leishmania classification has been focused on geographical distribution, clinical manifestations and biomolecular analysis. Thus, this study aims to characterize parasites of the genus Leishmania using scanning electron microscopy (SEM).

Methods: Leishmania species that cause cutaneous leishmaniasis (L. amazonensis, L. guyanensis and L. shawi) and zoonotic visceral leishmaniasis (L. infantum) were assessed. Metacyclic-liked promastigotes of axenic cultures were fixed, contrasted, dried, coated with gold palladium and mounted on stubs. Parasites were then observed by SEM at low (3500x), medium (5000x) and high (>7500x) magnification and the images captured by photography.

Results: Axenic Leishmania promastigotes have distinguishable morphologies by SEM. On the third day of growth, promastigotes of different species showed variation in size, flagellum development and in the density of exosomes on the surface of the cell membrane. L. amazonensis promastigotes were smaller than the other species and L. infantum presented fewer vesicles on the surface than L. amazonensis or L. guyanensis. L. guyanensis flagellum evidenced a greater length than the other species. In turn L. shawi promastigotes exhibited spiral groves covering the entire body of the cell.

Conclusions: Promastigotes of different species of Leishmania do not differ by optical microscopy, however by SEM parasites exhibit different morphological characteristics. These characteristics might be associated with virulence, infectivity, parasite differentiation and also can reflect adaptations to diverse sand flies. This work was supported by the Portuguese Foundation for Science and Technology (PTDC/CVT/113121/2009) and São Paulo Research Foundation (FAPESP 2013/16297-2).

\section{P-11}

\section{D Canine hepatocyte culture system modeling the Leishmania infantum liver infection}

Rodrigues $\mathrm{A}^{* *}$, Pires $\mathrm{GA}^{*}$, Mateus $\mathrm{DS}^{* *}$, Bolas $\mathrm{AV}^{* *}$, Fernandes $\mathrm{MF}^{* *}$, Pereira $\mathrm{M}^{* *}$, Da Fonseca IP*, Gomes GS**

*CIISA-FCT-Faculty of Veterinary Medicine, University of Lisbon, Lisbon, Portugal; ** Unidade De Ensino E Investigação De Parasitologia Médica, Global Health and Tropical Medicine, Instituto De Higiene E Medicina Tropical, Universidade Nova De Lisboa, Lisbon, Portugal

Introduction: Implementation of culture systems that can mimic the liver are useful tools to better understand the dynamics of Leishmania infection. Canine leishmaniosis is a zoonotic disease caused by L. infantum, a protozoan parasite transmitted by sand flies. The liver constitutes a preferential target organ for the parasite. However, it remains the less studied organ, as it is very difficult to assess and has a complex metabolism. This work aimed to establish and characterize a 3D canine hepatocyte culture to be used in L. infantum infection studies.

Methods: Liver samples from healthy stray dogs captured and euthanized by official entities were collected. Hepatocytes were isolated by a two-step perfusion technique and separated in a Percoll $^{\circledR}$ gradient. Cells were cultured in a spinner vessel and generation of cell aggregates was followed by optical, confocal and scanning electron microscopy. After $72 \mathrm{~h}$, virulent L. infantum promastigotes were added to culture. Results: Twostep perfusion technique followed by a Percoll ${ }^{\circledR}$ gradient separation proved to be an effective way to isolate viable hepatocytes. The $3 \mathrm{D}$ culture system was successfully established. After $72 \mathrm{~h}$ in a spinner vessel, hepatocytes constituted several cell aggregates with visible extracellular matrix. The addition of L. infantum showed that the parasite is attracted to hepatocytes but does not penetrate the cells, although remaining attached to them.

Conclusions: The 3D canine hepatocyte model allowed cells to recreate their natural tissue distribution and environment, as well to stimulate the production of extracellular matrix. This $3 \mathrm{D}$ culture system proved to be useful in the study of L. infantum infection, as for the first time, there are evidences of a close interaction of the parasite with hepatocytes. 
Work supported by the Portuguese Foundation for Science and Technology (FCT) and European Union (FEDER) - project PTDC/CVT/118566/2010. Armanda Rodrigues holds PhD scholarship SFRH/BD/73386/2010 from FCT.

\section{P-12}

\section{Leishmania infantum does not induce neutrophil extracellular traps in dogs}

Pereira $M^{* *}$, Pires GA* ${ }^{*}$, Bolas $\mathrm{AV}^{* *}$, Camara $M^{* * *}$, Mateus DS** , Rodrigues $\mathrm{A}^{* *}$, Santos $\mathrm{M}^{*}$, Da Fonseca IP*, Gomes $\mathrm{G}^{* *}$

*CIISA-FCT-Faculty of Veterinary Medicine, University of Lisbon, Lisbon, Portugal; **Unidade De Ensino E Investigaçao De Parasitologia Médica, Global Health and Tropical Medicine, Instituto De Higiene E Medicina Tropical, Universidade Nova De Lisboa, Lisbon, Portugal; ***Évora City Council, Municipal Veterinary Office, Évora, Portugal

Introduction: Leishmania infantum causes severe disease in humans and dogs. Canids are the main reservoir maintaining parasite transmission cycle. L. amazonensis, L. shawi and L. guyanensis found in Central and South America cause human cutaneous leishmaniosis. After parasite introduction into mammal dermis through the bite of sand fly, neutrophils are the first cells recruited to the inoculation site. Beyond its classical role as phagocytic cells, neutrophils are able to fight pathogens outside the cell by releasing Neutrophil Extracellular Traps (NET), limiting their spread, reducing its virulence or eliminating them completely. Previous studies have demonstrated that Leishmania induce the release of NET by human neutrophils. Thus, this study investigated whether neutrophils extracellular activity can be modulated by Leishmania.

Methods: Neutrophils isolated from peripheral blood of healthy dogs and from peritoneal exudate of BALB/c mice were incubated with $\mathrm{L}$. infantum promastigotes. Mice neutrophils were also exposed to L. amazonensis, L. shawi or L. guyanensis. Phorbol 12-myristate 13-acetate (PMA), an inductor of NET, was added to parallel cultures. NET releasing were observed under a scanning electronic microscope and photographed. Results: L. infantum, L. amazonensis, L. shawi and L. guyanensis induced mouse neutrophils to release NET. However, when exposed to L. infantum dog neutrophils did not produce NET despite the exuberant NET formation observed in PMA stimulated cultures. These results indicate that L. infantum exerts a different effect on dog neutrophils, preventing NET release.

Conclusions: When in the presence of different species of Leishmania, mouse neutrophils maintain the ability of release NET, promoting extracellular parasite capture and infection control. However, L. infantum modulates negatively NET formation in dog neutrophils, favouring parasite spreading and survival and contribute in making the dog a good reservoir.

Study supported by the Portuguese Foundation for Science and Technology (FCT, PTDC/CVT/113121/2009 and PTDC/ CVT/118566/2010). M. Pereira holds a FCT-PhD scholarship (SFRH/BD/77055/2011).

\section{P-13}

Localization of fast blue- and Dil-labelled efferent sympathetic neurons projecting to the thymus of the pig

$\underline{\text { Siembida }}^{\mathrm{AZ}^{*}}{ }^{* *}$ Kulik $\mathrm{P}^{* * *}$, Arciszewski $\mathrm{M}^{* * *}$

*Department of Animal Anatomy and Histology, Faculty of Veterinary Medicine, University of Life Sciences, Akademicka, Lublin, Poland; **University of Life Sciences, Faculty of Veterinary Medicine, Department of Animal Anatomy and Histology, Lublin, Poland, $* * *$ Department of Animal Anatomy and Histology, Faculty of Veterinary Medicine, University of Life Sciences, Akademicka, Lublin, Poland

It has been postulated that autonomic nervous system is functionally interconnected with both hormonal and lymphatic systems. Thymus is one of the most important primary lymphoid organs playing a role in developing of the juvenile's immune system mainly by maturation, development and migration of T-cells (T lymphocytes). However, till now no studies concerning autonomic innervation of the thymus of higher mammals (including the pig) have been undertaken. Therefore, in the present work retrograde tracing technique of Fast Blue (FB) and DiI was used to investigate the sources of sympathetic efferent supply to the porcine thymus. After FB injection into right cranial lobe of the thymus the presence of FB-positive neurons was found in unilateral cranial cervical ganglion (CCG) $(82.8 \pm 3.0 \%$ of total FB-positive neurons) as well as middle cervical ganglion (MCG) $(17.2 \pm 3.0 \%)$. No presence of FB was detected in contralateral CCG, MCG as well as in both left and right cervicothoracic ganglion (CTG), Th2-Th14 sympathetic chain ganglia and coeliac-mesenteric ganglion. Injection of DiI into the left cranial lobe of the thymus resulted in the presence of retrograde tracer in neurons of unilateral CCG $(80.4 \pm 2.3 \%$ of total amount of DiI-labeled neurons), MCG (18.4 $\pm 1.9 \%)$ and CTG (1.2 \pm 0.8$)$. No DiI-labelled sympathetic neurons were found in contralateral CCG, MCG and CTG and Th2-Th14 sympathetic chain ganglia and coeliacmesenteric ganglion. The distribution patterns of sympathetic neurons projecting to the porcine thymus, usefulness of FB and DiI in neuroanatomical experimental studies as well as the role of biologically active substances in regulation of thymus function(s) were discussed.

\section{P-14}

Nuclear morphometry of the steatotic hepatocytes and hepatocyte stimulated steatotisc with infrared laser

\section{Cornejo $\mathrm{R}^{*}$, Silva $\mathrm{H}^{*}$, Matamala $\mathrm{F}^{*}$}

*Universidad De La Frontera, Temuco, Chile

A total of 24 female rats, aged 4 months and weighing approximately 250 grams, and they given a solution of $40 \%$ alcohol dissolved in water, leading to alcoholic multivesicular steatosis and 12 of rats was given and infrared laser with dose of $8 \mathrm{~J} / \mathrm{cm}^{2}$ during 15 days. The rats were then killed and samples of steatosis and stimulated and were taken and processed for examination by 
transmission electron microscope. Transmission electron microscope microphotographs steatotic hepatocytes and stimulated steatotic were btained with final magnification of 9,500 X. They were subjected to morphometric studies to determine the volumetric fractions and areas the following components: cellular and nuclear area, volumetric fractions of nucleus, nucleolus, eu and heterochromatin, nucleocytoplamic ratio of each cell type was determined. Analysis of the results between alcoholic hepatocytes and stimulate alcoholic shows that noticeable differences exist in all the cell components quantified. It is concluded that the effects of the stimuli of laser infrared provoke in the hepatocytes, a drastic transformation of their ultrastructure and morphology. This finally leads to functional variations, representing the effects produced by this stimulate in the hepatocytes. Palabras Clave: esteatosis, alcohol, morfometria, hepatocito

Keywords: Steatosis, alcohol, morphometry, hepatocytes

\section{P-15}

\section{The microenvironment of the thymus of the Egyptian water buffalo (Bos bubalis)}

\section{Zidan M}

Department of Histology and Cytology, Faculty of Veterinary Medicine, Alexandria University, Egypt

Understanding morphological features of the immune system is the first step to assessing its function. The thymus is the central lymphoid organ which is essential for development of $\mathrm{T}$ cells from their progenitor and is essential for development of other lymphoid organs. Many studies have been published on the structure of the thymus of different species but not, the Egyptian water buffalo, therefore this study described the basic structure of the thymus of the buffalo Egyptian water buffalo. Specimens from the thymus of 10 male buffalo calves (45-60 days old) were studied by light and transmission electron microscopes. A thin connective tissue capsule and trabeculae enclosed the thymus and divided it into lobules and sublobules. Adipose tissue was found between the lobules. Each lobule was formed from peripheral dark cortex and central lighter medulla. Several characteristic extensions of the medulla into the cortex were prominent. The epithelial reticular cells showed variable morphological appearance and form a meshwork with its long cytoplasmic processes. The cortex appeared darkly stained in contrast to lighter medulla. The cortex was rich in thymocytes supported with reticular epithelial cells. Groups of cortical thymocytes were enclosed within nurse cells. The cortical capillary was enveloped with reticular epithelial cells forming blood thymic barrier. The medulla had fewer thymocytes supported with epithelial reticular cells. Different shapes and numbers of Hassall's corpuscles were prominent. Few apoptotic lymphocytes were observed free or within the macrophage. This study may be a base for farther immunological and physiological studies as will as the possible age related involution.

\section{P-16}

Protective effects of melatonin against mitochondrial injury in a mouse model of multiple sclerosis

$\underline{\text { Rajabi } Z^{*}}$, Kashani IR*, Akbari $\mathrm{M}^{*}$, Hassanzadeh $\mathrm{G}^{*}$, Mohseni $\mathrm{A}^{*}$, Eramsadati $\mathrm{MK}^{*}$, Rafiee $\mathrm{K}^{* *}$, Beyer $\mathrm{C}^{* *}$, Kipp $M^{* * *}$, Zendedel $A^{*}$

*Department of Anatomical Sciences, Faculty of Medicine, Tehran University of Medical Sciences, Tehran, Iran; **Department of Biochemistry, Faculty of Medicine, Tehran, University of Medical Sciences, Tehran, Iran; **Faculty of Medicine, Institute of Neuroanatomy, RWTH Aachen, University, Aachen, Germany

Introduction: Multiple sclerosis (MS) is the most prevalent inflammatory demyelinating disease of the central nervous system. Besides other pathophysiological mechanisms, mitochondrial injury is crucially involved in the development and progression of this disease. Mitochondria have been identified as targets for the peptide hormone melatonin.

Objectives: In the present study, we sought to evaluate the impact of oxidative stress on mitochondrial density and enzyme transcription during experimentally induced demyelination and the protective influence of melatonin.

Methods: Adult male mice were fed with cuprizone for 5 weeks which caused severe demyelination of the corpus callosum (CC). Animals were simultaneously treated with melatonin by daily intra-peritoneal injections.

Results: Melatonin exposure reversed cuprizone-induced demyelination and axon protection. Transmission electron microscopy demonstrated significantly increased mitochondrial numbers and slightly increased mitochondrial size within CC axons after cuprizone exposure. Melatonin antagonized these effects and, in addition, induced the expression of subunits of the respiratory chain complex over normal control values reflecting a mechanism to compensate cuprizone-mediated down-regulation of these genes. Similarly, melatonin modulated gene expression of mitochondrial fusion and fission proteins. Biochemical analysis showed that oxidative stress induced by cuprizone was regulated by melatonin.

Conclusion: The data implicate that melatonin abolishes destructive cuprizone effects in the CC by decreasing oxidative stress, restoring mitochondrial respiratory enzyme activity and fusion and fission processes as well as decreasing intra-axonal mitochondria accumulation

\section{P-17}

Anatomically-anthropological study of cranial capacity from prehistoric to recent times at area of present-day Bosnia

Hadžihalilović $\mathrm{AS}^{*}$, Kulenović $\mathrm{J}^{*}$, Kulenović A*, Busuladžić A***, Hasanović $\mathrm{A}^{*}$

*Department of Anatomy, Faculty of Medicine, University of Sarajevo, Bosnia \& Herzegovina; **Department of Neurology, Public Institute General Hospital "Prim. Dr. AbdulahNakaš", Sarajevo, Bosnia and Herzegovina; ***Department of History-Archeology, Faculty of Philosophy, University of Sarajevo, Bosnia \& Herzegovina 
Objectives: The development of bipedal position anatomically changes the entire skeleton, not only the legs and pelvis, but also the spine, feet and the skull. By assesment of changes using the appearance of the skull and calculations of its volume we are able to track changes of brain volume and intelligence development as part of human evolution.

Methods: The research was conducted on a specimen of 196 macerated and degreased skulls, 82 of which belong to the osteological collection of the Department of Archeology - Chair of Prehistory, Antique Period and The Middle Ages - of the State Museum of Bosnia and Herzegovina in Sarajevo. From the osteological collection of the department of Anatomy of the Medical Faculty in Sarajevo, a total of 114 skulls have been examined, 57 of which were skulls of male gender and 57 of female gender. We estimated cranial capacityon each of the examined skulls applying craniometrical method.

Results: Regardless the period that was questioned, we proved the highest variability in the category of cranial capacity. It is actually a natural sequence due to the impact of all three linear dimensions (length, width and height) of the skull at its formation.

Conclusions: Cranial capacity shows a growing tendency going to younger period of time from which the skull encouraged, except for a slight decline during the Middle Ages.

Keywords: Skull, cranial capacity, craniometry, prehistoric skull, antic period skull, middle ages skull, recent period skull

\section{P-18}

\section{Expression of calretinin in the claustrum and} endopiriform nucleus of the chinchilla

Matysek M, Szalak R, Arciszewski MB

Department of Animal Anatomy and Histology, Faculty of Veterinary Medicine, University of Life Sciences, Akademicka, Lublin, Poland

Claustrum $(\mathrm{Cl})$ and endopiriform nucleus $(\mathrm{EN})$ are telencephalic structures, reciprocally connected with the cerebral cortex. Calretinin (CR) is calcium-binding protein $(\mathrm{CaBP})$ widely present in the central nervous system (CNS) and regulating many important cellular processes. In the present study the localization and the distribution pattern of neurons expressing CR were studied in the chinchilla $\mathrm{Cl} / \mathrm{EN}$. Formalin-fixed, paraffin-embedded mature chinchilla brains were cut into $10 \mu \mathrm{m}$ sections. Sections were immunohistochemically stained with antibodies raised against calretinin (CR) according to the peroxidase-antiperoxidase (PAP) method. Stained slides were subjected to histomorphometric analysis. Immunoreactivity for CR was observed within neurons in the nucleus, cytoplasm and neuronal processes. Numerous and evenly distributed CR-immunoreactive (ir) neurons were commonly found in $\mathrm{EN}$. In $\mathrm{Cl}$ CR-ir neurons were relatively scarce and predominantly localized in the marginal zone. The vast majority of CR-ir neurons in $\mathrm{Cl}$ were oval or round in shape, however CR-ir fusiform perikarya were also occasionally found. Numerous CR-ir round and oval bipolar cells were detected in EN, however a substantial numbers of CR-ir pyramidal, fusiform and multipolar in shape neurons were also present. The present study revealed for the first times the distribution patterns and cellular localization of $\mathrm{CR}$ in $\mathrm{Cl}$ and $\mathrm{EN}$ of the chinchilla. In conclusion, the chinchilla can be used as an animal model to study CaBP-related functional disorders of the mammalian forebrain.

\section{P-19}

Immunoreactivity to calcium/calmodulin-dependent protein kinase II (CaMKII) in the hippocampus of the chinchilla

\author{
Mozel S, Matysek M, Szalak R, Arciszewski MB \\ Department of Animal Anatomy and Histology, Faculty of Veterinary \\ Medicine, University of Life Sciences, Akademicka, Lublin, Poland
}

Calcium/calmodulin-dependent protein kinase II (CaMKII) is well known regulator of excitatory synaptic transmission playing a role in neurotransmitter release. The expression of CaMKII is widely found in neurons of the central nervous system (CNS), mainly in the forebrain. The hippocampus plays important role in memory shaping, formation of addiction and in the pathogenesis of many diseases of the CNS. In the present study immunohistochemical technique (peroxidase-antiperoxidase method) was used to study the localization, distribution pattern and morphology of CaMKII-immunoreactive (IR) neurons in the chinchilla hippocampus. Formalin-fixed, paraffin-embedded mature chinchilla brains were cut into $10 \mu \mathrm{m}$ sections. Sections were immunochistochemically stained with antibodies raised against CaMKII according to the peroxidase-antiperoxidase method. Stained slides were subjected to histomorphometric analysis. In neurons of the pyramidal layer and granular layer positive immunoreaction to CaMKII was noted in neuroplasm (but not in neuronal nucleus). CaMKII-IR neurons were found in pyramidal layer of the hippocampus from CA1 to CA4 fields as well as in the granular layer of the dentate gyrus (DG). In the CA1-CA4 fields immunoreactivity to CaMKII was observed in neurons of different shapes including pyramidal, fusiform, oval, round, multipolar cells. In the granular layer of DG single CaMKII-IR neurons oval and round in shape were occasionally found. In neurons of the pyramidal layer of the hippocampus and granular layer of DG the intensity of CaMKII immunoreaction generally ranged from weak $(+)$ to moderate $(++)$, whereas only scarce neurons showing intense (+++) immunoreaction to CaMKII were found. The present study revealed relatively moderate expression of CaMKII in neurons of the hippocampus and DG in chinchilla. The obtained results suggest that $\mathrm{CaMKII}$ in chinchilla hippocampus may play a similar role(s) to those previously described in other species.

\section{P-20 \\ Variations of sinoatrial and atrioventricular nodal arteries of the cardiac conduction system}

Hasanović A, Kulenović A, Hadžihalilović AS

Department of Anatomy, Faculty of Medicine, University of Sarajevo, Bosnia \& Herzegovina

Objectives: The objective of the study was to determine angiographically the origins of the sinoatrial and atrioventricu- 
lar nodal arteries and the correlation with the coronary dominance in patients with coronary disease.

Methods: The study included 200 patients (70 females, 130 males, mean age 55 years) who underwent coronary angiography in Heart Centre of the Clinical Center University of Sarajevo in period from July 2013 to July 2014, either for the symptoms of chest pain, angina pectoris etc. Coronary angiography was performed in the right and left anterior oblique position. The origin of sinoatrial and atrioventricular nodal artery was observed and correlated with the arterial dominance.

Results: In the present study 110 patients (55\%) of our 200 patients had the right coronary dominance, 40 patients $(20 \%)$ had the left coronary dominance and in 50 patients (25\%) were detected coronary codominance. The sinoatrial (SA) nodal artery originated from the right coronary artery (RCA) in 110 patients $(55 \%)$, in 80 patients $(40 \%)$ from the circumflex branch (CX) of the left coronary artery (LCA), and in 10 of cases $(5 \%)$ from the both coronary arteries (RCA-CX). The atrioventricular (AV) nodal artery originated from the RCA in $150(75 \%)$ patients, in 50 patients (25\%) from the CX branch, and in none of the cases was this node supplied by both coronary arteries. There were statistically significant differences between the origin of the nodal arteries in relationship to the type of dominance of coronary arteries, in favor atrioventricular nodal artery.

Conclusion: Knowledge of variation in the blood supply of conducting system may help cardiac surgeons, particularly during surgical correction of certain valvular disorders and congenital malformations of the coronary arteries.

Keywords: Sinoatrial nodal artery, atrioventricular nodal artery, coronary angiography

\section{P-21}

\section{Index by Dancmajer's analysis dermatoglyphic pattern on the finger}

Macut ND ${ }^{*}$, Malobabic $\mathrm{S}^{* *}$, Mandic $\mathrm{P}^{*}$, Filipovic T*, Saranovic $\mathrm{M}^{*}$

*Department of Anatomy, Faculty of Medicine, University of Pristina and Kosovska Mitrovica, Serbia; ${ }^{*}$ Department of Anatomy, Faculty of Medicine, University of Belgrade, Serbia

Objectives: Demonstrate some phenotype (dermatoglyphic) features of Serbs and Montenegrins, and comparative findings demonstrate biological closeness of the Yugoslav peoples.

Methods: As materials we have used prints digitopalmarnih dermatoglyphics, in subjects from 14 to 20 years. Overall there was 297 respondents, of which 230 Serbs, (113 men and 117 women) and 67 Montenegrins (35 men and 32 women) .For taking fingerprints We used standard method-taking prints using color (link method). When we want to emphasize the proportion obtained tread on the toes, there are many different values expressed as indices. Index by Dacmajr-in reflects the relationship arcs and coils.
Results: We observed a significant difference between the sexes as well as between groups. The index ratio of the total number of pattern on the left hand between the Serbs and Montenegrins is $45.20 \%: 33.98 \%$ and $28.95 \%$ on the right: $15.50 \%$. The total number of pattern both hands in both sexes the index stood at $36 \%$ for the Serbs, and $23 \%$ for Crnogorce. Our data differ significantly from data from areas of Montenegro (N. Boljeviç), where the relationship of the Montenegrins and Albanians was 17\% 80: 14.90\%.

Conclusion: Different populations differ in the number and type of patterns. Based on the analysis of dermatoglyphics can be praite migration, isolated populations, as islands, reserves, because due to genetic "drift" may differ from the general population from which they arise.

Keywords: Man, leather, patterns of the fingers.

\section{P-22}

\section{Study on variant anatomy of sciatic nerve and their clinical implications}

İkiz ZAA, Bilge O, Üçerler H, Çelik S, Boduc E

Department of Anatomy, Faculty of Medicine, Ege University, Izmir, Turkey

Although the classical description, sciatic nerve and piriformis muscle variations are relatively common. The variations of piriformis muscle, sciatic nerve and its branches have clinical importance during surgical interventions and some other clinical approaches. The aim of present study was to present these variations and discuss their clinical importances. Twenty six formalin fixed adult male cadavers with no evidence of lower limb surgery or pathology were used for this study. The anatomical relationship between the sciatic nerve and and the piriformis muscle, and division level of sciatic nerve was observed and recorded. In 44 of 52 lower limbs, the sciatic nerve exited the pelvis as a single branch through the infrapiriform foramen with no variation (84.62\%). In 12 of 52 gluteal regions or lower limbs, division variations of sciatic nerve was determined $(23.08 \%)$. In 8 of 12 high divisions were observed in gluteal regions (15.38\%). In one left side lower limb and three right side lower limbs, the common peroneal nerve and the tibial nerve entered deep gluteal region via the greater sciatic foramen below piriformis muscle separately (7.69\%). Two separate nerve divisions passed above and through (or below) the piriformis muscle and then combined together to form common peroneal nerve while tibial nerve passing below the piriformis muscle in 4 lower limbs (7.69\%). In 4 of 12, high division occured in the $1 / 3$ upper portion of the thigh unilaterally $(3.85 \%)$. The sciatic nerve separation into two divisions was above the piriformis and continued distally as two peripheral nerves. The division of sciatic nerve in the popliteal fossa was seen in two lower limbs (3.85\%). The clinical importance of these anatomical variations of the sciatic nerve around the piriformis muscle or in the thigh was discussed for piriformis syndrome, palsy after total hip arthroplasty, intramuscular injections in gluteal region and failed popliteal nerve block. 


\section{P-23}

Predictor models based on dermatoglyphic features in patients with schizophrenia

Popova FA*, Mantarkov $\mathrm{M}^{* *}$, Sivkov $\mathrm{S}^{*}$, Akabaliev V**

*Department of Anatomy, Histology and Embryology, Faculty of Medicine, Medical University of Plovdiv, Plovdiv, Bulgaria;

**Department of Psychiatry and Medical Psychology, Faculty of Medicine, Medical University of Plovdiv, Plovdiv, Bulgaria

Introduction: Over the last years a tendency of using biological markers in mental disorders has been observed, focusing on early prenatal brain damages and caused by the influence of a certain static agent. An important approach to the neurodevelopmental etiology of mental disorders is the assessment of dermatoglyphic patterns in patients with schizophrenia.

Objectives: The aim of this study was to determine the predictor dermatoglyphic features of independent contribution to the membership status "schizophrenia patient - control subject".

Methods: The study included 141 patients with schizophrenia (76 males, 65 females) and 120 mentally healthy subjects (54 males, 66 females) of Bulgarian origin. Fingerprints were obtained using an ink method and were read with light (6D) magnification in accordance with the methods given by Cummins, Midlo. The data were analyzed with SPSS 17.0 using logistic regression.

Results: Differences between the patients and control groups were statistically significant for five dermatoglyphic features in males and two in females that made significant contribution to the prediction of the patient-control status in a step-wise logistic regression model.

Conclusions: The logistics model defines a set of dermatoglyphic features that well enough distinguish patients with schizophrenia from healthy controls and thereby contributes to the validation of dermatoglyphics as biological markers in the development of schizophrenia.

Keywords: Dermatoglyphics, schizophrenia, logistic regression, predictor models

\section{P-24}

Slit1 expression in satellite glial cells is induced by neuronal contact

Zhao JH, Dong X, Zhang XF, Zhang HY, Zhang QP, Luo G, Ma ZJ, Yi XN

Department of Anatomy, Hainan Medical College, Haikou, China

Slit is known to be an axon guidance factor, but the pattern and significance of Slit1 expression in satellite glial cells (SGCs) of the dorsal root ganglion (DRG) is unclear. In this study, we hypothesized that Slit1 expression in SGCs may be affected by neuronal contact. Immunofluorescence double labeling technique was applied to the neuron-SGC co-culture and DRGs from sciatic nerve crush (SNC) models. The results showed that all SGCs wrapped around the neuronal soma at 12 hours; a part of SGCs still wrapped around the neuronal soma, while the others had separated at 24 hours, and all SGCs were off neuronal soma at 72 hours in the neuron-SGC co-culture. Slit1 was no or weakly expressed in SGCs with no neuronal soma contact by immunofluorescence. However, Slit1 was strongly expressed in SGCs which surround neurons soma. In SNC animal models, Slit1 only expressed in SGCs that surrounded living neuronal soma not in those whose parent neurons died. Combining with our another research result that Slit1 expression was up-regulated in the SGCs which wrapped around the injured neuronal soma in vivo, we confirmed that neuronal soma affect Slit1 expression in SGCs and the damage signal may raise its expression. Because Slit1 associated with nerve regeneration, our results imply that Slit1 expression up-regulation in SGCs, possibly facilitate post-injury nerve regeneration.

\section{P-25}

Anatomic study and clinical significance of the extraforaminal ligaments at the cervical level

Ding Z, Shi B

Anatomical Institute of Minimally Invasive Surgery, Southern Medical University, Guangzhou, China

Introduction: A dissection-based study of 6 embalmed cadavers.

Objective: To identify and describe the EFLs in relation to the area of the cervical intervertebral foramina (IVF) and to evaluate their clinical significance. Summary of Background Data. EFLs between the lumbar spinal nerves and the tissues surrounding the intervertebral foramens have been well established. However, rare research work has been undertaken to describe the local anatomy of the extraforaminal part of the cervical spine, detailed anatomic studies of the EFLs of cervical nerves have not been performed.

Methods: Eighty-four cervical IVFs from 6 adult embalmed cadavers were studied, and the existence and type of the EFLs were identified. The morphology, quantity, origin, insertion, and the spatial orientation of the EFLs in the cervical region were observed, and the length, width, or diameter and thickness of the ligaments were measured with a vernier caliper.

Results: The EFLs could be found from the second cervical to the first thoracic spinal nerve. These ligaments could be divided into 2 types: Radiating ligaments, which connected the nerve root sleeves radiated to the transverse processes, the wall of the IVF and even the adjacent nerve root through the small transverse foramen; Transforaminal ligaments (TFLs ), which originated from the anteroinferior margin of cranial transverse process and inserts in the superior margin of the anterior tubercle of caudal transverse process crossing the spinal nerve ventrally.

Conclusion: Between the cervical spinal nerves and nearby structures, there are two types of the EFLs. The radiating lig- 
S138 XXIV International Symposium on Morphological Sciences, 2nd-6th September, 2015, Istanbul, Turkey

aments may serve as a protective mechanism against traction and play an important role in the positioning of the nerves in the intervertebral foramen. However, in all probability, the transforaminal ligaments may be the underlying cause of the cervical radiculopathy.

\section{P-26}

Infrared laser effect on GAP-43 expression in compression-related injury rat sciatic nerve

Matamala $\mathrm{F}^{*}$, Paredes $\mathrm{M}^{* *}$, Lafuente $\mathrm{L}^{* *}$, Iturriaga $\mathrm{D}^{* *}$, Montiel E**, Cornejo $\mathrm{R}^{* *}$

* Laboratorio De Biotecnologia Animal Departamento De Ciencias Bàsicas Facultad De Medicina. Universidad De La Frontera; **Universidad De La Frontera, Temuco, Chile

Objectives: Laser irradiation of biological tissue is being studied for the terapeutic purposes. As a hypothesis it is proposed that treatment of previously injured sciatic nerve axon compression with infrared laser induces increased GAP-43 expression.

Methods: 24 Sprague Dawley rats weighing $300 \mathrm{~g}$ were used. And divided into four groups: intact nerve, irradiated nerve, damages irradiated nerve and damages unirradiated nerve. After application of anesthesia the nerve was surgically isolated and compressed with a pressure clamp controlled by exerting a constant force of $40 \mathrm{~N}$ for 3 minutes on a $10 \mathrm{~mm}$. Twenty-four hours post-injury, the sector corresponding to the nerve pathway was irradiated transcutaneously with a gallium arsenide laser $(904 \mathrm{~nm} ; 10 \mathrm{~mW})$ at an energy density of 2 and $10 \mathrm{~J} / \mathrm{cm}^{2}$. This treatment was applied for $10 \mathrm{~min} /$ day for 10 consecutive days. Once the treatment was complete, the rats were euthanized at 17 and 24 days post-injury. The nerve was extracted and sectioned in distal and proximal portions at the injury site and frozen immediately at $-80^{\circ} \mathrm{C}$ until processing for RNA extraction... Total RNA was extracted from the distal and proximal portions of the injured nerve using the "AxyPrep Multisource Total RNA Miniprep Kit” (Axygen Biosciences, USA) following the manufacturer's instructions. The statistical significance of the differences in the values of GAP-43 transcriptional expression between control and treatments was evaluated by Student's test

Results: The data showed GAP-43 expression in the all the samples of nerves treated with infrared low energy laser. From the quantitative point of view the expression levels of the nerves treated with low energy laser increased the level of the transcripts more than 2000 times compared to the control, reaching máximum values around 5000 times.

Conclusion: The low intensity laser stimulates transduction pathways associated with GAP-43 expression (Stadler 2000). The potential therapeutic applications of low energy laser irradiation is discussed.

Keywords: Infrared Laser, GAP-43, Real-time qPCR, Sciatic nerve
P-27

Evaluation of morphologic and morphometric characteristic of foramen transversarium on 3-dimensional multidetector computed tomography angiography (MDCTA)

Tellioğlu AM${ }^{*}$, Durum $Y^{* *}$, Gök $M^{* *}$, Karakaş $S^{*}$, Polat AG*, Karaman $\mathrm{CZ}^{* *}$

*Department of Anatomy, Faculty of Medicine, Adnan Menderes University, Aydın, Turkey; ** Department of Radiology, Faculty of Medicine, Adnan Menderes University, Aydın, Turkey

Objectives: Foramen transversarium which is in the cervical vertebra, transmits the vertebral artery and ven, accompanied by a plexus of sympathetic nerves. The aim of this study is to investigate the size and variations of foramen transeversarium. To study the anatomical variation of vertebral artery entering the transverse foramen of cervical vertebrae.

Methods: 141 (90 male, 51 female) patients images, whose ages between 18-79 (mean 52.7) were analyzed. As a result, 987 cervical spine (C1-C7) and 1974 foramen transversarium were evaluated individually. Every single foramen transversarium's anterior-posterior (AP) and transverse diameters were measured bilaterally from multidetector CT images (80-Row detector 160 slice CT System). The absence of TF as agenesis, a diameter of less than $2 \mathrm{~mm}$ was considered to be hypoplastic. Double foramina and incomplete double foramina and their side was also noted. We evaluated the levels at which the vertebral arteries entered the transverse foramina.

Results: The most frequent variation was the duplication of TF, which was noted in $88(8.91 \%)$ cervical vertebrae in total. Agenesia of TF was seen in $37(3.74 \%)$, hypoplasia of TF was seen in $26(2.63 \%)$ cervical vertebrae. The vertebral artery in 4 $(1.84 \%)$ cases in this study entered into the transverse foramen of the 5 th cervical vertebra from both sides. In 4 (1.84\%) cases, the vertebral artery entered into the transverse foramen of the 7 th cervical vertebra. Only in $1(0.45 \%)$ case, the vertebral artery entered into the transverse foramen of the 4th cervical vertrebra. The 6 th cervical vertebra was the most common site of the entry, the vertebral artery entered the transverse foramen of the 6th cerevical vertebra in remaining 209 (95.87\%) cases.

Conclusion: We believe that determination of foraminal variations could be an important guide for surgeons and radiologist in the diagnosis and treatment.

Keywords: Foramen transversarium, morphometry, 3-dimensional multidetector computed tomography angiography (MDCTA)

\section{P-28}

Intensified expression of monocarboxylate transporter (MCT1) in the filopodia of growing endothelial cells in the brain

Kishimoto A, Zheng M, Iwanaga HT, Iwanaga T

Laboratory of Histology and Cytology, Hokkaido University Graduate Faculty of Medicine, Hokkaido, Japan 
Endothelial cells of the brain during developmental stage express more intensely monocarboxylate transporter (MCT1) than GLUT1 for the uptake of monocarboxylates such as lactate and ketone bodies derived from milk and circulating in blood. The present immunohistochemical study characterizes blood vessels expressing MCT1 in the brain of neonatal mice at light and electron microscopic levels. At the growing tips of blood vessels, many long filopodia were immunolabeled with MCT1 antibody; they often projected radially with distance longer than 30 micro meter. Immunostaining for GLUT1 was difficult to label such long and fine filopodia in the same specimens. SEM observation revealed detailed morphology of growing blood vessels: fine filopodia covered entirely the outer surface of endothelium as well as the tips of growing blood vessels. These vessels were associated with pericytes immunoreactive for smooth muscle actin but growing tips were devoid of pericytes. The MCT1-expressing filopodia were not visible after weaning (20 days after birth). In contrast, the vascular endothelium changed to show an intense immunoreactivity for GLUT1 after weaning. Double staining of MCT1 and GLUT1 in blood vessels was also carried out in other tissues of neonates, and the staining results were compared with those in adult tissues. The functional significance of MCT1 expression in the growing blood vessels is discussed concerning the development of blood vessels in the brain.

\section{P-29}

\section{Macroscopic and microscopic alterations in TNBS- induced colitis adult female rats}

\section{Karaboğa İ*, Karaca T**, Demirtaş $\mathrm{S}^{* *}$}

*Department of Histology and Embryology, Faculty of Medicine, Trakya University, Edirne, Turkey; Faculty of Health, Namık Kemal University, Tekirdağ, Turkey; **Department of Histology and Embryology, Faculty of Medicine, Trakya University, Edirne, Turkey

Objectives: The present study was aimed to evaluate microscopic and macroscopic changes in 2, 4, 6- trinintrobenzene sulfonic acid (TNBS)-induced colitis in female Wistar rats.

Methods: In this study, 16 adult female Wistar rats were used. Rats divided two groups randomly; control group $(n=8)$ and colitis group $(n=8)$. Experimental colitis was induced by intraclonic administration of 2,4,6-trinitrobenzene sulfonic acid (TNBS). $25 \mathrm{mg} / \mathrm{kg}$ of TNBS (diluted \% $50 \mathrm{v} / \mathrm{v}$ ethanol, totally $1 \mathrm{ml})$ under anaesthesia with ketamine $(50 \mathrm{mg} / \mathrm{kg})$ and xylazine $(10 \mathrm{mg} / \mathrm{kg}$ ) for sixty second was applied. Control animals received only rectal saline for same time. During the study, the weights and feces status were recorded as a marker of clinical condition. The animals were sacrified fifteenth day after TNBS administration under anaesthesia. Macroscopic examination was performed according to McCaferty et al. (1994). For microscopic examination, colon tissues were stained hematoxyline-eosin and histopathologic features were scored according to Obermeier et al. (1999). Besides, body and colon weights were determined at the end of the experimental period.

Results: Body weights of colitis group reduced when compare with control groups. Increased colonic weight was observed in colitis group. Macroscopic and microscopic damage score was observed higher in colitis group compared with the control group. Meanwhile, histopathological changes clearly observed in colitis group such as epithelial degenerations, Goblet cells loss, inflammatory cell infiltration, oedema and ulcerations in colonic mucosa and submucosa.

Conclusion: In the present study evaluated morphological and histopathological changes of ulcerative colitis model in TNBSincuced rats. This TNBS-induced chemical model is cited by many studies as an important experimental model of ulcerative colitis, which is characterized by an acute inflammatory response, followed by thickening of the colon wall, ulcerations and immune cell infiltration.

\section{P-30}

\section{Expression of vascular endothelial growth factor (VEGF), transforming growth factor (TGF-b) and vascular density in hyperthyroidism-induced pregnant Wistar rats}

\section{$\underline{\text { Demirtaș }^{*}}$, Karabacak R*, Çiçek AÇ* Karaboğa İ**,} Karaca $T^{*}$

*Department of Histology and Embryology, Faculty of Medicine, Trakya University, Edirne, Turkey; **Department of Histology and Embryology, Faculty of Medicine, Trakya University, Edirne, Turkey, Faculty of Health, Namık Kemal University, Tekirdağ, Turkey

Objectives: Hyperthyroidism is a medical condition that results from an excess of thyroid hormone in the blood. This study was designed to investigate the expression of VEGF, TGF-B and vascular density in maternal rat adrenal glands with hyperthyroidism in late gestation.

Methods: Twelve mature female Wistar albino rats with the same biological and physiological features were used for this study. Rats were divided into control and hyperthyroidism groups. Hyperthyroidism was induced by daily subcutaneous injections of L-thyroxine $(250 \mu \mathrm{g} / \mathrm{kg})$ for 21 days. After thyroxin administration for 21 days, vaginal smears were taken from each animal, and animals in the oestrus phase were placed in a cage attached to another cage with male rat. All females were met with males (ratio 1:1;n = 6). In the next morning, the animals who were seen spermatozoa in their vaginal smear and it was considered day-zero of pregnancy. At the twentieth day, animals were sacrified. The tissues were stained immunohistochemically with Anti-CD 31, Anti-VEGF and Anti-TGF-B. Immunohistochemical stain density respectively graduated as 0; none, +; weak, ++; moderate, +++; high.

Results: VEGF, TGF-B and CD31 expressions were increased statistically in maternal hyperthyroid group compared with control group. Immunoreactivity has detected especially on zona glomerulosa and zona fasciculata.

Conclusion: VEGF is a signal protein produced by cells that stimulates vasculogenesis and angiogenesis and CD31 is known to have various roles in vascular biology including angiogenesis. TGF-Bs can act as local mediators of the various actions of steroid 
hormones such as steroids, retinoids, androgens, etc. Therefore, it is proposed that TGF-B is bifunctional regulator of cell growth and adrenocortical differentiation. It is known that thus proteins play a potent role of vascularization and differentiation of adrenal cortex tissue. This study shows that hyperthyroid stimulates VEGF, TGF-B and CD31 expression on adrenal cortex.

\section{P-31}

The protective effect of erdostein in experimental ovarian ischemia-reperfusion injury

Ciçek AÇ ${ }^{*}$, Uğurel V**, Demirtaş $\mathrm{S}^{*}$, Cemek $\mathrm{M}^{\star * *}$, Karaca $T^{*}$

*Department of Histology-Embryology, Faculty of Medicine, Trakya University, Edirne, Turkey; **Department of Obstetrics and Gynecology, Faculty of Medicine, Trakya University, Edirne, Turkey; $* * *$ Department of Bioengineering, Faculty of Chemical and Metallurgical Enginnering, Yıldız Technical University, istanbul, Turkey

Objectives: To investigate the protective effect of erdosteine, an anti-apoptotic and anti-oxidant agent, on torsion-detorsion induced histopathological changes in experimental ovarian ischemia-reperfusion (IR) injury.

Methods: A total of 18 female Wistar albino rats were used. Animals were divided into three groups: Control (sham operation), IR (torsion-retorsion), and IR+Erdosteine (torsion/retorsion plus Erdosteine). The IR+Erdosteine group received the erdosteine for seven days before the induction of torsion/retorsion, (orally, $10 \mathrm{mg} / \mathrm{kg} /$ days). IR and IR+Erdosteine were exposed to right unilateral adnexal torsion for $3 \mathrm{~h}$. After a 3 -h period of ischemia, re-laparotomy was performed, and the right ovaries were surgically excised. Then, the rats were euthanized by a high dose of anaesthetic. At the end of experiment, Intracardiac blood samples were collected for biochemical analysis. The ovaries tissues were collected and ?xed with $10 \%$ neutral buffered formalin. Paraffin sections were stained H\&E, anti-PCNA and TUNEL.

Results: The IR group were severe acute inflammation, polynuclear leukocytes and macrophages, also degenerative cells, stromal oedema and haemorrhage. Treated of erdosteine in rats significantly retained degenerative changes in the ovary. PCNA positive cells were detected in the oocytes, follicle epithelium, theca follicle, and stromal cells in all groups. PCNA positive cell numbers were significantly decreased IR and IR+Erdosteine groups compared with control rats. However, its numbers were significantly increased IR+erdosteine group compared with IR group. TUNEL positive cell numbers were significantly increased IR group compared with control and IR+Erdosteine groups. In erdosteine treated group, TUNEL positive cells were seen to a significantly lesser degree than IR group $(\mathrm{p}<0.05)$.

Conclusion: Present study demonstrates the protective effect of erdosteine treatment against to ovary I/R injury in rats. Erdosteine treatment decreased IR injury in an experimental rat ovarian torsion model. This protective effects is likely to protect against IR by avoiding scavenging of free radicals, release of oxidant free radicals, and through prevention of proinflammatory processes.

\section{P-32}

Comparative and corralative evaluation of dynamic and static balance with anthoropometric measurements

Uzun $A^{*}$, Şahin B*, Özdemir F ${ }^{*}$, Kabadayı $M^{* *}$, Gölpınar $M^{*}$, Nahir $M^{*}$, Birinci $\mathrm{MC}^{* *}$

*Department of Anatomy, Faculty of Medicine, Ondokuz Mayıs University, Samsun, Turkey; **Faculty of Yaşar Doğu Sport Sciences, Ondokuz Mayıs University, Samsun, Turkey

Introduction: Anthropometric parameters is one of the most important factors affecting the human balance system.

Objectives: The aim of this study is to describe and correlate between dynamic and static balance as opened and closed eyes performance in anthropometric measurements (height, weight, body mass index, thigh, leg, foot, lower limb length, foot width and height, sitting height) and foot base area.

Methods: The study included 37 volunteer male students of Ondokuz Mayis University. Taking measurements of the isokinetic balance measurement instrument (CSM brand Prok tecnobody) was used. The dynamic balance and static balance test were applied in double-leg stance position. The data was analysed using independent sample t test and pearson correlation test; $p$ value at 0.05 or less was accepted as significant difference.

Results: The average track error in dynamic balance test was $25.05 \pm 14.11 \%$. The static balance test scores in static balance as opened and closed eyes were $13.02 \pm 7.56$ and $10.51 \pm 3.31$ in subjects. There was no significant difference in static balance between opened and closed eyes $(p>0.05)$. The dynamic balance was negative correlated with height $(\mathrm{r}=-0.30, \mathrm{p}<0.05)$, right $(\mathrm{r}=-0.35, \mathrm{p}=0.01)$ and left $(\mathrm{r}=-0.31, \mathrm{p}=0.03)$ thigh length, respectively. The static balance test as closed eyes was negative correlated with the height $(r=-0.43, p=0.01)$, weight $(r=-0.29$, $\mathrm{p}=0.04), \operatorname{leg}(\mathrm{r}=-0.30, \mathrm{p}=0.04)$ and foot $(\mathrm{r}=-0.37, \mathrm{p}=0.01)$ length, foot width $(\mathrm{r}=-0.30, \mathrm{p}=0.04)$ and sitting height $(\mathrm{r}=-0.40$, $\mathrm{p}=0.01$ ), respectively.

Conclusion: The human height and thigh length affect the dynamic balance negatively and height, weight, leg and foot length, foot width and sitting height have a negative effect on static balance as closed eyes but anthropometric measurements have no correlation with static balance as opened eyes.

\section{P-33}

Quercetine ameliorates methotrexate induced renal damage, apoptosis and oxidative stress in rats

Erboğa $M^{*}$, Aktaş $C^{*}$, Erboğa $\mathrm{ZF}^{*}$, Dönmez YB*, Gürel A**

*Department of Histology and Embryology, Faculty of Medicine, Namık Kemal University, Tekirdağ, Turkey; **Department of Biochemistry, Faculty of Medicine, Namık Kemal University, Tekirdağ, Turkey

Introduction: Methotrexate (MTX) is not only used as a chemotherapeutic agent for the treatment of cancer types. 
Despite this wide range of indications, MTX therapy is associated with a number of adverse reactions, particularly pneumonia, nephrotoxicity, and hepatotoxicity.

Objectives: In the present study, the protective and therapeutic effects of quercetin $(\mathrm{QE})$ on renal injury induced by MTX have been examined. Methods: A total of 24 male rats were divided into the following 3 groups: control group, MTX group, and MTX+QE (15 mg/kg) group. Rats in MTX group received 20 $\mathrm{mg} / \mathrm{kg}$ of single dose of MTX, while those in MTX+QE group received $20 \mathrm{mg} / \mathrm{kg}$ of single dose MTX, in addition to $15 \mathrm{mg} / \mathrm{kg}$ of QE administered 30 minutes prior to MTX and in the following 5-day period as a single daily dose. At the end of the experimental period, kidney tissues were removed for histopathological, apoptotic and biochemical assessments.

Results: Light microscopic examination showed a disruption of the renal structure in rats in MTX group in the form of tubular degeneration and dilation, with shedding of the tubular epithelial cells into the lumen. QE treatment was associated with less marked degenerative changes. Furthermore, QE treatment resulted in decreased the number of apoptotic cells. Biochemical assessments showed significantly higher MDA levels in MTX group as compared to control and MTX+QE groups, with no significant differences between MTX+QE and controls in this regard. SOD, GSH-Px and CAT levels showed a significant decrease in MTX group as compared to controls. QE was associated with elevated levels of SOD, GSH-Px, and CAT enzyme levels, which were reduced by MTX.

Conclusion: Our results demonstrate that oxidative stress plays an essential role in MTX-induced renal toxicity in rats. The findings of this study show that QE is capable of reducing MTXinduced renal oxidative injury through its antioxidant and antiapoptotic effects, which were evaluated both biochemically and histologically.

\section{P-34}

Thymoquinone ameliorates cadmium-induced nephrotoxicity, apoptosis and oxidative stress in rats is based on its antioxidant properties

Erboğa $M^{*}$, Kanter $M^{* *}$, Aktaş $C^{*}$, Şener $\ddot{U}^{* * *}$, Erboğa $Z F^{*}$, Dönmez YB*, Gürel A****

*Department of Histology and Embryology Faculty of Medicine, Namık Kemal University, Tekirdağ, Turkey ; ${ }^{*}$ Department of Histology and Embryology, Faculty of Medicine, Istanbul Medeniyet University, Istanbul, Turkey; ***Department of Physiology, Faculty of Medicine, Namık Kemal University, Tekirdağ, Turkey; $* * \star \star$ Department of Biochemistry, Faculty of Medicine, Namık Kemal University, Tekirdağ, Turkey

Introduction: Cadmium (Cd), an environmental and industrial pollutant, generates free radicals responsible for oxidative stress. $\mathrm{Cd}$ can also lead to various renal toxic damage such as the proximal tubules and glomerulus dysfunction. Thymoquinone (TQ) is the main constituent of the essential oil obtained from black seeds (Nigella sativa) and has various pharmacological effects.

Objectives: The aim of the present study was to examine the nephroprotective, antioxidant and anti-apoptotic effect of the TQ against Cd- induced renal nephrotoxicity.

Methods: A total of 24 male Wistar albino rats were divided into three groups: control, Cd-treated and Cd-treated with TQ; each group contain 8 animals. The Cd-treated group was injected subcutaneously with $\mathrm{CdCl} 2$ dissolved in saline in the amount of $2 \mathrm{ml} / \mathrm{kg} /$ day for 30 days, resulting in a dosage of 1 $\mathrm{mg} / \mathrm{kg} \mathrm{Cd}$. The rats in TQ treated groups were given TQ (50 $\mathrm{mg} / \mathrm{kg}$ body weight) once a day orally together with first $\mathrm{Cd}$ injection during the study period.

Results: The histopathological studies in the kidney of rats also showed that TQ markedly reduced the toxicity of $\mathrm{Cd}$ and preserved the normal histological architecture of the renal tissue. Immunohistochemical analysis revealed that TQ significantly decreased the $\mathrm{Cd}$-induced over expression of nuclear factor- $\kappa \mathrm{B}$ in renal tissue. Furthermore, TQ treatment resulted in decreased the number of apoptotic cells. TQ significantly suppressed lipid peroxidation, compensated deficits in the antioxidant defenses [reduced superoxide dismutase, glutathione peroxidase and catalase activities] in renal tissue resulted from Cd administration.

Conclusion: These findings suggest that the nephroprotective potential of TQ in Cd toxicity might be due to its antioxidant and anti-apoptotic properties, which could be useful for achieving optimum effects in Cd-induced nephrotoxicity.

Keywords: Cadmium, thymoquinone, nephrotoxicity, apoptosis, rats

\section{P-35}

The immunohistochemical analysis of SPARC and VEGF in uterine and placenta tissues of hyperthyroid rats

Karabacak R, Karaca T

Department of Histology and Embryology, Faculty of Medicine, Trakya University, Edirne, Turkey

Introduction: Hyperthyroidism is defined with high blood level of thyroid hormone. As for reproductive system, hyperthyroidism causes various complications such as miscarriage and pre-eclempsia. In addition, SPARC is a matricellular protein to regulate the organization of extracellular matrices and cell-matrix interactions. It binds directly to VEGF which the expression level also changes in hyperthyroidism, in addition to various growth factors.

Objectives: In our study, we aim to contribute to the underlying mechanism of the complications because of hyperthyroid during pregnancy in different stages.

Methods: Twenty four female rats are divided into four groups (control 5thday, control 20thday, hyperthyroid 5thday and hyperthyroid 20thday; $\mathrm{n}=6$ for each). L-thyroxine were administered to animals for 21 days to establish hyperthyroid and then mate with male rats to develope pregnancy. With vaginal smear analysis, the pregnancy were identified. In the 5thday and 20thday of pregnancy, the animals were sacrificed; the 
immunoreactivity of SPARC and VEGF protein expression were analysed in placenta and uterine tissues. 20thday placenta sections were analysed by counting the number of positive cells per $\mathrm{mm} 2$ whereas 5 thand 20thday uterine sections were analysed with semiquantitative scoring system as described. The intensity of staining with antibodies was subjectively scored; $0=$ nonreactivity; +=weak, individualized cell reactivity in $\leq 25 \%$ of magnified area; $++=$ mild to moderate reactivity in $\leq 50 \%$ of magnified area; $+++=$ strong reactivity in $\leq 75 \%$ of islets; and $++++=$ very strong reactivity in $>75 \%$ of magnified area.

Results: As a result, we observed that the expression of SPARC and VEGF decrease in 5th day and 20th day uterine tissues and placenta of hyperthyroid groups comparing to control groups. The results were statistically evaluated.

Conclusion: In conclusion, SPARC and VEGF are two of underlying proteins of the complications of hyperthyroid in uterine and placenta tissues during pregnancy. Understanding the interactions between them and the downstream and upstream mechanisms contributes the treatments to prevent fertility dysfunctions because of hyperthyroidism.

\section{P-36}

\section{The soleus muscle with three heads}

Durmaz MT, İkiz ZAA, Çelik S

Department of Anatomy, Faculty of Medicine, Ege University, Izmir, Turkey

Classically the soleus arises from the posterior surface of the head of the fibula, the soleal line and the middle third of the medial border of the fibula; some fibers also arise from a tendineus arch between the tibial and fibular origins of the muscle that arches over the popliteal vessels and tibial nerve. The fibers end in an aponeurosis which covers the posterior surface of the muscle, and gradually becoming thicker and narrower. It joins with the tendon of the gastrocnemius, and forms with the tendocalcaneus. The gastrocnemius and soleus together form a muscular mass which is occasionally described as the triceps surae; its tendon of insertion is the tendo calcaneus (Tendo Achillis), the common tendon of the gastrocnemius and soleus is the thickest and strongest in the body. If an accessory head is present, its lower and inner part usually ends in the calcaneal tendon. During routine dissection, the variation in the soleus muscle was observed. The soleus muscle of the cadaver had three origins. The lateral and medial parts combined together to form tendo calcaneus downwards. The intermediate part mostly originated from the posterior head of the fibula and joined the lateral and medial parts of the muscle in the middle one third of the leg. Tendineus arch was between the tibial and fibular origins of the muscle that arches over the popliteal vessels and tibial nerve. The innervation and arterial patterns were normal. The soleus muscle with three heads has clinical importance for surgeons and radiologists since anatomical relationship between the tibial nerve and muscles may be the reason of entrapment of the tibial nerve. In addition, exis- tence of such variation of the soleus may complicate surgical repair and diagnostic procedures.

\section{P-37}

\section{A pilot study of neo-angiogenesis and invasion of small cell lung cancer cell line ( $\mathrm{NCl}-\mathrm{H} 146)$ using chicken embryo chorioallantoic membrane}

Preisegolaviciute L, Valančiūtė A, Mozuraitò R

Department of Histology and Embryology, Lithuanian University of Health Sciences, Kaunas, Lithuania

Introduction: Small cell lung carcinoma is a frequent neoplasm of cancer and has a high rate of metastasis with poor prognosis. Neoangiogenesis of tumors is particularly important in prognosis and treatment. Commercial cell line NCI-H146 was used for studying tumor associated neoangiogenesis on chicken embryo chorioallantoic membrane (CAM).

Objectives: To evaluate invasion of small cell lung cancer cell line (NCI-H146) into the CAM, neo-angiogenesis and mitotic activity of the cells using two tumor models.

Methods: NCI-H146 cell line was grafted onto the CAM on embryonic day 7 . Tumor cells $(1$ million cells in $10 \mu$ growth medium) were incorporated with either matrigel or I type rat tail collagen $(10 \mu \mathrm{l})$ and drop onto absordable gelatin sponge $\left(3 \times 3 \mathrm{~mm}^{2}\right)$. Formed tumor cells $(20 \mu \mathrm{l})$ were then grafted on CAM. After 3rd, 4th and 5th day of cell grafting histological and imunohistochemical evaluation was made. Tumor cells were visualized using Ki67, vimentin and cytokeratin.

Results: Cells of H146 cell line mixed with type I rat tail collagen appeared as visible tumor on CAM, forming clear spoked wheel pattern of neoangiogenesis. Neo-angiogenesis starts at the second day after tumor cell grafting and is strongly expressed on 5 th day of tumorigenesis. Angiogenesis of H146 cells inoculated with matrigel was not as strong. Thickness of CAM and its epithelium is increased under the tumor transplant and on the distinct sides as well. Using Ki67 marker of proliferation, mitotic figures in the tumor are seen. Angiogenesis was shown macroscopically using stereomicroscope each day after tumor transplant. No statistical assessment was yet performed.

Conclusion: H146 cell line formed tumor on CAM using type I collagen. H146 cells expressed strong neo-angiogenic reaction in the CAM and invaded the tumor. H146 cells induced thickness of CAM and its upper epithelium. Ki67 showed strong proliferative trace of tumor cells. Regarding to strong angiogenesis induced by tumor cells, further studies with antiproliferative drugs are going to be performed in the future.

\section{P-38}

Histological effects of linear alkylbenzene sulphonanic acid (LABSA) on ovary tissue of zebrafish (Danio rerio)

Öztürk B, Işel Z, Fidan B, Güneş S, Alsaran M, Akbulut C, Genç H, Zengin M, Yön ND

Sakarya University 
Linear alkylbenzene sulphonanic acid (LABSA) is surface active agent which widely used in synthetic laundry detergent formulation and household cleaning products. They are discharged into the environment via industrial/household or launderette effluent into waterways. They affect the aquatic environment and aquatic organisms. In our study, examination the histopathological effects of LABSA on ovary tissue of zebrafish were aimed. Sexually mature zebrafish were maintained under standardises laboratory conditions $\left(28^{\circ} \mathrm{C} \pm 1^{\circ} \mathrm{C}\right.$ and $14 \mathrm{~h} / 10 \mathrm{~h}$ light dark cycle). We created one control and two $(0.5 \mathrm{mg} / \mathrm{L}, 1$ $\mathrm{mg} / \mathrm{L})$ experiment groups concerning their different LABSA doses. After $48 \mathrm{~h}$ adaptation, different concentrations of LABSA were added to the aquarium. After exposure, on the fifth day of the study zebrafish individuals were anaesthetized with ice water and intestine tissues were dissected. For the histological analysis, tissues were fixed with $10 \%$ neutral buffered formalin for $24 \mathrm{~h}$. Fixed tissue was dehydrated with ascending series of ethanol (70\%-100\%) and embedded in the parafin wax and sectioned transversely at $5 \mu \mathrm{m}$ thickness and stained with Hematoxylin-Eosin staining. In control group normal ovary histology was observed. Primary oocytes, cortical alveolus stage oocytes, vitellogenic oocytes and mature oocytes were monitored clearly. In $0.5 \mathrm{mg} / \mathrm{L}$ exposure group, openings between zona radiata and vitelline envelope were detected Disintegration were monitored at follicle epithelium. Integration of cortical alveoli were also monitored at cortical alveolus stage oocytes. Degenerations were detected at primary oocyte nucleus. Vacuolization were observed in the oocyte structures. In $1 \mathrm{mg} / \mathrm{L}$ exposure group, increase in the number of degenerated and atretic oocytes were detected. Integration of cortical alveoli were also monitored at this group too. Significantly decrease in the number of primary oocyte were monitored. Deformation were observed at cortical alveoli structure. Degeneration and shrinking nucleus were also detected. As a result, LABSA has led to a deterioration in fish ovary tissues. In this study, statistical methods were not used.

\section{P-39}

Histological effects of tribenuron methyl on gill tissue of zebrafish (Danio rerio)

Öztürk B, Şaş E, Alsaran M, Güneş S, Akbulut C, Yön ND Sakarya University

Introduction: Tribenuron methyl is an herbicide that used to control dicots in cereals and fallow land. It is applied as a spray and it access to the soil directly and transferred to the roots. Objectives: Pesticides access aquatic ecosystems via ground waters and affect water quality and aquatic life.

Methods: After one-week adaptation period adult zebrafish individuals divided into four groups $(\mathrm{n}=10)$ as one control and 3 experimental groups ( $40 \mathrm{mg} / \mathrm{l}, 80 \mathrm{mg} / \mathrm{l}, 120 \mathrm{mg} / \mathrm{l})$. For investigating the effects of tribenuron methyl, gill tissues were dissected after 5 days of the exposure. Tissues were fixed with $10 \%$ neutral buffered formalin and a routine histological process was applied. The sections were stained with Hematoxylin-Eosin and results were evaluated with the light microscope.

Results: In the control group, normal gill histology was observed. Primary lamellae, secondary lamellae, epithelial cells and mucous cells monitored. Mucous cells were detected at the edge of primary lamellae. Secondary lamellae, separated from central primary lamellae which supported by cartilage and connective tissue were detected clearly. In $40 \mathrm{mg} / \mathrm{L}$ exposure group, hyperplasia at and vacuolization were detected at primary lamellae structure compared with control group. Hyperplasia at secondary lamellae and pillar cells and hypertrophy at mucous cells was monitored. In $80 \mathrm{mg} / \mathrm{L}$ exposure group; fusion at the apical side of gill tissue was monitored. Vacuolization and hyperplasia were detected at the connective tissue of primary lamellae. Dysmorphism and total fusion at secondary lamellae and vacuolization at pillar cells was observed in $120 \mathrm{mg} / \mathrm{L}$ exposure group, fusion at the apical side of gill tissue was monitored similarly with $80 \mathrm{mg} / \mathrm{L}$ exposure group. Hyperplasia and total fusion at secondary lamellae, vacuolization at secondary epithelium, hyperplasia and dysmorphism at pillar cells were detected. As a result, tribenuron methyl has led to a deterioration in fish gills tissues. In this study, statistical methods were not used.

\section{P-40}

\section{Histological effects of tribenuron methyl on ovary tissue of zebrafish (Danio rerio)}

Öztürk B, Öztürk C, Alsaran M, Güneş S, Akbulut C, Yön ND

Sakarya University

Introduction: Examination the histopathological effects of tribenuron methyl on ovary tissue of zebrafish were aimed.

Objectives: Tribenuron methyl is an herbicide that used to control annual and perennial dicots in cereals and fallow land. It is generally applied as a spray and it access to the soil directly and transferred to the roots. Pesticides access aquatic ecosystems via ground waters and affect water quality and aquatic life.

Methods: After one week adaptation period adult zebrafish individuals divided into four groups $(\mathrm{n}=10)$ as one control and 3 experimental groups (40 mg/l, $80 \mathrm{mg} / \mathrm{l}, 120 \mathrm{mg} / \mathrm{l})$. For investigating the effects of tribenuron methyl, ovary tissues were dissected after 5 days of the exposure. Tissues were fixed with $10 \%$ neutral buffered formalin and routine histological process was applied. The sections were stained with HematoxylinEosin and results were evaluated with the light microscope.

Results: In the control group, normal ovary histology was observed. All oocyte stages were monitored clearly. In all exposure groups, decrease in the number of primary oocytes was observed. In $40 \mathrm{mg} / \mathrm{L}$ exposure group, openings between zona radiata and vitelline envelope were detected. Disintegration was monitored at follicle epithelium. Fusion at cortical alveoli 
was also monitored at cortical alveolus stage oocytes. In 80 $\mathrm{mg} / \mathrm{L}$ exposure group, shrinking oocytes were detected. Integration at cortical alveoli was also monitored. In $120 \mathrm{mg} / \mathrm{L}$ exposure group, the increase in the number of degenerated and atretic oocytes was detected. Deformation was observed at cortical alveoli structure. As a result, tribenuron methyl has led to a deterioration in fish ovary tissues. In this study, statistical methods were not used.

\section{P-41}

Histological effects of linear alkylbenzene sulphonanic acid (LABSA) on liver tissue of zebrafish (Danio rerio)

Öztürk B, Güneş S, Alsaran M, Akbulut C, Genç H, Zengin M, Yön ND

Sakarya University

Linear alkyl benzene sulphonanic acid (LABSA) is surface active agent which widely used in synthetic laundry detergent formulation and house hold cleaning products. They are discharged into the environment via industrial/househ old or launderette effluent into water ways. They affect the aquatic environment and aquatic organisms. Inourstudy, examinationthe histopathological effects of LABSA on liver tissue of zebrafish were aimed.Zebrafish are maintained at standardized conditions of $27^{\circ} \mathrm{C} \pm 1{ }^{\circ} \mathrm{C}$ temperature, $95 \%$ dissolved oxygen saturation, $61 \%$ moisture and photoperiod (12:12-h light: dark). We created one control and two $(0.5 \mathrm{mg} / \mathrm{L}, 1 \mathrm{mg} / \mathrm{L})$ experiment groups concerning their different LABSA doses. After $48 \mathrm{~h}$ adaptation, different concentrations of LABSA were added to the aquarium. After exposure, on the fifth day of the study zebrafish individuals were anaesthetized with ice water and intestine tissues were dissected. For the histological analysis, tissues were fixed with $10 \%$ neutral buffered formalin for 24h. Fixed tissue was dehydrated with a scending series of ethanol (70\%-100\%) and embedded in the parafin wax and sectioned transversely at $5 \mu \mathrm{m}$ thickness and stained with Hematoxylin-Eosin staining. In control group normal liver histology was monitored. Parenchyma cells, hepatocytes and their cytoplasm were observed clearly. Sinusiodis and nuclei were easily monitored. In the $0.5 \mathrm{mg} / \mathrm{L}$ exposure group, degeneration, contraction at vacuoles in hepatocyte cytoplasm, hypertrophy and increase in the number of hepatocyte cells were detected. Further more some Kupffer cells were observed more elliptical form. In $1 \mathrm{mg} / \mathrm{L}$ exposure group, vacuoles in hepatocyte cytoplasm were reduced to almost nothing. Hypertrophy and increase in the number of hepatocyte cells were also monitored at this group too. Decrease in the number of Kuppfer cells were observed. Also changes in cell shape, vascular and sinusoidal degeneration and steatozis were detected. As a result, LABSA has led to a deterioration in fish liver tissues. In this study, statistical methods were not used.

\section{P-42}

Anatomical description of arterial segments in the Mazama gouazoubira kidney

Pereira $\mathrm{KF}^{*}$, Martins TMM*, Santos $\mathrm{AQ}^{* *}$, Pereira DKS*, Cardoso $\mathrm{JR}^{* * *}$, Malysz $\mathrm{T}^{* * * *}$, Lima $\mathrm{FC}^{*}$

*Human and Comparative Anatomy Laboratory. Federal University of Goiás - Regional Jataí, Jataí - GO, Brazil; **Wild Animal Teaching and Researching Laboratory. Federal University of Uberlândia, UberlândiaMG, Brazil; ***Wild Animal Researching Laboratory. Federal University of Goiás, Goiânia - GO, Brazil; ****Human and Comparative Anatomy Laboratory, Federal University of Rio Grande Do Sul, Porto Alegre - RS, Brazil

Introduction: The brocket deer (Mazama gouazoubira Fischer, 1814) is a deer that lives in South America, particularly in Brazil and nearby countries such as Uruguay and Argentina. Brazil in regions with predominantly savannah vegetation, fields and barns, this ruminant has as part of its fauna. This species is frugivorous, not presents branching horns and has ungulate hoof.

Objectives: This study aimed to describe the topography, wraps and renal arterial segments of brocket deer.

Methods: Used two specimens of M. gouazoubira, one female and one male, collected in the BR-364 highway, near JataíGoiás. Through dissection, the skin was completely removed and later scored the arteries of animals with homogenized stained latex red later the animals were fixed in a formaldehyde solution $10 \%$. Sequentially through a ventral access block was removed from the animal gut and kidneys disjoint this block.

Results and Conclusion: The kidneys were found arranged in parallel. The right kidney located at the level of the vertebrae L1-L3 and the left kidney at the level of vertebrae L2-L4 were presented rounded with smooth convex faces without lobation, wrapped sequentially by a thin fibrous capsule, the renal fat and fascia capsule. Medial to each kidney, the adrenal glands were. Renal artery forked in the hilar region in the cranial and caudal artery sectoral also observed in ovine and crab-eating raccoon, and one of these sectoral arteries (cranial and caudal) originated five main segments directed to the ventral region and the dorsal region each kidney, these segments are again bifurcated arterial segments totaling 14. The kidneys of brocket deer resemble the kidneys of other ruminants in general, and to carnivores. So its morphology, topography and renal arterial segments and consequently the anatomicosurgical territories of the species studied have been shown to be unique.

\section{P-43}

Effect of oral administration of Salvia rhytidea extract on histomorphometric changes of the hippocampus following experimental ischemia-reperfusion in rat

Tadjalli $M$, Haghjou R

Department of Anatomical Science, Faculty of Veterinary Medicine, Shiraz University, Shiraz, Iran 
Objectives: The Salvia is one of the membranes of the Laminaceae family that its pharmacologic effects is associated to Phenolics. According to its antioxidative effect, it can remove the free $\mathrm{O} 2$ radicals which produced during ischemia reperfusion.

Methods: 35 adult male rats were randomly divided into 7 groups of 5 rats: Group1 (control -):. Group 2 (control +): Cerebral ischemia. Group 3 (sham): Manipulation of the left common carotid and the left vertebral arteries. Groups (4, 5, 6 and 7): Cerebral ischemia induced by the left common carotid and the left vertebral arteries occlusion. Group 4 received $3.2 \mathrm{mg} / \mathrm{kg}$ aquaous and alcoholic salvia extract $2 \mathrm{~h}$ after ischemia. group 5 received $50 \mathrm{mg} / \mathrm{kg}$ silymarin drug, $2 \mathrm{~h}$ after ischemia. Group 6 received aquaous and alcoholic salvia extract $(3.2 \mathrm{mg} / \mathrm{kg}) 72$, 48,24 and 0 h before ischemia. group 7 received silymarin drug (50 mg/kg), 72, 48, 24 and $0 \mathrm{~h}$ before ischemia. $24 \mathrm{~h}$ following reperfusion, the rats were euthanized, and after blood collection, samples of the cerebrum were obtained. By using routine histological technique, the sections were stained by H\&E. The measurement of the thickness of the layers and cell count in cerebral cortex and different regions of hippocampus were accomplished

Results: Following cerebral and hippocampal ischemia, the superoxide dismutase enzyme (SOD) activity and thickness and neuronal cells of cerebral cortex and thickness of all layers of CA1 and CA2 regions of hippocampus and pyramidal cells of these 2 regions of hippocampus decreased. But pretreatment with Salvia rhytidea extract and silymarin did not change these factors. But administration of salvia extract and silymarin after ischemia, does not affect improving the thickness, neuronal cell numbers and SOD enzyme activity.

Conclusion: Therefore pretreatment with Salvia rhytidea extract similar to silymarin drug improves the cerebral and hippocampal injuries following ischemia.

Keywords: Salvia extract, histomorphometric study, silymari, hippocampus

\section{P-44}

\section{Chondroitin sulfate proteoglycan expression in the lesioned goldfish spinal cord}

\section{Takeda A, Funakoshi K}

Department of Neuroanatomy, Faculty of Medicine, Yokohama City University, Yokohama, Japan

Chondroitin sulfate proteoglycans (CSPGs) upregulated in the scar are a major inhibitory factor for regenerating axons after the central nervous system injury in mammals. In contrast to mammals, spontaneous nerve regeneration beyond the scar frequently occurs after spinal cord injury in fish. However, the mechanism allowing the axons to pass the scar is not clear. Here we examined the expression of CSPGs with immunohistochemistry for CS-56 antibody in the lesioned spinal cord of the goldfish. One week after the spinal hemisection, many CS-
56 immunoreactive cells were observed just around the fibrous scar. Some CS-56 cells appeared doubly positive for glial fibrillary acidic protein (GFAP), although the majority of them were distinct from the GFAP immunoreactive glial processes. CS-56 immunoreactivity is not observed in the extracellular matrix (ECM). The regenerating axons positive for acetylated tubulin run among the CS-56 cells to enter the fibrous scar. CS-56 immunoreactivity is not found in or around the lesion site 2 weeks after hemisection and thereafter. On the other hand, NG2-immunoreactive deposits were observed 3 weeks after the hemisection, but not within 1 to 2 weeks after the hemisection, suggesting that CS-56 cells negative for NG2. NG2 immunoreactivity were present mainly in the fibrous scar, but also in the surrounding glial tissue at a significantly lower level. The present findings suggest that the cellular structure expressing CSPGs does not make an impenetrable barrier for regenerating axons in the goldfish. It is possible that the lack of intense deposition of CSPGs in the ECM allows regenerating axons to pass among the CSPG cells. Alternatively, CSPGs might not play an inhibitory role for regenerating axons.

\section{P-45}

Navigational area of the cranio-orbital foramen and its significance in orbital surgery

\author{
Celik S* Kazak Z** Özer MA*, Gövsa F*
}

*Department of Anatomy, Faculty of Medicine, Ege University, Izmir, Turkey; **Department of Anatomy, Faculty of Dentristy, Kırıkkale University, Kırıkkale, Turkey

Objectives: The cranio-orbital foramen $(\mathrm{cOF})$ is located on the lateral wall of the orbit. It is a potential source of hemorrhage during deep lateral orbital dissection, since it functions as an anastomosis between the lacrimal artery and the middle meningeal artery. The aim of this study was to guide and facilitate the surgical procedures in the orbit, so as to determine a navigational area and the precise location of the $\mathrm{cOF}$ and to standardize certain anatomical marks.

Methods: The navigational area of the $\mathrm{cOF}$ and topographical features were studied in 75 craniums with presented cOF. 33 bilateral main cOFs, 41 (18 on the right, 23 on the left) unilateral main cOFs at the main cranium and 19 accessory cOFs were studied for their navigational features on the orbit. The distances between the $\mathrm{cOF}$ and the fronto-zygomatic suture, supraorbital notch, lateral angle of the superior orbital fissure (SOF) and Whitnall's tubercle were measured via flexible ruler and photogrammetrically.

Results: The mean distance of the cOF from the fronto-zygomatic suture, supraorbital notch, lateral angle of the SOF and Whitnall's tubercle was 26.3, 37.3, 92 and $27.1 \mathrm{~mm}$, respectively. For the navigational area signs of the $\mathrm{cOF}$, areas of the orbit that form the transversal and vertical lines are generated on the reference points. 
Conclusion: Whilst the upper outer area of the orbit contains a potential bleeding risk, the bottom section of the outer column is identified as safe for the surgical operations of the lateral orbital wall. The fronto-zygomatic suture and Whitnall's tubercle are recommended as the most reliable navigational landmarks for identifying the cOF. Hence, the transversal and vertical orientation of the cOF should be mastered by the surgeons reconstructing the anterior base of the skull and the orbit.

\section{P-46}

Coronary arterial anatomy of the one-humped camel (Camelus dromedarius)

Tadjalli M, Ghazi SR

Department of Anatomical Science, Faculty of Veterinary Medicine, Shiraz University, Shiraz, Iran

Objectives: To study the detailed coronary anatomy of camels and compare them with that of horses and cattle.

Methods: Seven normal hearts from one humped camels (Camelus dromedaries) of either sex were collected fresh from the abattoir. The pericardium was incised and the coronary arteries in all the hearts were exposed and cannulated. They were then flushed with heparinized saline solution several times. The following techniques described by Hildebrand (1968) were employed to study the details of the distribution of these arteries. 1-Injection for subsequent corrosion. 2Coloured latex injection.

Results: The results indicated that the left and right coronary arteries of camel heart originated from left and right aortic sinus respectively. The left coronary artery divide into interventricularis paraconalis branch and left circumflexe branch. The ramus collateralis proximalis in camels separates off some distance away from the origin of the ramus interventricularis paraconalis, as in horses, whereas it separates off very quickly in cattle. The ramus collateralis distalis has two branches in camels, whereas the left distal ventriculi ramus does not branch. The ramus angularis is also absent but the left distal atrii ramus is present.

Conclusion: In camels, there is a bilateral coronary supply, the right coronary artery being the larger. The left coronary artery follows the same pattern as that in horses. It differ with that of the cow and dog which have left coronary supply.

\section{P-47}

Morphology of skeletal muscle-derived mesenchymal stem cells from humans and rats and their possible application in regenerative medicine

Varga I $^{*}$, Miko M*, Polak S*, Danisovic L*

*Institute of Histology \& Embryology, Faculty of Medicine, Comenius University in Bratislava, Slovakia; **/nstitute of Medical Biology and Clinical Genetics, Faculty of Medicine, Comenius University in Bratislava, Slovakia
Skeletal muscle contains populations of myogenic cells (satellite cells) which are capable of differentiation into myoblasts and to a population of multipotent stem cells also referred as multipotent muscle-derived stem cells. The aim of this study was to isolate and culture in vitro human and rat muscle-derived stem cells and perform morphological analysis of their ultrastructure. Muscle-derived stem cells were isolated from biopsy specimens of femoral muscle of humans and rats. The cells were cultured in Dulbecco's modified Eagle's minimal essential medium supplemented with $10 \%$ fetal calf serum and gentamycin. When they reached $80 \%$ confluence, they were sub-passaged up to the third passage. Cells from the last passage were prepared for TEM analysis. Expression of alphaactin and desmin was confirmed by immunohistochemistry. Moreover, the phenotypic characterization was performed. TEM analysis showed typical ultrastructural morphology of mesenchymal stem cells. We didn't find remarkable differences in ultrastructural morphology of stem cells between humans and rats. Cells from both sources had large euchromatic nuclei. Nuclei were irregular with deep cleft and contained well developed nucleoli of compact type. Dilated cisterns of rough endoplasmic reticulum were present in cytoplasm. In certain parts of the cytoplasm there were aggregates of granules of glycogen (in human cells) and lipid droplets (in rats). Under plasma membrane were localized numerous small endocytotic vesicles, caveolae. Cells expressed alpha-actin and desmin. The results of phenotypic characterization showed that almost all analyzed cells were CD34 and Sca-1 positive and CD45 negative. Muscle-derived stem cells exhibited characteristics typical for mesenchymal stem cells. After analysis of their differentiation potential they could be used in tissue engineering and regenerative medicine. The study was supported by Grant of Slovak Research and Development Agency No. APVV-0434-12.

\section{P-48 \\ Scanning electron-microscopic study of the lingual papillae in newborn lambs}

Tadjalli $M$

Department of Anatomical Science, Faculty of Veterinary Medicine, Shiraz University, Shiraz, Iran

Objectives: To study the tongue papillae of the newborn lamb by scanning electron-microscopy.

Methods: Tongues were collected from 6 newborn lambs (1 to 5 days) immediately after slaughter. The samples were collected from the dorsal surface fo apex, body and torus linguae of the tongue. The samples were rinsed with $0.1 \mathrm{M}$ phosphate buffer ( $\mathrm{pH} 7.4$ ) and prefixed in $4 \%$ glutaraldehyde solution, washed with $0.1 \mathrm{M}$ phosphate buffer and post fixed with $1 \%$ osmium tetroxide. After dehydration through a graded ethanol series and infiltration by hexamethyl disilazan, the specimens were mounted on aluminum stubs and coated with gold- palladium. The specimens were observed at various angles under a scanning electron-microscope (Stereoscan 360). 
Results: The rose bud shaped filiform papillae had a primary papillae and 6 to 8 slender secondary papillae. The convex surfaced fungiform papillae were raised above the lingual mucosa. The conical papillae had a sharp tip and were arranged regularly. Lenticular papillae revealed a hempisphere protrusion appearance. The circumvallate papillae were round to oval shaped and were characterized by a papillary groove and a thick annular pad or valium of lingual mucosa.

Conclusion: These findings indicated that the tongue of the lamb is similar to that of the other ruminants.

\section{P-49}

Immunocytochemicals melanocytic markers expression on nevos and melanomas of human skin biopsies

$\underline{\text { Molina B*}}{ }^{*}$, Paredes $M^{* * *}$, Vasconcello $A^{* * *}$, Pellon $M^{* * *}$, Navarrete $\mathrm{F}^{* * *}$, Quilaqueo $\mathrm{N}^{* * *}$

*Universidad De La Frontera, Dpto De Ciencias Basicas, Facultad De Medicina, Temuco, Chile; **Universidad De La Frontera; $* * *$ Universidad De La Frontera, Temuco, Chile

Melanoma is a kind of skin cancer that surges when melanocytes turn to a malign pathway as a consequence of several exogenous as well as endogenous physicochemial and cell environmental factors. Histopathological diagnostic is generally based on a traditional biopsy, nevertheless this method does not allow to distinguish different cell lines involved in tumour development, for such a reason to complete this task it is needed the use of several molecular markers expressed at the cell surface. On this respect the protein HMB45 was one of the first markers to be studied. This marker allows the differentiation between melanocytic nevus and melanoma and also to discriminate between active, inmature and intraepidermic melanocytes. Notwithstanding, give negative results on quiescent melanocytes. Another marker is the Calcium binding protein $(\mathrm{S}-100)$ isolated from bovine brain. It possesses 18 isoforms and each one of them has a characteristic tissue expression, it has been usefull as a marker for studies on primary melanoma and also to study their subsequent metastasis. A third marker protein is MART-1, which is expressed on cell surfaces and it is usefull as a melanocytes tumour marker, but is also found on benign nevus. The objective of this work was to evaluate the immunohystochemical expression of S100, MART-1 and HMB-45 on biopsies samples and to evaluate their cell expression on the hystopathological diagnostic. To achieve this task it was employed Anti-s100, MAT-1, HMB45 antibodies on 40 human skin biopsies by the ABC-DAB detection method. The results obtained represent a sustained association between S100, SMAR-1, HMB-45 on characterization of pathological states of tumoral cells. S100 marker is clearly expressed on the nevum cells, specially the on round cells and the on fusiformes cells and finally in multinucleated atypical cells. Expression level for MART-1 was somewhat scarce, together at fibroblasts and macrophage demarcation. HMB

\section{P-50}

Quantitative inmunohistochemical study of estrogen receptor in endometrial tissue from high and low prolificacy sheep breeds

Paredes $M^{*}$, Vasconcellos $A^{* *}$, Cisternas $C^{* *}$

*Research Laboratory Animal Biology, Faculty of Medicine, Universidad De La Frontera, Temuco, Chile; **Reproductive Biotechnology Center, CEBIOR, Universidad De La Frontera, Temuco, Chile

The association of genetic, nutritional and environmental conditions directly affects the fertility of ewes. There are high fertility sheep breeds (Texel, Suffolk) and other standard fertility breeds as (Romney, Araucana). Animal reproduction is modulated by sex hormones that act by binding to specific receptors. In sheep, alpha (ER-a) estrogen receptor is widely distributed in the reproductive system, modulating several mechanisms associated with reproductive system function. One factor possibly related to the difference in fertility between sheep breeds, is the differential expression level of these receptors in the reproductive system. In the present study a quantitative comparison of the immunohistochemical expression of ER- $\alpha$ was carried out in pre-pubertal sheep endometrium in high fertility (Texel breed) versus low fertility (Araucana breed), by measuring the immunohistochemical integrated optical density in specific areas of the endometrium. Results indicate a significant difference between ER- $\alpha$ expression in endometrium of Texel breed and Araucana breed, and registered higher levels in all areas of evaluated Texel breed prepubertal. This differential expression suggests a posible link between ER- $\alpha$ expression intensity and fertility in the breeds studied in this work.

Keyword: Alpha estrogen receptor, prolificity, Texel, Criolla araucana

Support: Proyecto DIUFRO DI12-0027, Universidad de La Frontera

\section{P-51 \\ Congenital atlantoaxial fusion in camel (Camelus dromedarius): a case report \\ Paryani $\mathrm{MZ}^{*}$, Raad SB* \\ *Department of Basic Sciences, Faculty of Veterinary Medicine, Karaj Branch, Islamic Azad University, Karaj, Iran}

The skeleton of a camel aged above 6 years old, which used for the students of anatomy has an anomaly in cervical vertebrae. The skeleton of this camel were cleaned and prepared using appropriate techniques. The vertebral column in this camel was studied and showed malformation in cervical region. The normal vertebral formula is $\mathrm{C} 7, \mathrm{~T} 12, \mathrm{~L} 7, \mathrm{~S} 5$ and in this case there is no difference in the number of vertebrae, but a fusion was seen between atlas and axis. This fusion shows remnant fused between caudal arch of the atlas and cranial arch and odontoid process of axis. In order to the classification of 
atlantoaxial fusions into three groups, this form belong to the complete fusion between these two vertebrae. To my knowledge, There are no more reports regarding this type of fusion in vertebral column of the camel.

Keywords: Anatomy, vertebrae, cervical, camel, atlantoaxial fusion

\section{P-52}

Role of accumbens BDNF in heroin-induced conditioned-place preference and naloxone precipitated withdrawal in rats

Li YX, Liang WM

Guizhou Medical University, Guiyang, China

Objectives: Heroin dependence is a major public health problem in worldwide. BDNF was previously shown to be involved in cocaine reward and relapse. The goal of this research is to explore the role of BDNF in the rat nucleus accumbens (NAc) in heroin-induced conditioned-place preference and naloxone precipitated withdrawal.

Methods: We developed a lentiviral-mediated gene transfer approach to knockdown (LV-siBDNF) or overexpress ( $\mathrm{LV}$ $\mathrm{BDNF}$ BDNF in the nucleus accumbens (NAc). Behavioral experiments consisted of heroin-induced conditioned-place preference (CPP) and withdrawal syndromes observation.

Results: LV-BDNF-treated rats display suppressed heroininduced CPP, and blocked CPP reinstatement. In contrast, LV-siBDNF-treated rats display enhanced heroin-induced CPP, and increased CPP reinstatement. The withdrawal score of LV-BDNF-treated rats was significantly lower than LVsiBDNF-treated rats.

Conclusion: These results indicate that BDNF plays an important role in heroin reward in the mesolimbic dopaminergic pathway in rats and suggest that a key threshold range of BDNF levels is associated with impaired heroin abuse.

\section{P-53}

The relationship between the proximal femoral morphometric measurements and hip osteoarthritis

$\underline{\text { Karabulut Ö}}^{*}$, Karabulut Z**, Hatipoğlu EŞ*, İskenderoğlu $\mathrm{AY}^{*}$, Keskin I*

*Department of Anatomy, Faculty of Medicine, Dicle University, Diyarbakır, Turkey; **Private Fizyopolitan Physiatry Branch Center, Diyarbakır, Turkey

Objectives: To evaluate if the morphology of the femoral head and neck, was a risk factor for radiographic hip osteoarthritis.

Methods: The study group included 25 female and 25 male patients with hip osteoarthritis (OA). The patients were enrolled at age 40 years or greater. The control subjects were 20 female patients with lumbago. Postero anterior (PA) pelvic radiographs of all patients were taken. We calculated body mass index for each subject. Dual x-ray absorptiometry (DXA) was carried out for femoral neck bone mineral densiy (BMD) and intertrochanteric bone mineral density measurements in all subjects. Femoral neck length, femoral neck width, the acetabular angle and femoral head-neck shaft angles were measured geometrically from the PA pelvic radiographs.

Results: The radiographic hip osteoarthritis cases had significantly higher body mass index and higher bone mineral density than control subjects $(\mathrm{p}<0.05)$. In radiographic measurements we detected 10 hips with small osteophytes and 40 hips with definite osteophytes. Definite osteophytes were more frequently recorded in male hip joints compare to female hip joints. Femur heads were closer to acetabulum than that of the distance in a normal hip in 22 cases. Reduced joint space was significant for severe hip OA. Anterosuperior segment of femur neck had a compression effect on anterosuperior segment of acetabulum. Femur neck diamaters were relatively wide when compared with femur head diamaters. We determined reduction in the femoral head-neck shaft angle and increase in acetabular angle measurements in all OA subjects. Bone sclerozis and trabecular bone changes were existing in 20 OA subjects.

Conclusion: Assesment of the proximal femur morphology helps the early identification of radiographic osteoarthritis of the hip.

\section{P-54}

The morphological assesment of talocalcaneal joint in rheumatoid arthritis, based on MRI

$\underline{\text { Karabulut Ö}}^{*}$, Keskin I*, İskenderoğlu AY*, Hatipoğlu EŞ*, Karabulut $Z^{* *}$

*Department of Anatomy, Faculty of Medicine, Dicle University, Diyarbakır, Turkey; **Private Fizyopolitan Physiatry Branch Center, Diyarbakır, Turkey

Objectives: To analyse the architecture of the talocalcaneal joint in rheumatoid arthritic feet, using magnetic resonance (MR) imaging.

Methods: Our study group included 20 female patients affected by romatoid arthritis (RA) and 10 control subjects who applied with traumatized feet. Coronal and sagittal T1-weighted turbo spin echo sequences were carried out for the morphological evaluation of talus, calcaneus, navicular and cuboid bones. The bones affected by rheumatoid arthritis were measured with regard to standard parameters and analysed by comparing with the images of control subjects. Paired t-tests were used for statistical analysis.

Results: All RA patients in our study group had subtalar synovitis and tenosynovitis with the existence of pain. Bone marrow edema with hypointense areas and bone erosion were detected in all MR images of affected ankles. The images demonstrated tissue loss in cortex of all measured bones. The talocalcaneal (subtalar) joint space was narrowed and degener- 
ated. Both of the joint spaces formed by talus and calcaneus with the cuboid bone were narrowed evidently. There was a volume increase in hypointense synovial membrane. When the tendon structures were analysed, a homogeneous and hypointense thickening was marked in tendon sheath. 8 RA patients had feet with normal consruction but 12 RA patients had feet with complete abnormal construction.

Conclusion: Therefore the evaluation of MR imaging findings could be detected earlier than the complete structure abnormalization in affected joints, these analyses were valuable in terms of early diagnosis of the disease. Such an extensive analysis that contains examining of whole tissues in the joint formation will shed light on the differantial diagnosis and the therapy process of the disease.

\section{P-55}

\section{Histopathological effects of linear alkyl benzene sulfonic acid (LABSA) on zebrafish (Danio rerio) gills \\ Öztürk B* ${ }^{*}$ Güneş $S^{*}$, Alsaran $M^{*}$, Akbulut $C^{*}$, Genç $H^{*}$, Zengin $\mathrm{M}^{*}$, Yön ND* \\ Sakarya University}

Linear alkyl benzene sulfonic acid is one of the most important raw materials in detergent production process. It is widely used in in industry as the active component of synthetic detergents, being frequently found in domestic cleaning products. Once used and disposed, linear alkyl benzene sulphonic acid (LABSA) can enter natural water sources by discharge of domestic sewage and industrial waste. In this study, investigation the histological effects of LABSA on gill tissue of zebrafish was aimed. Zebrafish were maintained under the standardized conditions at $28^{\circ} \mathrm{C} \pm 1$ oC. The light / dark cycle was $14 \mathrm{~h} / 10 \mathrm{~h}$. In the study, two dose groups $(0.5 \mathrm{mg} / \mathrm{L}, 1 \mathrm{mg} / \mathrm{L}$ LABSA $)$ and one control group were determined. After 48 hours of adaptation LABSA were added to the aquarium. For the histological analysis, at the end of 5 days, gill tissues were dissected. Tissues were fixed with $10 \%$ neutral buffered formalin fluid for $24 \mathrm{~h}$. Tissues were dehydrated and embedded in the parafin wax and sectioned at $5 \mu \mathrm{m}$ thickness and stained with Hematoxylin Eosin. The samples were evaluated by examining under the light microscope. In control group normal gill histology was observed. Primary lamellae, secondary lamellae, epithelial cells and mucus cells monitored. Mucus cells were detected at the edge of primary lamellae. Secondary lamellae, seperated from central primary lamellae which supported by cartilage and connective tissue were detected clearly. In the $0.5 \mathrm{mg} / \mathrm{L}$ LABSA exposure group, opening at central vena was detected. Thinning was observed in epithelium of primary lamellae. Dysmorphism, hyperplasia and hypertrophy were monitored at secondary lamellae. Aneurism was observed at secondary lamellae. In $1 \mathrm{mg} / \mathrm{L}$ LABSA exposure group, increase in diameter of central vena was detected. Thickening at the epithelium of central vena was observed. Dysmorphism, thickening and deformation were monitored at secondary lamellae. As a result,
LABSA has led to a deterioration in fish gills tissues. In this study, statistical methods were not used.

\section{P-56}

Determination of the density of neovascularization in invasive front in skin squamous cell carcinoma, with varying degrees of histological differentiation

Mazhenkovska LK ${ }^{*}$, Janevska $\mathrm{V}^{* *}$, Basheska $\mathrm{N}^{* * *}$, Crvenkova $\mathrm{S}^{* * * *}$, Petrushevska $\mathrm{G}^{* *}$, Spasevska $\mathrm{L}^{* *}$, Milenkova L*, Kostovska N*

*Institute of Histology and Embryology, Faculty of Medicine, "Ss. Cyril and Methodius" University, Skopje, Republic of Macedonia; **Institute of Pathology, Faculty of Medicine, "Ss. Cyril and Methodius" University, Skopje, Republic of Macedonia; $* * *$ Department of Histopathology and Clinical Cytology, University Clinic Of Radiotherapy and Oncology, Faculty of Medicine, "Ss. Cyril and Methodius" University, Skopje, Republic of Macedonia; $\star * \star *$ University Clinic of Radiotherapy and Oncology, Faculty of Medicine, "Ss. Cyril and Methodius" University, Skopje, Republic of Macedonia

Introduction: Angiogenesis is a process of formation of new blood vessels from the existing normal capillaries. Tumors with higher angiogenesis activity belong to the category of very aggressive tumors. Aim of this paper is determination of the density of the blood vessels, i.e. neovascularization in the invasive front of the tumor stroma in skin squamous cell carcinoma (SCC) in relation to the healthy skin and the ratio of the density of the blood vessels in the skin SCC with a different degree of histological differentiation.

Methods: The material is consisted of operational materials from 30 patients with skin SCC. Neoplasms were classified according to the degree of histological differentiation (G). Histological sections of the invasive front of the neoplasms, the tumor tissue and the surrounding healthy skin were stained immunohistochemically using specific primary monoclonal antibodies SMA and CD34 and analyzed by light microscopy. The density of the blood vessels in the skin SCC in relation to the dermis of the healthy skin was determined, and the density of the blood vessels in neoplasms in relation to the degree of histological differentiation $(\mathrm{G})$.

Results: The histological analysis has shown high statistical difference in the density of the blood vessels in the invasive front of skin SCC compared to the healthy skin (MannWhitney $\mathrm{U}, \mathrm{p}=0.0000001$ ). The difference found in the density of neovascularization in the neoplasms with different degree of differentiation (G1, G2, G3) is statistically significant for (Kruskal-Wallis, $\mathrm{p}=0.0003$ ).

Conclusion: The density of the neovascularization changes considerably compared to the healthy skin. Depending on the degree of histological differentiation of the neoplasms, the found differences in the density of the blood vessels are statistically significant and there is positive correlation between 
them. The increase of vascularity in the invasive front of the neoplasm in a higher grade cancers suggest about its possible role in the progression of neoplasm.

\section{P-57}

Weight of the liver, spleen and kidneys regard to ethnic groups and body mass index in chilean individuals over 60 years old

Torrez I*, RiquelmeN**, Torrez F**, Binvignat $\mathrm{O}^{*}$, Olave $\mathrm{E}^{*}$ *Universidad De La Frontera, Temuco, Chile; **Universidad Mayor, Santiago, Chile

Several factors can influence the weight of the thoracic and / or abdominal viscera. In order to know the average weight of the liver, spleen and kidneys and of Chilean individuals, the information in these organs from 218 necropsies on fresh male cadavers between 2009-2013 was obtained, considering her ethnicity (Mapuche and non-Mapuche), age and body mass index (BMI). Data of bodies without apparent injury or disease of these viscera were studied. The subjects were divided in three groups of age, 60-69 (l), 70-79 (II) and 80-89 (III). In non-Mapuche individuals, in the group I the liver weighed $1.679 \mathrm{~g}$, the spleen $121 \mathrm{~g}$, the right kidney $151 \mathrm{~g}$ and the left kidney $153 \mathrm{~g}$; in group III these organs weighed $1.388 \mathrm{~g}, 93 \mathrm{~g}$, $142 \mathrm{~g}$ and $137 \mathrm{~g}$, respectively. In Mapuche individuals, the weight of these organs was lower. Regarding BMI, considering the total number of individuals, the weight of liver was $1.156 \mathrm{~g}$ in the low weight group, in the normal group was $1.391 \mathrm{~g}$ and in the overweight group was $1,581 \mathrm{~g}$; the spleen weighed $75 \mathrm{~g}$, $98 \mathrm{~g}$ and $108 \mathrm{~g}$; the right kidney $103 \mathrm{~g}, 137 \mathrm{~g}$ and $151 \mathrm{~g}$, and the left kidney $109 \mathrm{~g}, 136 \mathrm{~g}$ and $153 \mathrm{~g}$, respectively. These results are compared with other population groups in the world and are a contribution to morphological knowledge of the abdominal viscera.

Keywords: Anatomy, abdomen, weight viscera, body mass index

\section{P-58}

\section{Morphological characteristics of recurrent artery of Heubner}

Papazova $\mathrm{M}^{*}$, Dodevski A*, Lazarova $\mathrm{D}^{*}$, Zhivadinovik J*, Matveeva $\mathrm{N}^{*}$, Petrovska $\mathrm{S}^{* *}$, Zafirova B*

*Institute of Anatomy, Medical Faculty, "Ss. Cyril and Methodius University", Skopje, Republic of Macedonia; **Institute of Medical Physiology and Anthropology, Medical Faculty, "Ss. Cyril and Methodius University", Skopje, Republic of Macedonia

Introduction: Cerebral circulation, especially arterial, in recent decades has attracted the interest of anatomists and clinicians. Recurrent artery of Heubner is the largest and most constant perforating branch of anterior cerebral artery (ACA). The aim of this study was to determine the morphological and topographic characteristics of the Heubner artery.
Methods: The investigations of anatomical characteristics of the recurrent artery of Heubner are made on 133 human brains without cerebrovascular pathology, from both sexes at age from 23 to 68 . Brains were fixed in a $10 \%$ solution of formaldehyde, and the obtained material was analyzed using a stereoscopic light microscope.

Results: Of the brains examined, $94 \%$ of the brains had a recurrent artery of Heubner while in $6 \%$ of the brains we could not identify the artery. The recurrent artery of Heubner was present as a single vessel in $76 \%$ of cases, as a double vessel in $24 \%$ of cases. It originated from the junction of the ACA and the anterior communicating artery in $60 \%$, this was the most common type, from the A2 segment of the ACA in $21 \%$ of the specimens, and from the A1 segment of the ACA in 19\%. The length of the recurrent artery of Heubner is in range from 14 to $46 \mathrm{~mm}$, with mean value of $24 \mathrm{~mm}$. The diameter of the artery is in range from 0.9 to $2.8 \mathrm{~mm}$, with a mean value of 1.3 $\mathrm{mm}$.

Conclusion: Detailed anatomical knowledge of the recurrent artery of Heubner is important when considering vascular surgery in the area of the anterior portion of the circle of Willis since improper clip placement can result in occlusion producing neurological deficits.

Keywords: Recurrent artery of Heubner, brain, anatomy, origin, diameter

\section{P-59}

\section{Comparison of preoperative temporal bone CT with intraoperative findings in patients with chronic otitis media}

Madan $\mathrm{G}^{*}$, Turamanlar $\mathrm{O}^{*}$, Bucak $\mathrm{A}^{* *}$, Acay $\mathrm{MB}^{* * *}$, Yıldız E**, Gönül Y*, Yusuf Gülsarı*

*Department of Anatomy, Faculty of Medicine, Afyon Kocatepe University, Afyonkarahisar, Turkey; **Department of Otolaryngology, Faculty of Medicine, Afyon Kocatepe University, Afyonkarahisar, Turkey; $* * *$ Department of Radiology, Faculty of Medicine, Afyon Kocatepe University Afyonkarahisar, Turkey

The aim of our study was to investigate the diagnostic confidence level of the presurgical 160-slice CT findings compared with perioperative observational findings about anatomic variations in the structure of the facial canal, lateral semicircular canal and dural plate. 55 patients admitting with middle ear pathology to Afyon Kocatepe University Department of Otolaryngology were included in the study and the mean age was $42( \pm 15.55)$. Preoperative images of the temporal bone of the patient was obtained by tomography Toshiba Aquilion One (Toshiba Medial System, Oktavana, Japan). In determining of the facial nerve canal dehiscence sensitivity $52 \%$, specificity $88 \%$, positive predictive value $73 \%$, negative predictive value $75 \%$, in determining of the dehiscence in the tympanic segment sensitivity $50 \%$, specificity $89 \%$, positive predictive value $71 \%$, negative predictive value $76 \%$, in determining of the lateral semicircular canal dehiscence sensitivity $71 \%$, specificity 
$96 \%$, positive predictive value $71 \%$, negative predictive value $96 \%$ and to detecting the presence of dural plate defect Sensitivity $100 \%$, specificity $96 \%$, positive predictive value $50 \%$, negative predictive value of $100 \%$ were found. In determining the presence of dehiscence of the facial nerve canal and the tympanic segment by HRCT were observed moderate compatible with surgery. In determining the lateral semicircular canal dehiscence and the presence of defect of the dural plate were observed a good level compatible with surgery.

\section{P-60}

\section{Culturing of primary chondrocytes and chondrosarcoma cells type SW 1353, in vitro}

Dontchev $\mathrm{V}^{*}$, Hayvazov E**, Vasev $\mathrm{S}^{* *}$, Konstantinov $\mathrm{S}^{* * *}$, Momekov $\mathrm{G}^{* * *}$, Ilieva J***, Stefanova $\mathrm{E}^{*}$, Landzhov B*

*Department of Anatomy and Histology, Faculty of Medicine, Medical University of Sofia, Bulgaria; **Department of Orthopaedics and Traumatology, Hospital; ***Department of Pharmacology, Pharmacotherapy, Toxicology, Faculty of Pharmacy, Medical University of Sofia, Bulgaria;

Chondrocytes are highly differentiated cells. They are surrounded by extracellular matrix which contain collagen type 2 and proteoglycans. Removed from their environment and cultured in vitro, cells reducing their differentiation and change the type of secreted collagen. Primary chondrocytes grown after trypsinization in DMEM with supplement. Then the cells have been fixed, observed and described. Part of the same cells were treated immunocytochemically for secretion of collagen and histochemically for the release of proteoglycans. Was used antibodies agains collagen 1 and 2. For demonstration of proteoglycans was applied toluidine blue in solution. Human chondrosarcoma cells from cell line SW 1353 also have been cultivated, and treated just the same way as the primary chondrocytes. Aim of the study was to cultivate cells with characteristics of chondrocytes, which in a subsequent stage can be incorporated in three-dimensional structure which can be developed to become the basis of implant for restoration of damaged articular cartilage. For this purpose, we are cultivating and using two biological models, namely: $1 /$ culturing of autologous primary chondrocytes and 2/culturing chondrosarcoma cells of a cell line SW 1353. Basic idea was to compare the parameters of both cell models. Both types of cells have the potential for formation of cartilage tissue. They synthesize collagen type 2 , but the secretion of chondrosarcoma cells is relatively constant over time, whereas in primary chondrocytes, it reduces significantly in result of cell passage and age of the cultures. In cultures of chondrosarcoma cells prove more proteoglycans. Ultimately, in order to assess which of the two models is promising, must be performed experiments to generate threedimensional structure of chondrocytes and its implantation.

The article was written as a result of research grant to the Council for Medical Science, Medical University - Sofia, under Contract No. 30/2012

\section{P-61}

Absence of right posterior communicating artery

Şekerci R* ${ }^{*}$, Sindel $M^{*}$, Özgür Ö ${ }^{* *}$, Aytaç $G^{*}$, Sindel T**

${ }^{*}$ Department of Anatomy; Faculty of Medicine, Akdeniz University, Antalya, Turkey **Department of Radiology; Faculty of Medicine, Akdeniz University, Antalya, Turkey

In human anatomy, the left and right posterior communicating arteries are arteries at the base of the brain that form part of the circle of Willis. The posterior communicating artery, embryologically, belongs to the anterior circulation, representing the "caudal ramus" of the internal carotid artery (ICA). The posterior communicating artery $(\mathrm{PCoA})$ is a principal collateral circulation pathway and the source of numerous penetrating arteries that supply the ventral lateral and dorsomedial thalamic nuclei, as well as the lateral aspect of the thalamic pole, tuber cinereum, mamillary bodies, and cerebral peduncle. During routine cadaver dissection for undergraduate students, we found absence of right posterior communicating artery in a male cadaver, about 55 years age. The anomalies of the posterior communicating artery (PCoA) have been described by many researchers. The posterior communicating artery is sometimes absent, or the branch representing it fails to join the posterior cerebral on the right side in $4.5 \%$, on the left side in $6.5 \%$ and on both sides in $1.5 \%$. Some authors have explained the absence of PCoA due to lack of formation of anastomosis between the ICA and basilar artery in the developmental stage. They stated that in the embryo this artery attains its maximum size by the time the primitive trigeminal artery disappears. In cases which the trigeminal artery remains, the PComA becomes narrow and in a few cases it might even disappear. Angiograpichally when the PCoA couldnt seen it usually is assumed that hypoplasia of the artery. But this case showed us it could be agenesis. A small or absent ipsilateral posterior communicating artery is a risk factor for ischemic cerebral infarction in patients with internal carotid artey occlusion.

\section{P-62}

\section{Histological effects of mancozeb on intestinal tissue of zebrafish (Danio rerio)}

Öztürk B $^{*}$, Güneş $S^{*}$, Alsaran $M^{*}$, Kaymak $G^{*}$, Kayhan FE ${ }^{* *}$, Akbulut $\mathrm{C}^{*}$, Yön ND*

*Sakarya University; ** Marmara University

Mancozeb is a synthetic pesticide which has been used since 1967. It has been used to hinder the growth of fungi and plants against damage. Pesticides access aquatic ecosystems via groundwaters and affect water quality and aquatic life. In our study, examine the histopathological effects of mancozeb on intestine tissue of zebrafish was aimed. Zebrafish were raised in a computer-controlled incubation chamber, and received 14 hours of daylight and 10 hours of darkness everyday. After one week adaptation period zebrafish divided into four group $(\mathrm{n}=10)$ as one control and 2 experimental groups (5 ppm, 7.5 $\mathrm{ppm})$. For investigating the effects of mancozeb, intestine tis- 
sues were dissected after 5 day of the exposure. Tissues were fixed with $10 \%$ neutral buffered formalin and dehydration were carried out in an ascending series of ethanol. After tissues were cleared in xylene, embedded in paraffin wax and cut into $5 \mu \mathrm{m}$ sections on a microtome. The sections were stained with hematoxylin (H\&E) and results were evaluated with light microscope. In control group normal intestine histology was observed. Serosa, submucosa and mucosa layers were monitored clearly. In 5 ppm exposure group, degeneration at villi structure and increase in the number of goblet cells were detected. Depending on the increased number of leukocytes enteritis was monitored. Degeneration and expansion in villi structure were also observed. In $7.5 \mathrm{ppm}$ exposure group, severe degenerations were detected compared with other groups. Mucus accumulation and expansion in muscular layer were monitored. Enteritis and increase in the number of goblet cells were also detected at this group too. Integration and plethora at villi structure were observed. As a result, mancozeb has led to a deterioration in fish intestine tissues. In this study, statistical methods were not used.

\section{P-63}

Double popliteal vein with double adductor hiatus: a case report

Candan B, Öztürk K, Sulak O, Albay S

Department of Anatomy, Faculty of Medicine, Süleyman Demirel University, Isparta, Turkey

Objectives: The purpose of this study is to determine variation in popliteal vein and its components.

Methods: During routine dissection of the lower limb a variant popliteal vein was observed in the left popliteal fossa of a 39 year old male cadaver.

Results: Lower extremities of embalmed cadavers were dissected, with the knees fixed in the extended position. The dissection was carried out for undergraduate medical students in the Department of Anatomy. After removal of the skin, superficial fascia and adipose tissue, the gastrocnemius, soleus and hamstring muscles were reflected to expose the arteries and veins within the popliteal fossa. We have found the popliteal vein is formed by a medial (MV) and lateral vein (LV) in left side. $M V$ received the vein of medial head of gastrocnemius and combined vein (CV) which consisting of the fibular and posterior tibial veins (this vein received the anterior tibial vein) and this MV located in the superficial according to LV. CV giving a small communicating branch to the LV. MV is divided into two branches. The first branch was course along adductor magnus and it received the inferior gluteal vein, veins of the semimembranosus and semitendinosus then it passed from the accessory adductor hiatus (on adductor minimus) which was located in more above normal adductor hiatus. The other branch which was thicker than first branch drained into the $\mathrm{LV}$. The first branch of the MV and LV were united and formed femoral vein on adductor longus. Distance between the midpoint of the popliteal fossa and first and second adductor hiatus is $17.5 \mathrm{~cm}$ and $30 \mathrm{~cm}$ respectively.

Conclusion: We believe that this report may provide detailed information for anatomists and surgeons performing procedures in the popliteal region.

Keywords: Popliteal vein, adductor hiatus, variation, popliteal fossa

\section{P-64}

\section{The effect of hyperbaric oxygen therapy on Fos expression in spinal cord neurons following spinal cord injury}

Ertürk $M^{*}$, Keser A**, Sengül G*, Dağc1 T**, Aydın F***

*Department of Anatomy, Faculty of Medicine, Ege University, Izmir, Turkey; ${ }^{*}$ Department of Physiology and Center for Brain Research, Faculty of Medicine, Ege University, Izmir, Turkey; ${ }^{* *}$ Neoks, Center for Hyperbaric Treatment, Izmir, Turkey

We investigated the effect of hyperbaric oxygen (HBO) therapy on Fos protein expression in spinal cord neurons in rats with neuropathic pain induced by spinal cord injury (SCI). Rivlin and Tator clip method was used to produce SCI at T9-T11 in Sprague-Dawley rats $(\mathrm{n}=16)$ divided into four groups. 1.Control (SCI, no HBO), 2. Preoperative HBO (HBO five days before SCI), 3. Postoperative HBO (HBO just after SCI for five days), 4. Pre- and postoperative $\mathrm{HBO}$ (HBO five days before SCI and after SCI for five days). Rats received HBO at 2.80 ATA 60 min daily. Fos immunohistochemistry was used to localize neurons involved in nociception following SCI and $\mathrm{HBO}$ therapy in spinal cord segments $1 \mathrm{~cm}$ proximal and $1 \mathrm{~cm}$ distal to SCI. HBO applied preoperatively, postoperatively and pre- and postoperatively significantly increased Fos expression in the spinal cord. The increase corresponded with the duration of HBO therapy. In all groups, Fos expression was mostly in laminae $1-2$, followed by $3-4$, and $7-8$. This is the first study showing the effect of $\mathrm{HBO}$ therapy on Fos expression in rats with SCI. In a previous study, we found that HBO decreased nNOS following SCI (Dayan et al., 2012). Nitric oxide is involved in nociception. In this study, we expected a decrease in Fos following HBO therapy in SCI accordingly; however, this was not the case, suggesting the increase in Fos is not related to pain, but possibly to mechanisms related to neuroprotection.

\section{P-65 \\ Comprehensive evaluation philtral morphology in Turkish young adults}

Bagheri H, Şirintürk S, Gövsa F, Pınar Y, Özer MA

Department of Anatomy, Faculty of Medicine, Ege University, Izmir, Turkey

Objectives: A study of the morphology and aesthetic preferences of philtral complex will provide a database that will serve 
as a guide for reconstruction. While philtral column contributes to facial beauty, the relative influence of this feature to whole facial appeal has not yet been established.

Methods: Standard photographs of the philtral column were obtained from Turkish two hundred young adults participated in this study. The shape of the philtral column was grouped into 4 distinct types as shown in Uemura's classification. A: parallel type, B: triangulat type, C: concave type, D: unclear type.

Results: Among young adults; in men, the incidence of the philtral column types was classified as $27 \%$ in $\mathrm{A}, 26 \%$ in $\mathrm{B}$, $33 \%$ in $\mathrm{C}$, and $14 \%$ in D. In women, the incidence of the philtral column types was seen as $28 \%$ in A, $28 \%$ in B, $24 \%$ in $\mathrm{C}$, and $20 \%$ in D. There were significant gender differences in the aesthetic rankings of philtral columns shapes.

Conclusion: To obtain an optimally natural philtral construction accompanied by a symmetrical lip in cleft surgery, it is critical to take into consideration both the geometric features of the philtral morphological features of the philtrum as objective criteria. These results suggest that the comprehensive evaluation of philtral morphologic features by three-dimensional analysis is a useful means of identifying the ideal parameters in philtral construction in cleft surgery.

\section{P-66}

The protective role of chrysin on harmful effects of formaldehyde on the development of kidney in rat fetuses; a histopathologic study

Cuglan $S^{*}$, Yıldız A**, Sapmaz HI ${ }^{* * *}$, Vardı ${ }^{* *}$, Köse E* Ekinci $\mathrm{N}^{* * * *}$

*Department of Anatomy, Faculty of Medicine, Inönü University, Malatya, Turkey; ${ }^{* *}$ Faculty of Medicine, Department of HistologyEmbriology, Inönü University, Malatya, Turkey; ***Department of Anatomy, Faculty of Medicine, Gaziosmanpaşa University, Tokat, Turkey; $* * * *$ Faculty of Medicine, Department of Anatomy, Karabük University, Karabük, Turkey

Objectives: In this study, we aimed to determine possible harmful effects of formaldehyde exposure on the kidney of fetuses. In addition, potential protective role of chrysin $(\mathrm{CH})$ against these effects.

Methods: 58 female rats were divided into 6 groups and were tried to become pregnant. 10 rats which were not pregnant excluded from the experiment. It was administered every other day during 7-20 days of pregnancy to group I intraperitoneally (i.p.) saline, group II by gavage $20 \mathrm{mg} / \mathrm{kg} \mathrm{CH}$, group III i.p. 0.1 $\mathrm{mg} / \mathrm{kg}$ FA, group IV i.p. $1 \mathrm{mg} / \mathrm{kg}$ FA, group V i.p. $0.1 \mathrm{mg} / \mathrm{kg}$ FA and by gavage $20 \mathrm{mg} / \mathrm{kg} \mathrm{CH}$ and group VI i.p. $1 \mathrm{mg} / \mathrm{kg} \mathrm{FA}$ and by gavage $20 \mathrm{mg} / \mathrm{kg} \mathrm{CH}$. CH was applied one day before application. Fetuses obtained by caesarean section. Histological staining was performed to determine the impact on the kidney development in fetuses. Leica DFC-280 stained preparations were examined with a study microscope. Statistical analysis was performed.
Results: The number of mature glomeruli in the FA groups were found to be significantly decreased compared to control group $(\mathrm{p}<0.05)$. In terms of changes in the number of immature glomeruli, difference between FA-1 groups and control group was found statistically significant $(\mathrm{p}<0.05)$. The number of mature glomeruli in FA groups treated with $\mathrm{CH}$ was higher than FA groups. This increase was found statistically significant $(\mathrm{p}<0.05)$. Also, the number of total glomerul in FA-1 group was significantly reduced compared to control groups $(\mathrm{p}=0.01)$. The total number of glomeruli in FA-1 + CH group were found to be significantly increased compared with the FA-1 group $(\mathrm{p}=0.02)$.

Conclusion: It was determined that low dose FA exposure leads to histological damages on the development of the kidney of the rat fetuses. $\mathrm{CH}$ reduced these effects on the kidney significantly.

Keywords: Formaldehyde, Chrysin, Rat fetus, Kidney

\section{P-67}

\section{The osteoclastic activity using local platelet-rich plasma (PRP) injection on the tooth movement in rats: a histomorphometrical study}

Kovan A** Cumbul A*, Uslu Ü*, Bölükbaşı BÇ**

*Department of Histology and Embryology, Faculty of Medicine, Yeditepe University, Istanbul, Turkey; ${ }^{*}$ Department of Orthodontics, Faculty of Dentistry, Marmara University, Istanbul, Turkey

Objectives: Platelet-rich plasma (PRP) is an autologous platelets. PRP does not cause direct differentiation of cells. It increases the response to morphogenic factors that induce differentiation of progenitor cells. These factors, some of which are thought to stimulate healing of bone and soft tissue [1]. This study was to histomorphometrically and clinically evaluate the effect of local PRP injection on tooth movement.

Methods: 24 Sprague-Dawley rats were subjected to moderate concentration PRP injection. Tooth movement was performed on the upper first molars using a 40-g nickel titanium closed coil spring activated across the span from the central incisors to the first molar on both sides of the rats [2]. PRP injection was done on the right side (mPRP-E) and the left side was kept as a splithmouth orthodontic tooth movement control (mPRP-C). Our study has three groups on days 3, 7 and 21. After the experimental days, the animals were sacrificed in ethics and two upper jaws were dissected. The samples were received in $10 \%$ neutral formaldehyde in $0.1 \mathrm{M}$ phosphate buffered saline $(\mathrm{pH}=7.4)$ for fixation, then submitted to histological evaluation. Paraffin blocks were sectioned to $10 \mu \mathrm{m}$. Physical fractionators and systematic sampling methods were used and stained with the tartrate resistant acid phosphatase (TRAP, an osteoclast marker). Histomorphometric analysis was used to quantify TRAP activity between the roots of first molar under a stereological station.

Results: The mean osteoclastic activities for mPRP-E/mPRP-C Trap $+/ \mathrm{mm}^{2}$ on day 3, 7, 21 were $16.81 \pm 2.27 / 14.05 \pm 0.46,22.94 \pm$ $0.68 / 33.64 \pm 1.02$, and $7.97 \pm 0.91 / 14.59 \pm 0.44$ respectively. On day 
3 osteoclastic activity of mPRP-E was higher than mPRP-C ( $\mathrm{p}=0.044)$. On day 21 the osteoclastic activity within the roots of molar of the mPRP-E was lower than mPRP-C ( $\mathrm{p}=0.0001)$.

Conclusion: The results suggested that use of PRP could increase the osteoclastic activity. It could decrease the percentage of alveolar volume on paradental tissues. It provides the bone remodeling during the orthodontic tooth movement.

\section{P-68}

Computerized analysis of anatomic landmarks bony palate for palatine nerves block anesthesia

Cagimni P* ${ }^{*}$ Gövsa $F^{*}$, Özer MA*, Kazak $Z^{* *}$

*Department of Anatomy, Faculty of Medicine, Ege University, Izmir, Turkey; ${ }^{*}$ Faculty of Dentistry, Kırıkkale University, Kırıkkale, Turkey

Objectives: Greater palatine nerve block anesthesia (GPNA) is used in oral surgeries, periodontics and donor of soft tissues graft. Investigation of the computerized dimensional anatomic location of the greater palatine foramen (GPF) is important for the greater palatine nerve block anesthesia and indicate site to collect palatal donor tissue. This study aim was to provide standards for anatomic structures in the oral cavity that can be easily referred to in GPNBA.

Methods: 120 bony palates were examined to detect the position of the GPF and lesser palatine foramina (LPF) related to adjacent anatomical landmarks. The GPFs were assessed regarding position, diameter and distances to the midline maxillary suture (MMS) and to alveolar ridge (AR).

Results: The perpendicular distance of the GPF to the midline sagittal suture was $16.2 \pm 1.6 \mathrm{~mm}$ on the right and $16 \pm 1.6 \mathrm{~mm}$ on the left side. The mean distance from the posterior palatal border was consistent $4.2 \mathrm{~mm}$ on the right and $3.9 \mathrm{~mm}$ on the left side. In the greater majority of the skulls (78\%), the GPF were between proximal-distal surfaces of the 3rd maxillary molar. A single LPF was observed in $41 \%$ of the skulls, two LPF were observed $33 \%$ of the skulls bilaterally and five LPF were rare (2\%). The commonest position of LPF was at the junction of the palatine bone and the inner lamella of the pterygoid plate (84\%).

Conclusion: Whenever the third molar is erupted, it could be used as landmark for successful GPNA block anesthesia. Our resaults can help clinicans localize the palatine foramina in patients with and without maxillary molars and to predict the depth of a needle to anaesthetise the maxillary nerve with greater success when performing surgical procedures in hard and soft palate.

\section{P-69}

Impaired presynaptic neurotransmitter release and synaptic plasticity in inducible inositol 1, 4, 5-triphosphate 3-kinase a-overexpressing transgenic mice

Choi B, Kim JY, Lee HW, Kim H

Department of Anatomy, College of Medicine, Korea University, Seoul, Korea
Inositol 1,4,5-trisphosphate 3-kinase A (IP3K-A) is a brain specific enzyme whose expression is regulated by altered neuronal activities such as learning and seizure. IP3K-A is known to control dendritic spine formation via modulation of actin or tubulin cytoskeletons. Our previous study showed that IP3K-A null mice exhibited defects in some forms of learning, impaired synaptic plasticity such as long-term potentiation, and changed actin dynamics in hippocampus. To further explore the function and mechanism of IP3K-A on the neuronal plasticity, we generated transgenic ( $\mathrm{Tg}$ ) mice whose expression of IP3K-A was inducible in the forebrain region including hippocampus using Tet-on system. As a result, IP3K-A Tg mice showed defects in spatial memory (WT, n=11; Tg, n=10) and higher efficacy of evoked synaptic transmission at CA1 synapses than wild type (WT) mice. In electrophysiology data, IP3K-A Tg mice showed enhanced early phase long-term potentiation and reduced metabotropic glutamate receptor-dependent long-term depression (WT, n=20 cells; Tg, n=18 cells). Interestingly, however, there was no change in dendritic spine density or synaptic molecule level in IP3K-A Tg mice. Instead, IP3K-A Tg mice had increased number of synaptic vesicles at CA1 synapses. Our data suggest that IP3K-A might regulate hippocampal CA1 synaptic plasticity by modulating presynaptic release process in hippocampus, rather than by changing cytoskeletons.

\section{P-70}

The role of dextrose solutions used for prolotherapy and platelet rich plasma in cell morphology and gene expression levels

Coban ZD ${ }^{*}$, Karasimav Ö**, Güran Ş*

*Department of Medical Biology, Gülhane Military Medical Academy, Ankara, Turkey; **Department of Sports Medicine, Gülhane Military Medical Academy Ankara, Turkey

Objectives: Dextrose solutions used in prolotherapy are the standart applications in regenerative medicine. Platelet rich plasma (PRP) applications are generally used in alternative and complementary medicine. In our study, the effect of these treatments were studied in cellular and molecular levels.

Methods: We used healthy fibroblast cell culture for this study. We composed 7 groups: one for control; 5 for 5\%, 10\%, $15 \%, 20 \%$ and $25 \%$ dextrose solutions and one for PRP solution. All groups were examined and photographed for morphological changes during 24 hours. Then the cells were harvested for RT-PCR. The gene expression levels of PDGFA, PDGFB, VEGFA, IGF for wound healing and Caspase 3, BAX and BCL-XL for apopitosis were analyzed.

Results: In 5\% and 10\% dextrose groups, the cells died and separated from the flask surface within 24 hours but in 15\%, 20\% and $25 \%$ dextrose groups the cells remained intact in flasks. In PRP group, because of platelets, nothing can be observed in microscopy. Due to RT-PCR results, all of the solution groups were statistically different from control group. But $25 \%$ dextrose and PRP groups had the most augmented levels. 
Conclusion: Due to our results, 5\% and 10\% dextrose solution caused cell death, but the cells remained alive in $15 \%$, $20 \%$ and $25 \%$ dextrose solutions. The intracellular effects of prolotherapy and PRP solutions occured over the genes; PDGFA, PDGFB, VEGFA, IGF which have important roles in wound healing. They also triggered the apoptosis pathway over the genes; Caspase 3, BAX and BCL-XL.

\section{P-71}

The cytotoxic effect of folic acid on prostate cancer cells

Çoban ZD*, Babayiğit MA**, Güran Ş*

*Department of Medical Biology, Gülhane Military Medical Academy, Ankara, Turkey ${ }^{* *}$ Department of Public Health, Gülhane Military Medical Academy, Ankara, Turkey

Objectives: This study aims to investigate the cytotoxic effect of folic acid at supra-physiological doses on cancer cells in association between normal cells and the stem cells.

Methods: The cultures cell of prostate cancer (ATCC ${ }^{\oplus}$ PC3), mesenchymal stem cells (ATCC ${ }^{\circledR}$ Bone Marrow-Derived Mesenchymal Stem Cell) and gingival fibroblasts (ATCC ${ }^{\oplus} \mathrm{HGF}-$ 1) were used for this study. The cells were treated with the supraphysiological doses of folic acid (10 mM, $1 \mathrm{mM}, 100 \mu \mathrm{M}$ and 10 $\mu M)$. Cell proliferation rates were examined by MTT assay.

Results: The mean absorbance values of control group was found as $1.69 \pm 0.1$ in gingival fibroblasts, while $1.4 \pm 0.08$ for 10 $\mathrm{mM}, 1.82 \pm 0.1$ for $1 \mathrm{mM}, 1.79 \pm 0.1$ for $100 \mu \mathrm{M}$, and $2.07 \pm 0.09$ in $10 \mu \mathrm{M}$ folic acid treated groups, respectively $(\mathrm{p}<0.001)$. In mesenchymal stem cells, mean of control group was $1.62 \pm 0.07$, whereas cells treated with folic acid by $10 \mathrm{mM}$ was $1.24 \pm 0.1,1$ $\mathrm{mM}$ was $1.56 \pm 0.07,100 \mu \mathrm{M}$ was $1.71 \pm 0.1$ and $10 \mu \mathrm{M}$ was $1.67 \pm 0.1(\mathrm{p}<0.001)$. In prostate cancer $(\mathrm{PC} 3)$ cells treated with folic acid, antitumor (cytotoxic) effect have been determined. Compared to the control group $(1,51 \pm 0.1)$, PC3 cells treated with $10 \mathrm{mM}$ folic acid was $0,28 \pm 0.05(\mathrm{p}<0.005)$. The other concentrations had no statistically significant differences with control group $(1.3 \pm 0.1$ for $1 \mathrm{mM}, 1.82 \pm 0.1$ for $100 \mu \mathrm{M}$ and 1.77 for $10 \mu \mathrm{M}$, respectively, $\mathrm{p}>0.005)$.

Conclusion: Due to our results, the supra-physiological doses of folic acid inhibit cancer cell proliferation whereas these doses do not inhibit normal and stem cell proliferation. There is a need for further research on this topic.

\section{P-72}

Molecular view to the effect of centaury oil on wound healing

Coban ZD* ${ }^{*}$ Yener $\mathrm{M}^{* *}$, Benli A.S ${ }^{* *}$, Amanov K**, Altaylı $\mathrm{E}^{*}$, Demir H* $^{*}$ Kilıçarslan Ö* Çavana $\mathrm{E}^{* * *}$, Güran $\mathrm{S}^{*}$

*Gülhane Military Medical Academy, Department of Medical Biology, Ankara, Turkey; **Gülhane Military Medical Academy, School of Medicine, Ankara, Turkey; ***Gülhane Military Medical Academy, Ankara, Turkey
Centaury oil (the extract of Hypericum perforatum L.) is traditionally used for the treatment of wounds in Turkey. It has analgesic and anti- inflamatuar properties beside wound healing effect. There are many studies about the positive effect of this extract on wound healing in literature. But there is no mention about the animal studies that show which pathways are involved in. In our study the effect of Hypericum perforatum in early stages (first 7 days) and late stages (second 7 days) of wound healing was showed on the mice (mus musculus) by creating sterile sutures on their back. We evaluated the wound healing stages of scars with an updated methodology of Toledo- Piza at al. Due to our results, Hypericum perforatum has positive effects on the surface of the wounds in early stages but has no effect on late stages. This information is explained as Hypericum perforatum can be useful if it is used in the first week of the wound. In addition, the gene expression profiles of VEGFA, VEGFB, VEGFC, PDGFB and FGF2 genes which have roles in wound healing via angiogenesis, were studied by RT-PCR method. As a result, the gene expression levels of VEGFA, VEGFB, VEGFC and FGF2 were increased in early stages of wound healing but only VEGFA gene expression level was increased in late stages. The gene expression findings were corralate with morphological findings in both early and late stages of wound healing. Our findings are important for exploring the effect of Hypericum perforatum on angiogenesis via VEGFA, VEGFB, VEGFC and FGF2 genes in early stages of wound healing.

\section{P-73}

Embryonic stem cell (ESC) differentiation pathway on ion implanted surfaces

Sokullu E* Öztarhan A**, Dağc1 T ${ }^{* * *}$, Keser A***, Gözen $\mathrm{O}^{* * *}$, Şengül $\mathrm{G}^{* * *}$, Ertürk $\mathrm{M}^{* * * *}$

*Department of Bioengineering, Izmir Katip Çelebi University, Izmir, Turkey; **Department of Electric and Electronics Eng., Izmir University, Izmir, Turkey; ***Department of Physiology, Faculty of Medicine, Ege University Izmir, Turkey; ****Department of Anatomy, Faculty of Medicine, Ege University, İmir, Turkey

Embryonic Stem Cells (ESCs) are cells which potentially maintain a normal karyotype infinitely on culture in vitro, and can differentiate into any cell type. In literature, their differentiation ability into neural lineages has been evaluated, both in vitro and in vivo. This differentiation pathway may serve as an in-vitro model for the study of early human neurogenesis. Furthermore it may enable development of in-vitro models of neurodegenerative disorders. Another use of ESCs may a source of neural cells for transplantation and gene therapy. All these potential applications promote attention to ESCs and their model experiments for in vitro studies. In this research work, we have evaluated ESCs' growth capacity on C- and Auion implanted surfaces. For this aim, we have evaluated and compare two types of treatment effects on the surfaces. The resulting materials are promoting cell attachments and also providing appropriate environments for co-culture studies. 
S156 XXIV International Symposium on Morphological Sciences, 2nd-6th September, 2015, Istanbul, Turkey

P-74

Anti-apoptotic and anti-oxidant effects of caffeic acid phenethyl ester on cadmium-induced testicular toxicity in rats

Dönmez $\mathrm{YB}^{*}$, Erboğa $M^{*}$, Kanter $\mathrm{M}^{* *}$, Aktaş $C^{*}$, Erboğa $\mathrm{ZF}^{*}$, Aktaş $\mathrm{E}^{* * *}$, Gürel $\mathrm{A}^{* * * *}$

*Department of Histology and Embryology, Faculty of Medicine, Namik Kemal University, Tekirdağ, Turkey; ${ }^{* \star}$ Department of Histology and Embryology, Faculty of Medicine, Istanbul Medeniyet University, Istanbul, Turkey; ***Department of Nutrition and Dietetics, Faculty of Health Sciences, Karabük University, Karabük, Turkey; ****Department of Biochemistry, Faculty of Medicine, Namık Kemal University, Tekirdağ, Turkey

Introduction: Cadmium $(\mathrm{Cd})$ is a serious environmental and occupational contaminant and may represent a serious health hazard to humans and other animals. $\mathrm{Cd}$ is reported to induce the generation of reactive oxygen species, and induces testicular damage in many species of animals. Caffeic acid phenethyl ester (CAPE) is an active component of honeybee propolis extracts and has been used for many years as a folk medicine.

Objectives: The aim of the present study was to investigate the anti-apoptotic effects of CAPE on Cd-induced apoptosis in rat testes. Methods: A total of 40 male Wistar albino rats were divided into four groups: control, CAPE alone, Cd-treated, and Cd-treated with CAPE; each group consisted of 10 animals. To induce toxicity, $\mathrm{Cd}(1 \mathrm{mg} / \mathrm{kg}$ body weight) was dissolved in $2 \mathrm{ml}$ normal saline and subcutaneously injected into rats. The rats in CAPE treated group was given a daily dose of $10 \mu \mathrm{mol} / \mathrm{kg}$ body weight of CAPE by using intraperitoneal injection. This application was continued daily for a total of 30 days.

Results: The mean seminiferous tubule diameter, mean testicular biopsy score values and serum testosterone levels were significantly decreased in Cd-treated groups were compared to the control and CAPE alone groups. Furthermore, the CAPEtreated animals showed an improved histological appearance and serum testosterone levels in Cd-treated group. Our data indicate a significant reduction in the number of TUNEL positive cells in testis tissues of the Cd-treated group with CAPE therapy. Moreover, CAPE significantly suppressed lipid peroxidation, compensated deficits in the antioxidant defenses [reduced superoxide dismutase, glutathione peroxidase and catalase activities] in testes tissue resulted from Cd administration.

Conclusion: These findings suggest that the protective potential of TQ in Cd toxicity might be due to its antioxidant and anti-apoptotic properties, which could be useful for achieving optimum effects in Cd-induced testicular toxicity.

Keywords: Cadmium; CAPE; testicular toxicity; apoptosis, oxidative stress

\section{P-75}

Thymoquinone as a nephroprotective agent against doxorubicin-induced renal toxicity in rats

Dönmez YB ${ }^{* * *}$, Erboğa $M^{* *}$, Aktaş $C^{* *}$, Erboğa $Z F^{* *}$, Aktaş $\mathrm{E}^{* * *}$, Gürel A****

*Department of Histology and Embryology, Faculty of Medicine, Namık Kemal University, Tekirdağ, Turkey; **Department of Histology and Embryology, Faculty of Medicine, Istanbul Medeniyet University, Istanbul, Turkey; ${ }^{* *}$ Department of Nutrition and Dietetics, Faculty of Health Sciences, Karabük University, Karabük, Turkey; $* * * \star$ Department of Biochemistry, Faculty of Medicine, Namık Kemal University, Tekirdağ, Turkey

Introduction: Doxorubicin (Dox) is among the most widely used anticancer drugs for solid tumors. In animal experimental models DOX action resulted in acute toxicity followed by chronic progressive toxicity mainly affecting heart and kidney. Thymoquinone (TQ) is the main constituent of the essential oil obtained from black seeds (Nigella sativa) and has various pharmacological effects.

Objectives: The aim of this study was to examine the nephroprotective, antioxidant and anti-apoptotic effect of the TQ against Dox-induced renal toxicity.

Methods: A total of 40 male Wistar albino rats were divided into four groups: control, TQ alone, Dox-treated and Dox-treated with TQ; each group contain 10 animals. The rats in TQ alone group was given TQ $(50 \mathrm{mg} / \mathrm{kg}$ body weight $)$ once a day orally for 10 days. To induce nephrotoxicity, DOX $(30 \mathrm{mg} \mathrm{kg} / \mathrm{body}$ weight) was injected intraperitoneally by a single dose and the rats were sacrificed after $48 \mathrm{~h}$. The rats in the Dox-treated with TQ group were given a daily dose of $1 \mathrm{ml}$ ACE for 10 days.

Results: The histopathological studies in the kidney of rats also showed that TQ markedly reduced the toxicity of Dox and preserved the normal histological architecture of the renal tissue. Immunohistochemical analysis revealed that TQ significantly decreased the Dox-induced over expression of nuclear factor- $\kappa \mathrm{B}$ in renal tissue. Furthermore, TQ treatment resulted in decreased the number of apoptotic cells. TQ significantly suppressed lipid peroxidation, compensated deficits in the antioxidant defenses [reduced superoxide dismutase, glutathione peroxidase and catalase activities] in renal tissue resulted from Dox administration.

Conclusion: These findings suggest that the nephroprotective potential of TQ in may represent a novel therapeutic approach for the prevention of DOX-induced nephrotoxicity through anti-oxidant, and anti-apoptotic effects which could be useful for achieving optimum effects in Dox-induced nephrotoxicity.

Keywords: Doxorubicin; thymoquinone; nephrotoxicity; apoptosis, oxidative stress

\section{P-76}

Baddeley's working memory model

Dönmez D, Taşkınalp O

Faculty of Medicine, Department of Anatomy, Trakya University, Edirne, Turkey 
Memory is the process of keeping information that the power of hiding in mind consciously: the events, learned topics and their relationship with the past. The informations transfer with synapses that have some components: dendrites, axons, synaptic cleft and neurotransmitter transporter. Changes in the synaptic strength can be short-term, lasting seconds to minutes, or long-term lasting hours (long term potention- LTP). Learning and memory are believed to result from long-term changes in synaptic strength. The storage of informations is the important and unilluminated topic that a matter of investigation for decades. There are three storage models for memory: William James (1890), Atkinson, Shiffrin (1968), Baddeley, Hitch (1974) Baddeley model is a model for short-term memory. It has three components to explain how to work shortterm memory: central executive, visuo-spatial sketchpad, phonological loop. Central executive: manages all system. It also separates the informations to subsystems. Visuo- spatial sketchpad: working like navigation. In 2000, Baddeley updated his model, added new component that called "episodic buffer" which is bridge long-term and short-term memory. In conclusion, the memory models promote each other. But, they are not enough by themselves. The models have to investigate extensively to obtain clear knowledge.

\section{P-77}

Investigation of the protective effect of acetylsalicylic acid against central nervous system damage caused by rotenone

$\underline{\text { Köse E }}^{*}$, Ekici $\mathrm{S}^{* *}$, Karademir $Z^{* *}$, Tanbek $\mathrm{K}^{* * *}$, Polat $\mathrm{A}^{* * * *}$, Parlakpinar $\mathrm{H}^{* * * * *}$

*Department of Anatomy, Faculty of Medicine, Inönü University, Malatya, Turkey; ${ }^{*} 6$. Class Student, Faculty of Medicine, Inönü University, Malatya, Turkey; ${ }^{* *}$ Department of Physiology, Faculty of Medicine, Inönü University, Malatya, Turkey; ${ }^{* * * * K a r a b u ̈ k ~ U n i v e r s i t y, ~}$ Karabük Education and Research Hospital, Karabük, Turkey; $* * * *$ Department of Pharmacology, Faculty of Medicine, Inönü University, Malatya, Turkey

Objectives: Rotenone has been used an insecticide and piscicide that as a mitochondrial toxin. Recently, it has been used for central nervous system (CNS) damage modelling. In our study, protective effect of the acetylsalicylic acid against CNS damage modelling with rotenone was investigated.

Methods: For this purpose, 28 Wistar albino female rats diveded into 4 groups. Group I: Control; Group II (Rotenon): s.c. $3 \mathrm{mg} / \mathrm{kg}$ rotenone for 7 days; Group III (ASA): p.o. $10 \mathrm{mg} / \mathrm{kg}$ ASA for 7 days; Group IV (ASA+ rotenon): $10 \mathrm{mg} / \mathrm{kg}$ ASA applied before (30-45 minutes) s.c. $3 \mathrm{mg} / \mathrm{kg}$ rotenone injection for 7 days. At the end of the experiment, all rats were sacrificied by high-dose anesthesia and cerebrum and cerebellum tissues removed for biochemical examination (MDA, GSH, SOD, TOS, TAS, OSI).

Results: Biochemically, in the cerebrum tissues, MDA, TOS and OSI levels increased in the group II (only treated rotenone) when compared to control group. In addition, the levels of these markers of group IV (treated with ASA+rotenone) decreased when compared group II. The cerebrum GSH levels of the only rotenone treated group decreased when compared to control. In the cerebellum tissues, SOD levels of the only rotenone treated groups, decreased when compared to control group. However, TAS levels incresed in the group II when compared to control. There were no statistically significance for other biochemical markers for cerebellum.

Conclusion: It can be concluded that, rotenone caused an oxidative injury in the cerebrum tissues. And also, ASA, which has anti-inflammatory and antioxidant properties, shows beneficial effects on cerebral damage due to oxidative stres induced by rotenone.

Keywords: Rotenone, CNS damage, acetylsalicylic acid

\section{P-78 \\ Effects of selenium and melatonin on cadmium-induced damage in rat lungs}

Candan İA, Bayram D, Özgöçmen M

Department of Histology and Embryology, Faculty of Medicine, Süleyman Demirel University, Isparta, Turkey

Objectives: Cadmium (Cd) is a metal that is highly toxic to humans. Cd exposure has been associated with lung cancer incidence in a population living in a Cd-polluted area. Melatonin (MLT) is the major secretory product of the pineal gland. It has been shown to possess chemotherapeutic potential in human cancers and to be capable of modulating several signal transduction pathways associated with cell survival, proliferation, apoptosis and invasion. Selenıum (Se) is an essential trace element for antioxidant enzymes. A detoxification mechanism underlying Cd-Se effect hasn't yet been entirely clarified, but it may include beneficial antioxidative effect of Se and the formation of a Cd-Se complex. Our study performed to determine the protective effects of melatonin, selenium and their combination against cadmium induced oxidative damage in rat lungs.

Methods: Study was composed of 40 female rats were equally divided into five groups, group 1 (control), group $2(\mathrm{Cd}, 2$ $\mathrm{mg} / \mathrm{kg}$ by gavage), group 3 (Cd+MLT, $2 \mathrm{mg} / \mathrm{kg}$ Cd by gavage, $3 \mathrm{mg} / \mathrm{kg}$ MLT ip ), group $4(\mathrm{Cd}+\mathrm{Se}, 2 \mathrm{mg} / \mathrm{kg}$ Cd by gavage, $1 \mathrm{mg} / \mathrm{kg}$ Se ip.), group 5 (Cd+MLT+Se, $2 \mathrm{mg} / \mathrm{kg}$ Cd by gavage, $3 \mathrm{mg} / \mathrm{kg}$ MLT, $1 \mathrm{mg} / \mathrm{kg}$ Se ip.). After 1 month, rats were killed then lungs were excised for histopathological and immunohistochemical analyses.

Results: When control group was compared with other groups, we found significant histopathological findings such as; hemorrhagic areas, mononuclear cell infiltrations, bronchioles degeneration, alveolar dilatation and vascular congestion. We found that Se was more protective effects against cadmiuminduced oxidative damage than MLT. When we applied combine treatment, we found slight protective effect against cad- 
mium-induced damage. Immunohistologically, intense staining was observed in the cadmium while weak staining was observed in the MLT and SE groups.

Conclusion: In conclusion, cadmium caused significant structural changes in rat lungs. Our findings suggest that SE may be more protective effect than MLT.

Keywords: Cadmium, melatonin, selenium, oxidative damage, lung

\section{P-79}

The role of alpha lipoic acid as an antioxidant on smoking-induced skin damage

Baş F**, Bayram D*, Arslan B**, Arma ğan Iं*, Yeşilot Ş***, Çiçek $\mathrm{E}^{* * * *}$, Yorgancıgil $\mathrm{E}^{* * * * *}$

*Department of Histology and Embryology, Faculty of Medicine, Süleyman Demirel University, Isparta, Turkey; ${ }^{* *}$ Department of Family Medicine, Faculty of Medicine, Süleyman Demirel University, Isparta, Turkey; ***Department of Pharmacology, Faculty of Medicine, Süleyman Demirel University, Isparta, Turkey; $* * *$ Department of Biochemistry, Faculty of Arts and Sciences, Süleyman Demirel University, Isparta, Turkey; *****Faculty of Medicine, Süleyman Demirel University, Isparta, Turkey

Objectives: Smoking causes structural and compositional changes on the skin. These changes link to carcinogenic, oxidative and inflammatory reactions. Smoking increases degradation of collagen and elastic fibres, forming the basic skin structure. This way it contributes to premature skin aging and wrinkle formation. Exposed to cigarette leads to the formation of reactive oxygen species and the generation of bioactive molecules that can damage skin cells. This investigation was carried out to study possible effects of Alpha Lipoic Acid (ALA) on smoking- induced rat skin injury.

Methods: 28 Spraque-Dawley female rats were divided to three groups: control group $(\mathrm{n}=8)$, smoking group $(\mathrm{n}=10 ; 12$ cigarettes/day, 8 weeks) and smoking+ALA group $(\mathrm{n}=10 ; 12$ cigarettes/day+100 mg/kg ALA, 8 weeks). The concentration of carbonmonoxide in the cigarette smoke condensates was 310-380 ppm. Experiment group animals were sacrificed under anesthesia with $10 \%$ ketamine+2\%xylasine at the end of second mounth and then skin examples were taken from the epigastric area. Histochemical (Hematoxylin-Eosin and Masson's trichrome, immunohistochemical (TNF- $\alpha$ ) and biochemical analysis were performed on these skin tissues. Histological findings were performed semi- quantitative evaluations.

Results: Histologically, skin were distinguished normal structure in the control group. In the smoking group, collagen bundles and hair follicle degradation/reduction, sweat gland degeneration, mononuclear cell infiltration in dermis were encountered. In ALA-treated group, all of these changes were improved. Collagen bundles structures were appearanced more regular than the smoking group. Immunohistologically, intense staining was observed in the smoking group, while very weak staining was observed in control group, weak staining was observed in the ALA-treated group. Biochemically, MDA levels and protein carbonylation in the control and ALA-treated groups were lower, While it was observed higher in smoking group. CAT activity was observed higher than control group in experiment groups.

Conclusion: Smoking showed significant structural changes in rat skin. All the results suggest that ALA may be protective effective against smoking- induced skin damage.

Keywords: Alpha Lipoic Acid; smoking; skin damage; reactive oxygen species

\section{P-80}

The accessory pectoral muscle variation during cadaver dissection: a case report

Onur $S^{*}$, Akdoğan I*, Güngör A**

*Department of Anatomy, Pamukkale University, Faculty of Medicine, Denizli, Turkey; **Department of Neuroscience, Pamukkale University, Institute of Health Science, Denizli, Turkey

The pectoralis muscle variation have described in literature many times. However an accessory muscle of pectoral region have described in a case report. During cadaver dissection on a 77-year-old male cadaver, a accesory pectoral muscle was detected under the pectoralis major muscle on the left pectoral region. It was in mediolateral position and parallel to the pectoralis major muscle. The accesory pectoral muscle which is shape trigonal was approximately $15 \mathrm{~cm}$ in length and $5 \mathrm{~cm}$ in widest diamater. The origin of the muscle started from the 5 th and 6 th ribs at costo-chondral junction. From the origin it travelled supero-laterally and inserted by fusing with tendon of pectoralis major muscle. This accessory muscle was found only on left side. Muscular variations and their identification are important for procedure through the dissection planes in breast reconstruction surgery and also this would shed a light on the outcomes of the surgery.

Keywords: Accessory pectoral muscle, pectoralis major, variation

\section{P-81}

Protective role of alpha lipoic acid against polychlorobipheniyl (Aroclor 1254)-induced testicular toxicity in rats

\section{Gules O, Eren U}

Department of Histology-Embryology, Faculty of Veterinary Medicine, University of Adnan Menderes, Aydın, Turkey

The present study was aimed to investigate the antioxidant, biochemical and histological effects of alpha lipoic acid (ALA) against polychlorinated biphenyl (PCB)-induced testicular toxicity in male rats. Group I (control) rats weren't administered any chemicals for 30 days. Group II (sham) rats were administered with corn oil (2 ml/kg body weight (bw)/day) for 30 days. 
Group III (PCB) rats were treated with Aroclor 1254 (5 mg/kg bw/day) for 30 days. Group IV (ALA) rats were administered with ALA (25 mg/kg bw/day) for 30 days. Group V $(\mathrm{ALA}+\mathrm{PCB})$ rats were treated with ALA $(25 \mathrm{mg} / \mathrm{kg}$ bw/day) 24 hours prior to the administration of Aroclor $1254(5 \mathrm{mg} / \mathrm{kg} \mathrm{bw})$ for 30 days. Body and testis weights, total oxidant status (TOS) and total antioxidant status (TAS) levels in serum and testis samples, serum testosteron levels, heme oxygenase-1 (HO-1) band (Western blot) and staining intensity, histological appearance, histological alterations, apoptotic cells, seminiferous tubule diameter (STD) and seminiferous epithelium height (SHE) at stage VII-VIII and stage XII-XIV, incidences of stage XIV tubules and count of abnormal spermatozoa were investigated. TOS level in serum and testis, number of apoptotic cells, sub-basal vacuolization, desquamation of germinal cells in epithelium, germ cells across to tubular lumen, capillary congestion, HO-1 staining density and count of abnormal spermatozoa were significantly increased in PCB group. Moreover in PCB group, STD were decreased at stage VII-VIII and stage XII-XIV tubules. TOS level in serum and testis, sub- basal vacuolization, desquamation of germinal cells in the epithelium, germ cells across to tubular lumen, capillary hyperemia and apoptosis were significantly decreased in ALA+PCB groups. These findings suggested that ALA has protective role against PCB-induced testicular toxicity.

Keywords: Polychlorinated biphenyl, Aroclor 1254, alpha lipoic acid, histomorphometry, TUNEL, heme oxygenase-1, testosterone, total oxidant status, total antioxidant status, abnormal spermatozoa

\section{P-82}

\section{The effect of vitamin E on kidney in bisphenol} A-exposed rats

$\underline{\text { Y1ldız }}^{*}$, Güleş Ö**, Boyacıŏglu $M^{* * *}$, Kum Ş**

*Gynecology-Obstetrics and Pediatrics Hospital, Aydın, Turkey; **Department of Histology and Embryology, Adnan Menderes University, Aydın, Turkey; ***Department of Pharmacology and Toxicology, Adnan Menderes University, Aydın, Turkey

In the present study, we were aimed to determine the protective effects of vitamin $\mathrm{E}$ on the kidney in bisphenol A (BPA)exposed rats. A total of 30 three-month-old adult male Wistar albino rats were randomly divided into 5 groups consisting of 6 animals each. Group I (control) rats weren't administered any chemicals. Group II (sham) rats were administered with corn oil $(0,5 \mathrm{ml} /$ day) for three weeks. Group III (BPA) rats were treated with bisphenol A $(10 \mathrm{mg} / \mathrm{kg} / \mathrm{day})$ for three weeks. Group IV (Vitamin E) rats were administered with vitamin E (300 IU/kg/day) for three weeks. Group V (Vitamin E+BPA) rats were treated with both vitamin $\mathrm{E}(300 \mathrm{IU} / \mathrm{kg} / \mathrm{day})$ and bisphenol A (10 mg/kg/day) for three weeks. At the end of experimental period, the animals were sacrificed and right kidneys of the rats were removed. Kidney samples were fixed in 10 $\%$ neutral buffered formalin. The tissue sections that obtained after routine tissue processing were stained by Crosman's triple stain for histomorphometrical analysis. Also, superoxide dismutase (SOD) and malondialdehyde (MDA) levels were measured in kidney tissue. In the study, it was determined that the number of corpusculum renis, widths of proximal tubule, distal tubule, ascending limb of Henle's loop, collecting duct and SOD level significantly decreased in BPA group compare with the control group. Besides, the number of corpusculum renis, widths of ascending limb of Henle's loop, collecting duct and SOD level significantly increased in Vitamin E+BPA group compare with BPA group. On the other hand, MDA level significantly increased in BPA group compare with control group and it significantly decreased in Vitamin E+BPA group compare with BPA group. It was concluded that vitamin $\mathrm{E}$ has some protective effects on the kidney in bisphenol A- induced toxicity.

Keywords: Bisphenol A, vitamin E, histomorphometry, kidney, rat

\section{P-83}

\section{Investigation of Vaccinium myrtillus's antioxidant effects in experimental diabetic rat model}

${\underline{\text { Savaş } \mathrm{HB}^{*}}}^{*}$ Türkkan $\mathrm{A}^{* *}$, Yavuz B**, Yiğit $\mathrm{A}^{* * *}, \mathrm{Uz} \mathrm{E}^{*}$, Bayram NA****, Kale B****

*Department of Medical Biochemistry, Faculty of Medicine, Süleyman Demirel University, Isparta, Turkey; ${ }^{*}$ Faculty of Medicine, Süleyman Demirel University, Isparta, Turkey; ***Department of Medical Genetic, Faculty of Medicine, Süleyman Demirel University, Isparta,

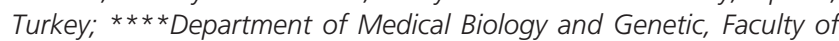
Medicine, Süleyman Demirel University, Isparta, Turkey; $* * * * *$ Department of Internal Medicine, Faculty of Medicine, Süleyman Demirel University, Isparta, Turkey

Objectives: Vaccinium myrtillus is a species of shrub with edible fruit of blue color, commonly called "bilberry", or blueberry. It has much in common with the American blueberry (Vaccinium cyanococcus) but it is also common in Turkey. This project was aimed to investigate the effects of Viscum myrtillus (VM) which believed has therapeutic properties for several disease including diabetes, and for this purpose a common format conventionally used, particularly in Turkey, on lipid peroxidation and antioxidant system, in experimental diabetic rat model induced by streptozotocin (STZ).

Methods: 32 Sprague-Dawley adult male rats were divided into 4 groups with 8 rat, respectively as follows: control, STZ, VM, VM + STZ. STZ citrate buffer (0.1 M, pH 4.5) contains $55 \mathrm{mg} / \mathrm{kg}$ after preparation so that to create a diabetes model, a single dose was administered intraperitoneally to rats. (Deliorman et al. 1995). VM extract relevant groups; $100 \mathrm{mg} /$ $\mathrm{kg} 1$ times / day by gavage. Catalase (CAT) which is an antioxidant enzyme, activity was studied according to the method of Aebi. Lipid peroxidation product that malondialdehyde (MDA) content were studied by spectrophotometric method according to Draper and Hadley method. Protein determination was performed using the supernatant and the extract of tissue 
homogenates by the method of Lowry. Data was assessed by ANOVA test and $<0.05$ was regarded as statistically significant.

Results: Application of VM increased in liver catalase activity was significantly in diabetic rats which were created by the application STZ $(\mathrm{p}<0.05)$.

Conclusion: Oxidative stress increases in diabetic rats. Giving of Vaccinium myrtillus to diabetic rats to reduce oxidative stress will be useful for the general condition of the rats. Further studies are needed for the treatment to reduce oxidative stress in diabetic rats.

Keywords: Vaccinium myrtillus, diabetes, MDA, CAT, antioxidant

\section{P-84}

The prophylactic effects of viscum album in streptozotocin-induced diabetic rats

Türkkan $\mathrm{A}^{* *}$, Savaș $\mathrm{HB}^{*}$, Yavuz B**, Yiğit A**, $\mathrm{Uz} \mathrm{E}^{*}$, Bayram NE****, Kale B ${ }^{* * * * *}$

*Department of Medical Biochemistry, Faculty of Medicine, Süleyman Demirel University, Isparta, Turkey; **Faculty of Medicine, Süleyman Demirel University, Isparta, Turkey; $* * *$ Department of Medical Genetic, Faculty of Medicine, Süleyman Demirel University, Isparta, Turkey; ${ }^{* * *}$ Department of Medical Biology and Genetic, Faculty of Medicine, Süleyman Demirel University, Isparta, Turkey; $\star * * * \star$ Department of Internal Medicine, Süleyman Demirel University, Isparta, Turkey

Objectives: Viscum album is a species of mistletoe in the family Santalaceae, commonly known as European mistletoe, common mistletoe or simply as mistletoe (Old English mistle). It is native to Europe and Turkey. This project was aimed to investigate the effects of Viscum Album (VA) which believed has therapeutic properties for several disease including diabetes, and for this purpose a common format conventionally used, particularly in Turkey, on lipid peroxidation and antioxidant system, in experimental diabetic rat model induced by streptozotocin (STZ).

Methods: 32 Sprague-Dawley adult male rats were divided into 4 groups with 8 rat, respectively as follows: Control, STZ, VA, VA + STZ. STZ citrate buffer (0.1 M, pH 4.5) contains 55 $\mathrm{mg} / \mathrm{kg}$ after preparation so that to create a diabetes model, a single dose was administered intraperitoneally to rats. (Deliorman et al. 1995). VA extract relevant groups; 100mg / $\mathrm{kg} 1$ times / day by gavage. Catalase (CAT) which is an antioxidant enzyme, activity was studied according to the method of Aebi. Lipid peroxidation product that malondialdehyde (MDA) content were studied by spectrophotometric method according to Draper and Hadley method. Protein determination was performed using the supernatant and the extract of tissue homogenates by the method of Lowry. Data was assessed by ANOVA test and $<0.05$ was regarded as statistically significant.

Results: Giving of VA has a significant decrease in renal MDA levels was significantly in diabetic rats which were induced by the application STZ $(\mathrm{p}<0.05)$.
Conclusion: Oxidative stress increases in diabetic rats. Giving of Viscum Album to diabetic rats to reduce oxidative stress will be useful for the general condition of the rats. Further studies are needed for the treatment to reduce oxidative stress in diabetic rats.

Keywords: Viscum Album, diabetes, MDA, CAT, antioxidant. Note: This project was made with the support by TÜBİTAK 220919198011303600 project number, 2013/2 term

\section{P-85}

\section{Carnosine effects to the number of Purkinje cells in cerebellum following exposure to $900 \mathrm{MHZ}$ electromagnetic field}

$\underline{\operatorname{Arslan}}^{*}$, Acer $\mathrm{N}^{*}$, Ocak $\mathrm{H}^{* *}$, Sönmez $\mathrm{MF}^{* * *}$, Kesici $\mathrm{H}^{* * * *}$, Ertekin T* , Akargöl S*, Dağdelen $\mathrm{U}^{* * * * *}$, Saracoğlu ÖG ${ }^{* * * * *}$

*Department of Anatomy, Faculty of Medicine, Erciyes University, Kayseri, Turkey; **Department of Oral and Maxillofacial Surgery, Faculty of Dentistry, Erciyes University, Kayseri, Turkey; $* * \star$ Department of Histology and Embryology, Faculty of Medicine, Erciyes University; ****Department of Histology and Embryology, Faculty of Medicine, Department of Electrical and Electronic Engineering, Gaziosmanpaşa University, Tokat, Turkey; *****Erciyes University Faculty of Engineering, Kayseri, Turkey

The application of carnosine in medicine has been discussed since several years. In studies have shown that the neuroprotective properties of carnosine. In the last few years, studies have indicated that electromagnetic field (EMF) emitted by mobile phones could affect the brain activity. In the presented study, the effects on the number of Purkinje cells in the cerebellum of 16-weeks old female rats were investigated carnosine and following exposure to $900 \mathrm{MHz}$ EMF. Four groups of rats, a control group (KG), sham group (SG), electromagnetic field exposed and injected carnosine $(\mathrm{EMF}+\mathrm{CG})$ and an electromagnetic field exposed group (EMFG), were used in this study. SG was placed in the exposure assembly but not exposed to EMF. EMFG group rats were exposed to $900 \mathrm{MHz}$ EMF (1 h/day for 20 days) in an exposure assembly. EMF+CG group rats were exposed to $900 \mathrm{MHz}$ EMF $1 \mathrm{~h}$ /day for 28 days and injected 2 $\mathrm{mg}$ and $20 \mathrm{mg}$ carnosine, carnosine was injected intraperitoneally before 30 minutes ago. The CG wasn't placed into the exposure assembly but it was exposed to EMF, only injected SF intraperitoneally during the study period. The specific energy absorption rate (SAR) varied between 0.008 . At the end of the experiment, all of the female rats were sacrificed and the number of Purkinje cells was estimated using a stereological counting tecnique. Histopathological processes were also done on sections of the cerebellum.

Results: Showed that no significant difference between EMF and $(\mathrm{EMF}+\mathrm{CG}) \mathrm{EMF}$ and injected $2 \mathrm{mg}$ carnosine. In addition total number of Purkinje cells in the cerebellum of (EMF+CG) EMF injected $20 \mathrm{mg}$ carnosin was higher than EMF group. Therefore, it is suggested that exposure to $900 \mathrm{MHz}$ EMF leads to decreases of Purkinje cell numbers and Carnosine administration increases the number of Purkinje cells in the rat cerebellum. 


\section{P-86}

Quantitative evaluation of normal aqueductal cerebrospinal fluid flow using phase-contrast cine MRI according to age and sex

Öner $Z^{*}$, Kahraman $\mathrm{AS}^{* *}$, Köse $\mathrm{E}^{* * *}$, Öner $\mathrm{S}^{* * * *}$

*Department of Anatomy, Faculty of Medicine, Inönü University, Malatya, Turkey; **Department of Radiology, Faculty of Medicine, Inönü University, Malatya, Turkey; $* * \star$ Department of Anatomy, Faculty of Medicine, Inönü University, Malatya, Turkey; $* * * \star$ Department of Radiology, Faculty of Medicine, Karabük University, Karabük, Turkey

Objectives: The aim of this study was cerebrospinal fluid (CSF) flow quantification in the cerebral aqueduct using phase-contrast cine magnetic resonance imaging (MRI) according to both sexes and three different age groups to obtain normative data.

Methods: 72 volunteers with no cerebral pathology were included in this study. Subjects were divided into three age groups: 20-34 years, 35-49 years and ?50 years including equal gender groups. CSF flow's quantitatively evaluation was performed with images that were obtained by $1.5 \mathrm{~T} \mathrm{MR}$ unit from cerebral aqueduct level on the semi-axial plan. Between groups, peak velocity $(\mathrm{cm} / \mathrm{s})$, average velocity $(\mathrm{cm} / \mathrm{s})$, forward volume $(\mathrm{mL})$, reverse volume $(\mathrm{mL})$, net forward volume $(\mathrm{mL})$, and average flow over range $(\mathrm{mL} / \mathrm{min})$ values of current flowing through aqueduct and average aqueductal areas were compared.

Results: There were no statistically significant differences in CSF flow parameters among different age groups and between sexes $(p>0.05)$. There was a statistically significant difference in average cerebral aqueduct area between the age group of $\geq 50$ years and the other age groups $(\mathrm{p}=0.002)$. The average aqueductal area was higher in the age group of $\geq 50$ years.

Conclusion: Normal aqueductal CSF flow parameters evaluated with phase-contrast cine MRI don't show a significant difference by age and sex. We have achieved the lower and upper values of these parameters would be useful in future clinical studies. The size of aquduktal area may also be explained by atrophy-dependent ventricular system dilatation in the elderly.

\section{P-87}

Anti-inflammatory, antioxidant and anti-apoptotic effects of interleukin-18 binding protein pretreatment on remote organ kidney damage induced by hepatic ischemia reperfusion injury

Gönül Y* ${ }^{*}$ Özsoy $M^{* *}$, Koçak A**, Özkeçeci ZT**, Karavelioğlu A****, Bozkurt M.F****, Çartıllı O*, Keleş $\dot{I}^{* * * * *}$, Koçak H H****** , Çelik $\mathrm{S}^{* * * * * * * *}$

*Department of Anatomy, Faculty of Medicine, Afyon Kocatepe University, Afyonkarahisar, Turkey; **Department of General Surgery, Faculty of Medicine, Afyon Kocatepe University, Afyonkarahisar, Turkey;

***Department of Histology and Embryology, Faculty of Medicine, Dumlupınar University, Kütahya, Turkey; $* * * *$ Department of Children Surgery, Faculty of Medicine, Afyon Kocatepe University, Afyonkarahisar, Turkey; $* * * *$ Department of Pathology, Faculty of Veterinary Medicine, Afyon Kocatepe University, Afyonkarahisar, Turkey; $* * * * * \star$ Department of Urology, Faculty of Medicine, Afyon Kocatepe University,
Afyonkarahisar, Turkey; $* * * * * * *$ Department of Medical Biochemistry, Faculty of Medicine, Dumlupınar University, Kütahya, Turkey; $\star * * * * * * \star$ Department of Medical Biochemistry, Faculty of Medicine, Afyon Kocatepe University, Afyonkarahisar, Turkey

Objectives: We aimed to investigate the relationship between liver ischemia and reperfusion (I/R) achieved by pringle maneuver and kidney damage in an experimental rat model and to reveal whether interleukin 18 binding protein (IL-18BP) pretreatment.

Methods: A total of 21 Wistar albino rats were included in this study. Animals were equally and randomly seperated into three groups as follows: Sham $(n=7),(I / R)$ group $(n=7)$ and IR + IL$18 \mathrm{BP}$ group $(\mathrm{n}=7)$. In study groups, clamps were removed after 60 minutes of ischemia process and livers were perfused for two hours. After sacrification, serum aspartate aminotransferase (AST), alanine aminotransferase (ALT) and lactate dehydrogenase (LDH) activities and serum urea (URE) and creatinine (CRE) levels were determined in serum samples. Pro-inflammatory cytokine levels including tumor necrosis factor (TNF)- $\alpha$, interleukin (IL)6 , IL-1 $\beta$, interferon (IFN) $-\gamma$, total protein, total oxidant status (TOS), total antioxidant status (TAS) and oxidative stress index (OSI) were measured in kidney tissue homogenate samples. In histopathological examinaiton, collected tissue samples were processed, stained and examined under light microscope.

Results: Renal TOS, OSI, IL-18 levels, serum AST, ALT, $\mathrm{LDH}$ activities and CRE levels were significantly lower in IR+IL-18BP groups, when compared with the I/R group $(\mathrm{p}<0.05)$. Beside this, TAS levels were remarkably higher in I/R+IL-18BP group, when compared to I/R group ( $<<0.05)$. In immunohistochemical analysis, there was a statistically significant difference between the sham and I/R groups $(\mathrm{p}=0.04)$ and between the I/R and I/R+IL18BP groups $(\mathrm{p}=0.026)$ in terms of apoptotic signal staining degree.

Conclusion: Acute kidney injury (AKI) may develop after pringle maneuver intervention. It has also been demonstrated that IL-18BP pretreatment may have anti-inflammatory, antioxidant and anti-apoptotic effects against this damage.

\section{P-88}

The effects of IL-18BP on mRNA expression of inflammatory cytokines and apoptotic genes in renal ischemia reperfusion injury

Gönül Y* ${ }^{*}$, Genç A**, Ahsen A***, Bal A****, Hazman Ö ${ }^{* * * * *}$, Toktaş $M^{* * * * *}$, Ulu MS ${ }^{* * *}$, Özdinç $S^{* * * * * * *}$, Songur $A^{*}$

*Department of Anatomy, Faculty of Medicine, Afyon Kocatepe University, Afyonkarahisar, Turkey; ${ }^{* *}$ Department of Physiology, Faculty of Medicine, Afyon Kocatepe University, Afyonkarahisar, Turkey; $* * *$ Department of Nephrology, Faculty of Medicine, Afyon Kocatepe University, Afyonkarahisar, Turkey; ****Department of General Surgery, Faculty of Medicine, Afyon Kocatepe University, Afyonkarahisar, Turkey; $\star * * * \star$ Department of Biochemistry, Faculty of Science and Arts, Afyon Kocatepe University, Afyonkarahisar, Turkey; $* * * * * *$ Department of Anatomy, Faculty of Medicine, Turgut Özal University, Ankara, Turkey; $* * * * * * *$ Department of Emergency, Faculty of Medicine, Afyon Kocatepe University, Afyonkarahisar, Turkey 
S162 XXIV International Symposium on Morphological Sciences, 2nd-6th September, 2015, Istanbul, Turkey

Aortic ischemia-reperfusion (IR) damage, which is related to aortic cross-clamping, is frequently observed during the surgery of abdominal aorta (AA) and is considered not only in lower extremities but also in remote tissues and organs. IL18BP has a well-known antioxidant properties and anti-inflammatory effect. In the present study we aimed to assess these effects of IL-18BP in preventing kidneys from this kind of damage. Thirty adult male Wistar albino rats were randomized to three groups: sham laparotomy, IR and IR+ IL-18BP. We applied $30 \mathrm{~min}$ infrarenal aortic occlusion then 2 hour reperfusion. We investigated the tissue and serum levels of cytokins, oxidative stress parameters. Additionally ure, creatinin levels, histopathological kidney evaluation, mRNA expression levels of inflammatory cytokines and apoptotic genes were investigated. We have found that; Urea and creatinin, tissue and serum levels of TNF- $\alpha$, IL- 6 , IL-18, IFN- $\gamma$ and TOS, oxidative stress index (OSI) were significantly lower in IR+ IL-18BP group than IR group. Moreover, mRNA expression levels of inflammatory cytokines and apoptotoc genes were clearly depressed in IR+IL-18BP pretreatment group. This improvements were demonstrated with total score of histopatological damage (TSHD). As a result we demonstrated IL-18BP can preclude renal damage in infrarenal AIR models of rats by showing antioxidant, antiapoptotic and antienflamatuar effects.

\section{P-89}

Effects of electromagnetic radiation exposure on bone mineral density, thyroid nodule and oxidative stress index in electrical workers

Kunt $\mathrm{H}^{*}$, Şentürk Iं**, Gönül Y***, Ahsen $\mathrm{A}^{* * * *}$, Korkmaz $\mathrm{M}^{* * * * *}$, Hazman $\mathrm{O}^{* * * * * *}$, Bal $\mathrm{A}^{* * * * * * *}$, Genç $\mathrm{A}^{* * * * * * *}$, Songur A***

*Department of Science Teacher, Faculty of Education, Dumlupınar University, Kütahya, Turkey; **Department of Orthopedics and Traumatology, Faculty of Medicine, Afyon Kocatepe University, Afyonkarahisar, Turkey; ***Department of Anatomy, Faculty of Medicine, Afyon Kocatepe University, Afyonkarahisar, Turkey; $* * * \star$ Department of Nephrology, Faculty of Medicine, Afyon Kocatepe University, Afyonkarahisar, Turkey; $* * * * \star D e p a r t m e n t$ of Radiology, Faculty of Medicine, Dumlupınar University, Kütahya, Turkey; $* * * * * *$ Department of Biochemistry, Faculty of Science and Arts, Afyon Kocatepe University, Afyonkarahisar, Turkey; $* * * * * * *$ Department of General Surgery, Faculty of Medicine, Afyon Kocatepe University, Afyonkarahisar, Turkey; $* * * * * * * *$ Department of Physiology, Faculty of Medicine, Afyon Kocatepe University, Afyonkarahisar, Turkey

In the literature, there are publications reporting that many diseases increase in those who live near high voltage electric transmission lines (HVETL) or are exposed professionally. However, it was not investigated whether HVETL affect bone metabolism, oxidative stress and prevalence of thyroid nodule. To search this effect, DEXA bone density measurements, serum FT3, FT4, RANK, RANKL, osteoprotegerin (OPG), alkaline phosphatase (ALP), Phosphor (P), Total antioxidant capacity (TAC), Total oxidant capacity (TOA), Oxidative stress
Index (OSI) levels were analyzed. Bone mineral density levels of L1-L4 vertebra and femur were observed significantly lower in the electrical workers. ALP, P, RANK, RANKL, TOA, OSI and anteroposterior diameter of the left thyroid lobe (LAPD) levels in the study group were significantly higher when compared with the control group whereas OPG, TAS, FT4 levels were detected significantly lower $(\mathrm{p}<0.05)$. Consequently, replacement of construction-destruction balance in the bone metabolism towards destruction of electrical workers employed in HVETL and in relation to this, decrease in OPG levels whereas increase in RANK and RANKL levels were observed. These data, consistent with previous studies reporting that long term electromagnetic field exposure causes disorders in many organs and systems, set to think that affects bone and thyroid metabolism and also increases oxidative stress index by increasing the total oxidant capacity and decreasing the antioxidant capacity.

\section{P-90 \\ Evidences of attenuation of remote organ kidney injury induced by hepatic ischemia reperfusion with Pringle maneuver following interleukin-18 binding protein pre-treatment}

Gönül $Y^{*}$, Kazandı $S^{*}$, Koçak A**, Ahsen A***, Bal A****, Karavelioğlu $\mathrm{A}^{* * * *}$, Hazman $\mathrm{Ö}^{* * * * * *}$, Turamanlar $\mathrm{O}^{*}$, Kokulu $\mathrm{S}^{* * * * * *}$, Yüksel $\mathrm{S}^{*}$

*Department of Anatomy, Faculty of Medicine, Afyon Kocatepe University, Afyonkarahisar, Turkey; ${ }^{* *}$ Department of Histology and Embryology, Faculty of Medicine, Dumlupınar University, Kütahya, Turkey; ${ }^{* *}$ Department of Nephrology, Faculty of Medicine, Afyon Kocatepe University, Afyonkarahisar, Turkey; $* * * *$ Department of General Surgery, Faculty of Medicine, Afyon Kocatepe University, Afyonkarahisar, Turkey; ${ }^{* * * *}$ Department of Children Surgery, Faculty of Medicine, Afyon Kocatepe University, Afyonkarahisar, Turkey; $\star * * * * *$ Department of Biochemistry, Faculty of Science and Arts, Afyon Kocatepe University, Afyonkarahisar, Turkey; $* * * * * * *$ Department of Anesthesia and Reanimation, Faculty of Medicine, Afyon Kocatepe University, Afyonkarahisar, Turkey

Objectives: Liver ischemia/reperfusion (IR) injury occurs in case of occlusion of the portal vein, especially during major resection surgeries and liver transplantation. Liver damage is not the only result of hepatic IR and distant organs such as heart, lung and kidneys can also be affected. We aimed to investigate the considerable effect of Interleukin-18 Binding Protein (IL-18 BP) against acute kidney injury induced by hepatic IR.

Methods: A total of 24 male Wistar albino rats were included in this study. Animals were equally and randomly seperated into three groups as follows: I- Sham group, II- IR group (1 hr ischemia and $4 \mathrm{hr}$ reperfusion) and III- IR+IL-18BP group (50 $\mu \mathrm{g} / \mathrm{kg}$ IL-18BP was intraperitoneally administered 30 minutes before surgery). Blood, liver and kidney samples were collected for histopathological and biochemical [hepatic and renal function parameters, nitric oxide (NO), malondialdehyde (MDA) 
and glutathione (GSH) levels)] analysis. Also, pro- inflammatory cytokines including tumor necrosis factor- $\alpha$ (TNF- $\beta)$, interleukin-1, (IL-1,) and interleukin-6 (IL-6) levels were measured in kidney tissues.

Results: IL-18 BP has improved liver and kidney functions in acute kidney damage, restored structural changes, exhibited anti-inflammatory effects by decreasing pro-inflammatory cytokines and regulated the oxidative stress parameters by antioxidant effect.

Conclusion: Current study would be the first to evaluate the protective, antioxidant and anti-inflammatory effects of IL$18 \mathrm{BP}$ on renal damage induced by hepatic IR. Our results may contribute to reveal the full mechanism underlying acute kidney injury induced by liver IR and thereby can represent an important step for the current transplantation surgery practice.

Keywords: Liver, ischemia/reperfusion, IL-18BP, kidney, injury

\section{P-91}

Histology in examination of three-dimensional cultures

Mokry I, Hrebikova H, Cizkova D, Chvatalova J, Pisal R

Department of Histology and Embryology, Medical Faculty in Hradec Kralove, Charles University in Prague, Hradec Králové, Czech Republic

The cell culture became an invaluable tool for studying cell behaviour, development, function, gene expression, toxicity of compounds and efficacy of novel drugs. Although most results were obtained from cell cultivation in two-dimensional systems, in which cells are grown in a monolayer, three-dimensional cultures are more promising as they correspond closely to the native arrangement of cells in living tissues. In our study, we focused on three types of three-dimensional in vitro systems used for cultivation of one cell type. Cell morphology, their spatial distribution inside of resulting multicellular structures and changes in time were analysed with histological examination of samples harvested at different time periods. In multilayered cultures of WRL 68 hepatocytes grown on semipermeable membranes, the cells were tightly apposed, showed features of cell differentiation. After 6 weeks in vitro, the the cells accumulated in 8-9 layers; the number of viable cells decreased while eosinophilic cells with condensed chromatin and karyorrhexis increased in numbers. Eosinophilic cells expressed M30 neoepitope confirming that cells were dying by apoptosis. They were of different sizes and bizzare shapes whereas negative cells were oval or polyedric. Non-passaged neurospheres generated by proliferation of neural progenitor cells showed similar features including cell differentiation as well as cell death from the second week in vitro. After one and two months of cultivation the neuropheres were still viable, showed similar microscopic features with characteristic zonal distribution. Biogenic scaffolds composed of extracellular matrix of the murine tibial anterior muscle were colonized with $\mathrm{C} 2 \mathrm{C} 12$ myoblasts in vitro. Although the recellularized scaffolds did not reach high cell densities comparable with the former systems they supported well cell anchorage and migration without any signs of cell regression. This work was supported by Prvouk P37/06.

\section{P-92 \\ Localization of p62 and NBR1 in cathepsin $D$ - deficient neurons}

Yamaguchi $J^{*}$, Nanao $T^{*}$, Shibata $M^{* *}$, Uchiyama $Y^{*}$

*Department of Cellular and Molecular Neuropathology, Juntendo University, Tokyo, Japan; **Department of Neuroanatomy, Kagoshima University Graduate Faculty of Medicine and Dental Sciences, Kagoshima, Japan

Cathepsin D deficiency is known to induce autophagy and accumulate abnormal lysosomes called granular osmiophilic deposits in mouse brain neurons. Dysfunctional lysosomes contain undigested materials that are substrates for cathepsin $\mathrm{D}$, while they are often found within autophagic vacuoles with double membranes. Here we show the localization of p62 and NBR1, adapter proteins for selective autophagy, in neurons. Immunoreactivity for p62 and NBR1 was diffuse in the perikarya of neurons in wild-type mouse brain, while punctate immunoreactivity was localized only in somatodendrites but not in axons and their terminals of neurons in cathepsin Ddeficient brains. This localization pattern of p62 and NBR1 was confirmed in primary cultured neurons obtained from the cerebral cortex of wild-type and cathepsin D-deficient mouse embryos at embryonic day 16 or 17 , while the amino-terminal specific domains of these proteins were responsible for the polarized localization. We further examined the intraneuronal target components of p62 and NBR1; they were co-localized with LC3 and ubiquitin in neuronal cell bodies, while immunoreactivity for p62 and NBR1 were co-localized with those for ubiquitin on the limiting membrane of dysfunctional lysosomes. These results suggest that the limited localization of p62 and NBR1 contributes to the selective autophagy in the somatodendrites of neurons.

\section{P-93}

\section{The investigation of the role of KCBJ11 gene in the development of gestational diabetes}

Bozkurt $\mathrm{S}^{*}$, Arıkoğlu H $\mathrm{H}^{*}$, Baldane $\mathrm{S}^{* *}$, İşcioğlu $\mathrm{F}^{* * *}$

*Department of Medical Biology, Faculty of Medicine, Selçuk University, Konya, Turkey; **Department of Endocrinology, Faculty of Medicine, Selçuk University, Konya, Turkey; ***Department of Statistics, Faculty of Science, Ege University, Izmir, Turkey

Objectives: Gestational Diabetes Mellitus (GDM) is impaired glucose tolerance at any level, which began during pregnancy or was noticed for the first time during pregnancy resulting in varying degrees of hyperglycemia and predispose to the development of Type 2 Diabetes Mellitus (T2DM). In this study, we aimed to scan the KCNJ11 gene encodes Kir6.2 channel pro- 
S164 XXIV International Symposium on Morphological Sciences, 2nd-6th September, 2015, Istanbul, Turkey

tein, thought to make susceptible to GDM which is important determinant of T2DM transition, and to evaluate the associations with the disease by determining the single nucleotid polymorphisms (SNP).

Methods: Seventy four pregnant diagnosed as GDM and 49 healthy pregnant for control group from Department of Endocrinology Faculty of Medicine Selçuk University were included in our study. The whole coding region of the KCNJ11 gene was amplified by Polymerase Chain Reaction (PCR) and was evaluated by two-way DNA sequencing analysis. In statistical analyzes, $\mathrm{p}<0.05$ was considered significant.

Results: In our study, 5 of the 15 SNPs reported in the literature were determined. We did not find any new SNP specific to our population. No significant association was determined between SNP E23K, A190A, I337V with GDM under additive, dominant and recessive models $(\mathrm{p}>0.05)$. Statistical analysis could not be performed for L267L and L270V polymorphisms because of their low frequency.

Conclusion: No association was determined between GDM and the polymorphisms in KCNJ11 which have shown in the genetic basis of T2DM in many populations until today. With this study for the first time the effect of KCNJ11 gene on genetic basis of GDM was investigated and presented a basic work for subsequent studies.

\section{P-94}

Protective effects of urtica dioica on testicular torsion/detorsion-induced ischaemia-reperfusion injury in rats

$\underline{\text { Aktaș }} C^{*}$, Erboğa $M^{*}$, Topçu B**, Erboğa ZF*, Dönmez YB* *Namık Kemal University, Faculty of Medicine, Department of Histology and Embryology, Tekirdağ, Turkey; **Namık Kemal University, Faculty of Medicine, Department of Biostatistics, Tekirdağ, Turkey

Introduction: Testicular torsion/detrorsion is a urologic emergency that requires immediate surgical intervention to prevent testicular damage. Testicular torsion/detrorsion is identified as a typical case of ischemia-reperfusion (I/R) injury. Urtica dioica L. (UD) is a multipurpose herb in traditional medicine for which some anti-oxidative and anti-inflammatory properties have been identified.

Objectives: The aim of this study was to investigate the protective effect of UD on testicular torsion/detorsion-induced I/R injury. Methods: A total of 24 Sprague Dawley male rats were divided into three groups: control, I/R and I/R treated with UD; each group contain eight animals. Testicular torsion was created by rotating the left testis $720^{\circ}$ in a clockwise direction. The ischaemia period was $4 \mathrm{~h}$ and orchiectomy was performed after $4 \mathrm{~h}$ of detorsion. UD ( $2 \mathrm{ml} / \mathrm{kg}$, i.p.) was administered only once, 30 min prior to detorsion.

Results: To date, no histopathological changes on testicular torsion/detorsion-induced I/R injury in rats by UD treatment have been reported. Spermatogenesis and mean seminiferous tubule diameter were significantly decreased in I/R groups were compared with the control group. Furthermore, UD treated animals showed an improved histological appearance in $\mathrm{I} / \mathrm{R}$ group. It was also observed that treatment with UD increased the activity of proliferating cell nuclear antigen (PCNA), which decreased as a result of $\mathrm{I} / \mathrm{R}$, and this treatment also reduced the number of TUNEL-positive cells.

Conclusion: We believe that further preclinical research into the utility of UD may indicate its usefulness as a potential treatment on testes injury after I/R in rats.

Keywords: Ischemia and reperfusion, thymoquinone, testis, apoptosis, oxidative stress

\section{P-95}

Protective effects of curcumin on formaldehyde induced hepatotoxicity and oxidative stress in rats

Aktaş $C^{*}$, Bali I ${ }^{* *}$, Yıldırım $\mathrm{O}^{* * *}$, Gelincik $\mathrm{I}^{* * * *}$, Bali $\mathrm{C}^{* * * * *}$, Emir $\mathrm{S}^{* *}$, Turan $\mathrm{CB}^{* * * * * *}$, Erboğa $M^{* * * * * * *}$, Aktaş $\mathrm{C}^{* * * * * * *}$, Gürel $\mathrm{A}^{* * * * * * * *}$

*Namık Kemal University, Faculty of Medicine, Department of Histology And Embryology, Tekirdağ, Turkey; **Namık Kemal University, Faculty of Medicine, Department of General Surgery, Tekirdağ, Turkey; ***inönül University, Faculty of Medicine, Department of Internal Medicine, Malatya, Turkey; $* * * *$ Namık Kemal University, Faculty of Medicine, Department of Pathology, Tekirdağ, Turkey; *****Baskent University, Faculty of Medicine, Department of Anesthesiology and Reanimation, Ankara, Turkey; $* * * * * *$ Namık Kemal University, Faculty of Medicine, Department of Anesthesiology and Reanimation, Tekirdağ, Turkey; $* * * * * *$ Namık Kemal University, Faculty of Medicine, Department of Histology and Embryology, Tekirdağ, Turkey; $* * * * * * *$ Namık Kemal University, Faculty of Medicine, Department of Biochemistry, Tekirdağ, Turkey

Introduction: Curcumin (Cur), a polyphenolic antioxidant derived from the turmeric root has undergone extensive preclinical development, showing remarkable efficacy in wound repair, cancer and inflammatory disorders. Formaldehyde (FA) is a colorless toxic chemical. It is used as a disinfectant in medicine, in addition to its use as a fixative in anatomy, pathology, and histology laboratories.

Objectives: The aim of this study was to investigate the protective effect of Cur on FA induced hepatotoxicity and oxidative stress. A total of 21 Sprague dawley male rats were divided into three groups: control, FA and FA treated with Cur. FA and FA + Cur treated groups received i.p injection of $10 \mathrm{mg} / \mathrm{kg} \mathrm{FA}$ for 30 days. The rats in the Cur-treated group were given Cur $(100 \mathrm{mg} / \mathrm{kg})$ once a day intra gastric for 30 days starting with FA administration. Liver tissues and blood samples were collected for histopathological and biochemical analysis.

Results: In FA group, a significant increase in MDA, NO and $\mathrm{XO}$ levels was observed in liver tissues and a significant decrease in the levels of CAT, SOD and GSH-Px activities in liver tissues was determined when compared with control group. In addition, a significant decrease in MDA, NO and XO levels was observed in curcumin treated rats and a significant increase was determined in the levels of CAT, SOD and GSH-Px activities in liver tissues, when compared with FA group. FA caused significant increases in TNF, YKL-40, IL-6, TGF- $\beta$, periostin levels in 
plasma. In the FA group, degeneration in hepatocytes, cytoplasm vacuolization, cytoplasm losses and hypertrophic hepatocytes were observed. The Cur treated rats in the FA group showed an improved histological appearance and serum TNF, YKL-40, IL6 , TGF- $\beta$, periostin (p küçük harf) levels.

Conclusion: It was concluded that Cur treatment might be beneficial in preventing FA-induced hepatotoxicity and oxidative stress in liver tissue damage, therefore, shows potential for clinical use.

\section{P-96}

Effect of calcium dobesilate on the expression of vascular endothelial growth factor (VEGF) in a rat model of ovarian hyperstimulation syndrome (OHSS)

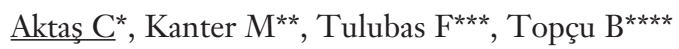

*Namık Kemal University, Faculty of Medicine, Department of Histology and Embryology, Tekirdağ, Turkey; **istanbul Medeniyet University, Faculty of Medicine, Department of Histology and Embryology, Istanbul, Turkey; $* * *$ Namık Kemal University, Faculty of Medicine, Department of Biochemistry, Tekirdağ, Turkey; $* * *$ Namık Kemal University, Faculty of Medicine, Department of Biostatistics, Tekirdağ, Turkey

Introduction: Ovarian hyperstimulation syndrome (OHSS) resulting from supraphysiological stimulation of the ovaries, usually during ovulation induction by gonadotropins. vascular endothelial growth factor (VEGF), also referred to as vascular permeability factor, is a potential mediator in the development of OHSS. Calcium dobesilate $(\mathrm{CaD})$ is considered a vasoprotective drug.

Objectives: To date, no examinations of the protective effects of $\mathrm{CaD}$ on OHSS have been reported. Our aim was to investigate the effects of calcium dobesilate on vascular permeability and the expression of VEGF in a rat model of OHSS.

Methods: The immature female rats (21 days) were randomly allocated to six groups, including Control-1, Control-2, Control-3, Control-3 + CaD, OHSS, OHSS + CaD. Ovaries from rats in each of the all groups were removed and weighed. The ovaries from one side were of each rat were used for VEGF analysis by specific ELISA kits, and the other ovary was used for immunohistochemically analysis of VEGF, oestrogen and proliferating cell nuclear antigen (PCNA). Oestrogen concentrations in serum and ovary tissue were determined and VEGF concentration in the serum and abdominal fluid were determined by specific ELISA kits.

Results: We have demonstrated that there was an increase in vascular permeability and OHSS developed by measurement of hematocrit and evans blue. CaD significantly protected vascular permeability. The HSCORE of PCNA, estrogen, VEGF and VEGF levels in peritoneal fluid, serum and ovarian tissues in ovary tissues significantly increased in OHSS groups. Treatment of $\mathrm{CaD}$ markedly decreased the reactivity of VEGF in OHSS.

Conclusion: The present study shows that the $\mathrm{CaD}$ protect vascular permeability reducing the expression of VEGF in experimentally induced OHSS. This is the first study which shows for preventive effects of $\mathrm{CaD}$ in OHSS. Therefore, we believe that further extensive studies supporting our results will contribute to the find an effective method to treat and prevent this disease and support the clinical managements.

Keywords: OHSS, VEGF, Calcium dobesilate

\section{P-97}

Morphological study of the muscle content in persian leopard (Panthera pardus saxicolor) esophagus

Sadeghinezhad $\mathrm{J}^{*}$, Ashgriz $\mathrm{N}^{*}$, Babaei $\mathrm{M}^{*}$, Hamid $\mathrm{N}^{*}$, Memarian I**

*Department of Basic Sciences, Faculty of Veterinary Medicine, University of Tehran, Tehran, Iran; **Pardisan Park, Tehra, Iran

The esophagus shows different morphological features especially for the muscle content in different species. The Persian leopard is a carnivore belonging to the felidae family and constitutes the largest leopard subspecies. We carried out this study with the aim of providing information on the morphological features of the muscle fibers of the esophagus in Persian leopard. Two Persian leopards with severe injuries were used in this investigation. After euthanizing, esophagus of each animal was removed. The samples were collected and fixed in $10 \%$ neutral buffered formalin. The tissues were routinely processed for light microscopy and embedded in paraffin. The paraf?n-embedded blocks were cut into $6 \mu \mathrm{m}$ sections and stained using hematoxylin and eosin (H\&E) and Masson's trichrome The wall of the Persian leopard esophagus was composed of the typical layers seen in other species. Muscularis mucosa which was a smooth muscle appeared from the caudal half of the esophagus. Tunica muscularis with internal circular and external longitudinal muscle layers which were separated with connective tissue have been seen in the beginning of esophagus. From the beginning of the second half of the esophagus, smooth muscle fibers were observed between the internal circular striated muscles and then gradually increased to completely constituted the inner muscle layer in four fifth part of the esophagus. Finally in gastroesophageal junction, the longitudinal muscle was also substituted with smooth muscle and the smooth muscle of the esophagus continued to the stomach. This study which is the first description of the histological features of the esophagus of the leopard draws attention to the different morphological profiles of esophagus among different species.

\section{P-98}

\section{The effects of omega-3 on the renal parenchyma unilateral ureteral obstruction in rat}

Firat $\mathrm{F}^{* *}$, Kesici $\mathrm{H}^{*}$, Karaca $\mathrm{ZI}^{*}$, Kesici E***, Karaca MO****, Aslan $\mathrm{H}^{*}$

*Department of Histology and Embryology, Faculty of Medicine, Gaziosmanpasa University, Tokat, Turkey; ${ }^{* *}$ Department of Urology, Tokat State Hospital, Tokat, Turkey; ***Department of Physical Medicine and Rehabilitation, Faculty of Medicine, Gaziosmanpasa

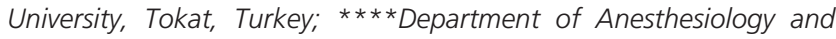
Reanimation, Niksar State Hospital, Tokat, Turkey 
Objectives: Partial Unilateral Ureteral Obstruction (PUUO), is a common clinical urology practice. Ureteral obstruction, which may occur due to compression of the ureter from inside or outside. In adults, stones, ureteropelvic junction obstruction and ureteral strictures are the most common cause PUUO. In our study, created as a result of unilateral ureteral obstruction in rats aimed to investigate the effect of omega 3 on oxidative damage and apoptosis in the systemic circulation and renal tissue.

Methods: After local ethics committee approval study, weights ranging from 280-330 g 5-6 months total of 24 male Wistar albino rats were used. The rats were divided into 3 groups. The first group includes nephrectomy was performed in the control group. The second group consisted of partial ureteral obstruction group held for 28 days. The third group, formed after partial urethral obstruction, is the group given the omega- 3 in $5 \mathrm{ml}$ dose orally for 28 days. Once created ureteral obstruction, nephrectomy specimen and systemic serum from malondialdehyde (MDA), superoxide dismutase (SOD), carbonic protein (PC) and nitric oxide (NO) levels were evaluated. Furthermore, histological, cell counts in kidney tubular and glomerular diameter, was evaluated in terms of parameters such as apoptosis rate.

Results: It was detected that serum SOD, MDA and Creatinine levels were significantly higher in group 2 when compared to the control group $(\mathrm{p}<0.05)$. It was observed that omega 3 decreased the SOD, MDA and Creatinine levels closer to the level of the control group. Histopathological damage was not seen in Group 1, and bax, bcl2 and caspase3 immunoreactivity was found to be minimal. Histopathological damage was seen in the Group2 and Group3, and bax, bcl2 and caspase 3 immunoreactivity was found to be higher.

Conclusion: This study showed us that an anti-inflammatory, antioxidant, anti-apoptotic agents as omega-3 fame kidney damage that occurs due to partial ureteral obstruction can be used in clinical practice for the prevention of thought.

\section{P-99}

\section{Evaluation of adrenal glands volume in acromegalic} patients: a stereologic study

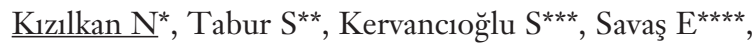
Korkmaz $\mathrm{H}^{* *}$, Erbağg1 $\mathrm{H}^{* * * * *}$

*Department of Anatomy, Faculty of Medicine, Gaziantep University, Gaziantep, Turkey; **Department of Endocrinology, Faculty of Medicine, Gaziantep University, Gaziantep, Turkey; ***Department of Radiology, Faculty of Medicine, Gaziantep University, Gaziantep,

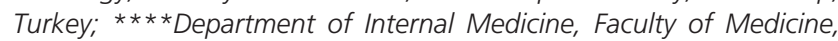
Gaziantep University, Gaziantep, Turkey;*****Department of Anatomy, Faculty of Medicine, Zirve University, Gaziantep, Turkey

It is known that visseromegaly is a common clinical feature of acromegali due to the growth-promoting chronic effects of growth hormone $(\mathrm{GH})$ on many tissues. It is also acknowledged that growth hormone-insulin- like growth factor (IGF) system plays an important role in the regulation of adrenocortical function. The aim of this study was to evaluate adrenal gland volume in acromegalic patients. Nine acromegalic patients (4 males, 5 females) were included in this study. For control group nine normal patients( 6 males, 3 females) were included. All of adrenal gland volume were measured with computerized axial tomography (CT). Measurements were made in $1.25 \mathrm{~mm}$ CT sections of adrenal gland with Cavelier Principle (as stereologic method). To compare the two implementation groups, for continuous variables Mann Whitney U test (for non-normal data), was used. Mean and standard deviations were given as descriptive statistics. All analyses were performed in SPSS for windows version 22.0. A two sided $\mathrm{P}$ value $<0.05$ was defined as statistically significant. Mean values; in acromegalic patients: $17.95 \mathrm{~cm} 3( \pm 5.78)$, in control group $22.56 \mathrm{~cm}^{3}( \pm 11.36)$ There was no significant difference between groups according to volume of adrenal glands $(\mathrm{p}=0.372)$. The study will continue by adding new acromegalic patients. Cortisol and IGF levels of the patients will be mesured for evaluation of the adrenal function.

\section{P-100 \\ Investigation of effect of BQ123 on testis in rats after ischemia-reperfusion injury}

Karaca $\mathrm{ZI}^{*}$, Kesici $\mathrm{H}^{*}$, Karaca $\mathrm{MO}^{* *}$, Kesici E**, Erdemir $\mathrm{F}^{* * * *}$, Aslan $\mathrm{H}^{*}$

*Department of Histology and Embryology, Faculty of Medicine, Gaziosmanpaşa University, Tokat, Turkey; **Department of Anesthesiology and Reanimation, Niksar State Hospital, Tokat, Turkey; ***Department of Physical Medicine and Rehabilitation, Faculty of

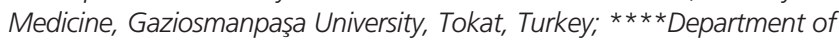
Urology, Faculty of Medicine, Gaziosmanpasa University, Tokat, Turkey

Objectives: Testicular torsion occurs more commonly during puberty newborn and is an emergency situation. As a result of this event, and the time depending on the degree of torsion from ischemia progressing to necrosis, histopathological changes occur in testis. Torsion, constituting while ischemia period, detorsion, constitutes reperfusion period. In our study, we aimed to evaluate the effectiveness of BQ-123 on the tissue damage after testicular torsion which is a very common pathological condition especially in children. Since our research conducted using stereological technique, has a different significance.

Methods: In our study, male rats into three groups consisting of five rats (Group I, Group II, Group III) were separated. Spermaticus funiculus in rats' has been reached with a cut left inguinoscrotal incision. After the separation of the left spermatic cord and testis in first and second group, the left testis was rotated 720 degrees for 120 minutes. A single dose BQ-123 $(1.6 \mathrm{mg} / \mathrm{kg}$ ) were given tail vein 30 minutes before detorsion to the rats of third group. One week later the rats were sacrificed. Spermatogonia and spermatocyte cells which is in the seminiferous tubules of rats, were examined by optical fractionation method.

Results: When we compared spermatogonia and spermatocytes cells in testicular tissue of groups, the average number of cells in the second group were found to be more than the first group and 
this was considered statistically significant. Group III and Group I in comparison to the average number of cells in Group I and found less was considered significant $(\mathrm{p}<0.001)$. Group III and Group II compared to the average number of cells in Group III in cell number was higher but not significantly.

Conclusion: Consequently, testicular torsion applications with BQ-123 in the seminiferous tubules, in the spermatogonia and spermatocytes, and both cells have been shown to reduce damage both.

Keywords: Testicular torsion, BQ-123, ET antagonist, optical fractionator

\section{P-101}

\section{Morphological and topographical anatomy of nutrient foramina in human patella bones and their surgical importance}

\section{Oğuz N, Gürçay S, Önder M}

Department of Anatomy, Faculty of Medicine Akdeniz University, Antalya, Turkey

Objectives: To study the morphology and topography of nutrient foramina and to determine the foraminal index of the Patella bones.

Methods: In our study, 50 os patella obtained from the bone collection of Department of Anatomy, Akdeniz University, Faculty of Medicine were evaluated. The bones had gross morphologic deformities were excluded from the study. The dominant NF determined and the bones were macroscopically observed under the stereomicroscope according to the number, location and direction of the NF. The total patella length (TL; maximum distance from proximal to distal end of the bone) and the distance from the proximal end of the bone to the NF (DNF) were measured.

Results: According to measurements of TL and DNF at left and right patella averages were $3.97 \pm 0.24 / 3.78 \pm 0.49$ and $2.12 \pm$ $0.50 / 9.089 \pm 0.48$ respectively. The mean foraminal index for the patella bones were $57.25 \%$ for the right patella, whereas the left patella of the foraminal index were $\% 52.42$.

Conclusion: The study has provided additional information on the morphology and topography of nutrient foramina in patella bone. The knowledge about these foramina is useful in certain surgical procedures to preserve the circulation.

Keywords: Nutrient foramina, foraminal index, morphology, patella

\section{P-102}

\section{Gastrosplenic trunk and anatomic variations of the} hepatic arterial system

Fazlığulları $Z^{*}$, Gün $C^{*}$, Nayman A**, Koplay $M^{* *}$, Uysal İं*

*Department of Anatomy, Faculty of Medicine, Selçuk University Konya, Turkey; ${ }^{* *}$ Department of Radiology, Faculty of Medicine, Selçuk University, Konya, Turkey
Objectives: The celiac trunk and superior mesenteric artery are the two widest vessels arising from the ventral aorta. The celiac trunk then branches into the left gastric artery, the common hepatic artery and the splenic artery. Four primitive ventral branches which are associated with each other branch from the abdominal aorta during embryogenesis. Developmental variations of the celiac trunk and superior mesenteric artery appear as a result of over growth or regression of these primitive branches during development.

Case Report: A celiac trunk variation was detected in CT angiography evaluation of a 65 -year old male patient who referred our hospital because of abdominal pain. Common hepatic artery branches from the abdominal aorta by oneself in the case. Left gastric artery and splenic artery branch from the abdominal aorta in a single root (gastrosplenic trunk) concurrently. In addition to these variations, an accessory right hepatic artery branches from the superior mesenteric artery. In the sagittal section measurments; a gastrosplenic trunk originated $43.44 \mathrm{~mm}$ below the hiatus aorticus and a common hepatic artery originated from aorta just in the right side of this trunk. Superior mesenteric artery branches from the abdominal aorta as $45.13 \mathrm{~mm}$ below hiatus. In the coronal section, diameter of common hepatic artery was measured as $3.21 \mathrm{~mm}$; length and diameter of gastrosplenic trunk were measured as $11.28 \mathrm{~mm}$ and $4.20 \mathrm{~mm}$, respectively.

Conclusion: Variations of the celiac trunk are reported within a range of $11 \%$ and $37.5 \%$. Gastrosplenic trunk variation is observed approximately by $7 \%$ whereas the most common variation of the hepatic artery is branching of the hepatic artery from superior mesenteric artery. Recognition of anatomic variations of the celiac trunk and hepatic artery is important for liver transplantation, laparoscopic surgery, radiological abdominal procedures and clinical conditions such as penetrating abdominal traumas.

Keywords: Celiac trunk, gastrosplenic trunk, common hepatic artery, CT angiography, variation

\section{P-103}

\section{Brain tissue plasticity in high fat diet fed and fasting rats}

Yay A*, Gölgeli A**, Yalçın B* ${ }^{*}$ Yazgan K** Keklik E**, Başaran $\mathrm{KE}^{* *}$, Hatipoğlu $\mathrm{N}^{* * *}$

*Department of Histology, Faculty of Medicine, Erciyes University, Kayseri, Turkey; ${ }^{* *}$ Department of Physiology, Faculty of Medicine, Erciyes University, Kayseri, Turkey; $* * *$ Department of Pediatric Endocrinology, Faculty of Medicine, Erciyes University, Kayseri, Turkey

In this study, we investigated changes of some cell groups especially wall and the base of the 3 rd ventricle which are suggested that associated with hypothalamus hunger and satiety centers depending on the short- and long-term alteration in metabolism in rats. This work was supported by TUBITAK Project No: 114S009. In the study, 36 male Wistar albino rats were used. The rats fed with high-fat diet $(\mathrm{n}=12)$, the rats formed starvation 
S168 XXIV International Symposium on Morphological Sciences, 2nd-6th September, 2015, Istanbul, Turkey

models $(\mathrm{n}=12)$ and the control grup $(\mathrm{n}=12)$ normal feding during 14 weeks. At the end of the study, brain tissues removed from rats which are decipitated divided into two hemisphere and routine tissue follow-up were done. Obtained each tissue sections were stained with hematoxylin-eosin. To determine of immunoreactivity intensity of MAP-2 and vimentin, immunohistochemical staining method and immunofluoresan method for nestin immunofluoresan immunoreactivity was performed in the brain tissues. In the both cases which changes of the metabolism, a high-fat diet and starvation, MAP-2, vimentin and nestin immunoreactivity intensity was increased compared to the control group in the section of the choroid plexus. The immunoreactivity intensity of MAP-2 and vimentin significantly increased in the high-fat diet fed group compared to the control group, while nestin immunoreactivity was not significantly different. MAP-2, vimentin and nestin immunoreactivity intensity was higher than other groups in the starvation group $(\mathrm{p}<0.001)$. Changes in metabolizm caused to increase of MAP-2 and vimentin, particularly in starvation models. Nestin is used as an embriyonic stem cell marker and adult neurogenesis marker. İncrease in expression of this protein may be a sign to neurogenesis in chroid plexus. Although high-fat diet increase the expression of nestin, this is not significant as a starvation model. This result reinforces to understand of the relation between high-fat diet and neurogenesis.

\section{P-104}

\section{The ameliorating effect of Saint John's wort on 5-fluorouracilinduced ileum and colon}

\section{Bülbül N* , Aykaç A** , Cetinel S*, Şehirli A.O***}

*Department of Histology and Embryology, Faculty of Medicine, Marmara University, Istanbul, Turkey; ${ }^{*}$ Department of Biophysic, Faculty of Medicine, Near East University, Lefkosia, Turkish Republic of Northern Cyprus; ***Department of Pharmacology, Faculty of Pharmacy, Marmara University, İstanbul, Turkey

Introduction: 5-Fluorouracil is a commonly used chemotherapeutic agent for cancer treatment. However, it has some side effects including ileitis and colitis. St. John's Wort (SJW) is an herbal medicine that is used for therapy of mild depression mainly besides it has antioxidant, anti-inflammatory, and antibacterial properties.

Objectives: The aim of this study is to investigate the possible preventive effect of Saint John's Wort against ileitis and colitis which are induced by 5 -Fluorouracil.

Methods: Female Sprague Dawley rats (220-300 g) were used in the study and three experimental groups $(n=5)$ : Group (1) -saline treated control group, Group (2) 5-fluorouracil (5-FU) treated group, and Group (3) 5-FU + Saint John's Wort treated group. Following $150 \mathrm{mg} / \mathrm{kg}$ dose of 5 -FU, either saline or Saint John's Wort $(300 \mathrm{mg} / \mathrm{kg}$ ) were administered in each day during 4 days. After decapitation, ileum and colon tissues of the intestine were removed for histopathology. The ileum and colon tissues were fixed in $10 \%$ formaldehyde, then processed routinely for light microscope. Apoptotic injury (cas-3,cas-9, bcl 2/bax) was shown via Western Blot method. Data were analyzed statistically.

Results: In the colon and ileum tissues, the control groups demonstrated regular epithelial and glandular morphology with abundance of goblet cells. In the colon tissue, 5-FU treatment degenerated the glands prominently and had led to epithelial disorganization along with a high degree of accumulation of leukocytes in lamina propria. The SJW treatment appeared to have reduced the degeneration of glands and concentration of leukocytes. In the ileum, there was degeneration with severe desquamation in the epithelium, and accumulation of leukocytes in the lamina propria, SJW treatment led to a moderate regeneration in the epithelium and glands. Western Blot method results confirmed histology.

Conclusion: Treatment with SJW ameliorated the tissue degeneration by reducing the glandular hyperplasia and congestion besides epithelial reorganization.

\section{P-105}

Effects of the monosodium L-glutamate BMI and visceral organs in male and female rats

Yazgan K**, Gölgeli A**, Yalçın B*

*Department of Histology and Embryology, Faculty of Medicine, Erciyes University, Kayseri, Turkey; ** Department of Physiology, Faculty of Medicine, Erciyes University, Kayseri, Turkey

The prepared food in the most commonly used food additive, monosodium L-glutamate (MSG) is thought to cause obesity by increasing the desire to eat. Body mass index (BMI), obesity is the most frequently used method of identification. BMI = weight $(\mathrm{kg}) /$ height $\left(\mathrm{m}^{2}\right)$ is calculated by formula. Cholesterol and triglycerides in obesity can lead to increased liver damage. In our study, long-term use of monosodium L-glutamate in male rats $\mathrm{BMI}$ and visceral organs (heart, liver, kidney) were investigated in weight in female and male rats. Wistar albino adult rats were used in this study. The control group and MSG in male and female was formed four groups including 10 animals. MSG is added to drinking water provided take for 12 weeks, in a week three days. MSG calculated joined 0,5 liters drinking water $250 \mathrm{mg} / \mathrm{kg}$ will have based total weight animals in cage. Study of body weight before and at the end of the experimental group were measured heights, intracardiac blood glucose received, triglycerides and cholesterol. The heart, liver and kidney weights were weighed abdominal opening. Data were analyzed with the Mann- Whitney-U test IBM statistic program. The $\mathrm{BMI}$ values were significantly reduced by the use of MSG in both sexes at the end $(\mathrm{p}<0.05)$. When were evaluated between body weight and liver weight was observed the groups was significantly reduced in MSG groups $(\mathrm{p}<0.05)$. Blood chemistry analysis increased glucose and cholesterol levels in MSG group and the male MSG group is significantly higher than female MSG group $(\mathrm{p}<0.05)$. Long time using did not cause obesity in MSG consumption in drinking water. But 
the increase in the reduction of cholesterol levels and liver weight in rats suggests that MSG can cause liver damage. This work was supported project No. TYL-2014-5462 by Erciyes University Research Fund.

\section{P-106}

\section{Morphometric investigation of the orbitofrontal cortex}

\section{Güner N, Kafa İM}

Department of Anatomy, Faculty of Medicine, Uludag University, Bursa, Turkey

The frontal lobe is one of the main four lobes of the brain of the mammalians. The orbitofrontal cortex, which is located ventrally to aspect of thee frontal lobes, is involved in the cognitive processing of decision-making and it is a key system in the regulation of emotional, behavioral, endocrine, and innate immunological responses to stress. Thus, we aimed to investigate this important brain region morphologically. Twenty six adult, male, fixed brain hemispheres were used in this study, which are obtained from the brain collection of Anatomy Department of the Uludag University. We measured twelve parameters, including the length of the frontal fissure, length of the orbitofrontal cortex, width of orbitofrontal cortex, length of the olfactory sulcus, length of the gyrus rectus, width of the gyrus rectus and other important and predefined areas. We also investigated all sulci, which are located in the orbitofrontal region limits in the terms of length, ramification and varieties. Cerebral hemispheres were photographed from the ventral aspect digitally and images transferred to computer. Measurements were performed on these digital images using ImageJ software after the carefully calibration which is done for the accuracy and precision of the study. Then, we compared of right and left cerebral hemispheres for acquired measurements. Our results showed that no significant differences between the right and left hemispheres, which are indicating that the orbitofrontal cortices are mostly symmetrical between right and left sides. We believe that these findings including the sulcal features may contribute to our understanding regarding to the morphological features of the orbitofrontal cortex.

\section{P-107}

\section{The effect of estrogen treatment on eccentric exercise-induced damage in rat kidneys}

Gerek $Z^{* *}$ Malkoç I* , Parlak SN***

*Department of Anatomy, Faculty of Medicine, Atatürk University, Erzurum, Turkey; **Department of Physical Education and Sports Sciences, Physical Education and Sport Academy, Atatürk University, Erzurum, Turkey; ***Department of Embryology and Histology, Faculty of Medicine, Atatürk University, Erzurum, Turkey

Objectives: The aim of this study was to examine the effects of anabolic steroids on eccentric exercise-induced kidney tissue, using biochemical and histological methods.
Methods: Sprague Dawley male rats $(n=36)$ were equally divided into the estrogen (17-, Estradiol, $10 \mathrm{mg} / \mathrm{kg}$ per day)induced and nonestrogen-induced groups. Both groups were subdivided into as rest groups $(n=6)$, and as exposed to the eccentric exercise groups $(n=12)$ that were also subdivided into decapitated one hour $(n=6)$ and 48 hour $(n=6)$ groups after eccentric exercise.

Results: Histopathological effects of estrogen treatment on eccentric exercise-induced rat kidney tissues were seen. The histopathological results revealed that hemorrhage, endothelium-dependent vasodilatations and degenerative changes in proximal tubules, glomeruli, and connective tissue in the estrogen-given and estrogen-given plus eccentric exercise groups. Distal tubule degenerations and dilatations with peritubular capillary dilatations and polymorphonuclear leucocyte (PMNL) infiltrations to connective tissue in eccentric exercise and eccentric exercise plus estrogen-given groups were detected but also it was noticeable decreases in PMNL infiltrations in estrogen-given groups. Biochemical results showed oxidative stress in experimental groups except Control group.

Conclusion: It has been concluded that estrogen could not have been effective for preventing eccentric exercise-induced kidney damage and might have toxic effects on kidney tissues on clinical practice however anti-inflammatory effect on kidney tissue detected. Further investigation is required.

Keywords: Eccentric exercise, estrogen, kidney, rat

\section{P-108}

\section{JNK and IL-6 interaction in diabetic rat testes}

Dönmez YB $^{*, * *}$, Kizilay $G^{* * *}$, Metin MS**** Kara $M^{* * * * *}$

*Department of Histology and Embryology, Faculty of Medicine, Namık Kemal University, Tekirdağ, Turkey; **Department of Histology and Embryology, Faculty of Medicine, Namik Kemal University, Tekirdağ, Turkey; ***Department of Histology and Embryology, Faculty of Medicine, Trakya University, Edirne, Turkey; ****Health Services Vocational College, Trakya University, Edirne, Turkey; $* * * * *$ Department of Histology and Embryology, Faculty of Medicine, Yüzüncü Yıl University, Van, Turkey

Introduction: Infertility is a widespread complication in diabetic men. The increase of c-Jun N-terminal kinase (JNK) levels leads to release various cytokines such as IL-6 and thus induced apoptosis in the diabetic testes.

Objectives: It is aimed to reveal the interaction between IL-6 which synergistically interacts with hyperglycemia and with/ without inhibition of JNK that involved in diabetic male fertility.

Methods: Forty Sprague-Dawley male rats were divided into; control $(\mathrm{n}=8)$, second group $(60 \mathrm{mg} / \mathrm{kg}$ streptozotocin, 15 days; $\mathrm{n}=8)$, third group (60 mg/kg streptozotocin,30 days; $\mathrm{n}=8)$, fourth group $(60 \mathrm{mg} / \mathrm{kg}$ streptozotocin-15 mg/kg SP600125,15 days; $\mathrm{n}=8$ ) and fifth group (60 $\mathrm{mg} / \mathrm{kg}$ streptozotocin $-15 \mathrm{mg} / \mathrm{kg}$ SP600125,30 days; $\mathrm{n}=8$ ).

Results: The Johnsen scores of diabetic groups decreased significantly when compared to control $(\mathrm{p}<0.001)$. In pairwise 
comparison of third and fifth groups, a significant increase was shown in the fifth group ( $\mathrm{p}=0.01)$. Regarding to apoptotic tubules and apoptotic cells indices; there were significant increase in second, third and forth groups as compared with control for second and third groups $(\mathrm{p}=0.021, \mathrm{p}=0.020$; for forth group $\mathrm{p}=0.043, \mathrm{p}=0.020$, respectively). Whereas, significant decreased was shown in the indices of apoptotic tubules $(\mathrm{p}=0.021)$ and cells $(\mathrm{p}=0.021)$ in fifth group compared to third group. When phospho-JNK immunoreactivity results of all groups were analyzed in reference to control group; it was revealed an increase in the second and third groups $(\mathrm{p}=0.003$, $\mathrm{p}=0.01$; respectively) but there was no difference in forth and fifth groups $(\mathrm{p}=0.248, \mathrm{P}=0,141$; respectively). The IL-6 immunoreactivity was higher in diabetic groups as compared to control group $(\mathrm{p}=0.006, \mathrm{P}=0.004, \mathrm{p}=0.010$ ve $\mathrm{p}=0.004$, respectively), however, in pairwise comparison of third and fifth groups, it was significantly lower in the fifth group $(\mathrm{p}=0.010)$.

Conclusion: We concluded that the interaction between IL-6 and JNK will be revealed more clearly by using various doses and time protocols of SP600125 in diabetic testis tissues, and thereby, it may be contribute to the development of new treatment protocols related to diabetic male infertility.

Keywords: Diabetes mellitus, testes, SP600125

\section{P-109}

Evaluation of the diameters of common carotid artery and internal carotid artery by angiography

Karacan $\mathrm{K}^{*}$, Akcaalan $\mathrm{M}^{*}$, Karacan $\mathrm{A}^{* *}$, Acar BA***,

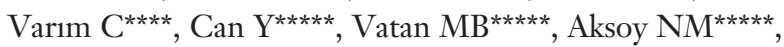
Mansiz $\mathrm{S}^{* *}$, Akdemir $\mathrm{R}^{* * * * *}$

*Department of Anatomy, Faculty of Medicine, Sakarya University, Sakarya, Turkey; **Department of Radiology, Faculty of Medicine, Sakarya University, Sakarya, Turkey; $* * \star$ Department of Neurology, Faculty of Medicine, Sakarya University, Sakarya, Turkey; ***Department of Internal Medicine, Faculty of Medicine, Sakarya University, Sakarya, Turkey; $* * * * *$ Department of Cardiology, Faculty of Medicine, Sakarya University, Sakarya, Turkey

Objectives: Common carotid artery (CCA) is the largest artery located in the neck which originates from trunchus brachiocephalicus on the right side and aorta directly on the left side and arises up trigonum caroticum. CCA is divided into 2 branches upper edge level of cartilago thyroidea (level of $\mathrm{C} 4$ vertebra). The bifurcation of the CCA has tendency to the development of atherosclerotic lesions. Age, endothelial dysfunction, endothelial shear stress, and genetic profile, smoking habit, diabetes mellitus, hypertension, dyslipidemia and obesity are risk factors of atherosclerosis. Geometric parameters such as branch angles, curvature and diameters are risk factors for atherosclerosis development. The plaques can demonstrate intramural hemorrhage, which may bring about thromboemboli, concluding in transient ischemic attacks and/or stroke. The aim of this study is to evaluate the diameter of CCA and internal carotid artery (ICA) by angiography.
Methods: In our study, conventional angiography images of 284 patients (182 males, 102 females) were retrospectively evaluated in terms of diameters of CCA and ICA in Sakarya University Training Research Hospital Department of Cardiology.

Results: While diameter of right CCA was $7.13 \pm 1.18 \mathrm{~mm}$, diameters of left CCA was $6.93 \pm 1.14 \mathrm{~mm}$. Also, diameters of right ICA and left ICA were $5.08 \pm 0.98 \mathrm{~mm}, 5.09 \pm 1.01 \mathrm{~mm}$, respectively. When diameters were compared in term of gender, difference wasn't significant.

Conclusion: Diameter of CCA and ICA should be taken into consideration in medical material production such as stent, balloon.

\section{P-110}

\section{In-vivo examination of the relationship between estrogen receptors and microtubule dynamics}

Karameşe $\mathrm{SA}^{* *}$, Karameşe $\mathrm{M}^{* * *}$, Karakuş $\mathrm{E}^{* * * *}$, Malkoç I', Bünyami $\ddot{U}^{* * * * *}$, Cemal $\mathrm{G}^{* * * * * *}$

*Department of Anatomy, Faculty of Medicine, Atatürk University, Erzurum, Turkey; ${ }^{* *}$ Department of Histology and Embryology, Faculty of Medicine, Kafkas University, Kars, Turkey; $* * *$ Department of Microbiology, Faculty of Medicine, Kafkas University, Kars, Turkey; $* * * *$ Department of Pharmacology and Toxicology, Faculty of Veterinary, Atatürk University, Erzurum, Turkey; $* * * * \star$ Department of Histology and Embryology, Faculty of Medicine, Atatürk University, Erzurum, Turkey; $* * * * * \star$ Department of Pathology, Faculty of Medicine, Atatürk University, Erzurum, Turkey

Introduction: Estrogen is one of the most important regulators of neuronal function. There is a broad consensus that loss of estrogen is associated with neurodegeneration in the hippocampus that leads cognitive impairments. Hematopoietic$\mathrm{Pbx}$-Interaction-Protein (HPIP) is a novel scaffolding protein interacts with estrogen receptors and microtubule. In this study, it was aimed to explain the possible mechanism of neurodegeneration, determine the presence and the possible role of HPIP on hippocampal neurons and examine the relationship between estrogen receptors and microtubule damage which was seen on the rat brains that were on post-menopausal term.

Methods: A total of 80 female Wistar albino rats, 12 weeks old divided into 10 groups: Control, Control+17- $\beta$-estradiol, Control + Tamoxifen, Control + MAPK inhibitor, Control + PI3-K inhibitor, Ovariectomized, Ovariectomized $+17-\beta-$ estradiol, Ovariectomized + Tamoxifen, Ovariectomized + MAPK inhibitor, Ovariectomized + PI3-K inhibitor groups. Light and electron microscopic examinations were performed. Real- Time PCR method was used to determine the expression level of HPIP in experimental groups.

Results: Some interesting morphological changes in neuronal axons of hippocampus samples were detected in both light and electron microscopic examinations. Severe fluctuations and shrinkages in axons were detected in all ovariectomized groups. On the other hand, HPIP was first detected in neurons in all groups' hippocampal neurons with difference expression levels. 
Conclusion: A new focus on neurodegeneration in postmenopausal women may arise through the proof of that the HPIP protein can be found on hippocampal neurons. Future molecular and pharmacologic studies in this area should be performed to reduce the rate of cognitive symptoms resulting from hippocampal neurodegeneration.

Keywords: Axonal neurodegeneration, estrogen receptors, HPIP, microtubule, ovariectomy

\section{P-111}

The protective effects of beta-carotene against ischemia/reperfusion injury in rat ovarian tissue

Karameşe SA**, Toktay E*** Ünal D***, Selli ${ }^{* * *}$, Karameşe $M^{* * * *}$, Malkoç I*

*Department of Anatomy, Faculty of Medicine, Atatürk University, Erzurum, Turkey; **Department of Histology and Embryology, Faculty of Medicine, Kafkas University, Kars, Turkey; ${ }^{* *}$ Department of Histology and Embryology, Faculty of Medicine, Atatürk University, Erzurum, Turkey; $* * * *$ Department of Microbiology, Faculty of Medicine, Kafkas University, Kars, Turkey

Objectives: Beta-carotene, is a well-known antioxidant and precursor of Vitamin A, has a preventative role in oxidative damage process. Our aim was to investigate the possible preventive effects of beta-carotene on the oxidative damage occurred by experimentally ischemia and ischemia-reperfusion models in rat ovaries.

Methods: Atraumatic vascular clamps were used for 3 hours to induce ischemia (Group 2, 3, 4, 5, 6, 7) and the clamps was then removed to allow reperfusion for three hours (Group 3, 6, 7). Sham-operated rats (Group 1) underwent laparotomy without doing anything. RT- PCR was performed by detecting IL-1beta, IL-6 and iNOS expression levels. Histopathological (H\&E) and immunohistochemical staining (NF-k $\beta$ p65) methods was performed. Finally, SOD, GSH and MDA levels were detected in the current study.

Results: Dense hemorrhagic areas were observed in both in ischemia and ischemia/reperfusion groups whereas minimal hemorrhage was observed in treatment groups. Ischemia and ischemia/reperfusion groups was extremely observed immunoreactivity by NF-k $\beta$ p65 staining, this reactivity reduced after beta-carotene application. The expression of IL1-beta, IL-6 and iNOS in injury groups significantly increased, whereas a dose-dependent improvement was observed in treatment groups. Finally, MDA levels significantly increased and SOD and GSH level extremely reduced in injury groups whereas these values detected as too close to optimal values after the administration of beta- carotene.

Conclusion: As a result, we have shown by molecular and biochemical parameters that the protective effect of beta-carotene, which is a potent antioxidant, on ischemia-reperfusion model that was made by experimental ovarian.

Keywords: Beta-carotene, ischemia, reperfusion, ovary, rat

\section{P-112}

Tractography of the brain white matter tracts with DTI Studio method

Soysal H${ }^{*}$, Acer $\mathrm{N}^{* *}$, Öktem H*, Kürkcüoğlu A*, Pelin $\mathrm{C}^{*}$

*Department of Anatomy, Faculty of Medicine, Başkent University, Ankara, Turkey; ${ }^{*}$ Department of Anatomy, Faculty of Medicine, Erciyes University, Kayseri, Turkey

Objectives: Tractography based on diffusion tensor imaging (DTI) allows visualization of white matter tracts. In this study four major white matter tracts were reconstructed and evaluated using DTIStudio. The protocols were applied to a DTI database of adult normal subjects to study size and fractional anisotropy (FA) of four white matter tracts; Cortico-spinal tract (CST), forceps major (Fmajor), forceps minor (Fminor), superior longitudinal fasciculus (SLF).

Methods: MR images of 10 adults aged between 18-49 years were evaluated. Individuals who had any medical disorders had been excluded from the study. Using DTIStudio white matter tracts were determined by fiber tracking. Fiber size and FA values were evaluated.

Results: No significant hemispheric asymmetry related with FA values and average sizes of fibers (the number of pixels that contain the fibers multiplied by the nominal size of the pixel) was observed.

Conclusion: DTIStudio provides guidelines for DTI-based tract-specific quantification.

Keywords: Diffusion tensor imaging, DTIstudio, Tractography, White matter.

\section{P-113}

Morphometric analysis of the tibial plateau in Turkish population

Babacan S, Kafa I

Department of Anatomy, Faculty of Medicine, Uludağ University, Bursa, Turkey

Fractures, osteoarthritis or other sort of traumas or pathological conditions may deform the knee joint. One of the surgical treatments of such disorders is total or unicompartmental knee replacement. The prosthesis and the resected bone should match perfectly to have an accomplished outcome. The aim of current study is to demonstrate the morphometric anatomy of the tibial plateau and provide the Turkish population specific features. The study was conducted on 159 tibias. The tibial plateau were photographed digitally and transferred to computer. Sixteen different parameters related to the tibial plateau were measured. The measurements were obtained using ImageJ software and SPSS was used for the statistical analyses. Various differences revealed between the Byzantine and contemporary groups, right and left sides and between condyles. In descriptive statistics, eight of the measurements were statisti- 
cally significant (six parameters were greater in Byzantine; two were greater in contemporary groups). In comparison of right and left tibias, seven measurements were showed significance. Five measurements showed greater results in Byzantine period, and two in the contemporary period. In addition, one measurement was greater on medial condyle on the right side in contemporary period; four measurements on lateral condyle on the right side and three measurements on medial condyle on the left side were greater in Byzantine period. In comparison of the lateral and medial condyles' area, breadth and length were greater in medial condyles in contemporary period. In fact that, knee prosthesis techniques are designed based on anthropometric measurements especially obtained from the European and North American populations, it is necessary to accomplish population specific values regarding to the knee anatomy. We believe that our results are valuable for the surgical reconstructions or replacement procedures related to this region.

\section{P-114}

Caecum location in laboraty rats and mice: an anatomical and radiological study

Uysal M*, Gül SS ${ }^{* *}$, Karaman S***, Taş U*, Sapmaz HI*, Uysal $\mathrm{F}^{* * * *}$, Aytekin $\mathrm{K}^{* * * *}$, Tümer MK $\mathrm{MK}^{* * * * *}$

*Department of Anatomy, Faculty of Medicine, Gaziosmanpaşa University, Tokat, Turkey; **Department of Nuclear Medicine, Faculty of Medicine, Gaziosmanpaşa University, Tokat, Turkey; $* * *$ Department of Emergency Medicine, Faculty of Medicine, Gaziosmanpaşa University, Tokat, Turkey; $* * * \star$ Department of Physiology, Faculty of Medicine, Uludağ University, Bursa, Turkey; $\star * * * \star$ Department of Orthopaedics and Traumatology, Faculty of

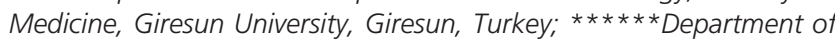
Oral and Maxillofacial Surgery, Faculty of Dentistry, Gaziosmanpaşa University, Tokat, Turkey

Objectives: Intraperitoneal (i.p.) injection is the mostly used parenteral administration way in rats and mice. Caecum is a big abdominal organ and for that reason it's prone to be pierced by pinpoint during i.p. injection. It's not clear whether caecum located in the right lower quadrant or left lower quadrant in the ventral cavity. For that reason, we try specify the localization of the caecum in rats and mice and thus revealing the most reliable localization for i.p. injection in rats and mice.

Methods: In this study, 200 Wistar albino rats and 100 $\mathrm{BALB} / \mathrm{C}$ mice have been used. The localization of the caecum has been specified by making the intra-abdominal organs visible right after the euthanasia and photographed with the help of digital photography machine. CT has been performed for 100 animals before euthanasia (50 rats and 50 mice). Both digital photography images and CT sections have been analyzed in terms of caecum morphology and localization.

Results: Caecum was highly on the left side both in rats and mice. It's less frequently monitoring in the right and middle side. Caecum was mostly in comma shape but it was round and $\mathrm{S}$ shape in some animals. The direction of rotation of caecum from the basis to apex was mostly counter clockwise.
Additionally, it's specified that apex usually been shown orientation toward the midline.

Conclusion: In the light of the findings of our study, caecum was mostly indicated in the left side in rats and mice and for that reason, it's understood that the most suitable localization for i.p. injection was right lower quadrant. Furthermore, when we compare the CT images and autopsy findings, it can be seen that caecum does not change location in the abdominal cavity in postmortem.

Keywords: Caecum, rat, mice, computed tomography, intraperitonel injection

\section{P-115 \\ Effects of modafinil on learning and memory in Wistar albino and WAG/Rij rats}

Özyurt B*, Çakıl D**, Uysal M${ }^{*}$, Aygün $\mathrm{H}^{* * *}$, Özsoy Ş**, Çiçek $M^{*}$

*Department of Anatomy, Faculty of Medicine, Gaziosmanpaşa University, Tokat, Turkey; ${ }^{*}$ Department of Physiology, Faculty of

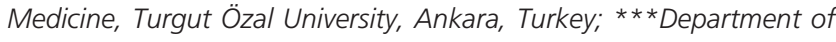
Physiology, Faculty of Medicine, Gaziosmanpaşa University, Tokat, Turkey

Modafinil is a drug used in narcolepsy and sleep-apnea syndrome. Although its mechanism of action is not fully clear, studies have shown modafinil increases glutamate release and inhibit the activity of GABAergic neurons. Childhood absence epilepsy (CAE) accounts for approximately $8 \%$ of epileptic patients among schoolaged children. Although CAE previously was considered as a benign syndrome, significant cognitive and behavioral disabilities have been reported in many of these children. The strain of genetic absence epilepsy rats from WAG/Rij provides a validated genetic model of human generalized idiopathic absence epilepsy. In this study, we aimed to investigate the effect of low and high dose modafinil on learning and spatial memory in Wistar albino and WAG/Rij strain rats. Forty-two rats were randomly divided into 6 groups: Wistar albino control group ( $\mathrm{n}=7)$, Wistar albino low dose (40 $\mathrm{mg})$ modafinil group, Wistar albino high dose $(120 \mathrm{mg})$ modafinil group $(n=7)$, WAG/Rij control group $(n=7)$, WAG/Rij low dose (40 mg) modafinil group $(\mathrm{n}=7)$, and WAG/Rij high dose $(120 \mathrm{mg})$ modafinil group $(\mathrm{n}=7)$. Rats were administered modafinil (40 mg and $120 \mathrm{mg}$; i.p.) 30 minutes before Morris water maze test. In the probe trial carried out on the 5th day of Morris water maze test, Wistar albino rats administered with $40 \mathrm{mg}$ modafinil were observed to spend more time in target quadrant $(\mathrm{p}<0.05)$. Whereas $120 \mathrm{mg}$ modafinil did not have a statistically significant effect on probe trial in both Wistar albino and WAG/Rij rats. These findings indicate low dose modafinil have significant improving effect on spatial memory in Wistar albino rats.

Keywords: Morris water maze, modafinil, absence epilepsy, WAG/Rij rat, spatial memory 


\section{P-116}

Immunohistochemical investigation of aquaporin 3 expression in meniscus tissue

$\underline{\text { Uysal M}}^{*}$, Sapmaz HI*, Ocaklı $\mathrm{S}^{* *}$, Bıçakçı $\mathrm{H}^{* * *}$, Açıkgöz R***, Taş U*

*Department of Anatomy, Faculty of Medicine, Gaziosmanpaşa University, Tokat, Turkey; **Department of Histology and Embryology, Faculty of Medicine, Gaziosmanpaşa University, Tokat, Turkey; *** Department of Anatomy, Faculty of Medicine, Şifa University, Izmir, Turkey

Objectives: Meniscus is a dynamic and flexible tissue that tolerates between the bone surfaces unconformity in the knee joint. Its structures and water content gives flexibility to the meniscus. Aquaporins are the water channel proteins expressed in the several cells and tissues that serve as a gateway for water and small solution materials. Aquaporin 1 (AQP1) has been studied in meniscus tissue. However, we cannot find any studies regarding the aquaporin 3 (AQP3) in the literature. The aim of this study is to examine the expression of AQP 3 that is belongs to the aquaglyceroporin group in rat meniscus tissue.

Methods: Seven Wistar albino rats were used in this study. The rats were sacrificed, and knee joints were removed. Tissues were embedded in paraffin after routine histological procedures AQP1 and AQP3 were stained immunohistochemically. Also hematoxylin- eosin (H\&E) were stained and all sections were examined under the light microscope.

Results: The AQP1 and AQP3 expression was shown with immunohistochemistry in meniscus tissue of rats. Staining was more intense in the periphery of the meniscus.

Conclusion: The expression of AQP3 has been shown in the rat meniscus tissue. It is important to show AQP3 expression besides the existence of AQP1 in the meniscus tissue for explaining the pathophysiology of the meniscus degeneration.

Keywords: Rat, meniscus, immunohistochemistry, aquaporin 1 , aquaporin 3

\section{P-117}

Investigation of the spinal perineural (Tarlov) cyst incident by magnetic resonance imaging method

Taş $\mathrm{AD}^{*}$, Uysal $\mathrm{M}^{*}$, Aytekin $\mathrm{K}^{* *}$, Altunkaş $\mathrm{A}^{* * *}$, Sapmaz HI*, Taş U*, Özyurt B*

*Department of Anatomy, Faculty of Medicine, Gaziosmanpaşa University, Tokat, Turkey; **Department of Orthopedics and Traumatology, Faculty of Medicine, Giresun University, Giresun, Turkey; ***Department of Radiology, Faculty of Medicine, Gaziosmanpaşa University, Tokat, Turkey

Objectives: Perineural cyst, also known as Tarlov cyst, rarely gives symptoms by pressuring on the nerve. They are generally determined incidentally, and usually found in the sacral section. The studies showing perineural cyst incidents in our country are few and most of them are case reports. The objec- tive of this study is to investigation of the spinal perineural cyst incident in Turkish population.

Methods: This study has been carried out by examining the magnetic resonance imaging (MRI) reports of the 11781 patient that had a cervical, thoracic, lomber, and sacral MRI.

Results: There are 188 (1.5\%) perineural cysts have been observed in 11781 patients in total. The prevalence of the cyst has been respectively $7 \%$ in sacral, $5.3 \%$ in thoracic, $0.6 \%$ in cervical and $0.2 \%$ in lomber. The cyst has been observed more in S2 and T1 spinal nerve roots. While the size of perineural cyst was $10.25 \mathrm{~mm}$ average for all patients, it was $9.80 \mathrm{~mm}$ in male patients and $10.43 \mathrm{~mm}$ in female patients. Average size of cyst was the highest in sacral nerve $(12.14 \mathrm{~mm})$ and was lowest in cervical nerve $(6.31 \mathrm{~mm})$.

Conclusion: The findings in our study were consistent with the literature in general. The findings may contribute to better understanding of the location and frequency of Tarlov cysts.

Keywords: Magnetic resonance imaging, Tarlov cyst, perineural cyst, spinal nerve

\section{P-118}

\section{The comparative effects of low and high doses of melatonin on spatial memory in Wistar albino and WAG/Rij rats}

Özyurt B, Uysal M, Çiçek M, Sapmaz HI, Taş U

Department of Anatomy, Faculty of Medicine, Gaziosmanpaşa University, Tokat, Turkey

Absence epilepsy is sudden onset childhood age epilepsy characterized with loss or alteration in consciousness. Although regarded as a benign condition in terms of cognitive functions and behavioral properties, increasing number of studies suggest significant differences in that respect. WAG/Rij strain rats are genetical models with epilepsy which are raised inbred for animal studies of absence epilepsy, and provide important data about the pathophysiology of absence epilepsy. In this study, we aimed to investigate the effect of low and high dose melatonin on learning and spatial memory in Wistar albino and WAG/Rij strain rats. Fortytwo rats were randomly divided into 6 groups: Wistar albino control group ( $\mathrm{n}=7)$, Wistar albino low dose $(1 \mathrm{mg})$ melatonin group, Wistar albino high dose $(10 \mathrm{mg})$ melatonin group $(\mathrm{n}=7)$, WAG/Rij control group (n=7), WAG/Rij low dose (1 mg) melatonin group $(\mathrm{n}=7)$, and WAG/Rij high dose $(10 \mathrm{mg})$ melatonin group $(\mathrm{n}=7)$. Rats were administered melatonin $(1 \mathrm{mg}$ and $10 \mathrm{mg}$; i.p.) 30 minutes before Morris water maze test. In the probe trial carried out on the fifth day of Morris water maze test, Wistar albino rats administered with $1 \mathrm{mg}$ melatonin were observed to spend more time in target quadrant $(\mathrm{p}<0.05)$. Whereas $10 \mathrm{mg}$ melatonin did not have a statistically significant effect on probe trial in both Wistar albino and WAG/Rij rats. These findings indicate low dose melatonin have significant improving effect on learning and spatial memory in Wistar albino rats.

Keywords: Morris water maze, melatonin, absence epilepsy, WAG/Rij rat, spatial memory 


\section{P-119}

The effect of septal deviation that affects the palatum durum on the maxillary sinus volume

Sapmaz E* Kavaklı A** Sapmaz HI ${ }^{* * *}$, Ögetürk $M^{* *}$

*Department of Otorhinolaryngology, Faculty of Medicine, Gaziosmanpaşa University, Tokat, Turkey; **Department of Anatomy, Faculty of Medicine, Firat University, Elazığ, Turkey; ${ }^{* * *}$ Department of Anatomy, Faculty of Medicine, Gaziosmanpaşa University, Tokat, Turkey

Objectives: To research the effect of septal deviation that causes angling in the palatum durum on the maxillary sinus volume.

Methods: Paranasal sinus tomography taken in the coronal plan of the 1568 patients between the age of 18 and 60 has been examined. In total, 402 tomographies have been included in the study. Maxillary sinus volume has been measured by using stereological method on the CT scans. Palatum durum angle has been measured with the angle of septal deviation and results were compared statistically. $\mathrm{p}<0.05$ was considered as statistically significant.

Results: Septal deviation that causes or does not cause angling in the palatal bones has affected the maxillary sinus volume both in male and female patient group. Maxillary sinus volume at the opposite side of the deviation was significantly higher than at the same side. There is a positive correlation between the septal deviation angle and palatum durum angle.

Conclusion: Whether septal deviation affects the palatum durum or not, it decreases the maxillary sinus volume at the side of the deviation but it does not affect the total maxillary sinus volume.

Keywords: Maxillary sinus volume, stereology, palatum durum, septum deviation

\section{P-120}

\section{The effect of use of hesperidin and diosmin on} necrotizing enterocolitis: an experimental study

Şenel U*, Tanrıverdi Hï* Sapmaz HI ${ }^{* *}$, Akbaş A**, Gevrek $\mathrm{F}^{* * * *}$, Uysal $\mathrm{M}^{* *}$, Taş $\mathrm{U}^{* *}$

*Department of Pediatric Surgery, Faculty of Medicine, Gaziosmanpaşa University, Tokat, Turkey; **Department of Anatomy, Faculty of Medicine, Gaziosmanpaşa University, Tokat, Turkey; $* * *$ Department of Medical Biochemistry, Faculty of Medicine, Gaziosmanpaşa University, Tokat, Turkey; $* * * *$ Department of Histology and Embryology, Faculty of Medicine, Gaziosmanpaşa University, Tokat, Turkey

Objectives: Necrotizing enterocolitis (NEC) is an intestinal disease observed in new born. It's thought that uncontrolled activation of inflammatory pathways and free oxygen radicals play a role in pathogenesis of necrotizing enterocolitis. Flavanoids are phenolic compounds and commonly found in plants. Diosmin and hesperidin (Hsd) as that are the flavonoid glycosides have antiapoptotic, antioxidant and anti- inflammatory characteristics.

Methods: In this study, 30 newborn rats have been divided into three equal groups as control $(\mathrm{n}=10)$, NEC group $(\mathrm{n}=10)$ and treatment group $(\mathrm{n}=10)$. Animals in the treatment group have been given $100 \mathrm{mg} / \mathrm{kg}$ flavonoid with oral gavage twice a day. NEC scoring has been histopathologically performed by taking tissue from the seven different points of the gastrointestinal tract (duodenum, proximal small intestine, jejunum, distal small intestine, caecum, proximal colon and distal colon) after laparotomy by euthanizing under anesthesia at 5 th day. Apoptotic changes have been evaluated with TUNEL staining. The levels of oxidant and antioxidant in tissues have been determined by biochemical analysis. IBM-SPSS 21.0 has been used in statistical analysis and $\mathrm{p}<0.05$ has been adopted as statistically significant.

Results: The levels of MDA and NO in distal ileum have been significantly low in control group when compared to NEC and treatment group. The levels of MDA and $\mathrm{NO}$ in proximal jejunum have been significantly high in NEC group when compared to control and treatment group. Apoptosis and NEC scoring in all tissues have been significantly increased in NEC group than the control group. Furthermore, apoptosis in proximal colon, caecum, jejunum, proximal jejunum and stomach has been significantly decreased in treatment group when compared to NEC group.

Conclusion: These findings support that hesperidin and diosmin treatment noticeably decrease the severity of the gastrointestinal injuries related to NEC.

Keywords: Necrotizing enterocolitis, flavonoid, apoptosis, hesperidin, diosmin

\section{P-121}

The effects of carvedilol on ischemia/reperfusion model: biochemical, histopathological and immunohistochemical evaluation

Özsoy AZ*, Nursal AF ${ }^{\star *}$, Arıc1 A***, Bütün $\dot{I}^{* * * *}$ Uysal $M^{\star * * * *}$, Sapmaz HI***** İşgüder ÇK $\mathrm{K}^{*}$, Doğru $\mathrm{HY}^{*}$, Taş $\mathrm{U}^{* * * * *}$

*Department of Obstetrics \& Gynecology, Faculty of Medicine, Gaziosmanpaşa University, Tokat, Turkey; ${ }^{*}$ Department of Medical Genetics, Faculty of Medicine, Giresun University, Giresun, Turkey; $* * *$ Department of Medical Pathology, Faculty of Medicine, Gaziosmanpaşa University, Tokat, Turkey; ${ }^{* * *}$ Department of Mical Biochemistry, Faculty of Medicine, Gaziosmanpaşa University, Tokat, Turkey; $* * * * *$ Department of Anatomy, Faculty of Medicine, Gaziosmanpaşa University, Tokat, Turkey

Objectives: The aim of this study was to investigate the possible protective effect of carvedilol (CVD) on ovarian ischemia/reperfusion (I/R) injury.

Methods: We organized a model of ovarian I/R by using Wistar albino rats. Ischemia was induced by unilateral occlusion of the tubo- ovarian vessels for $3 \mathrm{~h}$. Reperfusion was carried out by releasing the occlusion and restoring the circulation for $3 \mathrm{~h}$. In group III, CVD was administred intraperitonally before reperfusion. A total of twenty-one female rats were randomly seperated into three equal groups. The groups were classified as group I: $(n=7)$ sham operated control, group II: (n=7) I/R, group III: (n=7) $\mathrm{I} / \mathrm{R}+\mathrm{CVD}$. Superoxide dismutase (SOD)/ and glutathione peroxidase (GSH-Px) activities and malondialdehyde levels (MAD) in ovarian tissues and serum were measured. Histopathological 
and immunhistochemical evaluation were performed by a scoring that evaluation of tissue damage and expression of Bax protein in ovarian tissues.

Results: MDA levels in ovary tissues were significantly increased group II (I/R) when compared to group I (control) $(\mathrm{p}=0.048)$ and group III $(\mathrm{I} / \mathrm{R}+\mathrm{CV})(\mathrm{p}=0.038)$. GSH-Px levels in serum were lower in group II (I/R) than in the group III (I/R+CVD) and difference between the two groups was statistically significant $(\mathrm{p}=0.010)$. SOD activities in serum were found significantly decreased in group II $(\mathrm{I} / \mathrm{R})$ than group I (control) $(\mathrm{p}=0.010)$. Histopathological examination showed a significant improvement in ovarian tissue in the CVD-treated rats $(\mathrm{I} / \mathrm{R}+\mathrm{CVD})$ compared with the group II (I/R). The expression of Bax protein has found higher in group II (I/R) than group III (I/R+CVD) (p=0.037).

Conclusion: According to our results, we can suggest that CVD had reducing effect on damage of ovarian $\mathrm{I} / \mathrm{R}$ model in rats.

Keywords: Ovarian torsion, ischemia/reperfusion injury, carvediol

\section{P-122}

\section{Natural eyebrow profile to be used in reconstructive surgery}

Atalay $\mathrm{FN}^{* *}$, Arga Ö**, Tektas B**, Bülbül YE*, Yazıcı $\mathrm{M}^{* *}$, Turgut $\mathrm{S}^{* *}$, Öztuna $\mathrm{D}^{* * *}$, Esmer $\mathrm{AF}^{*}$

*Ankara University Faculty of Medicine, Department of Anatomy, Ankara, Turkey; **Ankara University Faculty of Medicine, Ankara, Turkey; ${ }^{* *}$ Ankara University Faculty of Medicine, Department of Biostatistics, Ankara, Turkey

Of this study determining the natural eyebrow profile young population to be used in reconstructive surgery. Measurements were taken of 50 women and 50 men aged 18-25 years old. Individuals who have permanent operations on their eyebrows were not included. Measured values were separated as male and female as well as right and left eyebrow. Parameters used were: medial edge of eyebrow (MEB), lateral edge of eyebrow (LEB), peak of eyebrow (PB), nasal ala (NA), medial canthus (MC), lateral canthus (LC), distance between MEB and PB (a), distance between LEB and PB (b), thickness of the eyebrow at the medial edge (TEME), thickness of the eyebrow peak (TEP), length of face (LF), and pupil of eye. Descriptive statistics were obtained. The mean TEME $\backslash L F, T E P \backslash L F$, a/b ratios were investigated. LEB for \% 68 MLE was on the NA-LC line, while for MRE it was \%72. LEB for \%24 MRE was lateral to the NA-LC line while for MLE it was \%28. LEB for \%98 FLE was on the NALC line, while for FRE it was \% 96. PB for \%62 MLE was on the P-NA line, while for MRE it was $\% 60$. PB for $\% 78$ was on the P-NA line while for FRE it was \% 80. MEB for \%68 MLE was on the MC-NA line, while for MRE it was \% \%66. MEB for $\% 86$ FLE was on the MC-NA line, while for FRE it was \%84.To avoid unnatural looking eyebrows; MEB must not be lateral to MC-NA line; PB mustn't be medial to P-NA line; male's eyebrows must be thicker than female's eyebrows; female's LEB mustn't be lateral to the LEB-LC line. Also we saw that eyebrows can be asymmetric.

\section{P-123}

The histological effect of Cnidoscolus aconitifolius aqueous leaf extracts on the archetecture of the ovary, testis and sperm cells of adult Wistar rats

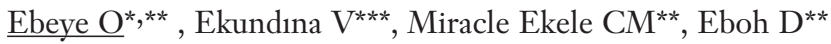

*Department of Anatomy, Delta State University Abraka, Nigeria; **Department of Anatomy, Delta State University Abraka, Nigeria; $* * *$ Department of Medical Laboratory Science, Afe Babalola University, Ado-Ekiti, Nigeria

This study accessed the effects of Cnidoscolus aconitifolius on some reproductive organs (testis and ovary) and semen analysis cells of adult Wistar rats. Twenty four (24) adult Wistar rats Weighing $170 \mathrm{~g}-215 \mathrm{~g}$ were used for this study; the animals were weighed and sorted into control and three treatment groups of six rats each. The control received feed mash and water liberally, while the treated groups 2-4 were given $200 \mathrm{mg}, 400 \mathrm{mg}$ and $600 \mathrm{mg}$ of Cnidoscolus aconitifolius aqueous extract respectively, also they received feed mash and water liberally. At the end of the four weeks experiment animals were sacrificed, organs harvested and fixed in 10\% formal saline for histological studies and sperm cells were placed in normal saline for semen analysis. The extract has no effect on body weight as gradual increase in body weight was observed in all the groups. Microscopic examination of the testis and ovary showed a dose dependent effect, for treated groups; testis revealed spermatogenesis arrest, the ovary revealed lutienization of the ovarian stroma and semen analysis for motility, morphology, viability and sperm count showed significant differences when compared to control group. Therefore caution should be taken in the use of Cnidoscolus aconitifolius.

Keywords: Cnidoscolus aconitifolius, medicinal plant, phytochemical screening, anti-fertility agents, reproductive organs

\section{P-124}

\section{Morphometry of the transverse foramina}

İstanbulluoğlu $\mathrm{S}^{*}$, Öztürk $\mathrm{K}^{*}$, Coskun $\mathrm{G}^{*}$, Eker $\mathrm{C}^{*}$, Sakal B*, Yavuz $\mathrm{Y}^{* *}$, Esmer TŞ ${ }^{*}$ Esmer $\mathrm{AF}^{*}$

*Department of Anatomy, Faculty of Medicine, Ankara University, Ankara, Turkey; ${ }^{* *}$ Department of Biostatistics, Faculty of Medicine, Ankara University, Ankara, Turkey

The transverse foramina existing on the transverse process of cervical vertebrae are known as a hole for vertebral artery, vertebral vein and sympathetic nerves. These foramina have variations on their shape, size and numbers. An accessory transverse foramen usually exists to posterior of the main one and smaller than it. Vertebral artery is major structure in transverse foramen and it feeds spinal cord, brain stem, cerebellum and occipital zone of cerebrum. Showing variations of the transverse foramen is important to evaluate radiological scanning and neurosurgery. In this study 269 cervical vertebrae (62-the first cervical vertebrae (atlas), 70-the second cervical vertebrae(axis) and 137 other cervical vertebras) which has no surgical operation and trauma were examined. Based on the results; the mean diameter of transverse line were $6,03 \pm 0.58 \mathrm{~mm}$ and $6.05 \pm 0.52 \mathrm{~mm}$ on the left and right side 
respectively. The mean diameter of the left sagittal line was $5.76 \pm 0.62 \mathrm{~mm}$ and $5.81 \pm 0.62 \mathrm{~mm}$ on the right. Additively, we analyzed the variations of transverse foramina. We found significant narrowing of the transverse foramina in one side at 3 specimens (\% 1.11) and bone septa separated the foramen at 45 vertebrae. 15 of them (\% 5.57) were unilateral and 30 of them (\%11.15) were bilateral. And we recorded 1 cervical vertebrae (\%0.37) without any transverse foramina on one side which has not been mentioned before. Eventually, absence of transverse foramina may cause the course of vertebral artery and the surgeons should be careful on it. Also, it must be kept in mind the narrowing transverse foramen might cause vertebrobasilar insufficiency.

\section{P-125}

\section{Elbow pain during coughing}

Aytekin $\mathrm{K}^{*}$, $\underline{\mathrm{Uysal}}^{* *}$, Balta $\mathrm{O}^{* * *}$, Kurnaz $\mathrm{R}^{* * * *}$, Sapmaz $\mathrm{HI}^{* *}$, Taş $\mathrm{U}^{* *}$

*Department of Orthopedics and Traumatology, Faculty of Medicine, Giresun University, Giresun, Turkey; ${ }^{* * D e p a r t m e n t ~ o f ~ A n a t o m y, ~ F a c u l t y ~}$ of Medicine, Gaziosmanpaşa University, Tokat, Turkey; ${ }^{* * *}$ Department of Orthopedics and Traumatology, Zile State Hospital, Tokat, Turkey; $* * * \star$ Department of Orthopedics and Traumatology, Acıbadem Eskişehir Hospital, Eskşehir, Turkey

Perineural cysts originate from the junction of the dorsal root ganglion on the radix posterior of the nerve root. They are usually asymptomatic and do not lead to complaints. The lesions are usually seen in the sacral region in these patients in whom the cyst is suggested to form as a result of the hydrostatic and pulsatile pressure of the cerebrospinal fluid (CSF). Rarely, compressive symptoms may be observed. Herein, a 50-year-old female patient who had been admitted to the orthopedics clinic with complaint of pain beginning from the medial of the left elbow and spreading to the forearm (T1 dermatome) only while couching has been presented. The magnetic resonance imaging (MRI) scan of the patient revealed bilateral perineural cyst at the 1 . thoracic spine level.

Keywords: Elbow pain, coughing, perineural cyst, spinal nerve, dermatome

\section{P-126}

The impact of curcumin, resveratrol and the combination on experimental myringosclerosis development

Gürbüzler L*, Kesici H' ${ }^{* *}$, Somuk BT*, Karaca Ziं**, Soyalıc $\mathrm{H}^{*}$, Akbaş $\mathrm{A}^{* * *}$, Doğru $\mathrm{S}^{* * * *}$, Aksakal C*****, Aslan $\mathrm{H}^{* *}$

*Department of Otolaryngology, Gaziosmanpasa University Faculty of Medicine, Tokat, Turkey; ${ }^{*}$ Department of Histology and Embryology, Gaziosmanpasa University Faculty of Medicine, Tokat, Turkey;

***Department of Biochemistry, Gaziosmanpasa University Faculty of Medicine, Tokat, Turkey; $* * * *$ Department of Anesthesiology and Reanimation, Gaziosmanpasa University Faculty of Medicine, Tokat, Turkey; $* * * * *$ Department of Otolaryngology, Tokat State Hospital, Tokat, Turkey
Objectives: Tympanosclerosis (TS) is a clinical entity characterized by, hyaline degeneration and calcification and connective tissue growth in the middle ear mucosa and tympanic membrane. Sklerotik plaque development, if only limited by the eardrum, it is called a myringosclerosis (MS). Two important factors that influence the development of the TS and MS; increased oxygen radical production and the inflammatory reaction and collagen layer of the tympanic membrane.

Methods: In the study, Wistar Wistar albino rats were used. Consisting of eight rats were divided into five groups. Including the control group all rats the right tympanic membranes evaluated under a microscope and made myringotomy in posterosuperior region. Group 1: Myringotomy in the right tympanic membrane was performed. Ve myringosclerosis development process is monitored by the control group. Group 2: $5 \%$ ethanol for 10 days was given alone (IP) group. Group 3: $30 \mathrm{mg} / \mathrm{kg} /$ day of curcumin is dissolved in $5 \%$ ethanol and was given (IP) a period of 10 days. Group 4: $25 \mathrm{mg} / \mathrm{kg} / \mathrm{day}$ of resveratrol is dissolved in $5 \%$ ethanol and was given (IP) a period of 10 days. Group 5: $30 \mathrm{mg} / \mathrm{kg} /$ day of curcumin and 25 $\mathrm{mg} / \mathrm{kg} /$ day of resveratrol is dissolved in $5 \%$ ethanol and was given (IP) a period of 10 days.

Results: According to the assessment we made using stereological methods, we have seen that curcumin prevents myringosclerosis development by reducing the number of fibroblasts alone. Resveratrol has been found to have no effect on this issue. According to the results of the examination made under a microscope, we observed that myringosclerosis score of curcumin group was statistically significantly lower than scores of myringosclerosis enthanol group.

Conclusion: We think that there was no effect of resveratrol prevent myringosclerosis development and the only effect of curcumin in preventing the development of myringosclerosis.

Keywords: Myringosclerosis, resveratrol, curcumin, stereology, fibroblast

\section{P-127}

Investigation of volumetric changes of the thalamus in schizophrenia patients by stereological method

Songur A*, Özbulut $\mathrm{H}^{*}$, $\underline{\text { Cartıllı Ö}}^{*}$, Coşkun K.Ş**, Gönül Y*, Kaçar $\mathrm{E}^{* * *}$

*Department of Anatomy, Faculty of Medicine, Afyon Kocatepe University, Afyonkarahisar, Turkey; **Department of Psychiatry, Faculty of Medicine, Afyon Kocatepe University, Afyonkarahisar, Turkey; $* * *$ Department of Radiology, Faculty of Medicine, Afyon Kocatepe University, Afyonkarahisar, Turkey

Objectives: Schizophrenia (SCZ) is one of the most common psychotic disorders and affects $1 \%$ of the population. Thalamic and hippocampal volume reduction was detected in SCZ. We believe that the elaboration of the research on this topic shed light on literature. This study was planned in order to reveal the volume changes may occur in the thalamus in SCZ by stereological methods. 
Methods: In our study, 21 patients in the 19-81 age group diagnosed with SCZ in Afyon Kocatepe University Psychiatric Clinic, and 21 volunteers in the 20-45 age group's MRI was examined without any pathological findings were included. In each section of MRI images, point field measurements were placed randomly on the thalamus and the spots were counted. The data obtained after count was used to calculate the thalamic volume.

Results: The patient group was composed of 11 men and 10 women and the control group was 12 men, 9 women. The mean age of the control group was $36.0 \pm 7.5$ years and the patient group was $37.4 \pm 16.0$ years and there was no statistical difference comparison of the age and sex between the groups. Right, left and total thalamic volumes were detected as $5965.2 \pm 285.4 \mathrm{~mm}^{3}, 5746.8 \pm 282.4 \mathrm{~mm}^{3}$ ve $11711.0 \pm 541.3 \mathrm{~mm}^{3}$ respectively in control group and $5214.2 \pm 315.0 \mathrm{~mm}^{3}$, $5089.5 \pm 352.9 \mathrm{~mm}^{3}$ ve $10303.0 \pm 651.3 \mathrm{~mm}^{3}$ in patient group. According to the evaluations it was seen in all three parameters was significantly reduced in the patient group $(\mathrm{p}<0.01)$.

Conclusions: Although early studies on this subject do not mention the thalamic volume reduction in SCZ; recent studies supports the thalamus volume reduction in patients with SCZ. Thus, it will be beneficial to know the volumetric changes of thalamus in SCZ and to associated it with the clinical signs of the illness.

Keywords: Schizophrenia, stereology, thalamus volume

\section{P-128}

\section{An easy and effective methodology in defining the estrous cycle in mice}

Çoban ZD* ${ }^{*}$ Kayır H**, Baykal B***, Altaylı E*, Güran Ş* *Department of Medical Biology, Gülhane Military Medical Academy, Ankara, Turkey; **Department of Medical Pharmacology, Gülhane Military Medical Academy, Ankara, Turkey; $* * \star$ Department of Histology and Embryology, Gülhane Military Medical Academy, Ankara, Turkey

Estrous cycle is a recurring physiologic change in libido, which is induced by reproductive hormones in rodents. This cycle is very short in rodents including mice. It persists for 4 or 5 days. Defining the estrous cycle is important for specifying the exact time of the mating period. It represents the beginning of the pregnancy in mice. Having short time period in estrous cycle makes the rodents in advantage in reproductive studies. Because of the difficulties in uses of this technique in mice, generally rats are preferred. The uses of genetically engineered mice increase in recent medical research studies. So, it makes important the determining of estrus cycle in mice. The main steps of the procedures are the obtaining of vaginal samples for having vaginal smear and the determination of the cell types in vaginal smear under the microscope. There are many different studies about this topic in the literature. Vaginal swap methodologies are favorable for the first step of these procedures. In the determination of the cell types from vaginal smears, some methodologies prefer the analyses of cell types under microscope without any staining. In other methodologies, some chemicals prefer for the staining of the cells obtained from vaginal smears. In our lab, a rapid, simple and effective methodology was established in the determination of estrous cycle in mice. Vaginal washing methodology was applied in first step instead of vaginal swap methodology. In the preparation of mouse vaginal smear, toluidine blue was used for staining. In the evaluation of cell types, light microscope was used. By this methodology, the pregnancy ratios were increased statistically and it is possible to obtain the oocyte and embryo of desired date by knowing the exact time of pregnancy. These findings represented that our modified methodologyismore rapid and easierin the application and more effective intheevaluationthan other methodologies in defining the estrous cycle in mice.

\section{P-129}

\section{Architectural comparison of jaw muscles in three species of raptors}

Wang H, Liu X, Liu W, Zhang Z

College of Life Sciences, Capital Normal University, Beijing, China

Jaw muscles of birds are closely related to diet choice and feeding behaviors. Muscle architecture can reflect the functional properties and specializations of different muscles. The two most important architectural parameters are physiological cross-sectional area (PCSA) and muscle fiber length. PCSA of a muscle is directly proportional to force generation, whereas muscle excursion and velocity are directly proportional to muscle fiber length. In the present study, Accipiter gularis (Accipitriformes), Falco tinnunculus (Falconiformes) and Otus scops (Strigiformes) were selected for comparison. Three main jaw muscles, M. depressor mandibulae, M. adductor mandibulae externus and M.pterygoideus, were dissected and detailed measurements were made including muscle mass, muscle belly length, fascicle length. From these values, muscle volume, physiological cross-sectional area and maximum isometric force were derived. The results show that $M$. depressor mandibulae, the opener of the lower jaw, was characterized by relatively long fascicle length, small PCSA, and thus suggest a design for excursion and velocity. Both the muscle mass and PCSA of Mm. musculus adductor mandibulae externus and pterygoideus in Falco tinnunculus were highest and yielded significant differences $(\mathrm{p}<0.05)$ when comparing with other two species; these features indicate that Falco tinnunculus is able to produce highest bite force among studied species. M. pterygoideus in Accipiter gularis and Otus scops play more important role than $M$. adductor mandibulae externus in jaw closing, because of its larger PCSA and longer fascicle length. Architectural comparisons demonstrate mechanism difference in catching and processing prey among raptors. 
S178 XXIV International Symposium on Morphological Sciences, 2nd-6th September, 2015, Istanbul, Turkey

\section{P-130}

Protective effects of propolis on methotrexate-induced testis injury in rat

Sönmez MF*, Çilenk KT*, Karabulut D*, Ünalmış S, Deligönül E, Öztürk İ, Kaymak E*

*Department of Histology and Embryology, Erciyes University Faculty of Medicine, Kayseri, Turkey; **Department of Histology and Embryology, Fatih University Faculty of Medicine, Istanbul Turkey; ${ }^{* * *}$ Department of Food Engineering, Erciyes University, Faculty of Engineering, Kayseri, Turkey

Propolis is a potent antioxidant and a free radical scavenger. This study was designed to determine whether propolis could protect against dysfunction and oxidative stress induced by metotrexate-induced injury in rat testis. A total of 40 male Wistar albino rats were divided into four groups: Group 1 was the untreated control, group 2 was injected with $20 \mathrm{mg} / \mathrm{kg}$ methotrexate, group 3 was injected with with $20 \mathrm{mg} / \mathrm{kg}$ methotrexate plus $100 \mathrm{mg} / \mathrm{kg} /$ day propolis, and group IV was injected with $100 \mathrm{mg} / \mathrm{kg} /$ day propolis. The rats were decapitated under ketamine anesthesia and their testes tissues were removed. Tissue malondialdehyde, xanthine oxidase levels, HSP-70 and PCNA expression, apoptosis and histopathological damage scores were then compared. Methotrexate caused increases in malondialdehyde level and number of apoptotic cells and caused a decrease in MSTD and JTBS, PCNA and HSP-70 expression and xanthine oxidase levels in group 2. Propolis prevented the rise in malondialdehyde, xanthine oxidase levels and HSP-70 expression and improved testicular morphology and JTBS. As a result, methorexate gives rise to serious damage in testes and propolis is a potent antioxidant agent in preventing testicular injury.

\section{P-131}

Effects of 17-beta estradiol on the endothelial function in the male rats; immunohistochemical study

Dursunoğlu D* ${ }^{*}$ Erdoğan E*, Ayaz $M^{* *}$

*Department of Histology-Embryology, Selçuk University, Faculty of Medicine, Konya, Turkey; **Department of Biophysics, Selçuk University, Faculty of Medicine, Konya, Turkey

Objectives: Premenopausal women have lower risk of coronary heart disease (CHD) than men or postmenopausal women, suggesting that estrogens have protective effects and/or androgens have promoter effects against the CHD. However, in the literature, there is very little information about the cardiovascular effects of sex hormones on the opposite sex and the underlying mechanism of these effects remains unknown. Vascular endothelial function disorders play an important role in the pathogenesis of intimal hyperplasia and atherosclerosis. The aim of this study is to investigate the effects of female sex hormone 17-beta estradiol (E2) in the male rats on the molecules regulate the endothelial functions that play a key role in atherosclerosis.
Methods: For the planed purpose, 28 male Wistar albino rats were used and four experimental groups were organized: Control (Con), Castrated (M-), vehicle given to castration (MT) and E2 given to castration (MX). Sacrification was done on the 30th day. Heart tissues of rats were excised. After standard procedures, sections were taken from the tissues. The sections were stained with primary antibodies for endothelial nitric oxide sentaz (eNOS), endothelin-I (ET-I) and vascular endothelial growht factor (VEGF) as indicators of the endothelial function. The expressions of molecules were scored according to the intensity of the staining.

Results: The expression of eNOS increased in MX group compared to Con and M- groups. The expressions of ET-I and VEGF did not change in MX group compared to M- group.

Conclusion: Previous studies have shown that E2 have protective cardiovascular effects in the female. Our study shows that E2 may have protective effects also on the male cardiovascular system. Increased eNOS by E2 might be one of the mechanisms of cardioprotective effects of E2. Consequently, in the treatment and prevention of CHD in men, estrogen therapy may have beneficial effects.

Keywords: 17-beta estradiol, orchiectomy, cardiovascular system, eNOS, ET-I, VEGF, immunohistochemistry

\section{P-132 \\ 3D (3-Dimensional) evaluation of the ischemic penumbra \\ Çıtışlı $V^{* *}$, Onur $S^{*}$, Acar $F^{* *}$, Özdemir $M^{* *}$, Kıroğlu $Y^{* * *}$, Erdoğan $\mathrm{C}^{* * * *}$, Özdemir MB* \\ *Department of Anatomy, Pamukkale University, Faculty of Medicine, Denizli, Turkey; **Department of Neuro Surgery, Pamukkale University, Faculty of Medicine, Denizli, Turkey; ${ }^{* * *}$ Department of Radiology, Pamukkale University, Faculty of Medicine, Denizli, Turkey; $* * * \star$ Department of Neurology, Pamukkale University, Faculty of Medicine, Denizli, Turkey}

The ischemic penumbra is an enigma and is the key to stroke pathophysiology and treatment. The aim of this study was to evaluate ischemic penumbral area by $3 \mathrm{D}$ technology in damaged brain from the images obtained from patient's magnetic resonans (MR) and computional tomography (CT). For this purpose, 3D reconstruction of the ischemic brain area were done from diffusion weighted images (DWI) and perfussion weighted images (PWI). 3D is more estimable than 2D in ischemic penumbra. Paradoxically, ischemic penumbra is still poorly understood. 3D will will improve the understanding of the it. Ischemic penumbra in the brain can be detected by performed cranial imaging methods. Generally, it can be estimated by substracting ischemic area in DWI from ischemic area in PWI. It gives only 2D approachs to the penumbra. In present study, 3D evaluation of the ischemic penumbra was done in the first time. Present study results can improve the understanding of the ischemic penumbra. By the way, it can help to explain stroke pathophysiology then open the way of new treatment.

Keywords: Brain, ischemia, penumbra MRI, CT, 3D, compututional neuroscience 


\section{P-133}

\section{Myocardial bridging}

$\underline{\text { Kara }}^{*}$, Karaca L** Keleş $\mathrm{P}^{*}$, Fil F***

*Department of Anatomy, Faculty of Medicine, Atatürk University, Erzurum, Turkey; **Department of Radiology, Faculty of Medicine, Atatürk University, Erzurum, Turkey; ***Department of Radiology, Regional Training and Research Hospital, Erzurum, Turkey

Myocardium needs oxygen more than other organs except brain. Myocardium is fed from coronary arteries, located in the groves on the surface of the heart. The movements of heart during systole and diastole do not prevent the coronary arteries from feeding the heart. Coronary arteries rise from enlargement areas, on the root of ascending aorta, named right and left aortic sinus. Since the opening of coronary arteries are over aortic valve, the blood supply during systole and diastole is not affected. There are two coronary arteries, left and right. Right and left coronary arteries and large branches originating from these arteries are distributed on the heart surface through the subepicardial connective tissue. Although, coroner arteries lie through the subepicardial layer, sometimes a segment of these vessels lies into the myocardial layer. This is called as myocardial bridging. Myocardial bridging causes atherosclerosis in arterial lumen since it changes arterial wall structure and arterial angle. This may lead to angina, myocardial ischemia, myocardial infarction, left ventricular dysfunction, and sudden cardiovascular death in patients. In our study, we found myocardial bridging in two cases. We discovered myocardial bridging in left anterior descending artery (LAD), which is a branch of left coronary artery, in 59-year-old female, who consulted for atypical chest pain, and in right coronary artery in 39-year-old male who also consulted for atypical chest pain. If there is an atypical chest pain in a patient, it should remaind us this abnormality.

Keywords: Myocardial bridging, coronary artery, atherosclerosis

\section{P-134}

\section{Chemo brain; Pamukkale University Pilot Study I}

Doğu GG**, Herek D***, Demiray AG**, Taskoylu BY**, Yaren $\mathrm{A}^{* *}$, Citıslı $\mathrm{V}^{* * * *}$, Onur $\mathrm{S}^{*}$, Özdemir MB*

*Department of Anatomy, Pamukkale University, Faculty of Medicine, Denizli, Turkey; **Department of Medical Oncology, Pamukkale University, Faculty of Medicine, Denizli, Turkey; ${ }^{* *}$ Department of Radiology, Pamukkale University, Faculty of Medicine, Denizli, Turkey; $* * * *$ Department of Neurosurgery, Pamukkale University, Faculty of Medicine, Denizli, Turkey

After cancer treatment, there is alterations in memory, concentration and the way of think in patients. It may a link between the loss of function and alteration in morphology of the brain structures. The aim of this study is to evaluate the volume of the cerebrum, ventricles cerebellum, right hippocampus and left hippocampus before and after the chemotherapy from the images obtained from patient's magnetic resonans (MRI). For this purpose, $3 \mathrm{D}$ reconstruction of the brain area were done by
Surf Driver program and volume was estimated on 3D structure. Cavalliari method was also used for volume estimation. We compare the means statistically on SPSS program (T test). The mean volume of the cerebrum before chemotherapy was $912827 \mathrm{~mm} 3$, after chemotherapy was $899576 \mathrm{~mm}^{3}$; for ventricles was $8728 \mathrm{~mm} 3$ to $8506 \mathrm{~mm}^{3}$; for cerebellum was 8319 $\mathrm{mm} 3$ to $8293 \mathrm{~mm}^{3}$; for right hippocampus was $4306 \mathrm{~mm}^{3}$ to $2978 \mathrm{~mm} 3$ and for left hippocampus it was $4192 \mathrm{~mm}^{3}$ to 2885 . There was no change statistically between before and after chemotherapy in cerebrum, ventricles and cerebellum volumes ( $>0.05)$. But, There was alteration statistically between before and after chemotherapy in right and left hippocampus $(\mathrm{p}<0.05)$. It is not clear exactly what causes these memory and concentration changes in patient under cancer therapy. Present study may explain the morphological alteration in hippocampus, but not in the other part of the brain. In degenarative brain disease like Alzheimer' and so, cerebrum and ventricles are affected together. But in this study, hippocampus volume were only changed. Cerebellum was the another structure that we would like to examine. It is possible to explain the loss of memory in chemo brain. But we need more studies for the others.

Keywords: Chemo brain, cerebrum, ventricles, cerebellum, volume, MRI, 3D

\section{P-135}

The regional and temporal characters of de- and remyelination processes in cuprizone induced demyelination animal model

\author{
Zhang Y, Fan K, Ma J \\ Department of Anatomy of Dalian Medical University, Dalian, China
}

Cuprizone (CPZ), which is copper chelator, has been wildely used to induce mouse demyelination model for understanding the pathogenesis of demyelination diseases, such as multiple sclerosis (MS). However, the spatial and temporal characters of $\mathrm{de}-$ and remyelination process in this model are rarely reported. In the present study, $24 \mathrm{C} 57 \mathrm{BL} / 6 \mathrm{~J}$ mice were treated by $0.5 \%$ cuprizone feeding and were sacrificed at different de- and remyelinating time points, then the myelin statuses in different brain regions were valued by image analysis after Black-Gold myelin staining and $\mathrm{MBP}$ (myelin basic protein, MBP) IHC, respectively. The results showed that the demyelination speed was not equal in different brain area even at the same time point after CPZ administration. The lateral septal area (LSA) and hippocampus exhibited more sensitive to the toxicity of $\mathrm{CPZ}$. However, the fimbria of hippocampus, the anterior commissure and the fornix were significantly resistant to the toxicity of CPZ. More interestingly, the demyelination speed in the corpus callosum (CC) also was not equal that the caudal part was faster than that in the cranial part. Similar with demyelination process, the remyelination also showed variable speed in different brain area after $\mathrm{CPZ}$ withdrawal. Significant remyelination occurred in $\mathrm{CC}$ even in 3 days and the myelin status almost restored to the normal level in 2-3weeks. However, in LSA and hippocampus significant remyelination occurred in 3 
weeks after $\mathrm{CPZ}$ withdrawal and not yet restored to the normal level. These regional and temporal characters may help to further understand the processes of de- and remyelination in this classical and popular animal model.

\section{P-136}

Short and long effects of mesenchymal stem cell administrations on the experimental cirrhosis model

$\underline{\text { Vatansever HS }}^{* *}$, Akmaz B*, Bilekyiğit C***, Aslan E*, Gökalp S*, Mansoub NH****, Ercan $\mathrm{G}^{* * * *}$

*Department of Histology and Embryology, Faculty of Medicine, Celal Bayar University, Manisa, Turkey; ${ }^{* \star D}$ Department of Histology and Embryology, Faculty of Medicine, Celal Bayar University, Manisa, Turkey \& Research Center of Experimental Health Science, North Cyprus; ***Faculty of Medicine, Celal Bayar University, Manisa,

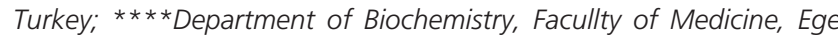
University, Izmir, Turkey

Objectives: To use and evaluate short and long affects of different mesenchymal stem cell sources on experimental rat cirrhosis model.

Methods: The experimental cirrhosis model was obtained using $0.3 \mathrm{~g} / \mathrm{L}$ Thioacetamide (TAA) for three months. The mesenchymal cells were collected from bone marrow, adipose tissue and liver. They were cultured in $\alpha$-MEM medium with $15 \%$ fetal bovine serum, $1 \%$ L-glutamine, $1 \%$ penicillin-streptomycin, $1 \%$ gentamycin and $0.1 \%$ Amphotericine B. Identification of mesenchymal cells, distribution of CD44, CD90, ICAM-1 for bone marrow; CD44 for adipogenic; $\alpha$ smooth muscle actin for liver mesenchymal cells were analyzed using indirect immunoperoxidase. CD14, CD19, CD34, CD44, CD45, CD73, CD90, CD103 and CD326 analyses were also using flow cytometry. Group I was healty control group and had no any treatment. Group II had cirrhosis group and had no any treatment. Group III, IV, V and VI were bone marrow, adipogenic or hepatic mesenchymal cells, respectively, after TAA administration for 4 weeks. The liver tissues were collected at 4 and 12 weeks. After routine paraffine embedding protocols, histochemical (hematoxylen-eosine, Masson Trichrome), TUNEL, immunohistochemical (bcl-2, sitochrome-C, caspase 3,8,9) and biochemical analyses (MDA, ALT, AST) were investigated.

Results: Bone marrow, adipogenic and hepatic adult mesenchymal cells identification were positive using their specific markers in both immunohistochemically and flow cytometric analyses. After administration with TAA for three months, the liver cells were hypertrophic and also some fibrotic tissue was observed in control II and III. In groups IV, V and VI the morphology of hepatocytes were similar healty control group (groupI) and in addition the decreased liver cell damage, accumulation of fibrotic tissue were also detected. The more effectively less cellular damage and normal ALT, AST and albumin levels were detected in adipogenic mesenchymal stem cell treatment.
Conclusion: In our study, the experimental cirrhosis TAA model which only liver damage occurred that was not in other tissues shown any negative effects. The curative effect of the stem cells treatment was detected using adipose tissue-derived mesenchymal stem cells and it may be preferred autologous stem cell sources.

\section{P-137}

Topographic anatomy of the azygos venous system: a cadaveric study

Kılıç C ${ }^{* *}, \underline{\text { Fazlıoğulları Z }}$, Yazar F** Karabulut AK*

${ }^{*}$ Department of Anatomy, Selçuk University Faculty of Medicine, Konya, Turkey; ${ }^{*}$ Department of Anatomy, Faculty of Medicine, Gülhane Military Medical Academy, Ankara, Turkey

Objectives: The azygos system consists of the remaining part of posterior cardinal veins. This system is composed of the azygos, hemiazygos and accessory hemiazygos veins. The azygos system drains blood from the back and from the thoracic and abdominal walls. The azygos venous system is variable in drainage and branching pattern. In the present study, we aimed to investigate the branching patterns of azygos system in human cadavers.

Methods: The study was performed on 20 adult human cadavers (16 males, 4 females) with ages varying between 23 and 76 who were obtained from anatomy laboratory and fixed by formaline. Anterior chest wall and anterior abdominal wall were removed first; after the organs on the area were taken away, dissection was continued and azygos, hemiazygos and accessory hemiazygos vein were exposed. Branching patterns of the azygos system were grouped through classifications reported in the literature.

Results: All of the cases were determined as transition type (Type II) according to Anson's classification which evaluates the connections between the vessels. Five subgroups including 7 cases in group 2, 3 cases in group 4, 5 cases in group 5, 1 case in group 6 and 4 cases in group 7 were determined. The flowing site of the azygos vein into the caval system was determined at T3 vertebra level in $50 \%$ of the cases.

Conclusion: Identifying of anatomic variations and branching patterns of the azygos venous system is important during imaging this region and surgical operations of mediastinum.

Keywords: Azygos vein, hemiazygos vein, accessory hemiazygos vein, cadaver

\section{P-138}

Double superior vena cava: a demonstration with MDCT

Fazlığulları $Z^{*}$, Koplay $M^{* *}$, Nabi $G^{*}$, Gün $C^{*}$

*Department of Anatomy, Faculty of Medicine, Selçuk University Konya, Turkey; **Department of Radiology, Faculty of Medicine, Selçuk University, Konya, Turkey

Objectives: The most common congenital anomaly of the superior vena cava (SVC) is a double SVC. This situation also 
called persistent left superior vena cava (PLSVC). This variation occurs in $0.3 \%$ of the general population, but in patients with congenital heart disease is more common. PLSVC results from the embryological failure of regression of the left anterior cardinal vein. PLSVC is usually asymptomatic and haemodymically insignificant. The presence of the vessel is identified incidentally during CT scanning of the chest, or during cathetarization procedures.

Case Report: Thoracic CT scan was performed on a 40 years old female patient with a history of surgery due to renal tumor to scan metastasis. In the examination, right superior vena cava was on the normal location whereas left superior vena cava discharging into the right atrium through coronary sinus was observed. Both vena cava were connected with a tubular vessel with a diameter of $5 \mathrm{~mm}$ on upper mediastinal area. Current findings were presented with axial images as well as multiplanar reconstruction and three dimensional images in detail.

Conclusion: Knowledge about these kinds of variations is important for surgeons especially thoracic surgeons, interventional radiologists, and other physicians whom are actively involved in central venous access device placement in patients.

Keywords: Double superior vena cava, MDCT, variation

\section{P-139}

\section{Nanoparticulate BDNF protective effect in modeled Parkinson's disease}

Syazli RWAKW*, Alyautdin R** Kapitonova $M^{*}$, Ullah $M^{* * *}$, Khlebnikov $\mathrm{V}^{* * * *}$, Halim ST*, Ahmad A*, İsmail N*

*Faculty of Medicine, Universiti Teknologi MARA (UiTM), Shah Alam, Selangor, Malaysia; **Scientific Centre For Expertise of Medical Application Products, Moscow, Russia; ***Faculty of Dentistry, Universiti Teknologi MARA (UiTM), Shah Alam, Selangor, Malaysia; $* * * *$ Kulliyyah of Medicine, IIUM, Kuantan, Malaysia; Volgograd State Medical University, Russia

Parkinson's disease (PD) is the most common age-related motoric neurodegenerative disorder in which degeneration of dopaminergic neurons is recognized as the underlying pathophysiology of the motor dysfunction. BDNF is a member of the neurotrophin family that has been well-established as a key regulator of neuronal survival and plasticity. BDNF, as a large protein, does not cross the blood-brain barrier (BBB) (J. Kreuter, 2013; R. Alyautdin et al., 2014). Synthetic polymeric nanoparticles overcoated with surfactant providing "Trojan horse" effect deliver different compounds into the brain through the BBB (K. Kurakhmaeva, 2009). The effectiveness of exogenous BDNF applied for treatment of the neurodegenerative diseases remains unknown. The objective of the study was to evaluate neuroprotective effects of nanoparticulate BDNF in the animal model of PD induced by 1-methyl- 4-phenyl1,2,3,6-tetrahydropyridine (MPTP). C57BL mice were used for modeling of PD. All animals received i.p. injections of MPTP followed by i.v. injection of BDNF absorbed on the commercial PLGA nanoparticles with F68 surfactant (group 1) or pure PLGA nanoparticles (group 2) or pure PLGA nanopar- ticles with surfactant (group 3) with total of 36 mice. Behavioral tests were performed 90 min, 24 hours, 3 and 7 days after MPTP injection. Thereafter mice were sacrificed, brain was sampled for ELISA and histological evaluation of changes in the substantia nigra and the striatum. The results showed that the nanoparticulate BDNF significantly relieved behavioral disorders (oligokinesia, rigidity, tremor) as shown by open field test (distance walked, rearings), rotarod performance, stride space test, body length measurement and tremor evaluation. Histological examination of the midbrain and basal ganglia showed that treatment with nanoparticulate BDNF with surfactant decreased loss of the neurons, inhibited microglia activation and reduced astrocyte hypertrophy in the substantia nigra and the striatum. This research provides the prospective medication for treatment of the PD using BDNF and gives a clue to understanding of mechanisms of its penetration through the BBB and neuroprotective effect.

\section{P-140}

The incidence of zygomatico-facial, zygomatico-orbital and zygomatico-temporal foramina

Babacan S, Güner N, Tuncel N, Kafa I

Department of Anatomy, Faculty of Medicine, Uludag University, Bursa, Turkey

The zygomatic bone contains some small openings, termed as the zygomatico-facial, zygomatico-orbital and zygomatico- temporal foramens, which serve as the passages for the branches of the maxillary nerve. These branches are important structures for the maxillofacial and orbital procedures due to their anatomical locations. Although several authors suggested that these foramens might be useful as anatomical landmarks for the dissections during surgeries, in fact the variability including the quantity and localization makes them unreliable. The aim of this study was to obtain data including the classification of these foramens and conducted on 50 dry human skulls. The criteria of excluding from study were fractures and other sort of deformities on the bones. Total sixty-nine zygomatic bones studied and foramens were counted carefully, noted and classified in six groups (type 0 to 5; absence of foramen, being one, two, three, four and five foramina) considering their quantities. We found that type 1 appearance is the most observed type for all foramens on the three different faces of the zygomatic bone. Our results for the zygomatico-facial foramen $(\mathrm{n}=69)$ were: type $1, \% 40.58$; type 2 , $\% 23.18$; type 0 , \%21.74; type 3, \%8.69; type 4, \%2.89 and type 5, \% 2.89; for the zygomatico-orbital foramen ( $\mathrm{n}=55)$ were: type 1, \%32.73; type 0, \%27.27; type 2, \%21.81; type 3, \% 16.36; type 4, \% 1.81 and type 5, \% 0 ; and for the zygomatico-temporal foramen ( $\mathrm{n}=62$ ) were: type $1, \% 37.10$; type $2, \% 30.64$; type 0 , $\% 19.35$; type 3 , \%11.29; type 5, \%1.61and type 4, \%0, in descending order. In this study, we classified the quantity and investigated the locations of the foramens related to the zygomatic bone and the maxillary nerve to show their varieties, in order to obtain more knowledge of this area and gain benefits to surgeries related to the zygomatic bone and neighboring regions. 
S182 XXIV International Symposium on Morphological Sciences, 2nd-6th September, 2015, Istanbul, Turkey

\section{P-141}

Oct 3/4 gene silencing in mouse embryonic stem cell and the effects of this gene silencing on embryonic stem cells

Coban ZD*, Güran Ş*, Ural AU**

Department of Medical Biology, Gülhane Military Medical Academy, Ankara, Turkey; ${ }^{*}$ Department of Hematology, Gülhane Military Medical Academy \& Bayındır Hospital Bone Marrow Transplantation Unit, Ankara, Turkey

Stem cells are undifferentiated or poor differentiated cells with important characteristics like self renewal and differentiation. Embryonic stem cells are the only one that can regenerate a living organism among all stem cells. The mouse embryonic stem cells used in laboratories are obtained from trophoblastic inner cells mass in early embryonic stages. Oct $3 / 4$ gene is the most important stemness gene in obtaining induced pluripotent stem cell (IPSC) from mature differentiated cell. The transfer of Oct $3 / 4$ gene activates the differentiated cell in IPSC technology. The silencing of this gene with molecular biology techniques is important in application of gene therapies and the other advanced genetic techniques. Generally, the silencing of selected mRNA is used in this technology in cytoplasm. So, small interference RNAs (siRNA) are widely used in silencing of targeted RNAs. In our study, we aimed to transfer the specific siRNA for Oct $3 / 4$ gene into the embryonic stem cells and inhibit the effects of this gene in mouse embryonic stem cells. Mouse embryonic stem cells were obtained from American Type Culture Collection-ATCC and co-cultured with mouse embryonic fibroblast-MEF, mitotically inactivated. Leukemia inhibiting factor-LIF was added for inhibiting the differentiation. For detecting the possible cell differentiation, Oct 3/4 gene mRNA expression was studied by Reverse Transcriptase PCR (RT-PCR) technique. The complementary siRNAs of Oct $3 / 4$ gene were transferred to the cytoplasm of embryonic stem cells and Oct? gene mRNA was silenced. For detecting the inhibition of this gene, the mRNA expression profiles were detected again with RTPCR. The results were compared with the results before the gene silencing procedure. The persistence of the stemness characteristics in embryonic stem cells was found. A significant inhibition in mRNA levels of Oct 3/4 gene was observed. We observed that the inhibition of an targeted gene is possible in uses of this technique. It is possible to manipulate a targeted gene or a gene product with this technique.

\section{P-142}

Which method is gold standard for determination of thyroid volume?

Imre $\mathrm{N}^{*}$, Acer $\mathrm{N}^{* *}$, Apaydın $\mathrm{N}^{* * *}$, Güvenç I $\mathrm{I}^{* * * *}$, Zararsiz $\mathrm{G}^{* * * *}$

*Department of Anatomy, Gülhane Military Medical School, Ankara, Turkey; **Department of Anatomy, Faculty of Medicine, Erciyes University, Kayseri, Turkey; ***Department of Anatomy, Faculty of Medicine, Ankara University, Ankara, Turkey; $* * * *$ Department of Radiology, Medicalpark Hospital, Ankara, Turkey; *****Department of Biostatistics, Faculty of Medicine, Erciyes University, Kayseri, Turkey
The accurate estimation of the thyroid volume is very important for the evaluation and management of thyroid disorders. There are various methods for estimating thyroid volume using different imaging techniques; however a gold standard has not yet been determined. The aim of the study therefore is to compare different techniques and to define an optimal correction factor for to determine the volume of thyroid gland, specifically by CT, linear measurement and fluid displacement method. Three methods were compared linear measurement, the stereological (point-counting) method using CT and fluid displacement technique. Correction factors from 0.450 to 0.600 in steps of 0.001 were modeled separately and mean squared errors were calculated for each model to find an optimal correction factor for thyroid volume estimation. The thyroid volumes were calculated in a total of 16 specimens. The mean \pm SD thyroid volumes of the fluid displacement, point counting and ellipsoid were $14.58 \pm 9.84,15.28 \pm 9.38$, and $14.97 \pm 8.35 \mathrm{~cm}^{3}$, respectively. No significant difference was found among the methods ( $p>0.05)$. It can be concluded that acceptable correction factor is situated in 0.523 using linear measurement according to gold standard.

\section{P-143}

\section{Measurement of the aortic root in young healthy Turkish men}

İmre $\mathrm{N}^{*}$, Tunç $\mathrm{E}^{* *}$

*Department of Anatomy, Gülhane Military Medical School, Ankara, Turkey; **Deparment of Cardiology, Izmir Military Hospital, Izmir, Turkey

Aortic root diameter is a very important parameter for the diagnosis of vascular pathologies and can easily be detected by transtorasic echocardiography (TTE). For example, aortic root dilatation is one of the diagnostic criteria for Marphan Syndrome. The progression at the follow up can determine the indication for surgery. Aortic root dilatation is suggested with higher cardiovascular morbidity and mortality. By using TTE in our study, we have tried to measure the normal values of healthy young adults (whose ages are between 20 and 30 years old) aortic annulus, sinus valsalva, sinotubuler junction and ascendan aorta diameter and also to measure relation of body mass index (BMI) and body surface area (BSA). Healthy 200 young male individuals were included in the study. Mean age of the participants were 22 years old. Aort diameter has been measured on four different levels. The measurement levels were aortic annulus, sinus valsalva, sinotubuler junction and ascendan aorta. Aortic root diameter were measured $22.23 \pm 1.79 ; 30.8 \pm 2.22 ; 26.09 \pm 1.98 ; 28.17 \pm 2.14 \mathrm{~mm}$. respectively. The relationship of aortic root diameter with BMI and BSA has been calculated statistically. Especially, there has been a strong relationship with ascending aorta diameter with BMI.

Keywords: Aortic root diameter, normal values, transtorasic echocadiography 


\section{P-144}

The effect of cyclooxygenase system of acetaminophen induced hepatotoxicity development

Uslu Ü*, Kolgazi $M^{* *}$, Cumbul A* Özdağ $\mathrm{Y}^{*}$, Yüksel $M^{* * *}$, Öğ̈̈nç $\mathrm{AV}^{* * *}$, Erhan $\mathrm{F}^{* * *}$, Alican $\mathrm{I}^{* * * * *}$

*Department of Histology and Embryology, Faculty of Medicine, Yeditepe University, Istanbul, Turkey; **Department of Physiology, Faculty of Medicine, Acibadem University, Istanbul, Turkey; $* * \star$ Department of Biochemistry, Vocational Faculty of Health Related Professions, Marmara University, Istanbul, Turkey; $* * *$ Department of Histology and Embryology, Faculty of Medicine, Marmara University, Istanbul, Turkey; *****Department of Physiology, Faculty of Medicine, Marmara University, İstanbul, Turkey

Objectives: The purpose of this study was investigate the effect of cyclooxygenase (COX) system's on oxidative liver damage induced by acetaminophen (paracetamol $\mathrm{N}$-acetyl-p-aminophenol; APAP) in rats.

Methods: 35 rats (Sprague-Dawley) were separated into 5 groups. Experimental animals were divided into the control group $(\mathrm{C} ; \mathrm{n}=7)$, paracetamol control group (APAP; $\mathrm{n}=7$ ), nonselective COX inhibitors group (INDO; $n=7$ ), selective COX-1 inhibitors group (KET; $n=7$ ) and selective COX-2 inhibitors group (NIM; $n=7)$. The INDO group was injected intraperitoneally with $5 \mathrm{mg} / \mathrm{kg}$ indomethacin; the KET group, $5 \mathrm{mg} / \mathrm{kg}$ ketorolac; and the NIM group $10 \mathrm{mg} / \mathrm{kg}$ nimesulide The $\mathrm{C}$ and APAP were administered saline and vehicles by the same route. Twenty-four hours after the administration of acetaminophen, all animals were sacrificed in ethics. Their livers were removed and immunohistochemical analyze were performed. The obtained liver samples were fixed in neutral formaldehyde and embedded in parafine wax. The samples of $5 \mu \mathrm{m}$ in thickness were marked with COX-2 and NF kappa B p65 by immunoassay. These samples were examined with a Leica DM600 B microscope and immunohistochemical index was achieved.

Results: Expression of COX-2 was higher in the APAP group compared to control (respectively $21.475 \pm 1.15$ vs. $14.11 \pm 1.68$; $\mathrm{p}<0.05)$ COX-2 suppressed in all COX inhibitors groups. Immunohistochemical index values demonstrated that the NF kappa B p65 was decreased by KET, NIM and INDO by respectively.

Conclusion: Drugs of COX blocker may prevent hepatotoxicity by suppressing inflammation.

Keywords: Acetaminophen induced hepatotoxicity, cyclooxygenase, Immunohistochemistry

\section{P-145}

Morphometric study of the anatomic structures belong to vertebrae and clinical evaluation: an anatomical study

Desdicioğlu K, Öztürk KE, Çizmeci G, Malas MA

Department of Anatomy, Faculty of Medicine, Katip Çelebi University, izmir, Turkey

Objectives: Aim of this study was to investigate the morphometry of anatomical structures belong to vertebrae and to evaluate the clinical aspects.
Methods: This study was conducted on a hundred vertebrae (15 cervical, 49 thoracic, 36 lumbar) that in the student laboratory of anatomy department at İzmir Kâtip Celebi University Medical Faculty. Atlas, axis and disintegrated vertebrae were excluded from the study. The morphometric measurements such as length dimensions belong to corpus of vertebrae, transvers and sagittal diameters belong to corpus and foramen of vertebrae, height, thickness and length belong to pedicle of vertebrae and the distance between pedicles were carried out. Statistical analysis were performed using SPSS.

Results: The mean values of morphometric measurements and the standard deviation were determinated. The differences between measurements in the same region and the different regions were evaluated by the statistical analysis. As a result of evaluations, there was a statistical difference between parameters belong to cervical, thoracic and lumbar vertebrae $(\mathrm{p}<0.05)$ but no statistical difference in vertebrae belong to same region ( $>>0.05)$.

Conclusion: Transpedicular screw fixation is used increasingly common by neurosurgeons and orthopedists even though the long term outcomes and effectiveness are unknown. Additionally even though the vertebrae are not commonly used at sex determination, they are one of the most abundant bones in the crime scene. This event increases the importance of the vertebrae in determining gender. As a result, we suggest that the data which we obtained in the study would be useful to surgeons, forensic science experts and the clinicians who interested in this region.

\section{P-146}

The relationship between morphometry of internal auditory canal and temporal bone pathologies of the patients with chronic otitis media

${\text { Turamanlar } \mathrm{O}^{*}}^{*}$ Akbal I*, Acay $\mathrm{MB}^{* *}$, Ünlü E**, Gönül $\mathrm{Y}^{*}$, Cartıllı Ö*, Ulu Ş***

*Department of Anatomy, Faculty of Medicine, Afyon Kocatepe University, Afyonkarahisar, Turkey; ${ }^{* *}$ Department of Radiology, Faculty of Medicine, Afyon Kocatepe University, Afyonkarahisar, Turkey; ***Department of Otolaryngology, Faculty of Medicine, Afyon Kocatepe University, Afyonkarahisar, Turkey

Objectives: Radiological examination of the temporal bone must be done before surgical interventions for pioneering microsurgery methods. The aim of this study is to reveal the morphometric measurements of internal auditory canal (IAC) and association with neighbouring structures with thin slice computerized tomography (CT) images and also to evaluate the relationship between the intraoperative findings of the patients who underwent operation due to chronic otitis media (COM).

Methods: Morphometric evaluation were done in preoperative axial and coronal CT images. Intraoperative findings were recorded for the presence of cholesteatoma, ear pathology, ear drainage, lateral semicircular canal dehiscence, facial nerve dehiscence, dural defect and bony chain destruction.

Results: Configuration of a total of 98 IACs of 49 subjects were as follows: 51 funnel (55.10\%), 34 cylindirical (34.69\%), 13 bud (13.26\%) shaped. IAC opening width is narrower in the 
S184 XXIV International Symposium on Morphological Sciences, 2nd-6th September, 2015, Istanbul, Turkey

right compared to the left. There was a positive correlation between the left sinus sigmoideus and the left IAC longitudinal length with equal development of temporal bone.

Conclusions: We believe that such studies would shed light on $\mathrm{COM}$ diagnosis and surgical interventions and provide positive contributions to treatment.

\section{P-147}

The effect of post-fixation on visualization of vimentin in retina with immunofluorescence method

$\underline{\text { Baykal B }}^{*}$, Korkmaz $C^{* *}$, Kocabiyık N*** Ceylan OM****

*Department of Histology and Embryology, Gülhane Military Medical Academy, Ankara, Turkey; **Department of Obstetrics and Gynecology, Gülhane Military Medical Academy, Ankara, Turkey; ***Department of Anatomy, Gülhane Military Medical Academy, Ankara, Turkey; ****Ophtalmology Service, Medical Park Hospital, Ankara, Turkey

A tissue preservation method used in scientific studies is short term fixation by perfusion fixation with paraformaldehyde and preservation of tissue at $-80^{\circ} \mathrm{C}$. Sections taken from short term fixed tissues require to undergo post-fixation. We detected that the method of post-fixation is important for visualizing vimentin using immunofluorescence method in sections taken from archival eyes, which were fixed by perfusion fixation and preserved at $-80^{\circ} \mathrm{C}$. We detected that no post-fixation or post-fixation with paraformaldehyde prevent the visualization of vimentin as fibrils in the retina, they allow for visualization of vimentin in the surrounding connective tissue as fibrils. Post-fixation with methanol or Alcohol/Acetic acid allow for visualization of vimentin both in the retina and in the surrounding connective tissue as fibrils. We also performed densitometric analysis of images of Alcohol/Acetic acid and methanol post-fixed sections and detected that the peaks are more regular and the background noise is lower in AAA post-fixed sections. Thus, it is essential to choose the fixation and post-fixation method carefully for the antigen, which is targeted to be visualized. This project has been supported by TUBITAK with project number 110 S308. This presentation has also been presented in XI. National Histology and Embryology Congress, 16-19 May 2012, Pamukkale University, Denizli, Turkey and the abstract has been published in Cell \& Tissue Biology Research Volume 3 / 2012 Supplement.

Keywords: Alcohol/acetic acid, methanol, paraformaldehyde, post-fixation, retina, vimentin

\section{P-148}

The use of propidium iodide as a nuclear counter-stain for the marking of degenerated neurons with fluoro-jade

Baykal B $^{*}$, Arslan $\mathrm{M}^{* *}$, Kayır $\mathrm{H}^{* * *}$, Korkmaz $\mathrm{C}^{* * * *}$, Unay B $\mathrm{B}^{* *}$ *Department of Histology and Embryology, Gülhane Military Medical Academy, Ankara, Turkey; **Department of Pediatric Neurology, Gülhane Military Medical Academy, Ankara, Turkey; ${ }^{* *}$ Department of Medical Pharmacology, Gülhane Military Medical Academy, Ankara, Turkey; $* * *$ Department of Obstetrics and Gynecology, Gülhane Military Medical Academy, Ankara, Turkey
Fluoro-jade is an anionic tribasic fluorescein derivative, which was introduced on 1993 as a new technique for visualization of degenerated neurons. It has a peak excitation wavelength of $485 \mathrm{~nm}$ and a peak emission wavelength of $525 \mathrm{~nm}$ (green). DAPI is offered to be used as a nuclear counter-stain together with fluoro-jade. DAPI is a fluorochrome, which has a peak excitation wavelength of $358 \mathrm{~nm}$ and a peak emission wavelength of $461 \mathrm{~nm}$ (blue). When using fluoro- jade with DAPI, they cause a high level of overlap and they can usually be observed using the same filter cube. The proximity of blue and green colors made us think that these two molecules are not a proper couple. Thus, we aimed to find a nuclear counter-stain, which can generate higher contrast when used with fluorojade. We tried several nuclear counter-stains on sections from the brain of a rat, on which pilocarpin induced status epilepticus model was applied. We detected that propidium iodide provided higher contrast and lower overlap with its red color. In order to be able to use propidium iodide in fluoro jade method, propidium iodide has to be dissolved in phosphate buffered saline and applied to the brain section before fluoro jade step of the method. This presentation has also been presented in XII. National Histology and Embryology Congress, 27-30 May 2014, Ankara University, Ankara, Turkey and the abstract has been published in Cell \& Tissue Biology Research, Supplement, pp134, 2014.

Keywords: Fluoro-jade, neurodegeneration, propidium iodide

\section{P-149}

Investigation of the effect of kinesiology tape applied to gastrocnemius muscle on the vertical jump height

Köse $\mathrm{ED}^{*}$, Taş $\mathrm{U}^{*}, \underline{\mathrm{Uysal} \mathrm{M}^{*}}$, Aytekin $\mathrm{K}^{* *}$, Kılınç F***, Sapmaz $\mathrm{HI}^{*}$, Özyurt B*

*Department of Anatomy, Faculty of Medicine, Gaziosmanpaşa University, Tokat, Turkey; **Department of Orthopedics and Traumatology, Faculty of Medicine, Giresun University, Giresun, Turkey; ${ }^{* *}$ Faculty of Physical Education and Sport, Süleyman Demirel University, Isparta, Turkey

Objectives: Kinesiology taping technique is a treatment approach that increases the distance between skin tissue and muscle fascia by forming folding in skin and thus relaxing structures under the skin during the functional activities. Gastrocnemius muscle that located in the back of the leg enables vertical jump by making ankle plantar flexion during the vertical jump. Vertical jumping height is a factor that enhances the performance of the sports competition and affects the result of the competition. In this study, we have invastigated the effects of the kinesiology tape technique on the vertical jumping movement.

Methods: 50 males between 18-24 age ranges have been divided into two equal groups. Kinesiology tape has been applied to Group 1 for 10 minutes and to Group 2 for 30 minutes. Vertical jumping height has been measured before and after the kinesiology tape for the both group. Vertical jumping height has been 
measured both by marking the wall and jump-meter. A satisfaction test has been made by all of the individual participating in the study for them to set their satisfaction between 0 to 10 . $\mathrm{p}<0.05$ has been adopted as statistically significant.

Results: According to the both measurement method, kinesiology tape technique significantly increased the vertical jumping height for both Group 1 and Group 2 ( $\mathrm{p}<0.001)$. According to the measurement values conducted by the jump-meter, performance enhancement for Group 1 and Group 2 is $11 \%$ and $7 \%$ respectively. In the wall marking method, performance enhancements for both groups were $6 \%$. Satisfaction test scale was determined as 7 out of 10 for both groups.

Conclusion: Findings of our study support that kinesiology tape technique enhances the vertical jumping performance significantly and this increase particularly is clearer on the group that has been applied tape for 10 minutes.

\section{P-150}

Evaluation of structures and their interrelations associated with lateral epicondylitis: an anatomic study

Öztürk H, Gilan IY, Öztürk NC

Department of Anatomy, Mersin Faculty of Medicine, Mersin Universiy, Mersin, Turkey

Lateral epicondylitis or commonly termed as "tennis elbow" is hypothesized to occur as a result of repetitive, strenuous and abnormal postural activities of the elbow and wrist. Epicondylitis is an inflammatory process that may be more accurately described as tendinosis. In the lateral epicondylar region, this process mostly affects the common extensor tendon. There is still a lack of understanding of how wrist and forearm positions and which anatomic structure(s) contribute most to this condition during common manual tasks. According to the literature, the problem mainly seems to manifest itself at the origin of the extensor carpi radialis brevis tendon. In this study, 25 (twenty-five) limbs from embalmed specimens were dissected to reveal all three extensors; extensor carpi radialis brevis, extensor carpi radialis longus and extensor digitorum communis muscles' musculotendon patterns, radial collateral ligament, capsule of the elbow joint, head of radius and their interrelations in the vicinity of the lateral epicondyle were investigated. Rotation of the head of radius anteriorly against $\mathrm{m}$. extensor carpi radialis brevis during pronation of the forearm due to its shape seems to be important in the kinetics of the associated structures at the region. It is believed that these factors may partly contribute to the onset of lateral epicondylitis as they seem to be responsible for the creation of microtears at the tendons' origins or elsewhere in the vicinity and progressive degeneration due to an immature reparative response. Familiarity with the normal anatomy of the associated structures at the region may help understand the pathophysiology better in lateral epicondylitis.

Keywords: Lateral epicondylitis (tenis elbow), anatomy

\section{P-151}

Evaluation of subcutaneous fat tissue (SFT)

Engin Y, Kapakin S

Department of Anatomy, Faculty of Medicine, Atatürk University, Erzurum, Turkey

SFT is one of the structures that determine the shape of the body. The accumulation and distribution of SFT is great importance on general health. It can alter the effectiveness of drugs and anesthetic substances, and can affect resistance to cold and hunger. Measurement of SFT can therefore provide very useful information. SFT is associated with diabetes, cancer, metabolic and cardiovascular diseases. We planned to measure SFT in various regions of the body and to compare the results in terms of age and sex. SFT thickness (T) was measured in nine areas using an ultrasound device. Body mass index (BMI) was calculated. Anthropometric circumference measurements were performed. 183 adult volunteers (100 women and 83 men) were enrolled. Mean age was $26.2 \pm 8.2$ years. Mean BMI was $23.3 \pm 3.7 \mathrm{~cm}$. Mean chest, waist, and hip circumference $89.3 \pm 9.6,79.4 \pm 13.6 \mathrm{~cm}$, and $98.1 \pm 8.2$, respectively. At ultrasound measurement, biceps SFTT was $2.69 \pm 1.37 \mathrm{~mm}$ on the right and $2.73 \pm 1.42 \mathrm{~mm}$ on the left, triceps SFTT was $5.02 \pm 2.21 \mathrm{~mm}$ on the right and $4.91 \pm 2.11 \mathrm{~mm}$ on the left, subscapular SFTT was $3.18 \pm 2.0 \mathrm{~mm}$ on the right and $3.2 \pm 2.01 \mathrm{~mm}$ on the left, suprailiac SFTT was $8.25 \pm 3.99$ $\mathrm{mm}$ on the right and $8.28 \pm 3.93 \mathrm{~mm}$ on the left, iliac crest SFTT was $6.11 \pm 2.61 \mathrm{~mm}$ on the right and $6.07 \pm 2.52 \mathrm{~mm}$ on the left, upper abdomen SFTT was $13.09 \pm 7.09 \mathrm{~mm}$, lower abdomen SFTT $12.44 \pm 7.03 \mathrm{~mm}$, medial thigh SFTT was $9.25 \pm 3.62 \mathrm{~mm}$ on the right and $9.36 \pm 3.42 \mathrm{~mm}$ on the left, and calf SFTT was $5.89 \pm 2.09 \mathrm{~mm}$ on the right and $6.07 \pm 2.15 \mathrm{~mm}$ on the left. Biceps, triceps, iliac crest, medial thigh, and calf measurements differed significantly between men and women. The increase in SFT after the age of 30 was significant in arm, chest, waist and hip circumferences. Increased age was significantly correlated with weight and increased BMI. In conclusion sex, age and weight increase are significantly correlated with SFTT.

Keywords: Subcutaneous fat tissue, Ultrasound, Anthropometry

\section{P-152}

\section{Morphological features of florid cemento-osseous dysplasia}

Köse TE*, Özcan I**, Balcığlu HA ${ }^{*}$

*Department of Anatomy, Faculty of Dentistry, Istanbul University, Istanbul, Turkey; **Department of Oral Radiology, Faculty of Dentistry, Istanbul University, Istanbul, Turkey

Florid cemento-osseous dysplasia is an uncommon variant of the cemento-osseous lesions which is usually diagnosed by chance during routine examination. Diagnosis is solely by typical radiological and clinical findings and a biopsy procedure is 
S186 XXIV International Symposium on Morphological Sciences, 2nd-6th September, 2015, Istanbul, Turkey

principally not needed. The term 'florid' here describes the wide spread, extensive manifestations of the disease in maxilla and/or mandible. A few postulations have been made on the etiology of the condition, however, the cause of the disease remains still uncertain. It affects mostly mature black women, yet caucasians have been reported to be effected. Since differential diagnosis with chronic diffuse osteomyelitis, Paget's disease of bone, Gardner's syndrome and familial gigantiform cementoma is essential, and the radiographs can produce many trap images to be recognized, radio-anatomical features of florid cemento-osseous dysplasia become more of an issue. This presentation aims to deal with the morphological charactheristics of Florid cemento-osseous dysplasia along with a series of cases.

\section{P-153}

\section{Can we estimate body mass index from the wrist anthropometric measurements?}

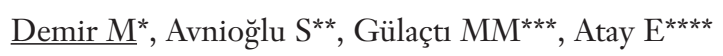

*Department of Anatomy, Faculty of Medicine, Sütçü Imam University, Kahramanmaraş, Turkey; **High Faculty of Health, Sütçü Imam University, Kahramanmaraş, Turkey; ***Vocational High Faculty of Health Services, Sütçü Imam University, Kahramanmaraş, Turkey; $* * * *$ Vocational High Faculty of Health Services, Kilis 7 Aralık University, Kilis, Turkey

In this study, the anatomic and physiological findings changes of the wrist (width, depth and circumference) and hand span lengths were established and correlated with certain parameters (e.g., sex, age and Body Mass Index (BMI)) in high Faculty students. This study has been realized on 189 students (44 (23.3\%) male and 145 (76.7\%) female). In this study, students hadn't got any complain or evidence of disease that could in volve the wrist and hand. The age of the students ranged from 17 to 28 years (mean age 19.2 years). Width and depth measurements of the wrist were obtained using a vernier caliper. Wrist circumference and hand span length measurements were taken with a soft tape measure. The average measurements of the male students were for BMI: $22.5 \pm 3.13 \mathrm{~kg} / \mathrm{m} 2$, circumference: $16.86 \pm 1.17 \mathrm{~cm}$, hand span length: $18.86 \pm 1.28 \mathrm{~cm}$, wrist width: $4.48 \pm 0.41 \mathrm{~cm}$, wrist depth:3.36 $\pm 0.40 \mathrm{~cm}$. Female students average measurements were for BMI: $22.43 \pm 3.28 \mathrm{~kg} / \mathrm{m}^{2}$, circumference: $15.56 \pm 0.88 \mathrm{~cm}$, hand span length: $16.45 \pm 1.29$ $\mathrm{cm}$, wrist width: $4.12 \pm 0.52 \mathrm{~cm}$, wrist depth: $2.99 \pm 0.33 \mathrm{~cm}$. We found that: correlation of wrist depth, width and circumference measurements with BMI is statistically very significant in all cases $(\mathrm{p}<0.01)$. Statistical significance is present in both sexes especially in overweight females the significance is much higher $(\mathrm{p}<0.001, \mathrm{r}=0.53)$. Circumference of the wrist had a positive corelation with depth and width measurements in both sexes $(p<0.01)$. When we evaluated the corelation of wrist width and depth with hand span length in both sexes. Wrist width showed a correlation with hand span length in all of the cases, but especially females had a much higher correlation. These results are a part of our continuing study. This study will be continued untill we get a large size of males and females with different BMI, for statistical analysis to represent the entire population. Keywords: Body Mass Index, wrist and anthropometric measurements

\section{P-154}

Cellular degeneration and apoptosis of the liver caused by chronic stress: an immunohistochemical study in a rat model

Özbek E**, Özbek A***, Demirci T*

*Department of Histology and Embryology, Faculty of Medicine, Erzurum, Atatürk University,Turkey; ${ }^{*}$ Department of Histology and Embryology, Faculty of Medicine, Sakarya University, Sakarya, Turkey; $* * *$ Department of Microbiology, Faculty of Medicine, Sakarya University, Sakarya, Turkey

Objectives: Nowadays, stress has become a part of our life. It causes to secrete glucocorticoids and catecholamines and also leads to both structural and functional changes in many organs. It has known that chronic stress triggers gastric ulcer, adrenal gland hypertrophy and disrupts the reproductive function. In this study, it was aimed to investigate the histopathological changes in the liver caused stress, formed using "chronic mild stress" (CMS) model in rats.

Methods: In our study, 4-5 week old twelve male rats were used. They were divided into two equal groups as control group (CG) and stress group (SG). During 12-week experimental period, rats were given standard rat chow. CG was not implemented any stress procedures, but SG was applied CMS procedure (applying some stressors such as immobilization, swimming in cold water and exposure to the noise in an order) the last 4 weeks of the study. At the end of the experiment, rats were sacrificed and their livers were removed. After routine histological procedure tissue samples were embedded in paraffin blocks. Sections were dyed with hematoxylin-eosin (HE) and were stained by the immunoperoxidase method using antiBax antibody for the purpose of detection of apoptosis. And they were examined with photomicroscope.

Results: While normal histological structure was seen in the livers of CG. In the HE stained sections of the livers of the SG, there were focally damaged areas exhibiting hepatocyte degeneration. Cytoplasmic swelling was seen in the damaged hepatocytes, and cell borders couldn't be distinguished. Additionally, in the SG, especially in the periportal areas, the hepatocytes with dark eosinophilic cytoplasm, pyknotic nuclei and apoptotic appearance were observed in abundance. In these cells, intensive Bax-positive immunostaining was observed. When compared with the CG, there were more apoptotic hepatocytes in the SG.

Conclusion: Our findings have shown that stress can cause liver damage and increased apoptotic activation in the hepatocytes. 


\section{P-155}

Histological effects of fluoxetine on intestinal tissue of zebrafish (Danio rerio)

Öztürk B* ${ }^{*}$, Güneş $S^{*}$, Alsaran $M^{*}$, Kaymak $G^{*}$, Kayhan FE**, Akbulut $C^{*}$, Yön ND*

*Sakarya University; **Marmara University

Fluoxetine is an antidepressant which generally used for major depressived is order, bulimianervos a (an eating disorder) obsessive- compulsived is order, panicd is order, and premen strualdy sphoricd is order. The bioavailability of fluoxetine is relatively high and peak plasma concentrations are reached in 6 to 8 hours. In our study, investigation the histopathological effects of fluoxetine on intestine tissue of zebrafish were aimed. Zebrafish were maintained under the standardized conditions at $28{ }^{\circ} \mathrm{C} \pm 1 \mathrm{C}$. The light / dark cycle was $14 \mathrm{~h} / 10 \mathrm{~h}$. In the study, one dose group (150 ng / L fluoxetine) and one control group were determined. After 48 hours of adaptation, fluoxetine were added to the aquarium. For the histological analysis, at the end of 15 minutes, 30 minutes, 4 days and 8 days intestine tissues were dissected. Tissues were fixed with $10 \%$ neutral buffered formalin fluid for $24 \mathrm{~h}$. Tissues were dehydrated and embedded in the parafin wax and sectioned at $5 \mu \mathrm{m}$ thickness and stained with Hematoxylin Eosin. The samples were evaluated by examining under the light microscope. In control group normal intestine histology was observed. Serosa, submucosa and mucosa layers were easily seen. In addition, laminapropria, villi and goblet cells were clearly monitored. In 15 and 30 minutes exposure groups, decrease in the number of goblet cells were detected when compared with control group. Expansion at villi structure were also observed. In 4 days exposure group, increase in the number of goblet cells towards to edge of villi were monitored. Degeneration were detected at villi structure. In 8 days exposure group, severe degeneration and displasia were observed at villi structure. Increase in the number of goblet cells were detected compared with other groups. Degredation were detected at cell boundaries were also monitored.

Keywords: Zebrafish (Danio rerio), fluoxetine, intestine, histology

\section{P-156}

\section{The effect of obesity on microscopic structure of the cornea}

\section{Özbek E ${ }^{* *}$, Demirci T* ${ }^{*}$, Salman $\dot{I}^{* * *}$}

*Department of Histology and Embryology, Faculty of Medicine, Atatürk University, Erzurum, Turkey; ${ }^{* *}$ Department of Histology and Embryology, Faculty of Medicine, Sakarya University, Sakarya, Turkey; $* * *$ Department of Eye Diseases, Faculty of Medicine, Atatürk University, Erzurum, Turkey

Objectives: Obesity is one of the most important and common health problems, and causes many disorders such as cardiovascular and metabolic diseases. It has been reported in a few stud- ies that feeding with cholesterol-rich diet can lead to lipid accumulation in the cornea and keratopathy. The aim of this study is to examine effects of obesity on the histological structure of cornea in rats fed a high fat diet .

Methods: Twelve adult male rats were divided into two equalsized groups. During twelve weeks, control-group (CG) was given a standard rat chow; fatty-diet-fed group (FDG) was fed a high-fat diet (30\% fat). The length and body weight of the animals were measured periodically. Body mass index (BMI) was calculated and recorded. At the end of the experiment, animals were sacrificed and their eyes were removed. One of them was embedded in paraffin after processing routine histological method; the other was embedded in Araldite ${ }^{\circledR}$ CY212 after \%3 glutaraldehyde and \% 1 osmium tetroxide fixation. Paraffin sections were stained Hematoxylin-Eosin stain and semi-thin sections were dyed toluidine-blue and were examined under a light microscope.

Results: At the beginning of the experiment, BMI values of CG and FDG were $5.723 \pm 0.232 \mathrm{~kg} / \mathrm{m}^{2}$ and $5.551 \pm 0.510 \mathrm{~kg} / \mathrm{m}^{2}$ respectively; at the end of the test $\mathrm{BMI}$ of them were $6.132 \pm 0.142 \mathrm{~kg} / \mathrm{m}^{2}$ and $6.701 \pm 0.415 \mathrm{~kg} / \mathrm{m}^{2}$ respectively. BMI of FDG was significantly higher than that of CG $(\mathrm{p}<0.01$; Mann Whitney-U). There were both intracytoplasmic and intranuclear vacuolar degeneration in the corneal epithelial cells. Picnotic nuclei and mitotic figures were observed in the basal layer of epithelium. In stromal layer of the cornea, edema and spaces found among the lamellar were seen. Descement membrane of obese rats were remarkably thicker when compared with the controls.

Conclusion: Our findings suggested that obesity can alter corneal structure by triggering cellular injury

\section{P-157}

Determination of GAT1 expression using immunocytochemical staining method on the stress-exposed rat sperm

\section{Demirci T $^{*}$, Özbek E**}

*Department ofHistology and Embryology, Faculty of Medicine, Atatürk University, Erzurum, Turkey; ${ }^{* *}$ Department of Histology and Embryology, Faculty of Medicine, Sakarya University, Sakarya, Turkey

Objectives: Gammaamino butyric acid (GABA) is a major inhibitory neurotransmitter and found in many tissues outside the central nervous system such as male genital tracts. GABA induces sperm acrosome reaction (AR). GABA uptake in to sperm, takes place via GABA transporters [GAT1, GAT2, GAT3 and GAT4] is located on the surface of sperm. GAT1 lead to sperm membrane depolarization and initiates the sperm AR. But, recent studies show that GAT1 over-expression can adversely affect the male reproductive system. In our study, we aimed to investigate whether stress may change GAT1 level in rat sperm.

Methods: Twelve male Sprague Dawley rats were randomly divided into two groups, stress-exposed and control 
S188 XXIV International Symposium on Morphological Sciences, 2nd-6th September, 2015, Istanbul, Turkey

(n=6/group). Stress group was exposed "Chronic-Mild-Stress" procedure for four weeks. At the end of the experiment, caudae epididymides were removed from the sacrificed animals. Then, they were minced in saline imidazole solution by scissors and the sperms were filtered. The obtained sperm suspension was used to prepare sperm smears. These smears were stained with immunoperoxidase method, in order to mark the GAT1 protein on the rat sperm at the light microscopic level. For each rat, 500 sperm were counted and the number of spermatozoa showing a positive immune reaction was recorded. The data were statistically compared between the groups.

Results: The number of GAT1-specific immunocytochemistry positive sperm in the stress-exposed group was significantly higher than the control group ( $\mathrm{p}<0.01$, Mann-Whitney U Test). Conclusion: According to our data, it has been concluded that stress increases the number of GAT1 protein (+) sperm, leading to male infertility.

\section{P-158}

\section{Morphological changes of the granulosa cells of mice under the of hexachlorocyclohexane (Lindane) influence}

$\underline{\text { Balmagambetova A }}^{*}$, Palmerine M.G**, Macchiarelli G**, Nottola SA ${ }^{* *}$, Zhurabekova $\mathrm{G}^{*}$

*Department of Normal and Topographical Anatomy, West Kazakhstan Marat Ospanov State Medical University, Aktobe, Kazakhstan; **Department of Life, Health and Environmental Sciences and Center of Electron Microscopies, University of L'Aquila, L'Aquila, Italy; $* * *$ Department of Anatomy, Histology, Forensic Medicine and Orthopaedics, Laboratory of Electron Microscopy "Pietro M. Motta", University La Sapienza, Rome, Italy

Lindane ( $\gamma$-hexachlorocyclohexane) is a persistent organic environmental pollutant. It has been widely used in agriculture as insecticide up to 2009, but still used as treatment for lices and scabies. Even if "moderately hazardous", in humans affects the nervous and immune systems, liver and kidneys. Lindane has toxic effects also on the reproductive systems, probably through endocrine-mediated mechanisms. In mice, Lindane induces embryo apoptosis, affects oocyte meiotic spindle morphology and impairs fertilization rate, even when used at very low concentrations. In this study, we evaluated the toxic effects of Lindane on mouse granulosa cells, matured in vitro with increasing concentration of Lindane. We examined structural and ultrastructural parameters connected to cellular, intracellular and intercellular morphology. Results showed a dose-dependant toxicity on granulosa cells, evidenced by reduction of microvillar extensions, reduction of cytoplasmic processes between adjacent cells, increase in the lysosomial compartment. Plasma membrane blebbing, apoptotic bodies and cellular debris increased with the dosage. No evident ultrastructural alterations were observed in mitochondria, endoplasmic reticula, Golgi apparatuses. These results showed a dose-dependant reproductive toxicity of Lindane on mouse granulosa cells, and should be taken into consideration in case of increased infertility and sterility connected to toxicant exposure.

\section{P-159}

What happens in pancreas morphology of the mice fed with high fat diet?

Ortadeveci A*, Topal F**, Peker $\mathrm{H}^{*}$, Aydar $\mathrm{Y}^{*}$, Yücel F* Peker $\mathrm{S}^{* * *}$

*Department of Anatomy, Faculty of Medicine, Eskişehir Osmangazi University, Eskişehir, Turkey; **Bilecik Şeyh Edebali Universty, Vocational High Faculty of Health Services, Bilecik \& Eskişehir Osmangazi University, Institute of Medical Sciences, Eskişehir, Turkey; $* * \star$ Department of Histology and Embryology, Veterinary Faculty, Uludağ University, Bursa, Turkey

Objectives: High fat diet is shown to deteriorate health condition in human and animals. The frequency of obesity and obesity-related disorders has been increasing throughout the world. Since it regulates carbohydrates, fat and protein metabolism, the pancreas is a crucial organ to carry out vital functions. The effects of obesity on the pancreas morphology have not been well studied. We planned to study the effects of high fat diet on the different morphological parameters of pancreas.

Methods: In the current study, we used female mice at age of eight weeks. The mice in experimental group fed with high fat for nine weeks while the mice in control group fed with control diet for nine weeks. The animals were anaesthetized and sacrificed by cervical dislocation. The pancreas was removed and processed with routine and standard paraffin embedding and serially sectioned in $5 \mu$ thickness. Then, every twentieth section was saved, stained with Crossman's triple stain. The sections were evaluated using unbiased stereological analyze methods. Length density of blood vessels per $\mu^{3}$ volume $(\mathrm{BVLv})$, volume fraction of connective tissue $(\mathrm{CTVv})$, volume fraction of blood vessels (BVVv) in exocrine pancreas and mean diameter of Langerhans islets in endocrine pancreas (MDLI) were stereologically counted.

Results: The body weight ( $\mathrm{p}=0.01)$ and the weight of adipose tissue ( $\mathrm{p}=0.001)$ of obese mice markedly increased when compared to those of the control mice. Mean diameters of Langerhans islets were not different between the experimental and the control groups $(\mathrm{p}=0.391)$. We observed that ' $\mathrm{BVVv}$ ' $(\mathrm{p}=0.042)$ and 'BVLv' $(\mathrm{p}=0.047)$ in exocrine pancreas decreased significantly in the experimental groups with respect to those of the control group. Moreover, we measured CTVv, but no difference was noted between the experimental and the control group $(\mathrm{p}=0.517)$.

Conclusion: Current findings suggest that high fat diet appear to affect several morphological parameters in the pancreas.

\section{P-160}

Growth impairment of renal and hepatic parenchymal cells in cathepsin D-deficient mice

Suzuki C $^{*}$, Yamaguchi J1, Shibata $M^{* * *}$, Uchiyama $Y^{* *}$

*Department of Cell Biology and Neuroscience, ${ }^{* *}$ Cell and Molecular Neuropathology, Juntendo University Graduate Faculty of Medicine, Tokyo, Japan, ${ }^{* *}$ Department of Morpohlogical Science, Kagoshima University Graduate. Faculty of Medicine, Kagoshima Japan 
Cathepsin D (CD) is a principal lysosomal aspartate proteinase and expressed in almost all tissue cells, in which the expression level varies considerably. Mice deficient in CD, generated by gene targeting, develop normally during the first 2 weeks, stop thriving in the third week and die in a state of anorexia or suffocation due to small intestinal necrosis or convulsive seizure at day $26 \pm 1$. An atrophy of the ileal mucosa first observed in the third week progresses towards widespread intestinal necrosis accompanied by thromboemboli. From these results, Saftig et al. (1995) suggested that vital functions of CD are exerted by limited proteolysis of proteins regulating cell growth and/or tissue homeostasis. However, the mechanism of growth disorder caused by CD deficiency is still unknown. We therefore investigated the relationship between CD deficiency and cell proliferation by using CD-knockout (CD-KO) mice. Positive immunoreactivity for ki67, a cellular marker for proliferation, was significantly less in number in renal tubular epithelial cells and hepatocytes of CD-KO mice at postnatal day (P) 18 than in those of wild-type (WT) littermate mice. Expression levels of p21 in CD$\mathrm{KO}$ mouse liver after p23 were significantly more increased than in control liver. Moreover, SA-,-galactosidase activity levels and the number of TUNEL-positive cells appeared high in CD-KO mouse livers. These results suggest that $\mathrm{CD}$ deficiency causes cell cycle arrest and early senescence in mouse liver and kidney.

\section{P-161}

An optimisation attempt for fiber dissection technique

Ilgaz $\mathrm{HB}^{*}$, Sargon $\mathrm{MF}^{*}$, Tatar I*, Urfalı B**, Özkan $\mathrm{S}^{* * *}$

*Department of Anatomy, Faculty of Medicine, Hacettepe University, Ankara, Turkey; **Department of Neurosurgery, Faculty of Medicine, Mustafa Kemal University, Hatay; *** Scientific and Technological Research Labs, Faculty of Medicine, Kırıkkale University, Kırıkkale, Turkey

Objectives: White matter fiber dissection technique is an important method for gaining neuroanatomic knowledge in surgical practice. Previous studies have defined and revisited the structures of the brain with their connections. In our study the object is to optimize the preparation processes of fiber dissection technique.

Methods: 108 sheep brain hemispheres were included in the study. We fixed at the same count (27) of the brains with \% 1 , $\% 5, \% 10$ and $\% 20$ formalin solution for $1,2,4$ and 8 weeks. And all of them were frozen at $-16^{\circ} \mathrm{C}$ for about $1,2,4$ and 8 weeks. We repeat this process for about 1,2 and 4 times. After that we dissected the brains from the medial aspect to take samples from corpus callosum. At the end we examined them with the microscopy to compare the differences about the counts of the nerve fibers. The technique is based on the expansion of water crystals to facilitate the separation of the fiber tracts.

Results: After freezing and thawing the axons of the nerve fibers swelling and inflating. Because of this there are no axons in the samples. Only the nuclei of the cells have seen. Counting the nuclei and the assessment the expansion of fibers in all groups would compared in the study.
Conclusions: The fiber dissection technique, although complex and time consuming, offers a three-dimensional knowledge of intrinsic brain anatomy. The present anatomic study provides saving time and quality for preparation of this technique.

\section{P-162}

The study of undifferentiated and differentiated gene expression in neonatal mouse spermatogonia stem cells followed by coculture with Sertoli cells

Khanehzad $M^{*}$, Abolhasani F* Koruji SM**, Kashani IR*, Fereshteh A*, Mohamadi $\mathrm{Y}^{*}$

*Department of Anatomy, Faculty of Medicine, Tehran University of Medical Sciences, Tehran, Iran ; **Department of Anatomy, Faculty of Medicine, Iran University of Medical Sciences, Tehran, Iran

Objectives: Spermatogenesis is a complex and highly organized process of proliferation and differentiation of spermatogonia stem cells (SSCs). SSCS with potential of self-renewal and differentiation, have a key role in the transfer of genetic material to the next generation. So they are unique among the different stem cells, and are important in public health. given the widespread use of SSCs, particularly in the treatment of infertility, creating a optimal environment for the prolifration of SSCs is very important.

Methods: After a two-step enzymatic digestion, SSCs were isolated from 6-3-day-old mice. Purity was assessed by flow cytometry and PLZF marker. Sertoli cells were separated using lectin-coated dishes and were confirmed by immunocytochemistry with vimentin marker. SSCs were placed in two groups 1control, 2- co-culture with Sertoli cells. undifferentiated (ID4) and differentiation (c-Kit) gene expression were evaluated by Real Time PCR technique.

Results: SSCs purity was obtained above 90\%. ID4 gene expression showed a significant increase toward the control group, while in the expression of c-Kit was observed a significant decrease compared with the control group at the end of each week.

Conclusion: Coculture with sertoli cells Maintains SSCs in the prolifration stage for long term, so can be used to optimize the culture medium at the clinic.

Keywords: Coculture, spermatogonia stem cells, sertoli cell

\section{P-163}

The anatomy of the fovea capitis femoris

Perumal V**, Woodley $\mathrm{S}^{* * *}$, Nicholson $\mathrm{H}^{*}$

*Department of Anatomy, Otago Faculty of Medical Sciences, University of Otago, New Zealand; **Department of Anatomy, Otago Faculty of Medical Sciences, University of Otago, New Zealand; $* * *$ Department of Anatomy, Otago Faculty of Medical Sciecnes, University of Otago, New Zealand

There is an ongoing debate about the clinical importance of the ligament of the head of the femur (LHF); whether it deliv- 
ers significant blood supply to the femoral head or contributes to the stability of the hip joint. However, there is little published information regarding the attachment sites of the LHF. This study explores the morphology of the femoral attachment, the fovea capitis femoris. Morphological observations and morphometry were performed on 125 dry isolated femora (61 right, 64 left) from the University's Anatomy Museum. All femora had a single prominent fovea capitis. The proximal part of the fovea had a rough floor indicating the attachment of LHF, while the distal part, the receptacle zone, was smooth. In 123 of 125 bones the fovea was located on the postero-inferior quadrant of the femoral head. The mean longitudinal and transverse diameters of the fovea were $1.77+0.4 \mathrm{~cm}(\mathrm{SD})$ and $1.38+0.3 \mathrm{~cm}$ (SD) respectively. The mean foveal area was $1.81+0.7 \mathrm{~cm}^{2}$ which represented $17 \%$ of the surface area of the femoral head. The shape of the fovea was oval in $73.6 \%$, circular $20 \%$ or triangular $8 \%$. The fovea was directed postero-inferiorly in $63.2 \%$, horizontally in $13.6 \%$ and antero-inferiorly in $9.6 \%$ of the femora and the mean angle of foveal inclination i.e. its deviation from the vertical plane was $70.9^{\circ}$. Multiple vascular pores were evident within the ligament attachment site in $76 \%$ of fovea, with pores commonly located centrally in this area. This study shows that the fovea consistently lies in the postero-inferior aspect of the femoral head, is usually oval with the attachment of the LHF located in the proximal part. The presence of patent vascular foramina within the LHF attachment site suggests that the ligament is able to provide some vascular supply to the head of the femur.

\section{P-164}

\section{The sphenoid emissary foramen}

\section{$\underline{\text { Parra JED }}^{*}$, Ríos JB**, Martínez DMS**}

*Universidad Autonoma De Manizales \& Departamento De Ciencias Basicas, Universidad De Caldas, Manizales, Colombia;

**Departamento De Ciencias Basicas, Universidad De Caldas, Manizales, Colombia

Objectives: The objectives were to quantify the presence of human venous foramen in a population sample of dry skulls and quantifying the values of the diameters thereof.

Methods: Based on the evaluation of 80 adult skulls of the Autonoma University of Manizales and Caldas University Colombia) with intact middle cranial fossae, the calvaria was withdrawal and inspecting the study area lateral to the sella turcica, ssessing the presence of foramina medial to foramen ovale neighborhood. Nylon tube was inserted through bore $0.25 \mathrm{~mm}$ and $20 \mathrm{~cm}$ length to notice continuity between the sphenoidal emissary foramen basal to the region of the posterior region of the scaphoid fossa or the surrounding trench, determining the course coinciding with emissary vessels.

Results: Of the 80 skulls studied, 37 of them had no foramen (46.25\%), 43 had it (53.75\%). Among the latter group, 27 skulls had bilaterally (64.2\%) and 15 unilaterally (35.71\%); 7 in the right (16.66\%) and 8 on the left (19.04\%). The diameters of the sphenoidal emissary foramen ranging from $1 \mathrm{~mm}$ the smallest to $0.5 \mathrm{~cm}$ greatest.

Conclusion: Studies made in Chile, with skulls of the second millennium B.C show the lowest frequency, only about $8 \%$ compared to the current studies, which may be the evolutionary result of increased cerebral specialization. According to our study, the incidence is higher bilateral than unilateral. Other studies also reported a mostly bilateral foramen incidence coincides with our work, as in the case of Kodama et al., 1997, which reports bilateral frequency of $16.5 \%$ compared to $5.5 \%$ of unilateral. Kale et al, 2009, found bilaterally $25.1 \%$ with a $19.9 \%$ one-sided.

\section{P-165}

\section{Canal for abducens nerve: a constancy in human gross anatomy}

$\underline{\text { Parra JED }}^{*}$, Ríos JB**, Zuluaga JM*

*Universidad Autonoma De Manizales \& Departamento De Ciencias Basicas, Universidad De Caldas, Manizales, Colombia;

**Departamento De Ciencias Basicas, Universidad De Caldas, Manizales, Colombia

Objectives: Quantify the constancy or inconstancy of the abducens nerve canal in humans and referral according to the results obtained proposal or refusal of the inclusion of this term in the Terminologia Anatomica.

Methods: 70 dry skulls full adult and six heads fixed with formalin $10 \%$ were selected without classifying them by ethnicity or gender. As inclusion criteria, were selected those who had in good condition the middle cranial fossa and the apex of the petrous temporal region.

Results: 55 skulls (85.94\%) showed the presence of canal bilaterally; $4(6.25 \%)$ showed it unilaterally to the right; 3 (4.68\%) were unilaterally at the left and $2(3.12 \%)$ had no canal.

Conclusion: The canal for abducens nerve referring to a small fibro osseous landmark, located at the apex of the petrous temporal region and for which the nerve abducens passes lying before reaching the cavernous sinus. This landmark is not referenced in the Terminologia Anatomica, even as fickle structure. In the literature are not many references to this specific area and even less detailed descriptions Dorello's canal, giving to understand it as if the canal does not exist, and the Terminologia Anatomica book not make mention of this fibro osseous landmark. Despite having been described in detail by Dorello in 1872, the few authors who referenced denominate the eponymous Dorello's canal. However, the data emerging from our study indicate that there is high constancy of this fibro-osseous canal located at the apex of the petrous temporal region, and that in most studied skulls presents bilaterally. It is necessary to confirm our results with other studies involving a larger sample, in order to corroborate this finding, and in those taking into account ethnicity and sex, and include this not ref- 
erenced foramen in the Terminologia Anatomica, and propose the term canal abducens nerve to avoid using.

\section{P-166}

The determination of correlation between stature and upper limb and hand measurements in Iranian adults

Mahakizadeh S, Moshkdanian G, Ghoroghi FM, Mokhtari T, Hassanzadeh $\mathrm{G}$

Department of Anatomy, Tehran University of Medical Sciences (TUMS), Tehran, Iran

Objectives: Estimation of stature is an important issue, which is significantly considered in forensic anthropology. It will be difficult to predict the identification of an individual when only some parts of dead body are discovered following disasters or criminal events. The aim of this study was to assess the relationship between stature and upper limb and hand length in Iranian adults to generate regression formulae for stature estimation.

Methods: Three anthropometric measurements; Stature, Upper Limb Length (ULL) and Hand Length (HL) were taken on the subjects, comprising 142 male students (18-25 years) using standard measuring instruments. The data were analyzed using SPSS 16. Then linear regression models were used to estimate stature.

Results: The results indicated a positive correlation between stature and upper limb and hand measurements. The correlation coefficient with upper limb length was $r=0.89 \& \mathrm{p}=0.0001$ and with hand length was $r=0.78 \& \mathrm{p}=0.0001$.

Conclusion: In conclusion, we found the strong correlation between stature and upper limb and hand length. The regression analysis also showed that the Upper Limb Length give better prediction of stature in compare to Hand length measurements.

Keywords: Stature, upper limb length, hand length, regression equation, forensic anthropology

\section{P-167}

Comparative anthropometric analysis of facial dimensions and shapes in Qazvin, Iran and Dera Ghazi Khan, Pakistan residents

Yousefi P, Hassanzaded G

Department of Anatomy, Tehran University of Medical Sciences (TUMS), Tehran, Iran

Objectives: This study sought to assess facial dimensions and determine the dominant face shape among adults residing in Qazvin and DG Khan.

Methods: In this cross-sectional study, a total of 300ethnic populations of Qazvin and 365 ethnic populations of DG Khan in the age range of 18-55years were randomly selected and evaluated. Facial length and breadth, nasion-prosthion height, upper facial index, total facial index, facial type and profile were evaluated. Descriptive statistics were used to determine the frequency. The t-test was applied to compare the mean values between males and females in Qazvin and DG Khan.

Results: The mean facial height in Qazvin males and females was longer than that of males and females in DG Khan; whereas, the mean facial breadth in both genders in DG Khan was greater than in Qazvin. The mean total facial index was $102.88 \pm 10.28$ in Qazvin males and 96.69 \pm 7.67 in Qazvin females. These rates were $90.55 \pm 7.6$ and $87.87 \pm 5.8$ in males and females of DG Khan, respectively. The most and the least common facial types in Qazvin were Hyperleptoprosopic and Euriprosopic, respectively; whereas, Leptoprosopic and Mesoprosopic were the dominant facial types among males and females of DG Khan. The dominant facial profile in Qazvin and DG Khan was orthognathic while the least common profile in both locations was prognathic. The difference in total facial index between males of Qazvin and DG Khan was statistically significant $(\mathrm{p}<0.005)$. This index was significantly different between Qazvin and DG Khan females as well $(\mathrm{p}<0.005)$.

Conclusion: Facial dimensions and shape are different among Qazvin and DG Khan ethnic residents and may be influenced by several factors such as age, sex, race and bioenvironmental factors. Facial index in both genders in Qazvin was significantly greater than that in populations of other countries and may be due to the long facial height and low facial breadth in this area.

Keywords: Facial index, facial profile, anthropometry, Iran, Pakistan

\section{P-168}

The effect of integrated teaching method on learning anatomy course in medical students of Guillaan University of Medical Sciences

Faghani $M^{*}$, Mesbah $M^{* *}$

*Anatomical Sciences Department, Faculty of Medicine, Guilan University of Medical Sciences, Rasht, Iran; **Faculty of Medicine, Guilan University of Medical Sciences, Rasht, Iran

Objectives: Due to the importance of teaching anatomy for medical students, instructors are always looking for employing appropriate educational practices to promote effective learning. This study investigated the impact of the two integrated and traditional approaches on learning anatomy in a practical course.

Methods: In this hemi experimental study, 30 medical students from the third semester of Guillan University of medical sciences were randomly divided in two experimental (15) and control (15) groups. In order to assess their information, a pretest was taken. Students were taught through traditional (lectures and practical work on cadaver) and integrated method (lectures, practical work on cadaver and educational CD).A post test was taken at the end of semester. Data analysis was done using SPSS (version 16) and T-test.

Results: The average scores of pre and post tests in traditional group were 9.75 and 12.66 and in integrated group 8.76 and 
16.42 respectively. There were no significant difference between pretest scores of traditional and integrated groups; however, a significant difference was observed in posttest scores $(\mathrm{p}<0.05)$.

Conclusion: Considering the effectiveness of active learning style, using various integrated methods like educational $\mathrm{CD}$ in teaching academic courses like anatomy is recommended.

\section{P-169}

Anatomic description of the apparent origin of the plexus braquial of the Cerdocyon thous: a preliminary study

$\underline{\text { Parra JED }}^{*}$, Giraldo JC**, Vélez GJF***

*Universidad Autonoma De Manizales \& Departamento De Ciencias Basicas, Universidad De Caldas, Manizales, Colombia; **Departamento De Ciencias Basicas, Universidad De Caldas, Manizales. Colombia; ***Departamento De Salud Animal, Facultad De Ciencias Agropecuarias, Universidad De Caldas En Convenio Con Corpocaldas, Manizales, Colombia

The Cerdocyon thous, also known as forest fox is a native Colombian species which is widely distributed throughout South America, whose nervous system anatomy has been subject to very few studies. This work includes an anatomical description of the apparent origin of the species' brachial plexus and also conducts, a comparative anatomy study against other mammals describing their similarities and differences. From the methodological viewpoint, five ethically-sourced animals from the wildlife care and assessment centers of CORPOCALDAS were used. They underwent a superficial to deep ventral dissection in the cervicothoracic region, to view the intervertebral foramina and establish the contribution of spinal nerves to the formation of the brachial plexus, together with the origin of the nerves that branch out there from. This research is the basis for subsequent functional studies of this species' brachial plexus and its applications in Medicine, surgery and Anesthesiology. It also becomes a tool for phylogenetic studies based on Comparative Anatomy. This study concludes that there are similarities between domestic dogs (Canis familiaris) and Cerdocyon thous as to the origin of the brachial plexus, its distribution and innervation, emphasizing the participation of the $\mathrm{T} 2$ in something brachial plexus, spinal segment as well as the absence of C5.

\section{P-170}

Quantitative assessment and description of apparent origin of glossopharyngeal, vagus and accessory nerve in the human brain stem

$\underline{\text { Parra JED }}^{*}$, Blanco YQ**, Pineda $\mathrm{EP}^{* * *}$, Ríos JB ${ }^{* * * *}$

*Universidad Autonoma De Manizales \& Departamento De Ciencias Basicas, Universidad De Caldas, Manizales, Colombia; **Departamento De Anatomía, Facultad De Medicina, Universidad De Ciencias Aplicas Y Ambientales, Bogotá, Colombia; ***Departamento De Anatomía. Facultad De Medicina. Universidad De Ciencias Aplicas Y Ambientales, Bogotá, Colombia; ****Departamento De Ciencias Basicas, Universidad De Caldas, Manizales. Colombia
Objectives: To establish the exact site of origin in the human brainstem of the glossopharyngeal, vagus and accessory nerves.

Methods: 80 human brain stems intact without gross pathologies or deformations of the Caldas and Cundinamarca (Colombia) departments, that had been fixed in formalin solution $10 \%$; they were evaluated. Meninges were removed to see the exact location of the emergency of these nerves in the medulla oblongata. The results were compared with reports of the literature consulted. In $100 \%$ of the human brain stems, found bilaterally to the glossopharyngeal, vagus, and accessory nerves emerge between 1.6 and $3.8 \mathrm{~mm}$ posterior to the retrogrove, matching with a work made on 68 brainstems as reported in the majority of the literature. Therefore, it is confirmed that the apparent origin of the glossopharyngeal, vagus and accessory nerves in humans, are in the medulla oblongata behind the retro-olive groove at a distance between 1.6 and 3.8 $\mathrm{mm}$ of the same retroolivary, not in the groove.

\section{P-171}

\section{Prevalence of pneumatized uncinate process and accompanying neighboring variations}

Yenigün $A^{*}$, Gün $C^{* *}$, Uysal İI***, Keleş MK****, Nayman $A^{*}$

*Department of Otorhinolaryngology, Faculty of Medicine, Bezmialem Vakıf University, Istanbul, Turkey; **Department of Radiology, Selçuklu Faculty of Medicine, Selçuk University, Konya, Turkey; $* * *$ Department of Anatomy, Selçuklu Faculty of Medicine, Selçuk University, Konya, Turkey; $* * * *$ Plastic and Reconstructive Surgery Clinic, Numune Hospital, Konya, Turkey

Objectives: The uncinate process of the ethmoid bone, in normal physiological conditions, plays a role in regulating the aeration and drainage of the sinuses. We sought to investigate the prevalence of uncinate process pneumatization and also to investigate the association of uncinate process pneumatization with concurrent morphological variations in neighboring structures and the presence of maxillary sinusitis.

Methods: This was a retrospective study where coronal computed tomography (CT) scans of 1500 uncinate processes in 750 patients were examined to determine the prevalence of uncinate process pneumatization and to assess any concurrent neighboring anatomical structures and the presence of maxillary sinusitis.

Results: Uncinate process pneumatization was identified in a total of $6.26 \%$ of our patients with $1.60 \%$ being bilateral, $2.53 \%$ on the right only, and $2.13 \%$ on the left side only. Other than concurrent occurrences of right maxillary sinus septa ( $\mathrm{p}=0.046)$, growth of the right ethmoidal bulla $(\mathrm{p}=0.044)$ and presence of maxillary sinusitis (right side: $\mathrm{p}=0.046$, left side: $\mathrm{p}=0.035$ ) were seen.

Conclusions: We detected a $6.2 \%$ prevalence of uncinate process pneumatization in our study group. An abnormally sized and over- pneumatized uncinate process can cause narrowing of the infundibulum and impaired sinus drainage. Such 
functional blockage can lead to recurrent maxillary sinusitis, stuffiness, and decreased olfaction.

Keywords: Paranasal sinus, uncinate process, pneumatized, sinusitis, computed tomography

\section{P-172}

The anatomical basis of the total pelvic exenteration

Nicolae $B^{*}$, Claudia $S^{* *, * * *}$, Radu $S^{* * *}$

*Department of Obstetrics and Gynecology, Clinical Hospital Cantacuzino Bucharest, University of Medicine and Pharmacy "Carol Davila" Bucharest, Romania; **Department of Anatomy- Clinical Anatomy and Surgical Techniques, University of Medicine and Pharmacy "Carol Davila" Bucharest, Romania; ***General Surgery Department, County Hospital Ilfov, Bucharest, Romania

Total pelvic exenteration should be regarded as a salvage intervention being the only curative option in loco-regionally advanced pelvic cancer and in pelvic tumor recurrences. The procedure involves "en bloc" resection of the internal female genital organs, total cystectomy and rectosigmoidectomy. From the first total pelvic exenteration carried out in 1948 by Brunschwig, the 5 year survival rate increased to about $50 \%$ due to improved therapeutic means (intensive care unit, oncology critical care) and to modern surgical techniques. The operatory steps are unfolded after an anatomical algorithm and every operatory step is based on an anatomical correspondent. These structures may represent either an important anatomical landmark or a "holy" space that allows tumor approach. The loco-regionally advanced pelvic tumor realizes a change in the normal pelvic anatomy due to its invasion of the avascular spaces and of the adjacent structures. Considering these, our work's purpose is to show the anatomical basis of each operatory step through images taken during total pelvectomy in a group of patients with pelvic cancer (loco- regionally advanced gynecological and rectal cancer). The knowledge of the surgical pelvic anatomy allows a curative tumor resection with good vascular pedicle control and digestive and urinary reconstruction.

Keywords: Total exenteration, anatomical landmark, operatory steps

\section{P-173}

The surgical importance of the paraaortic lymph node dissection in oncogynecological surgery

Nicolae B*, Claudia $\mathrm{S}^{* *, * * *}$, Radu $\mathrm{S}^{* *}$

*Department of Obstetrics and Gynecology, Clinical Hospital Cantacuzino Bucharest, University of Medicine and Pharmacy "Carol Davila" Bucharest, Romania; **Department of Anatomy-Clinical Anatomy and Surgical Techniques, University of Medicine and Pharmacy "Carol Davila" Bucharest, Romania; ** *County Hospital Ilfov - General Surgery Department, Bucharest, Romania

The para-aortic lymph node dissection represents an important and complementary surgical step during the surgical treatment for gynecological neoplasia. This contributes to a good staging of the disease and subsequently to the oncological therapeutic measures. For this reason, we have to perform an intraoperative dissection with very high accuracy based on anatomical knowledge of the retroperitoneal space. The "sharp dissection" is useful for removing the aortocaval lymph nodes and also for avoiding vascular injury. The aim of this dissection is to remove the all lymphatic tissue around the great vessels that may be a metastatic site in gynecological neoplasia. Considering this, the aim of this paper is to emphasize upon the intraoperative images, the anatomical landmarks and the surgical maneuvers that are useful for a good exposure of the aortocaval area and for a safe dissection. We used a group of patients with cervical cancer with various staging grades. The surgical technique performed was radical total hysterectomy. According to the oncogynecological surgery principles, the aortocaval lymph node dissection consists of removing of all nodal tissues. The uppermost limit is located to origin of the inferior mesenteric artery and the reflection of the duodenum, the inferior limit is the mid-portion of the common iliac artery, and the lateral limit is represented by the ureters. The final intraoperative result is given by the anatomical view of the retroperitoneal space and its landmarks and the postoperative result is reflected in the histopathological examination that holds prognostic value.

Keywords: aortocaval lymph nodes, retroperitoneum, gynecological cancer

\section{P-174}

\section{Variations in flow and branching of anterior and middle cerebral artery}

$\underline{\text { Kulenovic A }}^{*}$, Hadzihalilovic AS ${ }^{* *}$, Hasanovic A*** Spahovic $\mathrm{N}^{* * * *}$

*Department of Anatomy, Medical Faculty Sarajevo, University of Sarajevo, Sarajevo, Bosnia \& Herzegovina; **Department of Anatomy, Medical Faculty Sarajevo, University Sarajevo, Sarajevo, Bosnia \& Herzegovina; ${ }^{* \star}$ Department of Anatomy, Medical Faculty Sarajevo, University Sarajevo, Sarajevo, Bosnia \& Herzegovina; $* * * *$ Department of Radiology, University Clinical Center Sarajevo, Sarajevo, Bosnia \& Herzegovina

Objectives: We have investigated variations in flow caliber and branching anterior and middle cerebral artery, which supplies anterior two thirds of external and medial side of cerebrum and associated subcortical structures.

Methods: The examination were performed on 20 human brains using injection-corrosion method.

Results: While studying specimens, we observed that internal carotid artery constantly bifurcates, and gives anterior and middle cerebral artery. Precommunical segment of anterior cerebral artery has variable appearance. In $65 \%$ of cases, this segment is arch shaped with convexity anteriorly and laterally, and in $44 \%$ of cases it is straight and oblique in direction. In $1 \%$ of cases, precommunical segment of anterior cerebal artery has corrugated course. Anterior communical artery, in 1\% of cases, gives third pericallosal artery which is smaller than the 
other pair of pericallosal arteries. Initial segment, (sphenoidal parts), of middle cerebral artery displays usual course with average length of $2.5 \mathrm{~cm}$ in its. Terminal part of sphenoidal segment of this artery bifurcates in $70 \%$ of cases and in $30 \%$ of cases trifurcate occurs on our specimens. Insular segment of the middle cerebral artery branches into several segments which are narrower. When we studied our specimens we found one rare variation branching from middle cerebral artery where it bifurcated into anterior smaller and posterior larger branch, than they divide into multiple smaller branches in periinsular segment.

Conclusion: The knowledge of variations in flow, number and branching brains arteries is not only anatomical fact. They also represent a significant clinical fact especially in radiology and neurosurgery and help establishing a correct diagnosis and therapy.

Keywords: Brain, anterior cerebral artery, middle cerebral artery, variation

\section{P-175}

Morphological changes of the temporal lobe structures in schizophrenia and their link to the clinical symptomps: a comparative brain segmentation study

Elfaki A*, Ali TO**, Elsheikh A**, Güdek MA*, Gölpınar M*$^{*}$, Şahin B*

*Department of Anatomy, Faculty of Medicine, Ondokuz Mayıs University, Samsun, Turkey; **Departments of Anatomy and Psychiatry, Faculty of Medicine, National Ribat University, Burri 55 Khartoum, Sudan

Introduction: Temporal lobe dysfunction provides a model of the cognitive impairments observed in schizophrenia.

Objectives: The aim of this study was to evaluate changes in grey and white matter volume, cortical areas and cortical thickness of the temporal lobe in schizophrenic patients and to explore the functional significance of these changes through correlation with clinical symptoms of patients as measured with the positive and negative symptoms scale.

Methods: 88 control subjects and 57 chronic schizophrenics were participated in the study. Structural magnetic resonance imaging was performed and the DICOM images were evaluated using automatic brain segmentation software (BrainSuite). The volume and volume fraction of the region of interest were evaluated.

Results: The mean volume of temporal lobe, temporal grey and temporal white matter in controls and schizophrenics were $\left(174.85 \pm 17.70 \mathrm{~cm}^{3}\right.$ and $\left.154.20 \pm 16.49 \mathrm{~cm}^{3}\right),\left(124.14 \pm 13.15 \mathrm{~cm}^{3}\right.$ and $\left.110.41 \pm 12.25 \mathrm{~cm}^{3}\right)$ and $\left(50.71 \pm 6.29 \mathrm{~cm}^{3}\right.$ and $43.81 \pm 4.96$ $\mathrm{cm}^{3}$ ), respectively, however, the volumes of these structures were less in schizophrenics than that of the controls $(\mathrm{p}=0.000)$. The cortical areas of the temporal lobe of schizophrenics were less than that of controls $(\mathrm{p}<0.050)$. The male patients tend to have significant positive correlation between the volume of the temporal lobe and its structures and the positive symptoms.
Conclusion: The present study suggests that patients with schizophrenia have a generalized temporal deficit. Changes in specific areas may influence specific symptoms. Temporal grey and white matter disruption may play a crucial role in dysfunction of auditory, language processing, memory and in cognitive deficits of schizophrenics.

\section{P-176}

Distribution patterns of the median nerve in the superficial muscles of the anterior compartment of the forearm in Brazilian individuals

Olave E, Riveros A

*Universidad De La Frontera, Temuco, Chile; **Universidad San Sebastian, Concepcion, Chile

The median nerve is responsible for the innervation of the muscles of the superficial layer of the anterior compartment of the forearm, for it, originates along its course in the arm and forearm, branches to these muscles. Some branches originated from common trunks $(\mathrm{Ct})$. In order to obtain an anatomical and biometric description of the origin and distribution of the branches of the median nerve in the superficial muscles of the forearm: pronator teres $(\mathrm{Pt})$, palmaris longus (PL), flexor carpi radialis (FCR),flexor digitorum superficialis (FDS) and classify these branches (Br) according to their origin, 30 upper limbs of corpses of Brazilian adult fixed in $10 \%$ formaldehyde were studied, performing dissections and recording its origins in relation to the biepicondilar line (BEL). With the results obtained, a classification of the branches to the muscles of the superficial layer of the anterior compartment in 4 patterns (IA, IB, II, III, IV), including in the distribution analyzed the origin of the anterior interosseous nerve (AIN) was performed. In the pattern IA (29\%), branches originated from proximal to distal in the following sequence: CtPT, PL, FCR, AIN, Br1FDS, Br2FDS; in pattern IB (41\%) showed CtPT, TcFCR and PL, AIN, Br1FDS, Br2FDS; in pattern II (4\%) was CtPT and FCR, PL, AIN, Br1FDS, Br2FDS, Br3FDS; in pattern III (11\%) it was CtPT, Ct FCR and PL, AIN, Br1FDS, Br2FDS, Br3FDS and in pattern IV (15\%) was Br1Pt, Br2Pt, CtFCR and PL, Br1FDS, AIN, Br2FDS. In type pattern IB, the first muscle to receive branches from the median nerve in the forearm was the PT, who in $53 \%$ of cases originated from a common trunk. The PL was the second to be innervated, leaving third place to FCR, which $50 \%$ were supplied by a common trunk. The results are a contribution to the knowledge of the innervation of the muscles of the anterior compartment of the forearm.

\section{P-177}

\section{Morphological and biometric characteristics of the pectinate muscles of the right atrium in human heart}

Henriquez I*, Barriga A**

*Facultad De Medicina, Universidad De La Frontera, Temuco, Chile; **Programa De Magister En Ciencias Morfologicas. Universidad De La Frontera, Temuco, Chile; $* \star \star$ Profesor Programa De Magister En CS. Morfologicas, Universidad De La Frontera, Temuco, Chile 
Atrial structures are important in the cardiac interventions using percutaneous transcatheter procedures. Understanding their places and components helps reduce the risk of damage related to the procedure. The general arrangements of muscle fibers that make up the pectinate muscles are reviewed to provide a morphological basis for leading atrial and arrhythmias potential substrates. The right atrium is characterized of the pectinate muscles which are muscle aridges extending from the terminal crest anterolaterally to the lobby of the right atrioventricular foramen and into the atrium. These muscle fibers can take different directions, which mean that its length is variable. The purpose is to determine the distribution patterns, variations and length pectineal muscles in the right atrium. The dissection was a formed right atria 30; we proceeded to open its rear wall leading her side. This is kept open and the through a thin cooper wire is able to measure the length of the fibers do not maintain a straight line, we also measured the thickness of these muscles. We proceeded to take three measures pectineal muscles in each atrium. Since its length and thickness of them was variable in different areas of the atrium. The results showed four patters of distribution in the pectinate muscles which are perpendicular of the crista terminalis, Type I (65\%), Trabecular rborizados and Type II (15\%) without trabeculae and uniforms, Type III (15\%) special arborizados TS, Type IV (15\%) without trabeculae and uniforms. The fiber length varied between 32,95 to $37.32 \mathrm{~mm}$ and the thickness varied between 0.4 and $3.0 \mathrm{~mm}$. The atrium had different Type of distributions of the pectinates muscle. The more high of distribution patron is related with of the Type I.

\section{P-178}

\section{Morphometric studies of the nutrient foramen in lower limb long bones of adult black and white south Africans}

\section{Mazengenya $\mathrm{P}^{*}$, Fasemore MD**}

*Faculty of Anatomical Sciences, Faculty of Health Sciences, University of The Witwatersrand, Johannesburg, South Africa; ** Faculty of Anatomical Sciences, Faculty of Health Sciences, University of The Witwatersrand, Johannesburg, South Africa

The present study analysed the direction, number, location and position of the nutrient foramina in 1080 lower limb long bones of 20th century adult black and white South Africans. In each population 90 complete skeletons were used resulting in 360 femora, 360 tibiae and 360 fibulae being analysed. The majority of the nutrient foramina pointed away from the growing end of the diaphysis in the lower limb bones with a few pointing in the opposite direction in both black and white populations. A single nutrient foramen was common on the shafts of the tibia and fibula in both populations while in the femur double nutrient foramina were most frequent in the white population as opposed to one in the black counterparts. Nutrient foramina were located most frequently on the linea aspera of the femur and posterior surface of both the tibia and the fibula in both black and white populations. The mean foraminal indices were as follows: in the black population $41.87 \%$ for the femur, $31.66 \%$ for the tibia and $43.33 \%$ for the fibula and in white population $44.58 \%$ for the femur, $33.15 \%$ for the tibia and $46.86 \%$ for the fibula. These foraminal indices represent the relative positions of the nutrient foramina on the shaft of the bone. The information about direction, number, location and position of the nutrient foramina is important clinically during free vascularized bone grafting to preserve the blood supply of the graft, fracture repair, joint replacement surgeries and also in medico-legal cases.

Keywords: Foraminal index, fracture repair, long bone, nutrient artery, nutrient foramina

\section{P-179}

\section{The impact of gender and aging on the coronary arteries stenosis and obstruction}

Ece Y, Sağıroğlu AO, Akkoç RF

Department of Anatomy, Faculty of Medicine, Firat University, Elazığ, Turkey

Objectives: In the world, coronary artery diseases (CAD) are located the first lines among death reasons. Ischemic heart disease (IHD), is the most frequently seen condition of CAD because of atherosclerosis. Therefore is aimed what the designated male and female (different age groups) ischemic heart patients, distribution who past coronary angiography (CAG) are determined stenosis and obstructions right and left coronary arteries.

Methods: In this study, between 2007 january - 2013 february, we evaluated retrospectivaly, reports of patients who were made CAG. At the findings results, 300 women and 300 men total of 600 patients who had taken IHD were included in this study. To determine this, 25 - (69) 70 in the range of age male and famele patients according to gender: 25-39, 40-54, 55- (69) 70 were divided in to age groups. IHD grouping if anterior interventricular artery (AIA) and circumflex artery (CxA) which are main branches of left main coronary artery (LMCA) and right coronary artery (RCA) is plaque rate of below 30\%, occlusion no existent were considered. If plaque rate of over $30 \%$ occlusion in our study statistical analysis was done using chi-square test which is a parametric test.

Results: For the female patients who have RCA stenosis and occlusions according to gender is seen main full of difference $(\mathrm{p}<0.05)$. According to age group, with advancing age in RCA and main branches of LMCA seems to be increase stenosis and occlusions $(\mathrm{p}<0.05)$.

Conclusion: In light of the findings, according to gender, stenosis and occlusions of ACD is more common in women. Also with advanced age, has concluded that to increase stenosis and occlusions in RCA and main branches of LMCA.

\section{P-180}

The impact of smoking on the coronary arteries stenosis and obstruction based on gender

Ece Y, Sağıroğlu A.O, Ögetürk M, Özkan G, Deniz G

Department of Anatomy, Faculty of Medicine, Firat University, Elazığ, Turkey 
Objectives: Coronary artery diseases (CAD) not only advanced western countries but also in our country are the first place as a cause of mortality and morbidity. Ischemic heart disease (IHD) is the most common type of CAD because of atherosclerosis results. In both gender groups, the youth, the olds, and all racial groups a strong correlation was observed between the amount of cigarettes smoked with CAD. Therefore according to gender, smokers and non-smokers ischemic heart patient who is detected after coronary angiography (CAG) in right and left coronary artery stenosis and obstruction the incidence of it will be evaluated.

Methods: In this study between 2007 January - 2013 February, we evaluated retrospectively, reports of patients who were made CAG. At the findings results, 300 women and 300 men total of 600 patients who had taken IHD were included in this study. 300 female and 300 male patients as 150 smokers and 150 non-smokers were subdivided. IHD grouping if anterior interventricular artery (AIA) and circumflex artery (CxA) which are main branches of left main coronary artery (LMCA) and rigth coronary artery (RCA) are plaque rate of below 30\%, occlusion no existent were considered. If plaque rate of over $30 \%$ occlusion in our study statistical analysis was done using chi-square test which is a parametric test.

Results: In both gender groups, stenosis and occlusion of the RCA was determined to show a certain increase in smokers than non- smokers. This is a significant differance as statistically $(\mathrm{p}<0.05)$. According to gender female was seen to be more apt about RCA whit LMCA, AIA and CxA stenosis and occlusion $(\mathrm{p}<0.05)$.

Conclusion: In light of the findings, cigarette effecting coronary arteries as gender was concluded.

\section{P-181}

\section{Evaluation of the use of internet and computing resources as support in neuroscience and neuroanatomy courses}

Matamala $\mathrm{F}^{*}, * *$

*Neuroanatomia, Facultad De Medicina. Universidad De La Frontera, Temuco, Chile; **Universidada De la Frontera, Temuco, Chile

Objectives: Over the past 15 years the emergence of the Internet and computer education has had a profound impact on the thinking and learning processes of children, adolescents and adults. Since 2001 in the Neuroanatomy course subject the Universidad de La Frontera (UFRO) of Chile, has made continuous innovations in the teaching-learning process in order to adapt to the new thinking process of students, as well as new teaching trends. These innovations have also included changes in communications that are strongly influenced by the internet and the steady progress of computing resources. The objective of this research was to get the opinion of undergraduates enrolled in two different courses of neuroanatomy and neuroscience during 2014, with regard to assignment strategies and computer science content.
Methods: In the 2014 second semester courses we used 'test your mind' with answers based on the Likert scale, about resources installed on the virtual campus and Moodle platform: videos presented by the course subject teacher; class notes; an atlas on neuroanatomy with photographs of UFRO preparations and UFRO neuroanatomy web page.

Results: 1 . The greater valuation corresponds to the videos for their updated and applied contents. 2. Notes that complemented video themes. 3 . It is unclear if videos and notes can replace course lectures. 4. The atlas with photographs of the neuroanatomical preparations is an important support for practical tests, as are the web site with imaging software, auto evaluation software and links to research centers and medical and dental schools.

Conclusion: Computer education developed for this subject course is highly valued by the students of the Medicine and Education faculties compared with other universities, which is also reflected in the zero and/or minimum failed grades.

Keywords: Neuroanatomy, computing resources, opinion of undergraduate

\section{P-182}

\section{Sex determination of foramen magnum by multidetector computed tomography images}

Tellioglu AM, Durum Y**, Gök M**, Karakas S*, Polat AS, Karaman $\mathrm{CZ}^{* *}$

*Department of Anatomy, Faculty of Medicine, Adnan Menderes University, Aydın, Turkey; *Department of Radiology, Faculty of Medicine, Adnan Menderes University, Aydın, Turkey

Objectives: Foramen magnum provides transition between fossa cranii posterior and canalis vertebralis. Medulla oblongata, arteria vertebralis and nervus accessorius spinal part pass through the foramen magnum. In this study, we aimed to make the morphometric measurements of foramen magnum on CT and to determine the feasibility of sex determination based on this measurements.

Methods: In Adnan Menderes University Medical Faculty Hospital, Department of Radiology, head and neck CT with drawn from age 19-79 (52.7) ranging from 100 (50 male, 50 female) patients were included. To examine foramen magnum in each and every occipital bone, we measured foramen magnum's anterior - posteror (AP) diamater, transverse (T) diamater, the area of foramen magnum and it's circumference.

Results: From all of the variable measurements, we found that men have a higher average value than women $(\mathrm{p}<0.01)$. We measured FM area, FM circumference, FM AP diamater, FM T diamater in order that $8.17 \pm 1.09 \mathrm{~cm}^{2}, 120.22 \pm 11.04 \mathrm{~mm}$, $34.73 \pm 2.21 \mathrm{~mm}, 30.47 \pm 2.25 \mathrm{~mm}$ in male patients. In female patients $7.27 \pm 0.90 \mathrm{~cm}^{2}, 113.08 \pm 9.09 \mathrm{~mm}, 32.99 \pm 2.65 \mathrm{~mm}$, $28.48 \pm 2.72 \mathrm{~mm}$ was measured as mean \pm standard deviation. When we apply Fisher linear discriminant function test in our data, in single variable functions, the highest level in the correct gender separation was given by FM area $(68 \%)$. When all the variables considered at the same time, the proportion is $72 \%$. 
Conclusion: As a result of our study, the metric data we obtained will be useful in cases where the skeletons' gender could not be determined by any other methods. We believe that, our study can be useful for other studies to make the determination of sex from foramen magnum.

Keywords: Foramen magnum, sex determination, multidetector computed tomography images

\section{P-183}

The relationship between sitting height and some body measurements in university students

Uzun A, Gölpınar M, Özdemir F, Güdek MA, Altunsoy E

Department of Anatomy, Faculty of Medicine, Ondokuz Mayıs University, Samsun, Turkey

Introduction: Nowadays different cultures and ethnic groups live together in many countries. Changes in life styles, nutrition and ethnic composition of populations lead to changes in the distribution of body dimensions and require regular updating of anthropometric data collections.

Objectives: The aim of this study was to determine relationship between sitting height and some body measurements in university students.

Methods: Study group consisted of 40 young Turkish and 40 African students. Participants were students at University of Ondokuz Mayıs in Turkey. Sitting height, body height, weight and body mass index were measured by one researcher. The differences between groups and genders were analyzed by ttest. Comparison between parameters was performed using pearson correlation analysis.

Results: It was found that the mean sitting height, body height, weight and body mass index of Turkish students were $90.09 \mathrm{~cm}, 169.06 \mathrm{~cm}, 67.82 \mathrm{~kg}$ and $23.54 \mathrm{~kg} / \mathrm{mÇ}$ respectively while they were $86.22 \mathrm{~cm}, 169.33 \mathrm{~cm}, 63.78 \mathrm{~kg}$ and 21.87 $\mathrm{kg} / \mathrm{m}^{2}$ respectively for African students. There were significant differences between Turkish and African students with regard to sitting height.

Conclusion: Comparative anthropometric studies between different ethnic groups make up the population are need. Through anthropometric measurements are used for especially anthropology, ergonomy, healthy, engineering fields, it is important to determine anthropometric standards of ethnic groups in the population. Average values of the body measurements in the population may be used as a landmark for these fields.

\section{P-184}

The artistic phenomenon in the learning anatomical process

Pires GA

CIISA: UID/CVT/00276/2013 - Faculty of Veterinary Medicine, University of Lisbon, Lisbon, Portugal
Introduction: The pedagogy of Autonomy involves the creation of different possibilities for the construction of knowledge in opposition to the situation of a simple transference of Information towards the student. Learning Anatomy aims in generic terms to understand the Organization of systems and organs, its topography and the inherent functionality. To obtain skills underlying these learning process different methodologies can provide important contributions to the pedagogical process. One of the teachers' challenges is the promotion of opportunities for learning and consequently the reflection about the behavior of students towards the proposed methodology.

Methods: The construction of the anatomical knowledge does not preclude the introduction of the artistic phenomenon in the learning process, since the structure of mental representations through semiotics constitute a tool capable of providing consolidated knowledge. Students are challenged to work in small groups (3-7 students) and select an anatomical theme in order to represent it in an aesthetic work intended to be shown at the Veterinary Academy museum and evaluated.

Results: Classifications obtained by the different groups (6 semesters) involved in this learning methodology were 4.02 (from 0-5) and its own satisfaction in this learning methodology was also very high.

Conclusions: Art is a benchmark of reflection and production of knowledge. The construction of an anatomical-esthetic object presupposes the artistic construction based on investigation and the development of the cognitive process. This latter culminates with the working memory. The student is so much involved in the process that he remembers the data and he feels happy with the capacity of sharing its knowledge. We believe in the success of this methodology. There is a close relationship between the educational activity and joy and hope. The joy and hope that (together) teacher and students can learn, teach, produce, and also improve their skills.

\section{P-185}

\section{Changes in number of ethmoidal foramina}

Montoya STR* ${ }^{*}$ Parra JED*

* Universidad Autonoma De Manizales \& Departamento De Ciencias Basicas, Universidad De Caldas, Manizales, Colombia; **Departamento De Ciencias Basicas, Universidad De Caldas, Manizales. Colombia

Studies of human orbit indicating their individual characteristics are limited by the technique and application of treatment protocols in this region of the head, so it is necessary to identify the exact areas for further intervention and their characteristics to avoid encroaching into inappropriate areas. The ethmoidal foramina of the medial aspect of the orbit in humans beings have two foramina an anterior and posterior trough those homonym nerves go trough, but there is a variation in the number of this structures, which led to establish patterns of types of ethmoidal foramina. Fifty dry skulls of adult orbits for which no distinction 
S198 XXIV International Symposium on Morphological Sciences, 2nd-6th September, 2015, Istanbul, Turkey

of ethnicity or sex were selected. The existence of ethmoid foramen was assessed on the medial aspect of the orbit. Calvaria retired, probing with nylon $0.1 \mathrm{~mm}$ thick and $10 \mathrm{~cm}$ long, to observe the continuity of existing foramen into the nasal passages or to the cribriform plate. $68 \%$ of the studied population had 2 ethmoidal foramina into right and left, 14\% three bilaterally, $6 \%$ with 2 and 3 on the right orbit on the left, $2 \%$ to 3 foramina on the left and 4 on the right orbit $2 \% 2$ and 3 on the right in the left. These aspects of variability must be considered when performing surgical procedures and interventions nerve blocks.

\section{P-186}

\section{Distribution of dermatoglyphic patterns among} nationalities in Macedonia

Chadikovska E* ${ }^{*}$ Zivadinovic J*, Zafirova B*, Trpkovska B*, Bojadzieva B*, Matveeva $\mathrm{N}^{* *}$

*Medical Faculty, Institute of Anatomy; **Institute of Anatomy, Medical Faculty, Skopje

Population dermatoglyphic study has been conducted with purpose to determine and analyse individual dermatoglyphic characters in healthy individuals with Macedonian, Albanian and Roma nationality. Result comparison and present differences has been made. Dermatoglyphic parameters were read and classified on the 600 palm and 600 sole prints obtained from healty individuals of Macedonian, Albanian and Roma ethnic origin. Palm and sole prints were taken using Cummins and Midlo ink method. Dermathoglyphics were classified and comparison was made between three different ethnical groups. The results in population studies showed similarities in frequencies of dermatoglyphic data of all groups. Macedonians are with closer dermatoglyphic characters with Albanians and more different ones compared to Roma examinees. Those findings count for both sexes. Palmar prints are more specific for determining differences between ethnical groups than plantar dermatoglyphics in population studies. In conclusion, a precise definition of true dermatoglyphic patterns on the palms and first time soles in populations of Macedonian, Albanian and Roma nationality have been made. Normal variability of the dermatoglyphic patterns in different population have been presented. The results provide new possibilities for further investigation of dermatoglyphics and their biological and genetic properties. Findings from population studies can be compared with other authors' results.

Keywords: Dermatoglyphics, palms, soles, ethnical groups

\section{P-187}

Effect of cadmium on trophoblast cell proliferation and apoptosis in different gestation periods of rat placenta

Erboğa $M^{*}$, Kanter $M^{* *}$

*Department of Histology and Embryology, Faculty of Medicine, Namık Kemal University, Tekirdağ, Turkey; ${ }^{* *}$ Department of Histology and Embryology, Faculty of Medicine, Istanbul Medeniyet University, istanbul, Turkey
Introduction: Placenta is a transition place regulating many metabolic activities between mother and fetus and a critical organ affecting the result of gestation. In order for embryo to develop healthily placental development should be completed. Most important reason of early embryonic deaths is abnormal placental development.

Objectives: In this study, we aimed to show how cadmium affects the trophoblast proliferation and differentiation in the placenta and the apoptotic activity in different gestational days and hence, its effects of the placental development with immunohistochemical and TUNEL techniques.

Methods: Experimental model of our study consisted of placental development of control and cadmium groups on 15, 17, 19 and 21th days of the gestation. Female rats in cadmium groups were subcutaneously administered a single dose of 0.5 $\mathrm{mg}$ cadmium $/ \mathrm{kg} / \mathrm{d}$ dissolved in sodium chloride as $2 \mathrm{~mL} / \mathrm{kg}$ cadmium chloride until the day they sacrificed. Embryo and placenta of the female rats were seperately removed on 15,17 , 19 and 21th days of the gestation in which the placental development takes place and the placentas were processed for microscopic examinations.

Results: In the placentas of the control group, all the layers were observed to be formed on the 15 th gestational day and thereafter a continuous growth was monitored. In cadmium group also all the layers were existed from the 15 th gestational day. However they were smaller in size than the controls. Frequency of proliferating cell nuclear antigen positive cells was decreased and the number of apoptotic cells was increased in all the gestational days related to cadmium.

Conclusion: In conclusion, cadmium administered during the pregnancy was observed to cause abnormal placental development by disrupting the normal structure of placenta, inhibiting the proliferation of trophoblast and increasing the number of apoptotic trophoblast cells.

Keywords: Cadmium, placental development, trophoblast, apoptosis, PCNA, rat

\section{P-188}

\section{Anatomical variation in the origin of left vertebral artery: report of a rare case}

Yörük MD, Çagımnı P, Durmaz T, İkiz ZAA, Bilge O, Üçerler H

Department of Anatomy, Faculty of Medicine, Ege University, Izmir, Turkey

Classically, the vertebral artery arises from the superoposterior aspect of the first part of the subclavian artery as its first branch. It enters the transvers foramen in the transvers process of the 6th cervical vertebra and ascends through the foramina of the 6th to the 1st cervical vertebrae accompanied by a venous plexus and sympathetic nerves. It curves medially behind the lateral mass of atlas and lies in the groove on its posterior arch. It enters cranium via the foramen magnum. The 
vertebral artery provides the main arterial supply to the posterior fossa. During routine dissection of cadavers in Anatomy Department of Ege University Medicine Faculty, it was detected that a 72-year-old male cadaver had a anomalous origin of the left vertebral artery. It was originated from a trunk in common with the left subclavian artery from aortic arch. The length of the common trunk was $8.02 \mathrm{~mm}$. The diameter of this trunk at the origin was $13.54 \mathrm{~mm}$. The diameters of left vertebral artery and left subclavian artery were $6.72 \mathrm{~mm}$ and $9.20 \mathrm{~mm}$ sequentially. Additionally the thyroidea ima artery was arising from aortic arch between the left common carotid artery and the brachiocephalic trunk in present cadaver. Although the variations of the vertebral artery are uncommon, it has clinical importance to recognize the origin and course of the vertebral artery in the diagnostic procedures, catheterbased evaluation and treatment of patients suffering from cerebrovascular diseases. In order to understand this variation, the embryological development of the aortic system was also reviewed.

\section{P-189}

Bilateral double parotid ducts: a rare anomaly

Üçerler H, İkiz ZAA, Soylu F, Farajiband N, Yörük MD, Çă̆ımnı $\mathrm{P}$

Department of Anatomy, Faculty of Medicine, Ege University, Izmir, Turkey

The parotid duct is formed by the confluence of two ducts in the gland which ascend and descend at right angle to the main duct. While crossing masseter it can receive the accessory parotid duct. The lumen of the parotid duct is about 3 to $4 \mathrm{~mm}$ in diameter showing marked reduction approaching the oral cavity and its length is about $5 \mathrm{~cm}$. Although the anatomical course of this duct is well known, the reports on its normal anatomical variants and morphometry are very few. During routine dissection in the Department of Anatomy of Ege University Medicine Faculty, double parotid ducts bilaterally in a 72-year-old male cadaver were observed. Parotid ducts on both sides were traced carefully and neighbouring anatomic structures were demonstrated. The two ducts on both sides merged with each other and made the main parotid duct that had a straight course and ran horizontally towards the anterior border of masseter. The length of main parotid duct was 20.02 $\mathrm{mm}$ on the right side and $16 \mathrm{~mm}$ on the left side. The distance between tragus and union point of double ducts was $52.00 \mathrm{~mm}$ on the right side and $58.72 \mathrm{~mm}$ on the left side. Appreciation of the parotid duct variants have importance in assessment new diagnostic and therapeutic techniques. Beside this, the proper knowledge of the normal topographic anatomy and variations of the parotid duct provides easy understanding of the analysis of radiographic images and computerized tomographic scans used in sialography.

\section{P-190}

\section{Congenital anatomic abnormalities between mother asthma patients correlation}

$\underline{\text { Sar1 } C^{*}}$, Kalaycıŏglu A**, İmamoğlu $M^{* * *}$

*Department of Pediatric Surgery, Trabzon Faculty of Medicine, Karadeniz Technical University, Trabzon, Turkey; **Department of Anatomy, Trabzon Faculty of Medicine, Karadeniz Technical University, Trabzon, Turkey; ***Department of Pediatric Surgery, Trabzon Faculty of Medicine, Karadeniz Technical University, Trabzon, Turkey

The aim of this study is to shed light on factors that cause congenital malformations on babies whose mothers have asthma or allergic reactions, and also to raise awareness about protection from these types of risks. 100 mothers-whose babies were diagnosed with congenital anomalies- compared with other 100 mothers-whose babies were normal-. In this study there were 17 mothers who have asthma. These women were asked to answer some questions about possible risk factors which they had during their pregnancy. After getting ethical and other permissions participants were included in the study. In our study the ratio of mothers that have chronic diseases were $33 \% .8 .5 \%$ of these $(17$ patients) had been monitoring with asthma diagnosis. We observed that babies of these mothers had more ratio of having anomalies related with gastrointestinal system. $17.5 \%$ participants had atopic contexture. Nasal congestion is the most frequently observed symptom on mothers who have asthma and allergy. In this study we observed that mothers who have babies with anomalies had also nasal congestion during their pregnancy. The ratio of these mothers were $23 \%$ this result was statistically significant $(\mathrm{p}<0.05)$. In our opinion, our result has leading role for mothers (with atopic contexture)-who had asthma and nasal congestion during their pregnancy- about having babies with congenital anomaly. For this reason it is so important to reduce the risk of exposure to allergic factors and viral infections. In order to achieve this goal, during pregnancy more advanced techniques such as detailed diagnosis, scanning tests, genetic analysis and etc. should be performed on mothers with asthma.

\section{P-191}

\section{A questionnaire study; anatomy practical performance of assistances in faculty of medicine}

Emirzeoğlu M, Uzun A, Özdemir F, Gölpınar M, Nahir M, Altunsoy E

Department of Anatomy, Faculty of Medicine, Ondokuz Mayıs University, Samsun, Turkey

Introduction: Performances of assistances in anatomy practical lessons are important role for education of medical students.

Objectives: The aim of the present study is to describe and compare between the anatomy assistances performances of the preparing of lesson, knowledge of the practical guide and according to the practical purpose on faculty of medicine of first class students at the Ondokuz Mayis University. 
Methods: The questionnaire had 25 questions and 5 options were used in the present study. This study included 29 female and 25 male students, who were selected only in first laboratory group. The assistances have given lectures for 2, 3, 4, 5 years, respectively. In data analysis, the questionnaire items were divided into three groups; the preparing of lesson, knowledge of the practical guide and according to the practical purpose, respectively. The statistically test of ANOVA (analysis of variance) was performed, $\mathrm{p}$ value at or less than 0.05 was accepted as statistically differences.

Results: Our results revealed that the female students reported the most negative answers for the first assistance in knowledge of the practical guide and according to the practical purpose $(\mathrm{p}<0.05)$, fourth assistance took the most positive answers but there were no statistically differences with the second and third assistances $(\mathrm{p}>0.05)$. There were no statistically difference in the preparing of lesson about the female students answers ( $>0.05)$. The male students reported the same answers for the all assistances $(\mathrm{p}>0.05)$.

Conclusion: The conclusion of the present study, the experience has been observed that the students play an important role in reporting positive opinion.

\section{P-192}

\section{Students' views on methods of learning and teaching anatomy}

Yeğinoğlu G*, Uzun Ö**, Kalkısım S**, Öksüz CE**, Yeğinoğlu $\mathrm{Y}^{* * *}$

*Department of Anatomy, Faculty of Medicine, Karadeniz Technical University, Trabzon, Turkey; **Vocational Faculty of Healthy Science, Karadeniz Technical University, Trabzon, Turkey; ***Department of Statistics and Computer Science, Karadeniz Technical University, Trabzon, Turkey

Introduction: Medical education and the teaching of anatomy, one component of that training, are under discussion in Turkey and worldwide, and work intended to restructure these is ongoing. Every country needs to strive to improve and update the education of its students in the light of its own requirements in line with the changes taking place in education itself.

Objectives: The purpose of this study was to assess educational models applied in anatomy classes from the students' perspective. In addition to its guiding characteristics, the research is also valuable in terms of presenting ideas from other branches of science that can serve as models. This study was prepared in order to identify and discuss the current state of anatomy education at the Karadeniz Technical University Medical Faculty and problems concerned with education and teaching, and to assess the ideas, criticisms and recommendations of medical students in years 1, 2, 3 and 4 .

Methods: The research was performed at the Karadeniz Technical University Medical Faculty. First-, second-, thirdand fourth-year students (506 individuals) participated and gave informed consent. The questions we prepared in the light of the literature and our observations were administered in face-to-face survey form. Data were analyzed on SPSS software and were interpreted in percentage intervals on the basis of frequency distributions.

Results and Conclusion: Students agreed that anatomy is best learned in applied form. Models and cadavers are frequently preferred among applied teaching methods. Atlases of anatomy are of great importance among self-study methods. Participants thought that anatomy classes being harmonized with clinical practice would ensure permanent learning. Anatomy teaching is a visually-based process. Students thought that dissection is the best method if sufficient cadavers are available. This study, which shows that currently employed educational models can be improved by adopting the student perspective, will produce positive results by representing a model and a guide to anatomy teaching.

\section{P-193}

\section{Sodium valproate influence on urethane induced mouse lung tumors and it's cancerogenesis}

Mozuraite $\mathrm{R}^{*}$, Stakisaitis $\mathrm{D}^{* *}$, Didziapetriene $\mathrm{J}^{* *}$, Preisegolaviciute $\mathrm{L}^{*}$, Juodziukyniene $\mathrm{N}^{*}$, Valanciute $\mathrm{A}^{*}$

*Lithuanian University of Health Sciences, Kaunas, Lithuania;

**Institute of Oncology, Vilnius University, Vilnius, Lithuania

Introduction: Lung cancer is the leading couse of cancer related mortality in the world. Sodium valproate (NaVP), a histone deacetylase inhibitor, has been shown to display potent antitumor effects. In this research we evaluated NaVP influence on mice lungs cancerogenesis.

Objectives: To evaluate gender dependent effect on uterthane induced oncogenesis. To evaluate the effect of NaVP on tumorigenesis in ovariectomized and ovary-intact animals.

Methods: In the research $120 \mathrm{Balb} / \mathrm{c}$ line female mice were used, 60 non- sterilised and 60 sterilised. Sterilised and nonsterilized mice were treated with urethane only and urethane with NaVP. After 6 months lungs were collected for macroscopic and microscopic evaluation. Results were counted using Student's t and Mann - Whitney tests. Differences at the value of $\mathrm{p}<0.05$ were considered significant.

Results: Results showed that BALB/c line mice is sensitive to pulmotropic cancerogen urethane: tumors developed in all animals affected with urethane. Mice affected with urtehane develop maligant (adenocarcinoma) and benign (adenoma) tumors. A significant decrease of adenocarcinoma number in ovariectomized mice treated with a urethane-NaVP combination as compared with ovariectomized mice treated with urethane only was found $(\mathrm{p}=0.031)$.

Conclusions: In female mice, total number of urethaneinduced lung tumors was increased by NaVP treatment, in ovariectomized groups this increase was significant. NaVP together with low estrogen may have a protective effect on the malignization of adenomas in ovariectomized mice. 


\section{P-194}

Replaced right hepatic artery and its segmental distribution

Beger O, Elvan Ö, Kurtoğlu Z

Department of Anatomy, Faculty of Medicine, Mersin University, Mersin, Turkey

During the dissection of 66-year-old formalin-fixed male cadaver a hepatic arterial variation was encountered. In addition to proper hepatic artery which arose from the celiac trunk, there was an artery arising from the superior mesenteric artery and then entered into the right lobe of liver. This variant artery has been defined as replaced right hepatic artery or aberrant right hepatic artery in the literature. In this study, hepatic segmental distribution and the morphometric properties of both hepatic arteries were presented. Replaced right hepatic artery coursed behind the portal vein and then laterally to it and entered into the Calot's triangle. The length and width of this artery were measured as $63.10 \mathrm{~mm}$ and $3.6 \mathrm{~mm}$, respectively. After giving off cystic artery, it ramified into two branches. These branches were observed to enter into the right lobe of liver. Proper hepatic artery which arose from celiac trunk ramified into middle hepatic artery and left hepatic artery. The length and width of middle hepatic artery were $30.20 \mathrm{~mm}$ and $1.20 \mathrm{~mm}$, respectively. This artery gave a small branch to caudate lobe of liver. Later, it coursed towards quadrate lobe of liver, gave branches to medial superior and medial inferior segments. The length and width of left hepatic artery were 28.40 $\mathrm{mm}$ and $2.6 \mathrm{~mm}$, respectively. Before entering into left lobe of liver, it ramified into two branches then these branches entered into lateral superior and lateral inferior segments of the lobe. Replaced right hepatic artery is not rare in the literature. The size of arteries and their distribution to the liver segments were evaluated in terms of assessing the risk of complications with surgeries about pancreas, gall bladder and liver, especially liver transplantation.

Keywords: Replaced right hepatic artery, segmental distribution, liver, liver transplantation

\section{P-195}

Classification and clinical significance of the congenital anomalies of thymus

$\underline{\text { Varga I* }}^{*}$ Miko $M^{*}$, Polak S*, Mestanova $V^{* *}$

*Institute of Histology \& Embryology, Faculty of Medicine, Comenius University in Bratislava, Slovakia; **Institute of Histology \& Embryology, Jessenius Faculty of Medicine in Martin, Comenius University in Bratislava, Slovakia

Thymic morphology varies greatly even in the same age group. In young individuals, it is typically bilobed and $\mathrm{V}$-shaped, with two small processes extending into the neck. A great controversy about the terms being used in the classification of thymic anomalies arises in current literature. Most of the recent articles use the term "ectopic thymus" in a confusing way, because they call cervical extension of the thymus, accessory cervical lobes of the thymus or accessory thymic tissue also "ectopic thymus". All anomalies of the thymus are explained by the disruption of thymic primordia formation and/or disruption of its descent from the neck to the mediastinum. It may be found at any level of the pathway of normal thymic descent, from the angle of the mandible to the superior mediastinum. The "genuine" ectopic thymus is a distinct entity from accessory thymic tissue localized in the neck. The term "ectopic thymus" should be reserved for states where only ectopic thymic tissue is present, while no thymic tissue is localized in the normal position. A cystic version of accessory thymic tissue localized in the neck is called "congenital cervical thymic cyst". Thymopharyngeal duct cyst is another special and extremely rare variant of cystic accessory thymic tissue. Relatively common and asymptomatic anatomical variation is the fusion of thymus with one or more parathyroid glands. The most common thymic anomalies include anomalies in number of the thymic lobes, ectopic thymus, accessory thymic tissue, thymic aplasia, thymoparathyroid aplasia (DiGeorge syndrome), thymic hypoplasia and hyperplasia. In a case of ectopic thymus, the whole thymus is (most often) located in the midline neck, and no thymic tissue is located in the normal position in the mediastinum. The prevalence of foci of accessory thymic tissue varies according to several authors from $1.8 \%$ up to more than $50 \%$. The study was supported by Grant of Comenius University in Bratislava $\mathrm{UK} / 331 / 2015$.

\section{P-196}

The effect of long-standing time on foot morphology in the air transportation employees

\section{Şafak NK, Durgun B}

Department of Anatomy, Faculty of Medicine, Çukurova University, Adana, Turkey

In this study; it is aimed to investigate the effect of the longstanding time on foot structure, based on the hypothesis that the work at standing position for long time cause the deformations in the shape and dome structure of the foot comparing with work at sitting position for a long time. Foot measurements were obtained from 42 flight attendant (17 male, 25 female) aged between 21-43 years which spends most of their working time by standing. Control group contained 35 employees (17 male, 18 female) aged between 20-55 years who works in the office conditions at Adana Airport. After weight, height and lower limb height were measured, the foot measurements were performed by using tape measure and anthropometric set. These measurements were length, breadth, height and circumference of foot and foot segments. SPSS 17.0 proramme were used for statistical analysis. Taking all measurements together, There were significant differences between male and female $(\mathrm{p}<0.001-0.003)$ except leg lenght, all foot arches on right side and distance between Feiss line and navicula. Significantly differences was found between office workers 
and flight attendants for navicular height, tarsal height, foot breadth, ankle circumference, bimalleolar circumference, distance between Feiss line and navicula $(\mathrm{p}<0.05)$. These findings showed that working for long-standing time cause the change of foot structure because of static contraction of muscles. In conclusion, it is thought that the results of this first study about flight attendans'foot morphological analysis will make a contribution to understsanding of the effect of long-standing time on foot morphology and the foot dimensions in the air transportation employees.

Keywords: Foot, foot morphology, standing, air transportation employees

\section{P-197}

Estimation of height using percutaneous length of tibia and ulna in Iran

Broujeni FB, Hasanzadeh G

Department of Anatomy, Tehran University of Medical Science, Tehran, Iran

Objectives: Estimation of height based on bones of limb, sexuality and other human characteristics is one of main topic of research in forensic medicine and anthropology.

Methods: In this paper, the stature of 111 females and 71 males and their length of percutaneous of tibia and ulna were obtained from medical students in Tehran university of Iran. Using these data, three different models were applied. First, a single variable regression equation of percutaneous length of tibia is developed to determine the height of males and females. The second single variable regression model is based on the percutaneous length of ulna and the final model was a multivariable regression model of percutaneous length of tibia and ulna.

Results: The results show that the percutaneous length of ulna has superior effect on height $(\mathrm{R} 2=0.48)$ than the percutaneous length of tibia $(\mathrm{R} 2=0.31)$. Also the multi-variable regression which it is considered both of percutaneous length of ulna and tibia has better agreement with measured data $(R 2=0.58)$. Furthermore, the relative errors for the mentioned models were 2.8 to $3.8 \%$.

Keywords: Anthropology, percutaneous tibia, percutaneous ulna, multi-variable regression

\section{P-198}

Analysis of ganglion structures of the myenteric nervous plexus inferior part of the human duodenum during aging

Mandic P, Vitosevic Z, Macut ND, Filipovic T, Gasic M

Institute of Anatomy, Medical Faculty Pristina, Kosovska Mitrovica

Objectives: Aging is a process that affects the structure and function of the enteric nervous system, but available are few specific information on this theme, especially in humans. The aim of this study was to investigate the effect of age on the structure of the myenteric ganglions in the inferior part of the human duodenum. Specimens were obtained from 30 cadavers aged 20 to 84 years old.

Methods: Tissue samples of inferior part of the duodenum are divided by age into three groups (20-44, 45-64 and 65-84 years). After histological processing samples were stained with $\mathrm{HE}$, Cresyl violet and AgNO3 staining method. The morphometric analysis was performed using multipurpose test system M42. The results were analyzed by one-way ANOVA method with post hoc Tukey test.

Results: Number of neurons per unit of area of the myenteric plexus in all three age groups is expressed as mean $\pm \mathrm{SD}$. Noticeable is a significant decreasing $(\mathrm{p}<0.001)$ in the number of neurons at people aged between 65-84 years. Myenteric ganglions of very old people include areas where is a very small number of neurons. Evident was decreasing the number of neurons of $21.02 \%$ in the oldest (65-84 years) compared to the youngest (20-44 years). With aging comes to significant increase $(\mathrm{p}<0.05)$ surface of the neuronal soma and surface of their nuclei.

Conclusion: During the aging there is a significant loss in the number of neurons. There is a present tendency to compensate that loss of neurons by increasing the size of their soma and their nuclei.

Keywords: Myenteric plexus, duodenum, aging.

\section{P-199}

The incidence and topographic distribution of sutures including Wormian bones in human skulls

\author{
Çirpan S, Aksu F, Mas N \\ Department of Anatomy, Faculty of Medicine, Dokuz Eylul University, \\ izmir, Turkey
}

Objectives: The Wormian Bones (WB's) are accessory bones located within the cranial sutures and fontanelles. The present article examine the incidence of WB's and compare the number and topographic distribution between the sutures including WB's in skulls of West Anatolian Population.

Methods: 150 crania were examined. The parameters evaluated in the present study were as follows: the rate of skulls including WB's; the topographic distribution and frequencies of the sutures including WB's; the number of these sutures for each skull; the name and number of sutures which were bilaterally and symmetrically located on the right and left side of skull (paired sutures) and which coincidentally had WB's for each skull; the differences of frequencies between the paired sutures including WB's.

Results: The rate of skulls including WB's was determined as $59.3 \%$. The maximum and minimum number of sutures 
including WB's were six in one skull and one in each of 30 skulls, respectively. The maximum and minimum rates of sutures which had WB's were found in left lambdoid 40.7\% and right occipitomastoid $1.3 \%$ sutures, respectively. There was only a significant difference between the rate of right and left squamous sutures, $(\mathrm{p}=0.04)$. Forty-five skulls were including fifty-five pair of bilaterally and symmetrically located sutures which coincidentally had WB's in each pair. The each of thirty-five skulls had one pair of sutures including WB's and each of ten skulls had two pairs.

Conclusion: In the present study, the rate of WB's were determined as $59.3 \%$ in West Anatolian Population. This incidence rate is considerably lower than the other reports, and it may be as a result of racial variations. These divergent bones were more frequently found in left lambdoid sutures (40.7\%) and less frequently in right occipitomastoid sutures $(1.3 \%)$. This study may guide the investigators dealing with the neurosurgery, orthopedy, radiology, anatomy and anthropology in their practice.

\section{P-200}

\section{Coexistence of Wormian bones with metopism, and vice versa in adult skulls}

\section{Cirpan S, Aksu F, Mas N, Mağden AO}

Department of Anatomy, Faculty of Medicine, Dokuz Eylül University, izmir, Turkey

Objectives: To investigate coexistence of Wormian Bones (WB's) with metopism, and vice versa in adult skulls.

Methods: 160 dry adult human skulls were randomly selected from Dokuz Eylul University of Medical Faculty's Anatomy Laboratory. The skulls were examined for presence of metopism, WB's, coexistence of WB's with metopism and vice versa. Topographic distribution of the WBs were macroscopically evaluated within the skulls including metopism.

Results: The frequency of metopism and WBs in 160 skulls is as $7.50 \%(12 / 160)$ and $59.3 \%$ (95/160), respectively, $\mathrm{p}<0.05$. The incidence of coexistence of WB's with metopism was found as 11 of 12 skulls $(91.66 \%)$, whereas the incidence of coexistence of metopism with WB's was found as 11 of 95 skulls $(11.58 \%), \mathrm{p}<0.05$. There were totally 23 sutures including WB's in 11 skulls which had metopism. The number (\%) of metopic skulls for each specific suture including WB's were found as: 11 lamdoid sutures in 7/11 (63.63\%) skulls, 4 lamda in $4 / 11(36.36 \%)$ skulls, 2 asterion in $2 / 11$ (18.18\%) skulls, 1 squamous in $1 / 11$ (9.09\%) skull, 2 sagittal in $2 / 11(18.18 \%)$ skulls and 3 parieromsatoid sutures in $2 / 11$ (18.18\%) skulls. The distribution of these 23 WB's in sutures of 11 skulls including metopisms is determined as follows: 11/23 (47.82\%) WB's at lambdoid sutures; $4 / 23(17.39 \%)$ WB's at lambda; $2 / 23$ (8.69\%) WB's at asterion; $1 / 23$ (4.34\%) WB's at the left squamous suture; $2 / 23$ (8.69\%) WB's at sagittal sutures; $3 / 23$ (13.04\%) WB's at parietomastoid sutures.
Conclusion: There was a significant difference in rates between coexistence of WBs with metopism (91.66\%) and coexistence of metopism with WBs (11.58\%). The factors leading to metopism may also lead to WB's, whereas that the factors leading to WB's may not lead to metopism.

\section{P-201}

\section{Suprascapular foramen: a rare variation caused by ossified suprascapular ligaments}

Çirpan $\mathrm{S}^{*}$, Mas $\mathrm{NG}^{*}$, Aksu $\mathrm{F}^{*}$, Edizer $\mathrm{M}^{*}$, Karabekir $\mathrm{S}^{* *}$, Mağden $\mathrm{AO}^{*}$

*Department of Anatomy, Faculty of Medicine, Dokuz Eylül University, izmir, Turkey; ${ }^{*}$ Department of Neurosurgery, Faculty of Medicine, Dokuz Eylül University, Izmir, Turkey

Objectives: The aim of this study was to investigate the incidence of the suprascapular foramen in West Anatolian Population.

Methods: Eighty-one dried human scapulae of West Anatolian people of unknown ages and gender belonging to the Anatomy Department Laboratory of Dokuz Eylül University Medical Faculty were examined macroscopically. The vertical and transverse diameters of the suprascapular foramen and central tickness of the ossified ligaments were measured with caliper in milimeters and digital caliper, respectively.

Results: We observed the suprascapular foramen due to ossification of the suprascapular ligament only in 2 of 81 (2.47\%) scapulae. The vertical and transverse diameters of the suprascapular foramen and central thickness of the ossified ligaments (No-1 and 2) were measured as $0.80 \mathrm{~mm}$ versus $0.40 \mathrm{~mm}, 0.36$ mm versus $0.20 \mathrm{~mm}$ and $0.40 \mathrm{~mm}$ versus $0.14 \mathrm{~mm}$, respectively.

Conclusion: The suprascapular foramen caused by ossified suprascapular ligament is rarely observed variation in West Anatolian Population. Early diagnosis of the suprascapular nerve(SN) entrapment causing muscular atrophy is diffucult before the occurence of complaints such as deeply and poorly localized pain. The treatment of the $\mathrm{SN}$ compression starts with physical therapy for strengthening the rotator cuff muscles. The nerve blockage by a local anesthetic can relief the pain only for a short time and simultaneously provide diagnosis. It can be treated by surgical removal of the ossified transverse scapular ligament. The ossified suprascapular ligament narrows the suprascapular foramen; therefore, it causes compression of SN and complaints of SN entrapment. It is essential to define precisely the anatomical variations of scapular notch, scapular ligament, SN and vessels for surgeons performing SN decompression surgery, especially with arthroscopic techniques. We assume that the present study may guide the medical doctors and the surgeons during diagnostic and surgical procedures related with suprascapular notch. 


\section{P-202}

\section{Lymphoid formations of anal canal of rectum in middle fetal period}

$\underline{\text { Aubakirov }}^{*}$, Sagimova $\mathrm{G}^{*}$, Suleimenova $\mathrm{F}^{*}$, Yesbolatova $\mathrm{G}^{* *}$, Baryshev $\mathrm{B}^{*}$

*Astana Medical University, Astana, Kazakhstan; **Semey State Medical University, Semey, Kazakhstan

Objectives: Study of formation of the immune system of the digestive tract is important to find out the pathogenesis of immunodeficiency states and ways of their correction. The objective of our research is to study the development of lymphoid formations of the anal canal of rectum in middle fetal period.

Methods: the work was done on 32 preparations of the rectum of human fetuses obtained in the result of medical abortions in term of 21-28 weeks. The research was approved by the Ethical Committee. Macropreparations of mucous membrane on Hellmah's method were prepared. Histological sections stained by hematoxylin-eozin were prepared. Suspension of intestinal cells was studied by flow cytometry for determination of lymphocytes populations.

Results: on macropreparations of a mucous membrane of anal canal of rectum, lymphoid formations are located along the anal columns in the form of vertical chains. The average number of lymphoid formations on $0.25 \mathrm{cmC}$ is $8.4 \pm 0.82(7.1-10.7)$, the average size is $128.6 \pm 18.1$ microns (101.2-193.4). Microscopically in proper mucous plate of the anal canal lymphoid formations in the form of a lymphoid infiltration, prenodules and small solitary lymphoid nodules were observed. In the transition zone of anal canal, which is covered by not keratinizing stratified squamous epithelium, diffuse lymphoid cells around the veins are observed, which probably indicates that the lymphocytes migrating from the venules lumen are a source of lymphoid structures formation in the mucosa of the organ. According the data of flow cytometry, T-lymphocytes are predominate over B-lymphocytes. Subpopulations of T-lymphocytes are presented by helpers, cytotoxic cells and natural killer cells, also there is a large number of immature cells.

Conclusion: In the middle fetal period lymphoid tissue of the anal canal is presented by lymphoid formations of different stages of maturity that apparently indicate the continuing process of formation of immune system of the organ.

\section{P-203}

\section{Morphological characteristics of the anterior inferior cerebellar artery}

Dodevski A* Lazarova D*, Papazova $\mathrm{M}^{*}$, Matveeva N*, Zhivadinovik J*, Lazareska $M^{* *}$, Aliji $V^{* *}$

*Institute of Anatomy, Medical Faculty, "SS. Cyril And Methodius University", Skopje, Republic of Macedonia; **University Clinic of Radiology, Skopje, Republic of Macedonia
Introduction: The anterior inferior cerebellar artery (AICA) is one of the three vessels that provide arterial blood supply to the cerebellum. It has a variable origin, course and supply, with up to $40 \%$ of the specimens not having an identifiable standard AICA. The aim of this study was to investigate the origin and diameter of AICA in the Macedonian population and to emphasize clinical significance of this vessel.

Methods: We examined radiographs of 103 patients who had CT angiography undertaken for a variety of clinical reasons, performed as a part of their medical treatment at the University Clinic for Radiology in Skopje, R. Macedonia. The study population included 103 patients, 58 male and 45 females, age range from $25-82$, mean age 58.4 years.

Results: In $16.50 \%$ of the patients origin and course of AICA could not be visualized and in $83.49 \%$ of the patients the origin and course of AICA was visualized. In all of the patients in this study AICA arose as a single vessel from the proximal third of the basilar artery. The outer diameter of the left AICA was in range from 0.39 to $1.50 \mathrm{~mm}$ with a mean value of $0.89 \mathrm{~mm} \pm 0.30 \mathrm{~mm}$. The outer diameter of the right AICA was in range from 0.40 to $1.40 \mathrm{~mm}$ with a mean value of $88 \mathrm{~mm} \pm 0.26 \mathrm{~mm}$.

Conclusion: Although anatomically interesting, an awareness of the AICA anatomy and variations is clinically important for save performance of diagnostic and interventional procedures in radiology and for surgeons during planning and accomplishing surgical interventions.

Keywords: AICA, anatomy, variations, microsurgery, interventional procedures

\section{P-204 \\ Morphometric features of the ostium of the coronary sinus}

Zhivadinovik I, Lazarova D, Papazova M, Matveeva N, Dodevski A

Institute of Anatomy, Medical Faculty, "SS. Cyril and Methodius University", Skopje, Republic of Macedonia

Objectives: The ostium of the coronary sinus (OCS) is a significant landmark in the anatomy of the right atrium, forming one of the sides (the base) of the triangle of Koch. Part of the ostium is often covered with a valve named as Thebesian valve. The aim of this study was to present the diameter of OCS, its location inside the base of the triangle of Koch and the degree of development of the Thebesian valve.

Methods: The examination was made on 100 human hearts obtained after autopsies of patients died from no cardiac reasons and fixed in $10 \%$ formaldehyde.

Results: The post mortal measuring showed that the main value of the length of the base of the triangle of Koch (side b) was $20.2 \pm 3.6 \mathrm{~mm}$, of the segment b1 $-3.3 \pm 1.6 \mathrm{~mm}, \mathrm{~b} 2-9.3 \pm 1.8 \mathrm{~mm}$, b3 $-8 \pm 2.8 \mathrm{~mm}$. The diameter of the OCS was congruent to b2 segment.

Conclusion: The analysis of the degree of development of the Thebesian valve revealed that the valve was absent in 14 cases, 
residual - moderately developed covering less than $15 \%$ of OCS in 30 cases, partial - well developed covering more than $15 \%$ of OCS in 48 cases and fenestrated in 8 cases.

Keywords: Triangle of Koch, Thebesian valve, coronary sinus

\section{P-205}

\section{About what radiologic anatomy teaches the clinician: bilateral dens invaginatus}

Köse TE**, Uyanık $S^{* *}$, Büyükertan $M^{*}$, Özcan I** Balcığlu HA*

*Department of Anatomy, Faculty of Dentistry, istanbul University, istanbul, Turkey; **Department of Oral Radiology, Faculty of Dentistry, Istanbul University, Istanbul, Turkey

Dens invaginatus is a developmental malformation of the tooth. Dens invaginatus occurs in the dental papilla before the calcification process. Its etiology is unkonwn, however, several theories have been suggested. The incidence of dens invaginatus is reported to range from $0.04 \%$ to $10 \%$. Maxillary lateral incisors and central incisors are the most commonly seen teeth. Clinical and radiographic findings of dens invaginatus varies according to the location and extent of the invagination. Three types have been recognized to date. In this case, dens invaginatus was defined in bilateral maxillary lateral incisors of a healthy patient. This condition was detected by chance on panoramic radiography. A 31-year -old patient's panoramic radiography revealed ovoid radioopacities in the root canals of his upper lateral incisors. This image is similar to internal resorpsion. Conventional radiographies is not always possible to determine the canals and internal structure. CBCT images showed air filled cavity with enamel-like density borders. Clinically a deep foramen caecum was detected. Vitality test was performed on the teeht which gave positive response. After diagnosis, teeth with incisal invaginations was treated with fissure sealing before any caries occur. Morphological features of the case will be discussed in this presentation.

\section{P-206}

Anatomical and morphometric evaluation of the foramen transversarium of cervical vertebrae

Nurcan İ, Necdet K

Department of Anatomy, Gülhane Military Medical Academy, Ankara, Turkey

The purpose of this study is to reveal the incidence of double foramen transversarium constituting variational conditions at the foramen transversarium vertebrae cervicales located at the processus transversus of cervical vertebrae; and emphasize the dimensions, shape variations of foramen transversarium as well as the clinical symptoms that may be caused by these conditions. For this study, a total of 82 cervical vertebrae available at the laboratory of the Gülhane Military Medical Academy Department of Anatomy were studied. Variations of foramen transversarium on all vertebrae were recorded. Foramen transversarium was present in all vertebrae. Double foramen transversarium were observed in 18 of 82 cervical vertebrae. Of them, 6 vertebrae exhibited unilateral, and 12 vertebrae exhibited bilateral double foramen transversarium. Of the unilateral transversarium, 1 was on the right side and 5 were on the left side. Foramen transversarium observed were predominantly in round form. Anteriorposterior and medial-lateral diameters of right foramen transversarium was $5.7 \mathrm{~mm}$ and $6.5 \mathrm{~mm}$ respectively while the anteriorposterior and medial-lateral diameters of left foramen transversarium were $5.5 \mathrm{~mm}$ and $6.6 \mathrm{~mm}$ respectively. In this study, incidence of double foramen transversarium was found as $23.95 \%$. Among all double foramen transversarium, the incidence of the bilateral group was observed to be doubling the unilateral group. It's significant for clinicians to have a knowledge of this variation. Morphological variations in foreman transversarium are significant as they lead to neurological conditions due to the flow of blood passing through these openings except for C7.

Keywords: Vertebrae cervicales, double foramen transversarium, accessory foramen transversarium, morphometry

\section{P-207}

The effects of chronic renal failure on oxidant-antioxidant status

$\underline{\text { Savaş HB }}^{*}$, Altuntaş A**, Uz E*, Sezer MT**

*Department of Medical Biochemistry, Faculty of Medicine, Süleyman Demirel University, Isparta, Turkey; **Internal Medicine Clinic, Faculty of Medicine, Süleyman Demirel University, Isparta, Turkey

Objectives: Objectives: In living organisms, oxidant and antioxidant systems are in balance. Reactive products formed continuously by exogenous and endogenous sources which are rendered harmless by the antioxidant system. Oxidative stress is one of the etiological factors in aging and development of various diseases. In the present study, we aimed to investigate the effects of chronic renal failure on oxidant- antioxidant status in humans.

Methods: This study was investigated in Suleyman Demirel University Faculty of Medicine Clinical Internal Medicine, in 32 people with chronic renal failure and no renal failure, adults, men and women. People were divided into two groups as control $(n=11)$ and experiment $(n=21)$ groups. The remaining bloods after routine examination were collected. Serum was obtained by centrifugation of bloods. Total antioxidant and total oxidant status (TAS\&TOS) was determined by Erel's method using a commercial kit (Rel Assay Diagnostic Turkey) with a biochemical auto-analyser (Beckman Coulter AU 5800. USA). Oxidative stress index was calculated by using the following formula; OSI = TOS/TAS. Data was assessed by Anova test and $<0.05$ was regarded as statistically significant.

Results: There was a significant increase in TOS and OSI values in chronic renal failure group, compared to the control group $(\mathrm{p}<0.05)$.

Conclusion: Oxidative stress increases in chronic renal failure patients. To take measures to reduce oxidative stress in chronic renal failure patients will be useful for the general condition 
of the patient. Further studies are needed for the treatment to reduce oxidative stress in chronic renal failure patients.

Keywords: chronic renal failure, oxidant, antioxidant, human

\section{P-208}

Oviductal immunoexpression of sex steroid hormone receptors in the african giant rat (Cricetomys gambianus)

Madekurozwa $\mathrm{MC}^{*}$, Akinloye $\mathrm{AK}^{* *}$, Oke BO***

*Department of Anatomy and Physiology, Faculty of Veterinary Science, University of Pretoria, Private Bag X04, Onderstepoort 0110, Pretoria, South Africa; **Department of Veterinary Anatomy, Federal University of Agriculture, Abeokuta, Nigeria; $* \star \star$ Department of Veterinary Anatomy, University of Ibadan, Ibadan, Nigeria

The African giant rat (Cricetomys gambianus) is a large nocturnal rodent, which inhabits tropical forests and savannah grasslands of sub-Saharan Africa. This rodent is a popular food source in the western and eastern regions of the African continent. Despite general information being available on the reproductive biology of the giant rat, there is a paucity of information on changes in the immunolocalization of the androgen (AR), oestrogen $\alpha(\mathrm{ER} \alpha)$ and progesterone (PR) receptors in the oviduct of this rodent during the oestrous cycle. In light of the current lack of baseline information on the reproductive biology of this species, twenty-four female African giant rats, aged between 26 and 28 weeks and weighing 800-950 g, were used in the present study. Six animals were euthanized at pro- oestrus, oestrus, metoestrus and dioestrus. Tissue samples collected from the oviduct (infundibulum, ampulla and isthmus regions) were fixed by immersion in Bouin's fluid for 16 hours. Immunostaining was performed using a LSAB-plus kit (Dakocytomation, Denmark). Antibodies against receptors to AR, ER $\alpha$ and PR were used at dilutions of $1: 100,1: 50$ and 1:35 respectively. Simple ciliated columnar cells formed the luminal epithelium of the infundibulum, ampulla and isthmus. Underlying the luminal epithelium was a lamina propria which contained stromal cells. Circularly orientated smooth muscle cells formed the tunica muscularis. The intensity of AR, ER $\alpha$ and PR immunostaining in the luminal epithelium varied during the oestrous cycle. The intensity of the receptors' immunostaining was high during pro-oestrus, oestrus, and dioestrus. Weak to moderate immunostaining was exhibited during metoestrus. Immunostaining for AR, ER $\alpha$ and $\mathrm{PR}$ in stromal and smooth muscle cells did not vary in intensity during the oestrous cycle. The present findings are consistent with previous reports which have shown changes in the immunostaining intensity of sex steroid hormone receptors in relation to the various stages of the oestrous cycle.

\section{P-209}

Venous variations in neck region: external jugular vein and cephalic vein

Öztürk K, Dursun A, Albay S

Department of Anatomy, Faculty of Medicine, Süleyman Demirel University, Isparta, Turkey
External jugular vein gathers most of the blood from the deep part of the face and the exterior of the cranium. It is typically formed by the union of posterior division of retromandibular vein and the posterior auricular vein. On its course, it crosses superficial to the sternocleidomastoid muscle obliquely. Just above the midpoint of clavicle, it pierces the deep cervical fascia and opens into the venous angle or subclavian vein. The cephalic vein is the longest vein of the upper limb. It begins at the anatomical snuffbox as the continuation of the lateral end of the dorsal venous arch. It winds around the lateral border of the forearm and ascends lateral to biceps and between pectoralis major and deltoid muscles. It runs through the deltopectoral groove. At the deltopectoral triangle, it perforates the clavipectoral fascia and opens into the axillary vein. We report a unique bilateral combination of variations in neck region of 39 year old male cadaver. On the right side of neck, the external jugular vein constituted a common trunk with the cephalic vein crossing superficial to the medial third of the clavicle that opened into the subclavian vein after passed between the clavicle and the subclavius muscle. On the left side of neck, the terminal part of the cephalic vein drained into the subclavian vein after passed between the clavicle and the subclavius muscle in the medial third of the clavicle. Veins in the neck region are important during the surgical procedures, catheterization and pacemaker or defibrillator implantation. In addition to, external jugular vein is important for shunt procedures including the lateral ventricle in hydrocephalus surgery. The knowledge of these variations is important for general surgeons during the neck surgery, orthopedic surgeons during treatment of the clavicle fracture, anesthesiologists or cardiologists during the catheterization.

Keywords: External jugular vein, cephalic vein, variation, neck

\section{P-210}

\section{Anatomical variation in the origin of superior thyroid artery}

Özgür Ö**, Sindel $M^{*}$, Kaştan Ö$^{* * *}$, Aytaç $G^{*}$, Sindel T**

*Department of Anatomy, Faculty of Medicine, Akdeniz University, Antalya, Turkey; ${ }^{*}$ Department of Radiology, Faculty of Medicine, Akdeniz University, Antalya, Turkey; *** Department of Nursing, Faculty of Medicine, Akdeniz University, Antalya, Turkey

The superior thyroid artery arises from the anterior surface of the external carotid artery as a first branch, just below the level of the greater cornu of the hyoid bone and lies anterior and inferior. It gives the superior infrahyoid, sternocleidomastoid, superior laryngeal, cricothyroid, glandularis anterior, posterior and lateralis branches. Than it enters the superior pole of the thyroid gland anteromedially. Superior thyroid artery lies paralel to the external branch of the superior laryngeal nerve. This close relationship is important during the surgeries. The superior thyroid artery is generally considered to be always present. It was reported to arise from the common carotid in $18 \%$ of cases, the point of division of the common carotid in $36 \%$, or from the external 
carotid in $36 \%$ of cases. We reported a case that superior thyroid artery derives from the common carotid artery. A patient with possible transient ischemic attack had an digital substraction angiography. Angiography revealed superior thyroid artery derived from the common carotid artery in the right. The proximal part of external carotid artery had $95 \%$ preoclusive stenosis. Internal carotid artery was narrowed and had tandem stenosis in the cavernos segment. Middle cerebral artery seen narrowed. Vertebro-basilar system and left side was normal. Superior thyroid artery was protected because it derived before the oclusion. The common carotid artery has no branches and anatomic variations of this artery are rare. One of these variatios is the superior thyroid artery which arises from the common carotid artery. Knowledge of the variations of the superior thyroid artery is very important for neck dissections, surgeries, catheterizations, aneurysms, carotis endarterectomy surgeries.

\section{P-211}

\section{Occipital artery arising from internal carotid artery: a case report}

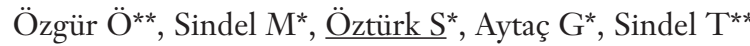

*Department of Anatomy, Faculty of Medicine, Akdeniz University, Antalya, Turkey; ${ }^{* *}$ Department of Radiology, Faculty of Medicine, Akdeniz University, Antalya, Turkey

Occipital artery $(\mathrm{OA})$ arises from the external carotid artery opposite the facial artery, its path is below posterior belly of digastric muscle to the occipital region. The OA may be a branch of the internal carotid artery (ICA), thyrocervical trunk, inferior thyroid artery or its ascending cervical branch. But its extremely rare for the OA to arise from ICA. During selective catheterisation of main carotid artery for vascular mapping of the cerebral vascular system angiography showed that OA artery was arising from ICA in a 42 year old male patient. Patient was suffered from subarachnoid haemorrhage with suspicious vascular anomalies. Imaging showed that the OA was arising from anterior espect of the right ICA with origin located $7.5 \mathrm{~mm}$ distal from the carotis bifurcation. Inner diameter of OA was $2.2 \mathrm{~mm}$. Diagnosis of the OA with anomalous origin is necessary before or during cerabral angiographies especially when selective catheterization required.

\section{P-212}

\section{Persistent carotid-vertebrobasilar anastomoses: proatlantal artery type I and type II}

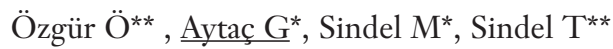

*Department of Anatomy, Faculty of Medicine, Akdeniz University, Antalya, Turkey; **Department of Radiology, Faculty of Medicine, Akdeniz University, Antalya, Turkey

Persistent carotid- vertebrobasilar anastomoses are occurs as a result of discontinuation in the development of the vertebrobasilar system. In the early stage of embryonic life longi- tidunal neural arteries supplies the hindbrain. Blood flows here by the posterior communicating, otic, hypoglossal, proatlantal and trigeminal arteries. These arteries forms anastomoses and named by the adjacent cranial nerves. These anastomoses called presegmental arteries. Presegmental arteries are provides connection between the longitidunal neural arteries and the internal carotid artery. These arteries disappears after the development of posterior communicating and vertebral arteries. Only the the posterior communicating artery is permenant after birth. Other presegmental arteries are shown $0.1-1 \%$ after birth. Persistent proatlantal artery is an anastomosis between the carotid and vertebrobasilar systems and typically classified type I and II. Both types are rare developmental anomalies. Both of them originate from the carotid artery and enter the cranium from the foramen magnum. Type I can originate from internal, external or common carotid arteries. Type II usually arises from the external carotid artery. We studied cerebral angiographies which have been performed to define subarachnoidal haemorrhagies retrospectively, to find carotidvertebrobasilar anastomoses. In our study we present two cases with type I proatlantal artery and with type II proatlantal artery respectively. Usually these anastomosis are detected incidentally during the research of other pathologies. It is important to know these anatomoses to avoid potential negatif effects to cerebral circulation before cranial surgeries or catheterizations.

\section{P-213}

\section{$25 \mathrm{OH}$ vitamin D levels of patients living in Isparta, Turkey}

Savaş HB, Ceyhan BM, Gültekin F

Department of Medical Biochemistry, Faculty of Medicine, Süleyman Demirel University, Isparta, Turkey

Objectives: Vitamin D levels influences the risk of fracture, rickets, osteomalacia, and osteoporosis. Vitamin D protects the body against muscle weakness; it helps regulate the heartbeat, strengthens the immune system, thyroid function and is necessary for normal blood clotting. Vitamin D increases calcium absorption from the digestive tract and helps the accumulation of calcium in the bones. Vitamin D increases calcium absorption and accelerates the active transport of calcium. Humans obtain vitamin $\mathrm{D}$ from exposure to sunlight and diet.

Methods: The patients who admitted to Süleyman Demirel University Faculty of Medicine Research and Application Hospital in last one year were examined about $25 \mathrm{OH}$ vitamin D levels. 12920 male and female patients were included in the study. Statistical analysis was performed with SPSS.

Results: There was a significant difference between $25 \mathrm{OH}$ vitamin $\mathrm{D}$ levels of patients in winter season and summerspring seasons $(<0.05)$. $25 \mathrm{OH}$ vitamin D levels of men were significant higher than women $(<0.05) .25 \mathrm{OH}$ vitamin $\mathrm{D}$ levels were found low in $72.48 \%$ of all patients $(<20 \mathrm{ng} / \mathrm{ml})$. The ratio of $25 \mathrm{OH}$ vitamin D levels was less than $10 \mathrm{ng} / \mathrm{ml}$ was found $40.92 \%$. 
Conclusion: When $25 \mathrm{OH}$ vitamin D levels assessing, the season of year and sex of patients should be taken into consideration.

Keywords: $25 \mathrm{OH}$ vitamin D

\section{P-214}

Investigation of relationship between the preoperative periappendicular CT values and appendicitis perforation in acute appendicitis

Horata $\mathrm{E}^{*}$, Turamanlar $\mathrm{O}^{* *}$, Celep RB***, Okur $\mathrm{N}^{* * * *}$, Acay $\mathrm{MB}^{* * * *}$, Güzel $\mathrm{H}^{* *}$, Gönül $\mathrm{Y}^{* *}$

*Atatürk Vocational Faculty of Health Services, Afyon Kocatepe University, Afyonkarahisar, Turkey; **Department of Anatomy, Faculty of Medicine, Afyon Kocatepe University, Afyonkarahisar, Turkey; ***Department of General Surgery, Faculty of Medicine, Afyon Kocatepe University, Afyonkarahisar, Turkey; $* * * *$ Department of Radiology, Faculty of Medicine, Afyon Kocatepe University, Afyonkarahisar, Turkey

Objectives: Acute appendicitis is a most common acute abdominal disease. Perforation rates of acute appendicitis are still around $20 \%$. In the literature count and scope of works, describing values that increase the perforation and relationship between appendicitis perforation with CT, aren't enuogh. The aim of our study is to evaluate this relationship and to identify surgical priorities and to prevent perforation and complications.

Methods: In our study 50 patients between 18-80 years of age was taken. Study examples were selected from people who admitted to Afyon Kocatepe University Hospital, abdominal CT scanned and verified the diagnosis of appendicitis and had an appendectomy. Patients with generalized peritonitis and perforated appendicitis were excluded. One wall thickness, lumen diameter and length of vermiform appendix, periappendicular inflammation, Hounsfield units value, appendicolith, increase in pericaecal fatty tissue, free fluid and localized lymphadenopathy were evaluated at the preoperative CT examination. Whether or not perforation during appendectomy is viewed postoperatively from the patient files.

Results and Conclusion: There was no postoperative perforation in 46 patients fulfilling the criteria of our study, while 4 had postoperative perforation. In our study, the mean values of some parameters of the non-perforated/perforated as has been looked; lumen diameter of vermiformis appendix $3.9 \mathrm{~mm} / 6.3$ $\mathrm{mm}, \mathrm{HU}$ value $-36.6 /-6$ was found. In addition, increase in pericaecal fatty tissue $73.9 \% / 100 \%$, periappendicular inflammation $73.9 \% / 100 \%$ and presence of free fluid $26.1 \% / 50 \%$ were found. Although the difference between the mean, statistically significant results weren't found between the preoperative CT values and postoperative perforation. When we look at the literature in the studies, appendiceal diameter, intraluminal fluid density, focal wall defect, appendicolith and periappendicular inflammatory changes have been associated with perforation. Perforation of acute appendicitis can lead to serious complications. We believe that the content of our study guide to the surgeon on urgent surgery of acute appendicitis.

\section{P-215}

Angiographic analysis of normal coronary artery lumen diameter in Turkish population and comparison with the previous studies

Turamanlar $\mathrm{O}^{*}$, Adalı $\mathrm{F}^{* *}$, Tor $\mathrm{O}^{* * *}$, Macar $\mathrm{O}^{* * *}$, Keş $\mathrm{H}^{* * *}$, Abbasoğlu $\mathrm{Y}^{* * *}$, Keskin $\mathrm{H}^{* * *}$

*Department of Anatomy, Faculty of Medicine, Afyon Kocatepe University, Afyonkarahisar, Turkey; ${ }^{* *}$ Department of Cardiovascular Surgery, Faculty of Medicine, Afyon Kocatepe University, Afyonkarahisar, Turkey; ${ }^{* * *}$ Faculty of Medicine, Afyon Kocatepe University, Afyonkarahisar, Turkey

Coronary artery disease increases recently and it is one of the health issues with the highest morbidity and mortality. Coronary artery diameters are one of the most effective factors on cardiac pathologies. When Turkey is compared to the European Union, it is the fourth in the mortality caused by cardiovascular diseases in men and the first in women pathologies. Frequently there is a correlation between the diameter of coronary arteries and clinical status. Even though it is known that coronary artery diameter is affected by physiological and pathological conditions, a very small number of studies regarding analysis of coronary artery diameter in the individuals without any coronary artery pathology are published so far. The images from 77 patients who presented to our hospital and have normal angiographic values were used in this study. Diameters of both two coronary arteries and the branches were measured in the following segments: 1- Medial part of the distance where LMCA arises at the level of aorta to the area that $\mathrm{Cx}$ and the LAD appear. 2- Medial of the distance from the beginning of the LAD to the region where diagonal ramus 1 leaves. 3- Medial of the distance from the beginning of $\mathrm{Cx}$ to the part where obtuse marginalis 1 leaves. 4- Proximal of the distance between where the RCA starts at the level of aorta and acute marginalis Measurements were performed using Image J program. In this program, coronary arteries were measured using the catheter diameter $(2 \mathrm{~mm})$ as a reference. Our study revealed that our results were parallel to those of the studies conducted in the United States. Significant correlation between certain coronary artery diameters has been demonstrated statistically. We conclude that these results will significantly contribute to the diagnosis and the treatment methods for the patients.

\section{P-216}

\section{Anatomical localizations of various structures in human body}

$\underline{\text { Pelin } C^{*}}$, Bor G**, Kürkcüoğlu A*, Oktem H*, Soysal H*

*Department of Anatomy, Faculty of Medicine, Başkent University, Ankara, Turkey; **Department of Radiology, Gazi Mustafa Kemal Memorial Hospital, Ankara Turkey

Objectives: Even though the importance given to surface anatomy decreases with the improvement in the field of radiol- 
ogy anatomical planes and the projection of the deeply localized structures are still important in medical education. The aim of the present study is to determine the levels of some anatomical structures according to vertebral column, to evaluate their localizations related with anatomical planes and to compare our results with the classical anatomy knowledge given in the text books.

Methods: In the present retrospective study 177 thoracic and 82 abdominal, a total of 259 CT images available in the archive of Gazi Mustafa Kemal Memorial Hospital Radiology Department were examined. The mean age of the patients was 51.11 for females and 50.08 for males. The levels of three anatomical planes and five anatomical structures were evaluated. The levels according to vertebral column were classified as below; at the middle of the body of the vertebra, at the upper half, at the lower half, at the upper end plate, at the lower end plate and at the disc level.

Results: In $51 \%$ of the images tracheal bifurcation was observed at T5 level. Azygos vein / superior vena cava junction was also observed at T5 level in $63.5 \%$ of the individuals. The highest point of aortic arc was at the level of T3 in $43.3 \%$, pulmonary trunk bifurcation was observed at the level of T6 in $59.3 \%$, and aortic bifurcation was at the level of L4 in $68.7 \%$ of the CT images. The localizations of the structures were also evaluated according to anatomical planes. The results of the study were similar with the anatomical knowledge reported in the text books. However there are still some important variations those should be emphasized.

Keywords: CT images, sternal plane, transtubercular plane, aortic bifurcation, aortic arch, tracheal bifurcation, pulmonary trunk bifurcation, azygos vein

\section{P-217}

\section{Morphology and motor innervation of hamstring muscles on fetal cadavers}

Candan B, Bilkay C, Sulak O, Albay S

Depertmant of Anatomy, Faculty of Medicine, Süleyman Demirel University, Isparta, Turkey

Objectives: The purpose of this study is to determine morphology and motor innervation of the hamstring muscles.

Methods: In this study 84 limbs from 42 embalmed fetuses aged between 18-40 weeks of gestation with no external pathology or anomaly were studied in our laboratory. Morphology of hamstring muscles and their motor nerve entry points were seen by dissection. Length, width and length of the tendon of biceps femoris, semitendinosus (St) and semimembranosus $(\mathrm{Sm})$ were measured. Distances to all motor nerve entry points to the hamstring muscles to tuber ischiadicum and lateral epicondyle of femur were measured and branching pattern of the sciatic nerve $(\mathrm{SN})$ were classified.

Results: We found that all parameters increase with age during the fetal period $(\mathrm{p}<0.05)$. Parameters do not show any sig- nificant differences between sexes $(\mathrm{p}>0.05)$. Branching pattern of the SN were classified into 4 main types: Type I in which all of the nerves to hamstring muscles arise from the SN separately $(82 \%)$. Type II in which nerve of the long head of biceps femoris (CL) and nerve of the St were arise in common trunk from SN (8\%). Type III in which nerve of the St and nerve of the Sm were arise in common from SN (7\%). And Type IV in which nerves of the CL, St and Sm were arise in common from $\mathrm{SN}(3 \%)$. The first branch of the sciatic nerve was innervated the CL in $87 \%$; St in $9 \%$ and $\mathrm{Sm}$ in $4 \%$ of the cases.

Conclusion: The present study has revealed that the morphology and motor innervation of the hamstring muscles during the fetal period. We hope that present results can be considered as providing some useful findings for clinical studies.

Keywords: Fetus, hamstring muscles, motor nerve point, sciatic nerve

\section{P-218}

The evaluation of the sphenoidal sinus and its related anatomical structuresby the cone-beam computed tomography

Yılmaz N*, $\underline{\text { Köse E }}$, Dedeoğlu N** Çolak C**, Özbağ D*

*Department of Anatomy, Faculty of Medicine, Inönü University, Malatya, Turkey; **Department of Oral and Maxillofacial Radiology, Faculty of Dentistry, Inönü University, Malatya, Turkey; $* * *$ Department of Biostatistics and Medical Informatics, Faculty of Medicine, Inönü University, Malatya, Turkey

Objectives: The anatomical structure of the sphenoid sinus, and due to the neighborhood with some important structures is important for transsphenoidal surgery. In this study, these structures were assessed by using cone-beam computed tomography (CBCT).

Methods: For this aim, our study was performed by using CBCT images of 200 (112 female, 88 male) patients who were applied Inonu University, Faculty of Dentistry, Oral and Maxillofacial Radiology Department between January 2012 and December 2014. At the same time, detailed analysis of ostium sinus sphenoidalis and sinus sphenoidalis were investigated using the same method.

Results: The bilateral sellar type was commonest type of pneumatization of the sphenoid sinus noted in this study with 194 (\%97) patients. 51 (\%25.5) patients has right C-type deviation of sphenoid sinus and 36 (\% 18) patients has left C-type of sphenoid sinus. S-shaped septum deviation of sphenoid sinus is noted 76 (\%38) patients. 21 patients are noted to have no septum of sphenoid sinus. Ostium sinus sphenoidalis was bilaterally shown 143 (\% 71.5) patients, was not bilaterally shown 20 (\%10) patients. The size of right ostium sinus sphenoidalis was measured $2.188 \pm 0.0996 \mathrm{~mm}$, the size of left ostium sinus sphenoidalis was measured $2.20 \pm 0.105 \mathrm{~mm}$ in female. The size of right ostium sinus sphenoidalis was measured $2.34 \pm 0.116 \mathrm{~mm}$, the size of left ostium sinus sphenoidalis was measured $2.39 \pm 0.129 \mathrm{~mm}$ in male. While the distance between two 
ostium sinus sphenoidalis was measured $7.30 \pm 0.333 \mathrm{~mm}$ in female, the distance between two ostium sinus sphenoidalis was measured $6.81 \pm 0.358 \mathrm{~mm}$ in male.

Conclusion: Presence or absence of the ostium sinus sphenoidales and other landmarks play a significant role for the neurosurgeons. So knowledge of these landmarks is important during the transsphenoidal surgery.

Keywords: Ostium sinus sphenoidalis, cone-beam computed tomography, transsphenoidal surgery

\section{P-219}

\section{Underweight and overweight in prefaculty children from R. Macedonia}

Trpkovska B, Lazarova D, Nakeva N, Papazova M, Zafirova B, Cadikovska E

Institute of Anatomy, Faculy Of Medicine, SS. Cyril and Methodius University of Skopje, Republic of Macedonia

Objectives: The aim of this research is to present body mass index (BMI) data in children 2-5 years of age from Skopje and provide the information on the prevalence of different categories of nutritional status during the early childhood.

Methods: The study included 400 prefaculty children (200 boys and 200 girls). Stature and body weight were measured, and the $\mathrm{BMI}$ value was calculated. Ten anthropometric parameters were measured using standard equipment and measurement technique according to the International Biological Programme.

Results: The majority of anthropometrical parameters have shown significant age and sex specific differences in favor of male subjects. Values at the 50th percentile in our male subjects for the weight-for-age index was $20 \mathrm{~kg}$, respectively whereas in the female subjects the corresponding value was the same 20 $\mathrm{kg}$. The height-for-age index value corresponding to the 50th percentile showed slightly higher values in our male subjects $111.3 \mathrm{~cm}$ than those in our female subjects $109.5 \mathrm{~cm}$. The values of 50th percentile of BMI in our males subjects was 16.3 $\mathrm{kg} / \mathrm{m}^{2}$, whereas in our females was $16.5 \mathrm{~kg} / \mathrm{m}^{2}$.

Conclusion: These results show that underweight is a health problem of the rising generation in Macedonia and needs to be considered along with the problem of overweight. It is recommended that the detected values could be applied for evaluation of deviations in growth and nutritional status in children aged 5.

Keywords: anthropometry, preFaculty children, underweight, overweight

\section{P-220}

\section{Computer - assisted analysis of lip proportions in Turkish young adults}

Bagheri H, Sirintürk S, Gövsa F, Pınar Y, Özer MA

Department of Anatomy, Faculty of Medicine, Ege University, Izmir, Turkey
Objectives: The lip complex contributes significantly to the concept of symmetry and proportion of the face. The aim of this study is to analyse in detail the morphological features of the lip and to clarify the objective parameters related to subjective regarding the lip augmentations and lips construction.

Methods: The standard lip photographs from 100 female and 100 male were calculated for the desired linear and angular components, defining the features of the lip with analysing software program.

Results: The lip shape was classified into five groups: thin, very thin, medium, full, and very full. In both genders, the medium shape of upper lip was the most common (45\% men and $61 \%$ women). The full shape and medium shape of lower lips were the most common as $48 \%$ men and $54 \%$ women, respectively. Rankings between lips and their corresponding whole faces differed greatly. Lips that were rated as the most attractive were smaller than average in midline upper lip surface heights, bilateral paramedian lip surface heights, upper lip angles and volume in the lower lip.

Conclusion: Tailoring lip enhancement treatment to each individual's anatomy, ethnic background and personal goals can optimise outcomes. These results suggest that the comprehensive evaluation of lips morphologic features by computerasisted analysis is a useful means of identifying the ideal parameters in lips construction in plastic surgery.

\section{P-221}

First whole exome sequencing in a patient with anomalous left coronary artery from the pulmonary artery (ALCAPA)

Cetin $Z^{*}$, Hekim N**, Batyraliev T***, Rolfs A***, Karben $Z^{* * * * *}$, Saygilı EI I******, Mihcioğlu D D $^{* * * * * *}$, Türkmen $\mathrm{S}^{* * * * *}$, İkidağ $\mathrm{MI}^{* * * * * * * *}$, Cüce MA*********

*Department of Medical Biology \& Genetics, Faculty of Medicine, SANKO University, Gaziantep, Turkey; **Department of Medical Biochemistry, Faculty of Medicine, Beykent University, Istanbul, Turkey; *** Kyrgizistan Minister of Health 720040, Moscow Str/148, Bishkek, Kyrgizistan; ****CENTOGENE, Centogene AG Schillingallee 6818057 Rostock/Germany; $* * * * *$ Department of Cardiology, Faculty of Medicine, SANKO University, Gaziantep, Turkey; $* \star \star * \star \star$ Department of Biochemstry, Faculty of Medicine, SANKO University, Gaziantep, Turkey; $* * * * * * *$ Institute of Science, Faculty of Medicine, SANKO University, Gaziantep, Turkey; ********Department of Radiology, Faculty of Medicine, SANKO University, Gaziantep, Turkey; $* * * * * * * *$ Department of Radiology, Sani Konukoğlu Hospital, Gaziantep, Turkey

Anomalous origin of left coronary artery from pulmonary artery (ALCAPA) also known as Bland-White-Garland syndrome is a rare congenital abnormality. In OMIM database there is no any gene description and no any molecular genetic study for BlandWhite-Garland syndrome. We present here an ALCAPA patient in which an whole genome sequencing analysis were applied. Our case was at 36 years old when he admitted to the 
Sani Konukoğlu Hospital Cardiology Clinic with a complaint of left-sided atypical chest pain. Electrocardiography (ECG) showed sinus rhythm without any pathological finding, and the TTE was suboptimal due to poor echogenicity and revealed normal systolic functions with normal-sized heart chambers, mild mitral and tricuspid regurgitation, and a pulmonary artery pressure (PAP) of 40/18 mmHg. In the parasternal short-axis view, an indefinite turbulent flow was detected in the pulmonary artery by color and continuous wave Doppler. In addition, there were no ischemic ST-T changes in the exercise stress test. Soon afterwards, the patient underwent cardiac catheterization, and since the left coronary artery (LCA) could not be seated by left Judkins and Amplatz catheters in the first step, the operator shifted to right coronary angiography in which the right coronary artery (RCA) was dilated. This detected well developed coronary collaterals filling the LCA leading to pulmonary artery washing by a reverse flow In coronary computed tomography (CT), the LCA was shown arising from the pulmonary artery and perfused by collaterals directly from the aorta and RCA. We detected 4 heterozygousalterations by using whole exome sequencing which weere also confirmed by Sanger sequencing. These alterations were (c.202A>G; p.Lys68Glu) and (c.2173G $>$ A p.Glu725Lys) mutations in Cystic Fibrosis Transmembrane Conductance Regulator (CFTR) gene, (c.1093A>G p.Met365Val) alteration in Plakophilin2 (PKP2) gene and (c.527G>A; p.Arg176Gln) alteration in MEN1 gene. We report here first whole exome sequencing analysis results detected in an ALCAPA patient.

\section{P-222}

\section{Evaluation of hip muscles with surface EMG in gait analysis}

\section{$\underline{\text { Karahan M}}$, Cigali BS}

Department of Anatomy, Faculty of Medicine, Trakya University, Edirne, Turkey

The aim of the study was to evaluate m.rectus femoris and $\mathrm{m}$. gluteus maximus in kinetic gait analysis with surface EMG. Also foot preasure and ground reaction forces were evaluated. Twenty women volunteers (Age 20.6+/-1.8, BMI 21.3+/-1.4) were accepted to our study after an elimination of the ones who had any physical trauma within the past one year or a permanent physical disorder. Measurements were made in the Gait Analysis Laboratory of the Anatomy Department of Basic Medical Sciences of Trakya University. "Zebris ${ }^{\circledR}$ Force Measurement System" was used to measure walking time, distance parameters and the ground reaction forces. "WinFDM" program which was compatible with the device was used to display and convert to numerical data of the measurement results. Muscles m. gluteus maximus and m.rectus femoris on both sides, in which to place the electrodes were selected according to criteria "Surface Electromyography for the Non-Invasive Assessment of Muscles". The highest value of EMG amplitude was considered to be a reference and values between 1 and 3 unit were given. Also, activation of the right and left extremities were compared with each other. Activation patterns of both muscle were similar in both groups. Muscles had been reached to the highest activation in the swing phase. The lowest activation was seen in the pre- swing phase. It was also observed that both the muscles were active in the loading and single support phase. Heel and metatarsal heads had been found to be exposed to the highest pressure in the foot. Force curves were observed twice at peak. The highest andthe lowest power in the majority of participants were identified in the single support phase. Our findings are consistent with the literature. According to that, surface EMG data can be used in the evaluation of muscle activation and Surface EMG seems to be a non- invasive method.

Keywords: Gait analysis, m. gluteus maximus, m. rectus femoris

\section{P-223}

\section{Variations of os sacrum and clinical significance}

Ulusoy $\mathrm{M}^{*}$, Bolatlı $\mathrm{G}^{*}$, Acar $\mathrm{M}^{* *}$, Zararsiz I*

${ }^{*}$ Department of Anatomy, Faculty of Medicine, Mevlana University, Konya, Turkey; **Department of Physiotherapy and Rehabilitation, Vocational Faculty of Health Services, Necmettin Erbakan University, Konya, Turkey

Sacrum which is located at the posterior of pelvis is formed from the union of five sacral vertebrae. Sacrum is the part of columna vertebrae which presents variations most frequently. Thirty two os sacrum available in Anatomy Laboratory of Mevlana University Medical Faculty were inspected and five different variations were determined. These were hiatus sacralis, foramina sacralia, cornu sacralia, processus spinosus and spina bifida. Although hiatus sacralis is the marking point of caudal epidural anesthesia and analgesia its variations should be under consideration. Variations of hiatus sacralis observed are as follows: Reverse $\mathrm{U}$ shaped hiatus (43.7\%), reverse $\mathrm{V}$ shaped hiatus $(18.7 \%)$, hiatus with tubercules $(21.9 \%)$, irregular shaped hiatus $(6,2 \%)$ and long hiatus $(9.4 \%)$. Variations of foramina sacralia which are critical in trans sacral block of sacral nerves in pelvic surgery were as follows: Bilateral (9.4\%), unilateral (3.1\%), five foramina and three foramina $(6.2 \%)$. Spina bifida occulta is a critical developmental defect of columna vertebralis and it is one of the most common causes of inefficient caudal epidural anesthesia. Spina bifida occulta was presenting as disunion of S1 vertebrae (6.2\%). Two processus spinosus join together at facies dorsalis and form crista sacralis mediana during fusion of sacral vertebrae. Processus spinosus was observed in S1 vertebrae (9.3\%), S1-S2 (9.3\%) and S1-S2S3 $(3.1 \%)$. Variations of cornu sacrale which is used for marking hiatus sacrale were identified as unilateral $(3.1 \%)$ and bilateral $(15.6 \%)$ cornu sacrale. We suggest that being aware of frequencies and types of sacrum variations is critical for safety of caudal epidural anesthesia and interventions of this anatomic localization. 


\section{P-224}

A rare case of arteria renalis detected in multislice computed tomography angiography

Ulusoy $M^{*}$, Bolatl $\mathrm{G}^{*}$, Koplay $M^{* *}$, Zararsiz I*

*Department of Anatomy, Faculty of Medicine, Mevlana University, Konya, Turkey; **Department of Radiology, Faculty of Medicine, Selçuk University, Konya, Turkey

Perfusion of kidneys is mainly supplied by arteria renalis which is derived from lateral wall of aorta at location of L1-2 vertebrae. Variations of arteria renalis are common. These variations are premature branching of renal artery and extra renal artery. Premature branching of A. renalis is described as branching before hilus. Extra renal artery is defined as hilar (accessory) renal artery which penetrates the kidney through hilus accompanying renal artery and polar (aberrant) renal artery which penetrates the kidney through the capsule. A variation of arteria renalis was randomly detected in 45 year old male patient with essential hypertension and right kidney rotation abnormality during routine abdominal multislice computed tomography angiography. Variations were observed in both of the renal arteries. Extra renal artery was deriving from aorta abdominalis at levels of arteria mesenterica inferior and penetrates the right kidney through right lower pole. Two extra renal arteries were detected at the left kidney one of which derives from aorta abdominalis at levels of lower border of renal artery and penetrates the kidney through hilus and the other deriving from superior mesenteric artery and penetrates the kidney through left lower pole. Being aware of the variations of renal artery is critical for renal transplantation surgery and evaluation of essential hypertension and renal diseases.

\section{P-225}

Anthropometry technique and historical development: literature review

Uzun A, Özel M, Özdemir F, Gölpınar M

Department of Anatomy, Faculty of Medicine, Ondokuz Mayıs University, Samsun, Turkey

Introduction: Anthropology is the scientific study of the origin, the behaviour, the physical, social, and cultural development of humans. The branch of anthropology is physical anthropology which examines the physical structure of human (nose, eye, ear etc.), measures, evaluates and use the anthropometry as a method. Anthropometry, objective characteristics of the human body, with specific measurement methods and principles; systematically according to the size and structure features techniques.

Objectives: The aim of this review is to provide information about the anthropometry place and usage of anatomy, studies from ancient to contemporary times. Methods: In the literature the anthropometric studies use direct and indirect methods. Direct anthropometric method use measuring tools and any tools have been developed by researcher. The indirect anthro- pometric methods are two-dimensional photographic evaluation, three- dimensional laser scanning

Results: In the world it's regarded that first modern research on anthropometric measurement was performed by Blumenbach in the 18th century. In Turkey, the most comprehensive anthropometric survey was carried out on total 59728 people, including male and female adults by Afet İnan and Şevket Aziz Kansu in 1937. In literature, there are studies on child, adult, elderly, male and female separately or together.

Conclusion: Measurements based on soft tissue landmarks may be more amenable to photogrammetry, while direct anthropometry is preferable for dimensions dependent on bony landmarks which are easily palpated but difficult to identify in the photographs. Three- dimensional anthropometry is designed to capture topographical surface data of the face. The technique is relatively non-invasive. Stereophotogrammetry, laser surface scanning and 3D photogrammetry are examples of these newer techniques. The laser surface scanner is the most common type of 3-dimensional technique, but this method is more expensive than others. It is important to look at previous studies to gain a wide perspective and to continue making positive advances in anthropometry.

\section{P-226}

\section{Development of the male external genital organs} during the fetal period

Özgüner G, Öztürk K, Dursun A, Bilkay C, Sulak O

Department of Anatomy, Faculty of Medicine, Süleyman Demirel University, Isparta, Turkey

Objectives: In the present study we aimed to determine normal size of the male external genital organs and anogenital distance in the human fetus by examining morphological structure and, to form a development scale during the fetal period.

Methods: This study was performed on 104 spontaneously aborted human male fetuses aged between 10 and 39 weeks of gestation. Fetuses were divided into groups according to gestational weeks, months and, trimester. Following parameters belong to the male external genital organs were than measured: - Penile length • Penile width • Transverse scrotal diameter • Anterior-posterior scrotal diameter • Height of scrotum • Distance from the anterior aspect of the penis to the center of the anus $\bullet$ Distance from the posterior aspect of the penis to the center of the anus $\bullet$ Distance from the posterior aspect of the scrotum to the center of the anus

Results: The mean of the each parameter was computed by gestational weeks, months and, trimester groups, and data were presented as mean \pm standard deviation. There were statistically significant differences between trimester groups. The transverse scrotal diameter was $3.76 \mathrm{~mm}, 8.95 \mathrm{~mm}, 23.35 \mathrm{~mm}$ and $29.75 \mathrm{~mm}$ in the first, second, third and full term fetuses respectively. Penile length was $3.19 \mathrm{~mm}, 10.08 \mathrm{~mm}, 21.54 \mathrm{~mm}$, and $28.50 \mathrm{~mm}$ in the first, second, third and full term fetuses respectively. Distance from the anterior aspect of the penis to 
the center of the anus was $7.46 \mathrm{~mm}, 21.06 \mathrm{~mm}, 41,87 \mathrm{~mm}$, and $56.50 \mathrm{~mm}$ in the first, second, third and full term fetuses respectively. In conclusion data obtained from this study, will be useful in the assessment of male external genital organ development in fetal period.

Keywords: fetus, male external genital, fetal development, morphometry

\section{P-227}

\section{Pterygomaxillary fissure}

Sindel A, Öğüt E, Gürçay S, Sindel M

*Department of Anatomy, Faculty of Medicine, Akdeniz University, **Department of Oral and Maxillofacial Surgery, Faculty of Dentistry, Akdeniz University, Antalya, Turkey

The pterygomaxillary fissure is formed by the divergence of the maxilla from the pterygoid process of the sphenoid bone. It connects the infratemporal fossa with the pterygopalatine fossa. During the osteotomies this area couldn't visualise and osteotomy is performed tactually. Osteotomy is performed by an osteotome or pterygoid chisel. Pterygomaxillary area is an important component of the orthognatic surgeries because during the Le Fort I osteotomies down fracture of maxilla is required. We studied 80 human dried skulls with undefined age and gender. We investigated the structure of the pterygomaxillary fissure and differences between the right and left sides. The lenght, width and range of pterygomaxillary fissure is measured in sagittal plane. Mean values, standart deviations are calculated and right and left sides are compared. The mean length, width and range of the pterygomaxillary fissure was $17.7( \pm 3.3)$, 6.6 $( \pm 1.4), 96.2( \pm 24.3)$ respectively. The measurements was significantly different from each others in right and left. $(\mathrm{p}<0.05)$ The last step of the Le Fort I osteotomies is seperation of the maxilla from the pterygoid plates. For this process the pterygomaxillary junction should be separated with curved osteotomes. By placing a finger inside the mouth and feeling the hamulus, the medial extent of the osteotome can be palpated to ensure the proper position. Once the osteotomies are completed, the down fracture is performed with digital pressure. Knowledge of the structure, mean values and distinctions of the pterygomaxillary fissure is important to prevent possible complications during the orthognatic surgeries.

\section{P-228}

\section{Septal accessory pathway}

Lazarova DT ${ }^{*}$, Zivadinovik J**, Papazova $M^{* *}$, Dodevski A**

*Institute of Anatomy, Medical Faculty, Skopje, Macedonia;

**Institute of Anatomy, Medical Faculty, Skopje Macedonia

Objectives: Accessory pathway (AP) ablation is one of the most satisfying invasive electrophysiology procedures associated with high success rates and relatively few complications. Nevertheless, when APs are found on the cardiac septum, ablative procedures become complex, and unique pitfalls need to be avoided. The most common conduction pathways in the septal place are Kent Bundles. They occupy an area along the right anterior A-V junction and than extended anteriorly and lateraly to the point where the right coronary artery enters the coronary sulcus.

Methods: The anatomical characteristics of the area were rewed by the dissection of 15 human hearts. In the dissection the anterior septal area was approached from thye superior aspect of the heart. The position of Kent Bundles was determined from a study of serial sections. A variety of stains were performed but the most useful proved to be hematoxillin-eosin and Masson trichrome.

Results: One important aqnatomical finding was the relationship of the atrial septum, the right fibrous trigone and the underlying membranous ventricular septum to the $\mathrm{A}-\mathrm{V}$ node and the bundle of His. This study indicates that the Kent Bundles and the bundle of His can be within $2 \mathrm{~mm}$ of each other. The other course from the right atrium to the right ventricle could be a point anterior to the right fibrous trigone to the arbitrary line separating the anterior septal area from the right free wall. The tendon of Todaro was found in 8 hearts and it was extended from the Eustachian valve of the coronary sinus to the right fibrous trigone.

Conclusion: This study indicates that the most common accessory pathway in the septal place are Kent Bundles. Kent Bundles and the bundle of His can be within $2 \mathrm{~mm}$ distance.

\section{P-229}

Unilateral atypic foramen ovale: a rare bone variation

Şenol D, Canbolat M, Köse E, Cay M, Yılmaz N, Özbă̆ D

Department of Anatomy, Faculty of Medicine, Inönü University, Malatya, Turkey

In routine anatomy laboratory studies, we observed that left foramen ovale (FO) located at the base of the skull was split two part by lateral plate of the pterygoid process of sphenoid bone. The other anatomical structures were normal. Divided FO variation is extremely rare. FO is considered as an important landmark for the brain surgeon during the surgery involving the trigeminal nerve. Mandibular nerve, accessor meningeal artery, emisserial vein and sometimes lesser petrosal nerve pass through this hole. The knowledge of these variations by clinicians is important for prevent the damage of the structures which located at the FO during surgery of this area.

Keywords: Foramen ovale, variation, anatomy

\section{P-230}

\section{Kimmerle's anomaly: posterior osseous bridging of atlas}

Şenol D, Canbolat M, Köse E, Ulutaş S, Cuglan S, Özbağ D

Department of Anatomy, Faculty of Medicine, Inönü University, Malatya, Turkey 
We observed variations of three atlas bone in routine anatomy laboratory studies. We found one partially unilateral, one bilateral complete with tunnel form and one bilateral complete Kimmerle's Anomaly. The knowlege of this rare variation is very useful for brain surgeons and radiologists. Ponticulus posterior makes pressure on atlantic part of vertebral artery. This variation should be considered in the patients with vertebrobasiler insufficiency symptoms such as headache, vertigo, shoulder and arm pain.

Keywords: Atlas, vertebral artery, Kimmerle's anomaly

\section{P-231}

\section{Morphological and radiological features of the mastoid foramen and mastoid emmissary vein and their anthropological and clinical significance}

$\underline{\text { Uyaroğlu FG }}^{*}$, Pekçevik Y**, Ertürk $M^{* * *}$, Şengül G***

*Department of Neurology, Izmir Tepecik Education and Research Hospital, Izmir, Turkey; ${ }^{*}$ Department of Radiology, Izmir Tepecik Education and Research Hospital, Izmir, Turkey; ***Department of Anatomy, Faculty of Medicine, Ege University, Izmir, Turkey

Objectives: The mastoid emissary vein (MEV) and its foramen have anthropological significance in transition to bipedalism and preferential intracranial venous flow into the vertebral plexus in the upright man. The aim of the study was to explore and delineate the morphological and radiological characteristics of MEV and mastoid foramen (MF) which is the mark of $\mathrm{MEV}$ on the temporal bone.

Methods: The present study included 200 adult human temporal bones of West Anatolian origin from the gross anatomy laboratory of Ege University, Department of Anatomy and CT data retrospectively reviewed from 182 patients referred to İzmir Tepecik Education and Research Hospital, Department of Radiology for CT angiography for various pathologies.

Results: The MF was observed single in 46 left (39\%) and 48 right (58\%) temporal bones, double in 45 left (\%38), 23 right (28\%) bones, triple in 17 left(14\%), 8 right (10\%) bones, quadruple 6 left (5\%) and none of right bones. The foramen was absent in 4 left (5\%) and 3 right (4\%) bones. The mean diameter of the MF was $2.04 \pm 0.76$ (range $0.6-4.8) \mathrm{mm}$ on the left and $2.08 \pm 2.03$ (range $0.5-6.2$ ) $\mathrm{mm}$ on the right side. The radiological data were different from anatomical results. Of 166 patients, the mastoid emissary vein $(\mathrm{MEV})$ was not identified in $37(22.3 \%)$ patients and only six patients had more than one $\mathrm{MEV}$ that were very small $(<2 \mathrm{~mm})$, and only five patients had very large $(>5 \mathrm{~mm})$ veins.

Conclussion: The emissary foramina allow the passage of emissary veins that connect the intracranial venous sinuses with extracranial veins. The frequency of emissary veins and related foramina has been found to be much higher in humans than in ape species. The results of this study will provide better understanding of the identification of structures for regulation of intracranial pressure, regulatory mechanisms in bipedality, and clinical cases of endolymphatic hydrops and pseudotumor cerebri in humans.

\section{P-232}

Morphometry of the condylar canal, condylar emmissary vein and occipital foramen with their evolutionary and clinical aspects

${\text { Özgören } \mathrm{O}^{*} \text {, Uyaroğlu FG }}^{* *}$, Pekçevik $\mathrm{Y}^{* * *}$, Şengül G*

*Department of Neuroscience, Institute of Health Sciences, Ege University, Izmir, Turkey; **Department of Neurology, Izmir Tepecik Education and Research Hospital, Izmir, Turkey; $* * \star$ Department of Radiology, izmir Tepecik Education and Research Hospital, Izmir, Turkey

Objectives: Emmissary veins and foramina on bony structure is better-developed in humans when compared to other primates. This is believed to be related to bipedality. In this study, we aimed to discuss the clinical and evolutionary significance of the occipital foramen (OF), condylar canal (CC) and condylar occipital vein $(\mathrm{COV})$ with their morphological and radiological analysis.

Methods: One hiundredoccipital bones from the collection of Ege University, Scchol of Medicine, Department of Anatomy were used for this study. Nine of these with damaged condylar canals were discluded from the study. Radiological data were retrieved from retrospective analysis of $182 \mathrm{CT}$ angiographies perfomed in İzmir Tepecik Research and Education Hospital.

Results: From 91 occipital bones, 6 had single OF, 2 had double and in the form of a canal in the median plane. The remaining 83 bones had $\mathrm{OF}$ on the left side of midline with an average number of $2.93(\min 1-\max 7)$ and on the right side average number 2.78 (min $1-\max 9)$. CC was absent on the left side in 31 bones and in 29 bones on the right side. The remaining 60 left and 62 right canals had an average length of $10.85 \mathrm{~mm}$ and $9.82 \mathrm{~mm}$, consequently. In CT angiographies, CV was not identified in 39 (23.5\%) patients. It was found bilaterally in 97 (58.4\%) and unilaterally in $30(18.1 \%)$. Patients. Only 15 patients had a very large (>5 mm) CV. The petrosquamosal sinus (PSS) was identified only in one patient $(0.6 \%)$ on the left side.

Conclussion: Posterior fossa emissary veins are important in complications following condylar fracture. Also, these structures have significance in evolutionary aspects related tobipedality. We believe the results of our study and other anatomical studies of the $\mathrm{OF}, \mathrm{CC}$ and $\mathrm{COV}$ and their relation to each other are of utmost importance in neurosurgery and evolutionary studies.

\section{P-233}

\section{Motor nerve entry points of the median nerve in the forearm muscles}

$\underline{\text { Uyaroğlu FG}}^{*}$, Özgören $\mathrm{O}^{* *}$, Şengül $\mathrm{G}^{* * *}$

*Department of Neurology, izmir Tepecik Education and Research Hospital, Izmir, Turkey; ${ }^{* *}$ Department of Neuroscience, Institute of Health Sciences, Ege University, Izmir, Turkey; ${ }^{* *}$ Department of Anatomy, Faculty of Medicine, Ege University, Izmir, Turkey 
Objectives: The functions of the human hand present an evolutionary unique structure. The research on its anatomic features are crucial not only for medical practice but also for evolution. This study aims to determine the features of the motor nerve points (MNP) of the median nerve innervating the forearm muscles.

Methods: 50 forearms of 23 male and 2 female cadavers were dissected and the motor branches of the median nerve in the forearms were examined and the muscles entrance points were analyzed.

Results: Mean number of MNP were found 2.76 \pm 1.35 (min 1$\max 4$ ) for pronotor teres, $2.08 \pm 0.9(\min 1-\max 4)$ for flexor carpi radialis, 3.9 2.17 (min 1-max 6) for musculus flexor digitorum superficialis muscles. Palmaris longus muscles were detected in 42 forearm and their mean MNP number was $1.32 \pm 0.77(\min 1-\max 2)$.

Conclusion: Compared to the other muscles, the forearm flexors indicate more MNP's to provide fine motor movement of hands. The results of our study have been discussed together with analysis of earlier studies.

\section{P-234}

A study of morphometric charateristics of Achilles tendon by using ultrasound imaging over 18-years-old healty population

Canbolat $M^{*}$, Özbağ D*, Özdemir Z**, Kafkas AS***, Şenol D*, Cay $M^{*}$

*Department of Anatomy, Faculty of Medicine, inönü University, Malatya, Turkey; ${ }^{* *}$ Department of Radiology, Faculty of Medicine, Inönü University, Malatya, Turkey; ***Faculty of Physical Education \& Sports, Inönü University, Malatya, Turkey

Objectives: In this study, we aimed to reveal normal values of achilles tendon with the ultrasound imaging. In addition to show changes in the size of Achilles Tendon depending on variables age, sex, weight, height, right foot-left foot, smokingnot smoking, engaging in sports-sedanteries. And also to demonstrate the relationship between anthropometric measurements and radiologic results.

Methods: This study performed on 171 healty volunteers over 18 years old. First we save the demographic characteristics. Then, we take some anthropometric measurements. After taking anthropometric measurements we take the tendon thickness, tendon width, tendon length and tendon area in axial plan with ultrasound imaging.

Results: The average of achilles tendon width, thickness, area and length of male volunteers were significantly higher than averages of female volunteers. Tendon width, thickness, area and length showed no significant difference between right and left tendon both of gender. For sizes of achilles tendon, there was significant difference between volunteers who engaged sports and sedanteries. For sizes of achilles tendon, there was significant difference between volunteers who smoking and no-smoking. Sizes of achilles tendon in 18-29 age group were significantly lower than age groups of 30-39, 40-49 and 50+. Only weight and BMI which including in the anthropometric measurements, were positively correlated with the achilles tendon sizes.

Conclusion: In the diseases of the ankle area, changes of tendon size has come to the fore. The ultrasound imaging is easy and inexpensive method for determine this changes.

Keywords: Achilles tendon, ultrasound imaging, anthropometry.

\section{P-235 \\ Left inferior phrenic artery originated from the celiac trunk: a case report}

Hamutoğlu R, Yıldırım M, Al-Talahma M, Şahin ZA, Cetinok H

Department of Anatomy, Cerrahpaşa Medical Faculty, Istanbul University, Istanbul, Turkey

The anatomical variations of the abdominal arteries are clinically important. The celiac trunk (CT) arises just below the aortic hiatus at the level of thoracic 12-lumbar 1 (T12-L1), and is the first anterior branch of abdominal aorta (AA). It is about $1.5-2 \mathrm{~cm}$ long and passes almost horizontally forwards. It divides into the left gastric (LGA), common hepatic (CHA) and splenic artery (SA). The inferior phrenic arteries (IPA) usually arise from the AA, just above the level of the CT. Each IPA gives origin to superior suprarenal artery. IPA is the most common source of extrahepatic collateral blood supply for hepatocellular carcinoma (HCC) and frequently supplies HCCs located in the bare area of the liver. One of the components of the AA usually arises directly from the CT. The aim of this study was to identify the origin and distribution of the left inferior phrenic artery (LIPA). During routine dissection of the upper abdominal cavity of a 38-year-old male cadaver, we observed a CT with four branches in the Department of Anatomy, Cerrahpaşa Medical Faculty. The CT gave rise to LIPA first and after it gave the three typical branches; CHA, SA and LGA. The LIPA also supplied few branches to suprarenal gland. CT was unusually lengthy. The anatomical variations of the CT are due to the the ventral splanchnic branches of dorsal aorta. Anatomical variations in the branching pattern of the CT are of considerable importance in the liver transplants, laparoscopic surgery, radiological abdominal interventions and penetrating injuries to the abdomen. Generally, additional branches of the CT other than its usual branches are referred as collaterals. Many authors stated that one of the additional variations included a LIPA originating from the CT in different rates. The knowledge of this type of variations are important for surgeons performing kidney transplants and suprarenal surgeries.

\section{P-236}

Two saphenous veins that drain femoral vein seperately: a case report

Yıldırım M, Hamutoğlu R, Peştemalcı ŞT, Yıldız YZ

Department of Anatomy, Cerrahpaşa Medical Faculty, Istanbul University, Istanbul, Turkey 
Venous system is more complex and important for clinical significance compared to the arterial system due to its frequent anatomical variations. Great saphenous vein (GSV), which is the longest in the body, is at the medial part of the dorsum of the foot the contuniation of the medial marginal vein. It passes anterior to medial malleolus and posterior to the medial tibial and femoral condyles and reaches the anterior thigh region. In subinguinal region its tribaturies are superficial epigastric, superficial circumflex iliac and external pudendal veins. The GSV passes through saphenous opening and enter the femoral vein (FV). Frequently medial accessory saphenous (MASV) or lateral accessory saphenous vein (LASV) may accompany the GSV. The morphology of these veins can show variations just as the other veins in the body. Because taking numerous branches throughout the course on the thigh and also relating with deep veins, VSM plays an important role in the venous transformation of lower extremity. During the dissection of the lower extremity of an adult cadaver in İstanbul University Cerrahpaşa Medical Faculty Anatomy Department, we observed two saphenous veins that drain to femoral vein seperately. The GSV is the most popular vein graft in coronary artery bypass surgery even though synthetic grafts and internal thoracic artery are becoming used more frequently. GSV is not only used in coronary by-pass surgery but also in the treatment of cerebrovascular diseases and as grafts in peripheric vascular surgery. Five anatomical GSV variations have been reported in the thigh region. An accessory saphenous vein (ASV) is often mistaken as a duplication of the GSV, but ASV is usually smaller in size and does not drain the same cutaneous territory as the GSV. Several authors found different kind of variations and rates about MASV/LASV in the literature. These variations will be discussed by using literature data.

\section{P-237}

\section{Brachial artery course variation with a deep brachial artery passing between the two roots of median nerve - a case report}

Taştan ÖA, Yıldırım M, Hamutoğlu R, Kaya YE, Al-Talahma M, Şahin ZA

Department of Anatomy, Cerrahpaşa Medical Faculty, Istanbul University, İstanbul, Turkey

Variations in the course and branching of the arteries in the upper limb are seen very frequently that one can call these variations are a rule than an exception. One such variation is superficial brachial artery (SBA) and its branching variations. To fully understand the anatomy and terminology of SBA, we should first evaluate the course and branching pattern of axillary artery (AA) and brachial artery (BA). Variations in the course and branching of these arteries must be taken into consideration when performing surgeries in axillary and arm region. During routine educational dissection in İstanbul University Cerrahpaşa Medical Faculty Anatomy Department, AA passing between the medial root of median nerve (MN) and a communicating branch from the lateral cord of brachial plexus to the medial root of $\mathrm{MN}$, from anterior to posterior, were found. After giving all its branches it becomes BA but BA continues superficial to $\mathrm{MN}$ after giving its deep brachial artery branch which passes between the two roots of $M N$ and continues in its normal course. SBA first defined by Adachi as the BA that runs superficially to MN. In our case, it seems to fit the definition of Adachi and Keen's Type 1 but main considerations in our case is that this variation is presented with a $\mathrm{MN}$ formation variation and that it's rather a main BA that runs superficially because AA gave all its normal branches before becoming BA and there isn't a second trunk that runs deeply as a normal BA, there is only a deep brachial artery (a. profunda brachii) that passes between the roots of MN. Also BA continues and gives its radial and ulnar branches normally in cubital region, so this variation can't be called a 'real' SBA.

\section{P-238}

Right inferior phrenic artery originating from a superior additional right renal artery: a case report

Al-Talahma M, Yıldırım M, Cetinok H, Hamutoğlu R

Department of Human Anatomy, Cerrahpaşa Medical Faculty, Istanbul University, Istanbul, Turkey

The inferior phrenic arteries (IPAa) are among the most common extrahepatic collateral vessels. They supply multiple organs such as the diaphragm, adrenal glands, esophagus, stomach, liver, inferior vena cava (IVC), and retroperitoneum. The right and left inferior phrenic arteries usually originate as separate arteries from the abdominal aorta (AA) or the celiac trunk (CT). Also seen with much less frequency to originate from a common trunk of the AA, the CT, or the left gastric artery. Surgical procedures involving the IPAa may lead to hemoperitoneum, hemoptysis, or gastroesophageal, diaphragmatic, or hepatic bleeding. For these reasons, reporting the origin and trajectory variations of the IPAa are clinically important. One formalinfixed adult male cadaver was used. The anterior abdominal wall and the upper abdomen internal organs were removed. Manual dissection was done to identify the origin and trajectory of the renal arteries, veins and the IPAa and veins. The right kidney receives triple separate renal arteries from different levels of the AA. The superior and middle renal arteries arose separately from the AA at the same level and passes as typically posterior to the inferior vena cava IVC. The inferior renal artery passes anterior to the IVC and posterior to the right testicular vein. The right inferior phrenic artery (RIPA) arose from the proximal third of the superior renal artery. Variations in the number of the renal arteries are common. RIPA is the most common extrahepatic collateral vessel that supplies hepatocellular carcinomas (HCCs). Therefore, awareness of the unique origin of the reported RIPA is clinically important to reduce the surgical risk of RIPA injuries. Particularly, in transcatheter embolization of HCC which often involves finding the root of the RIPA. These variants may potentially impact revascularization of the renal arteries. 


\section{P-239}

Studying with multidetector computed tomography angiography techniques of aortic arch branching variation

Bolatlı $\mathrm{G}^{*}$, Ulusoy $\mathrm{M}^{*}$, Koplay $\mathrm{M}^{* *}$, Çat $Z^{*}$, Zararsı $\mathrm{I}^{*}$

*Department of Anatomy, Faculty of Medicine, Mevlana University, Konya, Turkey; **Department of Radiology, Faculty of Medicine, Selçuk University, Konya, Turkey

Objectives: In this study, Multidetector Computed Tomography (MDCT) angiography technique with aortic arch branching variation types, frequency and clinical significance were investigated.

Methods: MDCT examinations carried out 83 men, 97 women were evaluated 180 patients. Arcus aorta types and frequency of the variation was detected.

Results: The aortic arch branching variations in images obtained from these patients were common. 26 A total of 50 men and 24 women $(27.7 \%)$ patients were detected variations. Brachiocephalicus trunk and carotid sinistra out variations on the same stem 23, a total of 44 men and 21 women (24.4\%) was seen in patients. Another variation type vertebral arteries under the two types of variation $(3.3 \%)$ were detected in the patient. The first vertebral artery from the aorta arch out of five people (two men, three women), the second type of variation vertebral artery subclavian artery to the aortic arch with sinistra output from the same root into a person was seen.

Conclusions: Clinically important aortic arch branching variation that can be detected by CT angiography technique. A good knowledge of these variations is important and instructive in terms of common invasive and surgical interventions.

\section{P-240}

\section{Variations of foramen transversarium of cervical vertebrae}

Ulusoy M, Bolatlı G, Acar S, Zararsız İ

Department of Anatomy, Faculty of Medicine, Mevlana University, Konya, Turkey

Objectives: Foramen transversarium which are the characteristic hall marks of cervical vertebrae are located on the transverse processes of cervical vertebrae. Vertebral arteries, veins and sympathetic nerve fibers pass through. Dimensional, formal and numerical variations are frequent due to developmental abnormalities. These variations lead to various clinical symptoms.

Methods: 86 cervical vertebrae available in Anatomy Laboratory of Mevlana University Medical Faculty are evaluated.

Results: Double foramen transversarium (17.4\%) and asymmetrical $(16.3 \%)$ foramen transversarium variations were observed. Unilateral double foramen transversarium were existed in $11.6 \%$ cases while $5.8 \%$ cases showed bilateral double foramen transversarium. The frequency of these variations was $10.5 \%$ in typical cervical vertebrae, and $15.1 \%$ in atypical cervical vertebrae.
Conclusion: Being aware of the variations of foramen transversarium is critical in characterization of vertebrobasilar insufficiency syndrome caused by compression of the anatomic components passing through. We also suggest that this would be useful in planning the cervical spine surgery and as well as avoiding postoperative complications.

\section{P-241}

Ankylosing spondylitis in an elderly patient followed up due to ischaemic heart disease: case report

İlgün E, Özdil H, Kayhan E

*Department of Anatomy, Faculty of Medicine, Mevlana University, Konya, Turkey; **Department of Physical Therapy and Rehabilitation, Faculty of Medicine, Mevlana University, Konya, Turkey; $* * \star$ Department of Cardiology, Faculty of Medicine, Mevlana University, Konya, Turkey

Objectives: Ankylosing spondylitis (AS) is a progressive disease, especially characterized by marked inflammation especially in spinal joints and notable peripheral joint stiffness as in hip and shoulder joints, in one third of the patients. During the course of AS, apart from stiffness in axial and peripheral bones, pathological conditions may also occur in cardiovascular systems.

Case: A 58 years old patient under consultation in the Physiotherapy and Rehabilitation department at Mevlana University having ischemic heart disease and hypertension was suffering from persistent chest pain inspite of undergoing an open heart surgery and a medical treatment. On examination, thoracic kyphosis was observed and lumbar lordosis was determined to have been flattened. Flexibility of his neck and lower back was found to be restricted in all direction. In radiologic assessment, it was revealed that there was grade 4 sacroilitis and fusion in his sacroiliac arthography. Furthermore, there was syndesmosis, bridges between vertebrae and bamboo spine, vertebral body squaring in lumbosacral arthrography. Based on these symptoms the patient was diagnosed as AS.

Conclusion: The risk of development of ischemic heart disease risk is higher in patients with AS as compared to ones who do not have it. In patients with chest pain, which fails to respond medical treatment, should be assessed with a multidisciplinary approach and not rule out the diseases causing chest pain similar to AS.

\section{P-242}

\section{Difference between objective and subjective} evaluations of facial asymmetry

Kürkçüoğlu A* , Ayan DM**, Baykan R**, Demirkan E**, Özkan Ö**, Özkubat I'**, Şimşek $M^{* *}$

*Department of Anatomy, Faculty of Medicine, Başkent University, Ankara, Turkey; ${ }^{*}$ Term II Students, Faculty of Medicine, Başkent University, Ankara, Turkey

In this study, it is aimed to objectively evaluate the facial asymmetry using anthropometric points and to demonstrate the differ- 


\section{S218 XXIV International Symposium on Morphological Sciences, 2nd-6th September, 2015, Istanbul, Turkey}

ence between objective and subjective evaluations. This study was conducted on 220 male and 220 female volunteers chosen among the students of the Başkent University, with ages between 18 and 25. All subjects were photographed in a standart position with a digital camera and all the images were stored in digital format. In each photograph, a vertical line over lapping with the midline of the face was drawn. Then, five anthropometric points were marked on both left and right sides of the face and the distance between these points and the midline were determined. The difference between the distances of each point on one side and the corresponding one on the other side were compared in order to determine the facial asymmetry. For subjective evaluation, a committee consisting of seven people was assembled. Each picture was shown to this committee for three seconds and they were asked to evaluate the faces as symmetrical or asymmetrical. Subjective values acquired from this practice was compared with the objective values acquired from the measurements described above and the difference between these two was demonstrated. After the measurements, it is concluded that the majority of faces are asymmetrical and the objective and subjective evaluations do not go together. It is also observed that in the faces subjectively evaluated as asymmetrical, asymmetries are concentrated in the middle third of the face. Data acquired from objective measurements and subjective evaluations of faces were presented and comparedin this study. Data were analyzed to determine if there is a statistically significant difference between objective measurements and subjective evaluations. We hope this study will be useful in better understanding of the relation between symmetry and perception of esthetics, and during the consultation of facial surgery patients.

\section{P-243}

Lip posture changes to Steiner's aesthetic line in Class I individuals

Kürkçüoğlu A* ${ }^{*}$ Karaca $Z^{* *}$, Oğuz Ö$^{* * *}$

*Department of Anatomy, Faculty of Medicine, Başkent University, Ankara, Turkey; **Department of Anatomy, Faculty of Medicine, Yeni Yüzyıl Üniversity, Istanbul, Turkey; $* * *$ Department of Anatomy, Faculty of Medicine, Çukurova University, Adana, Turkey

The soft-tissue profile and upper/lower lip posture play an important for forensic anthropologists, dentists and plastic surgeons. Compatible with plump and beautiful lips are the most important aesthetic facial harmony, especially in women's visual icon. We evaluated the upper and lower lip thickness in the Turkish race separately in groups consisting of boys and girls and compare the results between the groups and with other races. The study was carried out on 100 healthy individuals (50 girls and 50 boys) with Class I skeletal structure. The average age of the girls was $24.23 \pm 4.07$ years while the average age of the boys was $25.44 \pm 2.24$ years. The distance of three different points (Ls: labialis superior, Sto: stomion and Li: labialis inferior) to line $\mathrm{E}$ (Steiner's aesthetic line) determined on the lateral cephalometric X-ray images of the individuals was measured. All cephalometric images in Ls-E, Sto-E and Li-E distances are measured. The average value for the Ls-E distance was found as $-12.80 \mathrm{~mm}$ while the minimum value was -27.02 $\mathrm{mm}$ and maximum value: $-1.00 \mathrm{~mm}$; the average value for the Sto-E distance was $-21.94 \mathrm{~mm}$ while the minimum value was $38.02 \mathrm{~mm}$ and the maximum value was $-7.59 \mathrm{~mm}$; the average value for the $\mathrm{Li}-\mathrm{E}$ distance was $-8.07 \mathrm{~mm}$ while the minimum value was $-22.36 \mathrm{~mm}$ and the maximum value was $9.20 \mathrm{~mm}$. Lips are located in the midst of the face and are important to facial attractiveness. Lip thickness obtained in this study, we believe it would be useful to orthodontists in planning treatment of patients related to their facial profiles while it provides tips to the forensic anthropologists in the technique of facial reconstruction and to facial cosmetic surgeons in their evaluations as regards the harmony of the bottom part of the face.

\section{P-244}

\section{Is there any relationship flat foot and Achilles tendon?}

Gönül Y*, Yücel Ö*, Eroğlu $M^{* *}$, Şentürk I**, Eroğlu $S^{\star * *}$, Dikici $\mathrm{Ö}^{* * *}, \underline{\text { Cartıllı Ö}}^{*}$, Ulaşlı $\mathrm{AM}^{* * *}$

*Department of Anatomy, Faculty of Medicine, Afyon Kocatepe University, Afyonkarahisar, Turkey; **Department of Orthopedics and Traumatology, Faculty of Medicine, Afyon Kocatepe University, Afyonkarahisar, Turkey; ***Department of Physical Therapy and Rehabilitation, Faculty of Medicine, Afyon Kocatepe University, Afyonkarahisar, Turkey

Introduction: Flat foot $(\mathrm{FF})$ results from the loss or decrease of the medial longitudinal arch of the foot. It may lead to a higher risk in development of lower limb pathologies, but the precise mechanism(s) by which flat-arched feet may lead to an elevated risk of those is not completely understood. However, previous research suggests that there are systematic differences in lower limb kinematics, kinetics and muscle function comparing individuals with flat- and normal-arch feet. But there are few studies that have explored the association between foot posture and lower limb muscle and tendon morphology. Similarly, the connection between foot type and Achilles tendinopathy is not clear.

Objectives: Therefore, the aim of this study was to investigate the association between foot posture and morphology of Achilles tendon in pre-adolescent and adolescent subjects.

Methods: Fifty-nine subjects aged between 9-16 years were divided into two groups; A) the ones with flexible FF ( $n=30)$ and $\mathrm{B})$ the ones with healthy feet $(\mathrm{n}=29)$. For both groups age, height, weight, leg length and length, cross-sectional area and the site of insertion of the Achilles tendon were recorded. The measurements about the Achilles tendon were done with ultrasonography. The concept whether an asymmetry in the abovementioned features of the Achilles tendon exists between the two limbs was inspected. To analyze the data dependent and independent t-tests and backward multi regression method were used.

Results: According to the findings, the flexible FF has no relationship with the Achilles tendon length and cross- sectional area, age, height, leg and feet size; nevertheless it is highly possible to see flexible FF in overweight subjects. Besides, a negative correlation was found between the mean Achilles tendon 
cross-sectional area and age, and a positive correlation is found between FF and foot size. No differences were detected in the features of the Achilles tendon between the two limbs of the same subject.

\section{P-245}

Demostrating spatium parapharyngeum on plastinated specimens

Boduç E, Çelik S, Bilge O

Department of Anatomy, Faculty of Medicine, Ege University, Izmir, Turkey

Objectives: Tumors of the parapharyngeal space, despite constitute only $1 \%$ of head and neck neoplasms, anatomical complexity of this area still shrouds in mystery with its possible variation for the surgeons. Safe and complete removal of these tumors requires a perfect knowledge of the surgical anatomy and experience. With this study we aimed at to demostrate spatium parapharyngeum by using plastination technique in order to set light to the this area.

Methods: In this study we exposed the parapharyngeal spaces (spatium parapharyngeum) of two cadavers head. One head was dissected to show the parapharyngeal space three dimensionally. After dissection the head was plastinated. The other head was sliced transversely and the slices were plastinated.

Results: At the end, we were able to show the parapharyngeal spaces on different anatomical views. The examines several times of parapharyngeal space of the cadaver head is possible with this technique. At the same time for handling, the tissues protect their reality with their color and flexibility.

Conclusion: Through plastination technique which had been founded by Gunther Von Hagens in 1977 we have the opportunity to preserve the anatomic specimens clean, dry and odorless. Plastination technique is used not only in anatomy but also in pathology and histology. Plastinated specimens contributed to anatomy education and possible researches. We have Even three dimensional and also sectional plastinated specimens are useful for studying parapharyngeal space not only for students but also for the specialists.

\section{P-246}

Investigation of age and gender related alterations in nasopalatine canal morphometry and variations a preliminary study

Gönül Y, Bucak A**, Atalay $\mathrm{Y}^{* * *}$, Acay $\mathrm{MB}^{* * * *}$, Çalışkan $A^{* * * *}$, Sakarya $G^{* * * * *}$, Soysal $N^{* * * *}$,

Cimbar $M^{* * * * *}$, Özbek $M^{* * * * *}$

*Department of Anatomy, Faculty of Medicine, Afyon Kocatepe University, Afyon, Turkey; **Department of Ear, Nose and Throat, Faculty of Medicine, Afyon Kocatepe University, Afyon, Turkey; ${ }^{* * *}$ Department of Maxillofacial Surgery, Faculty of Dentistry, Afyon Kocatepe University, Afyon, Turkey; $* * * *$ Department of Radiology, Faculty of Medicine, Afyon Kocatepe University, Afyon, Turkey; ${ }^{* * * * * T e r m ~ I I I ~ M e d i c a l ~ S t u d e n t, ~}$ Faculty of Medicine, Afyon Kocatepe University, Afyon, Turkey
Objectives: Nasopalatine canal (NPC) connects the nasal and oral cavities. It includes nasopalatine nerve, descending branch of nasoplataine artery, fibrous connective tissue and minor seromucous salivary glands. These structures may be exposed to damage during surgical interventions and thus, one must bear in mind the morphometric variations of NPC. NPC also has crucial importance in anterior maxillary rehabilitation. In this study, we aimed to investigate the possible alterations in morphology of NPC due to age, gender and absence of anterior incisors via using multiple detector computed tomography (MDCT) imaging method.

Methods: A total of 100 (50 males and 50 females) between 18 and 90 years who have undergone MDTC imaging for head trauma, sinusoidal volume and headache and who do not have serious pathological condition that can affect the NPC were included. MDCT device with 80 detectors (160 slices) was used for imaging. Nasal foramen (P1) diameter, IF diameter (P2), NPC length (P3), distance between buccal wall of IF and facial side of the buccal bone (P4), distances: [between buccal bone wall of NPC and facial side of buccal bone (P5), distance between mid-NPC buccal wall and facial side of buccal bone (P6), distance between buccal wall of NPC and the apex of anterior central incisors (P7) and NPC angle (P8) were measured. Established data were statistically analyzed.

Results: Detected shape variations of NPC were as follows (total, male and female): Cylindirical, hourglass, banana, funnel; canal shapes in coronal plane: unilateral, Y-type and parallelized. Mean canal diameters were bigger in males except P1 and P3 ( $<<0.05)$. The morphology of NPC has exhibited difference with respect to age and gender.

Conclusion: NPC variations are important in aesthetical and functional aspects and must be entertained to decrease the incidence of complications related to facial or dental surgical interventions.

\section{P-247}

\section{Two lateral thoracic arteries originating from axillary artery}

Kaya YE, Yıldırım M, Şahin ZA, Hamutoğlu R, Taştan A

Cerrahpaşa Medical Faculty

Axillary artery (AA) takes a part in axilla as continuation of subclavian artery. Since in its branching there are many variations, AA is investigated in our study of artery researches. In 20142015 academic year, during dissection of a cadaver for mentioned study we see that there is a different branching pattern which tough superior thoracic artery, thoracoacromial artery, anterior humeral circumflex artery, posterior humeral circumflex artery and subscapular artery are in their normal places, beside main lateral thoracic artery (LTA) - emerges on the third part of AA- an additional LTA is also emerges. Picture is taken and diameter of additional branch is measured with others. According to literature LTA could be emerges all three 
parts of AA. However second part is the most. These emergences are reported by Huelke, Adachi, DeGaris, Swartey and Pelelgrini in $52.2 \%, 40 \%, 55.9 \%, 55.9 \%, 70 \%$ respectively. The variations in AA have importance in aspect of breast and axilla surgery furthermore in plastic surgery flaps, so we hope that this case will help clinicians and anatomists as well.

\section{P-248}

Age and sex related changes in the craniofacial region in 7-11-year-old Turkish children

Ulcay T ${ }^{*}$, Sarsılmaz $M^{* *}$, Uzun A***, Öğetürk $M^{* * * *}$

*Department of Anatomy, Faculty of Medicine, Ahi Evran University, Kırşehir, Turkey; **Department of Anatomy, Faculty of Medicine, Şifa University, Izmir, Turkey; ${ }^{* *}$ Department of Anatomy, Faculty of Medicine, Ondokuz Mayıs University, Samsun, Turkey; $* * *$ Department of Anatomy, Faculty of Medicine, Firat University, Elazığ, Turkey

Knowledge of the rate of the growth of the head and face in a healthy population can help the surgeon evaluate variations from normal, understand altered proportions, and select an optimal time for correction of cranial and facial deformity. Age-related growth changes in the face and relationships between its parts were calculated by using one vertical, two horizontal and three sagittal surface measurements from face. Five surface measurements of the head were taken between 7 year and 11 years of age in 265 (145 male, 120 female) Turkish children. A rapid rate of growth in face height was observed in both sexes between 10 and 11 years. The mandible width and the sagittal surface measurements (upper face depth, maxillary depth and mandibulary depth) showed a moderate level of development in both sexes. The height of the head showed the highest level of development in both sexes (male: 12.8\%, female: $12.1 \%$ ). Cranial base width of the head showed the lowest level of development in both sexes of this preadolescent period (male: $1.8 \%$, female: $2.1 \%$ ). Growth trends and relationships between aspects of the head and face based on anthropometric findings are predictable. The data obtained from this study can be used for planning the timing and type of reconstructive surgery in patients with cranial and facial growth disturbance.

Keywords: Craniofacial anthropometry, mandible depth, maxillary depth

\section{P-249}

The effect of coracoid process transfer on the surgical procedures of shoulder instability

\section{Çirpan $S^{*}$, Büdeyri A**, Özkan $M^{* * *}$, Kiray A*}

*Department of Anatomy, Faculty of Medicine, Dokuz Eylül University, Izmir, Turkey; **Department of Orthopedics and Traumatology, SANKO University Medical School, Gaziantep, Turkey; ***Department of Orthopedic Surgery, Faculty of Medicine, Dokuz Eylül University, Izmir, Turkey
Introduction: Bone loss of the anteroinferior rim of the glenoid is observed in shoulders with recurrent anterior dislocation. In such shoulders, the size of the defect affects the degree of shoulder instability. Thus, before coracoid process (CP) transfer procedures are attempted, the need for bone grafting is a major clinical concern and depends at least partly on the size of the glenoid defect. After the CP transfer is performed, the effect of this procedure on surface area of the glenoid cavity (GC) is need to be quantified. The purpose of this study, therefore, was to determine the effect of CP transfer on surface area of the GC of cadaveric shoulders in the surgical procedures of shoulder instability.

Study Design: A cadaveric laboratory study.

Methods: After the shoulder dissection, osseous defects of $21 \%$ and $34 \%$ of anteroinferior part of the glenoid surface area were created stepwise in 30 cadaveric scapulae, and Latarjet procedure was performed, respectively. Intact and postoperative surface area of the GCs were photographed, with Canon 400B (55 mm objective) and with a scale placed next to each specimen to indicate its relative size on the photographs. The images were loaded on a computer. The surface area of the photoghraphed GCs were calculated with use of UTHSCSA Image Tool 3.0 software.

Results: The mean surface area of intact GC was 969.98+ $129.95 \mathrm{~mm}^{2}$. The postoperative mean surface area of $21 \%$ and $\% 34$ defective GC were $1133.80+119.65 \mathrm{~mm}^{2}$ and $1006.50+$ $110.69 \mathrm{~mm}^{2}$, respectively. There was a statistically significant difference between the means of surface area of intact GC and postoperative surface area of $21 \%$ and $\% 34$ defective GC, ( $\mathrm{p}=0.000, \mathrm{p}=0.025$; respectively).

Conclusion: CP transfer (Latarjet procedure) significantly increases surface area of GC

\section{P-250}

\section{Rib aplasia: a case report}

Keskin İ $^{*}$ Ülkü R**, Tacar $\mathrm{O}^{*}$, İskenderoğlu $\mathrm{AY}^{*}$, Demirant $\mathrm{A}^{*}$, Karabulut $\mathrm{O}^{*}$

*Department of Anatomy, Faculty of Medicine, Dicle University, Diyarbakır, Turkey; **Department of Thorasic Surgery, Faculty of Medicine, Dicle University, Diyarbakır, Turkey

Objectives: Rib aplasia and hypoplasia is a rare deformity of the chest wall deformities. Rib fusions, bridging between two ribs, cervical and pelvic ribs, pseudoarthrosis of the first ribs, bifid ribs other rib abnormalities are known. These abnormalities and anatomic variations occur 0.15 to $0.31 \%$ in of the society. Rib variations may be member of components of the metabolic syndrome or a congenital disease. Particularly twelfth costal aplasias are common and the incidence of Down syndrome has been reported with aplasia in 11-33\%. Poland syndrome is characterized by unilateral aplasia or hypoplasia of the ribs. Besides, it is availiable unilateral of the absence the pectoralis major and minor muscles, lack of axillary hair, splint or 
amasti, the lack of subcutaneous fat tissue and syndactyly / brachydactyly syndrome. By 5 years of age, the thoracic volume increases to about $30 \%$ of the adult size, and by age 10 it reaches approximately $50 \%$ of adulthood. The remaining $50 \%$ develops during the prepuburtal period and early adolescence. Short ribs do not extend as far anteriorly as the sternum. The resultant diminished volume of the chest motion causes the respiratory insufficiency. Short ribs may be a part of several syndromes. It is present the rib aplasia in our case.

Case: A healty 5-year-old male patient was referred to the thoracic surgery clinic for the chest wall deformity. In physical examination; on the right side of the chest wall was identified that $1,2,3,4$, ribs were deformed and 5,6,7. ribs can not be manually palpated. In the X-ray images first four ribs in the rib arch showed significantly narrowed and 5, 6, 7. Ribs was identified as aplasia. However, the patient's lung structure and function fully normal and patients was discharged with control clinic proposal.

\section{P-251}

\section{Anatomical assessment of the complications in dental implant surgery}

$\underline{\text { Büyükertan } M^{*}}$, Üstünel $H^{* *}$, Bölükbaşi $N^{* * *}$, Balcığlu HA*

*Department of Anatomy, Faculty of Dentistry, Istanbul University, Istanbul, Turkey; **Senior Student, Faculty of Dentistry, Istanbul University, Istanbul, Turkey; ***Department of Oral Implantology, Faculty of Dentistry, Istanbul University, Istanbul, Turkey

Intra- and postoperative complications in oral implant surgery have been increasingly reported in recent years. Some of these complications are related with the skills and ability of the practitioners or medical issues of the patient, a part of them depends directly on. It is assumed that the oral surgeon has the adequate knowledge of anatomy to define and evaluate the unexpected pathology and morphological variations along with the surgical process. Knowledge of the headand neck anatomy and also the positions of the natural teeth within the jaw bones related with the biomechanics of the chewing system play a great role during the surgical process in order to achieve and maintain the optimum outcomes. The common complications of implant placement are anesthesia related complications, hemorrhages, nerve damage, damaging the healthy neighboring tooth, bone fractures, cortical layer perforations, primary stability failure related with misalignment of the implant, and complications with the maxillary sinus. An adequate knowledge of anatomy, careful planning and application of proper surgical technique and also usage of proper material would help to avoid these complications. During the planning step, the key issues are determining the anatomical features of the patient's mandibular canal and maxillary sinus, thickness of the cortical layer, density of the remaining bone and also selecting the suitable implant material to be installed in the right position.

\section{P-252}

Anomalous mandibular canal of a 51-year-old

Köse $T^{* *}$, Uyanık $S^{* *}$, Büyükertan $M^{*}$, Bölükbaşı $N^{* * *}$, Özcan I**, Yildırım $\mathrm{M}^{* * * *}$, Balcıŏglu HA*

${ }^{*}$ Department of Anatomy, Faculty of Dentistry, Istanbul University, istanbul, Turkey; **Department of Oral Radiology, Faculty of

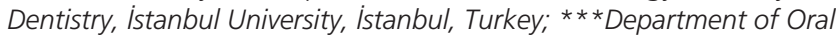
Implantology, Faculty of Dentistry, Istanbul University, Istanbul, Turkey; $\star * * \star$ Department of Anatomy, Cerrahpaşa Faculty of Medicine, Istanbul University, Istanbul, Turkey

Mandibular canal and medial sigmoid depression are important anatomical landmarks for dental practice. Local and generalized widenings of the mandibular canal may be signs of pathologies like hemangioma, arteriovenous malformation, peripheral nerve sheath tumour etc. A 51-year-old patient's panoramic radiography revealed widening of mandibular canal bilaterally and extraordinary radiolucent area between foramen mandible to sigmoid notch. Due to the image, possible bilateral peripheral nerve sheath tumour considered. With contrastenhanced MRI examination, extraordinary thinning of ramus mandible diagnosed and no other abnormality diagnosed. As this malformation is rare, in these cases beside peripheral nerve sheath tumour, also anatomical variations must be considered.

\section{P-253}

\section{Case report: tibial hemimelia with bilateral cleft hand}

Keskin $\dot{\mathrm{I}}^{*}$, Bulut $\mathrm{M}^{* *}$, Tacar $\mathrm{O}^{* * *}$, İskenderoğlu $\mathrm{AY}^{* * *}$, Demirant $\mathrm{A}^{* * *}$, Karabulut $\mathrm{Ö}^{* * *}$

*Department of Anatomy, Faculty of Medicine, Dicle University, Diyarbakır, Turkey; ${ }^{*}$ Departmet of Ortopedic Surgery, Faculty of Medicine, Dicle University, Diyarbakır, Turkey; ***Department of Anatomy, Faculty of Medicine, Dicle University, Diyarbakır, Turkey

Tibial displasia is as also known as tibial hemimelia. Tibial hemimelia and cleft hand are very rare disease in limb abnormalities. İncidance of Tibial hemimelia is in 1:1,000,000 live births, cleft hand occur in 1:90,000 in populotion. Tibial hemimelia is characterized incomplete tibia with relatively perfect fibula and in this anomalies is seen severe equinovarus deformity, deficiency of tibia could be complete or incomplete. As it may be part of another syndrome or it may be sporadik. Some of the reported associated anomalies include, facial cleft, oligodontia, deficiency of the radius or ulna, proximal focal femoral deficiency, congenital pyloric stenosis, GollopWolfang complex, hemivertebra, tracheoesophageal fistula, congenital heart disorders, sacral agenesis, anorectal atresia, , tibia hemimelia-split hand/foot syndrome (TH-SHFM), duplication foot or hand, absent digits. According to development of tibial cartilagineus and presence of fibula is seven type and five sub-grubs distribution tibial hemimelia in weber-classification. In cases of cleft hand; Starting in the middle of one or more fingers, metacarpal and / or metatarsal bones together, are kongenital aplazisia. It showed that approximately half and quarter of the cases with cleft hand and cleft foot are together. cleft 
hand and cleft foot may be associated with rare syndromes. EEC(ektrodaktili, ektodermal displasia, cleft) is the most common of these syndromes. We presented a case with unilateral tibial hemimalia and bilateral cleft hand. Case 5-year-old female child was admitted to ortopedics clinic with complaints of form and function disorders of right leg and bilateral cleft hand. In $\mathrm{x}$-ray examination; the right leg was observed in weber classification of tibia hemimelia type 7-b,complete absence of the middle finger of the right hand with cleft metacarpal bone hypoplasia and complete absence of the middle finger of the left hand with metacarpal bone aplasia. This case was planned to below-knee amputation and hand cleft correction.

\section{P-254}

Dimensions and pneumatization of the anterior clinoid process in middle Anatolian population

Sabancioğulları $\mathrm{V}^{*}$, Tastemur $\mathrm{Y}^{*}$, Salk $\mathrm{I}^{* *}$, Doğruyol G*

*Department of Anatomy, Faculty of Medicine, Cumhuriyet University, Sivas, Turkey; **Department of Radiology, Faculty of Medicine, Cumhuriyet University, Sivas, Turkey

Objectives: The anterior clinoid process (ACP) is usually removed during surgical procedures of the cellar region. The length and width of ACP may be different in people and it may be pneumatic, also. Because of clinical importance of ACP, we aimed to determined dimensions and rates of pneumatization of it in a large population study.

Methods: Magnetic resonance imaging (MRI) of cranium in individuals (592 female, 439 male) derives from the middle anatolian population were received in this study. The length and basal width of the ACP were measured on the cranial MRI. Also, incidence and degree of ACP pneumatization were identified.

Results: The width right and left of the ACP in female were $10.80 \pm 2.27 \mathrm{~mm}$ and $10.53 \pm 2.07 \mathrm{~mm}$, respectively. The width right and left of the ACP in male were $11.08 \pm 2.39 \mathrm{~mm}$ ve $10.98 \pm 2.35 \mathrm{~mm}$, respectively. The length right and left of the anterior clinoid process in female were $8.32 \pm 2.40 \mathrm{~mm}$ and $8.34 \pm 2.35 \mathrm{~mm}$, respectively. The length right and left of the anterior clinoid process in male were $8.87 \pm 2.62 \mathrm{~mm}$ and $8.93 \pm 2.64 \mathrm{~mm}$, respectively. There was statistically significant difference between men and women dimensions of ACP except for width right ACP. Pneumatization of the ACP was observed on the right side in 46 cases, on the left side in 53 cases, and bilaterally in 32 cases. Incidence of pneumatization of the ACP was lower in individuals under the age of 20 . While incidence of bilaterally pneumatization of the ACP was higher in individuals aged 21-40.

Conclusion: Radiologically recognizing pneumatization and anatomical variations of the ACP may be help in decreasing the incidence of surgical complications during anterior clinoidectomy.

Keywords: Anterior clinoid process, pneumatization, anatomical variations

\section{P-255}

Defining the hairline morphometrics of Turkish population

Evin $\mathrm{E}^{* *}$, Toruyenler $\mathrm{M}^{* *}$, Karaca $\mathrm{O}^{* *}$, Şahin $\mathrm{OB}^{* *}$, Darben $\mathrm{Y}^{* *}$, Ateş $\mathrm{C}^{* * *}$, Öztan Ö ${ }^{* * * *}$, Esmer TS̆ ${ }^{*}$, Esmer $\mathrm{AF}^{*}$

*Department of Anatomy, Faculty of Medicine, Ankara University, Ankara, Turkey; ${ }^{* *}$ Faculty of Medicine, Ankara University, Ankara, Turkey; $* * *$ Department of Biostatistics, Faculty of Medicine, Ankara University, Ankara, Turkey; ${ }^{* * *}$ ECM Aesthetics Plastic Surgery Clinic, Ankara, Turkey

Despite not being known as a life-threatening condition, hair loss has been considered to be one of the most prominent aesthetic problems known to mankind. Many individuals with hair loss experience lack of confidence and anxiety and fear their first impression on people are impacted by their problem. To this date, there have been no descriptive guidelines or researches regarding the hairline morphometrics of Turkish population. With the results of our research, we aim to aid hair transplantation operations with accurate information on hairline morphometrics, thus giving hair frontlines a more natural look. In this descriptive research, we have randomly chosen 138 Turkish males and 138 Turkish females aged 18 to 25 with no prominent hair loss (any hair loss score above ' 1 ' in Norwood and Ludwig scales for men and women respectively were considered prominent) and measured specific reference points of their hairlines. We have set our statistical significance level to 0,05 . Our most notable finding was the statistically significant difference of Trichion-Nasion/Trichion-Gnathion ratios between men and women. We have also determined the most prominent hairline type in women is round shaped, whereas men often possess rectangular and $\mathrm{m}$-shaped hairlines. We conclude that rectangular and $\mathrm{m}$-shaped hairlines resemble a more masculine appearance, whereas round shaped hairlines resemble femininity. Our findings also suggest that women's foreheads make up a larger percentage of their faces in comparison to men, and the more cornered the hairline is, the deeper frontotemporal recession gets. With the findings of our research, we believe hair transplants would be delivered more successfully on patients.

\section{P-256}

Variations of right and left tyrocervical trunks' branching in the same cadaver

Șahin ZA*, Yıldırım M*, Taştan ÖA*, Hamutoğlu R*, Kaya $\mathrm{YE}^{*}$, Al-Talahma $\mathrm{M}^{*}$

Department of Anatomy, istanbul University Cerrahpaşa Medical Faculty, Istanbul, Turkey

The subclavian artery takes an important place in anatomy education, radiological anatomy, variations anatomy and surgical anatomy. If the subclavian artery branches typically the branches are internal thoracic artery, vertebral artery, thyrocervical trunk and costocervical trunk. Branches of thyrocervi- 
cal trunk and costocervical trunk (inferior thyroid artery, transverse cervical artery - deep branch/dorsal scapular artery, suprascapular artery etc.) can arise from subclavian artery individually. During the dissection practice at the root of the neck in a 38-year-old male cadaver between 2014-2015, cases of right and left atypical thyrocervical trunks were observed. It was defined that bilateral inferior thyroid arteries arose from subclavian artery individually. On the left side transverse cervical artery and suprascapular artery arose from a common trunk with internal thoracic artery. In the same cadaver, another important variation was that deep branch (i.e. dorsal scapular artery) arose from third part of the subclavian artery. We know that, the deep branch is typically one of the branches of the transverse cervical artery. Even though many researchers reported that frequency of the dorsal scapular artery is almost $50 \%$, the inferior thyroid artery arising individually from the subclavian artery is significantly low (2\%-15\%). These interesting variations are very important for the operation of the root of the neck and thyroid gland, and also during the administration of local anesthetics to the brachial plexus. The literature was reviewed and the results were shared with the anatomists.

\section{P-257}

The variation about the course and communication of common fibular nerve in two cases

Arifoğlu $\mathrm{Y}^{*}$, Yıldırım $\mathrm{M}^{* *}$, Öztürk Ö$^{*}$, Kurbetli $\mathrm{N}^{*}$

${ }^{*}$ Department of Anatomy, Faculty of Medicine, Bezmialem Vakıf University, Istanbul, Turkey; ${ }^{* \star D e p a r t m e n t ~ o f ~ A n a t o m y, ~ C e r r a h p a s ̧ a ~}$ Faculty of Medicine, Istanbul University, Istanbul, Turkey

The sciatic nerve is the thickest and the longest branch of the sacral plexus. The nerve leaves the pelvis through the suprapiriform foramen whereas it descends on the posterior surface of the superior gemellus, internal obturator, inferior gemellus and quadratus femoris muscles. Generally sciatic nerve gives two terminal branches the common fibular nerve and tibial nerve near the superior angle of popliteal fossa. Sometimes this branching may occur between the popliteal fossa and pelvic cavity and one of the branches of sciatic nerve can follow a different course. In our cases, we observed variations regarding the branches of high divided sciatic nerve in both male cadavers which are dissected for the students of medical school. In the first case; the common fibular nerve pierced the piriform muscle while passing through it whereas the tibial nerve was on its normal course. The two branches of sciatic nerve coursed seperately towards the popliteal fossa. In the second case; we, unexpectedly, observed that the common fibular nerve passed through the suprapiriform foramen while the common fibular nerve connects with the tibial nerve. The anatomical variations which we described above are important for clinical and surgical perspectives due to the fact that these types of variations may lead to nerve compression which then may result in sciatica and piriformis syndromes.
Keywords: Common fibular nerve, tibial nerve, sciatic nerve, anatomical variation, piriform muscle

\section{P-258}

3D (Tree-dimensional) evaluation of ischemic damaged brain area and correlation of them with clinical symptoms

Kurbetli N* , Özdemir MB**, Arifoğlu Y*, Öncel C***, Yağc1 B ${ }^{* * * *}$, Citısli $\mathrm{V}^{* * * * *}$

*Department of Anatomy, Faculty of Medicine, Bezmialem Vakıf University, Istanbul, Turkey; **Department of Anatomy, Faculty of Medicine, Pamukkale University, Denizli, Turkey; ${ }^{* * \star}$ Department of Neurology, Faculty of Medicine, Pamukkale University, Denizli, Turkey; $* * * \star$ Department of Radiology, Faculty of Medicine, Pamukkale University, Denizli, Turkey; $* * * * \star$ Department of Neurosurgery, Faculty of Medicine, Pamukkale University, Denizli, Turkey

The relationship between ischemic damaged brain area and upon clinical symptoms on patients have been shown in previous studies but not in $3 \mathrm{D}$ evaluation. The aim of this study is to evaluate ischemic damaged brain areas in $3 \mathrm{D}$ obtained from patient's magnetic resonance (MR) and computational tomography (CT) images and correlate them with clinical symptoms of patients. For this purpose, 110 patients' images were evaluated in $3 \mathrm{D}$ by computer- aided soft ware program and results were correlated with reported clinical findings of patients. Correlation has already been detected between damaged areas and clinical symptoms significantly $\left.\mathrm{p}<0.05 \mathrm{R}={ }^{* *}\right)$. Damaged areas in the brain can be detected by performed cranial imaging methods. At the same time, localization of ischemic areas can be made by neurological examination. So far, many functional brain areas have been investigated by this way. But, this determination was usually made on $2 \mathrm{D}$ sections. In present study, correlation between $3 \mathrm{D}$ evaluation and clinical symptoms was made in the first time. Present study results can be source for the computational neuroscience. It can open the way of new researches in the clinical and basic sciences.

Keywords: Brain, ischemia, MRI, CT, clinics, neurology, symptoms, 3D, computational neuroscience

\section{P-259}

\section{Os coxae and the golden ratio}

Tuncel N, Ari İ, Kafa İM

Department of Anatomy, Faculty of Medicine, Uludağ University, Bursa, Turkey

In mathematics and art, golden ratio $(1.618 \ldots)$ is an ancient well-known numeric and geometric relationship, which is assumed that, gives us the most attractive formats of the perceived parts of whole things in spatial orientation. Even if the golden ratio long has been known and traditional in history, there is no certain information that when it discovered and began to be used by science and art. In our study, we investi- 
gated the existence of the golden ratio within the os coxae by predetermined landmarks and ratios. This study was carried out on twenty-five coxae belonging to the Late Byzantine period, which was preserved in the osteological collection of the Department of Anatomy of Uludag University. The measurements of the eighteen parameters from digital images obtained and thirty-eight ratios calculated from these parameters by the aid of computer software (Scion Image). After the calibrations and measurements, results were analyzed with SPSS software. We found that the length between incisura ischiadica - spina ischiadica and the length between incisura ischiadica - tuber ischiadicum were the closest parameter when compared to the golden ratio. The results from our study, that we investigated and found worthy to be presented, suggest that there is a golden ratio in human hipbone as another remarkable relationship of human body and the golden ratio besides the earlier wellknown and methodically documented anthropological ratios. Moreover, we also think that similar studies may be done on the other skeletal structures.

\section{P-260}

\section{Morphometric analysis of greater palatin foramen with MDCT}

$\underline{\text { Kabakçı ADA* }}^{*}$ Akın D*, Özen KE*, Çiçekcibaşı AE*, Yılmaz MT*, Kıreşi D**

*Department of Anatomy, Meram Medicine Faculty, Necmettin Erbakan University, Konya, Turkey; **Department of Radiology, Meram Medicine Faculty, Necmettin Erbakan University, Konya, Turkey

Introduction: Blockade of the maxillary division of the trigeminal nerve (TN) or its branches is common practice for local anaesthesia in maxillofacial and dental surgery. When determining the position of the needle for the injection of local anesthetic into the greater palatin foramen (GPF), referring to height, angle, or length relative to clinical anatomic structures in the oral cavity may increase the efficiency of anesthesia and reduce complications. Therefore, the aim of this study was to provide standards for anatomic structures in the oral cavity that can be easily referred to in anesthesia.

Methods: This study was conducted on carotid angiography, maxillofacial and sinus MDCT images of a total of 100 patients consisted of 62 male and 38 female who applied to Department of Radiology, Necmettin Erbakan University. Morphometric measurements were perfomed on inspace images related to GPF and neighboring structures. In addition to this, we classified the direction of the opening of the GPF into oral cavity and GPF's shape. The datas of this study were evaluated by using SPSS 21.0.

Results and Conclusion: As a result, it was found that mean left vertical diameter of the GPF $3.22 \pm 1.09 \mathrm{~mm}$ and left transverse diameter of the GPF $2.92 \pm 1.10 \mathrm{~mm}$; right vertical diameter of the GPF $3.01 \pm 1.26 \mathrm{~mm}$ and right transverse diameter of the GPF $2.67 \pm 0.96 \mathrm{~mm}$ in males. This measurements were $3.15 \pm 1.10, \quad 2.37 \pm 1.24, \quad 2.91 \pm 0.84,2.57 \pm 0.98$ respectively in females. We found that $33 \%$ of the GPF shape were round, $\% 58.5$ ovoid, while the remaining $8.5 \%$ were slit. The direction of GPF in the oral cavity was found anterior in \%32.5, anteromedial in \%54.5, anterolateral in \%2.5, inferior \%10.5. We believe that the knowledge of the morphometric data and anatomical variances of GPF can also be helpful in oral and maxillofacial surgery procedures.

Keywords: Greater palatin foramen, local anesthesia, MDCT

\section{P-261}

Anatomic measurement of splenic volume on CT in adult population with malignancy: is there any association between cancer type and volume?

Kalkan $\mathrm{H}^{* *}, \underline{\text { Akın D }}^{*}$, Kabakçı ADA** Keskin S**, Yılmaz M.T**, Çiçekcibaşı AE*

*Department of Anatomy, Meram Medicine Faculty, Necmettin Erbakan University, Konya, Turkey; **Department of Radiology, Meram Medicine Faculty, Necmettin Erbakan University, Konya, Turkey

Introduction: Splenic volume is nowadays can be measured with new softwares on CT. Because splenomegaly is associated with lots of clinical disorders, volume estimation is very important. There are so many studies that found relation between splenic volume and anthropometric datas, like age, gender, height, weight, body mass index. We differently aimed to find if there is relation between the splenic volume and the cancer type.

Methods: The study consists of 100 patients; 49 male and 51 female. Patients are randomized selected from the cases who had a type of cancer like lung(49\%), endometrium (10\%), breast (12\%) and colon (29\%) between 2011-2012 years. They were performed CT to investigate metastasis. The images were transferred to Leonardo Workstation. After manual contouring, the computer automatically calculates the volume and height. We also look for the relationship between splenic volume and body mass index, height, age, weight. All statics were generated by SPSS version 21.0.

Results and Conclusion: The average spleen volume for the males are $210.90 \pm 125.01$ and $189.87 \pm 108.11$ for the females. The height values are $9.55 \pm 2.11$ and $10.04 \pm 2.19$ respectively for the males and females. There was no correlation between splenic volume, height ant the other parameters $(p>0.05)$. Spleen volume was larger in the in colon cancer patients than the others. The largest volume was $554,18 \mathrm{~cm} 3$ for colon cancer in this study. There was no association between the anthropometric parameters and spleen volume. Cancer types can affect the splenic volume due to unknown mechanisms. Why colon cancer patients had the largest volume? It may be because of the chemotherapy agents that are used. This is a preliminary study that must be improved with the other cancer types and with larger population to reveal if every type of cancer leads to increase in splenic volume. What is the exact etiology, the chemotherapy agents or anything else?

Keywords: Spleen, CT, volume 


\section{P-262}

Avascular necrosis of the scaphoid bone: a case report Engin $\mathrm{Y}^{*}$, Karakaya AD**, Malkoç I' ${ }^{*}$, Gedik HT*, Kara ${ }^{*}$, Keleş $\mathrm{P}^{*}$

*Department of Anatomy, Faculty of Medicine, Atatürk University, Erzurum, Turkey; ${ }^{* *}$ Department of Radiology, Regional Training and Research Hospital, Erzurum, Turkey

Avascular necrosis is a bone necrosis resulting from inadequate blood supply from vessels that feed the bone. It is usually irreversible. Since it is a disorder of blood supply in bone it is known as ischemic or avascular necrosis, or as aseptic necrosis since no infection is involved. The bones most commonly affected are the femoral head, the wrist, the scaphoid bone and the talus. Avascular necrosis of the scaphoid bone may develop in association with fractures, repeated traumas, presence of systemic disease and chronic steroid use. Scaphoid fracture is the second most common wrist fracture after distal radius fracture. Scaphoid fracture is one of those types that occur due to falling with the hand open. Eighty percent of such fractures are seen in the middle portion. Sensitivity in the fossa radialis is seen at examination. Fractures in the proximal portion represent a small part of scaphoid fractures. However, they may lead to avascular necrosis due to interruption of blood supply. In that event, nonunion may occur and may progress to intercarpal arthrosis. Fragmentation of the scaphoid bone of the right hand was determined at antero-posterior hand radiography in a 25 -year-old patient presenting with a history of falling onto the right hand 2 months previously and with pain in that region. Signal loss in the scaphoid bone was determined in T1 weighted magnetic resonance imaging (MRI) of the right hand, while increased signal was present in right hand MRI proton density images. Avascular necrosis was diagnosed on the basis of these findings, and the case is discussed in the light of the current literature.

Keywords: Avascular necrosis, scaphoid, magnetic resonance imaging (MRI)

\section{P-263}

\section{Occipitocervical synostosis: case report}

$\underline{\text { Kayhan B* }}^{*}$, Illhan $\mathrm{P}^{*}$, Ertürk $\mathrm{M}^{* *}$, Şengül G**

${ }^{*}$ Department of Neuroscience, Institute of Health Sciences, Ege University, Izmir, Turkey; **Department of Anatomy, Faculty of Medicine, Ege University, Izmir, Turkey

Objectives: Fusion of atlas with occipital bone is a rare congenital anomaly known as occipitocervical synostosis, occipitalization of atlas, assimilation of atlas or atlanto-occipital fusion. The atlas partially or totally fuses with the occipital bone due to failure in segmentation and separation of the most caudal occipital sclerotome and first cervical sclerotome during the first week of intrauterine life.

Methods: One-hundred dry adult human occipital bones from the collection of Ege University, Faculty of Medicine,
Department of Anatomy were investigated for the presence of atlanto-occipital fusion. The anteroposterior and transverse diameters of the foramen magnum and diameters of the inferior articular facets were measured using a digital caliper.

Results: 2 skulls showed occipitalization of atlas (0.5\%). Both skulls showed complete occipitalization. For these cases, the sagittal and transverse diameters of foramen magnum were $35.70 \mathrm{~mm}$ and $30.32 \mathrm{~mm}$, respectively for the first case, and $35.33 \mathrm{~mm}$ and $26.56 \mathrm{~mm}$, respectively for the second case. The transverse diameter of the inferior articular facets were 16.50 and $13.84 \mathrm{~mm}$ on the right side, and $11,48 \mathrm{~mm}$ and $13.40 \mathrm{~mm}$ on the left side for these cases. The sagittal diameter was 22,97 $\mathrm{mm}$ and $21,18 \mathrm{~mm}$ on the right side and $19.64 \mathrm{~mm}$ and 19.11 $\mathrm{mm}$ on the left side.

Conclusion: The knowledge of bony fusion between the cranial base and the first cervical vertebra is important as it may cause narrowing of foramen magnum which may compress the brainstem, vertebral artery and cranial nerves, and also for surgeons approaching the craniovertebral region.

\section{P-264}

\section{Nutrient foramina in the os lunatum from the carpal bones: morphometric study}

Önder M, Gürçay S, Oğuz N

Department of Anatomy, Faculty of Medicine, Akdeniz University, Antalya, Turkey

Objectives: To study the morphology and topography of nutrient foramina (NF) and to determine the foraminal index of the lunatum bones.

Methods: In our study, 30 os lunatum obtained from the bone collection of Department of Anatomy, Akdeniz University, Faculty of Medicine were evaluated. The bones had gross morphologic deformities were excluded from the study. The dominant NF determined and the bones were macroscopically observed under the stereomicroscope according to the number, location and direction of the NF. The total lunatum length (TL; maximum distance from proximal to distal end of the bone) and the distance from the proximal end of the bone to the NF (DNF) were measured.

Results: According to measurements of TL and DNF at left and right os lunatum averages were $1.70 \mathrm{~cm} \pm 0.34 / 1.66$ $\mathrm{cm} \pm 0.31$ and $0.60 \mathrm{~cm} \pm 0.14 / 0.89 \mathrm{~cm} \pm 0.09$ respectively. The mean foraminal index for the lunate bones were $47.71 \%$ for the right patella, whereas the left os lunatum of the foraminal index were $44.28 \%$

Conclusion: The study has provided additional information concerning morphology and topography of nutrient foramina lunate bone. The knowledge about these foramina is useful in certain surgical procedures to preserve the circulation.

Keywords: Os lunatum, nutrient foramina, foraminal index, morphology 


\section{P-265}

\section{Anatomical study of the echocardiographic views in the dog}

Sadeghinezhad J*, Vajhi $\mathrm{A}^{* *}$, Goodarzi $\mathrm{N}^{* * *}$, ${\text { Ashgriz } \mathrm{N}^{*}}^{*}$ *Department of Basic Sciences, Faculty of Veterinary Medicine, University of Tehran, Tehran, Iran; **Department of Surgery and Radiology, Faculty of Veterinary Medicine, University of Tehran, Tehran, Iran; ***Department of Basic Sciences, Faculty of Veterinary Medicine, Razi University, Kermanshah, Iran

Echocardiography is a noninvasive technique that is an important tool in diagnosis of cardiac diseases. Interpretation of the results of the echocardiography is dependent on having anatomical knowledge of the heart. The aim of this study was to prepare the anatomical slices according to the related views in echocardiography. These sections can be used as a model for teaching of echocardiography. Six dogs euthanized for purposes unrelated to the present study were used. The hearts were removed and then dilated and fixed using formalin $10 \%$. Fixed hearts were then cut to obtain representative slices corresponding to long and short right parastenal standard echocardiographic views which were taken from three healthy dogs. After that, both anatomical and ultrasound images were compared. The external and internal morphology of the heart were suitable for this study. The comparison of the anatomical slices with related echocardiograms showed a high level of correlation. The slices of hearts which were fixed using formalin may be used for echocardiography interpretation.

\section{P-266}

\section{Right hepatic artery and left hepatic artery originated} from the coeliac trunk

$\underline{\text { Kara }}^{*}$, Karaca L ${ }^{* *}$, Keleş $\mathrm{P}^{*}$, Gedik T* Engin $\mathrm{Y}^{*}$, Malkoç İ*

*Department of Anatomy, Faculty of Medicine, Atatürk University, Erzurum, Turkey; ** Department of Radiology, Faculty of Medicine, Atatürk University, Erzurum, Turkey

The aim of our study, to show the vascular structures which are necessary for live liver transplantation. For transplation surgery to show hepatic vascular anatomy and variations of donor with vascular radiographs which taken from Multidetector Computed Tomography (MDCT) angiography is very important. Helping the surgery team to carry out appropriate anastomosis and reconstruction during the transplantation based on the parameters gained. In our study 47 years-old male, who admitted as a liver donor candidates, radyoraphies which shows hepatic vascular anatomy captured by MDCT angiography has been viewed. İn cases areteries, are called right hepatic artery and left hepatic artery, originated from the coeliac trunk were found. In addition to gastroduodenal artery took root from the left hepatic artery. Vascular variations type, number, diameter and length are very important in determining of the anastomosis and reconstruction techniques. Vascular imaging is required for the sender and receiver to reduce the possible complications that may arise, as well as to increase the success of the operation.

\section{P-267}

Right renal vein duplication as detected by computerized tomography: a case study

Gedik HT* ${ }^{*}$ Karaca L**, Kara A*, Engin $\mathrm{Y}^{*}$, Keleş $\mathrm{P}^{*}$, Malkoç I*

${ }^{*}$ Department of Anatomy, Faculty of Medicine, Atatürk University, Erzurum, Turkey; **Department of Radiology, Faculty of Medicine, Atatürk University, Erzurum, Turkey

Introduction: Carrying the blood filtered in the kidneys, the renal veins start from each kidney and drain into vena cava inferior at the L2-L3 level with a nearly perpendicular angle. As they complete a complex embryological process, anatomical variations of the renal veins are common. These variations usually do not present with clinical symptoms. They are often detected in dissections, abdominal imaging or surgical interventions to the area. The number of variations of the renal vein is less than that of the renal artery.

Case Report: In our case, using computerized tomography imaging (semi-axial and semi-coronal reformatted MIP imaging), we detected two renal veins that drain directly into vena cava inferior from the lower and upper poles of the right kidney in the 40 year old female patient who was referred to the general surgery department with complaints of abdominal and pelvic pain.

Conclusion: The clinician should keep renal vein variations in mind in cases such as tumor infiltration, renal vein thrombosis and retroperitoneal surgical interventions. The purpose of this study is to present duplication, which is a renal vein variation, in terms of embryological process, frequency and clinical value along with the literature.

Keywords: Renal vein, duplication, computerized tomography (CT)

\section{P-268}

Effect of shortly the ethyl alcohol consumption certain blood levels in female rats

Yazgan $\mathrm{K}^{*}$, Yildırım RT**, İnce $Z^{* *}$, Gölgeli $\mathrm{A}^{* * *}$, $\underline{\text { Yalçıı B }}^{* * * *}$, Gölgeli $A^{* * * * *}$

${ }^{*}$ Department of Physiology, Faculty of Medicine, Erciyes University Kayseri, Turkey; ${ }^{* *}$ Grade II, Faculty of Medicine, Erciyes University Kayseri, Turkey; $* * *$ Department of Physiology, Faculty of Medicine, Erciyes University, Kayseri, Turkey; $* * * *$ Department of Histology, Faculty of Medicine, Erciyes University, Kayseri, Turkey; $* * * * \star$ Department of Physiology, Faculty of Medicine, Erciyes University, Kayseri, Turkey

Alcohol consumption can cause changes on the short- and long-term effects many analytes, depending. Short-term effects 2-4 hours after ingestion with ethanol; reduce plasma glucose levels, increases uric acid and lactate levels. ln our study, we 
aimed, the effects of blood parameters to investigate of different doses of ethanol consumption in rats. Wistar albino 4 month old female rats were used. Three groups were formed control, $10 \%$ and $20 \%$ ethyl alcohol consumption $(n=7)$. The experimental group was made 10 days and the water was allowed to take the drinking water of $10 \%$ and $20 \%$ ethyl alcohol added. Intracardiac blood was taken and whole blood glucose analysis at the end. Data were analyzed IBM 21 programs with ANOVA-Tukey test. 20\% ethenol blood group HCT, $\mathrm{MCHC}, \mathrm{RBC}$ and glucose levels increased significantly $(p<0.05)$. Blood WBC, hemoglobin and platelet values decreased with increased alcohol consumption. The study, the experimental group compared weight were significant after prior to weight loss ethyl alcohol groups $(\mathrm{p}<0.05)$. The decrease in defense and immune cells of the body reduces the body's defenses WBC value $10 \%$ and $20 \%$ ethyl alcohol consumption of 10 days in female Wistar albino rats. Short term use of alcohol resulted in weight loss in female rats.

\section{P-269}

\section{Crossed renal ectopia: a case report}

Gün $C^{*}$, Fazlıŏulları $Z^{*}$, Sivri $M^{* *}$, Nabi $G^{*}$, Koplay $M^{* *}$

*Department of Anatomy, Faculty of Medicine, Selçuk University, Konya, Turkey; **Department of Radiology, Faculty of Medicine, Selçuk University, Konya, Turkey

Introduction: Development of the kidney includes 3 stages called as pronephrone, mesonephrone and metanephrone. Stage of metanephrone is associated with localization of the kidney and configuration of permanent kidney; at this stage, the kidney migrates from pelvis to retroperitoneal area as a result of caudal development. Positioning abnormalities may appear due to possible disorders during this migration. Crossed renal ectopia occurs when both kidneys are on the same side with two ureters inserting into the bladder. Blood supply of the ectopic kidney may originate from the aorta as well as both iliac arteries and medial sacral arteries.

Case Report: In MR-urography scan of a 51-year old male patient who referred our hospital because of pain on the lumbar area, the left kidney was not detected on normal location. Left kidney was determined as located ectopically adjacent to the right kidney and had malrotation. The right kidney was observed at L1-3 vertebra level and the ectopic kidney was detected at L3-5 vertebra level. Orifice of urether of the ectopic kidney was observed on normal location on the bladder by passing from the midline to the left. Arteries of both kidneys were detected to be originated from abdominal aorta.

Conclusion: Crossed renal ectopia is a rare congenital malformation. It is more common in men and left-to-right ectopia is three times more common than right-to-left ectopia. Majority of the patients are asympthomatic whereas some may have hypertension, urinary stone, infection and obstruction. Lesions of the ectopic kidney may be misdiagnosed such as appendicitis and abscess. Therefore, undefined formations should be approached carefully during the procedures and if ectopic kid- ney is detected, patients should also be investigated in terms of other mullerian anomalies.

Keywords: Crossed renal ectopia, MR-urography, variation.

\section{P-270}

\section{A case of hepatosplenomesenteric trunk with left gastric artery variation}

Nabi G*, Gün $C^{*}$, Fazlıŏulları $Z^{*}$, Sivri $M^{* *}$, Doğan $N^{*}$

*Department of Anatomy, Faculty of Medicine, Selçuk University Konya, Turkey; **Department of Radiology, Faculty of Medicine, Selçuk University, Konya, Turkey

Introduction: The celiac trunk is the first anterior branch and arises just below the aortic hiatus at the level of T12/L1 vertebral bodies. It divides into three branches; the left gastric artery, the common hepatic artery, and the splenic artery. In opposite of this classic trifurcation arrangement, many variations are exist. Variations in celiac trunk are usually asymptomatic but may become important in patients undergoing an upper abdominal operative procedure like liver transplantation, gastrectomy, bypass procedure etc. or an invasive arterial procedure. Earning knowledge about this variation enables clinicians to distinguish features, predict and avoid complications.

Case Report: We observed an atypical case of absent classic celiac trunk, despite a single left gastric artery and a hepatosplenomesenteric trunk presentation using multidetector computed tomography (MDCT) angiography in a 83 years old, male patient with a unrelated acute abdominal pain. The left gastric artery arose from the anterior wall of the abdominal aorta at $75 \mathrm{~mm}$ below the hiatus aorticus. Superior mesenteric artery (SMA) and two component of celiac trunk originated as a common trunk about $15 \mathrm{~mm}$ below the left gastric artery and totally $90 \mathrm{~mm}$ below the hiatus aorticus. This common trunk extended $25 \mathrm{~mm}$ and devided into superior mesenteric artery and a bifurcated celiac trunk. The diameter of hepatosplenomesenteric trunk is about 12 $\mathrm{mm}$, diameter of celiac trunk in origin is $10.3 \mathrm{~mm}$ and the diameter of superior mesenteric artery in origin is $6.3 \mathrm{~mm}$.

Conclusion: This variation might cause confusions in diagnostic and surgical procedures. Clinicians specially surgeons should keep in mind this variation to avoid complications.

Keywords: Celiac trunk, hepatosplenomesenteric trunk, left gastric artery, MDCT angiography.

\section{P-271}

\section{A case report: accessory spleen}

Karacan $\mathrm{K}^{*}$, Karacan $\mathrm{A}^{* *}$, Akcaalan $\mathrm{M}^{*}$

*Department of Anatomy, Faculty of Medicine, Sakarya University, Sakarya, Turkey; **Department of Radiology, Faculty of Medicine, Sakarya University, Sakarya, Turkey

Objectives: Accessory spleen is a variation including spleen tissue, generally locating in hilus, observed as normal histolog- 
ical appearance and forms during embryological evolution. As well as locating around splenopancreotic ligamentum, accessory spleen principally feeds from the branch of splenic artery. However, generally observed closer to hilum of spleen and tail of pancreas, it can be determined in many points from diaphragma level to scrotum. Due to having the same structure with normal spleen tissue, diseases related to spleen can be observed in accessory spleen. The prevelance of accessory spleen is \% 10-30 in the population. Accessory spleen, rarely diagnosed preoperative, is frequently asymptomatic. In this study, we aimed to present a case related to accessory spleen.

Methods: A 25 year old woman patient who applied to Sakarya University Training Research Hospital because of abdominal pain had a contrast enhanced computed tomography (CT) scan of abdomen. Contrast enhanced computed tomography scan is the best monitoring way to diognase the presence of accessory spleen.

Results: A nodular appearance which did not have any relation to vascular tissue or any organ showing similar contrast enhancement with spleen and anterior neigbourhood of spleen was monitored after CT. The described image belonged to incidentally detected accessory spleen.

Conclusion: Although accessory spleen appears frequently, it is incidentally diognased. Its presence gains importance for the patients who have hematologic pathology or need a splenectomy operation. Thus, it is important that knowing the prevelance of accessory spleen in population.

\section{P-272}

\section{Double left testicular artery and vein in cadaver}

\section{Karacan $\mathrm{K}^{*}$, Özbek E**, Akcaalan $M^{*}$}

*Department of Anatomy; Faculty of Medicine, Sakarya University, Sakarya, Turkey; **Department of Histology and Embryology, Faculty of Medicine, Sakarya University, Sakarya, Turkey

Objectives: Testicular veins are derived from pampiniform plexus and provide venous drainage with epididymis and testis. While right testicular vein drains inferior vena cava, left testicular vein drains left renal vein. Variation of the testicular veins includes duplication, different course and a typical drainage. These variations affect blood flow, temperature and spermatogenesis. Thus, they can cause varicocele or infertility. In this study, we aimed to present variations of the testicular artery and vein, detected in dissection of cadaver.

Methods: Variations were detected in the testicular artery and vein of male cadaver in Department of Anatomy. While the distance between renal vein juntion and anulus inguinalis profundus was used for measurement of testicular vein, the distance between output level and anulus inguinalis profundus was used for measurement of testicular artery. Measurements were performed by the use of a millimeter caliper.

Results: Double left testicular vein and double left testicular artery origining differently were detected in dissection. Width of medially localized left testicular vein was $4.17 \mathrm{~mm}$ and length of this vein was $250 \mathrm{~mm}$. Width of laterally localized left testicular vein was $3.59 \mathrm{~mm}$ and length of this vein was 240 $\mathrm{mm}$. While width of left testicular artery origining from renal artery origin was $3.73 \mathrm{~mm}$, length of it was $256 \mathrm{~mm}$, width of left testicular artery arising from the aorta was $1.74 \mathrm{~mm}$ and length of it was $243 \mathrm{~mm}$.

Conclusion: Consciousness about the variation of testicular artery-vein is very important in retroperitoneal surgical operation. The number, localization and drainage variations of vascular structure make varicocelectomy and retroperitoneal vascular operations difficult. These factors are some reasons of surgical failures. Atypical drainage of testicular veins increases the risk of renal transplantation. Also, knowing the variation of testicular vascular make diagnosis of urinary and genital pathology and interpretation of radiological examinations easier.

\section{P-273}

\section{Left pulmonary agenesis: a rare case}

Karacan $\mathrm{K}^{*}$, Karacan A**, Akcaalan $\mathrm{M}^{*}$

*Department of Anatomy, Faculty of Medicine, Sakarya University, Sakarya, Turkey; ${ }^{*}$ Department of Radiology, Faculty of Medicine, Sakarya University, Sakarya, Turkey

Objectives: Unilateral pulmonary agenesis (PA) is a rare condition and occurs due to the lack of unilateral lung buds during development of intrauterine resulting from failure of development of pulmonary including the carina. The etiology of PA that is seen approximately $1 / 100000$ is not known. It is seen with abnormalities of musculoskeletal, gastrointestinal, urogenital, especially cardiovascular system. If patients have no or mild related anomalies they can survive till adulthood. Since it is a rare condition we aimed to present an adult female case of left pulmonary agenesis.

Methods: A 54 year old woman patient who applied to Sakarya University Training Research Hospital by pre-diagnosis of myalgia had a contrast enhanced computed tomography (CT) scan of thorax. The patient had no surgical history.

Results: Left lung and left side bronchovascular structures were not observed in the patient after CT. The heart and mediastinal structures were located in the left hemithorax and there were significant increase in volume of the right lung lengthening to left hemithorax. Imaging findings are consistent with agenesis of the left lung.

Conclusion: Diagnosis of PA is usually made in neonatal period but also it can be detected in the coming years. However, co-existing anomalies and pulmonary function determine prognosis, morbidity and disease process. A considerable number of people apply to hospitals for respiratory distress and recurrent pulmonary infections reasons. Therefore, PA diagnosed people must be examined in terms of related anomalies and when lung infection occurs it must be treated carefully. 


\section{P-274}

Relationship with stylohyoid chain and internal carotid artery detected by 3D-angiography

Eraslan C**, Özer MA*, Gövsa F* ${ }^{*}$, Alagöz AK***, Callı C** *Department of Anatomy, Faculty of Medicine, Ege University, Izmir, Turkey; **Department of Radiology, Faculty of Medicine, Ege University, Izmir, Turkey; ${ }^{* *}$ Medical Student 4, Faculty of Medicine, Ege University, Izmir, Turkey

Objectives: The calcified stylohyoid chain (SHC), the elongated styloid process (SP), larger SP' angle and shortened contact distance of the internal carotid artery (ICA) are risk factors for stylocarotid artery syndrome. The aim of this study was to analyze SHC anatomy and its relations with ICA using threedimensional angiography (3D-CTA).

Methods: 3D-CTAs of 125 patients were analyzed in terms of SP length, its angulations, types of SHC and relationships between its proximity to the ICA.

Results: The mean length of the SP was measured as $31.3 \pm 7.4$ $\mathrm{mm}$, range (15.2-85 mm). It was found longer in males. Elongated SP was observed frequently in total both females (47\%) and males (55\%). The mean contact distance of the ICA to the SP was calculated as $8.2 \pm 3.3 \mathrm{~mm}$, range $(1.8-21.5 \mathrm{~mm})$. The frequency of the patients with that distance being less than $5 \mathrm{~mm}$ was $23.3 \%$. This short length between the ICA and the SP was observed more frequently in females $(10.7 \%)$ and males (28.1\%). The anterior and medial angulations of the SP were calculated as $71.2 \pm 4.3^{\circ}$, and $57.3 \pm 9.3^{\circ}$, respectively. In the present study SHC was determined as normal SP (54.2\%), absence of SP (1\%), duplication of the SP (1\%), elongated SP (26\%), complete ossification of SHC (1\%), and segmentation of SHC (9\%).

Conclusion: 3D-CTA is the most appropriate radiological investigation analyzing and measuring SHC anatomical variations and identifying types resulting from pressures on ICA. Our study also revealed the pressure on the artery not only arose from the tip of the SP but could also result from types stretching over artery wall. In those specimens, there is a higher probability of formation of stylocarotid syndrome due to the long term pressure on the sympathetic chain around the ICA.

\section{P-275}

\section{Anthropometric growth study of orbital region in Turkish children}

Ulcay $T^{*}$, Sarsilmaz $M^{* *}$, Uzun $A^{* * *}$, Ogetürk $M^{* * * *}$

*Department of Anatomy, Faculty of Medicine, Ahi Evran University, Kırşehır, Turkey; **Department of Anatomy, Faculty of Medicine, Şifa University, Izmir, Turkey; ***Department of Anatomy, Faculty of Medicine, Ondokuz Mayıs University, Samsun, Turkey; ****Department of Anatomy, Faculty of Medicine, Firat University, Elazığ, Turkey

Ocular measurements, including intercanthal width, biocular width, palpebral fissure length and canthal index are important in the evaluation of congenital deformities and posttraumatic telecanthus. The aim of the study was to describe the average values and age related changes of the orbital anthropometric measurements in Turkish children. Study group consisted of 265 (145 male, 120 female) Turkish children aged from 7 to 11 years. The average total increments achieved between ages 7 and 11 years were $1.7 \mathrm{~mm}$ in males and $1.5 \mathrm{~mm}$ in females in the intercanthal width and $5.9 \mathrm{~mm}$ in males and $3.4 \mathrm{~mm}$ in females in the biocular width. Palpebral fissure length showed a moderate level of development in both sexes. Age related changes of canthal index were minimal. But the average values of canthal indices in females were higher than males in all age groups. Data collected in the present study could serve as a data base for the quantitative description of child orbital morphology during normal growth and development.

Keywords: Intercanthal width, biocular width, canthal index

\section{P-276}

\section{Evaluation of the atherosclerosis in superior thyroid artery}

Karacan $\mathrm{K}^{*}$, Akcaalan $\mathrm{M}^{*}$, Karacan $\mathrm{A}^{* *}$, Acar BA**, Varım $\mathrm{C}^{* * * *}$, Can $\mathrm{Y}^{* * * * *}$, Vatan $\mathrm{MB}^{* * * * *}$, Aksoy $\mathrm{NM}^{* * * *}$, Mansiz $\mathrm{S}^{* *}$, Akdemir $\mathrm{R}^{* * * * *}$

*Department of Anatomy, Sakarya University, Faculty of Medicine, Sakarya, Turkey; **Department of Radiology, Sakarya University, Faculty of Medicine, Sakarya, Turkey; ***Department of Neurology, Sakarya University, Faculty of Medicine, Sakarya, Turkey; $* * *$ Department of Internal Medicine, Sakarya University, Faculty of Medicine, Sakarya, Turkey; ***** Department of Cardiology, Sakarya University, Faculty of Medicine, Sakarya, Turkey

Objectives: Superior Thyroid Artery (STA) is branch of external carotid artery and it feeds thyroid gland with inferior thyroid artery. Atherosclerosis which is the most common pathology of cardiovascular system is frequently in the origin location of the artery but it may be seen at other levels as well. Thyroid gland is fed by other side STA or both inferior thyroid arteries in total occlusion. The aim of this study is to evaluate frequency and degree atherosclerosis of STA in patients having carotid angiography.

Methods: In our study, conventional angiography images of 284 patients (182 males, 102 females) were retrospectively evaluated in terms of atherosclerosis of STA in Sakarya University Training Research Hospital Department of Cardiology. The patients who had stenosis in STA origin were divided into two groups in terms of stenosis ratio below $50 \%$ and above $50 \%$.

Results: In our study, while frequency of normal STA was 93.7 $\%$ in left side and it was $95 \%$ in right side. While frequency of cases stenosis ratio below $50 \%$ was $3.2 \%$, frequency of cases stenosis ratio above $50 \%$ was $1.8 \%$ in right STA. While frequency of cases stenosis ratio below $50 \%$ was $2.8 \%$, frequency of cases stenosis ratio above $50 \%$ was $3.5 \%$ in left STA. Total occlusion of STA was not determined in both sides.

Conclusion: Atherosclerosis of STA should be taken into consideration in all thyroid pathology especially causing volume reduction and hypothyroidism in addition to surgical intervention to thyroid gland. 


\section{P-277}

Evaluation of existence and frequency of lesion in left subclavian artery by angiography

Karacan $\mathrm{K}^{*}$, Akcaalan $\mathrm{M}^{*}$, Karacan $\mathrm{A}^{* *}$, Acar BA***, Varım $\mathrm{C}^{* * * *}$, Can $\mathrm{Y}^{* * * * *}$, Vatan $\mathrm{MB}^{* * * * *}$, Aksoy $\mathrm{NM}^{* * * * *}$, Mansiz $\mathrm{S}^{* *}$, Akdemir $\mathrm{R}^{* * * * *}$

*Department of Anatomy, Sakarya University, Faculty of Medicine, Sakarya, Turkey; **Department of Radiology, Sakarya University, Faculty of Medicine, Sakarya, Turkey; ${ }^{* *}$ Department of Neurology, Sakarya University, Faculty of Medicine, Sakarya, Turkey; $* * * *$ Department of Internal Medicine, Sakarya University, Faculty of Medicine, Sakarya, Turkey; ***** Department of Cardiology, Sakarya University, Faculty of Medicine, Sakarya, Turkey

Objectives: Left subclavian artery (LSA) originates directly from aorta and feeds upper extremity and neck. LSA does not branch in mediastinum. Many lesions abut LSA and limit the length of the proximal landing zone. Upper extremity blood pressure differences are generally because of atherosclerotic plaque, leading to a reduction in blood pressure to one upper extremity, or less ordinarily, both upper extremities. Although many kinds of pathology, and surely some aneurysms, can abut the left subclavian artery, some disease processes-for example, traumatic transection and dissection-almost always develop within close proximity to the origin of the great vessels. If significant left subclavian stenosis is not identified prior to bypass in these patients, it can lead to the coronary-subclavian steal syndrome. The aim of this study is to evaluate existence and frequency of lesion in left subclavian artery by angiography.

Methods: In our study, conventional angiography images of 284 patients (182 males, 102 females) were retrospectively evaluated in terms of stenosis of LSA in Sakarya University Training Research Hospital Department of Cardiology. The patients having stenosis in LSA origin were divided into four groups in terms of stenosis ratio below $50 \%$, between $50-70 \%$, $70-90 \%$ and total occlusion.

Results: Normal LSA was $95.1 \%$. Stenosis ratios of cases for below $50 \%$, between $50-70 \%$, between $70-90 \%$ and total occlusion were $1.8 \%, 0.7 \% 1.1 \%$ and $1.1 \%$ respectively. When results were compared in terms of gender, there is not any significant relation statistically.

Conclusion: Up to $90 \%$ of patients undergoing coronary artery bypass grafting (CABG) now receive a left internal mammary artery (LIMA) graft due to greater patient survival and conduit longevity when compared to saphenous vein grafts. Thus, knowing stenosis of left subclavian artery is highly important in left internal mammary artery (LIMA) graft.

\section{P-278}

Photographic periorbital soft tissue analysis in Turkish young adults: anthropometric measurements

\section{Işıklar S, Özdemir ST}

Department of Anatomy, Faculty of Medicine, Uludağ University, Bursa, Turkey
Objectives: Periorbital anthropmetric analysis is important in defining cosmetic, pathologic, and ethnic variation. The purpose of this study was to establish periorbital soft tissue analysis for Turkish young adults.

Methods: Anthropometric measurements of the periorbital soft tissue were taken from 167 female and 56 male Turkish adults aged between $18-24$ years $(19.18 \pm 1.22$ years). The linear inner intercanthal distance (IICD), interpapillary distance (IPD), outer intercanthal distance (OICD), horizontal palpebral aperture (HPA), medial canthal comissura (MCC), and lateral canthal comissura were digitally measured for the analysis of the periorbital soft tissue. Besides, palpebral slant angle was measured by means of standardized photographic records using TPSDIG 2.04 software. The obtained data were analyzed with SPSS 22.0 software programme with t test and Mann Whitney $\mathrm{U}$ statistics test.

Results: A statistically significant differences were found between males and females for outer intercanthal and interpupillary distance. In addiction horizontal palpebral apertura was found significantly different for left eye between female and male. Results were compared with other ethnic populations.

Conclusion: The periorbital soft tissue measurements of Turkish young adults were analyzed using 2D digital photogrammetry and average periorbital soft tissue data was obtained. The average values can be used in cosmetics and plastic surgery including periorbital region.

\section{P-279}

Three-dimensional analysis of facial shape in obstructive sleep apnea syndrome using geometric morphometric method

Özdemir ST $^{*}$, Ercan Iं** Ocakoğlu $\mathrm{G}^{* *}$, Çetinoğlu ED***, Ursavaş $\mathrm{A}^{* * *}$

*Department of Anatomy, Faculty of Medicine, Uludağ University, Bursa, Turkey; ${ }^{*}$ Department of Bioistatistics, Faculty of Medicine, Uludağ University, Bursa, Turkey; $* * *$ Department of Pulmonary Medicine, Faculty of Medicine, Uludağ University, Bursa, Turkey

Objectives: Studies of human face forms (size and shape) using traditional methods only show minimal variation. However, advanced image analysis and statistics such as geometric morphometric method have resulted to higly quantitative descriptions of detailed variation of human face. Obstructive Sleep Apnea Syndrome (OSAS) is a disorder charaacterized by apnea and hypopnea maintained for over 10 seconds and occuring at least 5 times per hour, or with at least 30 episodes during 7 hours of nocturnal sleep. Polysomnography (PSG) is the "gold standard" fort he identification of individuals with OSAS. OSAS has a significant relationship with the anatomic features of hard and soft tissues of face. The general aim of this study was to investigate the localize and quantify geometric morphometric differences in facial shape among obstructive sleep apnea patients and control subjects. 
Methods: 3D facial scans were obtained from 91 individuals (62 OSAS patients; 29 control group of non-OSAS). 3-D facial scan was used to capture facial soft tissues of both groups. In each image of the whole face thirthy craniofacial anatomic landmarks were identified and marked on the three dimensional images. Landmark data were used to determine differences of facial shape with OSAS and without OSAS. Facial shape was analysed using procructes analysis of landmark configurations (90 x, $y$ and $z$ coordinates of 30 facial landmarks). Mean facial shape were compared between OSAS and non-OSAS groups by statistical shape analysis. Shape deformations were investigated by Thin Plate Spline (TPS) analysis.

Results: No statistically siginificant differences in term of mean facial shape was found between the OSAS patinets and without OSAS $(\mathrm{P}=0.078)$.

Conclusion: Three-dimensional analyses revealed no significant differences in facial shapes of OSAS and without OSAS. This study have shown that the tools of geometric morphometrics can be used for detailed quantification of variations in shape of human face.

\section{P-280}

\section{Using Skype in anatomy education: two different approaches}

$\underline{\text { Yücel K}}^{*}$, Çelik B**, Aydıner IE ${ }^{* * *}$, Kaspar EÇ****, Özdemir $\mathrm{S}^{* * * *}$, Gergin $\mathrm{S}^{*}$

*Department of Anatomy, Yeditepe Faculty of Medicine, Yeditepe University, Istanbul, Turkey; **Second Year Medical Student, Yeditepe Faculty of Medicine, Yeditepe University, Istanbul, Turkey; $* * *$ First Year Medical Student, Yeditepe Faculty of Medicine, Yeditepe University, istanbul, Turkey; $* * * *$ Department of Biostatistics and Medical Informatics, Yeditepe Faculty of Medicine, Yeditepe

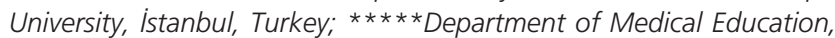
Yeditepe Faculty of Medicine, Yeditepe University, Istanbul, Turkey

Objectives: Skype is an application where people can make conference calls over the internet. It has been used by academicians for several reasons, e.g. residency interviews, exam remediation, problem-based learning, etc. Here we present two different approaches of using Skype in anatomy education.

Methods: Skype was used in anatomy education by two different ways. First, 5-10 students with the lowest academic performance were given an overview lecture through Skype before their next exams. The recorded $\mathrm{mp} 3$ files and power point slides of the Skype sessions were then shared by the entire class at a Wordpress blog this year. Second, clinicians talked about clinical anatomy during the last 10 minutes of some lectures through a Skype video conference call. An obstetrician for the "Pelvis" lecture and a neurosurgeon for the "Joints of the vertebral column" are examples for these Skype visits performed by clinicians in the class. A survey on social media through WhatsApp was given to 303 healthcare students. We collected data on the usage level of Skype and views on using Skype for education. We used Likert-type items in our survey.
Results: Skype was relatively popular among students. Half of the students ( $\mathrm{n}=147 ; 49 \%)$ had Skype accounts and were using Skype actively. 24 students (8\%) said they would use Skype classes if they were instructors. This year recorded Skype lectures were shared by the pharmacy students. We observed a $10 \%$ increase between the final exam marks of pharmacy first year students of this year and those of last year (from 61 to 67) $(p<0.05)$. Clinicians' visit to the anatomy lectures via Skype gets the full attention of the students.

Conclusion: We witnessed that clinicians were highly motivated during their Skype visits to the anatomy lectures. Voice delay is an important issue in Skype video conferences.

\section{P-281}

\section{Vine lab: using vine application in anatomy education}

Yücel K$^{*}$, Kaspar EÇ**, Özdemir S***, Aydıner İE****, Çelik B****, Gergin $\mathrm{S}^{*}$

*Department of Anatomy, Yeditepe Faculty of Medicine, Yeditepe University, istanbul, Turkey; **Department of Biostatistics and Medical Informatics, Yeditepe Faculty of Medicine, Yeditepe University, Istanbul, Turkey; ${ }^{* * *}$ Department of Medical Education, Yeditepe Faculty of Medicine, Yeditepe University, Istanbul, Turkey; ****First Year Medical Student, Yeditepe Faculty of Medicine, Yeditepe University, Istanbul, Turkey; $* * * * *$ Second Year Medical Student, Yeditepe Faculty of Medicine, Yeditepe University, İstanbul, Turkey

Objectives: Vine has been around since 2012, being a revolutionary micro-video application where one creates six-second videos. The shortness and infinite loop features are ideal for anatomy lab demonstrations. Here we present the usage of Vine videos in anatomy education and our students' feedback.

Methods: We prepared a survey via Google Docs to obtain information about the level of usage and contribution of the Vine Lab (https://vine.co/yeditepeanatomy) to anatomy education. The survey was sent to 71 second year medical students through Whatsapp. 60 students answered to our survey with a mean age of $20.6 \pm 0.9$ and a response rate of $86 \%$. We used Likert-type items in our survey.

Results: 82 vine videos were prepared for 7 labs during the two years. Vine lab videos were inserted in post at the E-lab blog. The average number of videos for each lab was 12 (6-22 videos). A total of 274 items were demonstrated in these vine videos (average; 39, 23-70). The average number of structures demonstrated per video was 3 (2-6). In the survey, 28 students (46.7\%) used Vine Lab often or very often. 41 (78\%) students reported that Vine Lab contributed to the learning processes. $23(38 \%)$ students thought that Vine Lab decreased the studying time more than $25 \% .26$ (43\%) students would prefer to see 3 items in one Vine video. "I do not want to see any educational material when using my Vine account" statement was objected by almost half of the students; $(n=29 ; 48 \%) .17$ (28\%) students were indecisive.

Conclusion: Short video applications like Vine and Instragram might be practical for lab demonstrations in anatomy. Short 
time period, infinite loop and easy access are three major advantages. The instructor can also track the students' progress in studying by the number of loops for each video.

\section{P-282}

The morphological and morphometrical evaluation the bones that transmit emissary veins in terms of surgery

Türk F* ${ }^{*}$, Akyer SP* Özdemir $M^{* *}$, Özdemir MB*, Akdoğan I*

*Department of Anatomy, Faculty of Medicine, Pamukkale University, Denizli, Turkey; **Department of Neurological Surgery, Faculty of Medicine, Pamukkale University, Denizli, Turkey

The complications such as bleeding, thrombosis and air embolism depend on injuries emissary veins is often encountered in surgery. Detailed descriptions of the mastoid foramen, occipital foramen, parietal foramen, posterior condylar canal and foramen vesalius are lacking in the literature. For this reason, the purpose of our study was to explore and represent the morphology and morphometry of these emissary foramina in order to prevent complications and to guide for surgeons. The present study was made on 60 dry human skull in the laboratories of Pamukkale University, Faculty of Medicine Department of Anatomy. After taken photograph of emissary foramens by Canon 650D professional camera, the evaluation and measurement's these foramens made with Matlab program by computer. The overall prevalence of mastoid foramen was $90.52 \%$, occipital foramen was $72.52 \%$, parietal foramen was $42.85 \%$, posterior condylar canal was $91.25 \%$ and foramen vesalius was $78.26 \%$. The mean diameter of the mastoid foramen was $1.81 \pm 0.76 \mathrm{~mm}$, occipital foramen was $1.20 \pm 0.25 \mathrm{~mm}$, parietal foramen was $1.49 \pm 0.46 \mathrm{~mm}$, posterior condylar canal was $2.83 \pm 1.33 \mathrm{~mm}$ and foramen vesalius was $1.74 \pm 0.60 \mathrm{~mm}$. Distances between emissary foramina and fixed bony landmarks were measured. Emissary veins are important in clinic practice and surgical procedures because they act a route of spread of exracranial infection to the intracranial structures and these veins may be a significant bleeding during surgery of the skull and they can be source of thrombosis and air embolism. The detailed anatomical knowledge of these veins and foraminas may help to prevent complications and to guide for surgeons.

Keywords: Emissary foramina, mastoid foramen, occipital foramen, parietal foramen, posterior condylar canal, foramen vesalius, morphology, morphometry

\section{P-283}

Morphometric investigation of tibia in dry bones

$\underline{\text { Akın D }}^{*}$, Çiçekcibaşı AE*, Kabakçı AD*, Kala Y**

*Department of Anatomy, Meram Faculty of Medicine, Necmettin Erbakan University, Konya, Turkey; **Institute of Health Sciences, Meram Faculty of Medicine, Necmettin Erbakan University, Konya, Turkey

Objectives: The knowledge of the morphometric values of tibia segments is important for forensic, anatomic and archeological case studies in order to identify unknown bodies and stature. Tibial measurements may help to indicate the characteristic morphological features of tibial segments in different population andalso the orthopedic surgeon place the various implants during the reconstruction of tibial fractures. Therefore, the aim of this study is to obtain anthropometric measurements from dry bones for the ideal design of prosthesis related to knee joint.

Methods: This study was conducted on 57 tibia (22 right, 35 left) in collections of Necmettin Erbakan University Meram Medicine Faculty Anatomy Laboratory. Digital calipers and osteometric board were used for the measurements of the tibia. Which were classified as measurements of upper end, body of the bone and lower end. Tibial mechanical axis (TMA): Tibial length of lines that was measured with a tape measure, drawn from the midpoint of the ankle to the midpoint of the body Tibia longest length (TL-I): The distance between lateral condyle and medial malleolus. Tibia shortest length of (TL-II): The distance between lateral condyle and fibular incisura Results were analyzed using the SPSS 21.0 Statistical Package Program.

Results and Conclusion: In this study, it was determined that mean of right TMA was $33.03 \pm 2.70 \mathrm{~cm}$, mean of left TMA was $33.77 \pm 2.5 \mathrm{~cm}$. Additionaly mean of right TL-I and TL-II were identified as $34.74 \pm 2.44 \mathrm{~cm}, 30.51 \pm 4.44 ; 35.47 \pm 2.65,33.25 \pm 2.62$ $\mathrm{cm}$ respectively in left. The success of total knee arthroplasty is dependent on the accurate measurements, cuts during the operation and the selection of suitable sized component. Therefore, morphological differences of the bones forming the knee joint should be taken into account. We believe that these measurements may help to orthopedic surgeon and provide data for designing of appropriate sized and shaped knee prosthesis.

Keywords: Tibia, morphometry, knee morphology

\section{P-284}

\section{Relationship between sleep disordered breathing and head-neck anthropometry}

Akçaalan $M^{*}$, Yılmaz $\mathrm{A}^{* *}$

*Department of Anatomy, Faculty of Medicine, Sakarya University, Sakarya, Turkey; ${ }^{*}$ Department of Anatomy, Faculty of Medicine, Trakya University, Edirne, Turkey

Objectives: Sleep is a reversible passivity situation that allows somebody to rest physically and psychologically, refreshes and makes one ready for a new day when human disassociates relations with the environment. Clinical charts are described as "sleep breathing disorders" which develope depending upon changes in breathing and cause to raise morbidity and mortality. The aim of this study is to investigate relationship between sleep disordered breathing and head-neck anthropometry.

Methods: 147 voluntary people were included in the research. The volunteers were grouped into two classes as diagnosed sleep breathing disorder or not diagnosed as sleep breathing disorder. Body height, sub-face length, interincisial distance, 
nose height, anterior, lateral and posterior neck length, thyromental distance, ramus mandibula length, corpus mandibula length, bigonial latitude, neck width, neck depth, neck circumference of the volunteers were measured metrically.

Results: Average values of the volunteers who were diagnosed as sleep breathing disorder and not diagnosed as sleep breathing disorder were measured respectively; body height, $168 \pm 6.64-166.97 \pm 5.72 \mathrm{~cm}$; sub-face length, 78.16 \pm 10.09 $105.06 \pm 15.4 \mathrm{~mm}$; interincisial distance, $56.74 \pm 13.08-58.13 \pm$ $10.55 \mathrm{~mm}$; nose length, $75.81 \pm 8.07-73.74 \pm 8.07 \mathrm{~mm}$; anterior neck length, $79.86 \pm 10.75-133.61 \pm 22.25 \mathrm{~mm}$; lateral neck length, $153.9 \pm 13.9-156.74 \pm 13.17 \mathrm{~mm}$; posterior neck length, $114.5 \pm 19.14-110.93 \pm 16.05 \mathrm{~mm}$; thyromental distance, $69.78 \pm$ $8.25-103.53 \pm 10.61 \mathrm{~mm}$; ramus mandibula length, $74.7 \pm 9.32$ 73.08-11.17 mm; corpus mandibula length, 88.73 \pm 7.79 $117.87 \pm 12.97 \mathrm{~mm}$; bigonial distance, $95.00 \pm 8.83-126.38 \pm 9.99$ $\mathrm{mm}$; neck width, 87.78 $\pm 6.85-88.83 \pm 9.99 \mathrm{~mm}$; neck depth, $126.18 \pm 12.00-126.89 \pm 12.78 \mathrm{~mm}$; neck circumference, $42.64 \pm$ $1.73-35.58 \pm 1.65 \mathrm{~mm}$.

Conclusion: In our study, it was identified that there was a significant relationship between body mass index (BMI), sub-face length, neck circumference, anterior neck length, bigonial distance, corpus mandibula length and sleep-disordered breathing. Thus, these parameters should be taken into consideration in diagnosing and treatment of sleep disordered breathing.

\section{P-285}

\section{Dimensional (3D) assesment of hippocampus in Alzheimer's disease}

Türk F* ${ }^{*}$ Özdemir MB**, Çă̆ırıı $\mathrm{S}^{* * *}$, Akyer SP**, Türk F** *Department of Anatomy, Faculty of Medicine, Pamukkale University, Denizli, Turkey; ${ }^{*}$ Department of Anatomy, Faculty of Medicine, Pamukkale University, Denizli, Turkey; ***Department of Neurology, Bakırköy Psychiatry Hospital, Istanbul, Turkey

Neuroanatomical appearance can be correlated with clinical or other characteristics of illness. With the introduction of diagnostic imaging machines, producing 3D images of anatomic structures, calculating the correlation between subjects and pattern of the structures have become possible. The aim of this study is to examine the $3 \mathrm{D}$ structure of hippocampus in cases with Alzheimer disease in different dementia severity. For this purpose, 62 female and 38 male- 68 patients's (age range between 52 and 88) MR scanning were imported to the computer. 3D model of each right and left hippocampus were developed by a computer aided propramme-Surf Driver 3.5. Every reconstruction was taken by the same investigator. There were different apperance of hippocampus from normal to abnormal. In conclusion, These results might improve the understanding of the correlation between the morphological changes in hippocampus and clinical staging in Alzheimer disease.

Keywords: Alzheimer disease, hippocampus, computer-assisted anatomy, 3D

\section{P-286}

Absence of the coeliac trunk in a rectal cancer patient

Tihan $\mathrm{D}^{*, * *}$ Babacan $\mathrm{S}^{*}$, Ergin $\mathrm{T}^{* * *}$, Teomete $\mathrm{U}^{* * * *}$, Dandin $\mathrm{O}^{* * * *}$, Kafa $\mathrm{I}^{*}$

*Department of Anatomy, Faculty of Medicine, Uludağ University, Bursa, Turkey; ${ }^{*}$ Department of General Surgery, Sevket Yılmaz Training and Research Hospital, Bursa, Turkey; ${ }^{* *}$ Department of Radiology, Faculty of Medicine, Gülhane Military Medical Academy, Ankara, Turkey; $* * * *$ Department of Radiology, Miller Faculty of Medicine, University of Miami, Miami, Florida, US; ${ }^{* \star \star * \star D e p a r t m e n t ~ o f ~ G e n e r a l ~ S u r g e r y, ~ B u r s a ~}$ Military Hospital, Bursa, Turkey

Haller described the trifurcation of the coeliac trunk firstly. Then, this important anatomical structure known as "Tripus Halleri". As well as the coeliac trunk shows itself in the normal anatomic form nearly in the $85 \%$ of human population, it also has variations just about in $15 \%$. Regarding the literature, rarest variation is the absence of the coeliac trunk that has a prevalence rate at approximately $0.4 \%$. Despite the fact that agenesis of the coeliac trunk is often asymptomatic, it should be considered during or planning the surgical interventions of the abdominal region. In this report, we present a case of the absence of the coeliac artery and independent originations of the left gastric, splenic and common hepatic arteries from the ventral wall of abdominal aorta. Forty-seven-year-old female patient was admitted to the general surgery clinic with a minimally acute rectal bleeding. To determinate of a distant metastasis, computed axial tomography scanning was performed and the lack of the coeliac trunk was noticed: patient's the left gastric artery, splenic artery and common hepatic artery had been originated independently from the anterior wall of abdominal aorta. The patient underwent an anterior resection with complete mesocolic excision. During the exploration, no visceral ischemia was observed due to anomalous pattern of the coeliac trunk, thus no intervention was performed to this vascular anomaly. Variations of the coeliac trunk (particularly the type of absence of the coeliac trunk) have no unfavorable consequences on the dependent visceral organs; moreover, the independent branches may even prevent a large visceral ischemia, which may be occurring in the situation of a massive thromboembolism of the coeliac trunk. However, the surgeons and interventional radiologist should consider the variational rooting patterns or absence of the coeliac trunk from the abdominal aorta.

\section{P-287}

\section{Thoughts of the students and the employees on medical terminology class}

\author{
Babacan S, Işıklar S, Kafa İ, Coşkun I \\ Department of Anatomy, Faculty of Medicine, Uludağ University, \\ Bursa, Turkey
}

It's essential to use common medical terminology to communicate and pursue the developments on medical platforms. Due to the medical terminology comprises many technical terms rooted from Greek, Latin, French, Arabic, Persian and English origins, medical professionals have difficulties to expressing themselves in their native languages. Current study aims to determine the 
thoughts of the students and employees (who have taken lessons previously) on medical terminology. This study was conducted on 209 students from Vocational Faculty of Health Services and 74 employees from the Hospital of Medical Faculty of Uludag University. The data were collected by a Likert's questionnaire form. Only the voluntary participants were included the study after getting the decision of the ethics committee. On the point of the question related to the necessity and the satisfactoriness of medical terminology classes, both of the students and the employees expressed that the medical terminology is necessary for their career (\% 78.5, \% 79.7, respectively) and the content is sufficient (\%51.2, \%65.2, respectively). On the notions of "consistency" and "having medical terminology classes one more time"; the students answered that the "consistency" isn't ample; however, the employees reflected the contrary (\%43.6, \%52.7, respectively). Students also stated that, they don't have to take the class again; but the employees stated that they should have the class one more time again (\%46, \%59.4, respectively). Both the students and the employees expressed that, they advance benefits from medical terminology classes at their works or internships (\%65.1, \% 71.6, respectively). Additionally, the employees expressed that, they sometimes have difficulty to understanding the written material (\% 41.9) and they usually ask for help from other personnel or staff (\% 56.7). Within these results, we think that the feedbacks and the evaluations obtained from our questionnaire are valuable for the development and the progress of the syllabus of the medical terminology.

\section{P-288}

\section{Prevalence of Eustachian valve in adult autopsy cases and fetal cadavers: morphometric and histological evaluation}

$\underline{\text { Gergin }}^{*}$, Özdoğmuş Ö* ${ }^{*}$ Şehirli ÜS*, Şirvanc1 $S^{* *}$, Verimli U*, Üzün I***, Ömeroğlu $E^{* * *}$

*Department of Anatomy, Faculty of Medicine, Marmara University, Istanbul, Turkey; ${ }^{* *}$ Department of Histology and Embryology, Faculty of Medicine, Marmara University, Istanbul, Turkey; ${ }^{* * *}$ Institute of Forensic Medicine, Ministry of Justice, Istanbul, Turkey

Objectives: Eustachian valve (EV) is also termed as inferior vena cava valve, one of the most variable structures in the body. EV has a large variability in shape, size, texture and thickness. The aim of the present study was to assess the prevalence, variations, and histological findings of the EV in adult and fetal hearts.

Methods: We observed EV in 50 adult autopsy cases at the Institute of Forensic Medicine and in 24 fetal cadavers at the Department of Anatomy, Faculty of Medicine, Marmara University. Measurements of the valves were made by the vernice milimetric caliper. The specimens were then fixed in $10 \%$ formalin, routinely processed for light microscopy, and embedded in paraffin. Five-micron-thick sections were stained with hematoxylin and eosin, and Masson's trichrome.

Results: The average valve length was $22 \pm 2 \mathrm{~mm}$ in adults and $5 \pm 1 \mathrm{~mm}$ in fetal cadavers. The average valve depth was $10 \pm 1.5$ $\mathrm{mm}$ in adults and $2 \pm 1 \mathrm{~mm}$ in fetal cadavers. In $32 \%$ of the adult cases, the EV was absent. Forty-two percent of the valves was small crescentic; and $71 \%$ of these were simple nonfenestrated and $29 \%$ were simple fenestrated. Sixteen percent of the cases had a large crescentic membrane, $75 \%$ of which were nonfenestrated and $25 \%$ were simple fenestrated. We observed that all of the fetal cadavers had a cresentic EV. Histological findings demonstrated that the valve was lined by endothelium, contained cardiac muscle tissue intermingled with connective tissue.

Conclusion: It is important to know the anatomic variations of the $\mathrm{EV}$, when the invasive procedures into the right atrium are considered. Besides, these variations may be associated with some common cardiac pathologies such as bacterial endocarditis, thomboembolism and atrial tumors.

\section{P-289 \\ New approach to microscopic morphology of the thoracic duct}

Türk F*, Kocamaz E**, Mete E***, Akyer SP*, Türk F*, Özdemir MB*

*Department of Anatomy, Faculty of Medicine, Pamukkale University, Denizli, Turkey; ${ }^{*}$ Department of Histology and Embriology, Faculty of Medicine, Pamukkale University, Denizli, Turkey; ${ }^{* * \star}$ Department of Microbiology, Faculty of Medicine, Pamukkale University, Denizli, Turkey

The informations known about the microscopic anatomy of the thoracic duct are less from the informations known about the macroscopic morphology of it. The aim of present study is to investigate microscopic patterns of the thoracic duct. For this purpose, a piece of thoracic duct was dissected from the level of arcus aorta with the related structures that surround it. After histological procedure, preparation was stained with Hematoxylin and Eosin. Sections were evaluated by light microscopy in different magnification. It was founded many vessels and nerves distrubeted on great vessels. Muscles of the wall might be included striated or heart muscle fibers with smoot muscle. Thoracic duct is a great lymphatic vessel, $38-45 \mathrm{~cm}$ in length and extends from the second lumbar vertebra to the base of the neck. It usually terminates at jugulo-subclavian junction of veins. There is no well known circulation system about lymphatics. The output of this study may open new fundamental knowledge for new research about circulation of lymphatics in basically and clinically.

Keywords: thoracic duct, morphology, microscopy, muscles, striated muscle, heart muscle

\section{P-290}

A case of bilateral shortness of the fourth metacarpal

$\underline{\text { Keleş }}^{*}$, Kara $A^{*}$, Karaca L** Engin $\mathrm{Y}^{*}$, Gedik T*${ }^{*}$, Keleş MS*** *Department of Anatomy, Faculty of Medicine, Atatürk University, Erzurum, Turkey; **Department of Radiology, Faculty of Medicine, Atatürk University, Erzurum, Turkey; ${ }^{* \star}$ Department of Biochemistry, Faculty of Medicine, Atatürk University, Erzurum, Turkey

Congenital shortening of metacarpal or metatarsal bones is seen particularly in females, and it usually effects fourth metacarpal bones, and mostly bilaterally. Congenital short metacarpal can be seen alone or together with certain syndroms, as turner syndrom, 
Freiberg Dissease, Pseudohypoparatyroidism. Acquired short metacarp can be seen due to the damage of the epiphysial plate, by the causes like enfections, posttravmatic avascular necrosis, or others. A case of idiopathic bilateral symmetrical shortening of the fourth metacarpal bones in a 20-year-old female is described. The deformity did not result from trauma or an endocrine disorder. There was no another abnormality in the physical examination. The condition of the patient's hand caused no discomfort. There is no family history. Blood test results including calcium, parathormone, thyroxin, thyrotropin stimulating hormone and sedimentation rate were normal. Radiological examination showed bilateral shortening of the fourth metacarpal bones.

Keywords: Short metacarpal, short metatarsal, Pseudohypoparatyroidism

\section{P-291}

Passive clearing trial in rat brain: is the stirring effective for the process?

Aydın $\mathrm{AG}^{*}$, Çonkur Ş**, Ak M*** Adıgüzel E $^{*}$

*Department of Neuroscience, Institute of Health Sciences, Pamukkale University, Denizli, Turkey; ${ }^{*}$ Department of Mechanical Engineering, Faculty of Engineering, Pamukkale University, Denizli, Turkey; ${ }^{* * *}$ Department of Chemistry, Faculty of Science and Art, Pamukkale University, Denizli, Turkey

Clearing in brain tissue is popular for brain research nowadays. Clearing method allows to researchers to obtain the more intact axonal branching and connections than the conventional histological methods. In this training we try to do fast clearing to use passive clearing method with stirring. Rat brain was perfused with acrylamide, bis-acrylamide and paraformaldehyde solution. Than the brain was removed and embedded in the same solution during 48 hours in anaerobe condition and $+4{ }^{\circ} \mathrm{C}$. The brain in anaerobe chamber was heated in water bath with $37^{\circ} \mathrm{C}$ for polymerization at the end of 48 hours. The polymerized brain was cleaned from polymer remaining around of it. It was put in sodium dodecyl sulfate solution with magnetic stirring on heat plate with $37 \mathrm{oC}$ for clearing. Clearing of the brain was evaluated day to day. Sodium dodecyl sulfate solution was replaced three times in a week. First evidence of clearing was observed in the fifth day after beginning of clearing. Protruding parts of the brain such as olfactory bulb and brain stalk were completely clear at the end of the nineteenth day. The process was going on during writing the abstract. This study supported by TUBITAK (Project number: 114S407).

Keywords: Clearing, brain, nervous system, visualisation

\section{P-292}

Investigation of the incidence of the accessory bones in the elbow joint

Yaylacı $\mathrm{S}^{*}$, Uysal $\mathrm{M}^{*}$, Sapmaz $\mathrm{HI}^{*}$, Aytekin $\mathrm{K}^{* *}$, Taş U*, Özyurt B*

*Department of Anatomy, Faculty of Medicine, Gaziosmanpaşa University, Tokat, Turkey; **Department of Orthopedics and Traumatology, Faculty of Medicine, Giresun University, Giresun, Turkey
Objectives: Elbow joint is a hinge type joint that has important functions and ensures mechanical connection between wrist joint and shoulder joint. In the event of not knowing elbow joint variations well, diagnosis of the acute or chronic cases in those regions gets hard. There are limited numbers of studies to inform about the incidents of the variations of the bone structures around the elbow joint in the literature. For that reason, we aimed to evaluate the accessory formations in the elbow joint and its surroundings over the wide bone serial radiography and research prevalence in a certain population.

Methods: In this study, antero-posterior and lateral joint graphs of the 4000 patients in total that have been treated as a result of the trauma or disease between May 2013 and January 2014 have been evaluated. Obtained data has been evaluated statistically and $\mathrm{p}<0.05$ has been accepted as significant difference in all comparisons.

Results: Foramen olecrani (2.4\%) was the most frequent type of accessory formation. Other accessory structures were respectively olecranon traction osteophyte (\% 1.3), ligamentous ossificiation (\%1.1), patella cubiti (\%0.8), processus supracondylaris (\%0.4) and congenital dislocation of the radial head (\%0.2). The number of the men were higher than those of the women in patients which were diagnosed as osteophytic olecranon and patella cubiti but this difference only shows statistical significance for osteophytic olecranon ( $\mathrm{p}=0.001)$.

Conclusion: Developmental variation of skeletal system is usually detected incidentally during anatomical and radiological studies. Knowing these variations well is important for differential diagnosis of clinical disease related with elbow joint. Furthermore, early radiological diagnosis of the congenital dislocation of the radial head bears clinical importance since it may be together with the thrombocytopenia and congenital anomalies of cardiovascular, central nervous and urinary systems.

Keywords: Elbow joint, variation, accessory bone, patella cubiti, supracondylar process

\section{P-293}

The evaluation of the therapeutic effect of intravenous lipid emulsion treatment against malathion induced liver injury in rats

Esen $M^{*}$, Sapmaz HI${ }^{* *}$, Uysal $M^{* *}$, Alıc1 Ö ${ }^{* * *}$, Ünsal V****, Bütün $\dot{I}^{* * * *}$, Ayan $\mathrm{M}^{*}$, Taş $\mathrm{U}^{* *}$

*Department of Emergency Medicine, Faculty of Medicine, Gaziosmanpaşa University, Tokat, Turkey; **Department of Anatomy, Faculty of Medicine, Gaziosmanpaşa University, Tokat, Turkey; ***Department of Medical Pathology, Faculty of Medicine, Gaziosmanpaşa University, Tokat, Turkey; $* * * *$ Department of Medical Biochemistry, Faculty of Medicine, Gaziosmanpaşa University, Tokat, Turkey

Objectives: Malathion is a lipid soluble synthetic organophosphorus insecticide widely used in agricultural for controlling insects on fruits and vegetables. Malathion exposure occurs through inhalation, ingestion and dermal contact. Intravenous lipid emulsion (ILE) has been shown to reduce toxicity caused 
by some lipid soluble drugs. In this study, we evaluated the therapeutic effect of intravenous lipid emulsion treatment on the liver tissue in malathion intoxicity.

Methods: 21 male Wistar albino rats randomly divided into three equal groups. Groups organized as; Group I (control), Group II (malathion) and Group III (malathion + lipid emulsion treatment). Group I, only $1 \mathrm{ml}$ corn oil was given by gavage. Group II, $100 \mathrm{mg} / \mathrm{kg}$ malathion were given by gavage. Group III, in addition to malathion 20\% lipid solution (3 $\mathrm{ml} / \mathrm{kg}$ ) were administered via tail vein into rats. At the end of the experimental period, liver and blood samples were taken from the decapitated animals. Tissue sections were examined for histopathological changes by hematoxylin and eosin (H-E) staining and for apoptotic changes by Bax and Bcl-2 immunohistochemical staining. The liver tissue activities of superoxide dismutase (SOD), glutathione peroxidase (GSH-Px), and malondialdehyde (MDA) levels were also measured.

Results: MDA levels in serum and tissues were significantly higher in the malathion group when compared with the control and treatment groups. Additionally, tissue SOD levels were significantly higher in the treatment group when compared with the control and malathion groups. An increase of Bax and Bcl-2 immunoreactivity was observed in the malathion group when compared with the control group. Furthermore, a decrease of Bax immunoreactivity was observed in the malathion + lipid emulsion treatment group.

Conclusion: The findings from our study suggest that lipid emulsion treatment has therapeutic effect on malathion induced liver toxicity.

Keywords: Malathion, liver, lipid emulsion treatment

\section{P-294}

Examination of anatomic formations and their variations located in sinonasal area by multislice computed tomography

Doğmus $\mathrm{S}^{*}$, Karabulut $\mathrm{AK}^{*}$, Fazlioğulları $Z^{*}$, Kıvrak AS ${ }^{* *}$

*Department of Anatomy, Faculty of Medicine, Selçuk University Konya, Turkey; **Department of Radiology, Faculty of Medicine, Selçuk University, Konya, Turkey

Objectives: Sinonasal area is one of the most common areas with anatomic variations in human; such anatomic variations are efficient factors in pathogenesis of sinus inflammation and some headaches. In this study, we aimed to compare metric measurements and variations according to the age groups, gender and lateralization.

Methods: CT images on axial, sagittal and coronal planes of 200 patients who had not any surgical procedure on the sinonasal area and trauma history were examined; lengths, heights and widths were measured between certain points. Furthermore, some variations were also detected and comparisons between the age groups and genders were performed.
Results: Anteroposterior length and maximum width of the nasal septum, anteroposterior length of (left) middle nasal concha (MNC), anteroposterior length of (left) anterior ethmoidal artery (AEA), the distance between anterior ethmoidal artery and inferior nasal concha (left and right) and the distance between inferior nasal concha and lamina cribrosa (left and right) were significantly longer in men. Mean values were generally found higher in men after morphometric measurements; no significant difference was found between right and left side and increase was detected in length of many of the parameters by age. The most common variation detected in the present study was agger nasi cells $(73.5 \%)$ and this is followed by septal deviation $(67.5 \%)$, pneumatization of MNC (47.5\%), frontal sinus septum (43.5\%), hypertrophy of AEA (42\%), onodi cell (36.5), Haller cell (29.5\%), septal spur (29.5\%), sphenoid sinus septum $(23 \%)$.

Conclusion: The sinonasal area is one of the most common areas with anatomic variations. Recognition of anatomic variations of the nose and paranasal sinus helps to prevent complications and achieve a better surgical result.

Keywords: Nasus, morphometry, sinus, variation

\section{P-295}

An unusual right vertebral artery variation accompanying to bovine arch: a case report

Nayman A**, Fazlıŏulları Z* ${ }^{*}$ Karabulut A.K*, Gün C*, Nabi G*

*Department of Anatomy, Faculty of Medicine, Selçuk University, Konya, Turkey; ${ }^{*}$ Department of Radiology, Faculty of Medicine, Selçuk University, Konya, Turkey

Objectives: Anatomic variations of the aortic arch and its branches are common. Although these variations are generally asymptomatic and detected randomly in radiological examinations, aberrant subclavian artery may cause symptoms such as dispnea and dysphagia. Vertebral arteries (VA) normally branch from subclavian artery. However, variation rates of the VA are reported between $4.2 \%$ and $6.1 \%$ in the literature.

Case Report: In a 74-year old male patient, bovine arch and right VA variation was detected incidentally during CT angiography for occlusion of common carotid artery. Right VA branches from the aortic arch (aberrant VA) whereas left VA branches from the subclavian artery. In the measurements on coronal slices, bovine arch was found $16.42 \mathrm{~mm}$, left common carotid artery was found $6.26 \mathrm{~mm}$, brachiocephalic trunk was found $13.33 \mathrm{~mm}$, right VA was found $3.12 \mathrm{~mm}$ and left vertebral artery was found $1.56 \mathrm{~mm}$. The right VA branches from the aortic arch after left subclavian artery and forwards retroesophageally and retrotracheally during its course. The patient has not any symptom such as dyspnea and dysphagia.

Conclusion: Variation frequency of the aortic arch in postmortem studies is reported in varying rates between $2.6 \%$ and $35.1 \%$. Bovine arch is the most common variation of the aortic 
arch and incidence is reported within a wide range in the literature $(0.9 \%-27.4 \%)$. In the literature, variations of the left VA are reported as observed more common than variations of the right VA. Variation of the right aberrant VA is very rare (1/2357 cases). Preoperative detection of these variations will provide a basis for the clinicians to plan treatment options better, to assess and prevent possible intraoperative complications.

Keywords: Aortic arch, bovine arch, vertebral artery, CT angiography, variation

\section{P-296}

A case study: unilateral absence of the musculocutaneous nerve and variational branching of the median nerve

Özkan $M^{*}$, Gergin $S^{* *}$, Y1ldız SD*, Borac1 $\mathrm{H}^{*}$, Altay $\mathrm{G}^{*}$, Verimli U*

*Marmara University Faculty of Medicine, Istanbul, Turkey; ** Yeditepe University Faculty of Medicine, Istanbul, Turkey

Objectives: The brachial plexus consists of ventral rami of C5T1 spinal nerves. Musculocutaneous nerve is a terminal branch of the plexus which commonly originates from the lateral cord of the plexus. However, there are many variations published in the literature concerning the musculocutaneous nerve.

Case Report: In a 85 years-old male cadaver, a unilateral variation based on the absence of the musculocutaneous nerve on the right-upper extremity was observed. The musculocutaneous nerve is a branch of the lateral cord, innervating the coracobrachialis (cb), biceps brachii (bb) and the brachialis (b) muscles. It courses distally as the lateral cutaneous nerve of forearm supplying a cutaneous region in the forearm. A regular course and distribution for the musculocutaneous nerve was not observed on the right-upper extremity of the cadaver, instead, an unusual small branch originating from lateral cord coursing towards the coracobrachialis muscle was encountered. Additonally a single trunk originating from the median nerve and separating into two distinct branches which course through the biceps brachii and brachialis muscles was observed respectively. Following the muscular course, the branches of the trunk continued as the lateral cutaneous nerve of forearm on the lateral aspect of the right-upper extremity.

Conclusion: Radunovic et al. described a classification of musculocutaneous nerve variations according to the course and origin of the nerve in which six major types of variations were mentioned. The variation observed in this study does not fit to any of the six variative types of the musculocutaneous nerve described by Radunovic et al. The aim of this study is to contribute to the literature by demonstrating a new type of variation observed in the musculocutaneous nerve. Additionally this new variation may contribute to the clinical aspects of the region since it may be taken into consideration in surgical interventions to prevent certain lesions in the region.
Keywords: Musculocutaneous nerve, variation, median nerve, brachial plexus

\section{P-297}

Comparison of bone morphogenic protein 7, osteoprotegerin or combination -via adenoassociated virus- on bone formation in the expanded inter-premaxillary suture in rats

$\underline{\text { Uslu U}}^{*}$, Ülkür F**, Yalvaç ME ${ }^{* * *}$, Cumbul A*, Uslu BG*, Özdemir $\mathrm{F}^{* *}$, Şahin $\mathrm{F}^{* * *}$

*Department of Histology and Embryology, Faculty of Medicine, Yeditepe University, Istanbul, Turkey; **Department of Orthodontics, Faculty of Dentistry, Yeditepe University, Istanbul, Turkey; $* * \star$ Department of Genetics and Bioengineering, Engineering and Architecture Faculty, Yeditepe University, Istanbul, Turkey

Objectives: The aim of this study is to compare the histomorphometric effects of adeno-associated virus (AAV) mediated bone morphogenic protein 7 (Bmp7) and osteoprotegerin (OPG) or combination on bone formation in rats after expansion of the inter-premaxillary suture.

Methods: Twenty-four male Sprague-Dawley rats were assigned to 4 groups. The expansion of the interpremaxillary suture was performed using helical springs, bonded to the maxillary incisor teeth; which generated a $50 \mathrm{cN}$ force, applied for 5 days. Then segmental wires stabilized the positions of the teeth. Each rat received $50 \mu \mathrm{l}$ of injection into inter-premaxillary suture. Group 1 received AAV-Bmp7, group 2 received $\mathrm{AAV}-\mathrm{OPG}$, group 3 received $\mathrm{AAV}-\mathrm{Bmp} 7+\mathrm{OPG}$, and group 4 received AAV only. After the 10th day of injection, rats were sacrificed and their maxillas were dissected. 10 days after injection rats were sacrificed. The obtained tissue samples were fixed in $10 \%$ neutral formaldehyde in $0.1 \mathrm{M}$ phosphate buffered saline (PBS; $\mathrm{pH}=7.4$ ) and submitted to histomorphometric evaluation. Paraffin-embedded upper jaw tissues were sectioned to $10 \mu \mathrm{m}$. Physical fractionators and systematic sampling methods were used and stained with Hematoxyline and Eosin technique. Stereo Investigator Version 7.0, a light microscope Leica DM 4000B and the Cavalieri principle was used to estimate bone volume using the previously described stereological workstation.

Results: According to the histomorphometric evaluation, bone volume increased statistically a significant difference in the AAV-OPG, group compared to AAV group $(\mathrm{p}=0.0001)$. This trend continued AAV-BMP7 ( $\mathrm{p}=0.008)$. Alveolar bone volume of AAV, AAV-OPG, AAV-Bmp7, and AAV-Bmp7-OPG was $38.8175 \pm 0.7 \mathrm{~mm}^{3}, 49.4025 \pm 4.7 \mathrm{~mm}^{3}, 46.9850 \pm 1.5 \mathrm{~mm}^{3}$, $42.5825 \pm 2.8 \mathrm{~mm}^{3}$, respectively.

Conclusion: After the expansition of the inter-premaxillary sutur the local injection of AAV-OPG and AAV-Bmp7 can increase new bone formation, which may shorten the retention period and may reduce the risk of relapse.

Keywords: Histomorphometry, BMP7, maxillary expansion, orthodontics, rat 


\section{P-298}

Study of anatomic variations of the hepatic artery in
live liver donors within East Anatolia region

$\underline{\text { Kara A* }}^{*}$ Malkoç İ $\dot{I}^{*}$ Kantarc1 A**

*Department of Anatomy, Faculty of Medicine, Atatürk University, Erzurum, Turkey; **Department of Radiology, Faculty of Medicine, Atatürk University, Erzurum, Turkey

Objective of our study is indicating anatomy and variations of arteria hepatica propria in live liver donors, and helping the surgery team to carry out appropriate anastomosis and reconstruction during the transplantation based on the parameters gained. The study was carried out on 78 healthy liver donors including 45 male and 33 female with ages ranging from 19 to 69 who applied at Transplantation Unit of Faculty of Medicine of Atatürk University between January 2009 and July 2013. Multislice Computed Tomography films shot in order to show hepatic vascular structure of the donor candidates (ÇKBT) were examined retrospectively. Classification by Michels was taken as reference in evaluation arteria hepatica propria variations pertaining to the candidates. Statistical evaluation has been conducted by using Kruskal Wallis Variance Analysis. In our study, it has been determined that out of 78 donor candidates, in 53 cases (67.9\%)arteria hepatica propria has been found to be in line with classic anatomy (Michels Type I classification). $25 \%$ of the cases $(32.1 \%)$ have showed arteria hepatica propria variation. Incidence of the variations observed was replase arteria hepatica sinistra stemming from arteria gastrica sinistra in 8 cases $(10.3 \%$ ) (Michels tyoe II), arteria hepatica dextra stemming from arteria (9\%) mesenterica superior in 7 cases (Michels type III). Out of the remaining cases, $1.3 \%$ was in compliance with Michels Type IV, $1.3 \%$ was in compliance with Michels Type V, 2,6\% was in compliance with Michels Type VIII and $1.3 \%$ was in compliance with Michels Type IX. Also, it has been found out that 5 cases $(6.4 \%)$ were not in compliance with Michels classification. No significant correlation has been encountered between incidence of variation and gender in the study conducted. Also, the highest number encountered within group with variation was Michel type II variation.

Keywords: Proper hepatic artery, arterial variation, transplantation, liver

\section{P-299}

Proximal femur and investigation its relations with acetabulum morphometrically with multidetector computed tomography

Zengin $\mathrm{F}^{*}$, Doğan $\mathrm{NU}^{*}$, Koplay $\mathrm{M}^{* *}$, Fazlığgulları $\mathrm{Z}^{*}$, Karabulut AK*, Uysal İं*

*Department of Anatomy, Faculty of Medicine, Selçuk University, Konya, Turkey; ${ }^{*}$ Department of Radiology, Faculty of Medicine, Selçuk University, Konya, Turkey

Objectives: Knowledge of the normal morphology of the proximal femur, physical properties angle and size changing with age and differences between the sexes is important in terms of preventing complications that might occur during surgical procedures. Therefore, we aimed to investigate the normal morphology of the proximal femur.

Methods: Computed tomography images of 200 patients with ages ranging from 10-86 years (99 men, 101 women) were evaluated in terms of morphometric characteristics. With the right and left hip measurements in terms of gender and age group; femoral neck axis length measurements between acetabulum and the femur were made.

Results: In the measurements, axis femur length, hip axis length, acetabular width, femoral head width, femoral neck width, femoral shaft width, they widths trochanters, inclination angle, the medial femoral shaft cortical thickness, the lateral femoral shaft cortical thickness were determined to be larger in females than males in both sides. Inclination angle were greater on the right and left in males than females. According to the analysis, there was no statistically significant difference between right and left sides and betweenmales and females ( $>0.05$ ).

Conclusion: The differences occurred between the studies reported in the literature and the comparison of the left and right side measurements of proximal femur, the differences in the inclination angle values and other measurements obtained. We think that these differences are due to gender and racial differences.

Keywords: Proximal femur, acetabulum, morphometry

\section{P-300}

\section{A rare case of tortuosity of the right renal vein}

$\underline{\text { Keleș }}^{*}$, Karaca L**, Kara A*, Gedik $\mathrm{HT}^{*}$, Engin $\mathrm{Y}^{*}$

${ }^{*}$ Department of Anatomy, Faculty of Medicine, Atatürk University, Erzurum, Turkey; **Department of Anatomy, Faculty of Radiology, Atatürk University, Erzurum, Turkey

Knowledge of renal vascular anatomy is important during operative interventions, involving the retroperitoneal region, like surgery, invasive and radiological procedures. Normally, a single renal vein is formed in the renal sinüs in front of the renal artery on each sides for per kidney. It emerges from the renal hilum anterior to the renal artery, lies horizontally and drains into the inferior vena cava, at the level of L2 vertebra. Because the inferior vena cava is on the right half of the body, the left renal vein is longer than right renal vein. This report describes the variation, found in the course of the right renal vein. The variation was found, during the computerised tomographic(CT) examination of a thirtyone-year-old male patient, who had urinary complaints. The right renal vein was extending through the right inferior abdominal region, Then the course progressed upward and showed tortuosity before joining to the inferior vena cava, in the superior part. Because of their embryological development, many variations of renal veins can be seen, but the tortuosity of the right renal vein reported in this case, doesn't seen to have 
been cited in the literature previously. During diagnostic and surgical approaches in the retroperitoneal region, vascular variants must be considered for choosing appropriate techniques. These anomalies can be identified in a rotuin CT examination, before the abdominal surgery.

\section{P-301}

\section{Bronchial atresia as detected by computerized tomography: a case study}

\section{Gedik HT${ }^{*}$, Güven F** Keleş $\mathrm{P}^{*}$, Malkoç I*}

*Department of Anatomy, Faculty of Medicine, Atatürk University, Erzurum, Turkey; ${ }^{*}$ Department of Radiology, Regional Training and Research Hospital, Erzurum, Turkey

Bronchial atresia is a congenital anomaly characterized by interruption and termination of segmental and subsegmental bronchia as a blind pouch within pulmonary parenchyma. It is accompanied by bronchocele without connection to the bronchial tree and hyperinflation in obstructed lung segment. As the segment is aerated with collateral ventilation it is radiologically observed as emphysematous. It most commonly occurs at the apico-posterior segment of the left upper lobe, which is followed by segmental bronchi of the right upper, middle, and lower lobes. It is usually asymptomatic and is often discovered incidentally in the chest roentgenograms of cases consulting for other complaints. Clinical findings in symptomatic cases involves a large spectrum from mild dyspnea and wheezing to recurrent pulmonary infections. In the 2-view chest radiography of 20year-old female patient, opacity was found in right lower zone. In the thorax CT angiography performed for advanced analysis, the local air trapping observed in lower lung lobe in lesion periphery was typical for bronchial atresia. Because radiologically it might be confused with mass, this case was presented by reviewing the literature. Bronchial atresia, which gives the impression of mass in thorax BT examination, but increases pulmonary aeration and has bulla appearance, can be diagnosed with clinical, bronchoscopic and thorax tomographic findings; therefore mass and vascular pathologies could ve excluded, and unnecessary invasive procedures are avoided.

Keywords: Bronchial atresia, thorax bt, atresia

\section{P-302}

\section{Aberrant right subclavian artery as detected by} computerized tomography: a case study

Gedik HT*, Güven F**, Kara A*, Diyarbakır S*

*Department of Anatomy, Faculty of Medicine, Atatürk University, Erzurum, Turkey; ${ }^{*}$ Regional Training and Research Hospital, Erzurum, Turkey

Aberrant right subclavian artery is most commonly confronted aortic arc anomaly and it is seen in the people with $0.5 \%$ to $1.8 \%$-ratio. In cases, right subclavian artery emerges from the left side of the aortic arch as the last branch instead of brachiocephalic artery. Abnormal arterial structure extends from the retroesophageal region toward the right axillary hole from the left to the right. Sometimes, it can occasionally extend between esophagus and trachea or in front of the trachea. Most of the patients have asymptomatic clinical course and they may be detected incidentally. In symptomatic cases, chronic coughing, dysphagia particularly with solid foods, regurgitation and chest pain have been reported. In the acquired thoracal computerized tomography angiography of the patient with cyst hydatid, aberrant right subclavian artery emerging as the distal branch of aortic arch and passing through the posterior of esophagus and trachea was observed. The aim of this presentation is to present the embryological process, the incidence in the companion of literature and radiologic images in terms of clinical values of the aberrant right subclavian artery which is an aortic arch anomaly.

Keywords: Aberrant, subclavian artery, CT angiography

\section{P-303}

Unilateral atresia of a pulmonary artery

$\underline{\text { Kara A*,** }}{ }^{*}$ Keleş $\mathrm{P}^{*}$, Karaca L**, Gedik HT*, Diyarbakır S*

*Department of Anatomy, Faculty of Medicine, Atatürk University, Erzurum, Turkey; ${ }^{* *}$ Department of Radiology, Faculty of Medicine, Atatürk University, Erzurum, Turkey

Atresia of pulmonary artery is a rare congenital abnormality with an estimated prevalence of 1 in 200,000 young adults. It can be isolated or associated with cardiac abnormalities such as tetralogy of Fallot, septal defects or pulmonary stenosis. Patients with isolated atresia of pulmonary artery can remain asymptomatic but usually reported with symptoms such as dyspnea, chest pain, hemoptysis, recurrent infections, pulmonary hypertension. Diagnosis atresia of pulmonary artery can be difficult because of the rarity of the condition and its nonspecific clinical presentation. We present a case of a 35-year-old man who applied to chest diseases clinic, suffer from dyspnea. On the computerised tomographic examination of the chest, tipical findings atresia of left pulmoner artery, was found. Although, there is currently no consensus concerning treatment of patients with atresia of pulmonary artery, the consequence complications like hemoptysis, recurrent infections, pulmonary hypertension, can be treated medically

Keywords: Atresia, pulmonary artery, dyspnea

\section{P-304}

\section{The meningo-orbital foramen}

\section{Babacan S, Kafa I}

Department of Anatomy, Faculty of Medicine, Uludağ University, Bursa, Turkey

The meningo-orbital foramen is an opening that links the cranial cavity and the orbit mostly located on the lateral wall of the orbit, nearby the superior orbital fissure. It contains a small branch of the middle meningeal artery, which anastomoses with the lacrimal and ophthalmic arteries. The existence of this foramen has clini- 
cal importance, because of the injuries to the arterial branch passing through this foramen contrary to the older textbooks of basic anatomy suggested that it has a rare occurrence. This study aims to provide the meningo-orbital foramen has more variations than mentioned. The current study was conducted on 22 orbits belong to the 13 th century and 35 belong to contemporary (total 57 orbits). The skulls were obtained from the bone collection of the Department of Anatomy, Uludag University. The orbits of each skull were observed carefully for meningo-orbital foramen, the results were noted, and other observer enumerated the meningoorbital foramens for the second time for reliability. As a result, 11 meningo-orbital foramen were observed in 22 orbits of Byzantine period (\% 50); 22 were observed in 35 orbits belong to contemporary (\%62.85) and totally 33 meningo-orbital foramen were noted in 57 orbits (\%57.89). While two bilateral meningo-orbital foramen were noted belong to the Byzantine period, seven bilateral meningo-orbital foramen were observed in the contemporary period group. While mostly only one meningo-orbital foramen was noted, two was noted in two orbits and three was noted in one orbit. We believe that the results were reported in our study will support the idea about the number of meningo-orbital foramen is more than mentioned in older anatomy books and also it may be useful for ophthalmologist, radiologist, anthropologist and neurosurgeons.

\section{P-305}

Intestinal malrotation

Karaca L*, Kara $A^{* *}$, Keles $\mathrm{P}^{* *}$

*Department of Radiology, Faculty of Medicine, Atatürk University, Erzurum, Turkey; **Department of Anatomy, Faculty of Medicine, Atatürk University, Erzurum, Turkey

Intestinal malrotation is a developmental anomaly caused by the failure of the midgut, which begins at the Treitz ligament and ends at the $2 / 3 \mathrm{rds}$ of the transverse colon, in completing its counter-clockwise rotation of $270 \mathrm{o}$ around arteria mesenterica superior. This rotation takes place at the 10th week. Before the rotates around arteria mesenterica superior, caecum is located at the midsection of the abdomen. If this rotational movement does not occur in embryological life, they will not be detected at their designated areas. Another characteristic finding of malrotation patients is the detection of vena mesenterica superior to the left of arteria mesenterica superior. In our study, we found that the caecum of the 44 year old male patient who was referred to our gastroenterology clinic with abdominal pain was located at the midsection of the abdomen and that the intestinal loops were located in the lower right quadrant using an axial contrast computerized tomography (CT) of the abdomen. $60 \%$ of the intestinal malrotation patients are detected during the first months following birth, $20 \%$ are diagnosed between the ages of 1 month-1 year and $25 \%$ between the ages of 1 year-adulthood. Distinct findings such as symptoms of intestinal obstruction make the diagnosis easier in the period following birth. During adulthood, although many patients are symptomatic, the atypical and non-disease-specific symptoms make the diagnosis harder. Because of these atypical symptoms, many malrotation patients are exposed to wrong diagnosis and treatment. In malrotation patients, the likelihood of intestinal obstruction, therefore necrosis is high. Intestinal malrotation in adults is an important anomaly because of the complications it may cause, though not a common one. Although the diagnosis is made difficult by the atypical characteristic of the symptoms, early diagnosis and treatment are highly beneficial. Intestinal malrotation must be among the.

\section{P-306}

A radiological anatomic analysis of the cribriform plate and it's relations with stationary structures

\author{
Çiftcioğlu E* ${ }^{*}$, Şentürk A**, Yıldırım Ö*** \\ *Department of Anatomy, Faculty of Medicine, Ondokuz Mayıs \\ University, Samsun, Turkey; **Department of Otorhinolaryngology, \\ Liman Hastanesi, Samsun, Turkey; ***Department of Radiology, Gato \\ Hospital, Samsun, Turkey
}

Objectives: Understanding of the anterior skull base anatomy is very important for the endoscopic sinus surgery. The aim of this study was to determine the data about cribriform plate depth, middle turbinate length, maximal vertical orbital height and distance between the ethmoid roof and the nasal floor.

Methods: Paranasal sinus scans of 34 patients obtained using computed tomography were evaluated retrospectively. This data is part of our ongoing work. The cribriform plate depth, middle turbinate length, maximal vertical orbital height and the distance between the ethmoid roof and the nasal floor were measured bilaterally.

Results: No statistical significant differences were found between the gender and the sides in cribriform plate depth, orbital height, distance between the ethmoid roof and the floor of nasal cavity and middle turbinate lengths after the Keros classification. The mean cribriform plate depth was $6.75 \pm 1.6$ $\mathrm{mm}$ on the right side, $6.75 \pm 1.5 \mathrm{~mm}$ on the left side. The length of the middle turbinate was $20.93 \pm 3.42 \mathrm{~mm}$ on the right side, $21.02 \pm 3.23 \mathrm{~mm}$ on the left side. In the Keros type I group the middle turbinate was significantly longer than in the other groups. In Keros type III the distance between the ethmoid roof and the nasal floor was statistically higher than the other groups. However we found a patient who had an extreme cribriform plate depth and very short middle turbinate.

Conclusion: The obtained data must be concidered to prevent probable complications by informing rhinologists performing endoscopic sinus surgery about preoperative and intraoperative processes from this study.

\section{P-307}

Body image, depression and quality of life among the 2nd grade student of medical faculty

\author{
Öztürk KE* ${ }^{*}$ Tozun $M^{* *}$, Sözmen MK**, Malas MA* \\ *Department of Anatomy, Katip Çelebi University, Faculty of \\ Medicine, Izmir, Turkey; ${ }^{*}$ Department of Public Health, Katip Çelebi \\ University, Faculty of Medicine, Izmir, Turkey
}


Objectives: Aim of this study was to evaluate the relationship between body image, depression and quality of life among Katip Çelebi University Medical Faculty 2nd Grade Students.

Methods: This study was conducted between January-June 2015. In total, 58 students completed the questionnaire (43.3\% of target). Body image was determined by Stunkard Body Image Scale. Students marked the most similar body shape and their ideal body shape to the questionnaire. Depression was determined by Beck Depression Inventory (BDI). WHOQOLBREF was used to assess quality of life. Body dysmorphic disorder was determined by Body Dysmorphic Disorder Questionnaire. Anthropometric measurements were made by using standardized techniques. Chi-square test, t-test, spearman correlation test were used for statistical analyses.

Results: In study group, 30(51.7\%) were male. The mean age was $20.03 \pm 0.94$ years. The mean body weight was significantly higher in males compared to females(respectively;78.76 \pm 12.66 $\mathrm{kg}, 56.52 \pm 7.77 \mathrm{~kg}),(\mathrm{p}<0.001)$. The mean height was significantly higher among males compared to females (respectively; $179.93 \pm 6.35 \mathrm{~cm}, 56.52 \pm 7.77 \mathrm{~cm}),(\mathrm{p}<0.001)$. The proportion of anxious individuals about their body shape was $31.0 \%$ (n:18), and no significant difference was found between genders (in males: $30.0 \%$, in females: $32.1 \%$ ).We found a strong positive relationship between rankings of ideal body image and perceived body image $(\mathrm{r}: 0.833 ; \mathrm{p}<0.001)$. Mild depression was detected in 5 students ( 1 male, 4 female). Mean quality of life score for physical domain was $62.61 \pm 6.92$ and it did not significantly differ between genders (males: 63.52 \pm 7.14 , females; $61.63 \pm 5.30),(p>0.05)$. While there was a positive relationship between being anxious about body shape and obesity (r:0.282; $\mathrm{p}<0.05$ ), no significant relationship was found between being anxious about body shape, depression and quality of life (p:0.057, p:0.073, respectively).

Conclusion: In this study, we found a positive correlation between ideal body image and perceived body image. Obesity and being anxious about body image seemed to be positively related. Although we cannot show exact relationship; perception of body image might have an impact on the depression level and the quality of life scores.

\section{P-308}

\section{Comparison of hand ratio in men with normal versus abnormal semen parameters}

Öztürk KE $^{*}$, Yılmaz B ${ }^{* *}$, Keskin MZ $Z^{* * *}$, Eginc E $\mathrm{E}^{* * * *}$, Malas MA*

*Department of Anatomy, Faculty of Medicine, Katip Çelebi University, izmir, Turkey; **Department of Obstetrics and Gynecology, Tepecik Education and Research Hospital IVF Unit, Izmir, Turkey; $* * *$ Department of Urology, Tepecik Education and Research Hospital, IVF Unit, Izmir, Turkey; ${ }^{\star \star \star *}$ Tepecik Education and Research Hospital, IVF Unit, Izmir, Turkey

Objectives: In some previous studies considered that there was a relation between second to fourth finger ratio in hand and infertility. We questioned whether there was a similar relation between hand ratio and infertility in our settings. The purpose of this study was to compare the hand ratio in men with normal and abnormal semen parameters.

Methods: A total of 128 men attending to in-vitro fertilization unit for semen analysis were enrolled prospectively between February and March 2015. Age, weight, height, body mass index, hand width and length measurements and seminal parameters from masturbatory semen samples were recorded. Anthropometric measurements were made by using standardized techniques. Hand ratios among men with normal versus abnormal semen parameters were compared. SPSS was used for statistical analysis.

Results: Mean age and body mass index were $33.37 \pm 5.86$ and $26.09 \pm 4.00$, respectively. Left and right hand ratio were $0.471 \pm 0.66$ and $0.472 \pm 0.66$, respectively. Normal semen parameters were present in 32 men while 96 men had abnormal semen parameters (oligospermia \pm asthenospermia \pm teratospermia) according to WHO 2010 criteria. Right hand ratio between men with normal versus abnormal semen parameters was similar like in the case of left hand ratio between men with normal versus abnormal semen parameters. Moreover, comparison of hand ratio between right versus left hand in men with normal or abnormal semen parameters regarding total sperm count, sperm concentration, progressive motility, total progressive motile sperm count (TPMSC) and sperm morphology showed no significant difference.

Conclusions: Neither right hand ratio nor left hand ratio differed between men with normal versus abnormal semen parameters. Moreover, there was no significant difference between mean right versus left hand ratio among men with normal or abnormal semen parameters.

\section{P-309}

Schizophrenics have smaller putamen and thalamus when it is compared to the healty subjects

Pirzirenli $\mathrm{ME}^{*}$, Elfaki A*, Ali TO**, Osman A***, Gudek MA*, Şahin B*

*Department of Anatomy, Faculty of Medicine, Ondokuz Mayıs University, Samsun Turkey; **Departments of Anatomy, Faculty of Medicine, National Ribat University, Khartoum, Sudan; $* * *$ Department of Psychiatry, Faculty of Medicine, International University of Africa, Khartoum, Sudan

Introduction: Schizophrenia is a kind of mental illness that manifests itself commonly in early adulthood period. Dopamine is the main neurotransmitter related to this disease and the basal ganglia are the area of dopamine receptors and the main target for antiphyschotic drugs in treatment. Neuropathological studies have shown anatomical structural changes in many parts of the cerebral areas as cerebral cortex, thalamus, basal ganglia, lymbic system and cerebellum.

Objectives: In the present study, the volumes of the thalamus and basal ganglia (caudate nucleus, putamen, globus pallidus, nucleus accumbens) have been examined depending on the right and left sides and sex in the schizophrenics and control.

Methods: 88 control and 57 schizophrenics were participated in the study. Structural magnetic resonance imaging was per- 
formed and the DICOM images were evaluated using automatic brain segmentation software (BrainSuite). The volume and volume fraction of the region of interest were evaluated.

Results: The mean volume of total putamen $\left(7.89 \mathrm{~cm}^{3}\right)$ was smaller in schizophrenics than the control group $\left(8.33 \mathrm{~cm}^{3}\right)$ $(\mathrm{p} \leq 0.05)$. The mean volumes of right and left putamen (4.26 and $\left.3.63 \mathrm{~cm}^{3}\right)$ also were smaller in schizophrenics than the control group (4.53 and $\left.3.80 \mathrm{~cm}^{3}\right)(\mathrm{p} \leq 0.05)$. The mean volume of total thalamus $\left(12.80 \mathrm{~cm}^{3}\right)$ was smaller in schizophrenics than the control group $\left(13.73 \mathrm{~cm}^{3}\right)(\mathrm{p} \leq 0.05)$. The mean volumes of right and left thalamus $\left(6.48\right.$ and $\left.6.32 \mathrm{~cm}^{3}\right)$ also were smaller in schizophrenics than the control group (7.00 and $\left.6.73 \mathrm{~cm}^{3}\right)(\mathrm{p} \leq 0.05)$.

Conclusion: There is a significant difference in thalamus and the putamen volumes of basal ganglia according to the side in schizohrenics and control groups. There were no differences in the volumes of caudate nucleus and nucleus accumbens of schizophrenics and control groups. Our findings indicate that the sizes of thalamus and basal ganglia in the putamen part with reduced volumes show a strong relationship according to the gender and side in schizophrenics.

\section{P-310}

Patent ductus arteriosus finding at axial contrast spiral thorax CT: a case report

$\underline{\text { Keleș }} \mathrm{P}^{*}$, Gedik HT**, Karaca L**, Malkoç İ

*Department of Anatomy, Faculty of Medicine, Atatürk University, Erzurum, Turkey; **Department of Radiology, Faculty of Medicine, Atatürk University, Erzurum, Turkey

Objectives: Patent ductus arteriosus (PDA) is a persistent opening between two major blood vessels leading from the heart. The opening, called the ductus arteriosus, is a normal part of a baby's circulatory system before birth that usually closes shortly after birth. If it remains open, however, it's called a patent ductus arteriosus. PDA is $10 \%$ of all congenital hearth dissease. The insidence of PDA increases in preterm infants and presence of some syndromes.

Case Report: We dedected PDA between Pulmonar artery and Aorta in an axial contrast-enhanced spiral chest CT of an infant patient with feding difficulties.

Conclusion: The ductus arteriosus arises from the left sixth primitive aortic arch and connects the proximal left pulmonary artery to the descending aorta, just distal to the left subclavian artery. In the fetus, $70 \%$ of right ventricular blood bypasses the lungs via the ductus to be directed to the placenta, where gas exchange occurs. In a full-term infant, functional closure of the ductus occurs within 24 hours after birth. Permanent closure takes place at 2 to 3 weeks and results in formation of a ligamentum arteriosum. The morbidity and mortality are releated with the decrease of Pulmonar artery pressure and the degree of shunt that occurs in the PDA, from Aorta to Pulmonar artery. A small patent ductus arteriosus often doesn't cause problems and might never need treatment. A small duct accompanied by a small shunt will not cause significant hemodynamic derangement but may predispose to endarteritis, espe- cially if accompanied by an audible murmur. However, a large patent ductus arteriosus will initially cause left ventricular volume overload and a progressive increase in pulmonary artery pressure, leading to lower pulmonary blood flow but higher pulmonary vascular resistance. On pathologic examination, this is seen as irreversible pulmonary vas- cular changes; clinically, it appears as systemic pulmonary pressure (Eisenmenger's syndrome). The increaded blood flow in Pulmonar artery can cause pulmonar oedema. The shunt from left to right in PDA can cause some problems like intraventricular bleeding, necrotising enterocolitis, cronic lung disseases, and increased mortality. And a large patent ductus arteriosus left untreated can allow poorly oxygenated blood to flow in the wrong direction, weakening the heart muscle and causing heart failure and other complications. The treatment of PDA depends on the patient' age, severity of postpartum complaints, size of PDA, and wheter the presence of another congenital cardiac disseases. The treatment options include monitoring, medications and closure by cardiac catheterization or surgery.

\section{P-311}

Investigation of relationship between midline brain developmental cysts and morphometry of aquaductus cerebri and corpus callosum

Turamanlar O*, Acay $\mathrm{MB}^{* *}$, Cartıllı $\mathrm{O}^{*}$, Ünlü E**, Horata $E^{*}$, Gülsarı $\mathrm{Y}^{*}$, Toktaş $\mathrm{M}^{* * *}$

*Department of Anatomy, Faculty of Medicine, Afyon Kocatepe University, Afyonkarahisar, Turkey; **Department of Radiology, Faculty of Medicine, Afyon Kocatepe University, Afyonkarahisar, Turkey; $* * *$ Department of Anatomy, Faculty of Medicine, Turgut Özal University, Ankara, Turkey

Objectives: Septum Pellucidum (SP), which is part of the limbic system, located in the medial wall of the lateral ventricles and has two thin membrane. Greater than $1 \mathrm{~mm}$ cavity between the SP leaves called as Cavum Septi Pellucidi (CSP). Closure defects of SP are generally called Cavum Verga (CV). Presence of CSP and CV is correlated with disruption of the midline brain development. In our study, we aimed to investigate the relationship between the presence of CSP, CV and the morphometric measurements of aquaductus cerebri (AC) and the corpus callosum (CC).

Methods: 79 men and women in the age range of 18- 80 were included in this study. Four groups were formed as CSP, CV, CSP \&CV and control group. Length and diameter of AC, length and area of the CC, width of the CC's genu (CC1), trunk (CC2), isthmus (CC3) and splenium (CC4) and skull diameter were measured from MR images for each groups.

Results and Conclusion: 45 women and 34 men participated in our study. There was a statistically significant difference between the groups about AC length and diameter, CC length, CC2, CC3, CC4. AC length was significantly longer in CSP $\mathrm{CSPeV}$ and $\mathrm{CV}$ groups, $\mathrm{AC}$ diameter was significantly longer in $\mathrm{CV}$ group, CC length, was significantly longer in $\mathrm{CSPeV}$ and $\mathrm{CV}$ groups than the control group. Measurements of CC2 and CC3; were significantly smaller in CSP, CSPeV and CV groups than the control group. Finally, CC4's value was statis- 
tically significantly larger in $\mathrm{CSPeV}$ group than the control group. We believe that, significant relationship between CSP, $\mathrm{CV}$, CSP \&CV and AC length and CC's morphometry will affect the the diagnosis, follow up and treatment of these cysts.

\section{P-312}

\section{Postnatal developmental changes of GAD67-GFP positive cells in mice pedunculopontine nucleus}

\section{Özkan M, Şehirli ÜS}

Department of Anatomy, Faculty of Medicine, Marmara University istanbul, Turkey

Objectives: Pedunculopontine nucleus (PPN) is a group of neurons located in mesopontine tegmentum and related with sleepwake cycle, locomotion and attention. Its importance revealed with the dense connections to basal ganglia, reciprocally. PPN has three major cellular groups; cholinergic, glutamatergic and gamma amino butyric acid (GABA)ergic. Despite the abundance of studies on developmental changes of the cholinergic and glutamatergic cells of PPN, there is not enough for the GABAergics. Two enzymes synthesizing GABA, glutamic acid decarboxylase $1(67 \mathrm{kDa}$ - GAD67) and, glutamic acid decarboxylase $2(65 \mathrm{kDa}$ - GAD65), are contained in GABAergic neurons in variable quantities and this fact may be due to functional distinctions of these cells containing different enzymes.

Methods: To evaluate distribution and the morphologic features of GAD-GFP positive GABAergic cells in PPN at the postnatal 14, 15, 21 and 25 days, we used GAD-GFP knock-in mice brains. Even though we had both knock-in types, only GAD67-GFP cells have been seen definitively. After getting sections, we labeled GAD-GFP positive cells with anti-GFP antibody. To limit PPN's area we also made choline-acetyl transferase immunohistochemistry to reveal cholinergic neurons.

Conclusion: We had similar results about caudal distribution of GABAergic cells in PPN as previous studies. As a result of our study, there is no significant differences between the numbers, diameters and neuronal distributions of GAD67-GFP cells in the all PPN, developmentally.

Keywords: Pedunculopontine nucleus, GAD67-GFP, GABAergic, Developmental

This study was financially supported by Research Fund of the Marmara University. Project Number: SAG-C-DRP-080415-0102.

\section{P-313}

\section{Is there a relationship between height of dens and lenght of apical ligament of dens?}

Kapakin S

Department of Anatomy, Faculty of Medicine, Atatürk University, Erzurum, Turkey

In anatomy, the second cervical vertebra of the spine is called the axis. It has an unusual structure and a tooth-like upward projection named dens due to its function. Apical ligament of dens is a ligament that extends from the tip of the dens to antri- or edge of foamen magnum. This study was carried out to determine the mean value of the height of dens and lenght of apical ligament of the dens and also whether there is a relationship between them in asymptomatic Turkish individuals relative to age and gender by using magnetic resonance imaging. A total of 105 asymptomatic individuals (44 males, age range $11-70$ years; mean $35.86 \pm 14.36$ and 61 females, range $13-$ 70 years; mean $37.57 \pm 13.43$ ) were selected during the study period. All the individuals underwent midsagittal magnetic resonance imaging for measuring the height of dens and lenght of apical ligament of dens. The mean value of the height of dens was $13.77 \pm 1.89 \mathrm{~mm}$ in female and $14.05 \pm 1.89 \mathrm{~mm}$ in male. The mean value of the lenght of apical ligament of dens was $8.08 \pm 1.94 \mathrm{~mm}$ in female and $8.93 \pm 2.16 \mathrm{~mm}$ in male. There is no correlation between height of dens and apical ligament of dens depending on age. Statistically, there was no difference in between female and male in terms of the height of dens. But, the lenght of apical ligament of dens in female was shorter than male. The data in this study should be useful to surgeons while determining the dens axis screw for fractures of dens.

Keywords: Axis, dens, apical ligament of dens, magnetic resonance imaging, morphometry

\section{P-314}

\section{Creating anaglyph form of the subfornical organ in rat brain}

$\underline{\text { Kapakin S, Kapakin KAT** }}$

*Department of Anatomy, Faculty of Medicine, Atatürk University, Erzurum, Turkey; ${ }^{*}$ Department of Pathology, Faculty of Veterinary Medicine, Atatürk University, Erzurum, Turkey

It is well known that circumventricular organs that are situated around the 3rd and 4th ventricles of the brain are outside the blood- brain barrier and responsible for maintaining osmotic, energy, and cardiovascular regulation. The subfornical organ is one of the brain's sensory circumventricular organs and lack of blood-brain barrier. It is situated on the ventral surface of the fornix at the level of interventricular foramen. There was no information about the morphology of the subfornical organ fulfilling these vital functions in literature. This study was undertaken to reveal the computer-assisted $3 \mathrm{D}$ reconstruction, photorealistic appearance, and anaglyph form of the subfornical organ. Anaglyph images are composed of two stereograms (anaglyphs) in which each image is displayed in a different color. Anaglyph images are extraordinary images perceived by using particular anaglyph glasses that permit only one stereogram to be viewed by each eye and stereoscopic depth perception occurs in different regions of cerebral cortex. A duo-chromatic digital image was generated and could be printed as a photorealistic image by using high-quality photo printers. The volume of the subfornical organ was $0.0891 \mathrm{~mm}^{3}$ and the surface area of the subfornical organ was $2.6256 \mathrm{~mm}^{2}$. The delineation of the subfornical organs in anaglyph form, providing a sense of depth, enabled students, researchers and surgeons to visualize, perceive, and interpret it and displayed three-dimensional spatial relationships better.

Keyword: Rat, subfornical organ, circumventriclar organs, anaglyph 


\section{P-315}

Is there a relationship between the cardiac volume and the thoracic cavity volume?

$\underline{\text { Kapakin S}}^{*}$, Kapakin KAT**

*Department of Anatomy, Faculty of Medicine, Atatürk University, Erzurum, Turkey; ${ }^{* \star}$ Department of Pathology, Faculty of Veterinary Medicine, Atatürk University, Erzurum, Turkey

Cardiomegaly has been increasing in recent years. One of the most commonly used methods to detect the cardiomegaly is cardiothoracic index. However, recent studies have claimed that this index is inaccurate. This study was undertaken to reveal the three-dimensional relationship between the cardiac volume and the thoracic volume in terms of cardiomegaly in the rabbit model. A total of ten adult healthy New Zealand Rabbits of both genders aged 1.5-2 years and weighing between 3.5 and $4 \mathrm{~kg}$ were used. Animals were intravenously anaesthetized with 5 $\mathrm{mg} / \mathrm{kg}$ of ketamine-HCl (KetamidorTM Richer Pharma AG, Wels, Austria) and $20 \mathrm{mg} / \mathrm{kg}$ propofol (PropofolTM amp., Fresenius Kabi, Austria). Under anaesthesia, in prone position the animal was scanned by a 16- slice computed tomography system (Aquilion, Toshiba Medical Systems). The coronal images obtained were transfered to a personal computer to perform surface-rendered reconstructions by using software SurfDriver 3.5.6. Those models are processed further to creat photorealistic images in the Cinema 4D software and transfered to 3D-Doctor software to measure the the cardiac volume and the thoracic volume. This approach took the ratio of the heart volume to thoracic cavity volume and it was called the three dimensional cardio thoracic ratio. The volume of the heart was 18913,564930 $\mathrm{mm}^{3}$ and the volume of the thoracic cavity was 120219,751719 $\mathrm{mm}^{3}$. Three dimensional cardio thoracic ratio was 0.15 in this study. It was thought that volumetric approach could be more reliable and feasible than surface approach in clinical practice.

Keywords: Rabbit, Three dimensional reconstruction, Cardio thoracic index, Three dimensional cardio thoacic ratio, Cardiomegaly

\section{P-316}

\section{MRI-based detailed evaluation of the anatomy of the human coccyx}

Tetiker $\mathrm{H}^{*}$, Koşar MI'* Çullu N**, Canbek U***, Otağ $\mathrm{I}^{* * * *}$, Taştemur $\mathrm{Y}^{* * * * *}$

*Department of Anatomy, Muğla Sıtkı Koçman University Faculty of Medicine, Muğla, Turkey; **Department of Radiology, Muğla Sıtkı Koçman University Faculty of Medicine, Muğla, Turkey; ${ }^{* *}$ Department of Orthopedics and Traumatology, Muğla Sıtkı Koçman University Faculty of Medicine, Muğla, Turkey; $* * * *$ Vocational Faculty of Health Services, Cumhuriyet University, Sivas, Turkey; $* * * *$ Department of Anatomy, Muğla Sıtkı Koçman University Faculty of Medicine, Sivas, Turkey

Objectives: The human coccyx varies considerably in shape and size. The objective of this study was to investigate the morphology and morphometry of the coccyx on pelvic MR imaging in asymptomatic individuals.
Methods: This study was conducted retrospectively on the pelvic MR images of 456 adult patients (122 male and 334 female patients with a mean age of 43.9 years) without a history of trauma in the coccyx region. The coccygeal vertebrae count, number of bone segments, and intercoccygeal and sacrococcygeal joint fusions were determined from the sagittal plane images. In addition, the length and angles (the sacrococcygeal angle, intercoccygeal joint angle and sacrococcygeal joint angle) were measured. The results were statistically analyzed.

Results: The coccyx is formed by four, five, or three vertebrae, in a decreasing ratio. The coccyx is composed of one to five bone segments; one bone segment was found in $2.8 \%$ of the cases. Intercoccygeal joint fusions have been observed predominantly in the last intercoccygeal joint, with or without sacrococcygeal joint fusion. The coccyx was found to be longer in men than in women. The sacrococcygeal angle might be antroverted or retroverted.

Conclusions: Knowledge of the anatomical variation of the coccyx would be useful for clinicians in diagnosis and treatment planning for patients presenting with conditions in the coccygeal region. We hypothesize that the detailed results of our study would contribute to future studies on coccyx morphology.

Keywords: Coccyx, sacrum, morphology, morphometry

\section{P-317}

The volumetric values of breast in adult women

$\underline{B a l c 1 ~ S}^{*}$, Göker P*, Binokay F**, Bozkır $M^{*}{ }^{*}$

*Department of Anatomy, Faculty of Medicine, Çukurova University, Adana, Turkey; **Department of Radiology, Faculty of Medicine Çukurova University, Adana, Turkey

Breast which is secondary sex organ varies by breadth, projection, tissue density, composition, shape and placement on thorax. The aim of this study was to investigate the breast volume of premenopausal adult women and correlation between breast volume and Body Mass Index (BMI). In this study, the age, height, weight and body size (circumference of waist and hip) were determined and the breast volume was measured with the GrossmanRounder Breast Measuring Device in 40 premenopausal adult women. The results obtained in the study showed that mean values of age, height, weight, BMI, circumference of waist and hip were found to be $31.69,1.64 \mathrm{~cm}, 60.5 \mathrm{~kg}, 22.29\left(\mathrm{~kg} / \mathrm{cm}^{2}\right), 79.95$ $\mathrm{cm}$ and $94.6 \mathrm{~cm}$, respectively. Right and left mean breast volumes were found to be $308.5 \mathrm{cc}$ and $309.3 \mathrm{cc}$, respectively. Statistical analyses showed that there was a positive correlation between the mean breast volume and BMI $(\mathrm{p}<0.05)$. There was no statistically (SPSS, Paired-samples T-test) difference between right and left breast volumes. The results of this study were expected to help surgeons for breast magnifying, reducing and reconstruction surgery and breast reconstruction as ideal dimensions.

Keywords: Anthropometric measurements, body mass index (BMI), breast, breast volume, premenopausal adult women 


\section{P-318}

An extremely rare anastomotic artery between right and left common iliac arteries

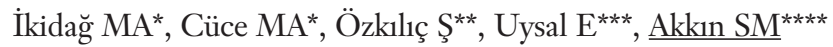

*Department of Radiology, SANKO University Medical School, Gaziantep, Turkey; **Department of Nuclear Medicine, SANKO University Medical School, Gaziantep, Turkey; ${ }^{* \star}$ Department of General Surgery, SANKO University Medical School, Gaziantep, Turkey; $* * * *$ Department of Anatomy, SANKO University Medical School, Gaziantep, Turkey

Congenital aortoiliac arterial abnormalities are not as common as aortic arch anomalies. Incidentally observed abdominal arterial and venous variations and anomalies are increasingly encountered due to widespread use of multislice CT for abdominal and vascular diseases. Knowledge of these variations or pathologies is crucial, especially before surgery to repair occlusive diseases, aneurysms and transplant surgeries, in order to reduce complications such as vascular injury and bleeding. Here we present an aortoiliac variation observed during abdominal CT examination of a 27 -year-old female. This variation appeared as an anomalous arterial structure coursing obliquely and transversely between the right and left proximal common iliac arteries and binding them just below the bifurcation. Physicians should also be familiar with such a case that appears as a triangular aortoiliac arterial window and case that has only been reported once in the old anatomical literature.

Keywords: Common iliac artery, anomaly, variation

\section{P-319}

\section{Scorpion-shaped pancreas together with an arterial variation complex: a case report}

İkidağ MA*, Cüce MA*, Özkılıç Ş**, Uysal E*** ${ }^{* k k ı n ~ S M}{ }^{* * * *}$ Department of Radiology, SANKO University Medical School, Gaziantep, Turkey; **Department of Nuclear Medicine, SANKO University Medical School, Gaziantep, Turkey; ***Department of General Surgery, SANKO University Medical School, Gaziantep, Turkey; $* * * *$ Department of Anatomy, SANKO University Medical School, Gaziantep, Turkey

There is a range of well-described anatomic variations and developmental anomalies of the pancreas in the literature. These include pancreas divisum, annular pancreas, ectopic pancreas, total or partial agenesis, and variations of the course and configuration of pancreatic ducts. Although some of these variations are extremely uncommon, knowledge of these abnormalities is essential for avoiding misdiagnosis and managing pancreatic pathologies clinically. Here we present an anatomical variation that we observed during thoracoabdominal CT examination of a 33-year-old female. This patient presented with the complaint of left chest pain and had undergone a splenectomy six years ago and a left thoracotomy one year ago, both for hydatid cyst. The case showed that the pancreatic tail raised upward and then anteriorly, resembling the tail of a scorpion, and was accompanied by a right hepatic artery that originated from the superior mesenteric artery, and a left accessory renal artery. Because no similar case has been reported in the available literature, our opinion is that physicians should also be familiar with such scorpion-shaped pancreas that can be termed as "pancreas scorpiforme" anatomically.

Keywords: Pancreas, anomaly, variation

\section{P-320}

The morphology of pterion and relation to frontal branch of the middle meningeal artery in adult dry skulls

$\underline{\text { Kocabiyık N*}}$, Hamzaoğlu V**, Özalp H*** Cömert A***

*Department of Anatomy, Gülhane Military Medical Academy, Ankara, Turkey; ${ }^{*}$ Department of Neurological Surgery Oregon Health and Science University Portland, USA; ${ }^{* *}$ Department of Neurosurgery, Faculty of Medicine, Mersin University, Mersin, Turkey; ****Department of Anatomy, Faculty of Medicine, Ankara University, Ankara, Turkey

Introduction: Pterion is an important anatomic landmark that is localized apparoximately $4 \mathrm{~cm}$ above the zygomatic arch. Aim of the study; to present the localization of pterion, to localize the place of it on cranium, to exhibit the specific anatomic landmarks in the surrounding structures on it and the distances between the pterion.

Methods: The study was executed on 24 adult skulls. Pterion and specified anatomic points interdistances are measured in milimeters by using digital calliper.

Results: Pterion is classified into 5 types as sphenoparietal, stellat and epipteric types with sphenoparietal, frontotemporal and wormian bone. Sphenoparietal type (85.3\%), epipteric type $(8.1 \%)$, stellate type $(5.2 \%)$ and frontotemporal type $(1.4 \%)$ are identified. The distances on the right side was found as $39.2 \pm 2.79$ from the superior side of the zygomatic arch to the center of the pterion, on the left side as $37.65 \pm 3.65 \mathrm{~mm}$. The horizontal distance on the right side was measured as $27.66 \pm 3.37$, on the left side as $28.25 \pm 3.48$; vertical distance on the right as $11.39 \pm 1.58$, on the left as $12.01 \pm 1.54 \mathrm{~mm}$. It was calculated on the right side as $31.39 \pm 5.73$, on the left side as $29.61 \pm 5.79$ from the superior aspect of the zygomatic arch to the frontal branch of the middle meningeal artery. The distance from the center of the pterion to the frontal branch of the middle meningeal artery was $5.82 \pm 1.29 \mathrm{~mm}$ on the left, on the right as $6.63 \pm 2.57 \mathrm{~mm}$.

Conclusion: Pterion is exactly in the intersection of parietal, frontal, greater wing of sphenoid and squamous part of temporal bone. Pterion is used in the surgical incisions because of its thin artitecture. The morphometric properties of the pterion and keeping the knowledge in mind that has relationship with the frontal branch of the middle meningeal artery, will be an important guidline for surgical plan and approach. At the same time it will be contributing to the studies in antropology.

Keywords: Pterion, morphometry, morphology, cranium, pterional approach 


\section{P-321}

Morphology of the first metatarsal bone an anatomical and clinical study

Demir BT*, Gümüşalan $Y^{*}$, Çağlar $V^{* *}$, Üzel $M^{* * *}$

*Department of Anatomy, Faculty of Medicine, Fatih University, Istanbul, Turkey; ${ }^{*}$ Deparment of Anatomy, Faculty of Medicine, Namık Kemal University, Tekirdağ, Turkey; *** Department of Orthopaedics and Traumatology, Faculty of Medicine, Kahramanmaraş Sütçü Imam University Kahramanmaraş, Turkey

Introduction: Structure most affected by the clinical deformities associated with lower extremity is the first metatarsal bone which may cause orthopedic problems and chronic pain on foot, because approximately $80 \%$ of the load from the talus and calcaneus is transmitted to the ground via medial arch. The load carried by the great toe exceeds twice the load carried by other fingers while standing and maximum load is transferred to the first metatarsus in the push phase of walking.

Objectives: Our study was performed on lower extremities of 32 adult patients without any foot deformity which were amputated due to circulation failure in the Department of Orthopaedics and Traumatology. Firstly, tibia length, foot length and foot width were measured. After then, the dimensions and length of first metatarsus as well as proximal and distal articular surface dimensions of the first metatarsus were measured.

Methods: The average values were calculated as tibia length:35,9 cm, foot length:22,7 cm, foot width: $8 \mathrm{~cm}$, length of first metatarsus: $5,8 \mathrm{~cm}$. Statistical significance was found among the dimensions of proximal end, dimensions of distal head and the thinnest site of the bone $(\mathrm{p} \leq 0,01)$. Similarly, the relation between dimensions of both distal and proximal articular surfaces was found statistically significant $(\mathrm{p} \leq 0.01)$. The shape of the head of first metatarsus was curved in $40,6 \%$, chevron in $28,1 \%$ and flat in $32 \%$ of the bones investigated.

Results: Various external deforming forces cause variations in the medial longitudinal arch and lead foot pain. The length, shape of the head as well as the dimensions of the proximal and distal joint surfaces of the first metatarsus are important factors in the development of foot deformities.

Conclusion: Since peroneus longus and tibialis anterior muscles insert onto the first metatarsus, the forces exerted by their tendons affect morphology of the bone. Therefore, dimensions of these muscles and their tendons in relation with the dimensions of first metatarsus are essential in the prevention and treatment of foot deformities.

\section{P-322}

A rare variation of the musculocutaneous nerve in human cadaver

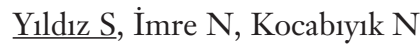

Department of Anatomy, Gülhane Military Medical Academy, Ankara, Turkey

Introduction: The musculocutaneous nerve, derived from the lateral cord, is one of the terminal branches of the brachial plexus. The classical pathway of this nerve is that it penetrates the coracobrachialis muscle, passes between the biceps brachii and the brachialis muscles, and terminates as the lateral antebrachial cutaneous nerve. The lateral cord is largely composed of fibers originating from the $\mathrm{C} 5$ and $\mathrm{C} 6$ ventral rami, thus accounting for the presence of these fibers in the musculocutaneous nevre.

Objectives: The course and branching anomalies of the musculocutaneous nerve and its relation to the coracobrachialis muscle have been documented in the literature. The change in the innervation of the coracobrachialis muscle is reported to be closely correlated with the change of course of the musculocutaneous nerve. Knowledge of these variations is critical to surgeons, radiologists and other clinicians working in the axillary region.

Methods: During the educational gross anatomy dissections of the axilla and brachium of a 65 -year-old male cadaver in our laboratory, we encountered a neurovascular variation.

Results: Musculocutaneus nerve was giving a muscular ramus in $1.5 \mathrm{~mm}$ thickness, $5-6 \mathrm{~cm}$ before entering corocobrachialis muscle. This muscular ramus was joining again to nerve itself at the entering point by covering vascular bandle going to corocobrachialis muscle from axillary artery and by reaching to $3 \mathrm{~mm}$ thickness. The course of the musculocutaneous nerve was normal in the forearm region. Other branches originating from the brachial plexus were also normal.

Conclusion: The distribution, course and branching of the musculocutaneous nerve is important from the clinical point of view, especially in compression neuropathies due to vigorous activity and stretch injuries seen in various surgical interventions. The variant course of the musculocutaneous nerve should be kept in mind as a possible way of treatment in recurrent compression neuropathies.

Keywords: Musculocutaneous nerve, brachial plexus, lateral cord, variation

\section{P-323}

Visual study about scientific illustrations of the human body in the University of Chile (1903-1956)

Valenzuela JLC ${ }^{*}$, Esterio MD**

*Assistant Professor, ICBM, Faculty of Medicine, University of Chile, Santiago, Chile; **Visual Designer, Santiago, Chile

Objectives: Perform a catalog of illustrations and visual studio with a historiographical and documentary approach focused on anatomical illustrations used for educational purposes of the Faculty of Medicine of the University of Chile during the first half of the twentieth century.

Methods: 475 anatomical illustrations used for teaching carried out between 1903 - 1956 are analyzed, measured, drawing techniques are described, analyzing the signatures of each of them, and the search for the sources of the same is made.

Results: These illustrations were made by students and their teachers, using various techniques such as ink, watercolor, oil, graphite on canvas brought from Germany in the period of World War pencil. Most of these jobs are direct copies of illustrations present in major anatomical textbooks were mainly 
Europeans who used to teach Bourgerie, Rouvière, Testut disciplinacomo. Others less, were original.

Conclusion: The study investigates the impact of these images on the transfer of foreign models for the development of modern thought anatomical knowledge. This paper proposes that anatomical illustrations operate as a transmission medium of the European vision of the human body in the teaching of anatomy in Chile. This approach is stated in a catalog that displays the relationships between the plates created at the Faculty of medicine of Chile and the original anatomical illustrations taken from the classic textbooks of anatomy.

\section{P-324}

First professional anatomy teacher in Turkish medical history

Yılmazer AZ*, Taskınalp O*, Uucam E*

Department of Anatomy, Faculty of Medicine, Trakya University, Edirne, Turkey

In the period of the Ottoman Empire, first medical Faculty was "Royal Faculty of Medicine and Surgery" established at 14 March 1827 by Sultan Mahmut II, in where students were educated as in west. In 1838, that Faculty was renamed as "Imperial Medical School" and moved to Galatasaray in 1839. Dr. C. A. Bernard was assigned as dean of the medical school, who had come from Europe. Then Dr. Sigmund Spitzer (1813-1895) came first among 25 participants in the exam that arranged to choose an anatomy teacher for that school. Dr. Spitzer came in İstanbul in 1839, as first professionally educated anatomy teacher who knew dissection techniques. Students of the Imperial Medical School, studied anatomy on models at the first few years. However using only models was not enough to learn effectively. In 1841 Sultan Abdulmecit gave permission to perform dissections in anatomy lessons by an edict. So medical students could observe dissections performed by Dr. Spitzer in his lectures. Dr. Spitzer was confronted by the people's negative reactions because of using cadevers, fortunately resistance suppressed by the support of sultan and other statesmen. Dr. Spitzer worked as anatomy teacher and practised in internal medicine clinic between 1839-1850. He was assigned as dean in 1845 Additionally he was sultan's private doctor. In Turkish medical history Dr. Spitzer is well known for his contributions to modernize and perform appropriate anatomy education. We remember Dr. Spitzer by respect and gratitude.

Keywords: Spitzer, anatomy education, anatomy history

\section{P-325}

The innervation pattern of the lumbrical muscles of the foot

Bozer $C^{*}$, Uzmansel D**, Dönmez D*, Parlak $M^{*}$, Beger O**, Elvan $\mathrm{Ö}^{* *}$

*Department of Anatomy, Faculty of Medicine, Trakya University, Edirne, Turkey; **Department of Anatomy, Faculty of Medicine, Mersin University, Mersin, Turkey
According to the standard text books of anatomy and past research reports, the first lumbrical is innervated by the medial plantar nerve and the remainder three lumbricals are innervated by the deep branch of the lateral plantar nerve. The typical function of the lumbricals is described as flexion of the proximal phalanx and extension of the proximal and distal interphalangeal joints. They have the unique ability to compress the metatarsal-phalangeal and interphalangeal joints. The lumbricals are most active from midstance to pre-swing. The lumbricals, along with the other intrinsic muscles of the foot, play a role in maintaining the medial longitudinal arch of the foot (6). Along with the interossei, they play a role in stabilization of the forefoot during stance phase and rearfoot during pre-swing $(7,8)$. In injuries of the tibial nerve and in conditions such as the hereditary motor-sensory neuropathies (e.g. Charcot-Marie-tooth disease) lumbrical dysfunction contributes to clawing of the toes (1). Although there are quite a number of studies that have reported variations about the origin of lumbricals, there are currently few studies that have reported variations about the innervation of the lumbricals of the foot. In the study of Brooks (1887), it was reported a variation in innervation of the foot lumbricals only 1 case in 10 cases. In that case, first and second lumbricals have innervated by the medial plantar nerve and third and fourth lumbricals have innervated by the lateral plantar nerve. In this study, we have investigated the innervation pattern of the lumbricals of the foot in 30 cadaveric feet (14 right and 16 left). We aimed to determine the innervation variations of the foot lumbricals and present the percentages of the innervation types. The types and their percentages are shown in diagrams and figures in the poster presentation of the study.

Keywords: Lumbrical muscles, foot, innervation, lateral plantar nerve, medial plantar nerve

\section{P-326}

Morphological characteristics of epithelium's proliferative activity in oviduct endometriosis, focused on different localization of endometriotic foci and influence of oviduct condition in endometriosis

Belyavskaya D, Sapargalieva A

Department of Pathology, Kazakh Medical University Named After S.D Asfendiarov, Almaty, Kazakhstan

Introduction: Endometriotic tissue, which is similar to endometrium and is present in oviduct as a result of retrograde throwing, retains hormonal activity. These ectopic lesins have hyperproliferative stromal and edometrial epithelium that are accompanied with inflammatory processes. So, it has significant value using both clinical and morphological analysis to determine if the oviduct condition in endometriosis influences on ectopic sites, especially on epithelial proliferative activity.

Objectives: To assess what features of oviduct condition in endometriosis impact on epithelium proliferative activity lining oviduct and to evaluate the nature of the proliferative activity.

Methods: The research investigation was hold in the framework of the intrauniversity grant "Morphological and clinical 
features of adenomyosis". We analysed surgical material derived from 17 patients (27-67 years old) who underwent hysterectomy for uterine leiomyomas (2013-2015) at the Research Center for Obstetrics, Gynecology and Perinatology of the Republic of Kazakhstan. Tissues were stained by hematoxylin and eosin, Van-Gieson stain.

Results: Endometriotic foci of different location were observed. Submucous location was characterized by the presence of chronic infiltration composed of cell-round elements. Also, there were pronounced sclerotic changes and obliteration of the lumen of the fallopian tube with a sharp gluing and deformated shape of the villi. In all cases there was a high proliferative activity of fallopian tube epithelium, which was characterized by the formation of true and false nipples. The proliferative activity in endometriotic foci of submucous location also remained.

Conclusion: Sclerotic changes developed in outcome of chronic inflamation are the typical factor that accomponies the oviduct endometriosis and creates favorable condition for the growth of ectopic lesions. Retaining the proliferative activity of endometriotic foci regardless the localization facilitates the proliferative activity of fallopian tube epithelium, and it confirms the maintaining of hormonal activity of ectopic site.

\section{P-327}

The effects of curcumin on sperm parameters and nitric oxide production in varicocelized rats

İzadpanah $M^{*}$, Abbasi $M^{*}$, Alizadeh $\mathrm{R}^{* *}$, Heydari $\mathrm{L}^{* * *}$

*Department of Anatomy, Faculty of Medicine, Tehran University of Medical Sciences, Tehran, Iran; **Department of Anatomy, Faculty of Medicine, Iran University of Medical Science, Tehran, Iran; ${ }^{* * *}$ Department of Anatomy, Faculty of Medicine, Tehan University of Medical Science, Tehran, Iran
Objective: One of the proposed mechanism by which varicocele induces its damage is excessive release of nitric oxide (NO). Several studies have shown the role of NO in poor sperm quality in infertile patients with varicocele. Scientific studies have demonstrated the beneficial effects of curcumin on the sperm parameters. Curcumin as an atoxic antioxidant can reduce production of NO. The aim of this study was to determine the effect of curcumin on NO level and investigate if curcumin can improve sperm parameters in varicocelized male rats.

Methods: Thirsty male Wistar rats were randomly divided into 5 groups, (V1 and V2 (varicocele), T (treatment), Sh (sham) and C was control). In groups V1, V2, T and Sh, the left renal vein was partially ligated to induce varicocele. In groups $\mathrm{V} 1$ and V2, sperm parameters and NO level were evaluated 8 and 16 weeks respectively after varicocele induction. Groups T and Sh received $100 \mathrm{mg} / \mathrm{kg}$ curcumin and placebo respectively, daily for 8 weeks after 2 months of induced varicocele. Sperm parameters (count, motility, viability and morphology), epididymis and testis weight and also NO concentration were measured.

Results: Sperm parameters (count, motility and viability) in groups V1, V2 and Sh were significantly low in comparison with control and treatment groups. The level of NO was significantly increased in serum of rats in groups $\mathrm{V} 1$ and $\mathrm{V} 2$, whereas group $\mathrm{T}$ rat serum in which curcumin was administered, showed decreased NO levels. The values of the epididymis and testis weight had no significant changes ( $\mathrm{P}$ ? 0.05) in all groups.

Conclusion: Administration of curcumin as a free radical scavenger, can decrease NO level and improve sperm parameters in varicocelized male rats.

Keyword: Varicocele, curcumin, sperm parameters, rat 